ORP-56503

Revision 12

\title{
Dynamic (G2) Model Design Document, 24590-WTP-MDD-PR-01-002, Rev. 12
}

Prepared for the U.S. Department of Energy

Assistant Secretary for Environmental Management

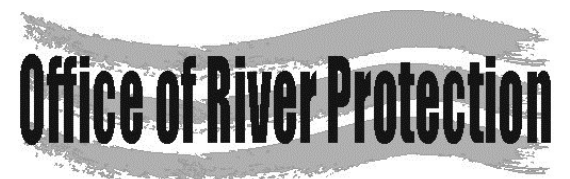

P.O. Box 450

Richland, Washington 99352 
ORP-56503

Revision 12

\section{Dynamic (G2) Model Design Document, 24590-WTP-MDD-PR-01-002, Rev. 12}

\section{Y. Deng}

Bechtel National, Inc.

Prepared for the U.S. Department of Energy

Assistant Secretary for Environmental Management

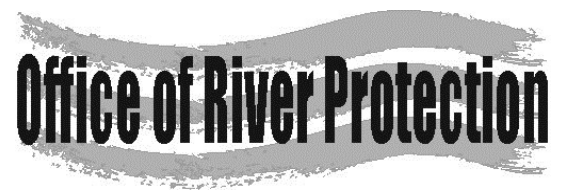

P.O. Box 450

Richland, Washington 99352

\section{APPROVED}

By Julia Raymer at 1:26 pm, Dec 16, 2013

Release Approval

Date 
ORP-56503

Revision 12

TRADEMARK DISCLAIMER

Reference herein to any specific commercial product, process, or service by tradename, trademark, manufacturer, or otherwise, does not necessarily constitute or imply its endorsement, recommendation, or favoring by the United States Government or any agency thereof or its contractors or subcontractors.

This report has been reproduced from the best available copy.

Printed in the United States of America 


\section{Dynamic (G2) Model Design}

Document titie: Document

Document number: 24590-WTP-MDD-PR-01-002, Rev 12

Contract number: $\quad$ DE-AC27-01RV14136

Department: $\quad$ Process Engineering

Author(s): $\quad$ Yueying Deng

Checked by: $\quad$ Shara L. Orcutt

Issue status: $\quad$ Approved

Approved by: $\quad$ Ernest D. Lee

Approver's position: Lead, Flowsheet Modeling

Approver's signature:
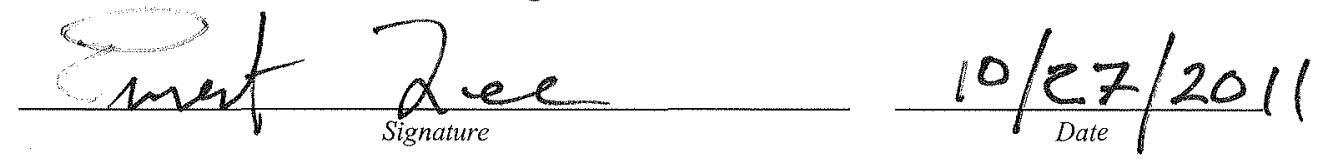

River Protection Project Waste Treatment Plant 2435 Stevens Center Place Richland, WA 99354

United States of America Tel: 5093712000
Some information within Appendix $F$ may appear to be illegible, however, the information necessary for assuring adequate design is legible. 


\section{History Sheet}

\begin{tabular}{lll} 
Rev & Reason for revision & Revised by \\
\hline 0 & Initial Issue & C Henley
\end{tabular}

1 Added Appendix G with references; updated FEP-VSL-00017A and FEP-VSL-00017B attributes in Appendix F.

C Henley

Updated Model Design Document to support version 1.2 of the model to incorporate MCRs 24590-WTP-MCR-PT-02-189,

24590-WTP-MCR-PT-02-190, 24590-WTP-MCR-PT-02-191, and 24590-WTP-MCR-PT-02-194. Document rewritten and changes are not tracked in this version. incorporate 63 MCRs. The implementation of the MCRs were required to incorporate the $2+2$ melter configuration, Waste Compliance Plan, Sample Analysis Plan, and update the Dynamic (G2) Model to reflect WTP design. This update comprises a major rewrite $(>20 \%)$ and therefore changes have not been tracked.

1) removal of the technetium ion exchange system,

2) removal of the technetium eluant recovery system,

3) addition of the cesium fresh resin system,

4) addition of the spent resin disposal system,

5) application of the rectifier model to the cesium nitric acid recovery process,

6) incorporation of the reconciliation data to the CNP evaporator,

7) incorporation of the reconciliation data to the HLW melter.

A total of 14 MCRs were implemented.

6

Updated to reflect the as-built design of the Dynamic (G2) Model Version 3.0. The major changes that affect the WTP chemical processes and operating logic included:

1) incorporate the Envelope C blending logic in the HLW Lag Storage Process System.

2) Allow batch specific Envelope C reactions in the Ultrafiltration Feed Preparation Vessels. 
3) Enable the Ultimate Overflow Vessel in the Plant Wash and Disposal System and the Eluate Contingency Storage Vessel in the Cesium Nitric Acid Recovery Process System.

4) Replace the oxide glass formers with mineral glass formers.

5) Include ammonia generation reactions in the HLW and LAW melters.

6) Include ammonia absorption reactions in the Submerged Bed Scrubbers in the HLW and LAW Offgas Systems.

7) Update sampling logic and provide sample composition at the SAP sample points.

8) Provide optional model features to store excess LAW feed and recycle from LAW Offgas Systems.

9) Update operating parameters.

A total of 13 MCRs were implemented. This update comprises a major rewrite $(>20 \%)$ and therefore changes have not been tracked.

1) Allow ultrafilters to operate at variable flux rates.

2) Include ultrafiltration solid heel ejection (PJM related).

3) Add the capability of performing oxalate liquid/solid phase equilibrium mass transfers in UFPVs and UFVs if oxalate is supplied in the ORP feed vector.

4) Update Cesium Nitric Acid Recovery Evaporator boil-off chemistry.

5) Adjust water contents at the LAW MFPVs to address rheology concerns.

6) Include dust control water for glass former hoppers.

7) Incorporate logic that restricts concentration of lithium in the LAW glass to limit corrosion of melter refractories.

8) Incorporate logic that limits the amount of sulfate in the LAW glass to that seen in pilot runs.

9) Incorporate HLW Glass Property Model that is based on HLW glass formulation developed by Vitreous State Laboratory for various tank wastes.

10) Incorporate HLW glass crystallization/liquidus models that adjust glass formers according to nepheline, spinel, and zirconium liquidus/ crystallization temperatures in the glass.

11) Include ultrafiltration recirculation loop flush.

12) Perform cesium ion exchange removal operation using overall removal efficiency control.

13) Improve operating logic and setpoint control for LAW Concentrate Receipt Process System and LAW Feed Process System.

14) Replace urea with ammonia for NOx reduction reactions in Catalytic Oxidizer Reducer.

15) Incorporate logic to meet Technical Specification TS-1.1, "Minimum Component Limits in HLW Glass" when the minimum silica (and glass former) concentration in the glass is also met.

16) Improve operating logic and setpoint control for HLW Concentrate Receipt Process System and HLW Feed Process System.

17) Reroute Waste Neutralization Vessel to HLW Acidic Waste Storage Vessel.

18) Update isotope molecular weight values. 
19) Update line flush volumes for vitrification systems.

20) Update HLW melter decontamination factor for sulfate.

21) Update HLW Lag Storage Vessel set volume when transition from commissioning to operation.

22) Apply vacuum eductor steam condensation to Treated LAW Evaporator Condensate Vessel when dual pumping to the Treated LAW Evaporator occurs.

23) Improve model efficiency including model maintainability, input flexibility, database initialization and configuration control, and data output.

This update comprises a major rewrite (more than $20 \%$ ) and therefore changes have not been tracked. parameters and logic included:

1) Change the Envelope $\mathrm{C}$ transuranic waste precipitation reactions to include actinides and birnessite.

2) Allow viscosity dependent variable flux rates through ultrafilters.

3) Apply variable caustic volume for leaching, feed forward leachate to the Ultrafiltration Permeate Collection Vessels.

4) Update cooling time after caustic leach in ultrafiltration.

5) Apply variable volumes for solid wash and add dilution capabilities to the Ultrafiltration Permeate Collection Vessels.

6) Add aluminum liquid/solid phase equilibrium capabilities in various Pretreatment vessels.

7) Expand oxalate liquid/solid phase equilibrium capabilities in various Pretreatment vessels.

8) Add blending logic for PWD-VSL-0044 and PWD-VSL-0015/0016 streams to produce evaporator concentrate of SpG 1.27 and/or solids concentration less than 8.0 percent.

9) Enable evaporator FEP-SEP-00001B.

10) Route Envelope $C$ through the FEP evaporator.

11) Add capabilities and switches to enable/disable ultrafilter caustic/acid cleaning, loop drain, and heel ejection.

12) Add capabilities to perform sequential and/or concurrent oxidative leaching.

13) Remove HLW Concentrate Receipt Vessels and revise HLW Melter Feed Preparation Vessel operating logic.

14) Include mineral impurities in glass former oxides.

15) Add viscosity constraints to the HLW glass model calculations.

16) Include glass former oxides and mineral impurities (the metal equivalent) to splits to SBS from melters.

17) Add iodine vaporization reaction in melters.

18) Allow variable $\mathrm{NH} 3$ gas productions in LAW and HLW melters so that near neutral solutions are achieved in LAW/HLW SBS Condensate Vessels.

19) Allow water to be vaporized or condensed in SBS, WESP, HEME, and Caustic Scrubber depending on moisture contents in the inlet.

20) Allow tritium and peroxide to follow water in SBS, WESP, HEME, and Caustic Scrubber.

21) Add acetonitrile and $\mathrm{PCB}$ production reactions in melters and $\mathrm{C} 2 \mathrm{H} 3 \mathrm{~N}$ and 
$\mathrm{C} 12 \mathrm{H} 4 \mathrm{Cl} 6$ phase change and destruction reactions in the offgas systems and Pretreatment Vessel Vent Process System. Also splits applicable equipment.

22) Update splits and chemical reactions in LAW melters, LAW Melter Offgas Treatment Process System, and LAW Secondary Offgas/Vessel Process System.

23) Update splits and chemical reactions in HLW melters and HLW Melter Offgas Treatment Process System.

24) Update operating logic, SpG and $\mathrm{pH}$ controls at the LAW Caustic Scrubber and the Caustic Collection Vessel.

25) Add halogen gas removal reactions to the HOP Silver Mordenite.

26) Update sample locations and hold times.

27) Add PJM/RFD dynamic air usage capabilities.

28) Include chloride impurity in all the caustic soda chemical reagents.

29) Allow waste sodium, process sodium, waste manganese, and process manganese to be tracked separately throughout the flowsheet. This includes the update of all calculations where $\mathrm{Na}+$ and $\mathrm{Mn}+4$ are used prior to the component list expansion, the addition of split factors where they are applicable, and the change of caustic soda reagents from $\mathrm{NaOH}$ to $\mathrm{NapOH}$ (except the caustic for leaching).

30) Update miscellaneous operating parameters in the LAW vitrification systems including line flush volumes, vessel volumes, and vessel vent air flowrates.

31) Update vessel vent air flowrates in the HLW vitrification systems.

In addition, several model perfection changes such as model maintainability, input flexibility, database initialization and configuration control, and data output were included:

32) Expand the flowsheet component lists (liquid, solid, oxide, gas) to include components that support additional chemical reactions. Glass former array and mineral array are replaced by the oxide array.

33) Develop a Visual Basic program that populates the new components in feed vector provided by the Tank Farm Contractor. This ensures the Tank Farm Contractor Operation and Utilization Plan is usable by the Dynamic (G2) Flowsheet.

34) Upgrade Oracle/MatLab post model run data processing utilities to accompany the expanded components.

35) Apply a customized coding technique to the Ultrafiltration Task Team related model changes that improves code reusability.

This update comprises a major rewrite (more than $20 \%$ ) and therefore changes have not been tracked. parameters and logic included:

1) Allow ultrafiltration trains to operate in parallel.

2) Update HLW Canister Decontamination Handling System operating logic.

3) Add dilution air to anhydrous ammonia streams connecting to HLW and LAW NOx scrubbers.

4) Update vessel volumes and maximum flowrates.

5) Modify HLW glass chemistry shell coefficients. 
6) Apply a new LAW glass chemistry shell.

7) Update Envelope $\mathrm{C}$ reaction cooling time.

8) 5.0. Major changes that affect the WTP chemical processes, operating parameters and logic included:

1) Perform caustic leach in UFP-VSL-00001A/B.

2) Apply ion exchange chemistry models based on Spherical ResorcinolFormaldehyde resin.

3) Add Glass Former Facility submodel.

4) Include end of mission waste handling operations.

5) Update line flushes for sampling, HPAV, and plugging.

6) Calculate hydrogen generation rates at vessels of interests.

7) Perform $\mathrm{pH}$ neutralization reactions for CNP evaporator bottoms.

8) Add rheology water for HLW feeds.

9) Add HEPAs to LVP and HOP.

10) Reroute PJV HEPA and add HLW PJV stack.

11) Update oxalate and aluminum solubility equations.

12) Update ultrafiltration variable flux rate equations.

13) Update sample hold time and locations.

14) Update vessel volumes and pump flows.

15) Update DFs and associated chemical reactions.

16) Add G2MBC.

Thirty three 33 MCRs have been incorporated. This version update comprises a major rewrite (more than $20 \%$ ) and therefore change bars were not provided. processes, operating parameters and logic included:

1) Remove PJV-VSL-00002.

2) Swap CXP-VSL-00001 and CNP-VSL-00003.

3) Combine UFP-VSL-00062A/B/C and CNP-VSL-00003.

4) Combine SBS columns with SBS condensate vessels in LOP and HOP.

5) Apply operating volumes to FEP-SEP-00001A/B and TLP-SEP-00001 evaporators.

6) Add process antifoam agent.

7) Add phosphate solubility.

8) Add caustic operation during solid wash.

9) Add IXC resin outlet flush operations.

10) Add fluid displacement operation during resin regeneration.

11) Incorporate caustic leach decision logic.

12) Incorporate oxidative leach decision logic.

13) Incorporate wash forward/backward decision logic.

14) Incorporate HLW Glass Shell Version 2.0.

15) Apply secular equilibrium for $\mathrm{mBa}-137$ and $\mathrm{Y}-90$.

16) Update DFs, lineflushes, volumes, flowrates, and other operating parameters in various systems. 
17) Update G2MBC, Oracle tables, and MatLab scripts.

A total of 26 MCRs have been incorporated. Significant details have also been added to this document which comprises more than $20 \%$ rewrites and therefore change bars were not provided. 7.0. Major changes that affect the WTP equipment configuration, chemical processes, operating parameters and logic included:

1) Add CXP guard filter.

2) Add IXC backflow operations.

3) Add UFP2 double batching operations.

4) Add flowsheet option for boehmite leaching.

5) Add vessel heel dilution and clean out operations.

6) Update CNP evaporator bottoms transfer, storage, neutralization, mixing, and cooling operations.

7) Update fresh resin preparation operations.

8) Update volumes, flowrates, and sample hold times.

9) Update LAW and HLW melter DFs.

10) Update phosphate solubility correlation.

11) Apply SpG and wt\% solid dual controls to FEP and TLP evaporators.

12) Model fate of mercury with composition dependent DFs.

13) Model fate of semi-volatiles with reactions and splits.

14) Add end of mission summary outputs.

15) Update Oracle/MatLab scripts and G2MBC.

A total of 20 MCRs have been incorporated. The document is updated to use the latest WTP template. 


\section{Contents}

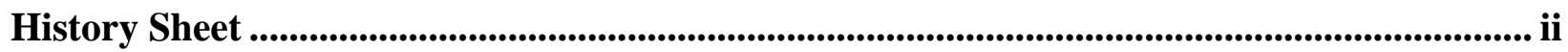

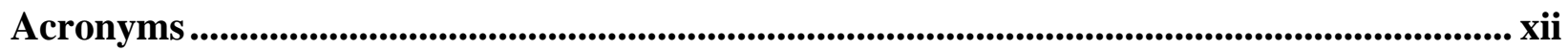

1 Introduction .........................................................................................................................

2 Purpose

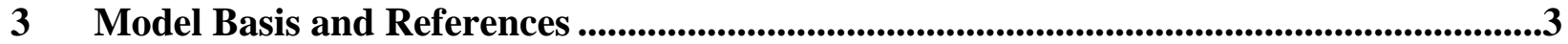

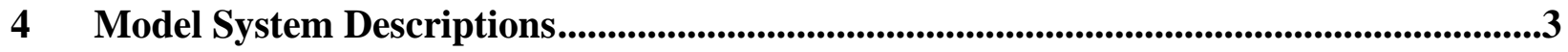

4.1 Time Steps and Dynamic Material Balance ....................................................................................................

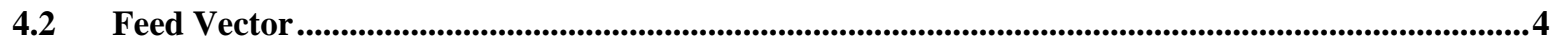

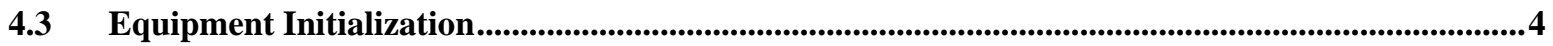

4.4 Component Lists.............................................................................................................................

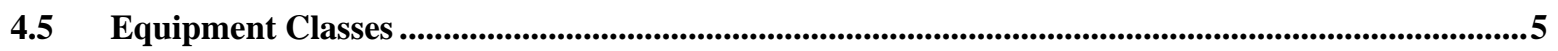

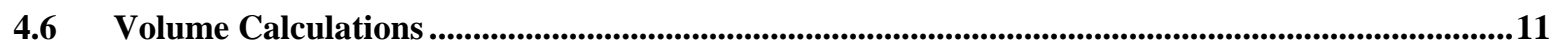

4.7 Common Operating Rules .........................................................................................................................12

4.8 System Specific Operating Logic ……..........................................................................................36

4.9 Workspace Description...............................................................................................................138

$5 \quad$ Model Construction.....................................................................................................139

5.1 Knowledge Base.......................................................................................................................139

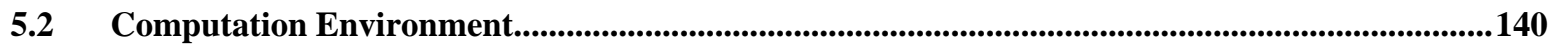

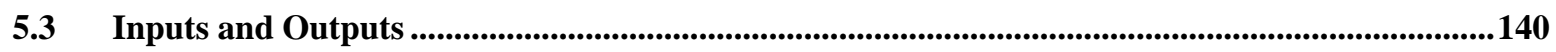

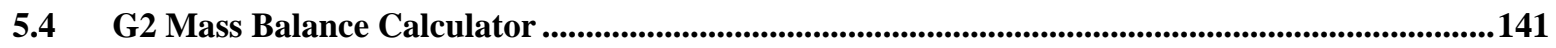

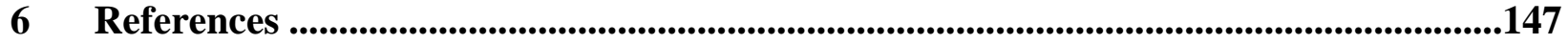

\section{Appendices}

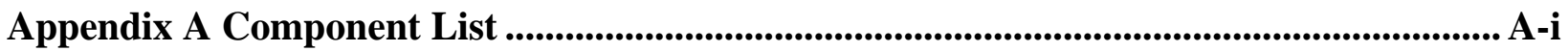

Appendix B Chemical Adds ................................................................................................... B-i

Appendix C Sample Identification and Logic ................................................................. C-i

Appendix D Component Splits ........................................................................................................ D-i

Appendix E Vessel Parameter Table................................................................................................ E-i

Appendix F Workspace Screenshots..........................................................................................

Appendix G Dynamic Air Utilization Modeling ..................................................................... G-i 
Appendix H Transfer, Sampling, and Time Triggered Line Flushes ..................................H-i

Appendix I Feed Vector Charge Balance ..................................................................................... I-i

Appendix J Mass Balance Flowsheet Diagrams and Volume Throughput ............................. J-i

Appendix K Envelope C Reactions ................................................................................................ K-i

Appendix L UFP2 Leaching with Boehmite................................................................... L-i

Appendix M UFP1 Leaching with Gibbsite ............................................................................. M-i

Appendix N Totalizer Files ...................................................................................................... N-i

Tables

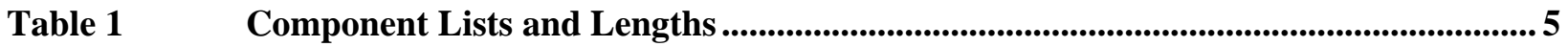

Table 2 Equipment Classes and Associated Component Lists ............................................... 8

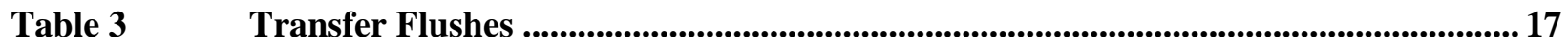

Table 4 Sample Flushes.............................................................................................................................. 19

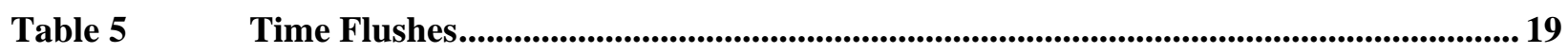

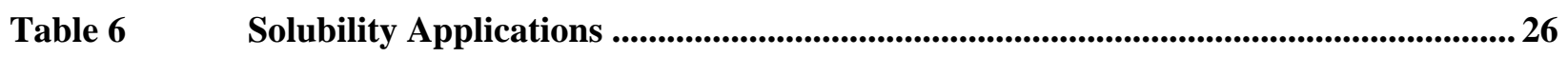

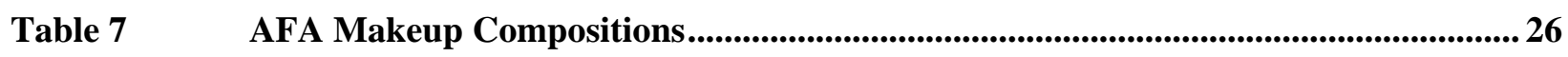

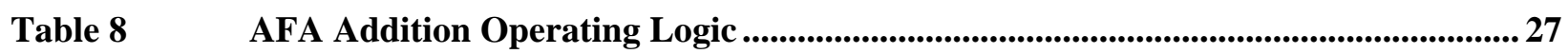

Table 9 Maximum Temperatures for HGR Calculations .......................................................... 28

Table 10 Hydrogen Contributing Organics and Constants ....................................................... 29

Table 11 Specific Activities and Decay Heat Rate of Alpha Emitting Radionuclides ............... 30

Table 12 Specific Activities and Decay Heat Rates of Beta/Gamma Emitting Radionuclides ................................................................................................................ 31

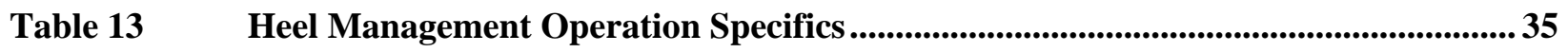

Table 14 Chemical Reagents for Resin Regeneration ..............................................................68

Table 15 Ion Exchange Operating Parameters...........................................................................6 68

Table 16 Glass Former Minerals............................................................................................ 130

Table 17 Makeup of Mineral Glass Formers _.................................................................. 131

Table 18 Names and Bulk Densities of Glass Former Stockpiles ........................................... 134

Table 19 Attributes of the Truck Class........................................................................................ 135

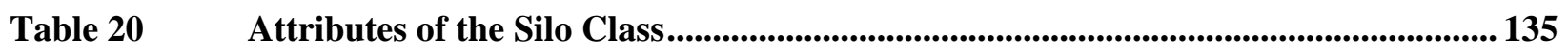

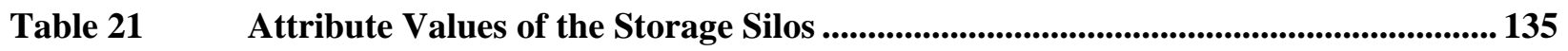

Table 22 Attribute Values of the Weigh Hoppers......................................................................... 136

Table 23 Attribute Values of the Transporters....................................................................... 137 
Table 24

Table 25

Table 26

Table 27

Table 28

Table 29
Attribute Values of the Blending Silos 137

Attribute Values of the LAW/HLW Transporters 137

Attribute Values of the LAW/HLW Glass Former Mixers 138

Workspace Description 138

General Data Output 140

G2MBC Programming Structures

\section{Figures}

Figure $1 \quad$ WTP Equipment Class Hierarchy................................................................................... 7

Figure 2 UFP2 Double Batching Operation (Part 1)............................................................ 45

Figure $3 \quad$ UFP2 Double Batching Operation (Part 2)................................................................ 46

Figure $4 \quad$ UFP2 Double Batching Operation (Part 3).......................................................................... 47

Figure 5 Caustic Leach Decisions Logic Diagram (Part 1) ........................................................49

Figure 6 Caustic Leach Decisions Logic Diagram (Part 2) .........................................................50

Figure 7 Caustic Leach Decisions Logic Diagram (Part 3) ..........................................................51

Figure 8 Caustic Leach Decisions Logic Diagram (Part 4) .........................................................52

Figure $9 \quad$ Oxidative Leach Decisions Logic Diagram (Part 1).................................................. 55

Figure 10 Oxidative Leach Decisions Logic Diagram (Part 2)................................................. 56

Figure 11 Oxidative Leach Decisions Logic Diagram (Part 3).................................................... 57

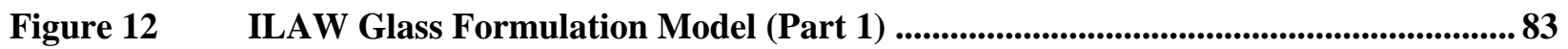

Figure 13 ILAW Glass Formulation Model (Part 2) ................................................................. 84

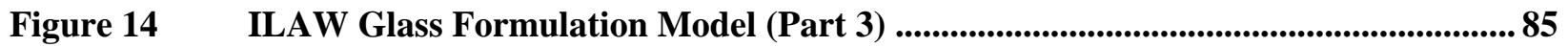

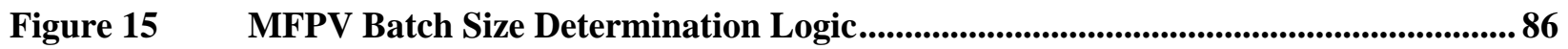

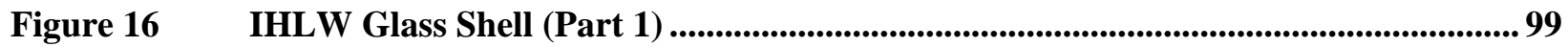

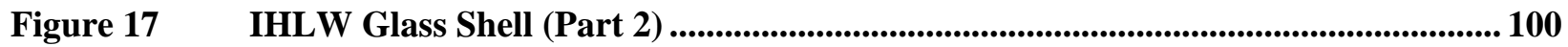

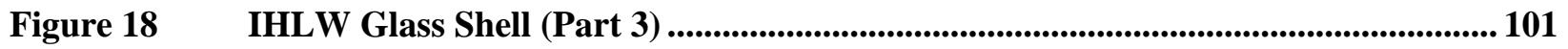

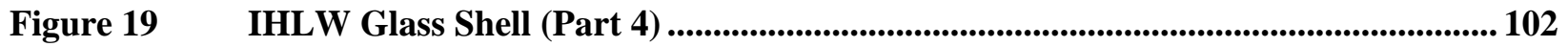

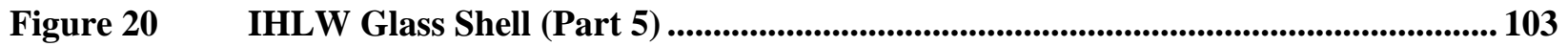

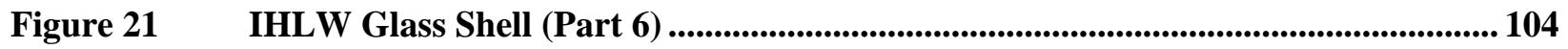

Figure 22 IHLW Glass Shell (Part 7) ....................................................................................................... 105

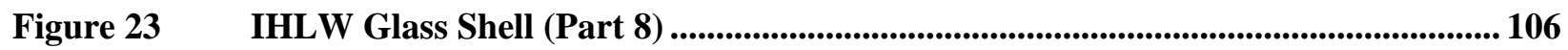

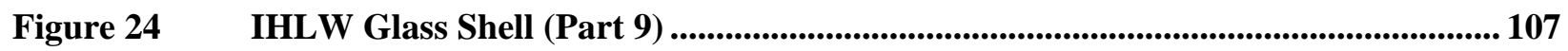

Figure 25 IHLW Glass Shell (Part 10) ............................................................................................ 108 
Figure $26 \quad$ IHLW Glass Shell (Part 11) ................................................................................................ 109

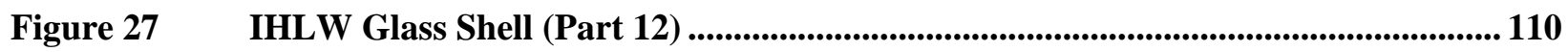

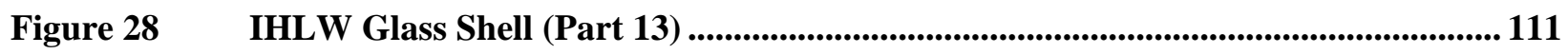

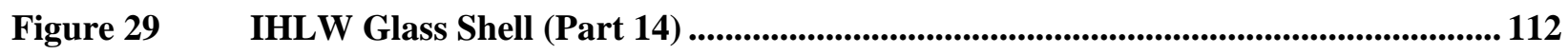

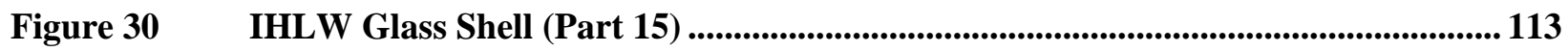

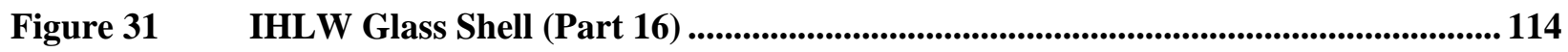

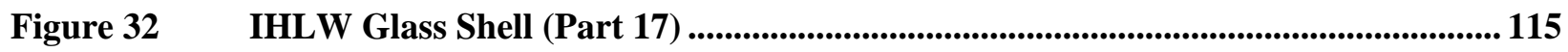

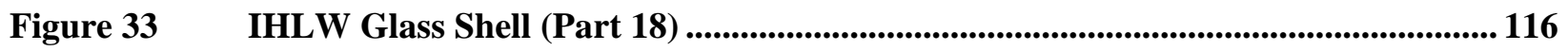

Figure $34 \quad$ IHLW Glass Shell (Part 19) ….............................................................................................. 117

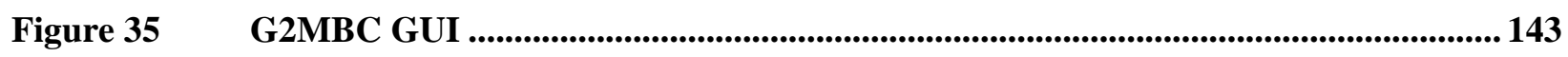




\section{Acronyms}

$\begin{array}{ll}\text { AES } & \text { Aspen Engineering Suite } \\ \text { AFA } & \text { Antifoaming Agent } \\ \text { ASCII } & \text { American Standard Code for Information Interchange } \\ \text { BARD } & \text { Flowsheet Bases, Assumptions, and Requirements Document } \\ \text { BMP } & \text { Bitmap File Format } \\ \text { BOF } & \text { Balance of Facilities } \\ \text { CF } & \text { Conversion Factor } \\ \text { CNP } & \text { Cesium Nitric Acid Recovery } \\ \text { CRP } & \text { Cesium Resin Addition } \\ \text { CRV } & \text { Concentrate Receipt Vessel } \\ \text { CSV } & \text { Comma Separated Value File Format } \\ \text { CXP } & \text { Cesium Ion Exchange Process System } \\ \text { CV } & \text { Column Volume } \\ \text { DF } & \text { Decontamination Factor } \\ \text { DIW } & \text { Demineralized Water } \\ \text { DOE } & \text { U.S. Department of Energy } \\ \text { DPPG } & \text { Decomposed Polypropylene Glycol } \\ \text { DPDMS } & \text { Decomposed Polydimethylsiloxane } \\ \text { EFRT } & \text { External Flowsheet Review Team } \\ \text { ETF } & \text { Effluent Treatment Facility } \\ \text { FEP } & \text { Waste Feed Evaporation Process } \\ \text { FRP } & \text { Waste Feed Receipt Process System } \\ \text { G2 } & \text { Gensym G2 Bundle Version 8.1 } \\ \text { G2MBC } & \text { G2 Mass Balance Calculator } \\ \text { GFR } & \text { Glass Formers Reagent System } \\ \text { GUI } & \text { Graphical User Interface } \\ \text { HDH } & \text { HLW Canister Decontamination Handling System } \\ \text { HEME } & \text { High Efficiency Mist Eliminator } \\ \text { HEPA } & \text { High Efficiency Particular Air Filter } \\ \text { HFP } & \text { HLW Melter Feed Process System } \\ \text { HGR } & \text { Hydrogen Generation Rate } \\ \text { HLP } & \text { HLW Lag Storage and Feed Blending Process System } \\ \text { HLW } & \text { High-Level Waste } \\ \text { HMP } & \text { HLW Melter Process System } \\ \text { HOP } & \text { HLW Melter Offgas Treatment Process System } \\ \text { HPAV } & \text { Hydrogen in Piping and Ancillary Vessels } \\ \text { ICD } & \text { Interface Control Document } \\ \text { IHLW } & \text { Immobilized High-Level Waste } \\ \text { ILAW } & \text { Immobilized Low-Activity Waste } \\ \text { ISARD } & \text { Integrated Sampling Analysis Requirements Document } \\ \text { IX } & \text { Ion Exchange } \\ \text { IXC } & \text { Ion Exchange Column } \\ \text { JPEG } & \text { Joint Photographic Experts Group File Format } \\ \text { KB } & \text { Knowledge Base } \\ \text { LAW } & \text { Low-Activity Waste } \\ \text { LCP } & \text { LAW Concentrate Receipt Process System } \\ & \end{array}$




$\begin{array}{ll}\text { LERF } & \text { Liquid Effluent Retention Facility } \\ \text { LFP } & \text { LAW Feed Preparation Process System } \\ \text { LMP } & \text { LAW Melter Process System } \\ \text { LOP } & \text { LAW Melter Offgas Treatment Process System } \\ \text { LVP } & \text { LAW Secondary Offgas/Vessel Process System } \\ \text { MCR } & \text { Model Change Request } \\ \text { MDD } & \text { Model Design Document } \\ \text { MDI } & \text { Multiple Document Interface } \\ \text { MRQ } & \text { Model Run Request } \\ \text { MFPV } & \text { Melter Feed Preparation Vessel } \\ \text { MFV } & \text { Melter Feed Vessel } \\ \text { NAPLs } & \text { Non-Aqueous Phase Liquids } \\ \text { ODBC } & \text { Open Database Connectivity } \\ \text { ORP } & \text { Office of River Protection } \\ \text { PCB } & \text { polychlorinated biphenyl } \\ \text { PDMS } & \text { Polydimethoylsiloxane } \\ \text { PE } & \text { Process Engineering } \\ \text { PJM } & \text { Pulse Jet Mixer } \\ \text { PJV } & \text { Pulse Jet Ventilation } \\ \text { PPG } & \text { Polypropyleneglycol } \\ \text { PVP } & \text { Pretreatment Vessel Vent Process } \\ \text { PWD } & \text { Plant Wash and Disposal System } \\ \text { RF } & \text { Spherical Resorcinol-Formaldehyde Resin } \\ \text { RDP } & \text { Spent Resin Collection and Dewatering Process System } \\ \text { RFD } & \text { Reverse Flow Diverter } \\ \text { RLD } & \text { Radioactive Liquid Waste Disposal System } \\ \text { SBS } & \text { Submerged Bed Scrubber } \\ \text { SDO } & \text { Supplemental Data Outputs } \\ \text { SP6 } & \text { TFC System Plan Revision 6 Feed Vector } \\ \text { SpG } & \text { Specific Gravity } \\ \text { STRN } & \text { Stream Number } \\ \text { TCP } & \text { Treated LAW Concentrate Storage Process System } \\ \text { TFC } & \text { Tank Farm Contractor } \\ \text { TLP } & \text { Treated LAW Evaporation Process System } \\ \text { TOC } & \text { Total Organic Carbon } \\ \text { TPA } & \text { Tri-Party Agreement } \\ \text { TRU } & \text { Transuranic Wastes } \\ \text { TUA } & \text { Tank Utilization Assessment } \\ \text { TXT } & \text { Text File Format } \\ \text { UFP } & \text { Ultrafiltration Process System } \\ \text { UFP1 } & \text { Ultrafiltration Feed Preparation Vessel } \\ \text { UFP2 } & \text { Ultrafiltration Feed Vessel } \\ \text { V\&V } & \text { Verification and Validation } \\ \text { WESP } & \text { Wet Electrostatic Precipitator } \\ \text { WITNESS } & \text { Lanner Group WITNESS } \\ \text { WT\% } & \text { Weight Percent } \\ \text { WTP } & \text { Hanford Tank Waste Treatment and Immobilization Plant } \\ & \end{array}$




\section{Introduction}

The Hanford Tank Waste Treatment and Immobilization Plant (WTP) Statement of Work (Department of Energy Contract DE-AC27-01RV14136, Section C) requires the contractor to develop and use process models for flowsheet analyses and pre-operational planning assessments. The Dynamic (G2) Flowsheet is a discrete-time process model that enables the project to evaluate impacts to throughput from eventdriven activities such as pumping, sampling, storage, recycle, separation, and chemical reactions. The model is developed by the Process Engineering (PE) department, and is based on the Flowsheet Bases, Assumptions, and Requirements Document (24590-WTP-RPT-PT-02-005), commonly called the BARD. The terminologies of Dynamic (G2) Flowsheet and Dynamic (G2) Model are interchangeable in this document.

The foundation of this model is a dynamic material balance governed by prescribed initial conditions, boundary conditions, and operating logic. The dynamic material balance is achieved by tracking the storage and material flows within the plant as time increments. The initial conditions include a feed vector that represents the waste compositions and delivery sequence of the Tank Farm batches, and volumes and concentrations of solutions in process equipment before startup. The boundary conditions are the physical limits of the flowsheet design, such as piping, volumes, flowrates, operation efficiencies, and physical and chemical environments that impact separations, phase equilibriums, and reaction extents. The operating logic represents the rules and strategies of running the plant.

The Dynamic (G2) Model is developed using the object-oriented programming platform of G2 (Gensym G2 Bundle Version 8.1). Equipment, unit operations, systems, and their connections are represented by objects graphically and are organized in knowledge base (KB) workspaces. Programming techniques such as modularization, class hierarchy and inheritance, encapsulation and information hiding, multi-thread and parallel computing, and process synchronization are utilized. Interprocess connectivity between Microsoft Access to/from G2, and G2 to Oracle databases, are enabled through Open Database Connectivity (ODBC) and G2-Oracle bridge products. Animations with layered and vector-based graphics are programmed for visualization of the plant operation. Graphical user interfaces (GUIs) including pull-down/pop-up menus, spreadsheet input/output, selection/type-in boxes, and action buttons are also provided.

The baseline version of the Dynamic (G2) Flowsheet contains input parameters such as vessel volumes, flowrates, sample hold times, chemical reactions and conversion factors (CFs), separation coefficients, feed vector, hot commissioning, hot operation, and ramp-up dates with their associated glass production rates. This baseline flowsheet is modified for scenario studies initiated by Model Run Requests (MRQs). Scenario runs could be as simple as a change to an input parameter listed above or as complicated as reconfiguring an entire unit operation/system.

Model runs can be performed for specific feed vector batches or for the entire feed vector. Under the PE's computing environment (24590-WTP-PL-TE-01-008, System Design for Process Modeling), a typical model run for an entire feed vector with full data collection will take up to 24 hours to complete and more than 10 gigabytes of data could be recorded in the Oracle databases. Automation tools such as MatLab scripts and Visual C\# programs are used to convert the Oracle data to more easily understandable tables and charts. 
Results of model runs generally include:

1) Feed vector batch required delivery dates

2) WTP simulation duration

3) Low-activity waste (LAW) glass container count and high-level waste (HLW) glass canister count

4) Volumes and compositions of treated LAW to the Supplemental LAW Facility

5) Volumes and compositions of WTP effluent to Liquid Effluent Retention Facility (LERF) and Effluent Treatment Facility (ETF)

6) Ultrafiltration Feed Preparation Vessel (UFP1) cycle time and associated operating parameters

7) Ultrafiltration Feed Vessel (UFP2) cycle time and associated operating parameters

8) Solubility constituent mass distribution diagrams

9) Solid wash and oxidative wash volumes

10) Caustic volumes for leaching and aluminum solubility

11) Volume history data for key process vessels

12) Sodium molarity and weight percent (wt \%) solids for key process vessels

13) Totalized mass transfer for every process stream

14) Waste loading of LAW and HLW glasses

15) Evaporator concentrate data including sodium molarity and Specific Gravity ( $\mathrm{SpG}$ )

16) Sample composition data at the Integrated Sampling and Analysis Requirements Document (ISARD) (24590-WTP-PL-PR-04-0001) locations and other locations of interests

17) Chemical reagent, process condensate, and demineralized water (DIW) consumption

18) Mineral glass former utilization

19) Mass balance of key species through the WTP

The 2010 WTP Tank Utilization Assessment (24590-WTP-RPT-PET-10-020) provides some comprehensive flowsheet analyses using the data described.

\section{Purpose}

Model Change Requests (MCRs) established by the PE department were incorporated in Version 7.0 of the Dynamic (G2) Flowsheet to: address the External Flowsheet Review Team (EFRT) issue resolutions and WTP's design evolution, align with research and technology results, and improve model fidelity, functionality, and performance. The MCRs were implemented based on the baseline Dynamic (G2) Flowsheet Version 6.0 released on August 17, 2010. Specific tasks that the Dynamic (G2) Flowsheet Version 7.0 supports include:

1) DOE Deliverable 2.6 - Tank Utilization Assessment (TUA)

a) Analyze Pretreatment and Vitrification performance

b) Analyze recycles and byproducts

c) Analyze LAW and HLW glass production and identify downtime due to Pretreatment and/or Glass Former Facility

d) Analyze sodium chemical additions

e) Analyze feed sequence and volume of waste received based on the feed vector 
f) Analyze effluent generation and distribution

g) Analyze variability of LAW and HLW vitrification feed and glass formulations

2) Tri-Party Agreement (TPA) Milestone M-062-049 (24590-WTP-RPT-PE-11-008)

a) Demonstrate WTP design capacity

3) Dynamic Flowsheet Developments

a) Evaluate process control strategies

b) Evaluate mixing uncertainties

c) Evaluate leaching kinetics

d) Evaluate design change alternatives

e) Evaluate hot commissioning alternatives

f) Evaluate Supplemental LAW Facility impacts

The WTP procedure Lifecycle Control of Process Models (24590-WTP-GPP-PT-013) requires that the dynamic flowsheet be summarized in a Model Design Document (MDD). The purpose of this document is to capture and describe the as-built design of the G2 model for Version 7.0. The Lifecycle Control of Process Models also stipulates that the model will undergo Verification and Validation (V\&V). The V\&V activities are specified in the Dynamic (G2) Flowsheet Version 7.0 Test Plan (24590-WTP-TP-PE11-001) and results are published in the Dynamic (G2) Flowsheet Version 7.0 Verification and Validation Report (24590-WTP-VV-PE-11-001).

\section{Model Basis and References}

The PE department has strategically applied the BARD as the basis for the development of process models, including the Steady-State Flowsheet (AES), Dynamic (G2) Flowsheet, and the WTP Operations Research (WITNESS) Flowsheet. The BARD provides the descriptions of plant layout, physical connections, systems, and unit operations and their design and operating parameters, governing equations, physical and chemical environment and reactions, and control logic that serve as the single-source reference for all the process models. The technical contents presented in this MDD are the implementation of the BARD in the Dynamic (G2) Flowsheet Version 7.0.

\section{Model System Descriptions}

\subsection{Time Steps and Dynamic Material Balance}

The Dynamic (G2) Model performs discrete-time material balance calculations over the WTP based on prescribed initial conditions, boundary conditions, and operating logic. The time steps are small enough to preserve the resolution of plant operations and large enough to avoid unacceptably long model run time. A 6-minute time step (i.e., every simulation clock tick represents $1 / 10$ of a simulated hour) is applied to the entire model except the Glass Former Reagent (GFR) System where a 1-minute time step (every clock tick represents $1 / 60$ of a simulated hour) is utilized. The 1-minute time step in the GFR is needed due to the large flowrates and small sizes of its equipment. 
Material balance is achieved by tracking the storage, pumping, separation, reaction, and phase change of chemical components in every unit operation over each time increment. Each unit operation is event driven as the status of that unit operation changes from time to time. An analyzer logic procedure controls the actions of each unit operation using pump procedures, reaction procedures, sample procedures, or other action procedures as appropriate depending on the current status of that unit operation. The simulation clocks are used to synchronize the actions of all the processes.

\subsection{Feed Vector}

The Dynamic (G2) Model processes a feed vector that is created by the Tank Farm Contractor (TFC) and provided by DOE-WTP. The feed vector consists of a waste quantity file and a leach factor file. The waste quantity file contains the batch names, proposed delivery dates, envelope types of the batches, and the quantities of liquid and solid components (in units of kg-moles) of the Hanford Tank Farm wastes. The leach factor file contains the leach factors for the constituents that will be leached out in the UFP2 vessels. Currently ten solid constituents, including $\mathrm{Al}^{+3}, \mathrm{Bi}^{+3}, \mathrm{Ca}^{+2}, \mathrm{Cr}($ Total $), \mathrm{Fe}^{+3}, \mathrm{Na}^{+}, \mathrm{PO}_{4}^{-3}, \mathrm{Si}^{+4}, \mathrm{U}$ (all isotopes), and $\mathrm{SO}_{4}{ }^{-2}$, are leached in the Dynamic (G2) Model (Note: leach factors for $\mathrm{Al}^{+3}$ and $\mathrm{PO}_{4}{ }^{-3}$ are not used in the model as the leaching of $\mathrm{Al}(\mathrm{OH})_{3}$ and "soft" $\mathrm{PO}_{4}^{-3}$ is driven by solubility correlations).

PE department personnel modify the TFC feed vectors to form components specific to the WTP operations. These components must be added to the feed vector arrays before the feeds enter the plant (Table I-1). Typically the components are created in a manner that maintains the feed vector's charge balance. Another modification to the TFC feed vector is the following application of secular equilibrium rules for $137 \mathrm{~m}-\mathrm{Ba}$ and $90-\mathrm{Y}$ (kg-moles):

$137 \mathrm{~m}-\mathrm{Ba}=1.526 \mathrm{e}-7 * 137-\mathrm{Cs}$

$90-\mathrm{Y}=2.54 \mathrm{e}-4 * 90-\mathrm{Sr}$

Appendix I further discusses the details of these feed vector operations.

After modifications have been made to the TFC feed vector, it is imported to the G2 model from an Access database through the ODBC bridge and is saved as part of the MRQ specific KB files. The feed vectors are updated periodically as a result of DOE-WTP studies intended to optimize the operations at both the Tank Farm, and the WTP, or as the result of other studies. The default in the Dynamic (G2) Model Version 7.0 is the TFC System Plan Revision 6 (SP6) Case 1 feed vector supplied by ORP.

\subsection{Equipment Initialization}

Some equipment must be filled with chemical reagents before they can be utilized. These include:

1) Ultrafiltration post-isolation valve pipes (UFP-02A-P2, UFP-02B-P2)

2) Cesium Reagent Vessel (CXP-VSL-00005)

3) Cesium Ion Exchange (CXP) Columns (CXP-IXC-00001/2/3/4)

4) Cesium Nitric Acid Recovery (CNP) Evaporator (CNP-EVAP-00001)

5) Cesium Resin Addition (CRP) Vessel (CRP-VSL-00001)

6) Recovered Nitric Acid Vessel (CNP-VSL-00004)

7) Spent Resin Slurry Vessel (RDP-VSL-00002A)

8) LAW Submerged Bed Scrubbers (SBS) (LOP-SCB-00001/2) 
9) HLW SBS (HOP-SCB-00001/2)

10) Vessel Vent Caustic Scrubber (PVP-SCB-00002)

11) Silica Storage Silo (GFR-TK-00001)

12) Zinc Oxide Storage Silo (GFR-TK-00002)

13) Titanium Dioxide Storage Silo (GFR-TK-00003)

14) Ferric Oxide Storage Silo (GFR-TK-00004)

15) Zirconium Silicate Sand Storage Silo (GFR-TK-00005)

16) Lithium Carbonate Storage Silo (GFR-TK-00006)

17) Boric Acid Storage Silo (GFR-TK-00007)

18) Aluminum Silicate Storage Silo (GFR-TK-00008)

19) Magnesium Silicate Storage Silo (GFR-TK-00009)

20) Calcium Silicate Storage Silo (GFR-TK-00010)

21) Sucrose Storage Silo (GFR-TK-00011)

22) Sodium Carbonate Storage Silo (GFR-TK-00012)

23) Borax Storage Silo (GFR-TK-00028)

24) Balance of Facilities (BOF)

Reagents for the WTP chemical processes are assumed to be readily available in the BOF before startup. Reagent types, volumes, and concentrations of the BOF utilized in the G2 model are presented in Appendix B. Reagent types, volumes, and concentrations added to the equipment listed above during model initialization are discussed in related sub-sections under Section 4.8.

\subsection{Component Lists}

The Dynamic (G2) Model utilizes four component lists for material balance calculations. These component lists and the associated number of constituents are given in Table 1.

Table 1 Component Lists and Lengths

\begin{tabular}{|c|c|}
\hline Component List & Number of Constituents \\
\hline Liquid & 162 \\
\hline Solid & 162 \\
\hline Gas & 16 \\
\hline Oxide & 73 \\
\hline
\end{tabular}

Appendix A presents the symbols, names, and molecular weights of these components. Radionuclides are tracked as liquid and solid components.

\subsection{Equipment Classes}

G2 is an object-oriented programming platform and each piece of WTP equipment is represented by a graphical object in the model (see figures in Appendix F for illustrations). Each object is an instance of an equipment class that is created according to the equipment's characteristics and functionalities.

Characteristics of an equipment class are implemented as attributes that represent the physical limits, control parameters, operating status, and contents of the equipment, while functionalities of an equipment class are implemented as methods that dictate what the equipment will do. Examples of characteristics are vessel volumes, pump rates, sample times, masses of various phases, pumping and receiving status, 
and others. Examples of functionalities include pumping, sampling, reacting, and others. Equipment of the same class possesses identical attributes and perform similar tasks. Figure 1 shows a WTP equipment class hierarchy where the parent-child relationships are presented. Major WTP equipment and associated equipment classes and component lists are given in Table 2. 
24590-WTP-MDD-PR-01-002, Rev 12

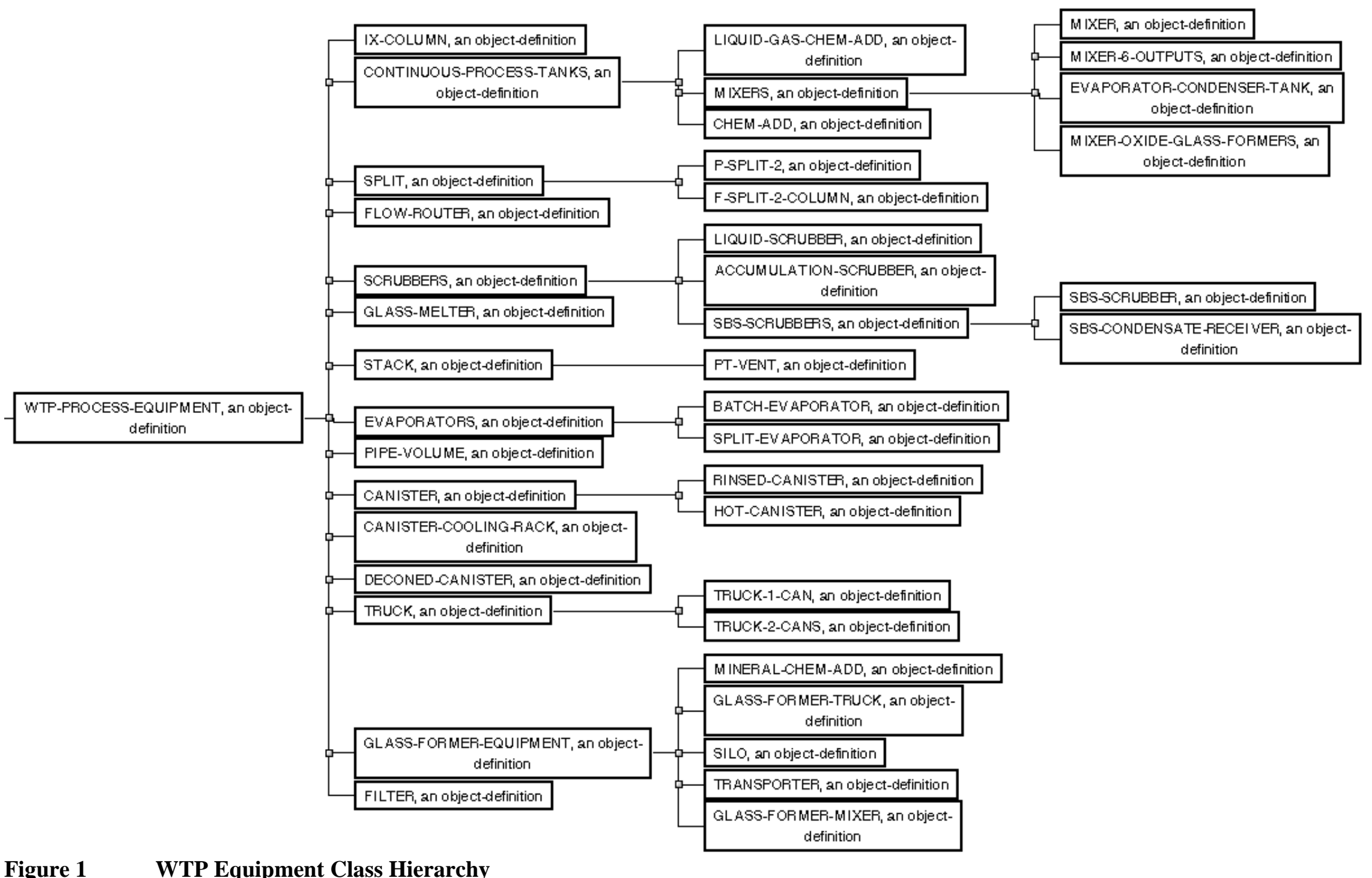

Figure 1 WTP Equipment Class Hierarchy 
Table 2 Equipment Classes and Associated Component Lists

\begin{tabular}{|c|c|c|}
\hline Equipment Name & Equipment Class & Component Lists \\
\hline CNP-DISTC-00001 & f-split-2-column & liquid, solid \\
\hline CNP-EVAP-00001 & batch-evaporator & liquid, solid \\
\hline CNP-VSL-00003 & mixer-6-outputs & liquid, solid, gas \\
\hline CNP-VSL-00004 & mixer-6-outputs & liquid, solid, gas \\
\hline CRP-VSL-00001 & mixer-6-outputs & liquid, solid, gas \\
\hline CXP-FILT-00001 & filter & liquid, solid \\
\hline CXP-IXC-00001 & ix-column & liquid, solid \\
\hline CXP-IXC-00002 & ix-column & liquid, solid \\
\hline CXP-IXC-00003 & ix-column & liquid, solid \\
\hline CXP-IXC-00004 & ix-column & liquid, solid \\
\hline CXP-VSL-00004 & mixer-6-outputs & liquid, solid, gas \\
\hline CXP-VSL-00026A & mixer & liquid, solid, gas \\
\hline CXP-VSL-00026B & mixer & liquid, solid, gas \\
\hline CXP-VSL-00026C & mixer & liquid, solid, gas \\
\hline ETF-1 & mixer-6-outputs & liquid, solid, gas \\
\hline FEP-DMST-00001A & evaporator-condenser-tank & liquid, solid, gas \\
\hline FEP-DMST-00001B & evaporator-condenser-tank & liquid, solid, gas \\
\hline FEP-SEP-00001A & split-evaporator & liquid, solid \\
\hline FEP-SEP-00001B & split-evaporator & liquid, solid \\
\hline FEP-VSL-00005A & mixer-6-outputs & liquid, solid, gas \\
\hline FEP-VSL-00005B & mixer-6-outputs & liquid, solid, gas \\
\hline FEP-VSL-00017A & mixer & liquid, solid, gas \\
\hline FEP-VSL-00017B & mixer & liquid, solid, gas \\
\hline FRP-VSL-00002A & mixer-6-outputs & liquid, solid, gas \\
\hline FRP-VSL-00002B & mixer-6-outputs & liquid, solid, gas \\
\hline FRP-VSL-00002C & mixer-6-outputs & liquid, solid, gas \\
\hline FRP-VSL-00002D & mixer-6-outputs & liquid, solid, gas \\
\hline GFR-TK-00001 & silo & oxide \\
\hline GFR-TK-00002 & silo & oxide \\
\hline GFR-TK-00003 & silo & oxide \\
\hline GFR-TK-00004 & silo & oxide \\
\hline GFR-TK-00005 & silo & oxide \\
\hline GFR-TK-00006 & silo & oxide \\
\hline GFR-TK-00007 & silo & oxide \\
\hline GFR-TK-00008 & silo & oxide \\
\hline GFR-TK-00009 & silo & oxide \\
\hline GFR-TK-00010 & silo & oxide \\
\hline GFR-TK-00011 & silo & oxide \\
\hline GFR-TK-00012 & silo & oxide \\
\hline GFR-TK-00013 & silo & oxide \\
\hline GFR-TK-00014 & silo & oxide \\
\hline GFR-TK-00015 & silo & oxide \\
\hline GFR-TK-00016 & silo & oxide \\
\hline GFR-TK-00018 & silo & oxide \\
\hline GFR-TK-00019 & silo & oxide \\
\hline
\end{tabular}




\begin{tabular}{|c|c|c|}
\hline Equipment Name & Equipment Class & Component Lists \\
\hline GFR-TK-00021 & silo & oxide \\
\hline GFR-TK-00022 & glass-former-mixer & liquid, oxide \\
\hline GFR-TK-00023 & glass-former-mixer & liquid, oxide \\
\hline GFR-TK-00025 & glass-former-mixer & liquid, oxide \\
\hline GFR-TK-00028 & silo & oxide \\
\hline GFR-TK-00031 & glass-former-mixer & liquid, oxide \\
\hline GFR-VSL-00002 & transporter & oxide \\
\hline GFR-VSL-00003 & transporter & oxide \\
\hline GFR-VSL-00004 & transporter & oxide \\
\hline GFR-VSL-00005 & transporter & oxide \\
\hline GFR-VSL-00006 & transporter & oxide \\
\hline GFR-VSL-00007 & transporter & oxide \\
\hline GFR-VSL-00008 & transporter & oxide \\
\hline HDH-TTBL-00001 & canister-cooling-rack & none \\
\hline HDH-VSL-00001 & mixer & liquid, solid, gas \\
\hline HDH-VSL-00002 & mixer & liquid, solid, gas \\
\hline HDH-VSL-00003 & mixer & liquid, solid, gas \\
\hline HDH-VSL-00004 & mixer & liquid, solid, gas \\
\hline HFP-VSL-00001 & mixer-oxide-glass-formers & liquid, solid, gas, oxide \\
\hline HFP-VSL-00002 & mixer-oxide-glass-formers & liquid, solid, gas, oxide \\
\hline HFP-VSL-00005 & mixer-oxide-glass-formers & liquid, solid, gas, oxide \\
\hline HFP-VSL-00006 & mixer-oxide-glass-formers & liquid, solid, gas, oxide \\
\hline HLP-VSL-00022 & mixer-6-outputs & liquid, solid, gas \\
\hline HLP-VSL-00027A & mixer-6-outputs & liquid, solid, gas \\
\hline HLP-VSL-00027B & mixer-6-outputs & liquid, solid, gas \\
\hline HLP-VSL-00028 & mixer-6-outputs & liquid, solid, gas \\
\hline HLW-CANISTER-COOLING-RACK & canister-cooling-rack & none \\
\hline HLW-CANISTERS-1 & mixer-oxide-glass-formers & liquid, solid, gas, oxide \\
\hline HLW-CANISTERS-2 & mixer-oxide-glass-formers & liquid, solid, gas, oxide \\
\hline HLW-PJV-STACK & stack & liquid, solid, gas \\
\hline HLW-STACK & stack & liquid, solid, gas \\
\hline HMP-MLTR-00001 & glass-melter & liquid, solid, gas, oxide \\
\hline HMP-MLTR-00002 & glass-melter & liquid, solid, gas, oxide \\
\hline HOP-ABS-00002 & accumulation-scrubber & liquid, solid, gas \\
\hline HOP-ABS-00003 & accumulation-scrubber & liquid, solid, gas \\
\hline HOP-ADBR-00001AB & accumulation-scrubber & liquid, solid, gas \\
\hline HOP-ADBR-00002AB & accumulation-scrubber & liquid, solid, gas \\
\hline HOP-HEME-00001 & liquid-scrubber & liquid, solid, gas \\
\hline HOP-HEME-00002 & liquid-scrubber & liquid, solid, gas \\
\hline HOP-HEPA-00001-2-AB & accumulation-scrubber & liquid, solid, gas \\
\hline HOP-HEPA-00007-8-AB & accumulation-scrubber & liquid, solid, gas \\
\hline HOP-SCB-00001 & sbs-scrubber & liquid, solid, gas \\
\hline HOP-SCB-00002 & sbs-scrubber & liquid, solid, gas \\
\hline HOP-SCR-00001 & accumulation-scrubber & liquid, solid, gas \\
\hline HOP-SCR-00002 & accumulation-scrubber & liquid, solid, gas \\
\hline HOP-VSL-00903 & sbs-condensate-receiver & liquid, solid, gas \\
\hline
\end{tabular}




\begin{tabular}{|c|c|c|}
\hline Equipment Name & Equipment Class & Component Lists \\
\hline HOP-VSL-00904 & sbs-condensate-receiver & liquid, solid, gas \\
\hline HOP-WESP-00001 & liquid-scrubber & liquid, solid, gas \\
\hline HOP-WESP-00002 & liquid-scrubber & liquid, solid, gas \\
\hline IHLW1 & hot-canister & liquid, solid, oxide \\
\hline IHLW2 & hot-canister & liquid, solid, oxide \\
\hline ILAW1 & hot-canister & liquid, solid, oxide \\
\hline ILAW2 & hot-canister & liquid, solid, oxide \\
\hline LAW-CONTAINERS-1 & mixer-oxide-glass-formers & liquid, solid, gas, oxide \\
\hline LAW-CONTAINERS-2 & mixer-oxide-glass-formers & liquid, solid, gas, oxide \\
\hline LAW-STACK & stack & liquid, solid, gas \\
\hline LCP-VSL-00001 & mixer-6-outputs & liquid, solid, gas \\
\hline LCP-VSL-00002 & mixer-6-outputs & liquid, solid, gas \\
\hline LFP-VSL-00001 & mixer-oxide-glass-formers & liquid, solid, gas, oxide \\
\hline LFP-VSL-00002 & mixer-oxide-glass-formers & liquid, solid, gas, oxide \\
\hline LFP-VSL-00003 & mixer-oxide-glass-formers & liquid, solid, gas, oxide \\
\hline LFP-VSL-00004 & mixer-oxide-glass-formers & liquid, solid, gas, oxide \\
\hline LMP-MLTR-00001 & glass-melter & liquid, solid, gas, oxide \\
\hline LMP-MLTR-00002 & glass-melter & liquid, solid, gas, oxide \\
\hline LOP-SCB-00001 & sbs-scrubber & liquid, solid, gas \\
\hline LOP-SCB-00002 & sbs-scrubber & liquid, solid, gas \\
\hline LOP-VSL-00001 & sbs-condensate-receiver & liquid, solid, gas \\
\hline LOP-VSL-00002 & sbs-condensate-receiver & liquid, solid, gas \\
\hline LOP-WESP-00001 & liquid-scrubber & liquid, solid, gas \\
\hline LOP-WESP-00002 & liquid-scrubber & liquid, solid, gas \\
\hline LVP-ADBR-00001AB & accumulation-scrubber & liquid, solid, gas \\
\hline LVP-HEPA-00001AB & accumulation-scrubber & liquid, solid, gas \\
\hline LVP-SCB-00001 & liquid-scrubber & liquid, solid, gas \\
\hline LVP-SCR-00001-2 & accumulation-scrubber & liquid, solid, gas \\
\hline LVP-TK-00001 & mixer & liquid, solid, gas \\
\hline PJM-RFD-VENT & accumulation-scrubber & liquid, solid, gas \\
\hline PJV-HEPA & accumulation-scrubber & liquid, solid, gas \\
\hline PJV-HEPA-00004-5 & accumulation-scrubber & liquid, solid, gas \\
\hline PJV-VSL-00001 & liquid-scrubber & liquid, solid, gas \\
\hline PVP-ABS-00001AB & accumulation-scrubber & liquid, solid, gas \\
\hline PVP-HEME-00001 & liquid-scrubber & liquid, solid, gas \\
\hline PVP-HEPA-00001-2 & accumulation-scrubber & liquid, solid, gas \\
\hline PVP-OXID-00001 & accumulation-scrubber & liquid, solid, gas \\
\hline PVP-SCB-00002 & sbs-condensate-receiver & liquid, solid, gas \\
\hline PVP-STACK & stack & liquid, solid, gas \\
\hline PVP-VSL-00001 & evaporator-condenser-tank & liquid, solid, gas \\
\hline PWD-VSL-00015 & mixer-6-outputs & liquid, solid, gas \\
\hline PWD-VSL-00016 & mixer-6-outputs & liquid, solid, gas \\
\hline PWD-VSL-00033 & mixer-6-outputs & liquid, solid, gas \\
\hline PWD-VSL-00043 & mixer-6-outputs & liquid, solid, gas \\
\hline PWD-VSL-00044 & mixer-6-outputs & liquid, solid, gas \\
\hline RDP-VSL-00002A & mixer-6-outputs & liquid, solid, gas \\
\hline
\end{tabular}




\begin{tabular}{|l|l|l|}
\hline Equipment Name & Equipment Class & Component Lists \\
\hline RDP-VSL-00002B & mixer-6-outputs & liquid, solid, gas \\
\hline RDP-VSL-00002C & mixer-6-outputs & liquid, solid, gas \\
\hline RESIN-DISPOSAL & mixer-6-outputs & liquid, solid, gas \\
\hline RLD-TK-00006A & mixer-6-outputs & liquid, solid, gas \\
\hline RLD-TK-00006B & mixer-6-outputs & liquid, solid, gas \\
\hline RLD-VSL-00003 & mixer & liquid, solid, gas \\
\hline RLD-VSL-00004 & mixer & liquid, solid, gas \\
\hline RLD-VSL-00005 & mixer & liquid, solid, gas \\
\hline RLD-VSL-00007 & mixer & liquid, solid, gas \\
\hline RLD-VSL-00008 & mixer & liquid, solid, gas \\
\hline RLD-VSL-00017A & mixer & liquid, solid, gas \\
\hline RLD-VSL-00017B & mixer & liquid, solid, gas \\
\hline SUPPLEMENTAL-LAW-RECYCLE & mixer-6-outputs & liquid, solid, gas \\
\hline SUPPLEMENTAL-LAW-TREATED & mixer-6-outputs & liquid, solid, gas \\
\hline TANK-FARM-HLW & mixer-6-outputs & liquid, solid, gas \\
\hline TANK-FARM-LAW & mixer-6-outputs & liquid, solid, gas \\
\hline TCP-VSL-00001 & mixer-6-outputs & liquid, solid, gas \\
\hline TLP-DMST-00001 & evaporator-condenser-tank & liquid, solid, gas \\
\hline TLP-SEP-00001 & split-evaporator & liquid, solid \\
\hline TLP-VSL-00002 & mixer-6-outputs & liquid, solid, gas \\
\hline TLP-VSL-00009A & mixer-6-outputs & liquid, solid, gas \\
\hline TLP-VSL-00009B & mixer-6-outputs & liquid, solid, gas \\
\hline UFP-02A-P1 & pipe-volume & liquid, solid \\
\hline UFP-02A-P2 & pipe-volume & liquid, solid \\
\hline UFP-02B-P1 & pipe-volume & liquid, solid \\
\hline UFP-02B-P2 & pipe-volume & liquid, solid \\
\hline UFP-VSL-00001A & mixer-6-outputs & liquid, solid, gas \\
\hline UFP-VSL-00001B & mixer-6-outputs & liquid, solid, gas \\
\hline UFP-VSL-00002A & mixer-6-outputs & liquid, solid, gas \\
\hline UFP-VSL-00002B & mixer-6-outputs & liquid, solid, gas \\
\hline UFP-VSL-00062A & mixer-6-outputs & liquid, solid, gas \\
\hline & & \\
\hline
\end{tabular}

\subsection{Volume Calculations}

The volume of material in a unit operation is calculated by analyzing the amounts and types of components in the unit's component array. In most cases, the total volume involves the partial volumes of liquid and solid, but in some unit operations it also possesses the contribution from oxides. In equipment where oxide exists, its volume is added to the solid volume. Gas components exist in the form of mass in some equipment and do not contribute to the total volume of the equipment.

A liquid's SpG is calculated by summing the mass of all the dissolved constituents in the volume defined for the solution, calculating the weight percent, and then estimating the density from a correlation derived from $\mathrm{NaOH}$ and $\mathrm{NaNO}_{3}$ dissolved in water (Equations 3 and 4). Using the liquid $\mathrm{SpG}$, a volume for the liquid components is obtained.

$$
\mathrm{SpG}=0.00935 \mathrm{X}+0.996 \quad \text { if } \mathrm{X}>0
$$




$$
\mathrm{SpG}=1.0 \quad \text { if } \mathrm{X} \leq 0
$$

Where:

$\begin{array}{ll}\mathrm{SpG} & =\text { specific gravity of a solution }(\text { dimensionless }) \\ \mathrm{X} & =\left\{\sum(\text { liquid }[\mathrm{i}])-\text { liquid }[\text { water }]\right\} / \sum(\text { liquid }[\mathrm{i}]) \times 100 \text { (dimensionless) } \\ \text { liquid } & =\text { liquid components in the solution }(\mathrm{kg}) \\ \mathrm{i} & =0 \text { to } 161\end{array}$

This SpG correlation applies to every process equipment that requires the calculation of liquid volume except the Cesium Evaporator Separator Vessel (CNP-EVAP-00001), where a ternary correlation of $\mathrm{HNO}_{3}, \mathrm{NaNO}_{3}$, and $\mathrm{H}_{2} \mathrm{O}$ is utilized. Details of the CNP-EVAP-00001 SpG correlation are discussed in Section 4.8.5.

The solid and oxide components have constant density values. The overall density for solids is approximated at 3.0 grams per cubic centimeter $\left(\mathrm{g} / \mathrm{cm}^{3}\right)$. The LAW glass density is set at $2.58 \mathrm{~g} / \mathrm{cm}^{3}$ while the HLW glass density is set at $2.66 \mathrm{~g} / \mathrm{cm}^{3}$. Using the constant densities, the volumes of solids and oxides are determined.

\subsection{Common Operating Rules}

There are operating rules/programming conventions that are used repeatedly throughout the model. The purpose of this section is to document these common operating rules used in the development of the Dynamic (G2) Model so that recurring discussions are not needed for the unit operations. The most prevalent operating rules used in the model are specified in the following subsections.

\subsubsection{Basic Model Rules}

Components, in kg-moles, are tracked in the model using component arrays. Each unit operation (vessel, evaporator, column, scrubber, melter, condenser, etc.) has its own component arrays which contain the components at that time. When material is transferred out of the unit, the amount of material to be removed in that time step is calculated and subtracted from the arrays of the unit emptying and added to the arrays of the unit filling using a floating-point data type with a 16-digit precision. This ensures a conservation of mass within the model.

Feed-lists and output-lists are used for the determination of batch transfers. When an upstream unit is ready to transfer material out, the unit is placed on its output-list. When a downstream unit is ready to receive material, it is placed on its feed-list. The upstream analyzer logic procedure constantly scans for the status of both lists and triggers the upstream unit pump procedure for pumping when it discovers that both upstream and downstream units are ready. This methodology ensures that unwanted transfers do not occur. Multiple feed-lists and/or output-lists may exist for vessels having multiple inflow sources and/or outflow destinations, respectively.

When the unit transfers material, it transfers a fraction of kg-moles from the component arrays based on the fraction of the unit's volume that needs to be transferred. The fraction is constrained by the pump rate of the upstream unit, the remaining volume of the upstream unit that can be pumped, and the remaining space of the downstream unit that can be filled.

There are exceptions in some vessels where they can be emptied and filled at the same time. There are also exceptions in some vessels where contingency volumes exist such that the vessel can receive if it 
needs to and discharge if the downstream unit becomes available. These exceptions are discussed as appropriate in detail in Section 4.8.

\subsubsection{Batch Transfers}

The batch transfer process is the most commonly used vessel operation in the dynamic model. When the upstream vessel is full or reaches its pre-assigned setpoint, the upstream vessel is placed on its output-list. When the downstream vessel is empty or reaches its minimum volume, the vessel is placed on its input-list. The analyzer logic of the upstream vessel confirms that both vessels are ready. If there are no other conditions or control logic preventing the transfer, the transfer begins and the contents of the upstream vessel are transferred to the downstream vessel. The transfer volume is calculated for each 6minute, or 1-minute as appropriate, time-step by taking the minimum of the following: the volume available to pump from the upstream vessel, the available space in the downstream vessel, and the capacity of the pump. After each time-step transfer, both the upstream and downstream vessel volumes are updated, along with the vessel component arrays. The volumes of both vessels are checked again and either triggered to continue transferring or triggered to stop transferring because the volume constraints have been met. The batch transfer is terminated when the upstream vessel reaches its minimum volume or the downstream vessel reaches either its setpoint or maximum volume.

Transfer lines do not have volumes in the model so that materials out of the upstream vessels equal to materials into the downstream vessels. However, actual transfer line volumes are used external to the G2 model when line flush volumes are calculated. Line flush volumes are provided in Table H-1.

Exceptions exist for the ultrafiltration filter loops where the transfer lines contain volumes and flushing efficiencies are applied during power flush operations. Section 4.8.3 provides detailed discussions of the ultrafiltration filter loops.

\subsubsection{Continuous Transfers}

The continuous transfer process is used primarily in the melters, offgas systems, and the evaporators, but is also applied to the UFP-VSL-00002A/B vessels and the "Super Tank" (Note: "Super Tank" is the modeling simplification that combines the functions of UFP-VSL-00062A/B/C and CXP-VSL-00004). When a continuous transfer occurs, material enters the unit operation, reactions and/or splits are performed, and the remaining material exits the unit operation all in one single time step.

Some continuous transfer unit operations can be described as semi-continuous transfer unit operations. This is because they accumulate some of the material that continuously passes through and eventually empty the gathered material in a batch transfer. The Cesium Nitric Acid Recovery Evaporator, Submerged Bed Scrubbers, and Caustic Scrubbers are examples of semi-continuous transfers.

\subsubsection{Action Modes of Vessels}

Most of the process vessels operate in six action modes, "Empty", "Fill", "Filling", "Sampling", "Empty-Now", "Emptying". Definitions for these action modes are given below:

"Empty" = A vessel is empty or at minimum volume

"Fill" = A vessel is waiting to be filled

"Filling" = A vessel is currently being filled

"Sampling" = A vessel is being sampled 
"Empty-Now" = A vessel is waiting to be emptied.

"Emptying" = A vessel is currently being emptied.

The less common operation modes used in the model are not discussed in this MDD but can be obtained from the G2 codes. A vessel can only be filled when it is in the "Empty" or "Fill" modes. Only one source can be pumped into a vessel at a time. Exception exists in some vessels where they can be filled and emptied at the same time. Exception also exists in some vessels where contingency volume is utilized (e.g., a vessel can either be filled or discharged when it is in the contingency zone, depending on the conditions of the upstream or downstream vessels). Contingency volume is discussed in Section 4.7.5. Details of these exceptions are discussed as appropriate in Section 4.8.

\subsubsection{Contingency Volume}

Some vessels have multiple setpoint controls and contingency volumes. These vessels operate between the minimum and maximum volumes with two setpoints in between. A contingency vessel can be filled, and then continue to be filled until the current batch (after being completely transferred in) raises the volume to or above setpoint \#1. The mode of the contingency vessel is then changed to "Continuous". If the downstream vessel is not ready to receive a transfer, the contingency vessel continues to be filled until the volume is at, or above, setpoint \#2, then the mode of the contingency vessel is changed to "EmptyNow". The contingency volume is set between setpoint \#1 and the setpoint \#2. The contingency vessel can be emptied any time when it is in the contingency zone and the downstream vessel is available.

The list below provides the names of contingency vessels which are further discussed in Section 4.8 as appropriate.

\begin{tabular}{|l|}
\hline CNP-VSL-00003 \\
\hline HOP-VSL-000903 (modeled as part of HOP-SCB-00001) \\
\hline HOP-VSL-000904 (modeled as part of HOP-SCB-00002) \\
\hline LOP-VSL-00001 (modeled as part of LOP-SCB-00001) \\
\hline LOP-VSL-00002 (modeled as part of LOP-SCB-00002) \\
\hline PWD-VSL-00033 \\
\hline PWD-VSL-00043 \\
\hline PWD-VSL-00044 \\
\hline PVP-VSL-00001 \\
\hline TCP-VSL-00001 \\
\hline TLP-VSL-00009A \\
\hline TLP-VSL-00009B \\
\hline
\end{tabular}

\subsubsection{Chemical Additions}

All chemical additions to the processes are done through chem-add objects. A chem-add is normally dedicated to a unit operation and delivers a specific solution of chemicals with a preset concentration. The amount of chemical solution needed is either pre-defined or calculated to achieve the desired concentration of that chemical in the unit. Readers of this document will notice that $\mathrm{Nap}^{+}$and Mnp are used throughout the document. The purpose is to distinguish the process sodium and process manganese added as chemical reagents in the WTP from the waste sodium and waste manganese either received from the feed vector or classified as "waste" in the WTP contract. A detailed discussion of chemical additions is presented in Appendix B. 


\subsubsection{Neutralization Reactions}

When both $\mathrm{H}^{+}$and $\mathrm{OH}^{-}$are present in a vessel, they will react to form $\mathrm{H}_{2} \mathrm{O}$. The reaction occurs just before the unit operation receives chemicals, performs other reactions, or becomes ready for discharge, so it is possible to see $\mathrm{H}^{+}$and $\mathrm{OH}^{-}$residing in a unit at the same time when the unit is filling. This is acceptable for modeling purposes.

\subsubsection{Sample Points and Sample Hold Times}

Some vessels in the model require sampling based on the Integrated Sampling Analysis Requirements Document (ISARD). If a vessel has a sample hold time, the vessel will fill to a designated level and hold for the prescribed "sample" time. When the hold time has expired, the vessel, if required, will perform its chemical operations (chem-adds, reactions, and so on) and become ready for discharge. The sample hold times include the expected time required to pull a sample from the vessel, analyze the sample, and return the results back to operators that verifying the contents of the vessel can continue to the next unit operation. The current model is based on the assumption that the samples always meet specifications and rework of the vessel contents is not required.

Sample points and their sample logic are summarized in Appendix C while sample hold times are provided in Appendix E.

\subsubsection{Chemical Reactions}

Certain unit operations in the model undergo chemical reactions. The chemical reactions are described later in this document and are presented in the sequence in which they occur in each unit operation. A chemical reaction is made up of reactants and products and is based on a simple stoichiometric reaction balance. A reaction conversion is given for the reaction and is applied to the first reactant only. If the reaction cannot reach the desired conversion, then the reaction will continue until all of the limiting reactant has been used up. The reaction subtracts the reactants used from the component array and adds the products generated to the component array. If the reactions involve phase changes, reactants and products are moved to the corresponding liquid/solid/gas/oxide positions in the arrays. Volume is recalculated after the reactions.

\subsubsection{Splits}

Splits are used in the model to demonstrate the entrainment of components in certain processes and the separation of components in other processes. Split values are determined based on the decontamination factors (DFs) of the unit operation, e.g., split to overhead =1 / DF. Splits are performed in the evaporators, condensers, melters, and offgas systems using either percents or fractions. The splits are component based and are applied to the component array of the unit operation. The fraction or percent of that particular component is subtracted from the component array of the unit operation performing the splits and added to the component array designated by the split table. The remaining material in the unit operation performing the split exits out of the other exit stream.

If the unit operation is an accumulation-scrubber (see Table 2 for equipment classes), the material that is removed using the split is not transferred to another unit operation. Rather, it is added to an accumulating array where captured material is stored. Components cannot be subtracted from the accumulating array, only added to it.

The split constants for the unit operations applicable are summarized in Appendix D. 


\subsubsection{Primary and Secondary Process Waters}

Many processes such as line flush, sodium dilution, solid wash, and oxidative wash require the use of process waters. Process condensate from the Pretreatment Process Condensate Vessel (RLD-TK-00006A) is the preferred source (primary source) if sufficient volume is available at the time when it is needed. Process water is the secondary choice and is only used if the primary source is unavailable or insufficient. The contents of process condensate change constantly as RLD-TK-00006A receives condensates from the Waste Feed Evaporator Condensate Vessel and the Treated LAW Evaporator Condensate Vessel where the feeds to the evaporators vary.

\subsubsection{Inhibited Water}

Inhibited water is used for line flushes before and after wastes are transferred to the WTP from the Tank Farm. Inhibited water is also used for line flush into a UFP2 vessel after a UFP1 vessel empties its contents entirely (i.e., reach the heel), for power flushing, and for flushing the lines from UFP2 to HLPVSL-00027A/B after solid discharge. Inhibited water is a mixed solution of $0.01 \mathrm{M} \mathrm{NapOH}$ and $0.011 \mathrm{M}$ $\mathrm{NapNO}_{2}$ (i.e., $\left[\mathrm{Nap}^{+}\right]=0.021 \mathrm{M},\left[\mathrm{OH}^{-}\right]=0.01 \mathrm{M}$, and $\left[\mathrm{NO}_{2}^{-}\right]=0.011 \mathrm{M}$ ).

\subsubsection{Line Flushes}

Line flushes are applied to the Dynamic (G2) Flowsheet to mitigate: 1) precipitation of salts per the Interface Control Document (ICD) for Waste Feed (24590-WTP-ICD-MG-01-019), 2) hydrogen generation per the Hydrogen in Piping and Ancillary Vessels (HPAV) - Plant Availability Assessment (24590-WTP-RPT-M-06-002), and 3) plugging of process lines per the Hanford Waste Treatment and Immobilization Plant (WTP) Project Response Plan for Resolution of Issues Identified by the Comprehensive Review of the WTP Flowsheet and Throughput (24590-WTP-PL-ENG-06-0008).

The ICD flushes are conducted using inhibited water and include the lines from the Tank Farm to the HLP-VSL-00022 and FRP-VSL-00002A/B/C/D vessels as discussed in the following.

\section{Tank Farm to HLP-VSL-00022}

Prior to an HLW batch transfer, HLP-VSL-00022 receives 2,500 gallons of inhibited water. HLP-VSL-00022 receives an additional 2,000 gallons of inhibited water after the Tank Farm batch transfer is completed and before the vessel is sampled.

At the end of the post-transfer flush, PWD-VSL-00043 and PWD-VSL-00033 will simultaneously receive 2,900 gallons and 100 gallons of inhibited water, respectively. Due to the dynamic nature of the flowsheet, PWD-VSL-00043 and PWD-VSL-00033 vessels could be in different operating modes when flushing takes place. The flush water can only be pumped into the Plant Wash and Disposal System (PWD) vessels when they are in the "empty" or "fill" modes, or otherwise be delayed.

\section{$\underline{\text { Tank Farm to FRP-VSL-00002A/B/C/D }}$}

A LAW batch may fill one, two, or three Waste Feed Receipt Vessels depending on the size of the batch. The 2,500 gallons of pre-transfer inhibited water will be sent to the first receiving vessel prior to the batch delivery while the 2,000 gallons of post-transfer inhibited water will be sent to the last receiving vessel prior to sampling. 
At the end of the post-transfer flush, PWD-VSL-00043 and PWD-VSL-00033 will simultaneously receive 2,900 gallons and 100 gallons of inhibited water, respectively. The operating logic for PWD-VSL-00043 and PWD-VSL-00033 is the same as those discussed above.

The HPAV and line plugging flushes are conducted using process condensate (primary source) or process water (secondly source) for Pretreatment vessels, however only process water can be used for flushes to the LAW and HLW equipment. These line flush operations can be triggered by: 1) waste transfer, 2) sampling, and 3) time. Transfer flushes are conducted after a waste batch transfer from the upstream tank to the downstream tank; sampling flushes are made after a sample is pulled from the vessel; and time flushes are carried out regularly at the specified time interval. In most cases, an origin of flush is associated with multiple destinations of flushes as the results of dead legs and piping configurations. The triggers for the line flush operations are summarized in Table 3, Table 4, and Table 5. The full list of line flushes including the triggers and the associated dead lag and piping configuration flushes is presented in Table H-1.

Table 3 Transfer Flushes

\begin{tabular}{|l|l|l|}
\hline Stream Number (STRN) & Upstream Name & Downstream Name \\
\hline FEP-04 & FEP-VSL-00017A & FEP-SEP-00001A \\
\hline FEP-07 & FEP-VSL-00017A & FEP-SEP-00001B \\
\hline FEP-12 & FEP-VSL-00017B & FEP-SEP-00001A \\
\hline FEP-15 & FEP-VSL-00017B & FEP-SEP-00001B \\
\hline FRP-01 & FRP-VSL-00002A & FEP-VSL-00017A \\
\hline FRP-02 & FRP-VSL-00002A & FEP-VSL-00017B \\
\hline FRP-03 & FRP-VSL-00002A & UFP-VSL-00001A \\
\hline FRP-04 & FRP-VSL-00002A & UFP-VSL-00001B \\
\hline FRP-05 & FRP-VSL-00002B & FEP-VSL-00017A \\
\hline FRP-06 & FRP-VSL-00002B & FEP-VSL-00017B \\
\hline FRP-07 & FRP-VSL-00002B & UFP-VSL-00001A \\
\hline FRP-08 & FRP-VSL-00002B & UFP-VSL-00001B \\
\hline FRP-09 & FRP-VSL-00002C & FEP-VSL-00017A \\
\hline FRP-10 & FRP-VSL-00002C & FEP-VSL-00017B \\
\hline FRP-11 & FRP-VSL-00002C & UFP-VSL-00001A \\
\hline FRP-12 & FRP-VSL-00002C & UFP-VSL-00001B \\
\hline FRP-13 & FRP-VSL-00002D & FEP-VSL-00017A \\
\hline FRP-14 & FRP-VSL-00002D & FEP-VSL-00017B \\
\hline FRP-15 & FRP-VSL-00002D & UFP-VSL-00001A \\
\hline FRP-16 & FRP-VSL-00002D & UFP-VSL-00001B \\
\hline FRP-41 & Tank-Farm-HLW & HLP-VSL-00022 \\
\hline FRP-44 & Tank-Farm-LAW & FRP-VSL-00002A \\
\hline FRP-45 & Tank-Farm-LAW & FRP-VSL-00002B \\
\hline FRP-46 & Tank-Farm-LAW & FRP-VSL-00002C \\
\hline FRP-47 & Tank-Farm-LAW & FRP-VSL-00002D \\
\hline HLP-03 & HLP-VSL-00022 & UFP-VSL-00001A \\
\hline & & \\
\hline
\end{tabular}




\begin{tabular}{|l|l|l|}
\hline Stream Number (STRN) & Upstream Name & Downstream Name \\
\hline HLP-04 & HLP-VSL-00022 & UFP-VSL-00001B \\
\hline HLP-05 & HLP-VSL-00022 & FEP-VSL-00017A \\
\hline HLP-06 & HLP-VSL-00022 & FEP-VSL-00017B \\
\hline HLP-13 & HLP-VSL-00028 & HFP-VSL-00005 \\
\hline HLP-15 & HLP-VSL-00028 & HFP-VSL-00001 \\
\hline HLP-22 & HLP-VSL-00027A & HLP-VSL-00028 \\
\hline HLP-26 & HLP-VSL-00027B & HLP-VSL-00028 \\
\hline HLW-04 & HFP-VSL-00001 & HFP-VSL-00002 \\
\hline HLW-17 & HFP-VSL-00005 & HFP-VSL-00006 \\
\hline HLW-29 & HOP-SCB-00001 & RLD-VSL-00007 \\
\hline HLW-35 & HOP-VSL-00903 & RLD-VSL-00007 \\
\hline HLW-39 & HOP-SCB-00002 & RLD-VSL-00007 \\
\hline HLW-44 & HOP-VSL-00904 & RLD-VSL-00007 \\
\hline HLW-51 & RLD-VSL-00007 & PWD-VSL-00043 \\
\hline HLW-81 & HDH-VSL-00003 & RLD-VSL-00007 \\
\hline HLW-85 & HDH-VSL-00001 & HDH-VSL-00003 \\
\hline LCP-1 & LCP-VSL-00001 & LCP-VSL-00001 \\
\hline LCP-2 & LCP-VSL-00002 & LCP-VSL-00002 \\
\hline LFP-1 & UFP-VSL-00002B & PWD-VSL-00043 \\
\hline LFP-10 & UFP-VSL-VSL-000001 & LFP-VSL-00001 \\
\hline LFP-2 & UFP-VSL-00027A \\
\hline LFP-3 & LFP-VSL-00004 & LMP-MLTR-00002 \\
\hline LFP-4 & LCP-VSL-00001 & LFP-VSL-00003 \\
\hline LFP-5 & LCP-VSL-00002 & LFP-VSL-00001 \\
\hline LFP-6 & LCP-VSL-00002 & LFP-VSL-00003 \\
\hline LFP-7 & LFP-VSL-00001 & LFP-VSL-00001 \\
\hline LFP-8 & LFP-VSL-00003 & LFP-VSL-00003 \\
\hline LFP-9 & LFP-VSL-00001 & LFP-VSL-00002 \\
\hline TCP-03 & LFP-VSL-00003 & LFP-VSL-00004 \\
\hline TCP-04 & LFP-VSL-00002 & LMP-MLTR-00001 \\
\hline UFP-13 & TCP-VSL-00001 & LCP-VSL-00001 \\
\hline UFP-15 & HFP-V0001 & LCP-VSL-00002 \\
\hline UFP-16 & PWD-VSL-00043 \\
\hline UFP-20 & HFP-21 & \\
\hline
\end{tabular}


Table 4 Sample Flushes

\begin{tabular}{|l|l|l|}
\hline STRN & Sample Tank & Downstream Name \\
\hline FRP-17 & FRP-VSL-00002A & FRP-VSL-00002B \\
\hline FRP-19 & FRP-VSL-00002B & FRP-VSL-00002B \\
\hline FRP-21 & FRP-VSL-00002C & FRP-VSL-00002C \\
\hline FRP-23 & FRP-VSL-00002D & FRP-VSL-00002C \\
\hline HLP-02 & HLP-VSL-00022 & HLP-VSL-00022 \\
\hline FEP-02 & FEP-VSL-00017A & FEP-VSL-00017A \\
\hline FEP-10 & FEP-VSL-00017B & FEP-VSL-00017B \\
\hline CXP-43 & CXP-VSL-00026A & CXP-VSL-00026A \\
\hline CXP-44 & CXP-VSL-00026B & CXP-VSL-00026B \\
\hline CXP-45 & CXP-VSL-00026C & CXP-VSL-00026C \\
\hline RDP-21 & RDP-VSL-00002B & RDP-VSL-00002B \\
\hline TLP-07 & TLP-VSL-00009A & TLP-VSL-00009A \\
\hline TLP-08 & TLP-VSL-00009B & TLP-VSL-00009B \\
\hline HLP-20 & HLP-VSL-00027A & HLP-VSL-00027A \\
\hline HLP-24 & HLP-VSL-00027B & HLP-VSL-00027B \\
\hline HLP-11 & HLP-VSL-00028 & HLP-VSL-00028 \\
\hline HLW-06 & HFP-VSL-00001 & HFP-VSL-00001 \\
\hline HLW-19 & HFP-VSL-00005 & HFP-VSL-00005 \\
\hline HLW-55 & RLD-VSL-00007 & RLD-VSL-00007 \\
\hline & &
\end{tabular}

Table 5 Time Flushes

\begin{tabular}{|l|l|l|c|}
\hline STRN & Upstream Name & Downstream Name & Time between Flushes (hrs) \\
\hline FEP-17 & FEP-SEP-00001A & UFP-VSL-00001A & 450 \\
\hline FEP-18 & FEP-SEP-00001A & UFP-VSL-00001B & 450 \\
\hline FEP-20 & FEP-SEP-00001B & UFP-VSL-00001A & 450 \\
\hline FEP-21 & FEP-SEP-00001B & UFP-VSL-00001B & 450 \\
\hline UFP-02 & UFP-VSL-00001A & UFP-VSL-00001A & 1,965 \\
\hline UFP-08 & UFP-VSL-00001B & UFP-VSL-00001B & 1,965 \\
\hline UFP-05 & UFP-VSL-00001A & UFP-VSL-00002B & 524 \\
\hline UFP-06 & UFP-VSL-00001A & UFP-VSL-00002A & 524 \\
\hline UFP-11 & UFP-VSL-00001B & UFP-VSL-00002B & 524 \\
\hline UFP-12 & UFP-VSL-00001B & UFP-VSL-00002A & 524 \\
\hline TLP-10 & TLP-SEP-00001 & TCP-VSL-00001 & 164 \\
\hline HLW-37 & HOP-HEME-00001 & HOP-VSL-00903 & 4,380 \\
\hline HLW-47 & HOP-HEME-00002 & HOP-VSL-00904 & 4,380 \\
\hline LFP-11 & LFP-VSL-00002 & LMP-MLTR-00001 & 4 \\
\hline LFP-12 & LFP-VSL-00004 & LMP-MLTR-00002 & \\
\hline
\end{tabular}


It is important to note that a trigger from above can result in flushes into multiple destinations due to dead legs and piping configurations. Details of the flushing destinations and associated volumes are given in Table H-1.

\subsubsection{Supplemental LAW}

Two pseudo tanks are included in the model as optional features. The pseudo tanks are named SUPPLEMENTAL-LAW-TREATED and SUPPLEMENTAL-LAW-RECYCLE. SUPPLEMENTALLAW-TREATED is created to store the excess treated LAW feed due to the Pretreatment / LAW Vitrification throughput differential. SUPPLEMENTAL-LAW-RECYCLE is created to store recycles from the LAW SBS and Wet Electrostatic Precipitator (WESP). Switches are provided to turn on/off the features depending on model run scenarios. Features can be activated separately of one another. The default states of the switches are "on" for SUPPLEMENTAL-LAW-TREATED and "off" for SUPPLEMENTAL-LAW-RECYCLE. Details of the operating logic are described below.

\section{SUPPLEMENTAL-LAW-TREATED}

1) TCP-VSL-00001 can receive concentrate from TLP-SEP-00001 at all times.

2) TCP-VSL-00001 is filled to its set volume (75,000 gallons) initially and is maintained at, or above this level except when transferring out to LCP-VSL-00001 and LCP-VSL-00002.

3) TCP-VSL-00001 transfers material to LCP-VSL-00001 and LCP-VSL-00002 whenever they are ready to receive the transfer. During this transfer, the vessel volume of TCP-VSL-00001 can be pumped down to the minimum volume if needed (9,621 gallons).

4) Any excess treated LAW feed (contents that result in volume above 75,000 gallons) will be pumped to SUPPLEMENTAL-LAW-TREATED. The trigger volume is 80,000 gallons for pumping.

\section{SUPPLEMENTAL-LAW-RECYCLE}

Recycle from RLD-VSL-00005 is pumped to TLP-VSL-00009A/B. SUPPLEMENTAL-LAWRECYCLE is inactive in the Dynamic (G2) Flowsheet Version 7.0.

\subsubsection{Vessel Parameters}

Vessel parameters, including minimum (heel) volumes, set volumes, maximum (heel + batch) volumes, flowrates, and sample hold times, are listed in Appendix E.

\subsubsection{Solubility}

Significant quantities of oxalate, phosphate, and aluminum are brought in to the WTP for processing. These solubility limited constituents exist in the liquid and solid phases in the Tank Farms and are expected to undergo further phase changes in WTP due to various Pretreatment operations, such as feed blending, solids concentration, heating, leaching, cooling, solid wash, dilution, $\mathrm{pH}$ adjustment, and evaporation. Phase equilibrium is of paramount importance in determining the fate of these partial soluble constituents as whether they will be sent to the LAW Facility, Pretreatment recycle streams, or HLW Facility, which can have major impacts on WTP throughput and waste loading in IHLW.

It is desirable to send more of the solubility limited constituents to the LAW Facility but the large volume of wash forward solution required to do this may bog down the CXP system, which in turn extends the processing duration. Sending wash forward solution at low hydroxide concentration may also cause 
aluminum precipitation that clogs ion exchange columns and plugs transfer lines. In contrast, allowing too much oxalate and phosphate to the Pretreatment recycle streams from wash backward will create a flywheel effect that reduces the throughput of wastes at ultrafiltration (Note: flywheel effect is the process phenomenon within the Ultrafiltration Process (UFP) -> Plant Wash and Disposal Process (PWD) -> Waste Feed Evaporation Process (FEP) -> UFP loop where oxalate and phosphate dissolve in the UFPVSL-00002A/B vessels during wash backward, recycle to the PWD and FEP systems, re-precipitate in the FEP-SEP-00001A during evaporation, and accumulate again in the UFP-VSL-00002A/B vessels during solids concentration). Finally, sending more aluminate and gibbsite to the HLW Facility will produce more IHLW glasses.

Solubility correlations have been developed and their applications to the G2 model are discussed below. It is noted that these correlations were derived by the WTP as first order attempts to address the flowsheet concerns above. There are various uncertainties in these correlations as they were derived primarily based on literature values as Hanford tank waste specific experimental data seldom exists.

\subsubsection{Solubility Correlations}

$\underline{\text { Oxalate Solubility }}$

Oxalate $\left(\mathrm{C}_{2} \mathrm{O}_{4}{ }^{-2}\right)$ phase equilibrium is calculated with the following equations:

$$
\begin{aligned}
& \text { NaMF }=\text { LiqNaMoles } /[2 *(\text { LiqNaMoles }+ \text { LiqKMoles })+\text { LiqH 2OMoles }] \\
& \ln \left(\lambda_{C 2 O 4-2}\right)=\left(a+b(N a M F)+c\left(N a M F^{2}\right)+d\left(N a M F^{3}\right)\right) * T_{K} \\
& \ln \left(\lambda_{N a+}\right)=\left(e+f(N a M F)+g\left(N a M F^{2}\right)+h\left(N a M F^{3}\right)\right) * T_{K} \\
& \ln \left(C_{C 2 O 4-2}\right)=\ln (Q)-\ln \left(\lambda_{C 2 O 4-2}\right)-2 * \ln \left(C_{N a+}\right)-2 \ln \left(\lambda_{\mathrm{Na+}}\right)-\ln \left(T_{K}\right)
\end{aligned}
$$

Where:

NaMF = Sodium mole fraction (unitless),

$\lambda_{\mathrm{C} 2 \mathrm{O4}-2}=$ Activity coefficient of the oxalate ion (unitless),

$\lambda_{\mathrm{Na}+} \quad=$ Activity coefficient of the sodium ion (unitless),

$\mathrm{C}_{\mathrm{C} 2 \mathrm{O} 4-2}=$ Oxalate concentrate at equilibrium $(\mathrm{gmol} / \mathrm{kg} \mathrm{H} 2 \mathrm{O})$,

$\mathrm{C}_{\mathrm{Na}+} \quad=$ Sodium concentration at equilibrium $(\mathrm{gmol} / \mathrm{kg} \mathrm{H} 2 \mathrm{O})$,

$\mathrm{T}_{\mathrm{K}} \quad=$ Vessel temperature $(\mathrm{K})$,

The constants for Equations 5 through 8 are given as:

\begin{tabular}{|l|r|}
\hline Constant & Value \\
\hline $\mathrm{a}$ & -0.0220206 \\
\hline $\mathrm{b}$ & -0.1028143 \\
\hline $\mathrm{c}$ & -1.016929 \\
\hline $\mathrm{d}$ & 17.1877483 \\
\hline $\mathrm{e}$ & -0.0008179 \\
\hline $\mathrm{f}$ & -0.0134259 \\
\hline $\mathrm{g}$ & 0.92062137 \\
\hline
\end{tabular}




\begin{tabular}{|l|r|}
\hline $\mathrm{h}$ & -9.7239226 \\
\hline $\ln (\mathrm{Q})$ & -4.21128 \\
\hline
\end{tabular}

If the concentration of oxalate is below the calculated solubility, solid oxalate and associated solid sodium dissolve until:

1) Concentration of oxalate reaches the solubility (excess solid oxalate and solid sodium) or

2) Solid oxalate has completely depleted (solid oxalate limited)

If the concentration of oxalate is above the calculated solubility, liquid oxalate and associated liquid sodium precipitate until:

3) Concentration of oxalate reaches solubility or

4) Liquid oxalate has completely depleted (liquid oxalate limited)

The oxalate dissolution/precipitation reactions are given as:

$\mathrm{C}_{2} \mathrm{O}_{4}^{-2}(\mathrm{~s})+2 \mathrm{Na}^{+}(\mathrm{s}) \leftrightarrow \mathrm{C}_{2} \mathrm{O}_{4}^{-2}(\mathrm{l})+2 \mathrm{Na}^{+}(\mathrm{l})$

Oxalate concentration will not reach solubility when conditions 2 or 4 are encountered. Details related to the development of the oxalate solubility equations are given in CCN 160518.

$\underline{\text { Phosphate Solubility }}$

Phosphate is partitioned into "soft" and "hard" components, designated in the model as "PO4-3" and "PO4-3(HARD)", respectively. Partitioning is done in accordance with the phosphate leach factors given in the TFC feed vector, e.g., the unleachable phosphate is converted to "PO4-3(HARD)". The "soft" or soluble PO4-3 is treated as trisodium phosphate $\left(\mathrm{Na}_{3} \mathrm{PO}_{4}\right)$ and the solubility is a function of supernatant sodium, hydroxide, and fluoride concentrations, and temperature (24590-WTP-M4C-V37T-00009)

$\mathrm{C}_{\mathrm{PO} 4}=\exp \left(\mathrm{A} / \mathrm{T}_{\mathrm{K}}+\mathrm{B}\right) * \mathrm{C}_{\mathrm{Na}}^{\mathrm{C}} * \mathrm{C}_{\mathrm{OH}}^{\mathrm{D}} * \mathrm{C}_{\mathrm{F}}^{\mathrm{E}}$

Where:

$\mathrm{C}_{\mathrm{PO} 4}=$ Phosphate concentration at equilibrium $\left(\mathrm{gmol} / \mathrm{kg} \mathrm{H}_{2} \mathrm{O}\right)$,

$\mathrm{C}_{\mathrm{Na}} \quad=$ Sodium concentration $\left(\mathrm{gmol} / \mathrm{kg} \mathrm{H} \mathrm{H}_{2} \mathrm{O}\right)$,

$\mathrm{C}_{\mathrm{OH}} \quad=$ Hydroxide concentration $\left(\mathrm{gmol} / \mathrm{kg} \mathrm{H} \mathrm{H}_{2} \mathrm{O}\right)$,

$\mathrm{C}_{\mathrm{F}} \quad=$ Fluoride concentration $\left(\mathrm{gmol} / \mathrm{kg} \mathrm{H} \mathrm{H}_{2} \mathrm{O}\right)$,

$\mathrm{T}_{\mathrm{K}} \quad=$ Temperature $(\mathrm{K})$.

Lower bounds are set for $\mathrm{C}_{\mathrm{OH}}=0.01 \mathrm{gmol} / \mathrm{kg} \mathrm{H}_{2} \mathrm{O}$ and $\mathrm{C}_{\mathrm{F}}=0.007 \mathrm{gmol} / \mathrm{kg} \mathrm{H} \mathrm{H}_{2} \mathrm{O}$. The constants for Equation 9 are given as:

\begin{tabular}{|l|r|}
\hline Constant & Value \\
\hline $\mathrm{A}$ & -5298.7 \\
\hline $\mathrm{B}$ & 15.1368 \\
\hline $\mathrm{C}$ & -1.0050 \\
\hline $\mathrm{D}$ & -0.1280 \\
\hline $\mathrm{E}$ & -0.4030 \\
\hline
\end{tabular}


The phosphate dissolution/precipitation reactions are given as:

$\mathrm{PO}_{4}^{-3}(\mathrm{~s})+3 \mathrm{Na}^{+}(\mathrm{s}) \leftrightarrow \mathrm{PO}_{4}^{-3}(\mathrm{l})+3 \mathrm{Na}^{+}(\mathrm{l})$

If the concentration of $\mathrm{PO}_{4}^{-3}(\mathrm{l})$ is below the calculated solubility, $\mathrm{PO}_{4}^{-3}(\mathrm{~s})$ and associated $\mathrm{Na}^{+}(\mathrm{s})$ dissolve until:

1) Concentration of $\mathrm{PO}_{4}^{-3}$ (l) reaches the solubility [excess $\mathrm{PO}_{4}^{-3}(\mathrm{~s})$ and $\mathrm{Na}^{+}(\mathrm{s})$ ] or

2) $\mathrm{PO}_{4}^{-3}(\mathrm{~s})$ has completely depleted $\left[\mathrm{PO}_{4}^{-3}\right.$ (s) limiting $]$

If the concentration of $\mathrm{PO}_{4}^{-3}$ (1) is above the calculated solubility, $\mathrm{PO}_{4}^{-3}(1)$ and associated $\mathrm{Na}^{+}(\mathrm{l})$ precipitate until:

3) Concentration of $\mathrm{PO}_{4}^{-3}$ (1) reaches solubility or

4) $\mathrm{PO}_{4}^{-3}(1)$ has completely depleted $\left[\mathrm{PO}_{4}^{-3}(1)\right.$ limiting]

$\mathrm{PO}_{4}^{-3}$ (l) concentration will not reach solubility when conditions 2 or 4 are encountered. Details related to the development of the phosphate solubility equation are given in 24590-WTP-M4C-V37T-00009.

\section{Aluminum Solubility}

The TFC feed vector provides the quantities of aluminum in " $\mathrm{Al}(\mathrm{OH})_{4}$-" and " $\mathrm{Al}$ " " forms in the liquid and solid phases. To support aluminum solubility modeling, the PE flowsheet engineers perform feed vector charge balance calculations in accordance with methods discussed in Appendix I. The G2 model tracks aluminum in the liquid phase as " $\mathrm{Al}(\mathrm{OH})_{4}{ }^{-"}$, in the solid phase as " $\mathrm{Al}(\mathrm{OH})_{3}$ " and " $\mathrm{Al}^{+3}$ ". Only the easy to dissolve gibbsite and soluble phase aluminate will participate in the phase equilibrium calculations. The phase equilibrium is calculated using equations from CCN 160514 or CCN 221451, depending on the vessel temperatures. It shall be noted that the solubility corrections are provided in reversible forms at each temperature. The $\mathrm{OH}=$ Function $(\mathrm{Al})$ correlation is used in unit operations where caustic addition is required, e.g., to determine the caustic volume for gibbsite dissolution. The Al $=$ Function $(\mathrm{OH})$ correlation is applied to unit operations where aluminum phase equilibrium is calculated, e.g, to dissolve gibbsite or to precipitate aluminate.

$\underline{\mathrm{CCN}} 160514\left(\right.$ Temperature $\left.=25^{\circ} \mathrm{C}\right)$

$S_{O H}=e^{*} C_{A l}+f * C_{A l}^{2}+g * C_{A l}^{3}$

$S_{A l}=\sqrt[3]{\left(j+\frac{C_{O H}}{2 g}\right)+\sqrt{\left(j+\frac{C_{O H}}{2 g}\right)^{2}+k}}+\sqrt[3]{\left(j+\frac{C_{O H}}{2 g}\right)-\sqrt{\left(j+\frac{C_{O H}}{2 g}\right)^{2}+k}}+l$

or

$$
=3.0 \text { gmol } \mathrm{Al}(\mathrm{OH})_{4}{ }^{-} / \mathrm{kg} \mathrm{H}_{2} \mathrm{O} \text { at maximum solubility }
$$

Where:

$\mathrm{C}_{\mathrm{OH}} \quad=$ Hydroxide concentration $\left(\mathrm{gmol} / \mathrm{kg} \mathrm{H}_{2} \mathrm{O}\right)$

$\mathrm{C}_{\mathrm{Al}} \quad=$ Aluminate concentration $\left(\mathrm{gmol} / \mathrm{kg} \mathrm{H} \mathrm{H}_{2} \mathrm{O}\right)$ 
$\mathrm{S}_{\mathrm{Al}} \quad=$ Aluminate solubility $\left(\mathrm{gmol} / \mathrm{kg} \mathrm{H} \mathrm{H}_{2} \mathrm{O}\right)$

$\mathrm{S}_{\mathrm{OH}} \quad=$ Hydroxide concentration when aluminate is at solubility $\left(\mathrm{gmol} / \mathrm{kg} \mathrm{H}_{2} \mathrm{O}\right)$

The constants for Eq. 10 and Eq. 11 are given as:

\begin{tabular}{|c|r|}
\hline Constant & Value \\
\hline $\mathrm{e}$ & 12.298114 \\
\hline $\mathrm{f}$ & -7.502178277 \\
\hline $\mathrm{g}$ & 1.50964729 \\
\hline $\mathrm{j}$ & -2.20180336 \\
\hline $\mathrm{k}$ & $-2.3228 \mathrm{E}-05$ \\
\hline $\mathrm{l}$ & 1.656496924 \\
\hline
\end{tabular}

$\underline{\text { CCN } 221451\left(25^{\circ} \mathrm{C}<\text { Temperature } \leq 45^{\circ} \mathrm{C}\right)}$

$$
S_{\mathrm{Al}}=e^{*} C_{\mathrm{OH}}+f * C_{\mathrm{OH}}^{2}+g * C_{\mathrm{OH}}^{3}
$$

$S_{\mathrm{OH}}=\sqrt[3]{\left(j+\frac{C_{A l}}{2 g}\right)+\sqrt{\left(j+\frac{C_{A l}}{2 g}\right)^{2}+k}}+\sqrt[3]{\left(j+\frac{C_{A l}}{2 g}\right)-\sqrt{\left(j+\frac{C_{A l}}{2 g}\right)^{2}+k}}+l$

Where:

$$
\begin{aligned}
& e=(7.07864 e-6) * T^{4}-(9.206808 e-4) * T^{3}+(4.47814 e-2) * T^{2}-(9.586194 e-1) * T+7.680834 \\
& f=(-4.823189 e-6) * T^{4}+(6.268796 e-4) * T^{3}-(3.044321 e-2) * T^{2}+(6.521685 e-1) * T-5.197387 \\
& g=(7.259612 e-7) * T^{4}-(9.410837 e-5) * T^{3}+(4.5653 e-3) * T^{2}-(9.764447 e-2) * T+7.787686 e-1 \\
& j=\frac{-f^{3}}{27 g^{3}}+\frac{f e}{6 g^{2}} \\
& k=\left(\frac{e}{3 g}-\frac{f^{2}}{9 g^{2}}\right)^{3} \\
& l=-\frac{f}{3 g} \\
& \mathrm{C}_{\mathrm{OH}} \quad=\text { Hydroxide concentration }\left(\mathrm{gmol} / \mathrm{kg} \mathrm{H} \mathrm{H}_{2} \mathrm{O}\right) \\
& \mathrm{C}_{\mathrm{Al}} \quad=\text { Aluminate concentration }(\mathrm{gmol} / \mathrm{kg} \mathrm{H} \mathrm{O}) \\
& \mathrm{S}_{\mathrm{Al}} \quad=\text { Aluminate solubility }\left(\mathrm{gmol} / \mathrm{kg} \mathrm{H} \mathrm{H}_{2} \mathrm{O}\right) \\
& \mathrm{S}_{\mathrm{OH}} \quad=\text { Hydroxide concentration when aluminate is at solubility }\left(\mathrm{gmol} / \mathrm{kg} \mathrm{H} \mathrm{H}_{2} \mathrm{O}\right) \\
& \mathrm{T}_{\mathrm{T}} \quad=\text { Vessel temperature }\left({ }^{\circ} \mathrm{C}\right)
\end{aligned}
$$

Aluminum will precipitate/dissolve in accordance with the following reversible reactions

$$
\mathrm{Al}(\mathrm{OH})_{4}{ }^{-}(\mathrm{l}) \leftrightarrow \mathrm{Al}(\mathrm{OH})_{3}(\mathrm{~s})+\mathrm{OH}^{-}(\mathrm{l})
$$


Details related to the development of the aluminum solubility correlations can be found in CCN 160514 and CCN 221451.

\subsubsection{Assumptions}

Assumptions related to solubility calculations and phase equilibrium are summarized as follows:

1) Oxalate exists in the forms of liquid and solid $\mathrm{C}_{2} \mathrm{O}_{4}{ }^{-2}$ and phase equilibrium is reached between the two. Phase change reactions in the model ensure that one gram mole of solid $\mathrm{C}_{2} \mathrm{O}_{4}{ }^{-2}$ is associated with two gram moles of solid $\mathrm{Na}^{+}$.

2) Phosphate is partitioned into "soft" $\mathrm{PO}_{4}^{-3}$ and "hard" $\mathrm{PO}_{4}{ }^{-3}(\mathrm{HARD}) . \mathrm{PO}_{4}{ }^{-3}$ exists in both liquid and solid phases but $\mathrm{PO}_{4}^{-3}(\mathrm{HARD})$ only exists in the solid phase. Phase equilibrium is reached between liquid $\mathrm{PO}_{4}{ }^{-3}$ and solid $\mathrm{PO}_{4}{ }^{-3}$. The model ensures that one gram mole of solid $\mathrm{PO}_{4}^{-3}$ is associated with three gram moles of solid $\mathrm{Na}^{+}$.

3) Aluminum exists in the liquid phase as $\mathrm{Al}(\mathrm{OH})_{4}{ }^{-}$, and in the solid phase as $\mathrm{Al}(\mathrm{OH})_{3}$ and $\mathrm{Al}^{+3}$. Phase equilibrium is reached between $\mathrm{Al}(\mathrm{OH})_{4}{ }^{-}$and $\mathrm{Al}(\mathrm{OH})_{3}$.

4) Solid $\mathrm{C}_{2} \mathrm{O}_{4}{ }^{-2}, \mathrm{PO}_{4}{ }^{-3}$, and $\mathrm{Al}(\mathrm{OH})_{3}$ can dissolve in unsaturated solutions at ambient temperature without first being leached.

5) Phase equilibrium is reached instantaneously in unit operations where solubility is applied, e.g., kinetics are not considered.

\subsubsection{Applications}

Solubility is calculated in the model at selected locations where phase equilibrium will impact process operation decisions. The goal is to obtain sufficient resolution in phase equilibrium chemistry while still maintaining a reasonable computation time. Ideally, solubility would be applied continuously to all WTP equipment, but that would prolong the model run time to unacceptable levels.

Generally solubility is calculated in correspondence to batch operations, for example, phase equilibrium is applied after a vessel is filled and before its contents can be discharged. However there are exceptions in some vessels where phase equilibrium is applied multiple times in a batch due to various process operations. There are also exceptions in the evaporators where phase equilibrium is applied continuously as the control for wt\% solids in evaporator concentrates is important. It shall be noted that solubility is not applied to the Vitrification Facility equipment since these operations are not dependent on the phases of the soluble constituents.

Table 6 provides a summary of the equipment where solubility is applied, the temperatures when solubility is applied, and the logic for the solubility application. 
Table $6 \quad$ Solubility Applications

\begin{tabular}{|l|l|l|}
\hline Equipment & $\left.\mathbf{T} \mathbf{~}^{\mathbf{0}} \mathbf{C}\right)$ & Logic \\
\hline FRP-VSL-00002A/B/C/D & 25 & After filled and before sampling \\
\hline HLP-VSL-00022 & 25 & After filled and before sampling \\
\hline FEP-VSL-00017A/B & 25 & After filled and before discharge \\
\hline FEP-SEP-00001A & 42 & Continuous during boil-off \\
\hline UFP-VSL-00001A/B & 45 & After pre-heating steam condensate addition and before discharge \\
\hline UFP-VSL-00002A/B & 45 & $\begin{array}{l}\text { 1) At the peak of saw-tooth operation during solids concentration, } \\
\text { e.g., when a UFP-VSL-00002 vessel is filled to the upper set } \\
\text { volume }\end{array}$ \\
& & $\begin{array}{l}\text { 2) At the end of solids concentration } \\
\text { 3) After caustic leach } \\
\text { 4) At the peak of saw-tooth operation during solid wash } \\
\text { 5) At the peak of saw-tooth operation during oxidative wash }\end{array}$ \\
\hline Super Tank & 45 & Continuous when pumping (actual operating temperature $\left.=50^{\circ} \mathrm{C}\right)$ \\
\hline CXP-VSL-00026A/B/C & 45 & After filled and before sampling \\
\hline TLP-SEP-00001 & 50 & Continuous during boil-off \\
\hline TCP-VSL-00001 & 31 & Continuous when pumping \\
\hline PWD-VSL-00015/16 & 25 & After filled and pH adjustment \\
\hline PWD-VSL-00044 & 25 & After filled and pH adjustment \\
\hline HLP-VSL-00027A/B & 25 & After filled and before discharge \\
\hline HLP-VSL-00028 & 25 & After filled and before sampling \\
\hline
\end{tabular}

\subsubsection{Antifoaming Agent (AFA)}

Antifoaming agent is added to the FEP and Treated LAW Evaporation Process (TLP) evaporators to minimize foaming during evaporator campaigns. To prevent foaming due to spargers, AFA is also added to non-Newtonian vessels in the UFP and HLW Lag Storage and Feed Blending Process (HLP) systems. AFA is composed of $50 \mathrm{wt} \%$ of Polypropylene Glycol (PPG) and $50 \mathrm{wt} \%$ of Polydimethylsiloxane (PDMS), however, AFA is always added as a mixture of $20 \mathrm{wt} \%$ AFA and $80 \mathrm{wt} \%$ water. Table 7 shows the makeup compositions of AFA used in the G2 model.

Table $7 \quad$ AFA Makeup Compositions

\begin{tabular}{|c|c|c|c|}
\hline Liquid Component & $\begin{array}{c}\text { Molecular Weight } \\
\text { (g/gmol) }\end{array}$ & $\begin{array}{c}\text { Mole Fraction } \\
\text { (gmol/gmol) }\end{array}$ & $\begin{array}{c}\text { Liquid Molarity } \\
\text { (gmol/liter) }\end{array}$ \\
\hline H2O & 18.015 & 0.999292769 & 54.119 \\
\hline PPG & 3,503 & $6.42937 \mathrm{e}-4$ & 0.034 \\
\hline PDMS & 35,015 & $6.42937 \mathrm{e}-5$ & 0.003 \\
\hline
\end{tabular}

Assumptions and simplifications made for AFA modeling are as follows:

1) Heating and irradiation will cause the AFA to decompose (become ineffective in foam control). Decomposition products of PPG and PDMS are tracked in the model by Decomposed Polypropylene Glycol (DPPG) and Decomposed Polydimethylsiloxane (DPDMS), respectively.

2) Only DPPG and DPMS are present in recycle streams as residence times in the recycle loops have caused the AFA to decompose completely.

3) AFA aging are not considered, for example, residence time will not impact antifoam properties and decay rate is constant. In other words, fresh AFA and aged AFA have the same properties. 
4) PPG and DPPG exhibit high water solubilities and are assumed to exist in the dissolved phase.

5) PDMS and DPDMS are hydrophobic and therefore exist in the suspended phase, for example, as nonaqueous phase liquids (NAPLs). As these NAPLs tend to bind with solids in the wastes, it is assumed that PDMS and DPDMS follow solids in the plant and will contribute to wt $\%$ solids calculations.

6) AFA and decomposed products are uniformly distributed in WTP equipment as mixing is assumed to be ideal.

7) The small quantities of silica in AFA and their decomposed products will not impact glass formulation.

AFA can be added simultaneously to any evaporator that is operating, however, it can only be added to a non-Newtonian vessel at a time. Target concentrations are $500 \mathrm{ppm}$ (250 ppm PPG and $250 \mathrm{ppm}$ PDMS) for the evaporators and $350 \mathrm{ppm}$ (175 ppm PPG and $175 \mathrm{ppm}$ PDMS) for the non-Newtonian vessels. AFA addition is not needed if existing concentrations are equal to or greater than the target values. It shall be noted that concentrations of PPG and PDMS may be different in the same equipment due to liquid and solid partitioning of the AFA and by the mixing and separation operations. AFA is added in accordance with the operating logic in Table 8.

Table 8 AFA Addition Operating Logic

\begin{tabular}{|c|c|}
\hline Receiving Equipment & Addition Logic \\
\hline FEP-SEP-00001A/B & $\begin{array}{l}\text { - In the beginning of a new batch, and } \\
\text { - Every 1-hour after. }\end{array}$ \\
\hline UFP-VSL-00001A/B & $\begin{array}{l}\text { - At the end of feed blending, and } \\
\text { - Every 6-hours during pre-heating and discharging. }\end{array}$ \\
\hline UFP-VSL-00002A/B & $\begin{array}{l}\text { - In the beginning of a concentration cycle, } \\
\text { - Every 6-hours during solid concentration, heating, leaching, cooling, and } \\
\text { washing, } \\
\text { - AFA will not be added during oxidative leach, } \\
\text { - In the beginning of oxidative wash, } \\
\text { - Every 6-hours during oxidative wash and solid discharge. }\end{array}$ \\
\hline HLP-VSL-00027A/B & - Every 6-hours after initially filled to above the heel volume. \\
\hline HLP-VSL-00028 & - Every 6-hours after initially filled to above the heel volume. \\
\hline TLP-SEP-00001 & $\begin{array}{l}\text { - In the beginning of a new batch, and } \\
\text { - Every 1-hour after. }\end{array}$ \\
\hline
\end{tabular}

AFA decomposition occur in the evaporators and vessels once every hour. Decomposition reactions are given as:

PPG (1) $\rightarrow$ DPPG (1) $\quad$ CF $=0.009255 \mathrm{wt} \% /$ hour

PDMS (1) $\rightarrow$ PDMS (s) $\quad$ CF $=100 \mathrm{wt} \%$ (after every AFA addition)

PDMS (s) $\rightarrow$ DPDMS (s) $\quad$ CF $=0.009255$ wt $\%$ /hour

$C F=1-\left(\frac{\text { end mass }}{\text { current mass }}\right)^{1 / n}$ 
Where:

$$
\begin{array}{ll}
\text { end mass } & =80 \mathrm{wt} \%, \\
\text { current mass } & =100 \mathrm{wt} \%, \\
\mathrm{n} & =24 \text { hours }
\end{array}
$$

All the PPG and PDMS will be decomposed during oxidative leaching in the presence of sodium permanganate. Also, all the PPG and PDMS will be converted to DPPG and DPDMS, respectively, in FEP-VSL-00005A/B, TLP-VSL-00002, and PWD-VSL-00015, PWD-VSL-00016, and PWD-VSL-00044 to simulate the decomposition due to long residence times. This is a conservative assumption that ensures only decomposed products exist in the Pretreatment recycles. Split fractions for the AFA and decomposed products to FEP/TLP evaporator overheads are set to 1.00e-8. Split factions of the AFA and decomposed products to LAW and HLW melter overheads are set to 0.0 , for example, $100 \%$ will be combusted according to the reactions below:

$$
\begin{aligned}
& P P G+240 \mathrm{O}_{2}(g) \rightarrow 180 \mathrm{CO}_{2}(g)+181 \mathrm{H}_{2} \mathrm{O}(g) \\
& P D M S+1892 \mathrm{O}_{2}(g) \rightarrow 946 \mathrm{CO}_{2}(g)+1419 \mathrm{H}_{2} \mathrm{O}(g)+472 \mathrm{SiO}_{2}(\mathrm{o}) \\
& D P P G+240 \mathrm{O}_{2}(g) \rightarrow 180 \mathrm{CO}_{2}(g)+181 \mathrm{H}_{2} \mathrm{O}(g) \\
& D P D M S+1892 \mathrm{O}_{2}(g) \rightarrow 946 \mathrm{CO}_{2}(g)+1419 \mathrm{H}_{2} \mathrm{O}(g)+472 \mathrm{SiO}_{2}(\mathrm{o})
\end{aligned}
$$

\subsubsection{Hydrogen Generation}

Hydrogen generation rates (HGRs) can be calculated for the vessels and evaporator shown in Table 9. To reduce the amount of computations, only the worst case conditions are used for the calculations, for example, HGRs are determined for the vessels when they are filled to the upper operating volumes, for UFP-VSL-00002A/B at the end of solid concentration, and for CNP-EVAP-00001 at its highest concentration just before it can be discharged. Maximum operating temperatures are also applied. A switch is provided in the model that turns the HGR calculation feature on and off.

Table 9 Maximum Temperatures for HGR Calculations

\begin{tabular}{|l|c|}
\hline Equipment Name & $\begin{array}{c}\text { Maximum Operating Temperature } \\
\left({ }^{(} \mathbf{F}\right)\end{array}$ \\
\hline FRP-VSL-00002A/B/C/D & 120 \\
\hline HLP-VSL-00022 & 150 \\
\hline FEP-VSL-00017A/B & 122 \\
\hline UFP-VSL-00001A/B & 194 \\
\hline UFP-VSL-00002A/B & 194 \\
\hline CNP-EVAP-00001 & 140 \\
\hline CNP-VSL-00003 & 190 \\
\hline RDP-VSL-00002A/B/C & 123 \\
\hline PWD-VSL-00033/43 & 141 \\
\hline LFP-VSL-00001/2/3/4 & 140 \\
\hline HLP-VSL-00027A/B & 138 \\
\hline
\end{tabular}




\begin{tabular}{|l|c|}
\hline Equipment Name & $\begin{array}{c}\text { Maximum Operating Temperature } \\
\left({ }^{\circ} \mathbf{F}\right)\end{array}$ \\
\hline HLP-VSL-00028 & 141 \\
\hline HFP-VSL-0001/5/2/6 & 140 \\
\hline HOP-SCB-00001/2 & 140 \\
\hline RLD-VSL-00005 & 166 \\
\hline
\end{tabular}

Steps for the HGR calculations are described as follows.

$\mathrm{TOC}=100 \times \sum\left(\mathrm{C}_{\mathrm{i}} \times \mathrm{LOM}_{\mathrm{i}} / \mathrm{MW}_{\mathrm{i})} / \mathrm{LTM}\right.$

Where:

TOC $=$ Liquid weight percent Total Organic Carbon (dimensionless)

$\mathrm{C}_{\mathrm{i}} \quad=$ Constant of a hydrogen contributing organic $(\mathrm{kg} / \mathrm{kg}-\mathrm{mole})$

$\mathrm{LOM}_{\mathrm{i}} \quad=$ Liquid mass of a hydrogen contributing organic $(\mathrm{kg})$

$\mathrm{MW}_{\mathrm{i}} \quad=$ Molecular weight of a hydrogen contributing organic $(\mathrm{kg} / \mathrm{kg}-\mathrm{mole})$

LTM $\quad=$ Liquid total mass $(\mathrm{kg})$

The constants and molecular weights of the hydrogen contributing organics are presented in Table 10. Isodecyl Alcohol is excluded from Eq. 15 as this compound is not tracked in the G2 model and its quantity is not provided by the Tank Farms. Sugar will only be considered in the Melter Feed Preparation Vessels (MFPV) and Melter Feed Vessels (MFV).

Table $10 \quad$ Hydrogen Contributing Organics and Constants

\begin{tabular}{|l|l|c|c|l|}
\hline Name of Organics & Formula of Organics & $\mathbf{C}_{\mathbf{i}}$ & $\mathbf{M W}_{\mathbf{i}}$ & Applicable Equipment \\
\hline Oxalate & C2O4-2 & 24 & 88.02 & All \\
\hline Formate & CHO2- & 12 & 45.018 & All \\
\hline Acetate & C2H3O2- & 24 & 59.044 & All \\
\hline Glycolate & C2H3O3- & 24 & 75.044 & All \\
\hline Citrate & C6H5O7-3 & 72 & 189.101 & All \\
\hline HEDTA & C10H15N2O7-3 & 120 & 275.238 & All \\
\hline EDTA & C10H12N2O8-4 & 120 & 288.213 & All \\
\hline Sugar & C12H22O11 & 144 & 342.299 & MFPVs and MFVs only \\
\hline $\begin{array}{l}\text { Polypropylene } \\
\text { Glycol }\end{array}$ & PPG & 2,160 & 3,503 & All \\
\hline $\begin{array}{l}\text { Decomposed } \\
\text { Polypropylene } \\
\text { Glycol }\end{array}$ & DPPG & 2,160 & 3,503 & All \\
\hline
\end{tabular}




$$
\begin{aligned}
& G H 2 O \alpha=\frac{1.05}{(1+2.4 *[N O 3-]+0.63 *[N O 2-])}+\frac{0.35}{(1+3900 *[N O 3-]+1400 *[N O 2-])} \\
& G H 2 O \beta \gamma=\frac{0.34}{(1+2.4 *[N O 3-]+0.62 *[N O 2-])}+\frac{0.11}{(1+120 *[N O 3-]+43 *[N O 2-])}
\end{aligned}
$$

Where:

[NO3- $\quad=$ Nitrate molarity $(\mathrm{gmol} / \mathrm{L})$

[NO2-] = Nitrite molarity $(\mathrm{gmol} / \mathrm{L})$

$$
\begin{aligned}
& \text { GTOC } \alpha=0.5 * a_{0} * f * \text { TOC } * \exp \left[\frac{-Q_{\text {rad }}}{R * T}\right] \\
& \text { GTOC } \beta \gamma=a_{0} * f * \text { TOC } * \exp \left[\frac{-Q_{r a d}}{R * T}\right]
\end{aligned}
$$

Where:

$$
\begin{aligned}
& a_{0} \quad=2.49 \times 10^{6} \text { (dimensionless) } \\
& \mathrm{f} \quad=0.7 \text { (dimensionless) } \\
& \mathrm{Q}_{\mathrm{rad}} \quad=44,300(\mathrm{~J} / \mathrm{mole}) \\
& \mathrm{R} \quad=8.314(\mathrm{~J} / \mathrm{mole}-\mathrm{K}) \\
& \mathrm{T}=\text { Maximum operating temperature }(\mathrm{K})
\end{aligned}
$$

Specific activities and decay heat generation rates for the alpha emitting radionuclides are shown in Table 11 and the values for the beta and gamma emitting radionuclides are given in Table 12. These property values are used in Eq. 20 and Eq. 21 for decay heat calculations.

Table 11 Specific Activities and Decay Heat Rate of Alpha Emitting Radionuclides

\begin{tabular}{|c|c|c|}
\hline $\begin{array}{c}\text { Alpha Emitting } \\
\text { Radionuclides }\end{array}$ & $\begin{array}{c}\text { Specific Activity } \\
\text { (Ci/g) }\end{array}$ & $\begin{array}{c}\text { Decay Heat Rate } \\
\text { (W/Ci) }\end{array}$ \\
\hline $233-\mathrm{U}$ & 0.00968 & 0.0286 \\
\hline $238-\mathrm{Pu}$ & 17.1 & 0.0326 \\
\hline $239-\mathrm{Pu}$ & 0.0622 & 0.0302 \\
\hline $240-\mathrm{Pu}$ & 0.228 & 0.0306 \\
\hline $241-\mathrm{Am}$ & 3.43 & 0.0328 \\
\hline $244-\mathrm{Cm}$ & 80.9 & 0.0344 \\
\hline
\end{tabular}


Table 12 Specific Activities and Decay Heat Rates of Beta/Gamma Emitting Radionuclides

\begin{tabular}{|c|c|c|}
\hline $\begin{array}{c}\text { Beta/Gamma Emitting } \\
\text { Radionuclides }\end{array}$ & $\begin{array}{c}\text { Specific Activity } \\
\text { (Ci/g) }\end{array}$ & $\begin{array}{c}\text { Decay Heat Rate } \\
\text { (W/Ci) }\end{array}$ \\
\hline $60-\mathrm{Co}$ & 1,130 & 0.0154 \\
\hline $90-\mathrm{Sr}$ & 136 & -- \\
\hline $90-\mathrm{Sr} / 90-\mathrm{Y}$ & -- & 0.0067 \\
\hline $99-\mathrm{Tc}$ & 0.0169 & 0.000501 \\
\hline $137-\mathrm{Cs}$ & 86.7 & -- \\
\hline $137-\mathrm{Cs} / 137 \mathrm{~m}-\mathrm{Ba}$ & -- & 0.00495 \\
\hline $154-\mathrm{Eu}$ & 270 & 0.00908 \\
\hline $235-\mathrm{U}$ & $2.16 \mathrm{e}-6$ & 0.0271 \\
\hline
\end{tabular}

Decay heat rates for $90-\mathrm{Sr} / 90-\mathrm{Y}$ and $137-\mathrm{Cs} / 137 \mathrm{~m}-\mathrm{Ba}$ include those decay products of $90-\mathrm{Y}$ and $137 \mathrm{~m}-$ Ba.

$$
\begin{aligned}
& \text { decayHeat } \alpha=\sum\left(L S G_{i} * A_{i} * H_{i}\right) \\
& \text { decayHeat } \beta \gamma=\sum\left(L S G_{j} * A_{j} * H_{j}\right)
\end{aligned}
$$

Where:

decayHeat $\alpha=$ Decay heat rate from alpha radiation (W/kg of waste)

$\mathrm{LSG}_{\mathrm{i}} \quad=$ Grams of a liquid and solid alpha emitting radionuclide in a kilogram of waste $(\mathrm{g})$

$\mathrm{A}_{\mathrm{i}} \quad=$ Specific activity of an alpha emitting radionuclide $(\mathrm{Ci} / \mathrm{g})$

$\mathrm{H}_{\mathrm{i}} \quad$ = Decay heat rate of an alpha emitting radionuclide $(\mathrm{W} / \mathrm{Ci})$

decayHeat $\beta \gamma=$ Decay heat rate from beta/gamma radiations (W/kg of waste)

$\mathrm{LSG}_{\mathrm{j}} \quad=$ Grams of a liquid/solid beta/gamma emitting radionuclide in a kilogram of waste $(\mathrm{g})$

$\mathrm{A}_{\mathrm{j}} \quad=$ Specific activity of an a beta/gamma emitting radionuclide $(\mathrm{Ci} / \mathrm{g})$

$\mathrm{H}_{\mathrm{j}} \quad$ = Decay heat rate of a beta/gamma emitting radionuclide $(\mathrm{W} / \mathrm{Ci})$

$$
H G R_{\text {rad }}=f_{1} * F *(\text { decayHeat } \alpha *(G H 2 O \alpha+G T O C \alpha)+\text { decayHeat } \beta \gamma *(G H 2 O \beta \gamma+G T O C \beta \gamma))
$$

Where:

$$
\begin{array}{ll}
\mathrm{HGR}_{\mathrm{rad}} & =\mathrm{HGR} \text { by radiolysis (moles of } \mathrm{H}_{2} / \text { day } / \mathrm{kg} \text { waste), note: waste includes liquid and solid } \\
\mathrm{f}_{1} & =\text { Liquid waste weight fraction (dimensionless) } \\
\mathrm{F} & =0.00895 \text { (dimensionless). }
\end{array}
$$

The weight percent aluminate is calculated as follows:

$$
\text { wpctAl }=\frac{100 *\left(\frac{27 * \text { LMaloh4 }}{\text { MWaloh4 }}\right)}{\text { LTM }}
$$

Where: 
wpctAl = Weight percent of aluminate (dimensionless)

LMaloh4 = Liquid mass of aluminate $(\mathrm{kg})$

MWaloh4 = Molecular weight of aluminate $(\mathrm{kg} / \mathrm{kg}-\mathrm{mole})$

$H G R_{\text {therm }}=a T * f * T O C *(w p c t A l)^{0.4} * f_{1} * \exp \left(\frac{-Q_{\text {therm }}}{R * T}\right)$

Where:

$\mathrm{HGR}_{\text {therm }}=$ HGR by thermolysis (moles $\mathrm{H}_{2} /$ day $/ \mathrm{kg}$ waste)

aT $\quad=2.76 \times 10^{9}$ (dimensionless)

$\mathrm{Q}_{\text {therm }} \quad=89,300(\mathrm{~J} / \mathrm{mole})$

$H G R=H G R_{\text {rad }}+H G R_{\text {therm }}$

Where:

HGR = Total hydrogen generation rate (moles $\mathrm{H}_{2} /$ day $/ \mathrm{kg}$ waste)

Hydrogen generation rates are converted to liters of $\mathrm{H}_{2}$ per hour per tank at maximum temperature at $1 \mathrm{~atm}$ when data are recorded.

\subsubsection{End of Mission Waste Handling}

The common operating rules described in Section 4.7.2 are applied to the startup and operation phases of the mission. The pumping logic triggers a transfer when volume of an upstream vessel reaches above the vessel's upper set point. However, pumping will not start if the upper set volume is not reached at the end of mission when continuous feeds from the Tank Farms become unavailable. This results in some residual volumes of wastes (defined as the remaining waste above the heel and below the upper set point) in various vessels that could not be processed by the melters. The end of mission waste handling logic is implemented to force out these residual wastes sequentially downstream so that they can be processed. This ensures that canister counts from different scenario runs can be compared on equal basis.

The G2 implementation for the end of mission waste handling logic is described below:

1) The end of mission waste handling logic starts after the last batches of LAW and HLW feeds have left the Waste Feed Receipt Process (FRP) system and HLP-VSL-00022, respectively.

2) WTP line flushes are terminated (because they place waters in already empty vessels).

3) The 24-hour discharge cycle at PVP-SCB-00002 is stopped (because it generates large volume of recycle).

4) The water flow to PVP-HEME-00001 is discontinued (because it generates large volume of recycle).

5) Wait until the FEP evaporators have processed the original recycles in FEP-VSL-00017A/B, for example, these vessels are emptied and can be used for new recycles. Note: original recycles are generated during normal operations and new recycles are generated from residual waste processing.

6) Residual wastes are forced out of the HLP-VSL-00022 and FRP vessels until heel volumes are reached. 
7) Residual wastes are forced out of UFP-VSL-00001A/B to UFP-VSL-00002A/B where the wastes are treated according to normal process procedures, such as solids concentration, wash, oxidative leach and wash, solid discharge, and filter cleaning. UFP2 solid treatments will begin after all the UFP1 residual wastes have been processed and regardless if the solid concentration target ( $20 \mathrm{wt} \%$ solids) is achieved. Wash water is recycled (new recycles) to PWD.

8) Two sub- end of mission processes start simultaneously after the UFP2 vessels are clear of wastes, one process is to empty the vessels on the path to HLW Melter Process (HMP) and the other is to clear the vessels on the path to LAW Melter Process (LMP).

9) For the HLW sub-process, wait until HLP-VSL-00028 becomes ready to receive, empty residual wastes in HLP-VSL-00027A/B.

10) Sample and empty residual wastes in HLP-VSL-00028.

11) Operate HLW Melter Feed Preparation Vessels as normal regardless if set volume can be achieved for the last batch.

12) Empty HLW MFPVs and MFVs completely, shut down the HMP and HLW Melter Offgas Treatment Process (HOP) processes. Before HMP and HOP are shut down, new recycles from HOP and HLW Canister Decontamination Handling (HDH) are routed to PWD through RLD-VSL-00007 according to normal operation logic.

13) For the LAW sub-process, empty the residual wastes from vessels in the following order: Super Tank, CXP-VSL-00026A/B/C, TCP-VSL-00001, LCP-VSL-00001/2, LFP-VSL-00001/3, and LFP-VSL$00002 / 4$. Operate the LAW MFPVs as normal regardless if set volume can be achieved for the last batch.

14) Shut down LMP, LAW Melter Offgas Treatment Process (LOP), and LAW Secondary Offgas/Vessel Process (LVP) processes, and before that new recycles from LOP and LVP are routed to TLP according to normal operation logic.

15) Shut down FEP evaporators and store new recycles in the FEP-VSL-00017A/B vessels to prevent filling the already emptied UFP vessels. The volumes of new recycles will be recorded.

16) Shut down the entire WTP process when both HLW Melter Feed Process (HFP) and LAW Melter Feed Process (LFP) are clear of wastes.

Volumes in the key Pretreatment, LAW Facility, and HLW Facility vessels will be recorded before and after the end of mission logic is executed. It shall be noted that the end of mission waste handling logic described above may not represent the actual operations which will be determined by the WTP operations and maintenance contractor.

\subsubsection{Heel Management}

Heel management operations are performed in selected Pulse Jet Mixer (PJM) vessels to address concerns of solids accumulation in stationary heels due to inadequate mixing. Two types of heel management operations can be performed including: a) heel dilution, and b) heel cleanout. Heel dilution is a normal operation that is performed in Newtonian vessels at regular time intervals, while heel cleanout is an offnormal operation that is performed in non-Newtonian vessels as needed. For modeling purpose, heel cleanout is also performed at regular time intervals in G2. Both heel dilution and heel cleanout involve three steps: a) dilution, b) fluidization, and c) removal. Removal is carried out with a portable pump that is able to discharge to lower heel levels. The portable pump has a $50 \mathrm{gpm}$ capacity. General rules of the heel management operations are discussed as follows. 


\section{$\underline{\text { Heel Dilution }}$}

1) Continue normal operations until a scheduled date for heel dilution is reached

2) Discharge wastes with the vessel's fixed pump until the fixed pump heel level is reached

3) Configure jumpers/hoses and install a portable pump in parallel with Steps 1 and 2, preparation time is not added

4) Continue to discharge wastes until the portable pump heel level is reached

5) Fill vessel to PJM operation level with dilution fluid such as LAW feed from FRP-VSL$00002 \mathrm{~A} / \mathrm{B} / \mathrm{C} / \mathrm{D}$, process condensate, or DI water in priority order

6) Fluidize vessel contents for 1 hour

7) Discharge fluidized contents to portable pump heel level

8) Flush jumpers/hoses with inhibited water

9) Reconfigure jumpers/hoses and uninstall the portable pump, holdup time is not added

\section{$\underline{\text { Heel Cleanout }}$}

1) Continue normal operations until a scheduled date for heel cleanout is reached

2) Discharge wastes with the vessel's fixed pump until the fixed pump heel level is reached

3) Configure jumpers/hoses and install a portable pump, the preparation process takes 8 hours

4) Continue to discharge wastes until the portable pump heel level is reached

5) Fill vessel to PJM operation level with cleanout fluid, e.g., concentrated solids from upstream vessel(s) originated from UFP-VSL-00002A/B

6) Fluidize vessel contents for 1 hour

7) Discharge fluidized contents to portable pump heel level

8) Flush jumpers/hoses with inhibited water

9) Reconfigure jumpers/hoses and uninstall the portable pump, the process takes 8 hours

Table 13 provides the specifics for the heel management operations. The logic for triggering the heel management operations are discussed as appropriate in sub-sections under Section 4.8.

\subsubsection{Fate of Mercury}

Mercury undergoes complicate transformations in various WTP processes with reduction, oxidation, vaporization, and condensation reactions (24590-WTP-RPT-PR-01-011). For simplicity, mercury is tracked as " $\mathrm{Hg}^{+2}$ " in liquid and solid. A gaseous state mercury " $\mathrm{Hg}$ " is also used for material balance in the offgas systems. Systems that require special treatments for the fate of mercury modeling include the LMP, LOP, TLP, HMP, HOP, and PWD. Refer to these system sub-sections under Section 4.8 for details. 
Table 13 Heel Management Operation Specifics

\begin{tabular}{|c|c|c|c|c|c|c|c|}
\hline \multirow[t]{2}{*}{ Vessel } & \multirow{2}{*}{$\begin{array}{c}\text { Management } \\
\text { Type }\end{array}$} & \multirow{2}{*}{$\begin{array}{c}\text { Frequency } \\
\text { (times/yr) }\end{array}$} & \multirow{2}{*}{$\begin{array}{c}\text { PJM Operating } \\
\text { Level (gal) } \\
\end{array}$} & \multicolumn{2}{|c|}{ Pump Heel (gal) } & \multirow{2}{*}{$\begin{array}{c}\text { Lineflush } \\
\text { Volume (gal) }\end{array}$} & \multirow[t]{2}{*}{ Receipt Vessel } \\
\hline & & & & Fix & Portable & & \\
\hline HLP-VSL-00022 & Dilution & 1 & 18,478 & 29,486 & 1,144 & 210 & UFP-VSL-00001B \\
\hline FEP-VSL-00017A & Dilution & 1 & 5,720 & 5,692 & 1,817 & $\mathrm{n} / \mathrm{a}$ & FEP-SEP-00001A \\
\hline FEP-VSL-00017B & Dilution & 1 & 5,720 & 5,692 & 1,817 & $\mathrm{n} / \mathrm{a}$ & FEP-SEP-00001A \\
\hline UFP-VSL-00001A & Dilution & 2 & 5,757 & 7,704 & 1,191 & 150 & UFP-VSL-00002A \\
\hline UFP-VSL-00001B & Dilution & 2 & 5,757 & 7,704 & 1,191 & 150 & UFP-VSL-00002B \\
\hline HLP-VSL-00027A & Cleanout & 2 & $10,642-20,000^{\mathrm{a})}$ & 10,600 & 773 & 165 & HLP-VSL-00028 \\
\hline HLP-VSL-00027B & Cleanout & 2 & $10,642-20,000^{a)}$ & 10,600 & 773 & 165 & HLP-VSL-00028 \\
\hline HLP-VSL-00028 & Cleanout & 2 & $11,556-20,000$ & $27,000^{b)}$ & $770^{\mathrm{b})}$ & 165 & HFP-VSL-00001/5 \\
\hline
\end{tabular}

a) Desired volume range for PJM operation, however, the upper bound can be exceeded so that an entire UFP2 solid discharge batch can be received.

b) The lower bound may not be reached due to a restriction of not sending partial batches to the HLW Melter Feed Prep Vessels. 


\subsection{System Specific Operating Logic}

The following sections provide a system-by-system description of the operating logic used in the Dynamic (G2) Model. Graphical representations of the systems are found in Appendix F and can be used in conjunction with the reading for a better understanding of the model layout. All reactions provided in the descriptions occur in the order in which they are presented.

It is noted that Version 7.0 of the Dynamic (G2) Model is configured to perform: a) the baseline UFP2 leaching with gibbsite, or b) the optional UFP2 leaching with boehmite, or c) the optional UFP1 leaching with gibbsite flowsheet operations. The optional UFP2 leaching with boehmite flowsheet differs from the baseline flowsheet in how caustic leach reactions are performed, the optional UFP1 leaching flowsheet differs from the baseline flowsheet primarily in vessels where caustic leach reactions are performed, but also in feed blending, recycle handling, and solid processes. Operating logic and parameters applicable to the optional flowsheets are discussed in Appendices L and M.

\subsubsection{Waste Feed Receipt Process (FRP) System}

\begin{tabular}{|l|l|}
\hline Vessel Tag \# & Equipment Description \\
\hline FRP-VSL-00002A & Waste Feed Receipt Vessel \\
\hline FRP-VSL-00002B & Waste Feed Receipt Vessel \\
\hline FRP-VSL-00002C & Waste Feed Receipt Vessel \\
\hline FRP-VSL-00002D & Waste Feed Receipt Vessel \\
\hline
\end{tabular}

The Waste Feed Receipt Vessels receive batch transfers of LAW feed and associated pre-transfer and post-transfer line flushes of inhibited water from the Tank Farm. These vessels also receive line flush water from various operations shown in Table H-1. In general, the transfer lines are flushed after each use and the flush water goes to not only the pumping and receiving vessels, but also other FRP and FEP vessels as the result of piping configurations. Transfers from HLP-VSL-00022 to UFP-VSL-00001A/B will also lead to significant volume of flush water to the FRP-VSL-00002A vessel. Headspace, which is defined as the remaining usable volume after the vessel is filled with slurry wastes, is reserved in each vessel for the line flush water. No pumps can fill the vessels to above their maximum volumes.

LAW batches from the Tank Farm greater than 1 million gallons are commonly seen. Also, LAW feeds are generally high in sodium concentration and low in wt \% solids. Several rules that control the acceptance of batches into the plant exist in this system. The rules are narrated as follows:

1) At least three of the four Waste Feed Receipt Vessels must be empty before a new LAW batch can be received. This ensures that FRP is able to receive 1 million gallons of LAW feed, per the WTP contract requirement.

2) An input parameter is defined in the model that specifies the number of vessels that must be reserved as empty after a LAW batch has been received. The default for this parameter is zero.

3) Batches can be delivered sequentially or non-sequentially. Sequential delivery means that the LAW and HLW batches are delivered strictly in order based on the feed sequence in the feed vector. Non-sequential delivery means that if the plant needs a batch of LAW, then a batch of LAW can be delivered ahead of a batch of HLW that has not been delivered. The same logic applies to HLW batches. There is one restriction to non-sequential delivery: HLW batches are not allowed to get X number of days ahead of the last LAW batch that is processed. This is necessary to prevent the plant from processing HLW years ahead of schedule. This restriction is ignored by default as the value for $\mathrm{X}$ is set to 9999 days. 
4) Another rule/feature implemented in this system is batch delay. Each batch in the feed vector is associated with a forecasted delivery date by the Tank Farm. When batch delay is selected, a batch cannot be processed until the Tank Farm delivery date is reached. The application of this feature is set to false by default.

5) The first LAW batch cannot be received until the model reaches the initial LAW delivery date. The initial LAW delivery date can be changed based on the desired scenario.

6) The first HLW batch cannot be received until the model reaches the initial HLW delivery date. The initial HLW delivery date can be changed based on the desired scenario.

A vessel receives feed until the tank has reached its set volume or the full batch has been received from the Tank Farm. Separate batches will not mix in the same vessel except for the residual material remaining in the heel.

Oxalate, phosphate, and aluminum solubility are applied at $25{ }^{\circ} \mathrm{C}$ after the transfer is complete. Then the vessel is sampled and held for the prescribed hold time. When the hold time has expired, the vessel becomes available to be emptied to UFP. Besides normal waste processing, LAW feeds from the FRP vessels are also used for heel dilution operations as discussed in Section 4.7.20. It is assumed that vessel contents have cooled down to $25^{\circ} \mathrm{C}$ after transfer from the Tank Farm, held for sampling, and waiting to be discharged. Due to the large size of LAW batches, some wastes may be stored in the FRP vessels for months before they can be processed. Vessels will be pumped in the order in which they receive waste. Once a Waste Feed Receipt Vessel has reached its minimum volume, the vessel makes itself available to receive another batch. Once three of the four Waste Feed Receipt Vessels are empty, another batch is delivered from the Tank Farm.

Figure F-3 provides a graphical illustration of the FRP system where connections with upstream and downstream systems are shown. Table N-1 provides the totalizer files that are used to track the process streams and material flows into and out of the FRP.

\subsubsection{Waste Feed Evaporation Process (FEP) System}

\begin{tabular}{|l|l|}
\hline Vessel Tag \# & Equipment Description \\
\hline FEP-VSL-00017A & Waste Feed Evaporator Feed Vessel \\
\hline FEP-VSL-00017B & Waste Feed Evaporator Feed Vessel \\
\hline
\end{tabular}

The Waste Feed Evaporator Feed Vessels can receive feeds from the following vessels:

\begin{tabular}{|l|l|}
\hline Vessel Tag \# & Equipment Description \\
\hline FRP-VSL-00002A & Waste Feed Receipt Vessel (heel dilution) \\
\hline FRP-VSL-00002B & Waste Feed Receipt Vessel (heel dilution) \\
\hline FRP-VSL-00002C & Waste Feed Receipt Vessel (heel dilution) \\
\hline FRP-VSL-00002D & Waste Feed Receipt Vessel (heel dilution) \\
\hline PWD-VSL-00015 & Acidic/Alkaline Effluent Vessel \\
\hline PWD-VSL-00016 & Acidic/Alkaline Effluent Vessel \\
\hline PWD-VSL-00044 & Plant Wash Vessel \\
\hline RDP-VSL-00002B & Spent Resin Slurry Vessel \\
\hline
\end{tabular}

FEP-VSL-00017A and FEP-VSL-00017B operate in an alternating order and receive feeds from one of the vessels listed above in a first come, first serve fashion. A vessel receives feed until the tank has reached its set volume which may require multiple transfers from the upstream sources. Headspace is 
reserved as the vessel continues to receive line flush water (see Table H-1 for details of various sources of flushing). Solubility is applied at $25^{\circ} \mathrm{C}$ after the vessel is filled. The FEP-VSL-00017A/B vessels are restricted not to receive wastes with solids concentration greater than $2 \mathrm{wt} \%$. Flags will be raised if the $\mathrm{wt} \%$ solids of the upstream wastes exceed the target value. The contents of the vessels are pumped to FEP-SEP-00001A or FEP-SEP-00001B as appropriate.

Once a year, a heel dilution is performed in FEP-VSL-00017A/B to reduce solids concentration in the heel that may have accumulated. Fluids used for dilution are LAW feeds from FRP-VSL-00002A/B/C/D, process condensate, or DIW in preferred orders. The FEP-VSL-00017A/B heel dilution is scheduled to separate with other heel dilution operations in HLP-VSL-00022 and UFP-VSL-00001A/B. The portable pump connects FEP-VSL-00017A/B with FEP-SEP-00001A. Refer to Section 4.7.20 for further details on general rules of heel dilution and FEP-VSL-00017A/B specific volume parameters.

\begin{tabular}{|l|l|}
\hline Vessel Tag \# & Equipment Description \\
\hline FEP-SEP-00001A & Waste Feed Evaporator \\
\hline FEP-SEP-00001B & Waste Feed Evaporator \\
\hline
\end{tabular}

The Waste Feed Evaporator reboiler and separator vessel are modeled as one unit, for example, their volumes are combined. Evaporator volume is maintained at a constant level (set-volume) when operating. Concentrate will initially accumulate then get discharged as the volume rises above the setvolume. The evaporators receive feeds from FEP-VSL-00017A and FEP-VSL-00017B and associated line flush water. FEP-SEP-00001A is dedicated for processing recycles while FEP-SEP-00001B is used for processing the waste feeds through FEP. The FEP-SEP-00001B evaporator train is inactive in the baseline version of the model owing to the flowsheet design of waste feed bypassing FEP. Processing rates of the evaporators are limited by a boil-off rate of 30 gallons per minute (gpm), with 2 gpm used for the evaporator recycle spray resulting in a modeled boil-off rate of $28 \mathrm{gpm}$. The main purpose of the evaporator is to reduce volume by concentrating the recycle to a SpG of 1.27 and/or a weight percent solids of $8.9 \%$, whichever comes first. The evaporator feed pump and concentrate pump have maximum capacities at $50 \mathrm{gpm}$ and $34 \mathrm{gpm}$, respectively. These pump rates are adjusted dynamically at every time step so that the boil-off rate and the SpG and/or weight percent solids target can be met.

Oxalate and phosphate that come from recycles from the UFP operations tend to precipitate in the evaporator after boil-off. Solubility is applied continuously at the flash evaporation temperature $\left(42{ }^{\circ} \mathrm{C}\right)$ which may slightly alter the SpG and/or weight percent solids values of the FEP evaporator bottom product. Recycle concentrate is routed to a UFP1 vessel in accordance with the current UFP1 operating conditions as follows:

As soon as a UFP1 vessel finishes its feed blending and pre-heating operations, this vessel becomes a priority vessel for receiving evaporator bottom products, e.g., it will be used when evaporator starts the next batch operation regardless of the alternate UFP1 vessel mode.

However, if the evaporator is currently feeding the alternate UFP1 vessel, this batch operation will continue (e.g., will not stop due to a new UFP1 vessel comes on line) until either: 1) the feeding FEP17A/B vessel becomes empty, or 2) the receiving alternate UFP1 vessel is full.

Feeding can be switched from the current receiving UFP1 vessel when it gets full to the alternate UFP1 vessel if it still has storage volume available. Evaporator turns idle when both UFP1 vessels are not available. 
To minimize foaming, AFA is added to the evaporator when it starts receiving a new batch from a feed tank. AFA will be replenished after that in an 1-hour interval to make up losses due to decomposition and discharge. Target concentrations for PPG and PDMS are discussed in Section 4.7.17.

The offgas from FEP-SEP-00001A is routed to FEP-DMST-00001A and the offgas from FEP-SEP$00001 \mathrm{~B}$ is routed to FEP-DMST-00001B. Constituents are boiled off by the evaporators based on splits found in Table D-1.

\begin{tabular}{|l|l|}
\hline Vessel Tag \# & Equipment Description \\
\hline FEP-DMST-00001A & Waste Feed Evaporator Demister \\
\hline FEP-DMST-00001B & Waste Feed Evaporator Demister \\
\hline
\end{tabular}

The Waste Feed Evaporator Demisters, which for modeling purposes also perform the role of the condensers, are modeled as FEP-DMST-00001A and FEP-DMST-00001B. The demisters receive offgas from the Waste Feed Evaporators. The offgas is condensed based on splits that can be found in Table D-2. Condensates are sent to FEP-VSL-00005A. The offgas that is not condensed is sent to the Pretreatment Vessel Vent Process (PVP) system (PVP-SCB-00002).

\begin{tabular}{|l|l|}
\hline Vessel Tag \# & Equipment Description \\
\hline FEP-VSL-00005A & Waste Feed Evaporator Condensate Vessel \\
\hline
\end{tabular}

The Waste Feed Evaporator Condensate Vessel receives condensate from the demisters. Water, used to model condensate from the vacuum eductors, is also delivered from a steam header to FEP-VSL-00005A at a rate of $0.96 \mathrm{gpm}$ whenever the Waste Feed Evaporator is running. Once it reaches the maximum volume, it empties to RLD-VSL-00006A. FEP-VSL-00005A can always receive condensate unless it is at maximum volume and RLD-VSL-00006A cannot accept feed.

Figure F-4 provides a graphical illustration of the FEP system where connections with upstream and downstream systems are shown. Table N-1 provides the totalizer files that are used to track the process streams and material flows into and out of the FEP.

\subsubsection{Ultrafiltration Process (UFP) System}

The primary functions of the UFP are to: 1) separate dissolved and undissolved wastes by ultrafiltration, 2) caustic leach aluminum and other undissolved solids if necessary, 3) oxidative leach chromium if necessary, and 4) separate leachate from undissolved solids by washing.

\begin{tabular}{|l|l|}
\hline Vessel Tag \# & Equipment Description \\
\hline UFP-VSL-00001A & Ultrafiltration Feed Preparation Vessel \\
\hline UFP-VSL-00001B & Ultrafiltration Feed Preparation Vessel \\
\hline
\end{tabular}

The UFP1 vessels receive waste and recycle from the following equipment:

\begin{tabular}{|l|l|}
\hline Vessel Tag \# & Equipment Description \\
\hline FRP-VSL-00002A & Waste Feed Receipt Vessel \\
\hline FRP-VSL-00002B & Waste Feed Receipt Vessel \\
\hline FRP-VSL-00002C & Waste Feed Receipt Vessel \\
\hline FRP-VSL-00002D & Waste Feed Receipt Vessel \\
\hline HLP-VSL-00022 & HLW Feed Receipt Vessel \\
\hline FEP-SEP-00001A & Waste Feed Evaporator (default) \\
\hline
\end{tabular}




\begin{tabular}{|l|l|}
\hline Vessel Tag \# & Equipment Description \\
\hline FEP-SEP-00001B & Waste Feed Evaporator (inactive) \\
\hline HLP-VSL-00027A & HLW Lag Storage Vessel (UFP1 leaching) \\
\hline RLD-VSL-00006A & Process Condensate Vessel \\
\hline
\end{tabular}

Feed blending logic are implemented in the UFP1 vessels to: a) improve the uniformity of ultrafiltration feeds, b) better balance the overall utilization of LAW and HLW feeds, and c) alleviate the pressure of water lock on the recycle loop. Under normal operating conditions, feed blending is performed by filling a UFP1 vessel with a pre-determined volume of HLW feed then topping it off with LAW feed to reach the set volume. The blend ratio is determined based on solid contents of the feeds and the heel, i.e.,

$V_{H L W}=\left(\frac{\left(W P C T_{\text {goal }}-W P C T_{\text {law }}\right) * V_{\text {set }}+\left(W P C T_{\text {law }}-W P C T_{\text {heel }}\right) * V_{\text {min }}}{\left(W P C T_{\text {hlw }}-W P C T_{\text {law }}\right)}\right)$

$V_{L A W}=V_{\text {set }}-V_{H L W}$

Where:

$\mathrm{V}_{\mathrm{HLW}} \quad=$ Volume contribution from the HLW feed (gallons)

$\mathrm{V}_{\mathrm{LAW}} \quad=$ Volume contribution from the LAW feed (gallons)

$\mathrm{V}_{\text {set }} \quad=$ Set volume of UFP1 (18,300 gallons)

$\mathrm{WPCT}_{\text {goal }} \quad=$ Feed blending wt $\%$ solid goal $(7 \%)$

$\mathrm{WPCT}_{\text {law }} \quad=$ Current wt $\%$ solids of the LAW feed

$\mathrm{WPCT}_{\text {hlw }} \quad=$ Current $\mathrm{wt} \%$ solids of the HLW feed

$\mathrm{WPCT}_{\text {heel }}=$ Current $\mathrm{wt} \%$ solids of the heel in the blend tank

All Tank Farm batches, including LAW and HLW wastes, are limited to solid concentrations less than or equal to $10 \mathrm{wt} \%$ solids. Some specific rules are applied to the feed blending operations under off-normal conditions:

If not enough HLW feed is available for blending, e.g., HLP-VSL-00022 is reaching the heel, LAW feed will be used to make up the volume difference after the remaining HLW feed is received. In that case, the wt $\%$ solids of the blended feed will be below the $7 \%$ target.

In contrast, if not enough LAW feed is available for blending, the vessel will be made available for discharge after the remaining LAW feed is received. In this case, the wt \% solids of the blended feed will be above the 7\% target, but it prevents the UFP1 vessel from becoming idle when a new LAW batch is received by the FRP from the Tank Farm.

If the wt $\%$ solids of the HLW feed is below the $7 \%$ target, no blending will be required such that only the HLW feed will be used to fill to the set volume.

Also, if HLW or LAW feeds is not available, the only feed available will be used to fill to the set volume without blending.

Line flush is received in the UFP1 vessel after each batch transfer from HLP-VSL-00022 and FRP-VSL$00002 \mathrm{~A} / \mathrm{B} / \mathrm{C} / \mathrm{D}$. To prevent foaming from Pulse Jet Mixer operations, AFA is added immediately after feed blending. AFA is replenished every other 6-hours to make up losses due to decomposition during 
pre-heating, storage, and discharge. Target concentrations for PPG and PDMS are discussed in Section 4.7.17.

Steam will also be added to the vessel after feed blending. Vessel contents will be heated to $45{ }^{\circ} \mathrm{C}$ with a steam condensate volume and a heating time determined by Equations 28 and 29, respectively.

$V_{S}=\frac{2540 * V_{W}}{49562}$

$T_{H}=\frac{3 * V_{W}}{49562}$

Where:

$\mathrm{V}_{\mathrm{S}} \quad=$ Steam condensate volume (gallons)

$\mathrm{V}_{\mathrm{W}} \quad=$ Waste volume before heating (gallons)

$\mathrm{T}_{\mathrm{H}} \quad=$ Heating time (hours)

Solubility is applied at $45{ }^{\circ} \mathrm{C}$ after pre-heating. At this point the vessel becomes a priority vessel in receiving evaporator concentrate as discussed in Section 4.8.2, note: this vessel must wait if the alternate UFP1 vessel is currently receiving from the FEP evaporator. The wait time will last until the evaporator has finished the current batch (e.g., the evaporator feed tank becomes empty or the alternate UFP1 reaches the maximum volume), and when the evaporator has feed to start a new batch. During the wait, the UFP1 vessel can also discharge if the downstream UFP2 vessel is available to receive feed.

UFP-VSL-00001A and UFP-VSL-00001B operate in an alternating fashion, e.g., one will not discharge until the other is "empty". A UFP1 vessel will feed both UFP2 vessels as needed during the saw-tooth operation to support parallel operation of the ultrafilters.

Twice a year, heel dilutions are performed in UFP-VSL-00001A/B to reduce solids concentration in the heel that may have accumulated. Fluids used for dilution are LAW feeds from FRP-VSL-00002A/B/C/D, process condensate, or DIW in preferred orders. The UFP-VSL-00001A/B heel dilutions are scheduled to separate with other heel dilution operations in HLP-VSL-00022 and FEP-VSL-00017A/B. The portable pump connects UFP-VSL-00001A with UFP-VSL-00002A and UFP-VSL-00001B with UFP-VSL00002B. Heel dilution can only be performed when: 1) the receiving UFP2 vessel is in solids concentration mode, 2) the UFP2B solids have been split to UFP2A, and 3) the receivingUFP2 current solid concentration $\leq 15 \mathrm{wt} \%$ solids. These conditions ensure that: 1 ) unleached solids will not be added to the already leached contents, 2) the receiving UFP2 vessel has enough space for the dilution contents, and 3) heel dilution can be completed in the current UFP2 solids concentration cycle. Refer to Section 4.7.20 for further details on general rules of heel dilution and UFP-VSL-00001A/B specific volume parameters.

\begin{tabular}{|l|l|}
\hline Vessel Tag \# & Equipment Description \\
\hline UFP-VSL-00002A & Ultrafiltration Feed Vessel \\
\hline UFP-VSL-00002B & Ultrafiltration Feed Vessel \\
\hline
\end{tabular}

The UFP2 vessels and associated ultrafilters are designed to operate in parallel during solids concentration and reconcentration after caustic leach. During solids concentration, the UFP2 vessels receive feed from a UFP1 vessel currently emptying. The UFP1 to UFP2 line is flushed with 480 gallons 
of inhibited water at 90 gpm at the end of solids concentration or when the UFP1 vessel reaches the heel. Flush water goes to the receiving UFP2 vessel.

Filter loops are divided into pre-isolation valve and post-isolation valve sections. Filter loop volumes of the pre-isolation valve and post-isolation valve sections are:

UPF-02A-P1 $=$ UFP-02B-P1 $=500$ gallons

UFP-02A-P2 = UFP-02B-P2 = 1,576 gallons

The post-isolation valve sections will be filled with $0.1 \mathrm{M}$ caustic $(\mathrm{NapOH})$ during startup while the preisolation sections will be filled with wastes by drainage in the beginning of each ultrafiltration cycle. Inlets of the pre-isolation valve pipes are situated at the 15,000-gallon level in the UFP2 vessels, meaning that these vessels can only be concentrated to the 15,000-gallon level through the circulation pump. Permeate flux rates through the ultrafilters depend on temperature, weight percent solids, sodium molarity, and effective filter area. The permeate flux rate is calculated using the equations below:

$$
F=A_{\text {eff }} * O_{F} *\left[\frac{\exp (0.445 * 5)}{\exp \left(0.445 * C_{N a}\right)}\right] *\left(\frac{1}{\exp \left[2500 *\left(\frac{1}{T_{K}}-\frac{1}{298}\right)\right]}\right)
$$

Where:

$\mathrm{F} \quad=$ Filter flux per train $(\mathrm{gpm})$

$\mathrm{A}_{\text {eff }} \quad=$ Effective filter area per train $\left(\mathrm{ft}^{2}\right)$

$\mathrm{C}_{\mathrm{Na}} \quad=$ Sodium molarity $(\mathrm{gmol} / \mathrm{liter})$

$\mathrm{T}_{\mathrm{K}} \quad=$ Operating temperature $(\mathrm{K})$

$\mathrm{O}_{\mathrm{F}} \quad=$ Operation Factor $\left(\mathrm{gpm} / \mathrm{ft}^{2}\right)$

For solids concentration and reconcentration:

$$
O_{F}=\frac{2.1 e-4}{X^{1.3}}+0.112
$$

Where:

$\mathrm{X} \quad=$ Weight percent solids ( $\mathrm{kg}$ solids $/ \mathrm{kg}$ slurry)

For solid wash including wash forward and wash recycle:

$\mathrm{O}_{\mathrm{F}}=0.012$

For oxidative wash:

$\mathrm{O}_{\mathrm{F}}=0.008$

Effective filter area equals to $1,451 \mathrm{ft}^{2}$ per train, ultrafiltration operates at $45{ }^{\circ} \mathrm{C}$, and upper and lower bounds of the filter flux are set to $115 \mathrm{gpm}$ and $1 \mathrm{gpm}$, respectively. Flux rate is reduced to $10 \%$ of the 
filter capacity when both HLP-VSL-00027A/B vessels are filled with treated solids and are unable to receive, this is done to extend the UFP2 cycle time and therefore ease the pressure of recycle production on storage volumes for equipment in the recycle loop.

Two positive displacement pumps connect the UFP2 vessels to the following receiving vessels:

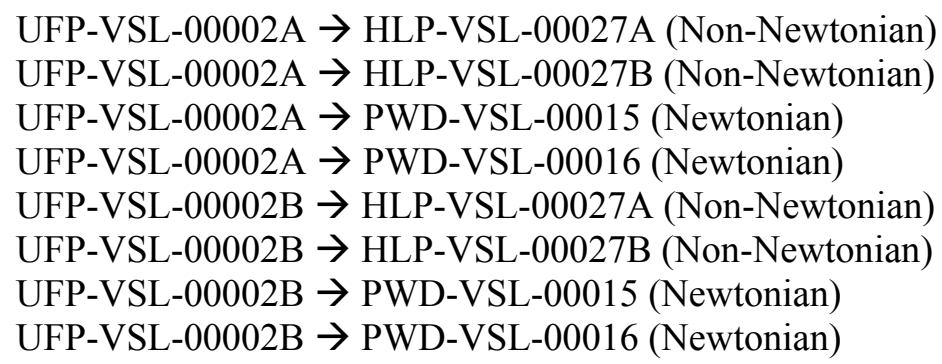

The positive displacement pumps will run at 94 gpm for Newtonian fluids and 55 gpm for Non-Newtonian fluids. The pumps can empty the UFP2 vessels to their minimum volumes of 500 gallons. The downstream vessel will receive 150 gallons of line flush with inhibited water at $90 \mathrm{gpm}$ after a Non-Newtonian fluid transfer. The operation sequence of UFP2 is discussed as follows.

Due to a Mechanical System design change that increases the minimum volume of ultrafilter circulation pump inlet level, a UFP2 double batching operation is introduced that enables: a) reasonable sized solid batches be leached, b) sufficient headspaces are available to support heating and leaching, and c) leached solids can be reconcentrated to target values before discharging. Details of the double batching operation are described as follows:

1) Set UFP2A/B maximum volume to 29,900 gallons

2) Start solid concentration in UFP2B, sawtooth at high set points between 26,000 - 25,000 gallons, concentration target $=20 \mathrm{wt} \%$ solid

3) In parallel to the UFP2B solid concentration, do wash forward, wash recycle, oxidative leach, oxidative wash, solid discharge, acid clean in UFP2A (Figures $2 \mathrm{a}$ and $2 \mathrm{~b}$ )

4) If UFP2B reaches the concentration target before UFP2A reaches the end of current cycle, hold UFP2B until UFP2A completes all the steps in Figure 2b

5) Else if UFP2A reaches the end of current cycle before UFP2B reaches concentration target, stop UFP2B solids concentration

6) Transfer half of the UFP2B contents to UFP2A, transfer rate $=94$ gpm (feed split, Figure 2c)

7) Restart solid concentration in UFP2A/2B, sawtooth at low set points between 16,000 - 15,000 gallons (Figure 2d). Continue solid concentration until both trains reach $20 \mathrm{wt} \%$ solid target concentration. Make caustic leach decisions at the same time

8) If both UFP2A/2B batches require caustic leach

- Caustic leach UFP2A/2B (Figure 3e)

- Reconcentrate UFP2A/2B to 15,000 gallons or $20 \mathrm{wt} \%$ solids, whichever comes first (initial recon, Figure 3f)

- Transfer UFP2B contents (excluding the 500 gallon heel) to UFP2A, transfer rate $=55 \mathrm{gpm}$ (feed combination, Figure $3 \mathrm{~g}$ )

- UFP2B goes back to Step 2, do filter clean if required. In parallel, reconcentrate UFP2A to 15,000 gallons or $20 \mathrm{wt} \%$ solids, whichever comes first (secondary recon, Figure $3 \mathrm{~h}$ )

- UFP2A goes back to Step 3 
9) Else if both UFP2A/2B batches do not require caustic leach

- Skip leaching, initial recon, feed combination, and secondary recon steps

- Wash, oxidative leach, oxidative wash, and discharge solids one train at a time. Start with the UFP2B train (Figure 4i)

- Do filter clean if required

- Go back to Step 2

10) Else if one train requires caustic leach and another train does not (possibility low)

- Apply Step 8 processes

The UFP2A/2B trains can operate in parallel during solid concentration, caustic leach, and initial reconcentration. Steam and cooling water supplies can support simultaneous operations. 


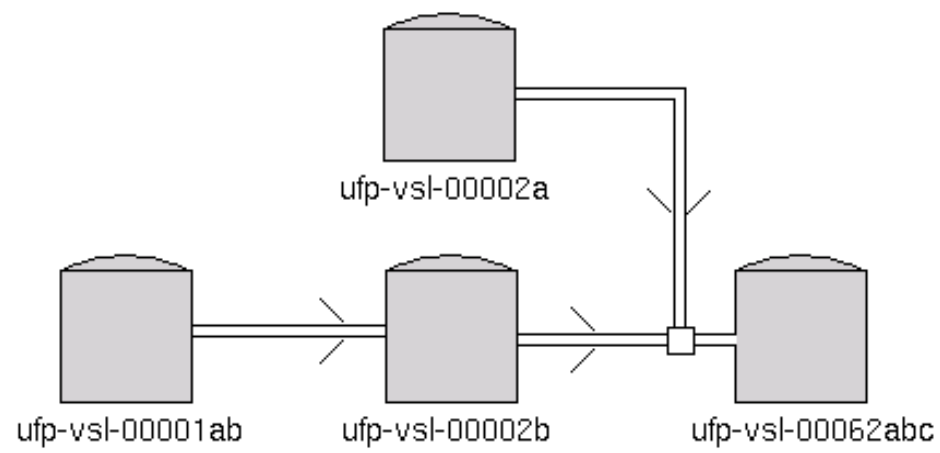

a) UFP2A in Wash Forward,

UFP2B in Solids Concentration at High Set Point

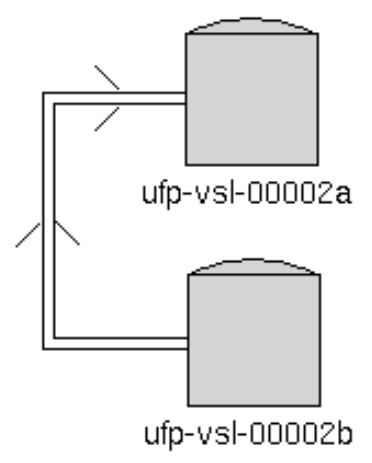

c) UFP2B -> UFP2A Feed Split

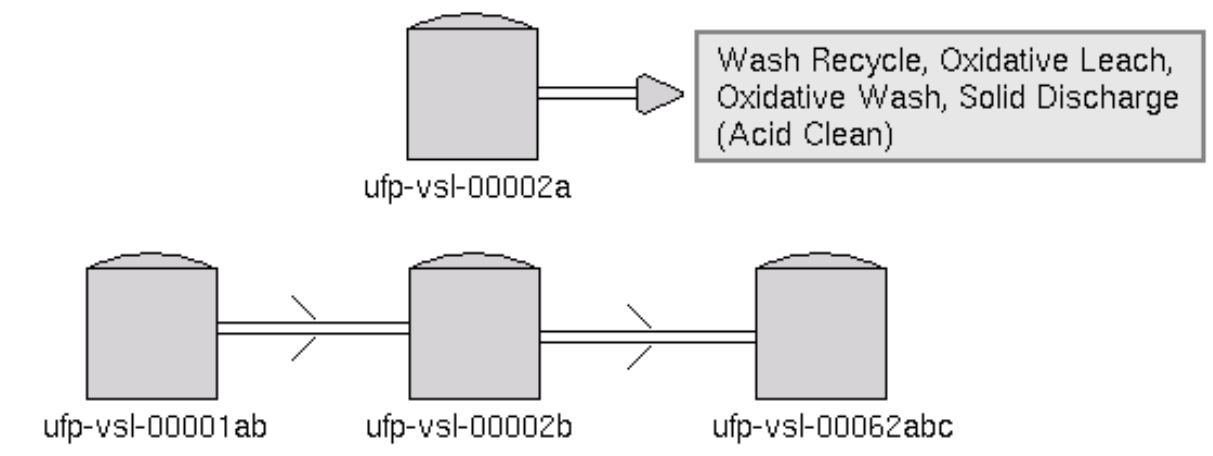

b) UFP2A in Post Wash Forward Steps, UFP2B in Solids Concentration at High Set Points

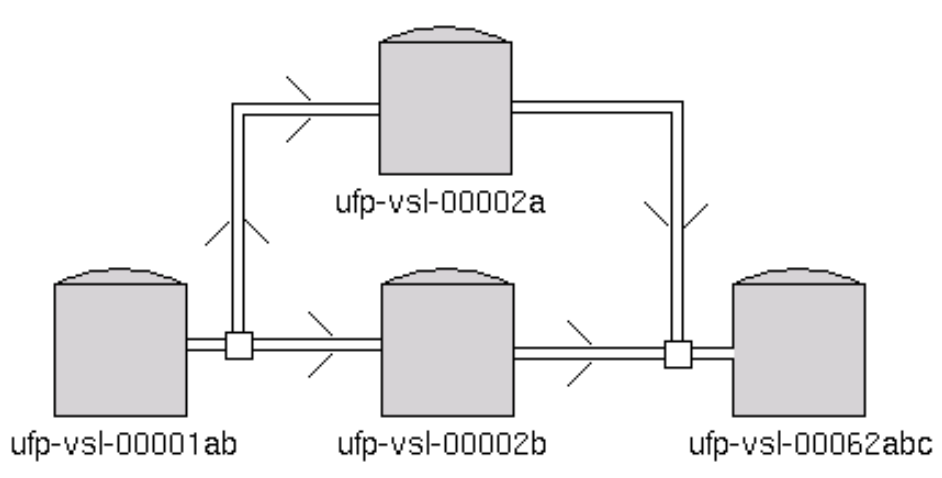

d) UFP2A/B in Solids Concentration at Low Set Points

Figure 2

UFP2 Double Batching Operation (Part 1) 

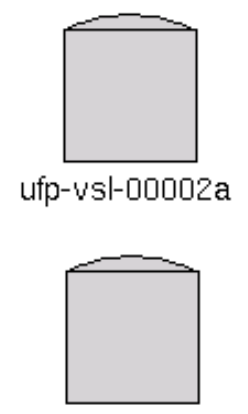

ufp-vst-00002b

e) UFP2A/B in Caustic Leach

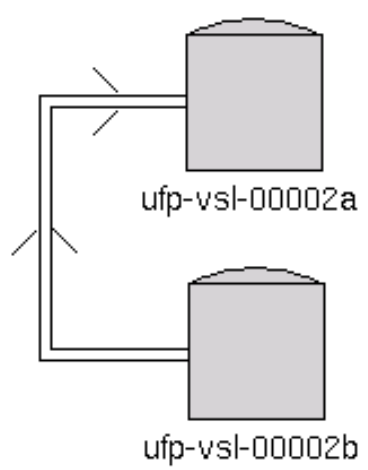

g) UFP2B -> UFP2A Recon Feed Combination

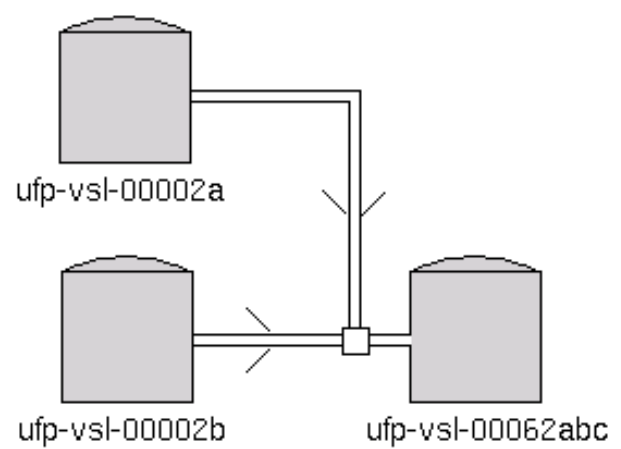

f) UFP2A/B in Reconcentration
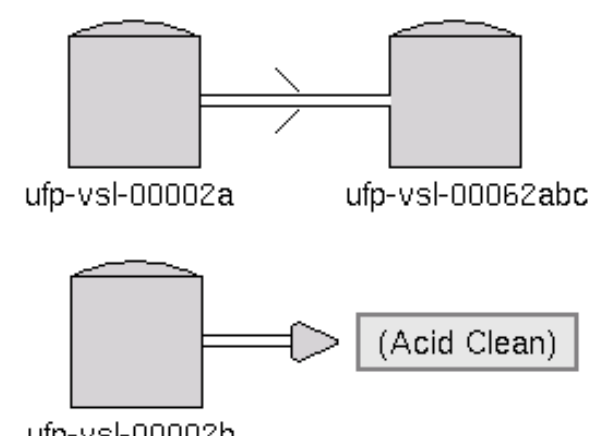

ufp-vst-00002b

h) UFP2A in Secondary Recon, UFP2B Do Acid Clean, Go Back a) When Finish

Figure $3 \quad$ UFP2 Double Batching Operation (Part 2) 


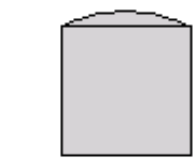

ufp-vsl-00002a

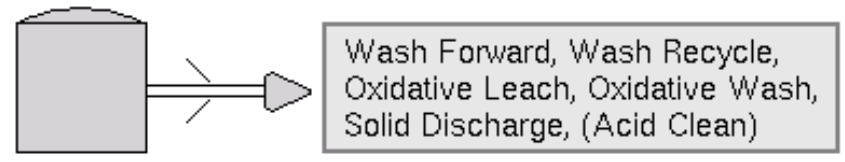

ufp-vst-00002b

i) UFP2A in Wait State,

UFP2B Do Wash and Post Wash Steps, Go Back a) When Finish

Figure $4 \quad$ UFP2 Double Batching Operation (Part 3) 


\section{$\underline{\text { Solids Concentration }}$}

AFA is added immediately after a UFP2 is filled to the set volume with feed from UFP1. Target concentrations for PPG and PDMS are discussed in Section 4.7.17. Solids concentration begins by pumping and filling (sawtooths) the UFP2 vessel between the upper set volume and the lower setpoint, the UFP2 vessel is allowed to receive before it reaches the lower setpoint to ensure an undisrupted ultrafiltration operation. Through the circulation pump, ultrafilter permeate is routed to the Ultrafiltration Permeate Collection Vessel (UFP-VSL-00062A/B/C) while concentrated slurry is returned to the UFP2 vessel. The solids concentration process continues until $20 \mathrm{wt} \%$ solids has been reached. Solubility correlations are applied at $45^{\circ} \mathrm{C}$ at the peak of the sawtooth during solids concentration. AFA is replenished every 6-hours to make up losses due to decomposition during the remaining UFP2 operations except during oxidative leaching.

\section{$\underline{\text { Caustic Leach }}$}

Generally caustic leach can reduce the HLW glass produced as most of the WTP solids are aluminum limiting. For waste batches that are limited by other Specification 1 species (see bounding condition flags in Figure 5 for details), caustic leach is unnecessary as it increases processing time and adds sodium to the waste. A caustic leach decision logic has been developed by PE that determines if a waste batch is aluminum limiting and consequently if the caustic leach can reduce HLW glass by $10 \%$ or more. Wash factors as well as the subsequent oxidative leach are also considered. Figures 5 through 8 provide logic diagrams that are implemented in G2 model, where:

- EG-Majors is the estimated glass amount expected if the glass limited by major glass constituents

- EG-Minors is the estimated glass amount expected if the glass limited by minor glass constituents

- EG-Cr is the estimated glass amount expected if the glass is chromium limited

- BC-i is the HLW glass oxide limiting value set in G2 as wt fraction units, not wt\%

- LF values are Tank Farm's given leach factor values for corresponding element, units in wtfraction

- $\quad$ PTWF-C2O4 is a wash factor for solid oxalate in Pretreatment

- PO4-3(Hard) is the insoluble form of phosphate in the batch, kilo-moles

- PO4-3 is the soluble form of phosphate in the batch, kilo-moles

- $\mathrm{P}_{\mathrm{pf}}$ is an estimated average of the soluble phosphate that will precipitate and report to HLW for vitrification

- OxLF is the oxidative leach factor for chromium

- WF-Ox is the estimated wash factor for removing dissolved chromium and other salts from the batch after oxidative leaching. Presently, three vessel washing are preformed

Details for the development of this caustic leach decisions logic can be found in 24590-WTP-MCR-PET09-0048. 


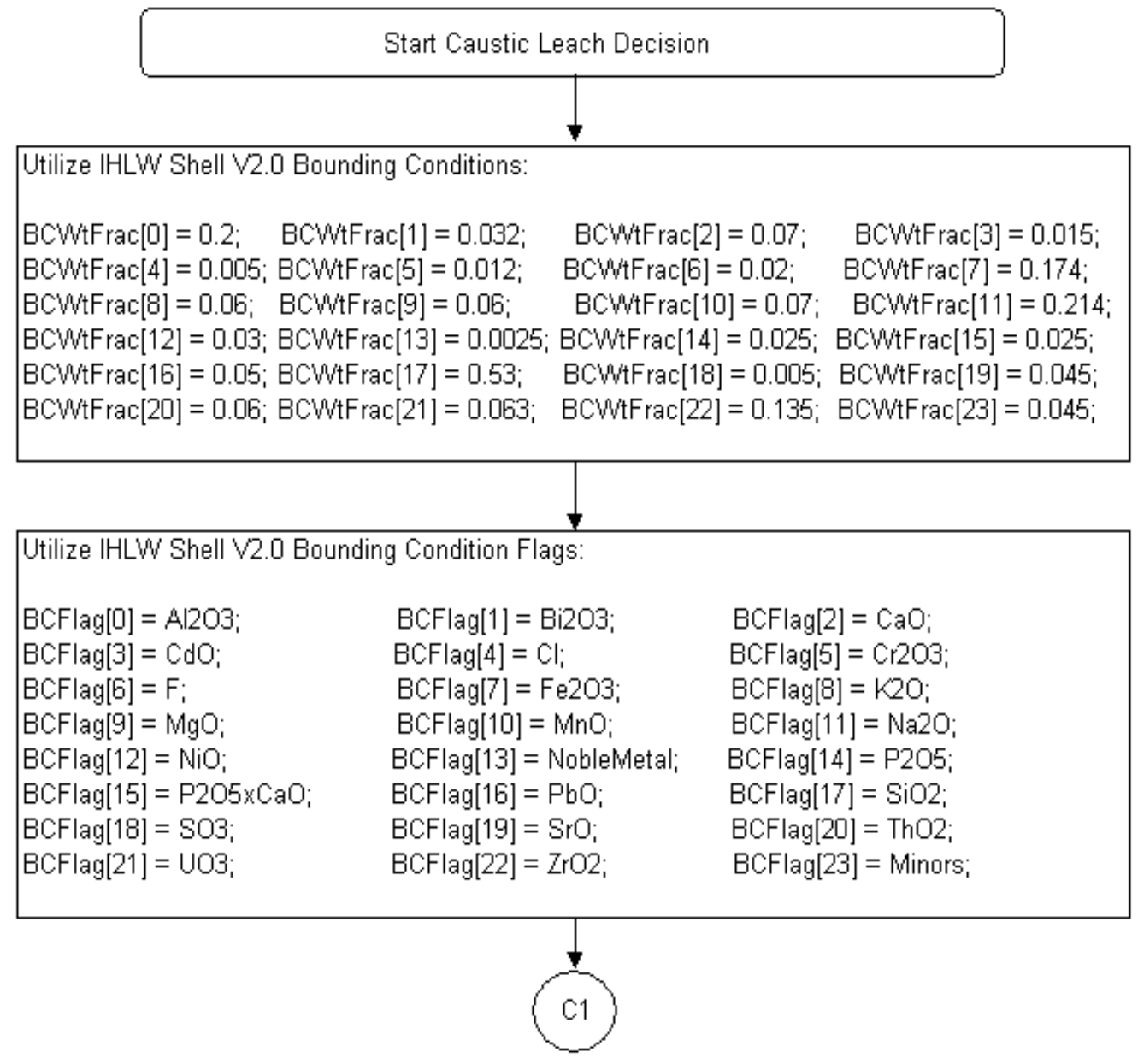

Figure 5 Caustic Leach Decisions Logic Diagram (Part 1) 


\begin{tabular}{|c|}
\hline 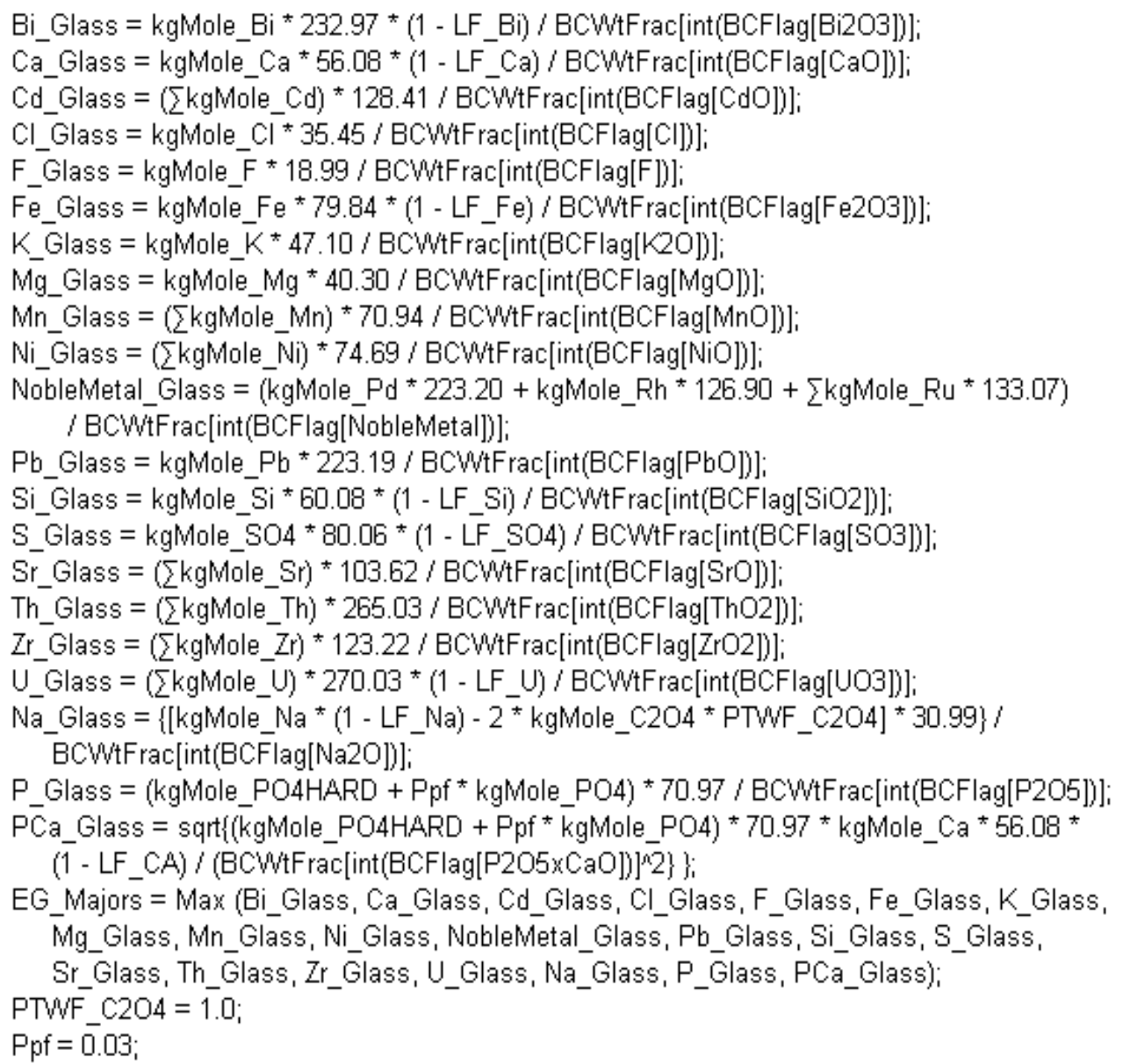 \\
\hline
\end{tabular}

Figure 6 Caustic Leach Decisions Logic Diagram (Part 2) 


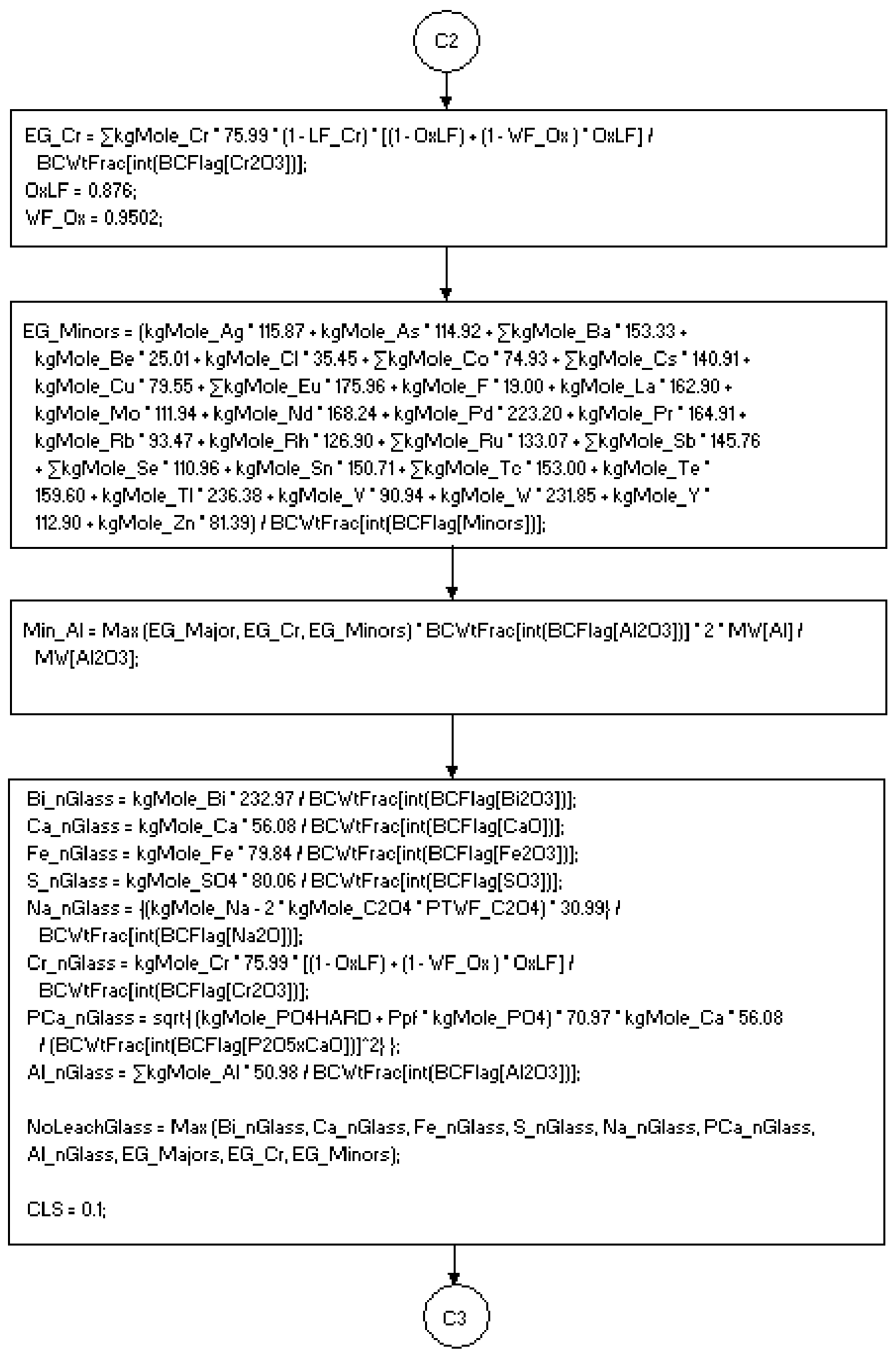

Figure $7 \quad$ Caustic Leach Decisions Logic Diagram (Part 3) 


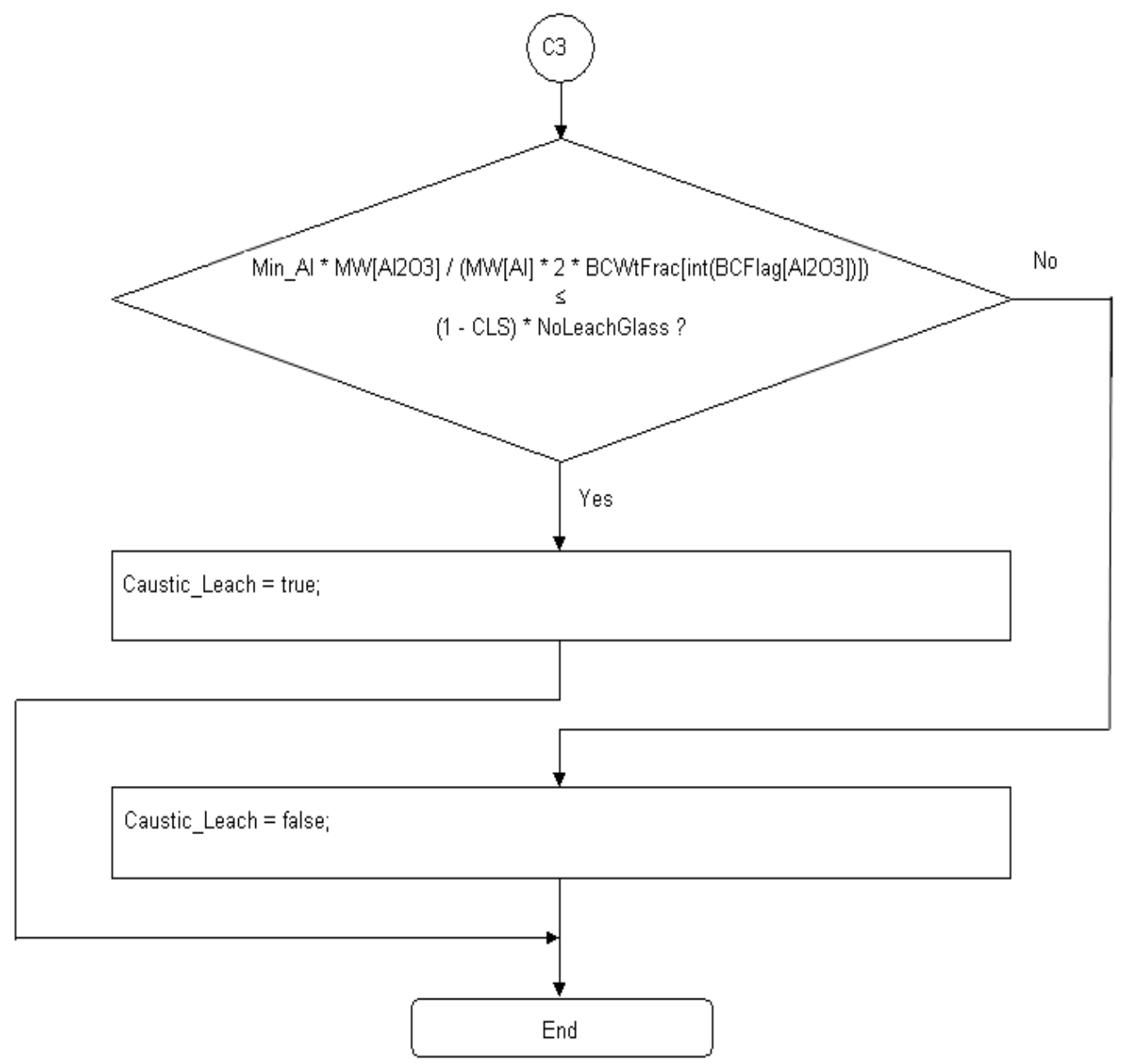

Figure 8 Caustic Leach Decisions Logic Diagram (Part 4)

The caustic leach decision is made based on UFP2 solids composition at the end of the solids concentration process. If caustic leach is not needed, the current UFP2 vessel will start solid wash, otherwise it will perform the caustic leach operations.

Before caustic leach operations can be performed, the filter loop is power flushed with one filter loop volume of inhibited water (2,076 gallons) which takes 0.183 hours. An $85 \%$ flushing efficiency is achieved and the slurry is pushed back into the UFP2 vessel.

Caustic leach operations include heating, reaction, cooling, and re-concentration. A pre-determined volume of $19 \mathrm{M} \mathrm{NaOH}(\mathrm{Na}=$ waste sodium) will be added during heating. Vessel contents will be heated to $85^{\circ} \mathrm{C}$ with steam. Condensate volume and heating time are determined by Equations 34 and 35, respectively. 
$V_{S}=\frac{5000 *\left(V_{W}+V_{A C V}\right)}{24500}$

$T_{H}=\frac{6 *\left(V_{W}+V_{A C V}\right)}{24500}$

Where:

$\mathrm{V}_{\mathrm{S}} \quad=$ Steam condensate volume (gallons)

$\mathrm{V}_{\mathrm{W}} \quad=$ Waste volume before heating (gallons)

$\mathrm{V}_{\mathrm{ACV}}=$ Average caustic volume (gallons)

$\mathrm{T}_{\mathrm{H}} \quad=$ Heating time (hours)

The average caustic volume (5,359 gallons) is obtained by averaging the values for the entire mission from a trial run. The caustic volume for leaching is determined based on Equation 11 with an additional $10 \%$ margin to account for dilution later during wash. The heated solutions are allowed to react for 8 hours. Leaching reactions include:

\begin{tabular}{|c|c|}
\hline Reaction & $\begin{array}{l}\text { Conversion } \\
\text { (Fraction) }\end{array}$ \\
\hline $\mathrm{Bi}+3(\mathrm{~s})+3 \mathrm{OH}-(\mathrm{l}) \rightarrow \mathrm{Bi}+3$ (l) $+3 \mathrm{OH}-(\mathrm{l})$ & Batch dependent \\
\hline $\mathrm{Ca}+2(\mathrm{~s})+2 \mathrm{OH}-(\mathrm{l}) \rightarrow \mathrm{Ca}+2(\mathrm{l})+2 \mathrm{OH}-(\mathrm{l})$ & Batch dependent \\
\hline $\mathrm{Fe}+3(\mathrm{~s})+3 \mathrm{OH}-(\mathrm{l}) \rightarrow \mathrm{Fe}+3$ (l) $+3 \mathrm{OH}-(\mathrm{l})$ & Batch dependent \\
\hline $\mathrm{Si}+4(\mathrm{~s})+4 \mathrm{OH}-(\mathrm{l}) \rightarrow \mathrm{Si}+4(\mathrm{l})+4 \mathrm{OH}-(\mathrm{l})$ & Batch dependent \\
\hline U(TOTAL) (s) + 2 OH- (l) $\rightarrow$ U(TOTAL) (l) +2 OH- (l) & Batch dependent \\
\hline $232-\mathrm{U}(\mathrm{s})+2 \mathrm{OH}-(\mathrm{l}) \rightarrow 232-\mathrm{U}(\mathrm{l})+2 \mathrm{OH}(\mathrm{l})$ & Batch dependent \\
\hline $233-\mathrm{U}(\mathrm{s})+2 \mathrm{OH}-(\mathrm{l}) \rightarrow 233-\mathrm{U}(\mathrm{l})+2 \mathrm{OH}(\mathrm{l})$ & Batch dependent \\
\hline $234-\mathrm{U}(\mathrm{s})+2$ OH- (l) $\rightarrow 234-\mathrm{U}(\mathrm{l})+2 \mathrm{OH}(\mathrm{l})$ & Batch dependent \\
\hline $235-\mathrm{U}(\mathrm{s})+2$ OH- (l) $\rightarrow 235-\mathrm{U}(\mathrm{l})+2 \mathrm{OH}(\mathrm{l})$ & Batch dependent \\
\hline $236-\mathrm{U}(\mathrm{s})+2$ OH- (l) $\rightarrow 236-\mathrm{U}(\mathrm{l})+2 \mathrm{OH}(\mathrm{l})$ & Batch dependent \\
\hline $238-\mathrm{U}(\mathrm{s})+2 \mathrm{OH}-(\mathrm{l}) \rightarrow 238-\mathrm{U}(\mathrm{l})+2 \mathrm{OH}(\mathrm{l})$ & Batch dependent \\
\hline $\mathrm{Cr}(\mathrm{TOTAL})(\mathrm{s})+3 \mathrm{OH}(\mathrm{BOUND})(\mathrm{s})+\mathrm{OH}-(\mathrm{l}) \rightarrow \mathrm{Cr}(\mathrm{OH}) 4-(\mathrm{l})$ & Batch dependent \\
\hline $\mathrm{Cr}(\mathrm{TOTAL})(\mathrm{s})+3 \mathrm{NO} 3-(\mathrm{s})+4 \mathrm{OH}-(\mathrm{l}) \rightarrow \mathrm{Cr}(\mathrm{OH}) 4-(\mathrm{l})+3 \mathrm{NO} 3-(\mathrm{l})$ & Batch dependent \\
\hline $\mathrm{Cr}(\mathrm{TOTAL})(\mathrm{s})+3 \mathrm{NO} 2-(\mathrm{s})+4 \mathrm{OH}-(\mathrm{l}) \rightarrow \mathrm{Cr}(\mathrm{OH}) 4-(\mathrm{l})+3 \mathrm{NO} 2-(\mathrm{l})$ & Batch dependent \\
\hline 2 Cr(TOTAL) (s) + 3 CO3-2 (s) + 8 OH- (l) $\rightarrow 2 \mathrm{Cr}(\mathrm{OH}) 4-(\mathrm{l})+3 \mathrm{CO} 3-2(\mathrm{l})$ & Batch dependent \\
\hline $2 \mathrm{Cr}(\mathrm{TOTAL})(\mathrm{s})+3 \mathrm{SO} 4-2(\mathrm{~s})+8 \mathrm{OH}-(\mathrm{l}) \rightarrow 2 \mathrm{Cr}(\mathrm{OH}) 4-(\mathrm{l})+3 \mathrm{SO} 4-2(\mathrm{l})$ & Batch dependent \\
\hline $\mathrm{Cr}(\mathrm{TOTAL})(\mathrm{s})+\mathrm{PO} 4-3(\mathrm{~s})+4 \mathrm{OH}-(\mathrm{l}) \rightarrow \mathrm{Cr}(\mathrm{OH}) 4-(\mathrm{l})+\mathrm{PO} 4-3(\mathrm{l})$ & Batch dependent \\
\hline Cr(TOTAL) (s) + 4 OH- (l) $\rightarrow$ Cr(OH)4- (l) & Batch dependent \\
\hline $\mathrm{Cr}(\mathrm{TOTAL})(\mathrm{l})+4 \mathrm{OH}(\mathrm{BOUND})(\mathrm{l}) \rightarrow \mathrm{Cr}(\mathrm{OH}) 4-(\mathrm{l})$ & 1.0 \\
\hline $\mathrm{SO} 4-2(\mathrm{~s})+2 \mathrm{Na}+(\mathrm{s}) \rightarrow \mathrm{SO} 4-2(\mathrm{l})+2 \mathrm{Na}+(\mathrm{l})$ & Batch dependent \\
\hline $\mathrm{Na}+(\mathrm{s}) \rightarrow \mathrm{Na}+(\mathrm{l})$ & Batch dependent \\
\hline $\mathrm{C} 2 \mathrm{O} 4-2(\mathrm{~s})+2 \mathrm{Na}+(\mathrm{s}) \leftrightarrow \mathrm{C} 2 \mathrm{O} 4-2(\mathrm{l})+2 \mathrm{Na}+(\mathrm{l})$ & Solubility driven \\
\hline $\mathrm{PO} 4-3(\mathrm{~s})+3 \mathrm{Na}+(\mathrm{s}) \leftrightarrow \mathrm{PO} 4-3(\mathrm{l})+3 \mathrm{Na}+(\mathrm{l})$ & Solubility driven \\
\hline $\mathrm{Al}(\mathrm{OH}) 3(\mathrm{~s})+\mathrm{OH}-(\mathrm{l}) \leftrightarrow \mathrm{Al}(\mathrm{OH}) 4-(\mathrm{l})$ & Solubility driven \\
\hline
\end{tabular}

Leach factors for the reactions are provided in the feed vector and vary from batch to batch. Due to feed blending and recycle operations, a single set of leach factor values from the Tank Farm is no longer 
representative to the mixed wastes, and weighted average values are calculated as wastes are transferred downstream.

$$
D L F_{i}=\frac{\left(U L F_{i} * U C M_{i}+D L F_{i}^{o} * D C M_{i}^{o}\right)}{\left(U C M_{i}+D C M_{i}^{o}\right)}
$$

Where:

$$
\begin{array}{ll}
\mathrm{DLF}_{\mathrm{i}} & =\text { Updated downstream leach factor for component } \mathrm{i} \text { (fraction) } \\
\mathrm{ULF}_{\mathrm{i}} & =\text { Upstream leach factor for component } \mathrm{i} \text { (fraction) } \\
\mathrm{UCM}_{\mathrm{i}} & =\text { Upstream mass for component } \mathrm{i} \text { (kg-moles) } \\
\mathrm{DLF}_{\mathrm{i}}{ }^{\circ} & =\text { Initial downstream leach factor for component } \mathrm{i} \text { (fraction) } \\
\mathrm{DCM}_{\mathrm{i}}{ }^{\circ} & =\text { Initial downstream mass for component } \mathrm{i} \text { (kg-moles) }
\end{array}
$$

The leach factors are tracked from the Tank Farm to FRP-VSL-00002A/B/C/D, HLP-VSL-00022, UFPVSL-00001A/B, and UFP-VSL-00002A/B vessels. It is assumed that solids in the recycle streams have already been caustic and/or oxidative leached, for example, solids from the PWD and FEP systems will not contribute to the weighted average leach factor calculation.

Reactions could be reactant limiting, and under such circumstances, the desired reaction extent will not be achieved. The $\mathrm{Cr}$ (Total) dissolution reactions take place in the order listed and stop when the Tank Farm leach factor has been reached, in other words, some of the listed chromium reactions may not be required. Gibbsite leaching is handled by the aluminum solubility equation (Eq. 12) at $45^{\circ} \mathrm{C}$. Oxalate and phosphate solubility correlations are also applied at the end of the reactions to ensure phase equilibrium. It is noted that the sodium dissolution reaction may not reach its desired leach factor as the G2 model enforces the sodium oxalate and sodium phosphate bounds in the solid phase. Vessel contents will be cooled to $45^{\circ} \mathrm{C}$, cooling takes 22 hours. Steam and chill water can be used for simultaneous heating or cooling both UFP2 trains.

UFP2 vessel contents will be re-concentrated to $20 \mathrm{wt} \%$ solids after cooling. Permeate from the reconcentration operation will be routed to the Supertank (UFP-VSL-00062A). For waste batches with large leachable solids, the $20 \mathrm{wt} \%$ solids re-concentration target may not be reached as the filter loop circulation pump limits the UFP2 minimum level to 15,000 gallons. Reconcentrated feeds in UFP2B will be transferred to UFP2A for secondary reconcentration.

Generally oxidative leach can reduce the HLW glass produced if the solids are chromium limiting. For waste batches that are limited by other Specification 1 species, oxidative leach is unnecessary as it increases processing time and adds sodium and manganese to the waste via the use of sodium permanganate. Similar to the caustic leach decisions discussed previously, an oxidative leach decision logic has been developed by PE that determines if a waste batch is chromium limiting and consequently if the oxidative leach can reduce HLW glass by $10 \%$ or more. Figures 9 through 11 provide logic diagrams that are implemented in the G2 model. Details for the development of this oxidative leach decisions logic can be found in 24590-WTP-MCR-PET-09-0049. 


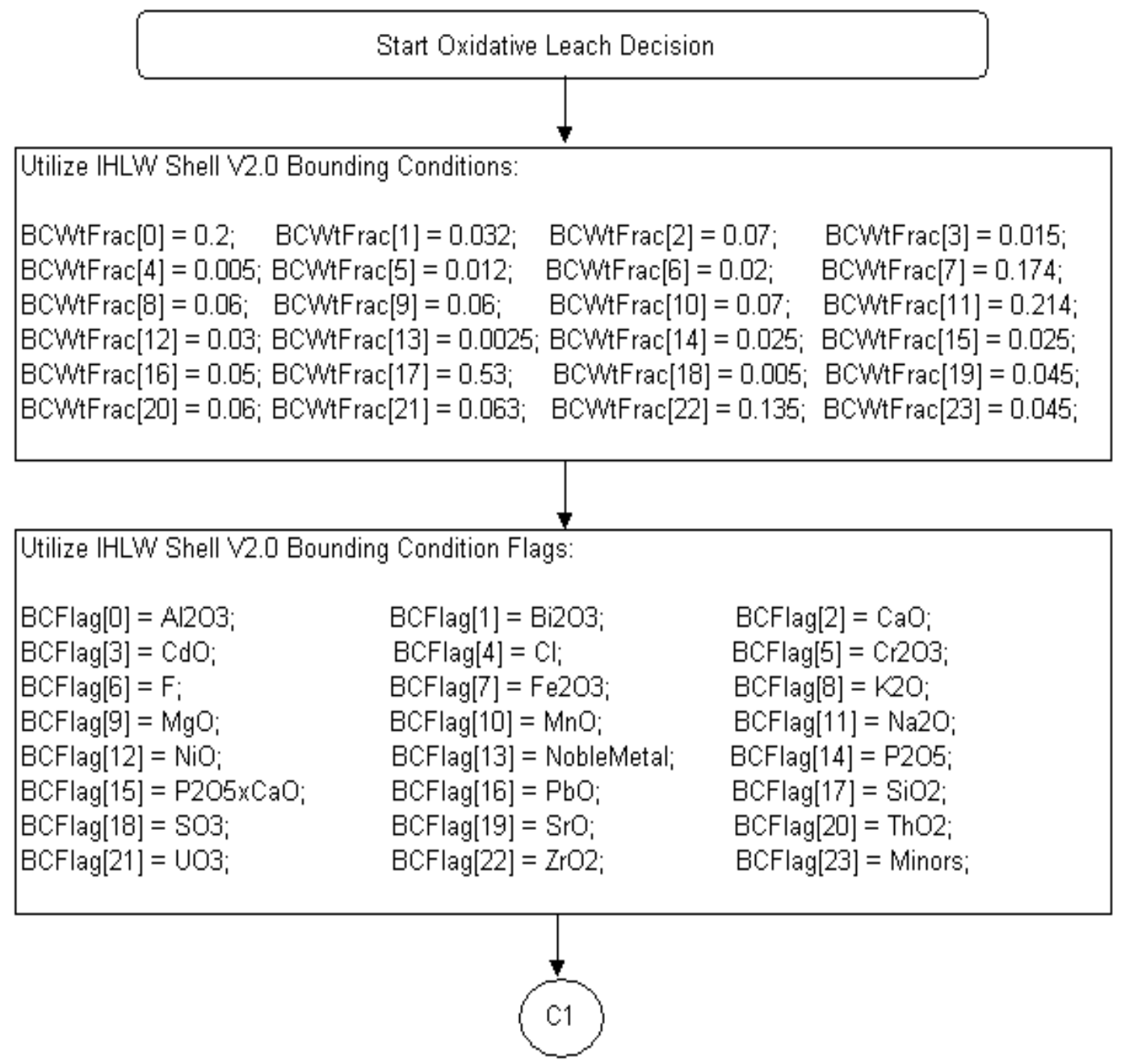

Figure 9 Oxidative Leach Decisions Logic Diagram (Part 1) 


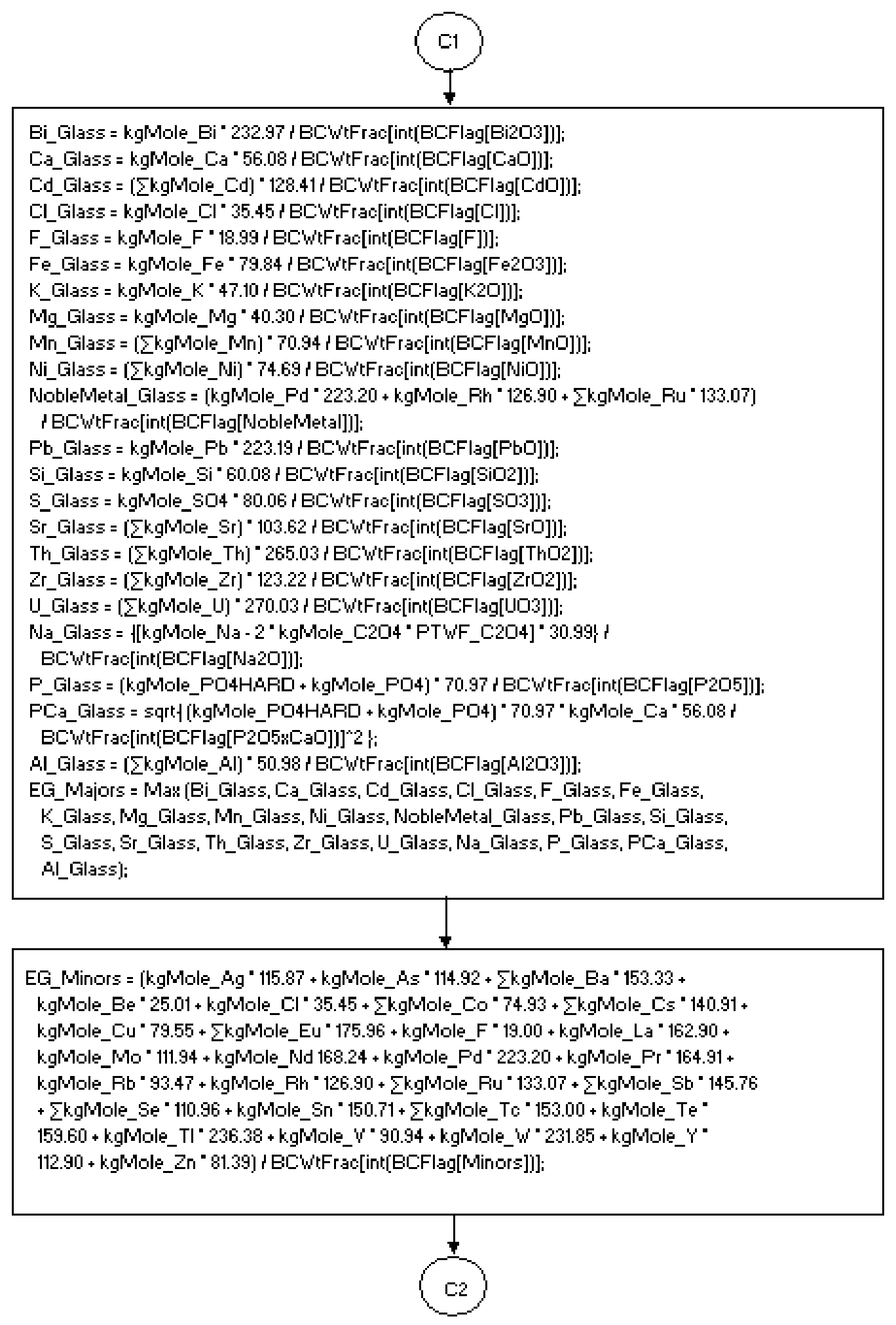

Figure 10 Oxidative Leach Decisions Logic Diagram (Part 2) 


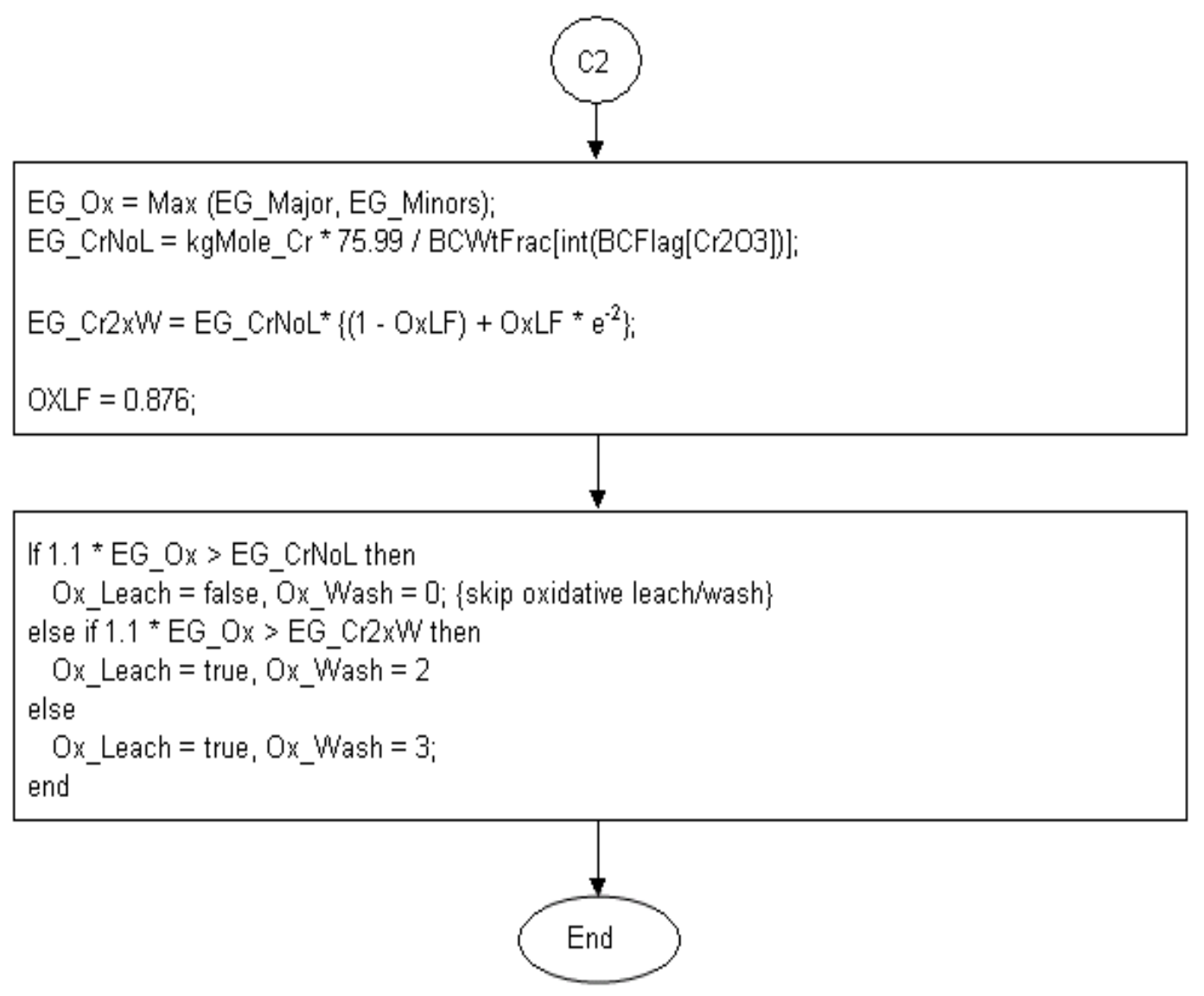

Figure 11 Oxidative Leach Decisions Logic Diagram (Part 3)

The oxidative leach decision is made based on UFP2 vessel contents at the end of re-concentration as this decision will also impact how solid wash will be performed.

\section{$\underline{\text { Solid Wash }}$}

Solid wash is achieved by pumping and filling the UFP2 vessel with process condensate (primary source) or process water (secondary source) in 1000-gallon increments based on the following scenarios:

1) If caustic leach is skipped, sawtooth between 16,000 and 15,000 gallons

2) If caustic leach is performed and re-concentration reaches $20 \mathrm{wt} \%$ solids, sawtooth between the volume at $20 \mathrm{wt} \%$ solids $\left(\mathrm{V}_{\mathrm{R}}\right)$ and $\mathrm{V}_{\mathrm{R}}+1000$ gallons

3) If caustic leach is performed and re-concentration could not reach $20 \mathrm{wt} \%$ solids, sawtooth between 16,000 and 15,000 gallons

UFP2 filling rates are 250 gpm with process condensate and 73 gpm with water. 
Solid wash is divided into two stages: 1) wash forward, and 2) wash recycle. Permeate is routed to the UFP-VSL-00062 vessels during wash forward and to the PWD-VSL-00015/16 vessels during wash recycle. Wash volumes depend on waste conditions at the end of solids concentration or re-concentration and if subsequent oxidative leach is required. The minimum volume for wash recycle is 1,000-gallons.

If subsequent oxidative leach is required:

- Total wash volume is set to wash until [OH-] reaches $0.25 \mathrm{M}$

- Permeate will be fed forward if all of the following are true:

$-[\mathrm{Na}+] \geq 3.8 \mathrm{M}$, and

- $[\mathrm{Na}+] \geq 0.5 *$ sodium concentration at end of solids concentration, and

- $[\mathrm{PO} 4-3] \geq 0.4 *$ peak phosphate concentration, and

- $[\mathrm{C} 2 \mathrm{O} 4-2] \geq 0.4 *$ peak oxalate concentration

- Permeate will be recycled until $[\mathrm{OH}-] \leq 0.25 \mathrm{M}$

- Wash recycle may or may not be needed, depending on the wash forward volume, e.g., skip wash recycle if wash forward volume equals to total wash volume

If subsequent oxidative leach is not required:

- $2.8 *$ V_slurry $\leq$ total wash volume $\leq \mathrm{V} \_$max

- Wash forward if all of the following are true:

$-[\mathrm{Na}+] \geq 3.8 \mathrm{M}$, and

- $[\mathrm{Na}+] \geq 0.5 *$ sodium concentration at the end of re-concentration, and

- $\quad[\mathrm{PO} 4-3] \geq 0.4 *$ peak phosphate concentration, and

- $\quad[\mathrm{C} 2 \mathrm{O} 4-2] \geq 0.4 *$ peak oxalate concentration

- Permeate will be recycled until total wash volume has been applied

- Skip wash recycle if wash forward volume equals to total wash volume

Where:

V_slurry = Slurry volume at the end of solids concentration (gal)

V_max = Maximum wash volume (gal)

Peak concentrations are defined as the highest dissolved phase oxalate and phosphate concentrations achieved during wash. It is not necessarily true that peak concentrations occur at the beginning of wash. In fact, dissolve phase oxalate and phosphate concentrations decline initially, then spike, and finally recede. This phenomenon occurs for batches with reasonable solid phase oxalate and phosphate at the beginning of wash, due to dilution and dissolution.

If the current batch is not phosphate limiting,

$$
\mathrm{V} \_\max =\text { least of }\left\{\left(4.5 * \mathrm{~V} \_ \text {slurry }\right),(0.25 \mathrm{M}[\mathrm{OH}-])\right\}
$$

otherwise

$$
\mathrm{V} \_\max =\text { least of }\left\{\left(4.5 * \mathrm{~V} \_ \text {slurry }\right),(0.25 \mathrm{M}[\mathrm{OH}-]),(0.1 * \text { peak }[\mathrm{PO} 4-3])\right\}
$$


The following method is applied to determine if the current batch will form phosphate limiting IHLW glass.

$\mathrm{EG}-\mathrm{P}=(\mathrm{PO} 4-3(\mathrm{HARD})+\mathrm{PO} 4-3(\mathrm{~s})+\mathrm{PO} 4-3(1)) * 70.97 /$ FLAG-P2O5

$\mathrm{EG}-\mathrm{O}=\mathrm{MAX}($ $\mathrm{Fe}+3 * 79.84$ / MAX(FLAG-FE2O3, 0.1375),
$\mathrm{Zr}+4 * 123.22$ / FLAG-ZrO2,
$\mathrm{U}(\mathrm{Total}) * 270.03$ / FLAG-UO3,
$\mathrm{Th}+4 * 265.03$ / FLAG-ThO2,
$\mathrm{Ca}+2 * 56.08$ / FLAG-CaO,
$\mathrm{Ni}+2 * 74.69$ / FLAG-NiO,
$\mathrm{Pb}+2 * 223.19$ / FLAG-PbO2,
$\mathrm{Bi}+3 * 232.97$ / FLAG-Bi2O3,
$\mathrm{F}-* 18.99$ / FLAG-F,
$\mathrm{SO} 4-2 * 80.06$ / FLAG-SO3,
$(\mathrm{Na}+-(2 * \mathrm{C} 2 \mathrm{O} 4-2(\mathrm{~s}) * \mathrm{PTWF}-\mathrm{C} 2 \mathrm{O} 4)) * 30.99+\mathrm{K}+* 47.09) /$ FLAG-Na2O,
$(\mathrm{AlOH} 3+\mathrm{Al}+3(\mathrm{~s})) * 50.98$ / FLAG-Al2O3,
$(\mathrm{Cr}(\mathrm{Total})+\mathrm{Cr}+3(1)) * 75.99$ / FLAG-Cr2O3,
)

Where:

EG-P = The estimated glass produced from phosphate $(\mathrm{kg})$

EG-O = The estimated glass produced from other wastes in solids $(\mathrm{kg})$

Individual element quanities (i.e., $\mathrm{Fe}+3$ ) units are in $\mathrm{kg}$-mole

FLAG-" $x$ " is the HLW glass limiting oxide value set in G2 as wt fraction units Multiplier values are in $\mathrm{kg}$ oxide / kg-mole element

PTWF-C2O4 is a wash factor for solid oxalate in Pretreatment

If EG-P $\geq 1.01 *$ EG-O and PO4-3 (s) $>0.01 *$ PO4-3(HARD) (s) then

phosphate limited glass $=$ true

else

phosphate limited glass $=$ false

To ensure phase equilibrium, solubility corrections are applied at $45^{\circ} \mathrm{C}$ during wash. It is important to note that oxalate and phosphate tend to dissolve as wash progresses when aqueous phase sodium is washed out, but aluminate tends to precipitate as hydroxide concentration also goes down. Section 4.7.16 states that it is desirable to send more of the soluble constituents to the LAW Facility with the goal of keeping a balance among the WTP mission, IHLW glass production, solids reprecipitation and line plugging factors.

To prevent aluminum precipitation, $19 \mathrm{M} \mathrm{NaOH}(\mathrm{Na}=$ waste sodium) is added to the UFP2 vessel during wash until the current aluminate concentration is less than / equal to $8 \%$ of its concentration at end of solids concentration or re-concentration, e.g., $92 \%$ of the aluminate has been washed out. The caustic is added on an as needed basis to keep aluminate unsaturated. Further discussions are provided later in this section for the sodium dilution and caustic addition operations in UFP-VSL-00062 to prevent solids precipitation. 
Slurries will be concentrated to $20 \mathrm{wt} \%$ solids if possible at the end of the wash. For batches not requiring oxidative leach, skip to solid discharge.

\section{Oxidative Leach}

The oxidative leach decision is made at the end of solids concentration or re-concentration. If "true", the solid wash operations above will continue until $[\mathrm{OH}-] \leq 0.25 \mathrm{M}$ is achieved. It is known that oxidative leach is most effective when $0.2 \mathrm{M} \leq[\mathrm{OH}-] \leq 0.25 \mathrm{M}$. The $\mathrm{G} 2$ model implements a caustic addition operation such that enough caustic $(2 \mathrm{M} \mathrm{NapOH})$ can be added to bring up the $[\mathrm{OH}-]$ concentration if it is below $0.2 \mathrm{M}$. Sodium permanganate ( $1 \mathrm{M}$ NapMnpO4) will be added to further dissolve chromium during oxidative leach. The volume of sodium permanganate added is based on a Mnp to Cr(Total) ratio equals to 1.1. Side reactions will take place that dissolve fractions of the plutonium and its isotopes. Oxidative leach reactions will take 6 hours to complete during which the recirculation loop will be run to enhance mixing, which also results in the contents of the loop being leached. Chromium, plutonium and its isotopes are leached according to the following reactions.

\begin{tabular}{|c|c|}
\hline Reaction & $\begin{array}{c}\text { Conversion } \\
\text { (Fraction) }\end{array}$ \\
\hline $\begin{array}{l}\mathrm{Cr}(\text { Total })(\mathrm{s})+3 \mathrm{OH}(\text { Bound })(\mathrm{s})+\mathrm{Mnp}(\mathrm{l})+4 \mathrm{O}-2(\mathrm{l})+\mathrm{OH}-(\mathrm{l}) \rightarrow \mathrm{Cr}(\text { Total })(\mathrm{l})+ \\
4 \mathrm{O}-2(\mathrm{l})+\mathrm{Mnp}(\mathrm{s})+2 \mathrm{O}-2(\mathrm{~s})+2 \mathrm{H} 2 \mathrm{O}(\mathrm{l})\end{array}$ & $0.876^{\text {a) }}$ \\
\hline $\begin{array}{l}\mathrm{Cr}(\text { Total })(\mathrm{s})+\mathrm{Mnp}(\mathrm{l})+4 \mathrm{O}-2(\mathrm{l})+4 \mathrm{OH}-(\mathrm{l}) \rightarrow \mathrm{Cr}(\text { Total })(\mathrm{l})+ \\
4 \mathrm{O}-2(\mathrm{l})+\mathrm{Mnp}(\mathrm{s})+2 \mathrm{O}-2(\mathrm{~s})+2 \mathrm{H} 2 \mathrm{O}(\mathrm{l})\end{array}$ & variable $^{\text {b) }}$ \\
\hline $\mathrm{Mnp}(\mathrm{l})+4 \mathrm{O}-2(\mathrm{l}) \rightarrow \mathrm{Mnp}(\mathrm{s})+2 \mathrm{O}-2(\mathrm{~s})+2 \mathrm{O}-2(\mathrm{l})$ & $1.0^{\mathrm{c})}$ \\
\hline $238-\mathrm{Pu}(\mathrm{s})+3 \mathrm{OH}($ Bound) (s) + OH- (l) $\rightarrow 238-\mathrm{PU}(\mathrm{l})+4 \mathrm{OH}-(\mathrm{l})$ & 0.01 \\
\hline $239-\mathrm{Pu}(\mathrm{s})+3 \mathrm{OH}($ Bound $)(\mathrm{s})+\mathrm{OH}-(\mathrm{l}) \rightarrow 239-\mathrm{PU}(\mathrm{l})+4 \mathrm{OH}-(\mathrm{l})$ & 0.01 \\
\hline $240-\mathrm{Pu}(\mathrm{s})+3 \mathrm{OH}($ Bound $)(\mathrm{s})+\mathrm{OH}-(\mathrm{l}) \rightarrow 240-\mathrm{PU}(\mathrm{l})+4 \mathrm{OH}-(\mathrm{l})$ & 0.01 \\
\hline $241-\mathrm{Pu}(\mathrm{s})+3 \mathrm{OH}($ Bound) (s) + OH- (l) $\rightarrow$ 241-PU (l) $+4 \mathrm{OH}-(\mathrm{l})$ & 0.01 \\
\hline $242-\mathrm{Pu}(\mathrm{s})+3 \mathrm{OH}($ Bound) (s) + OH- (l) $\rightarrow$ 242-PU (l) +4 OH- (l) & 0.01 \\
\hline $\mathrm{Pu}+4(\mathrm{~s})+3 \mathrm{OH}($ Bound) $(\mathrm{s})+\mathrm{OH}-(\mathrm{l}) \rightarrow \mathrm{PU}+4(\mathrm{l})+4 \mathrm{OH}-(\mathrm{l})$ & 0.01 \\
\hline
\end{tabular}

Notes:

a) Conversion reaches 0.876 if the batch is not $\mathrm{OH}(\mathrm{Bound})$ limiting.

b) Needed only if $\mathrm{OH}$ (Bound) is limiting.

c) Remaining permanganate is $100 \%$ oxidized.

AFA will not be added during oxidative leaching. Existing PPG and PDMS will decompose to DPPG and DPDMS completely.

\section{Oxidative Wash}

AFA will be added immediately after oxidative leaching and replenished every other 6-hours until the end of the current UFP2 cycle. Target concentrations for PPG and PDMS are discussed in Section 4.7.17. Oxidative wash is achieved by pumping and filling the UFP2 vessel with process condensate (primary source) or process water (secondary source) in a 1000-gallon increment in a region between the volume at the end of oxidative leach $\left(\mathrm{V}_{\mathrm{OX}}\right)$ and $\mathrm{V}_{\mathrm{OX}}+1000$ gallons. UFP2 filling rates are $250 \mathrm{gpm}$ with process condensate and $73 \mathrm{gpm}$ with water. Wash volume is either $2 \mathrm{x} \mathrm{V \_ slurry} \mathrm{or} 3 \times \mathrm{V}$ _slurry as determined when the oxidative leach decisions are made. Permeate is routed to the PWD-VSL-00015 and PWDVSL-00016 vessels. Solubility corrections are applied at $45{ }^{\circ} \mathrm{C}$ during oxidative wash. Slurries will be concentrated to $20 \mathrm{wt} \%$ solids at the end of oxidative wash if possible. 


\section{$\underline{\text { Solid Discharge }}$}

Concentrated solids will be discharged to the HLP-VSL-00027A/B vessels with the positive displacement pumps. At the end of solid discharge, e.g., vessel volume $=500$ gallons, the filter loop is power flushed with 2,076 gallons of inhibited water. Flushing efficiency equals to 0.85 , e.g., $85 \%$ of the slurry in the loop are displaced by the flush solution. Contents from the loop go back to the UFP2 vessel. The power flush operation takes 0.183 hours.

Contents of the UFP2 vessel (Non-Newtonian fluids) from the power flush operation will be ejected to HLP-VSL-00027A/B again by the positive displacement pumps. The transfer line is flushed with 150 gallons of inhibited water at $90 \mathrm{gpm}$ after the displacement pumping. Contents of the filter loop including the pre-isolation and post-isolation valve sections are drained to PWD-VSL-00033 at $50 \mathrm{gpm}$.

\section{$\underline{\text { Acid Clean }}$}

Ultrafilters will be acid cleaned after 500 hours of operation i.e., cumulative usage that excludes idle times, or after every four UFP2 cycles, whichever comes first (note: the filters will continue to be used if the UFP2 vessel has not finished wash). At the end of solid discharge, e.g., after the first power flush operation described above, the filter loop will be power flushed with 2,076 gallons of inhibited water a second time. Flushing efficiency equals to 0.85 , e.g., $85 \%$ of the slurry in the loop are displaced by the flush solution. Contents from the loop go back to the UFP2 vessel. The power flush operation takes 0.183 hours.

Contents of the UFP2 vessel (Non-Newtonian Fluids) from the power flush operation will be transferred to HLP-VSL-00027A/B by the positive displacement pumps. The transfer line is flushed with 150 gallons of inhibited water at $90 \mathrm{gpm}$ after the displacement pumping. Contents of the filter loop including the pre-isolation and post-isolation valve sections are drained to PWD-VSL-00033 at $50 \mathrm{gpm}$.

The UFP2 vessel will be filled with 17,076 gallons of 2M nitric acid which will then be circulated in the loop for 1.5 hours. The acid clean solutions (Newtonian Fluids) will be transferred to PWD-VSL$00015 / 16$ with the positive displacement pump.

Contents of the filter loop including the pre-isolation and post-isolation valve sections are drained to PWD-VSL-00033 at 80 gpm. Remaining acidic contents in the UFP2 vessel will be neutralized with 50 gallons of $19 \mathrm{M}$ caustic (NapOH) based on the reaction below:

$\mathrm{H}+(\mathrm{l})+\mathrm{OH}-(\mathrm{l}) \rightarrow \mathrm{H} 2 \mathrm{O}(\mathrm{l})$

After acid neutralization, the UFP2 vessel will be available to start the next cycle.

\begin{tabular}{|l|l|}
\hline Vessel Tag \# & Equipment Description \\
\hline UFP-VSL-00062A & $\begin{array}{l}\text { Ultrafiltration Permeate Collection Vessel - } \\
\text { Supertank combines UFP-VSL-00062A/B/C } \\
\text { and CXP-VSL-00004 operations }\end{array}$ \\
\hline
\end{tabular}

G2 model version 7.0 implements a WTP design modification that mitigates solids precipitation for feeds to the ion exchange columns (IXC). In this design modification ultrafiltration permeates are routed to the Permeate Collection Vessels (UFP-VSL-00062A/B/C), then to CXP-VSL-00004. Wash recycle and oxidative wash permeates are routed directly to PWD-VSL-00015 and PWD-VSL-00016. CXP-VSL00004 will discharge to the IXC through a guard file (CXP-FILT-00001). 
Permeate exiting CXP-FILT-00001 will be split into two streams, one is fed forward to the IXC while the other is recycled through a heat exchanger. The stream through the heat exchanger will be split again, one part goes directly to CXP-VSL-00004 and another part to UFP-VSL-00062A/B/C. The recycle operation mixes the UFP-VSL-00062A/B/C and CXP-VSL-00004 contents to virtually identical compositions and same temperature, as a result, a "Supertank" is created in the model to represent this group-of-four vessels.

The Supertank which combines the working volumes of UFP-VSL-00062A/B/C and CXP-VSL-00004 receives solids concentration, reconcentration, and wash forward permeates and discharges directly to the guard filter. When the ultrafilter trains are working in parallel, both permeate streams are placed in the "Super Tank" at the same time. It operates at $50{ }^{\circ} \mathrm{C}$ in a continuous mode (although solubilities are applied at $45^{\circ} \mathrm{C}$ due to lack of data for correlation curve fits), meaning that it can receive and discharge at the same time. The Supertank is named after the UFP-VSL-00062A vessel with the following working volumes:

$\mathrm{V}_{\min } \quad=11,755$ gallons

$\mathrm{V}_{\text {set }} \quad=11,800$ gallons

$\mathrm{V}_{\max }=78,775$ gallons

A headspace (15,000 gallons) is reserved for the sodium dilution and caustic addition operations below and the ion exchange displacement operations in Section 4.8.4.

\section{$\underline{\text { Sodium Dilution }}$}

The feed to CXP must meet a maximum sodium concentration target. Process condensate (primary source) or process water (secondary source) will be added for dilution if sodium concentration in the vessel is greater than $8 \mathrm{M}$. Filling rates are $250 \mathrm{gpm}$ with process condensate and $73 \mathrm{gpm}$ with water. Volumes for dilution vary from batch to batch depending on conditions of the ultrafilter permeates as well as the caustic addition operations below. Dilution will continue until $\left[\mathrm{Na}^{+}\right] \leq 8 \mathrm{M}$ or the maximum volume of the vessel has been reached.

Process condensate or process water will also be added to prevent oxalate and phosphate precipitation. The volume of water added is determined to ensure that dissolved $\mathrm{C}_{2} \mathrm{O}_{4}{ }^{-2}$ and $\mathrm{PO}_{4}{ }^{-3}$ are unsaturated or at equilibrium.

\section{Caustic Addition}

Aluminate tends to precipitate when $\mathrm{OH}^{-}$concentration is diluted as the result of sodium dilution above. Provided that the maximum volume of vessel is not reached, enough caustic $(19 \mathrm{M} \mathrm{NaOH}$, defined as waste sodium) will be added to prevent the following reaction from taking place:

$\mathrm{Al}(\mathrm{OH})_{4}^{-}(\mathrm{l}) \rightarrow \mathrm{Al}(\mathrm{OH})_{3}(\mathrm{~s})+\mathrm{OH}^{-}(\mathrm{l})$

This caustic addition may increase the sodium concentration to above the $8 \mathrm{M}$ target value.

An iterative approach is taken to continue the sodium dilution and caustic addition operations until sodium concentration $\leq 8 \mathrm{M}$ and $\mathrm{C}_{2} \mathrm{O}_{4}{ }^{-2}, \mathrm{PO}_{4}{ }^{-3}$, and $\mathrm{Al}(\mathrm{OH})_{4}{ }^{-}$do not precipitate or the maximum volume of the vessel is reached. Because the "Super Tank" is modeled as a continuous tank, dilution and caustic addition are evaluated continuously when it is running. 
The Supertank discharges to the ion exchange columns through CXP-FILT-00001 at a fixed flow rate at $30 \mathrm{gpm}$.

Figure F-5 provides a graphical illustration of the UFP system where connections with upstream and downstream systems are shown. Table N-1 provides the totalizer files that are used to track the process streams and material flows into and out of the UFP.

\subsubsection{Cesium Removal Using Ion Exchange Process (CXP) System}

The purpose of the Cesium Ion Exchange System is to remove cesium (Cs-134, Cs-137, and Cs+) that is in the solution. Because cesium is soluble, it cannot be removed by ultrafiltration and an ion exchange (IX) process is used. These elements need to be removed to meet LAW vitrification shielding and glass product requirements, and are sent to the HLW melter.

\begin{tabular}{|l|l|}
\hline Vessel Tag \# & Equipment Description \\
\hline CXP-VSL-00004 & Cesium Ion Exchange Caustic Rinse Collection Vessel \\
\hline CXP-FILT-00001 & Cesium Ion Exchange Guard Filter \\
\hline
\end{tabular}

The WTP has implemented design changes that remove CXP-VSL-00001 from the flowsheet, utilize CXP-VSL-00004 as the cesium ion exchange feed vessel, utilize CXP-VSL-00005 to supply caustic for IX displacement, add heating and recycling equipment for mixing of ion exchange feeds, and add an inline guard filter between CXP-VSL-00004 and the IX columns (see Figure F-8 for illustration). The Cesium Ion Exchange Caustic Rinse Collection Vessel now receives feeds from UFP-VSL-00062A, UFPVSL-00062B, and UFP-VSL-00062C. One main objective of the heating and recycling operations is to mix permeate from various UFP2 operations such as solids concentration, reconcentration, and wash forward in the group-of-four vessels. For modeling simplicity, the group-of-four vessels are combined into a large vessel called "Super Tank". For details of the "Super Tank" operations, refer to Section 4.8.3.

Feeds to the IX columns from the Super Tank are routed through CXP-FILT-00001. The CXP-FILT00001 filter housing has a 117-gallon holding capacity. The filter housing is empty before startup and after each back flush operation. It is assumed that $100 \%$ of the solids from the Super Tank is captured by CXP-FILT-00001. It is anticipated that filter cakes need to be removed by the back flush operations after 34 UFP2 batches have been processed (here a UFP2 batch is defined as the completion of a solids concentration cycle by an ultrafiltration train).

During model runs, the current-batch attribute of CXP-FILT-00001 increments after each UFP2 batch is processed. A filter back flush analyzer logic procedure keeps track of the current-batch value continuously. When current-batch reaches the target at 34, a back flush operation is triggered. During a back flush, all the liquid and solid in CXP-FILT-00001 are transferred to PWD-VSL-00016, along with 80 gallons of $0.1 \mathrm{M}$ NapOH. The back flush operation takes 0.25 hours. The transfer is made as long as PWD-VSL-00016 has sufficient storage to receive, regardless if the vessel is currently in receive or discharge mode. pH adjustment will not be made in PWD-VSL-00016 after a transfer if it receives in discharge mode due to the use of diluted caustic for back flush.

At the end of a back flush, the back flush clock starts to tick. If 24 hours have elapsed and current-batch is still at 34 (e.g., UFP2 has not finished the current solids concentration cycle), then a second back flush will be performed. This 24-hour interval back flush continues until current-batch do not equal to 34 (current-batch is reset to 0 at the end of batch 34). 


\begin{tabular}{|l|l|}
\hline Vessel Tag \# & Equipment Description \\
\hline CXP-IXC-00001 & Cesium Ion Exchange Column \\
\hline CXP-IXC-00002 & Cesium Ion Exchange Column \\
\hline CXP-IXC-00003 & Cesium Ion Exchange Column \\
\hline CXP-IXC-00004 & Cesium Ion Exchange Column \\
\hline
\end{tabular}

The cesium ion exchange columns operate in carousel patterned cycles such that the loading columns are in "lead", "lag", and "polishing" positions while the regenerating column goes through displacement, preelution rinse, elution, post-elution rinse, and regeneration. Once the loading is completed, the lead column enters regeneration, the lag column becomes the lead, the polishing column becomes the lag, and the regenerating column becomes the polishing column.

Each column consists of a 600-gallon resin bed and a 480-gallon headspace. The headspace is initially filled with regeneration solutions $(0.5 \mathrm{M} \mathrm{NapOH})$. It is assumed that plug flow occurs within the resin bed region while mixing can occur in the headspace.

Feed from the in-line guard filter is routed through the loading columns in the lead, lag, and polishing order. The feed pump runs at a fixed flow rate at $30 \mathrm{gpm}$. In the beginning of each loading cycle, 1,500 gallons, e.g., 2.5 column volumes (cv) of ion exchange effluent will be recycled to the supertank from the polishing column. After that, treated waste from the polishing column is sent to CXP-VSL-00026A, CXP-VSL-00026B, and CXP-VSL-00026C. Overall cesium removal efficiency of the loading columns equals to $99.9766 \%$. For modeling purpose, it is assumed that ion exchange only occurs within the lead column. Loading volume is determined based on the cesium and potassium concentrations of the feed at the start of a cycle (Equations 39, 40, and 41).

$\mathrm{A}=55214-80312 \times[\mathrm{K}+]$

$\mathrm{B}=342375-553228 \times[\mathrm{K}+]$

$\mathrm{V}_{\text {load }}=-\mathrm{A} \times \operatorname{Ln}([\mathrm{Cs}+])-\mathrm{B}$

Where:
A $\quad=$ Constant (gallons)
$\mathrm{B} \quad=$ Constant (gallons)
$[\mathrm{K}+] \quad=$ Waste feed potassium concentration $(\mathrm{gmol} / \mathrm{L})$
$[\mathrm{Cs}+] \quad=$ Waste feed overall cesium concentration $(\mathrm{gmol} / \mathrm{L})$

The loading cycle may terminate before $\mathrm{V}_{\text {load }}$ is reached if cumulated radioactivity contributed by 137-Cs reaches 75,000 curries.

The resin bed is packed with Spherical Resorcinol-Formaldehyde Resin (RF) that is saturated with process sodium $(\mathrm{Nap}+)$ initially and during regeneration. At saturation the column contains $4.63 \mathrm{~kg}-$ moles sodium which is equivalent to $6.8 \mathrm{mmole} / \mathrm{g}$ dry $\mathrm{H}$-form resin. Ion exchange takes place where cesium and potassium ions replace the sodium ion during the loading cycle.

$\{\mathrm{R}\}$-Nap (absorbed) + 134-Cs $(\mathrm{l}) \rightarrow\{\mathrm{R}\}$-134-Cs (absorbed) $+\mathrm{Nap}+(\mathrm{l})$

$\{\mathrm{R}\}$-Nap (absorbed) + 137-Cs $(1) \rightarrow\{\mathrm{R}\}$-137-Cs (absorbed) + Nap+ (1)

$\{\mathrm{R}\}$-Nap (absorbed) $+\mathrm{Cs}+(\mathrm{l}) \rightarrow\{\mathrm{R}\}$-Cs (absorbed) $+\mathrm{Nap}+(\mathrm{l})$ 
$\{\mathrm{R}\}$-Nap (absorbed) $+\mathrm{K}+(\mathrm{l}) \rightarrow\{\mathrm{R}\}-\mathrm{K}$ (absorbed) $+\mathrm{Nap}+(\mathrm{l})$

$\{\mathrm{R}\}$-Nap (absorbed) $+137 \mathrm{~m}-\mathrm{Ba}(\mathrm{l}) \rightarrow\{\mathrm{R}\}-137 \mathrm{~m}-\mathrm{Ba}$ (absorbed) $+\mathrm{Nap}+(\mathrm{l})$

Where:

$\{\mathrm{R}\} \quad=\mathrm{RF}$ resin (inert)

For every kg-mole of 134-Cs, 137-Cs, $137 \mathrm{~m}-\mathrm{Ba}, \mathrm{Cs}+$ and $\mathrm{K}+$ captured during ion exchange, $1 \mathrm{~kg}-\mathrm{mole}$ of Nap+ is released from the resin surface. The mass balances of the ion exchange within the lead column are given below:

\section{$\underline{\text { Start of Loading Cycle }}$}

Loading_Nap $+=4.63 \mathrm{~kg}$-moles $/$ column

Loading_Cs134 $=0.0 \mathrm{~kg}$-moles/column

Loading_Cs137 $=0.0 \mathrm{~kg}-\mathrm{moles} /$ column

Loading_Cs $+=0.0 \mathrm{~kg}-\mathrm{moles} /$ column

Loading_mBa137 $=0.0 \mathrm{~kg}-\mathrm{moles} / \mathrm{column}$

Loading_K $+=0.0 \mathrm{~kg}-$ moles $/$ column

\section{End of Loading Cycle}

Loading_Cs134 $=0.999766 \times$ Cs134_Input

Loading_Cs137 $=0.999766 \times$ Cs137_Input

Loading_Cs $+=0.999766 \times \mathrm{Cs}^{+}+$Input

Loading_mBa137 $=1.526 \mathrm{e}-7 \times$ Loading_Cs137

Loading_K $+=100$ percent removal until the end of loading cycle or $\mathrm{Q}_{\mathrm{K}}$ (Eq. 48) reaches Loading_Nap+

$=4.63-$ Loading_Cs134 - Loading_Cs137 - Loading_Cs+ - Loading_K+

The cesium loadings are determined based on the actual cesium input to the lead column from the Supertank. The $\mathrm{Q}_{\mathrm{Cs}}$ in Eq. 45 is not used as the value is estimated using the concentrations of cesium and hydroxide at the start of the loading cycle. This guarantees that the target removal efficiency can be achieved for the entire loading cycle.

Potassium is captured at a $100 \%$ removal efficiency until the end of loading cycle or the maximum loading for $\mathrm{K}+$ is reached whichever comes first. $\mathrm{Q}_{\mathrm{K}}$ is calculated as follows:

$$
\begin{aligned}
& \mathrm{F}_{\mathrm{NaHR}}=\frac{\left(\frac{\mathrm{K}_{\mathrm{w}}}{\left[\mathrm{OH}^{-}\right]}\right)\left(\frac{1}{\mathrm{~K}_{\mathrm{a} 2}}\right)}{\left[1+\left(\frac{\mathrm{K}_{\mathrm{w}}}{\left[\mathrm{OH}^{-}\right]}\right)\left(\frac{1}{\mathrm{~K}_{\mathrm{a} 2}}\right)+\left(\frac{\mathrm{K}_{\mathrm{w}}}{\left[\mathrm{OH}^{-}\right]}\right)^{2}\left(\frac{1}{\mathrm{~K}_{\mathrm{a} 1} \mathrm{~K}_{\mathrm{a} 2}}\right)\right]} \\
& \mathrm{F}_{\mathrm{Na} 2 \mathrm{R}}=\frac{1}{\left[1+\left(\frac{\mathrm{K}_{\mathrm{w}}}{\left[\mathrm{OH}^{-}\right]}\right)\left(\frac{1}{\mathrm{~K}_{\mathrm{a} 2}}\right)+\left(\frac{\mathrm{K}_{\mathrm{w}}}{\left[\mathrm{OH}^{-}\right]}\right)^{2}\left(\frac{1}{\mathrm{~K}_{\mathrm{a} 1} \mathrm{~K}_{\mathrm{a} 2}}\right)\right]}
\end{aligned}
$$


$\mathrm{F}_{\mathrm{SA}}=\frac{\mathrm{F}_{\mathrm{NaHR}}+2 \mathrm{~F}_{\mathrm{Na} 2 \mathrm{R}}}{2}$

$\mathrm{Q}_{\mathrm{Cs}}=\frac{\xi_{\mathrm{Cs}}[\mathrm{Cs}+] \mathrm{V}_{\text {load }}}{\rho_{\text {bed }} \mathrm{V}_{\text {bed }}}$

$\frac{\mathrm{Q}_{\mathrm{K}}}{\mathrm{Q}_{\mathrm{Na}}}=(1.52)\left(\left.\frac{[\mathrm{K}+]}{[\mathrm{Na}+]}\right|_{\text {Waste }}\right)^{0.696}$

$\mathrm{Q}_{\mathrm{Na}}=\frac{\left(\mathrm{F}_{\mathrm{SA}} \mathrm{Q}_{\text {total }}-\mathrm{Q}_{\mathrm{Cs}}\right)}{\left(\frac{\mathrm{Q}_{\mathrm{K}}}{\mathrm{Q}_{\mathrm{Na}}}+1\right)}$

$\mathrm{Q}_{\mathrm{K}}=\left(\mathrm{F}_{\mathrm{SA}} \mathrm{Q}_{\text {total }}-\mathrm{Q}_{\mathrm{Cs}}-\mathrm{Q}_{\mathrm{Na}}\right) * 2271 * 0.3 / 1000$

Where:

$\mathrm{K}_{\mathrm{a} 1} \quad=10^{-10.6}(\mathrm{gmol} / \mathrm{L})$

$\mathrm{K}_{\mathrm{a} 2} \quad=10^{-13.2}(\mathrm{gmol} / \mathrm{L})$

$\mathrm{K}_{\mathrm{w}} \quad=10^{-14}\left(\mathrm{gmol}^{2} / \mathrm{L}^{2}\right)$

$\mathrm{V}_{\text {load }} \quad=$ Calculated using Eq. 41

$\xi_{\mathrm{Cs}} \quad=$ Cesium removal efficiency $(0.999766)$

$\mathrm{F}_{\mathrm{SA}} \quad=$ Resin disassociation constant (unitless)

$\rho_{\text {bed }} \quad=0.3(\mathrm{~kg} \mathrm{H}$-form resin/L wet Na-form resin)

$\mathrm{V}_{\text {bed }} \quad=2271(\mathrm{~L})$

$\mathrm{Q}_{\text {total }} \quad=6.8(\mathrm{mmol} / \mathrm{g}$ dry H-form resin $)$

$[\mathrm{OH}-],[134-\mathrm{Cs}],[137-\mathrm{Cs}],[\mathrm{Cs}+],[\mathrm{K}+]$, and $\left[\mathrm{Na}^{+}\right]$are concentrations of feed at the start of a loading cycle.

At the end of a loading cycle, resins are regenerated following the steps below:

1) Displacement

2) Resin outlet flush \#1

3) Pre-elution rinse

4) Elution

5) Resin outlet flush \#2

6) Post-elution rinse

7) Resin outlet flush \#3

8) Regeneration

9) Regeneration fluid displacement

10) Resin outlet flush \#4

The displacement cycle is performed in order to prevent aluminum precipitation during the pre-elution rinse cycle. During the displacement cycle, $0.1 \mathrm{M}$ NapOH that comes from CXP-VSL-00005 is routed through the column to displace the pore liquids. Three column volumes of fluid are processed through the 
column at a rate of $3 \mathrm{CV} / \mathrm{hr}$. The displaced solution is routed to the Supertank. At the end of displacement, the lead column outlet is flushed the first time with 21 gallons of demineralized water. The outlet is flushed at 30 gpm flow rate and is routed to the Supertank.

The pre-elution rinse cycle is performed in order to prevent acidic eluant solution from reacting during elution with caustic waste solution residing in the column. During the pre-elution rinse cycle, demineralized water from a chem-add is routed to the column to remove the pore liquids. Two CV of DIW are processed at a rate of $3 \mathrm{CV} / \mathrm{hr}$. The exiting material is routed to PWD-VSL-00015 or PWDVSL-00016, depending on their availability. After $2 \mathrm{CV}$ of pre-elution rinse DIW is processed, the elution cycle starts.

During the elution cycle, $15 \mathrm{CV}$ of $0.45 \mathrm{M} \mathrm{HNO}_{3}$ from the Cesium Evaporator Recovered Nitric Acid Vessel (CNP-VSL-00004) is routed to the column at a rate of $1 \mathrm{CV} / \mathrm{hr}$. Ion exchange takes place where hydrogen ions replace cesium, potassium, and sodium ions. Ion exchange rates are charge balanced and assumed to be linear throughout the elution cycle.

$$
\begin{aligned}
& \{\mathrm{R}\}-134-\mathrm{Cs} \text { (absorbed) }+\mathrm{H}+(\mathrm{l}) \rightarrow\{\mathrm{R}\}-\mathrm{H}+134-\mathrm{Cs}(\mathrm{l}) \\
& \{\mathrm{R}\}-137-\mathrm{Cs}(\text { absorbed) }+\mathrm{H}+(\mathrm{l}) \rightarrow\{\mathrm{R}\}-\mathrm{H}+137-\mathrm{Cs}(\mathrm{l}) \\
& \{\mathrm{R}\}-\mathrm{Cs}(\text { absorbed) }+\mathrm{H}+(\mathrm{l}) \rightarrow\{\mathrm{R}\}-\mathrm{H}+\mathrm{Cs}+(\mathrm{l}) \\
& \{\mathrm{R}\}-\mathrm{K} \text { (absorbed) }+\mathrm{H}+(\mathrm{l}) \rightarrow\{\mathrm{R}\}-\mathrm{H}+\mathrm{K}+(1) \\
& \{\mathrm{R}\}-137 \mathrm{~m}-\mathrm{Ba} \text { (absorbed) }+\mathrm{H}+(\mathrm{l}) \rightarrow\{\mathrm{R}\}-\mathrm{H}+137 \mathrm{~m}-\mathrm{Ba}(\mathrm{l}) \\
& \{\mathrm{R}\}-\mathrm{Nap} \text { (absorbed) }+\mathrm{H}+(\mathrm{l}) \rightarrow\{\mathrm{R}\}-\mathrm{H}+\mathrm{Nap}+
\end{aligned}
$$

For every kg-mole of $\mathrm{H}+$ captured, $1 \mathrm{~kg}-$ mole of $134-\mathrm{Cs}, 137-\mathrm{Cs}, \mathrm{Cs}+, 137 \mathrm{~m}-\mathrm{Ba}, \mathrm{K}+$, and $\mathrm{Nap}+$ is released from the resin surface. The exiting material goes to CNP-EVAP-00001. At the end of elution, the lead column outlet is flushed the second time with 21 gallons of $0.45 \mathrm{M} \mathrm{HNO}_{3}$. The outlet is flushed at $30 \mathrm{gpm}$ flow rate and is routed to CNP-EVAP-00001.

The post-elution rinse cycle is performed in order to prevent caustic waste solution from reacting with acidic eluant solution residing in the column. During the post-elution rinse cycle, DIW is routed to the column to rinse off the pore liquids. Two and a half CV of DIW are processed at a rate of $1 \mathrm{CV} / \mathrm{hr}$. The rinse material is routed to CNP-EVAP-00001. At the end of post-elution rinse, the lead column outlet is flushed the third time with 21 gallons of $0.5 \mathrm{M} N a p O H$. The outlet is flushed at $30 \mathrm{gpm}$ flow rate and is routed to CNP-EVAP-00001.

During the regeneration cycle, $4.292 \mathrm{CV}$ of $0.5 \mathrm{M} \mathrm{NapOH}$ from a chem-add is fed to the column at a rate of $2.78 \mathrm{CV} / \mathrm{hr}$. Ion exchange takes place where sodium ions replace the hydrogen ions.

$$
\{\mathrm{R}\}-\mathrm{H}(\text { absorbed) }+\mathrm{Nap}+(\mathrm{l}) \rightarrow\{\mathrm{R}\}-\mathrm{Nap} \text { (absorbed) }+\mathrm{H}+(\mathrm{l})
$$

For every kg-mole of Nap+ captured, $1 \mathrm{~kg}$-mole of $\mathrm{H}+$ is released from the resin surface. The exiting material goes to PWD-VSL-00015 or PWD-VSL-00016, depending on their availability.

A regeneration displacement is performed after the regeneration cycle. This is accomplished by diverting the treated LAW products from the current polishing column outlet to the regeneration column. A total of 1,250 gallons of treated LAW will be pumped through the regeneration column with the first 1,050 gallons routed to the available PWD-VSL-00015/16 at $0.94 \mathrm{CV} / \mathrm{hr}$ and the remaining 200 gallons routed to "Super Tank" at $1.5 \mathrm{CV} / \mathrm{hr}$. A fourth resin outlet flush is performed at the end of regeneration displacement. This is accomplished by expanding the second part of the regeneration displacement with additional 54 gallons of treated LAW. The exiting material is routed to the "Super Tank" at $1.5 \mathrm{CV} / \mathrm{hr}$. 
At the end of the last resin outlet flush, the treated LAW is switched back to the CXP-VSL-00026A/B/C vessel currently available while the regeneration column must wait another 0.5 hours before it can be put on line for the next loading cycle. With the regeneration steps complete, the column is placed in stand-by until the "lead" column is ready for regeneration.

After a column has gone through the regeneration process 40 times, the resin must be changed out and two extra steps are added. In the first step, 7,500 gallons of resin transfer solution is used to flush the spent resin out of the regenerating column. The makeup of the resin transfer solution is discussed in Section 4.8.6. The spent resin, along with 7,085 gallons of transfer solution, is pumped to RDP-VSL-00002B while the remaining solution is delivered to RDP-VSL-00002A. In the second step, 1,140 gallons of fresh resin transfer solution is pumped through the regenerating column from CRP-VSL-00001. The fresh resin is deposited in the column while the transfer solution is sent to RDP-VSL-00002A. Further details regarding the fresh resin preparation and spent resin deposal are discussed in Sections 4.8.6 and 4.8.7, respectively.

Chemical reagent concentrations and effluent dispositions for the regenerating steps are presented in Table 14 and the ion exchange operating parameters are summarized in Table 15.

Table 14 Chemical Reagents for Resin Regeneration

\begin{tabular}{|l|c|c|c|}
\hline Cycles & Reagents & Concentrations & Effluent Disposition \\
\hline Displacement & $\mathrm{NapOH}$ & $0.1 \mathrm{M}$ & Supertank \\
\hline Pre-Elution Rinse & $\mathrm{DIW}$ & $\mathrm{N} / \mathrm{A}$ & PWD-VSL-00015/16 \\
\hline Elution & $\mathrm{HNO}_{3}$ & $0.45 \mathrm{M}$ & CNP-EVAP-00001 \\
\hline Post-Elution Rinse & $\mathrm{DIW}$ & N/A & CNP-EVAP-00001 \\
\hline Regeneration & NapOH & $0.5 \mathrm{M}$ & PWD-VSL-00015/16 \\
\hline Regeneration Displacement - Part 1 & Treated LAW & N/A & PWD-VSL-00015/16 \\
\hline Regeneration Displacement - Part 2 & Treated LAW & N/A & Supertank \\
\hline
\end{tabular}

Table 15 Ion Exchange Operating Parameters

\begin{tabular}{|l|c|}
\hline Parameter & Value \\
\hline Column Headspace Volume (gallons) & 480 \\
\hline Column Volume (gallons) & 600 \\
\hline Loading Volume (cv) & $\begin{array}{c}\text { Min (Eq. 41, cumulated } \\
\text { radioactivity } \geq 75,000 \\
\text { curries) }\end{array}$ \\
\hline Loading Rate (cv/hr) & 3 \\
\hline Displacement Volume (cv) & 3 \\
\hline Displacement Rate (cv/hr) & 2 \\
\hline Pre-Elution Rinse Volume (cv) & 3 \\
\hline Pre-Elution Rinse Rate (cv/hr) & $3.5)$ \\
\hline
\end{tabular}




\begin{tabular}{|l|c|}
\hline Parameter & Value \\
\hline Elution Volume (cv) & 15 \\
\hline Elution Rate (cv/hr) & 1 \\
\hline Post-Elution Rinse Volume (cv) & 2.5 \\
\hline Post-Elution Rinse Rate (cv/hr) & 1 \\
\hline Regeneration Volume (cv) & 4.292 \\
\hline Regeneration Rate (cv/hr) & 2.78 \\
\hline Regen Displacement Volume - Part 1 (gal) & 1,050 \\
\hline Regen Displacement Rate - Part 1 (cv/hr) & 0.94 \\
\hline Regen Displacement Volume - Part 2 (gal) & 200 \\
\hline Regen Displacement Rate - Part 2 (cv/hr) & 1.5 \\
\hline
\end{tabular}

\begin{tabular}{|l|l|}
\hline Vessel Tag \# & Equipment Description \\
\hline CXP-VSL-00005 & Cesium Reagent Vessel \\
\hline
\end{tabular}

The Cesium Reagent Vessel is initially filled up with $0.1 \mathrm{M}$ NapOH from a chem-add source. The solution is used for ion exchange column displacement as described previously. The vessel is replenished to its maximum level when the displacement solution is used.

\begin{tabular}{|l|l|}
\hline Vessel Tag \# & Equipment Description \\
\hline CXP-VSL-00026A & Treated LAW Collection Vessel \\
\hline CXP-VSL-00026B & Treated LAW Collection Vessel \\
\hline CXP-VSL-00026C & Treated LAW Collection Vessel \\
\hline
\end{tabular}

The Treated LAW Collection Vessels receive feed from the CXP column that is polishing. Once the set volume is reached, the vessel is sampled and held for the prescribed sample time. Solubility correlations are applied at $45^{\circ} \mathrm{C}$ before the vessel can be discharged. The Treated LAW Collection Vessels transfer feed to TLP-SEP-00001 one at a time. Flowrate is set to $34 \mathrm{gpm}$ for feed-only pumping or $30 \mathrm{gpm}$ for dual pumping. Feed-only pumping is performed when recycles from TLP-VSL-00009A and TLP-VSL00009B are not ready for discharging. Dual pumping is performed when both treated LAW feed and recycle are fed to the TLP evaporator. Details of the dual pumping logic are discussed in Sections 4.8.8 and 4.8.9.

Figures F-8 and F-11 provide graphical illustrations of the CXP system where connections with upstream and downstream systems are shown. Table N-1 provides the totalizer files that are used to track the process streams and material flows into and out of the CXP.

\subsubsection{Cesium Nitric Acid Recovery Process (CNP) System}

\begin{tabular}{|l|l|}
\hline Vessel Tag \# & Equipment Description \\
\hline CNP-EVAP-00001 & Cesium Evaporator Separator Vessel \\
\hline CNP-DISTC-00001 & Cesium Evaporator Nitric Acid Rectifier \\
\hline CNP-VSL-00003 & Cesium Concentrate Storage Vessel \\
\hline CNP-VSL-00004 & Cesium Evaporator Recovered Nitric Acid Vessel \\
\hline
\end{tabular}


The process steps for the operation of the CNP are presented below:

1) CNP-EVAP-00001 is charged with 2,128 gallons of $5 \mathrm{M} \mathrm{HNO}_{3}$ from a chem-add source.

2) Waste from the CXP column elution and post-elution rinse is routed to CNP-EVAP-00001.

3) The rectifier column is modeled based on a material balance typical for a simple distillation tower (i.e., the column performance is based on the nitric acid recovery efficiency and bottoms composition). The $\mathrm{HNO}_{3}$ recovery efficiency is applied to the $\mathrm{G} 2$ model at $99.5 \%$. The split of water in CNP-DISTC-00001 is dependent upon the amount of $\left[\mathrm{H}^{+}\right]$and $\left[\mathrm{NO}_{3}{ }^{-}\right]$in the column so that the resulting solution to CNP-VSL-00004 is $0.5 \mathrm{M} \mathrm{HNO}_{3}$. Distillate is recycled to PWD-VSL-00015 or PWD-VSL-00016 depending on their availability.

4) CNP-VSL-00004 provides the source of nitric acid for CXP column resin elution. The vessel receives recycle from the rectifier column and operates between the upper set volume at 7,342 gallons and the lower set volume at 5,342 gallons. The vessel is filled initially to the lower set volume with $0.45 \mathrm{M} \mathrm{HNO}_{3}$ from a chem-add source and is replenished when the volume reaches below the lower set volume.

5) Once the $Z$ value in Eq. 51 reaches above the $80 \%$ saturation limit, the evaporator makes itself available to empty (step 6), until then the evaporator continues to collect eluant and rinse solution (step 2).

6) The contents of the evaporator are discharged to CNP-VSL-00003, then to HLP-VSL-00028 or HLPVSL-00027B in priority order depending on the following conditions: a) If HLP-VSL-00028 is in the beginning of a "fill" cycle, for example the volume is at or slightly above the 27,000 -gallon heel, b) If HLP-VSL-00027B is in "fill" mode, and the volume of HLP-VSL-00027B is greater than 61,800 gallons, and the remaining volume of HLP-VSL-00027B can receive up to 2,615 gallons of CNP concentrate plus the associated caustic for neutralization. The maximum volume of CNP-VSL-00003 that can be discharged in one batch is 2,615 gallons, which limits the reaction heat generated from the neutralization. Before discharging, 19M NapOH will be added to the receiving HLW Lag Storage or Feed Blending Process System (HLP) vessel. The volume of caustic is determined based on an OHto $\mathrm{H}+$ ratio $=1.1$ to 1 , excluding the $\mathrm{OH}$ - already in the receiving HLP vessel. Refer to Section 4.8.12 for further details on acid neutralization.

7) The evaporator sets itself to recharge with acid (step 1).

Eluant is boiled off from CNP-EVAP-00001 and is routed to CNP-DISTC-00001 at the same rate that material enters CNP-EVAP-00001. The flowrate into and out of the evaporator will be the same, maintaining a constant level in the evaporator. Equations 46 through 49 are used to calculate the liquid-vapor equilibrium between the concentrate and overheads in CNP-EVAP-00001.

Acid activity coefficient for $\mathrm{HNO}_{3}-\mathrm{H}_{2} \mathrm{O}$ system

$$
-\log _{10}\left(\gamma_{A W}\right)=\left(\frac{1}{A\left(\frac{x_{c}^{a}}{x_{c}^{w}}\right)+B}\right)^{2}
$$

Influence of solute on acid activity coefficient

$$
\frac{\gamma_{a}}{\gamma_{A W}}=1+7.812 x_{c}^{n}
$$

Vapor-Liquid Equilibrium between concentrate and overheads

$$
x_{o}^{a}=\frac{\gamma_{a} x_{c}^{a} P_{A}^{s a t}}{P}
$$




$$
\mathrm{P}_{\mathrm{A}}^{\text {sat }}=2.9163 \times 10^{8} \mathrm{e}^{-4592.6 / \mathrm{T}}
$$

Where:

$$
\begin{aligned}
& \gamma_{\mathrm{AW}}=\text { Nitric acid activity coefficient in the binary HNO3-H2O system (dimensionless) } \\
& \mathrm{A} \quad=4.029672 \mathrm{e}^{-444.877899 / \mathrm{T}} \text { (dimensionless) } \\
& \mathrm{B}=0.002114 \times \mathrm{T}-0.093403 \text { (dimensionless) } \\
& \mathrm{X}_{\mathrm{c}}{ }^{\mathrm{a}}=\text { The mole fraction of acid in CNP-EVAP-00001 (the kg-moles of }\left[\mathrm{H}^{+}\right] \text {divided by the sum } \\
& \text { of kg-moles of } \left.\mathrm{H}_{2} \mathrm{O},\left[\mathrm{H}^{+}\right] \text {, and }\left[\mathrm{Na}^{+}\right]+\left[\mathrm{Nap}^{+}\right]\right) \\
& \mathrm{X}_{\mathrm{c}}{ }^{\mathrm{w}}=\text { The mole fraction of water in CNP-EVAP-00001 (the kg-moles of } \mathrm{H}_{2} \mathrm{O} \text { divided by the } \\
& \text { sum of kg-moles of } \mathrm{H}_{2} \mathrm{O},\left[\mathrm{H}^{+}\right] \text {, and }\left[\mathrm{Na}^{+}\right]+\left[\mathrm{Nap}^{+}\right] \text {) } \\
& \text { ya }=\text { Nitric acid activity coefficient relating acid composition of overhead to concentrate } \\
& \text { composition (dimensionless) } \\
& \mathrm{X}_{\mathrm{c}}{ }^{\mathrm{n}}=\text { The mole fraction of sodium in CNP-EVAP-00001 (the kg-moles of }\left[\mathrm{Na}^{+}\right]+\left[\mathrm{Nap}^{+}\right] \\
& \text {divided by the sum of } \mathrm{kg} \text {-moles of } \mathrm{H}_{2} \mathrm{O},\left[\mathrm{H}^{+}\right] \text {, and }\left[\mathrm{Na}^{+}\right]+\left[\mathrm{Nap}^{+}\right] \text {) } \\
& \mathrm{X}_{\mathrm{o}}^{\mathrm{a}}=\text { The mole fraction of acid in the offgas stream of CNP-EVAP-00001 (the kg-moles of } \\
& {\left[\mathrm{H}^{+}\right] \text {divided by the sum of } \mathrm{kg} \text {-moles of } \mathrm{H}_{2} \mathrm{O} \text { and }\left[\mathrm{H}^{+}\right] \text {in the offgas stream) }} \\
& \mathrm{P}=\text { Total system pressure (70 torr) } \\
& \mathrm{T} \quad=\text { Temperature }(328.15 \mathrm{~K})
\end{aligned}
$$

Once the mole fraction of acid $\left(\mathrm{X}_{\mathrm{o}}{ }^{\mathrm{a}}\right.$ for $\left[\mathrm{H}^{+}\right]$and $\left.\left[\mathrm{NO}_{3}{ }^{-}\right]\right)$in the offgas is determined, the kg-moles of $\left[\mathrm{H}^{+}\right]$, [ $\left.\mathrm{NO}_{3}{ }^{-}\right]$and $\mathrm{H}_{2} \mathrm{O}$ boil-off can be calculated. Equations 50 and 51 are used to determine the endpoint concentration equivalent to $80 \%$ saturation limit.

$$
\begin{aligned}
& \mathrm{S}_{\mathrm{sat}}=(0.00657[\mathrm{Al}+3]+0.01215[\mathrm{Ca}+2]+0.01655[\mathrm{Cs}+]+0.1046[\mathrm{Fe}+3]+ \\
& 0.00957[\mathrm{~K}+]+0.00586([\mathrm{Na}+]+[\mathrm{Nap}+])+0.0058[\mathrm{H}+]+ \\
&0.00454[\text { Others }]) \times 100(\mathrm{~g} / \mathrm{mL}) \\
&\left.\mathrm{Z}=\left(\mathrm{S}-\mathrm{H}^{\circ}\right) /\left(\mathrm{S}_{\mathrm{sat}}-\mathrm{H}^{\circ}\right) \times 100 \quad \text { (percent }\right)
\end{aligned}
$$

Where:

$$
\begin{array}{ll}
\mathrm{S}_{\text {sat }} & =\text { saturation at } 20^{\circ} \mathrm{C}(\mathrm{g} / \mathrm{mL}) \\
\mathrm{S} & =\text { saturation }(\mathrm{g} / \mathrm{mL}) \\
\mathrm{Z} & =\text { the approach to saturation (percent) } \\
\mathrm{H}^{\circ} & =\text { initial concentration }(\mathrm{g} / \mathrm{mL}) \\
{[\text { cation total }]} & =\text { the sum of all cations } \\
{[\mathrm{Al}+3]} & =([\mathrm{Al}(\mathrm{OH}) 4-]+[\mathrm{Al}+3]) /[\text { cation total }] \\
{[\mathrm{Ca}+2]} & =[\mathrm{Ca}+2] /[\text { cation total }] \\
{[\mathrm{Cs}+]} & =([134-\mathrm{Cs}]+[137-\mathrm{Cs}]+[\mathrm{Cs}+]) /[\text { cation total }] \\
{[\mathrm{Fe}+3]} & =[\mathrm{Fe}+3] /[\text { cation total }] \\
{[\mathrm{K}+]} & =[\mathrm{K}+] /[\text { cation total }] \\
{[\mathrm{Na}+]} & =[\mathrm{Na}+] /[\text { cation total }] \\
{[\mathrm{Nap}+]} & =[\mathrm{Nap}+] /[\text { cation total }]
\end{array}
$$


$[\mathrm{H}+] \quad=[\mathrm{H}+] /[$ cation total $]$

[Others $] \quad=1-([\mathrm{Al}+3]+[\mathrm{Ca}+2]+[\mathrm{Cs}+]+[\mathrm{Fe}+3]+[\mathrm{K}+]+[\mathrm{Na}+]+[\mathrm{Nap}+]+[\mathrm{H}+])$.

A density correlation specific to solutions dominated by the ternary system of nitric acid, sodium nitrate, and water is required for application in the cesium eluate concentration system. Eq. 52 was derived from binary density data that have been correlated from the $B A R D$. For application, $\mathrm{H}^{+}$is considered present as $\mathrm{HNO}_{3}$ with molecular weight of $63 \mathrm{~g} / \mathrm{gmol}, \mathrm{Na}^{+}$and $\mathrm{Nap}^{+}$is considered present as $\mathrm{NaNO}_{3}$ and $\mathrm{NapNO}_{3}$ with molecular weight of $85 \mathrm{~g} / \mathrm{gmol}$.

$$
\rho=\frac{\mathrm{a}_{0}}{1-\frac{1000\left(\mathrm{a}_{1} \mathrm{x}_{\mathrm{a}}+\mathrm{a}_{2} \mathrm{x}_{\mathrm{n}}\right)}{\left(63 \mathrm{x}_{\mathrm{a}}+85 \mathrm{x}_{\mathrm{n}}+18 \mathrm{x}_{\mathrm{w}}\right)}}
$$

Where:

$$
\begin{array}{ll}
\rho & =\text { Solution density at a specified temperature, } \mathrm{g} / \mathrm{cm}^{3} \\
\mathrm{a}_{0} & =\text { Function of temperature obtained from binary data correlations, } \mathrm{g} / \mathrm{cm}^{3} \\
\mathrm{a}_{1}, \mathrm{a}_{2} & =\text { Functions of temperature obtained from binary data correlations, } \mathrm{g}-\mathrm{L} / \mathrm{gmol}-\mathrm{cm}^{3} \\
\mathrm{x}_{\mathrm{a}} & =\text { Mole fraction of } \mathrm{HNO}_{3} \text { in solution } \\
\mathrm{x}_{\mathrm{n}} & =\text { Mole fraction of } \mathrm{NaNO}_{3} \text { and } \mathrm{NapNO}_{3} \text { in solution } \\
\mathrm{x}_{\mathrm{w}} & =\text { Mole fraction of water in solution }
\end{array}
$$

Data correlations for the temperature functions are shown by Eq. 53. Note that these data correlations were performed based on temperatures in ${ }^{\circ} \mathrm{C}$ and not based on absolute temperatures. The temperature range of applicability is $20^{\circ} \mathrm{C}$ to $100{ }^{\circ} \mathrm{C}$ for $\mathrm{a}_{0}$ and $\mathrm{a}_{1}$, but data limitations restrict $\mathrm{a}_{2}$ to a temperature range of $20^{\circ} \mathrm{C}$ to $55^{\circ} \mathrm{C}$. Therefore, the overall correlation temperature range of applicability is considered limited to $20^{\circ} \mathrm{C}$ to $55^{\circ} \mathrm{C}$.

$$
\begin{aligned}
& \mathrm{a}_{0}=1.0138-4.8625 \times 10^{-4} \mathrm{~T} \mathrm{~g} / \mathrm{cm}^{3} \\
& \mathrm{a}_{1}=0.032152-4.392 \times 10^{-5} \mathrm{~T} \mathrm{~g}-\mathrm{L} / \mathrm{gmol}-\mathrm{cm}^{3} \\
& \mathrm{a}_{2}=0.052057-4.2857 \times 10^{-5} \mathrm{~T} \mathrm{~g}-\mathrm{L} / \mathrm{gmol}-\mathrm{cm}^{3}
\end{aligned}
$$

Where:

$\mathrm{T} \quad=$ Solution temperature, ${ }^{\circ} \mathrm{C}$

Figure F-10 provides a graphical illustration of the CNP system where connections with upstream and downstream systems are shown. Table N-1 provides the totalizer files which that used to track the process streams and material flows into and out of the CNP. 


\subsubsection{Cesium Fresh Resin Addition Process (CRP) System}

\begin{tabular}{|l|l|}
\hline Vessel Tag \# & Equipment Description \\
\hline CRP-VSL-00001 & Cesium Resin Addition Vessel \\
\hline
\end{tabular}

After the resin goes through 40 loading cycles in the CXP columns, spent resin needs to be flushed out and fresh resin needs to be brought in. Resin capacity is set to $4.63 \mathrm{~kg}$-moles/column, equivalent to 600 gallons of $6.8 \mathrm{mmole} / \mathrm{g}$ dry H-form dry resin. The Cesium Resin Addition Vessel (CRP-VSL-00001) performs the following functions for cesium fresh resin preparation and addition.

1) Fill CRP-VSL-00001 with 1,140 gallons of DIW from FRESH-RESIN-WATER.

2) Transfer $4.63 \mathrm{~kg}$-moles of solid H+ RF resin to CRP-VSL-00001 from RF-RESIN, increment the conditioned-resin counter by 600 gallons.

3) Hold resins in CRP-VSL-00001 for 1 hour.

4) Drain CRP-VSL-00001liquid (1,140 gallons) to the first available RLD-VSL-00017A/B vessel.

5) Add 720 gallons of DIW to CRP-VSL-00001 from FRESH-RESIN-WATER.

6) Add 470 gallons of $2 \mathrm{M}$ caustic to CRP-VSL-00001 from FRESH-RESIN-CAUSTIC.

7) Hold resins in CRP-VSL-00001 for 1 hour for $\mathrm{H}$-form resin conversion as follows:

$\mathrm{H}+(\mathrm{s})+\mathrm{OH}-(\mathrm{l}) \rightarrow \mathrm{H} 2 \mathrm{O}(\mathrm{l}) \quad \mathrm{CF}=1.0(\mathrm{OH}-$ limiting, e.g., there are excessive $\mathrm{H}+)$

$\mathrm{Nap}+(1) \rightarrow \mathrm{Nap}+(\mathrm{s}) \quad \mathrm{CF}=1.0$

8) Drain 470 gallons of liquid from CRP-VSL-00001 to the first available RLD-VSL-00017A/B vessel.

9) Add 400 gallons of $2 \mathrm{M}$ caustic to CRP-VSL-00001 from FRESH-RESIN-CAUSTIC.

10) Hold resins in CRP-VSL-00001 for 1 hour for further H-form resin conversion as follows:

$\mathrm{H}+(\mathrm{s})+\mathrm{OH}-(\mathrm{l}) \rightarrow \mathrm{H} 2 \mathrm{O}$ (l) $\quad \mathrm{CF}=1.0(\mathrm{H}+$ limiting, e.g., there are excessive $\mathrm{OH}-)$

$\mathrm{Nap}+(\mathrm{l}) \rightarrow \mathrm{Nap}+(\mathrm{s}) \quad \mathrm{CF}=\mathrm{H}+/ \mathrm{OH}-$ from step 10)

11) Wait until 40 loading cycles have been reached in a cesium ion exchange column.

12) Pump solution to RDP-VSL-00002A through the ion exchange column and transfer the fresh resin to the ion exchange column where the resins are saturated with $4.63 \mathrm{~kg}$-moles of Nap+.

13) Repeat steps 1) through 12).

Figure F-9 provides a graphical illustration of the CRP system where connections with upstream and downstream systems are shown. Table N-1 provides the totalizer files which are used to track the process streams and material flows into and out of the CRP.

\subsubsection{Spent Resin Collection and Dewatering Process (RDP) System}

\begin{tabular}{|l|l|}
\hline Vessel Tag \# & Equipment Description \\
\hline RDP-VSL-00002A & Spent Resin Slurry Vessel \\
\hline RDP-VSL-00002B & Spent Resin Slurry Vessel \\
\hline RDP-VSL-00002C & Spent Resin Slurry Vessel \\
\hline
\end{tabular}

After the resin goes through 40 loading cycles in the IX columns, spent resin needs to be flushed out and fresh resin needs to be brought in. The spent resin slurry vessels perform the following functions: 
1) Fill RDP-VSL-00002A with 7,500 gallons of $0.25 \mathrm{M}$ NapOH from a chem-add initially.

2) Pump 7500 gallons from RDP-VSL-00002A to Cs IX column.

3) Route 7,085 gallons of solution from IX column to RDP-VSL-00002B.

4) Transfer 415 gallons spent resin to RDP-VSL-00002B.

5) Sample RDP-VSL-00002B (48 hours).

6) Pump fresh resin preparation solution (approximately 1,146 gallons) to IX column from CRP-VSL-00001.

7) Flush CRP-VSL-00001 to IX column line with 100 gallons of demin water.

8) Route IX column solution (approximately 415 [spent resin] + 1,146 [fresh resin preparation solution] +100 [DIW] gallons) from IX column to RDP-VSL-00002A.

9) Pump 5,495 gallons of solution from RDP-VSL-00002B to RDP-VSL-00002A.

10) Wait 6 hours.

11) Flush RDP-VSL-0002B to RDP-VSL-00002A line with 800 gallons of DIW.

12) If FEP-VSL-00017A/B is available, pump 2040 gallons of solution from RDP-VSL-00002B to FEP-VSL-00017A/B. If FEP-VSL-00017A/B is not available, pump the solution to RDP-VSL-00002C, wait until FEP-VSL-00017A/B is available, pump from RDP-VSL-00002C to FEP-VSL-00017A/B.

Figure F-9 provides a graphical illustration of the RDP system where connections with upstream and downstream systems are shown. Table N-1 provides the totalizer files that are used to track the process streams and material flows into and out of the RDP.

\subsubsection{Treated LAW Evaporation Process (TLP) System}

\begin{tabular}{|l|l|}
\hline Vessel Tag \# & Equipment Description \\
\hline TLP-VSL-00009A & LAW SBS Condensate Receipt Vessel \\
\hline TLP-VSL-00009B & LAW SBS Condensate Receipt Vessel \\
\hline
\end{tabular}

The two LAW SBS Condensate Receipt Vessels will alternate operating modes between "receive" and "discharge" (e.g., one is to be filled or being filled while the other is to be emptied or being emptied). One at a time, the LAW SBS Condensate Receipt Vessels fill to their set level, sample for the prescribed time, and perform the following reactions:

\begin{tabular}{|l|c|}
\hline Reaction & $\begin{array}{c}\text { Conversion } \\
\text { (Fraction) }\end{array}$ \\
\hline $\mathrm{H} 2 \mathrm{CO} 3(\mathrm{l}) \rightarrow \mathrm{CO} 3-2(\mathrm{l})+2 \mathrm{H}+(\mathrm{l})$ & 1.0 \\
\hline $\mathrm{HCO} 3-(\mathrm{l}) \rightarrow \mathrm{CO} 3-2(\mathrm{l})+\mathrm{H}+(\mathrm{l})$ & 1.0 \\
\hline $\mathrm{NH} 4+(\mathrm{l}) \rightarrow \mathrm{NH} 3(\mathrm{l})+\mathrm{H}+(\mathrm{l})$ & 1.0 \\
\hline $\mathrm{Hg}+2(\mathrm{l})+2 \mathrm{OH}-(\mathrm{l}) \rightarrow \mathrm{Hg}+2(\mathrm{~s})+\mathrm{O}-2(\mathrm{~s})+\mathrm{H} 2 \mathrm{O}($ Bound $)(\mathrm{s})$ & 1.0 \\
\hline $\mathrm{H}+(\mathrm{l})+\mathrm{OH}-(\mathrm{l}) \rightarrow \mathrm{H} 2 \mathrm{O}(\mathrm{l})$ & 1.0 \\
\hline
\end{tabular}

The $\mathrm{pH}$ of the vessels is adjusted to 12.0 with $5 \mathrm{M}$ NapOH after the reactions and before they can be emptied. The pumping rate for the vessel is set to $10 \mathrm{gpm}$. Under steady state operating conditions, the discharging vessel becomes "empty" before the receiving vessel gets ready for emptying because of the required sampling delay. As soon as the discharging vessel becomes "empty", it is made available to 
receive. This creates a condition that SBS recycles can be received continuously and is the preferred operating condition.

However, steady state conditions rarely exist in the dynamic model due to various factors. There are good possibilities that the receiving vessel reaches the set volume and the discharging vessel is still not "empty". Under such circumstance, the contingency volume of the receiving vessel will be utilized.

In the case that the contingency volume is used up in the receiving vessel and the discharging vessel is still not "empty" under abnormal operating conditions, the discharging vessel will be switched to receiving mode and the receiving vessel will be allowed to discharge.

Although not in the baseline flowsheet design, a supplemental LAW vessel (SUPPLEMENTAL-LAWRECYCLE) is included in the TLP for the option of modeling the additional capacity needed to process the LAW SBS/WESP condensate recycle. Details of the supplemental LAW vessel are noted in Section 4.7.14.

\begin{tabular}{|l|l|}
\hline Vessel Tag \# & Equipment Description \\
\hline TLP-SEP-00001 & Treated LAW Evaporator \\
\hline
\end{tabular}

The Treated LAW Evaporator is modeled to receive feeds from TLP-VSL-00009A or TLP-VSL-00009B, and/or CXP-VSL-00026A, CXP-VSL-00026B, CXP-VSL-00026C, defined as "feed-only", "recycleonly," and "dual-pumping". Feed-only is the operation when TLP-SEP-00001 receives only the treated LAW feed from CXP-VSL-00026A, CXP-VSL-00026B, or CXP-VSL-00026C. Recycle-only is the operation when TLP-SEP-00001 is fed with recycle from TLP-VSL-00009A or TLP-VSL-00009B. Dual-pumping is the operation when TLP-SEP-00001 receives both feed and recycle concurrently. Pump rates for the three operations are given below:

1) Feed-Only

- $\quad$ TLP-VSL-00009A/B = 0 gpm

- $\quad$ CXP-VSL-00026A/B/C = 34 gpm

2) Recycle-Only

- $\quad$ TLP-VSL-00009A/B = 10 gpm

- $\mathrm{CXP}-\mathrm{VSL}-00026 \mathrm{~A} / \mathrm{B} / \mathrm{C}=0 \mathrm{gpm}$

3) Dual-Pumping

- $\quad$ TLP-VSL-00009A/B = $10 \mathrm{gpm}$

- $\quad$ CXP-VSL-00026A/B/C = $30 \mathrm{gpm}$

Dominant operations are feed-only and dual-pumping, while recycle-only can also happen at times when HLW Facility backs up the UFP processes which stops the treated LAW production.

The target for evaporator concentrate is $\mathrm{SpG}=1.33$ and/or a $\mathrm{wt} \%$ solids $=3.4 \%$, whichever comes first. Oxalate and phosphate tend to precipitate in the evaporator after boil-off. Solubility is applied continuously at the flash evaporation temperature $\left(50{ }^{\circ} \mathrm{C}\right)$ which may slightly alter the $\mathrm{SpG}$ and/or wt $\%$ solids values of the TLP evaporator bottom product. The rate of the evaporator is limited by the boil-off rate of $30 \mathrm{gpm}-2 \mathrm{gpm}$ for the evaporator recycle spray to give a modeled boil-off rate of $28 \mathrm{gpm}$. Evaporator concentrate pump capacity is $34 \mathrm{gpm}$. The offgas from TLP-SEP-00001 is routed through TLP-DMST-00001. The splits for the evaporator are shown in Table D-10. 
To minimize foaming, AFA is added to the evaporator when it starts receiving a new batch from a feed tank. AFA will be replenished after that in 1-hour intervals to make up losses due to decomposition and discharge. Target concentrations for PPG and PDMS are discussed in Section 4.7.17.

\begin{tabular}{|l|l|}
\hline Vessel Tag \# & Equipment Description \\
\hline TLP-DMST-00001 & Treated LAW Evaporator Demister \\
\hline
\end{tabular}

The Treated LAW Evaporator Demister receives offgas from TLP-SEP-00001. The demister is also modeled as a condenser. The offgas is condensed based on splits as shown in Table D-11. The condensate is sent to TLP-VSL-00002. The offgas that was not condensed is sent to PVP-SCB-00002 in the Pretreatment Vessel Vent System.

\begin{tabular}{|l|l|}
\hline Vessel Tag \# & Equipment Description \\
\hline TLP-VSL-00002 & Treated LAW Evaporator Condensate Vessel \\
\hline
\end{tabular}

The Treated LAW Evaporator Condensate Vessel receives condensate from the demister, TLP-DMST-00001. Water, used to model condensate from the vacuum eductors, is also delivered from a chem-add to TLP-VSL-00002 at a rate of $0.96 \mathrm{gpm}$ whenever TLP-SEP-00001 is operating. As soon as the vessel reaches its maximum volume, it empties to RLD-VSL-00006A. TLP-VSL-00002 is available to receive condensate unless it is at maximum volume and RLD-VSL-00006A cannot accept feed.

Figure F-11 provides a graphical illustration of the TLP system where connections with upstream and downstream systems are shown. Table N-1 provides the totalizer files that are used to track the process streams and material flows into and out of the TLP.

\subsubsection{Treated LAW Concentrate Storage Process (TCP) System}

\begin{tabular}{|l|l|}
\hline Vessel Tag \# & Equipment Description \\
\hline TCP-VSL-00001 & Treated LAW Concentrate Storage Vessel \\
\hline
\end{tabular}

The Treated LAW Concentrate Storage Vessel receives the bottoms from TLP-SEP-00001. The vessel fills to its set level $(75,000$ gallons) and then makes itself available to deliver feed to LCP-VSL-00001 and LCP-VSL-00002. The Treated LAW Concentrate Storage Vessel can still receive feed while it is transferring out to another vessel. If the volume of TCP-VSL-00001 is above 80,000 gallons, then TLP-SEP-00001 is set to only process SBS condensate from TLP-VSL-00009A and TLP-VSL-00009B until the volume drops below 80,000 gallons (recycle-only operation as discussed in Section 4.8.8).

A supplemental LAW vessel (SUPPLEMENTAL-LAW-TREATED) is included in the TCP system for the option of modeling the additional capacity needed to process the treated LAW feed. Details of the supplemental LAW vessel operating logic are discussed in Section 4.7.14.

Figure F-11 provides a graphical illustration of the TCP system where connections with upstream and downstream systems are shown. Table N-1 provides the totalizer files that are used to track the process streams and material flows into and out of the TCP.

\subsubsection{Pretreatment Vessel Vent Process (PVP/PJV) Systems}

\begin{tabular}{|l|l|}
\hline Vessel Tag \# & Equipment Description \\
\hline PVP-SCB-00002 & Vessel Vent Caustic Scrubber \\
\hline
\end{tabular}


The Vessel Vent Caustic Scrubber receives offgas from FEP-DMST-00001 and TLP-DMST-00001. Air from air purge and air bubbler are blended into the offgas to the Vessel Vent Caustic Scrubber. The scrubber fills to its set volume with $0.01 \mathrm{M} \mathrm{NapOH}$ that is delivered through a chem-add at a rate of $70 \mathrm{gpm}$. As the offgas is received in the scrubber, components are collected in the $0.01 \mathrm{M} \mathrm{NapOH}$ solution based on splits. The splits of the Vessel Vent Caustic Scrubber are shown in Table D-3. The remaining gas is routed to PVP-SCB-00002. After 24 hours, the scrubber empties its contents at a rate of 50 gpm to PWD-VSL-00044. After emptying, it receives another batch of $0.01 \mathrm{M} \mathrm{NapOH}$ and continues to remove components.

\begin{tabular}{|l|l|}
\hline Vessel Tag \# & Equipment Description \\
\hline PVP-HEME-00001A & PVP High Efficiency Mist Eliminator (HEME) \\
\hline PVP-HEME-00001B & PVP HEME \\
\hline PVP-HEME-00001C & PVP HEME \\
\hline
\end{tabular}

PVP-HEME-00001 is used to model the combined functions of PVP-HEME-00001A, PVP-HEME-00001B, and PVP-HEME-00001C (see BARD for details). PVP-HEME-00001 receives offgas from PVP-SCB-00002. $\mathrm{H}_{2} \mathrm{O}$ is constantly fed to PVP-HEME-00001 by a chem-add at a rate of $0.133 \mathrm{gpm}$. Components from the offgas feed are captured in the liquid phase based on splits. The splits of the PVP HEME are shown in Table D-4. The remaining gas is passed on to the PVP-HEPA. The liquid from PVP-HEME-00001 is transferred to PVP-VSL-00001.

\begin{tabular}{|l|l|}
\hline Vessel Tag \# & Equipment Description \\
\hline PVP-VSL-00001 & Vessel Vent HEME Drain Collection Vessel \\
\hline
\end{tabular}

PVP-VSL-00001 constantly receives liquid from PVP-HEME-00001. This collection vessel fills to its set level and then transfers its contents to PWD-VSL-00044. If PWD-VSL-00044 is not available to receive material, then PVP-VSL-00001 can continue to fill to its maximum volume until PWD-VSL-00044 becomes available. If PVP-VSL-00001 reaches its maximum volume and still cannot empty, then the Pretreatment Vessel Vent Process will shut down.

\begin{tabular}{|l|l|}
\hline Vessel Tag \# & Equipment Description \\
\hline PVP-HEPA & High Efficiency Particular Air (HEPA) Filter \\
\hline
\end{tabular}

The PVP-HEPA receives offgas from PVP-HEME-00001. Liquid and solid components from the offgas feed are captured at the $100 \%$ level except for polychlorinated biphenyls ( $\mathrm{PCBs})\left(\mathrm{C}_{12} \mathrm{H}_{4} \mathrm{Cl}_{6}\right)$, which is captured at the $98 \%$ level, and acetonitrile $\left(\mathrm{C}_{2} \mathrm{H}_{3} \mathrm{~N}\right)$, which escapes totally to the downstream equipment. The gas is routed to the PVP-Thermal-Oxidizer.

\begin{tabular}{|l|l|}
\hline Vessel Tag \# & Equipment Description \\
\hline PVP-Thermal-Oxidizer & PVP Thermal Oxidizer \\
\hline
\end{tabular}

The PVP Thermal Oxidizer receives offgas from PVP High Efficiency Particular Air Filter. Acetonitrile and $\mathrm{PCB}$ are oxidized according to the following reactions. The gases are routed to the PVP-Carbon-Absorber.

\begin{tabular}{|l|l|}
\hline Reaction & $\begin{array}{l}\text { Conversion } \\
\text { (Fraction) }\end{array}$ \\
\hline $\mathrm{C} 2 \mathrm{H} 3 \mathrm{~N}(\mathrm{l})+3.75 \mathrm{O} 2(\mathrm{~g}) \rightarrow 2 \mathrm{CO} 2(\mathrm{~g})+1.5 \mathrm{H} 2 \mathrm{O}(\mathrm{g})+\mathrm{NO} 2(\mathrm{~g})$ & 0.757 \\
\hline $\mathrm{C} 12 \mathrm{H} 4 \mathrm{Cl} 6(\mathrm{l})+11.5 \mathrm{O} 2(\mathrm{~g})+\mathrm{H} 2 \mathrm{O}(\mathrm{g}) \rightarrow 12 \mathrm{CO} 2(\mathrm{~g})+6 \mathrm{HCl}(\mathrm{g})$ & 0.95 \\
\hline
\end{tabular}




\begin{tabular}{|l|l|}
\hline Vessel Tag \# & Equipment Description \\
\hline PVP-ABS-00001A & PVP Carbon Absorber \\
\hline PVP-ABS-00001B & PVP Carbon Absorber \\
\hline
\end{tabular}

PVP-ABS-00001A and PVP-ABS-00001B are modeled as one unit operation, the PVP Carbon Absorber. The PVP Carbon Absorber receives offgas from PVP Thermal Oxidizer. Acetonitrile and PCB gases are also captured at the $90 \%$ level. Remaining gases are routed to the PVP Offgas Stack.

\begin{tabular}{|l|l|}
\hline Vessel Tag \# & Equipment Description \\
\hline PJV-VSL-00001 & PJV Vessel Vent Demister \\
\hline
\end{tabular}

PJV-VSL-00001 in the Pulse Jet Ventilation (PJV) system is used to model PJV-DMST-00002A, PJV-DMST-00002B, and PJV-DMST-00002C. PJV-VSL-00001 receives gas from multiple sources of air as part of the Pulse Jet Mixer (PJM) and Reverse Flow Diverter (RFD). Components from the gas feed are captured by the demister based on splits. The splits of the PJV Vessel Vent Demister are shown in Table D-8. Cumulated captures are sent to PWD-VSL-00043 and the remaining gases are routed to the PJV HEPA.

\begin{tabular}{|l|l|}
\hline Vessel Tag \# & Equipment Description \\
\hline PJV-HEPA & PJV HEPA Filter \\
\hline
\end{tabular}

The PJV HEPA receives offgas from PJV-VSL-00001. Liquid and solid components from the offgas feed are captured at the $100 \%$ level. The splits of the PJV HEPA Filter are shown in Table D-9. The gas is passed out the PVP-Offgas-Stack.

Figure F-6 provides a graphical illustration of the PVP/PJV systems where connections with upstream and downstream systems are shown. Table N-1 provides the totalizer files that are used to track the process streams and material flows into and out of the PVP/PJV.

Dynamic air utilization modeling capabilities including the purge air, bubbler air, PJM air, and RFD air are discussed in Appendix G.

\subsubsection{Radioactive Liquid Waste Disposal (RLD) System}

\begin{tabular}{|l|l|}
\hline Vessel Tag \# & Equipment Description \\
\hline RLD-VSL-00017A & Alkaline Effluent Vessel \\
\hline RLD-VSL-00017B & Alkaline Effluent Vessel \\
\hline
\end{tabular}

The Alkaline Effluent Vessels receive fresh resin preparation solution from the Cesium Resin Addition Vessel (CRP-VSL-00001) and effluent from the Caustic Collection Vessel (LVP-TK-00001). When RLD-VSL-00017A/B reaches its set level, it will stop receiving feed, sample for a prescribed time, and then make itself available to empty to RLD-TK-00006B. The vessels are not allowed to collect at the same time. One of the vessels must fill and begin to sample before the other is allowed to start receiving feed.

\begin{tabular}{|l|l|}
\hline Vessel Tag \# & Equipment Description \\
\hline RLD-TK-00006A & Process Condensate Vessel \\
\hline
\end{tabular}

RLD-TK-00006A receives feed from the Waste Feed Evaporator Condensate Vessel (FEP-VSL-00005) and the Treated LAW Evaporator Condensate Vessel (TLP-VSL-00002). A portion of the process 
condensate will be recycled to various Pretreatment systems for washing, dilution, and line flush operations. Excessive process condensate will be sent to the ETF/LERF through RLD-TK-00006B.

When RLD-TK-00006A reaches above 200,000 gallons (upper set point), the vessel volume will be pumped down to 110,000 gallons (lower set point) by discharging to RLD-TK-00006B. The inventory between the heel and lower set point is reserved for the Pretreatment process condensate operations. It is possible for concurrent transfers from RLD-TK-00006A to RLD-TK-00006B and to the process condensate header because they are on separate pumping systems. The pump rate to RLD-TK-00006B is $100 \mathrm{gpm}$ and the pump rate to the process condensate header is $250 \mathrm{gpm}$.

\begin{tabular}{|l|l|}
\hline Vessel Tag \# & Equipment Description \\
\hline RLD-TK-00006B & Process Condensate Vessel \\
\hline
\end{tabular}

RLD-TK-00006B receives feed from the following vessels:

\begin{tabular}{|l|l|}
\hline Vessel Tag \# & Equipment Description \\
\hline RLD-VSL-00017A & Alkaline Effluent Vessel \\
\hline RLD-VSL-00017B & Alkaline Effluent Vessel \\
\hline RLD-TK-00006A & Process Condensate Vessel \\
\hline
\end{tabular}

When RLD-TK-00006B reaches its set level, the vessel will empty its contents to the ETF/LERF which is modeled as an infinite vessel collecting all the effluent transfers. The pump rate to ETF/LERF is 170 gpm.

\begin{tabular}{|l|l|}
\hline Vessel Tag \# & Equipment Description \\
\hline RLD-VSL-00004 & C3/C5 Drains/Sump Collection Vessel \\
\hline
\end{tabular}

RLD-VSL-00004 receives liquid continuously from the LAW WESP columns. When the level reaches the setpoint, the vessel empties its contents into RLD-VSL-00005.

\begin{tabular}{|l|l|}
\hline Vessel Tag \# & Equipment Description \\
\hline RLD-VSL-00005 & SBS Condensate Collection Vessel \\
\hline
\end{tabular}

RLD-VSL-00005 receives material from RLD-VSL-00004 and LAW-SBS. When the setpoint level of RLD-VSL-00005 has been reached, the vessel empties to either TLP-VSL-00009A or TLP-VSL-00009B depending on their availability.

\begin{tabular}{|l|l|}
\hline Vessel Tag \# & Equipment Description \\
\hline RLD-VSL-00007 & Acidic Waste Vessel \\
\hline
\end{tabular}

The Acidic Waste Vessel receives material from the HLW SBS Condensate Vessels (HOP-SCB-00001, HOP-SCB-00002) and the Waste Neutralization Vessel (HDH-VSL-00003) one at a time. A transfer can be made if RDL-VSL-00007 is not currently discharging and the volume is below the 8,662-gallon setpoint. If two or three of the upstream vessels need to be discharged at the same time (rarely occurs), the upstream vessel that reaches the upper setpoint first gets the priority to discharge, and the upstream vessel(s) with lower priority will continue to receive. RLD-VSL-00007 receives until the setpoint level is reached. Transfer lines are flushed with process water at the end of each batch and flush volumes can be found in Table $\mathrm{H}-1$. If necessary $5 \mathrm{M} \mathrm{NapOH}$ is added to bring the $\mathrm{pH}$ up to 8.0. After $\mathrm{pH}$ adjustment the vessel empties its contents into PWD-VSL-00043. 
If RLD-VSL-00007 is unable to discharge due to PWD-VSL-00043 vessel's unavailability to receive, the HLW melter feed pumps will be shut down temporary. This provides relief to RLD-VSL-00007 from the HLW SBS Condensate Vessels and the Waste Neutralization Vessel.

Figures F-16, F-22, F-24, and F-25 provide graphical illustrations of the RLD/Condensate and RLD/Effluent systems where connections with upstream and downstream systems are shown. Table N-1 provides the totalizer files that are used to track the process streams and material flows into and out of the RLD/Condensate and RLD/Effluent.

\subsubsection{HLW Lag Storage and Feed Blending Process System}

\begin{tabular}{|l|l|}
\hline Vessel Tag \# & Equipment Description \\
\hline HLP-VSL-00022 & HLW Feed Receipt Vessel \\
\hline
\end{tabular}

The HLW Feed Receipt Vessel receives batch transfers of HLW feed and associated pre-transfer and post-transfer line flushes of inhibited water from the Tank Farms. The vessel also receives line flush water from various operations shown in Table H-1. In general, the transfer lines are flushed after each use. Due to piping configurations, the destination of the flush water is not only to the pumping and receiving vessels, but may also include other FRP and FEP vessels. Headspace, as defined as the storage between the set volume and the maximum volume, is reserved in HLP-VSL-00022 for the line flush water. The vessel is constrained to not fill above its maximum volume.

Once a year, a heel dilution is performed in HLP-VSL-00022 to reduce solids concentration in the heel that may have accumulated. Fluids used for dilution are LAW feeds from FRP-VSL-00002A/B/C/D, process condensate, or DI water in preferred orders. The HLP-VSL-00022 heel dilution is scheduled to separate with other heel dilution operations in FEP-VSL-00017A/B and UFP-VSL-00001A/B. The portable pump connects HLP-VSL-00022 with UFP-VSL-00001B and will only discharge when UFPVSL-00001B is in a receiving mode. Refer to Section 4.7.20 for further details on general rules of heel dilution and HLP-VSL-00022 specific volume parameters.

Batches are not mixed in the vessel except for the waste in the heel from the previous batch. The vessel is filled to its set volume or until the full batch has been received. Solubility correlations are applied at $25^{\circ} \mathrm{C}$. A sample is taken and the vessel is held for the prescribed sample time. The vessel transfers waste feeds to either UFP-VSL-00001A or UFP-VSL-00001B, depending on their availability, according to the feed blending logic described in Section 4.8.3.

\begin{tabular}{|l|l|}
\hline Vessel Tag \# & Equipment Description \\
\hline HLP-VSL-00027A & HLW Lag Storage Vessel \\
\hline HLP-VSL-00027B & HLW Lag Storage Vessel \\
\hline HLP-VSL-00028 & HLW Feed Blending Vessel \\
\hline
\end{tabular}

The HLW Lag Storage Vessels and Feed Blending Vessel can receive feeds from the following vessels:

\begin{tabular}{|l|l|}
\hline Vessel Tag \# & Equipment Description \\
\hline FEP-SEP-00001A/B & Waste Feed Evaporator \\
\hline UFP-VSL-00002A/B & Ultrafiltration Feed Vessel \\
\hline RLD-TK-00006A & Process Condensate Vessel \\
\hline CNP-EVAP-00001 & Cesium Evaporator Separator Vessel \\
\hline CXP-VSL-00001 & Eluate Contingency Storage Vessel \\
\hline
\end{tabular}


When the backend leaching operations are chosen, both the HLP-VSL-00027A and HLP-VSL-00027B vessels are used to receive solids from UFP2. A HLP-VSL-00027 vessel can receive multiple batches of solids until the last batch raises the volume to above the upper set point. The vessel also receives 150 gallons of inhibited water at $90 \mathrm{gpm}$ from line flush at the end of each batch transfer. The two vessels work in an alternating fashion.

HLP-VSL-00028 receives feeds from HLP-VSL-00027A/B and associated line flush water as shown in Table H-1. HLP-VSL-00028 and HLP-VSL-00027B can also receive CNP evaporator concentrate from CNP-VSL-00003 in priority order based on the following conditions. A maximum of 2,615 gallons can be received in one batch.

- If HLP-VSL-00028 is in the beginning of a "fill" cycle, for example, volume is at or slightly greater than 27,000 gallons

- Else if HLP-VSL-00027B is in "fill" mode, and the volume of HLP-VSL-00027B is greater than 61,800 gallons, and the remaining volume of HLP-VSL-00027B can receive up to 2,615 gallons of CNP concentrate plus the associated caustic for neutralization

- Else wait until the above condition(s) become true

Before CNP evaporator concentrate can be transferred into either HLP-VSL-00028 or HLP-VSL-00027B, caustic (19M NapOH) is added to the receiving vessel. The volume of caustic is determined by calculating the amount of caustic necessary to achieve an $\mathrm{OH}^{-}$to $\mathrm{H}^{+}$ratio of 1.1 to 1 , excluding the $\mathrm{OH}^{-}$ already in the receiving vessel. There is a 30-minute wait after the CNP material is received to allow mixing and neutralization reaction to take place.

HLP-VSL-00028 will be topped off with wastes from HLP-VSL-00027A/B after neutralization. The minimum cool down time after neutralization and before discharging is 40 hours. HLP-VSL-00027B can be discharged after neutralization and cooling. The minimum cool down time after neutralization is 100 hours.

HLP-VSL-00028 is sampled, held for the prescribed time, then discharged. HLP-VSL-00028 discharges to the HLW MFPV in batches. Batch size is determined based on waste composition, glass formulation, glass former dusting water, water from line flush and rheology operations. All these operating variables are inter-related as:

glass formulation $=$ function (waste composition)

glass former dusting water $=$ function (glass formulation)

rheology $=$ function (waste composition, glass formulation, line flush, glass former dusting water)

The goal is to fill the HLW MFPV to the maximum volume after all of the above have been transferred in. An iterative approach is taken to solve for the batch size for waste (see Section 4.8.17 for further discussions). If the remaining volume of HLP-VSL-00028 is less than the calculated batch size, the HLPVSL-00028 vessel is switched from discharge to receive. Transfer lines are back flushed after each use with significant volumes of process condensate or water being placed in the HLP-VSL-00028 and PWDVSL-00043 vessels (Table H-1).

To minimize foaming, AFA is added to HLP-VSL-00027A, HLP-VSL-00027B, and HLP-VSL-00028 when the vessels have been filled to above the set volume. AFA will be replenished after that in 6-hour intervals to account for losses due to decomposition and discharge. Target concentrations for PPG and PDMS are discussed in Section 4.7.17. 
Twice a year, heel cleanout are performed in HLP-VSL-00027A/B and HLP-VSL-00028 to reduce solids concentration in the heel that may have accumulated. Fluids used for cleanout are concentrated solids from UFP-VSL-00002A/B for the HLP-VSL-00027A/B vessels and concentrated solids from HLP-VSL00027A/B for the HLP-VSL-00028 vessel. The HLP-VSL-00027A/B and HLP-VSL-00028 heel cleanout are scheduled to offset one with another. The portable pump connects HLP-VSL-00027A/B with HLP-VSL-00028 and connects HLP-VSL-00028 with HFP-VSL-00001/5. Refer to Section 4.7.20 for further details on general rules of heel cleanout and HLP-VSL-00027A/B and HLP-VSL-00028 specific volume parameters.

Figure F-17 provides a graphical illustration of the HLP system where connections with upstream and downstream systems are shown. Table N-1 provides the totalizer files that are used to track the process streams and material flows into and out of the HLP.

\subsubsection{LAW Concentrate Receipt Process (LCP) System}

\begin{tabular}{|l|l|}
\hline Vessel Tag \# & Equipment Description \\
\hline LCP-VSL-00001 & LAW Concentrate Receipt Vessel \\
\hline LCP-VSL-00002 & LAW Concentrate Receipt Vessel \\
\hline
\end{tabular}

The LAW Concentrate Receipt Vessels (CRV) receive material from TCP-VSL-00001 and alternate with each other in operation. When a LAW CRV has reached its maximum level, the vessel samples for a prescribed time, then becomes available to empty to either LFP-VSL-00001 or LFP-VSL-00003 depending on their availability. Based on the CRV sample compositions, batch size and glass former recipes will be determined. Batch size determines the number of transfers that can be made to the LAW MFPVs. Glass former recipes are determined using the Immobilized Low-Activity Waste (ILAW) glass formulation model shown in Figures 12 through 14 (refer to the BARD for further details). The batch size and glass former recipe decisions ensure that

1) LFP-VSL-00001 or LFP-VSL-00003 will not overflow

2) Rheology water target is met (36 wt \% water)

3) LAW glass quality specifications are met

4) Melter corrosion due to high lithium contents in the feed is prevented

5) Halide, chromium, and phosphate will not hinder melting process

The number of requests for LAW glass former recipes are equal to the number of batches to the LAW MFPVs. Glass formers can be prepared as soon as recipes exist in the LAW recipe queue given that the GFR is available. It shall be noted that GFR priorities are given to HLW glass formers, e.g., HLW recipes will be processed first if requests exist in both LAW recipe queue and HLW recipe queue (refer to Section 4.8.22 for GFR operatons). Due to the fact that LAW glass former recipies are known for future LAW CRV to LAW MFPV batches, LAW glass formers can be prepared in parallel to, or even ahead of, the CRV to MFPV waste transfers.

Transfer lines are flushed with process water after each use and both the CRV and MFPV will receive some flush water (Table H-1). Partial batch transfer to the MFPV is prohibited, meaning that the CRV will switch to filling mode if the remaining volume in the vessel is less than a full batch. After the predetermined number of batches have been transferred out, the CRV makes itself available to fill. 


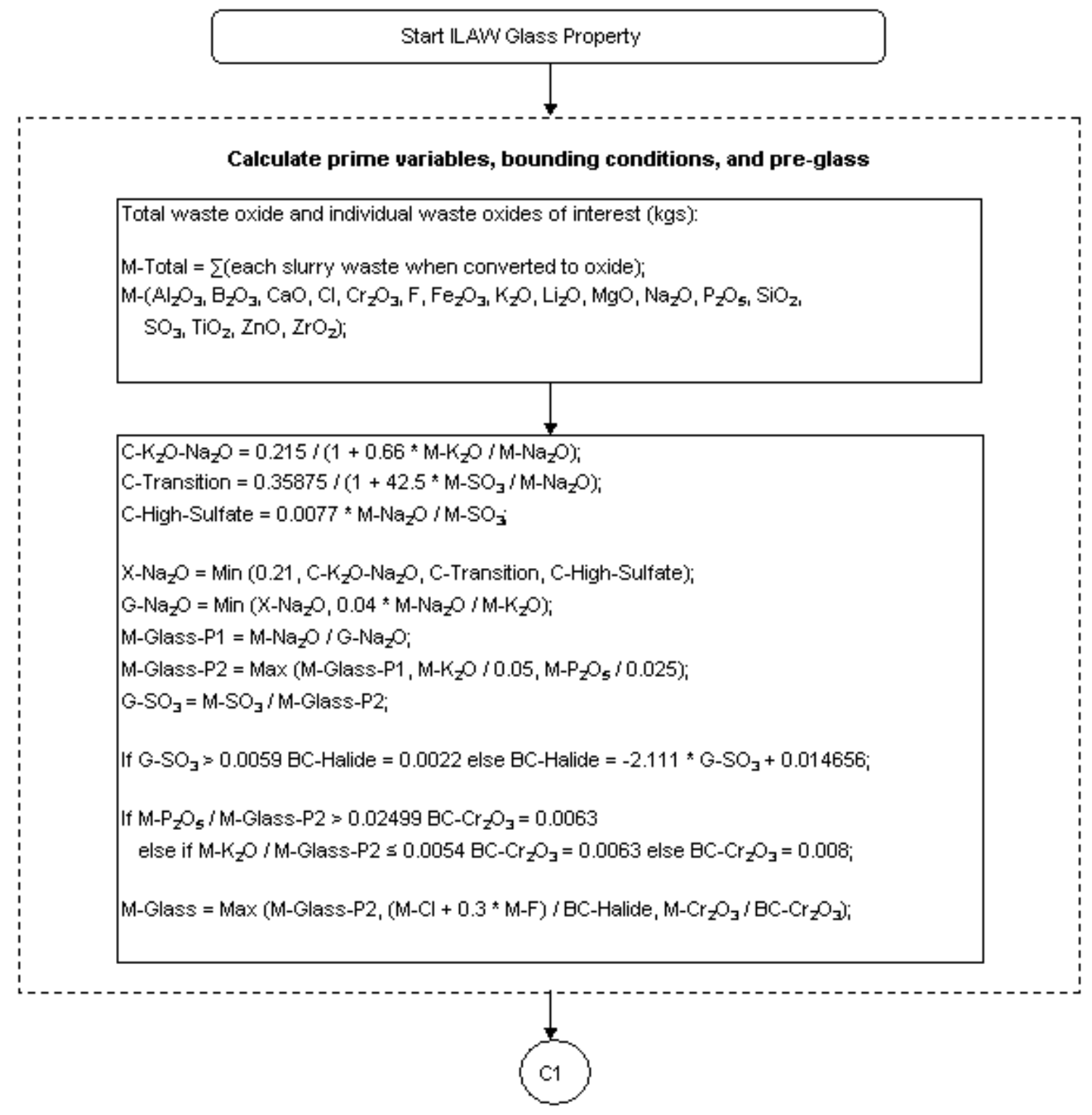

Figure 12 ILAW Glass Formulation Model (Part 1) 


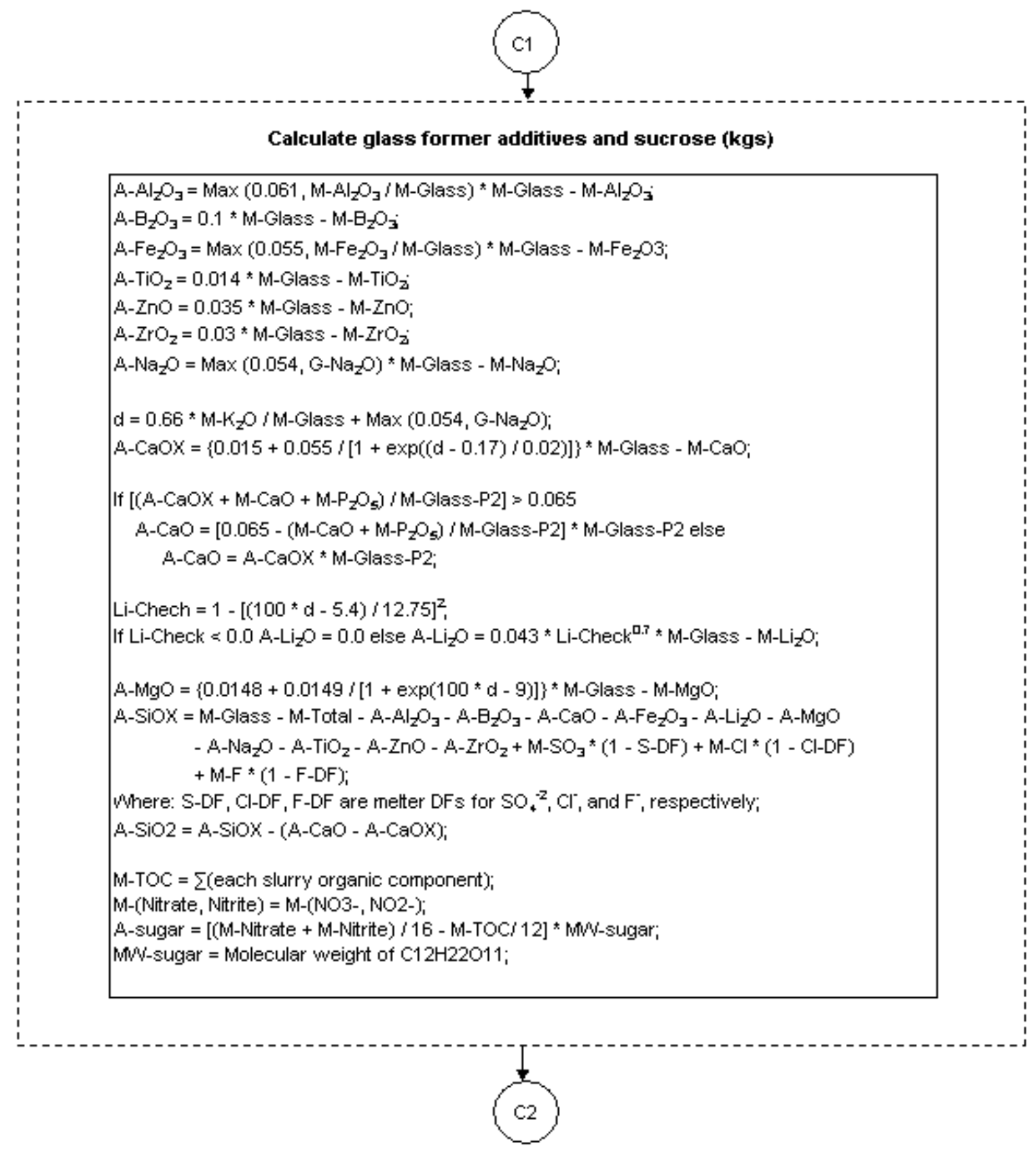

Figure 13 ILAW Glass Formulation Model (Part 2) 


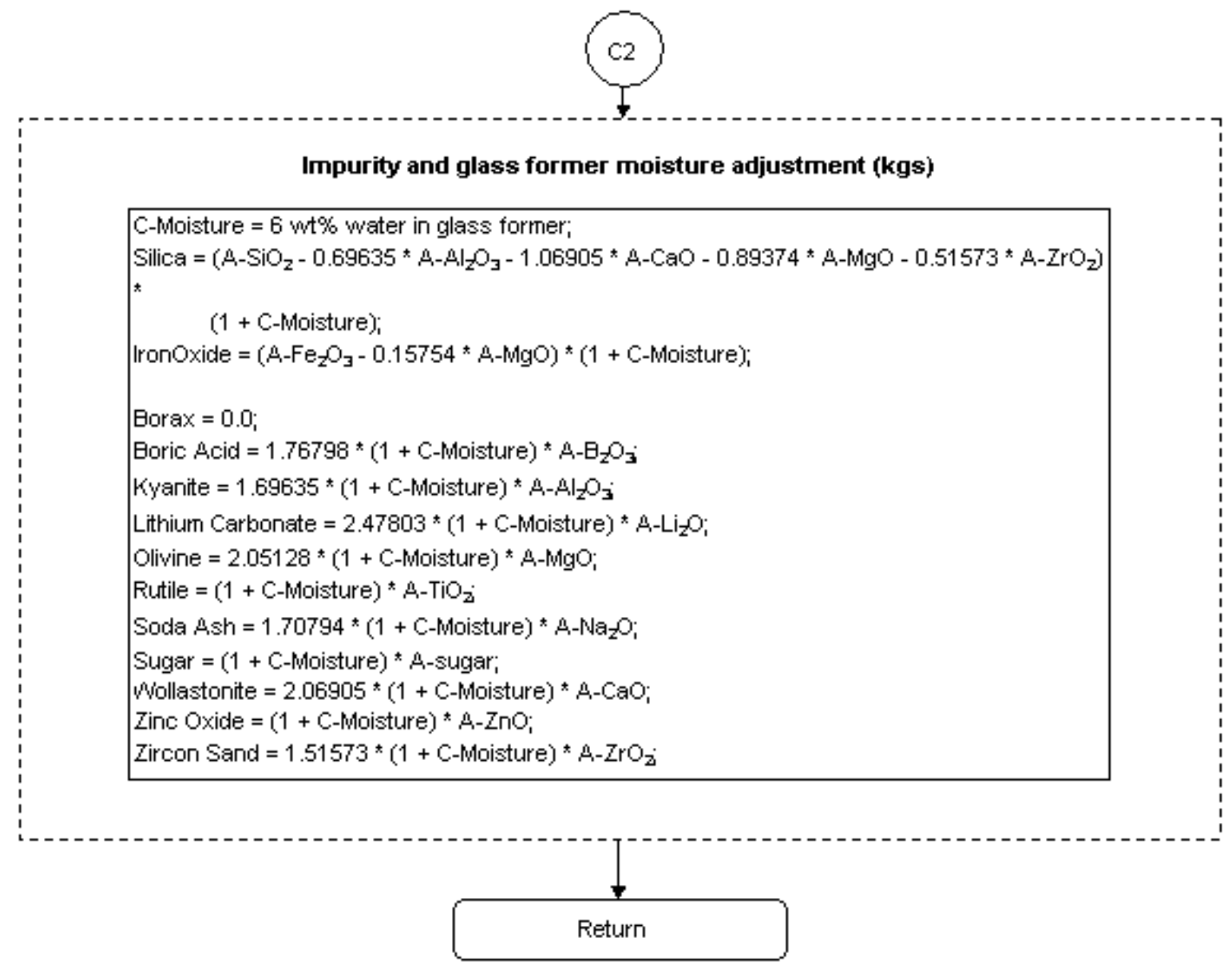

Figure 14 ILAW Glass Formulation Model (Part 3)

The glass former recipe, including the quantities of borax, boric acid, kyanite, lithium carbonate, olivine, rutile, soda ash, sugar, wollastonite, zinc oxide, and zircon sand, are returned from the ILAW glass formulation model.

\subsubsection{LAW Feed Preparation Process (LFP) System}

\begin{tabular}{|l|l|}
\hline Vessel Tag \# & Equipment Description \\
\hline LFP-VSL-00001 & LAW Melter 1 Feed Prep Vessel \\
\hline LFP-VSL-00003 & LAW Melter 2 Feed Prep Vessel \\
\hline
\end{tabular}

LFP-VSL-00001 receives waste feed from LCP-VSL-00001 or LCP-VSL-00002, and glass former from GFR-TK-00023. LFP-VSL-00003 receives waste feed from LCP-VSL-00001 or LCP-VSL-00002, and glass former from GFR-TK-00022. The LAW MFPV set volume is batch dependent and is associated with the current LAW glass former recipe, dusting water quantity, and rhelogy water. The goal is to fill the MFPV to a prescribed volume after the waste, glass formers, dusting water, and rheology water are received. Figure 15 shows the iterative approach for determining the MFPV set volume. The vessel is held 0.75 hours for a confirmation sampling before becomes available for discharge. A specified volume of flush water is placed in the MFPV after sampling (Table H-1). LFP-VSL-00001 empties its contents to 
LFP-VSL-00002 and LFP-VSL-00003 empties its contents to LFP-VSL-00004. Transfer lines are flushed with process water after each use and both the MFPV and MFV receive additional volumes of flush water. After emptying, the LAW MFPV starts a new cycle.

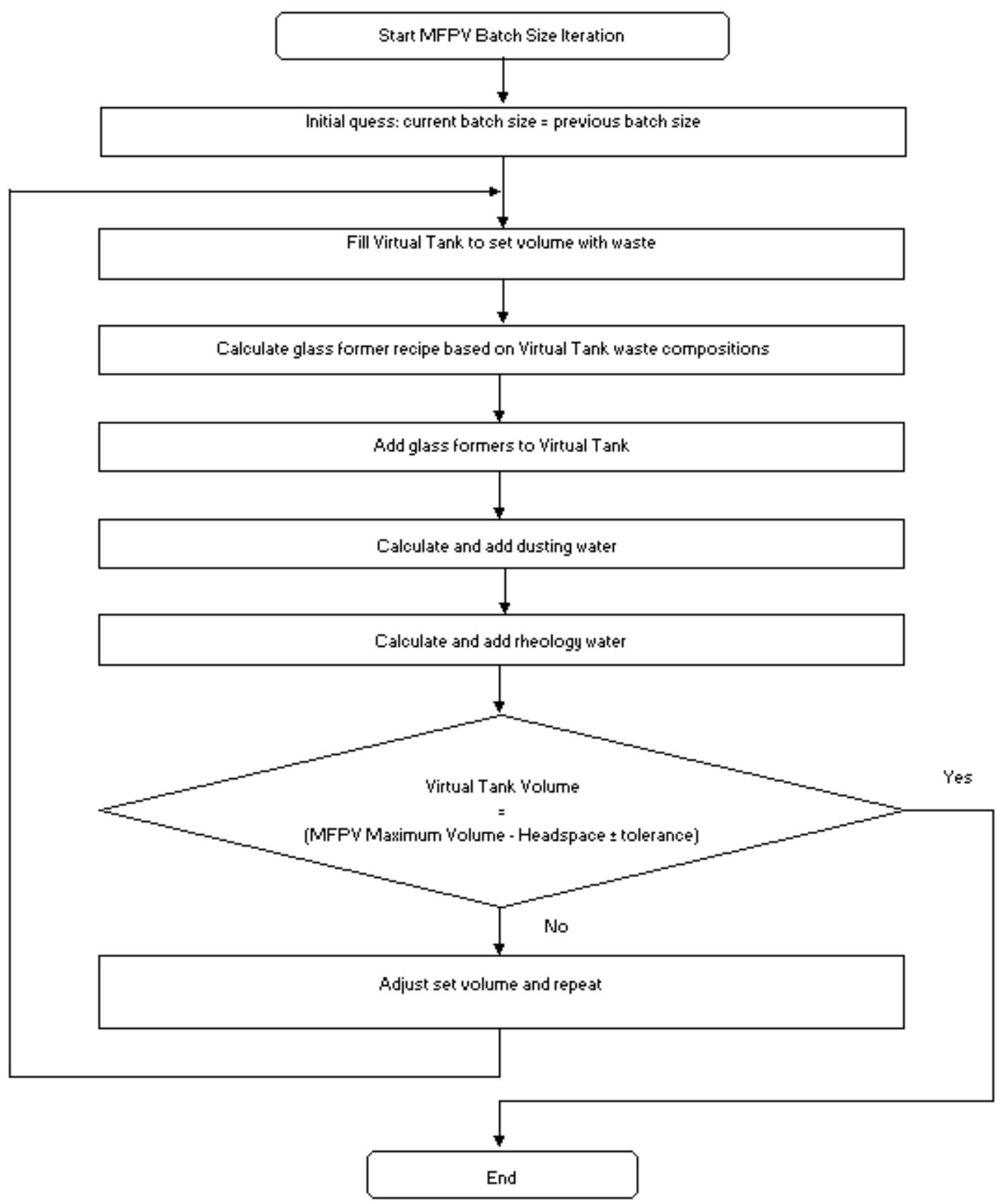

Figure 15 MFPV Batch Size Determination Logic 


\begin{tabular}{|l|l|}
\hline Vessel Tag \# & Equipment Description \\
\hline LFP-VSL-00002 & LAW Melter 1 Feed Vessel \\
\hline LFP-VSL-00004 & LAW Melter 2 Feed Vessel \\
\hline
\end{tabular}

The LAW MFVs receive feed from the corresponding LAW MFPVs. LFP-VSL-00002 transfers material only to LMP-MLTR-00001 and LFP-VSL-00004 transfers material only to LMP-MLTR-00002. Transfer rate is variable depending on the contents of the waste. A more diluted feed will have a higher transfer rate so that the target melter production rate can be maintained. When a LAW MFV reaches its lower setpoint (1,000 gallons) it becomes available to receive feed again while continuing to empty to its minimum volume (977 gallons). This prevents the LAW melter from becoming idle. The only time that an LAW MFV will stop pumping to the melter is when there is no feed available from the LAW MFPV and the LAW MFV is at its minimum volume.

After the MFV receives a batch transfer from the MFPV and after the MFV completes a batch discharge, process water are flushed into the MFV and associated melter (Table H-1). Process water is also flushed into the receiving melter once every 4 hours between the cycle.

Figure F-12 provides a graphical illustration of the LCP/LFP systems where connections with upstream and downstream systems are shown. Table N-1 provides the totalizer files that are used to track the process streams and material flows into and out of the LCP/LFP.

\subsubsection{LAW Melter Process (LMP) System}

\begin{tabular}{|l|l|}
\hline Vessel Tag \# & Equipment Description \\
\hline LMP-MLTR-00001 & LAW Melter 1 \\
\hline LMP-MLTR-00002 & LAW Melter 2 \\
\hline
\end{tabular}

LAW Melters (LMP-MLTR-00001 and LMP-MLTR-00002) are used to transform the LAW into glass. Liquid and solid wastes, oxide glass formers and impurities, lineflush water, air, and film cooler water rinse are delivered to the melters. The inflow of waste and glass formers depends on the availability of feed; however, the inflow of air is continuous once started. The air stream is made up of: 1) air inleakage, 2) bubbler air, 3) purge air, 4) control air, and 5) cooling air, while the water stream represents the film cooler water rinse. The combined air flowrate and the associated temperature and pressure are shown in Table B-15. The film cooler water rinse flowrate is shown in the same table. The air provides the source of oxygen for the melter reactions.

When feed enters the melter, a split is applied to the non-gas components including the liquid and solid wastes, and oxide glass formers and associated mineral impurities. The liquid split values apply to the liquid components that come into the melter while the solid split values apply to the combined solids of solid wastes, oxide glass formers, and impurities. The predefined split values are shown in Table D-12. Exceptions are applied to the following components of which melter reactions or a combination of melter reactions and splits are more appropriate:

\begin{tabular}{|l|l|l|l|}
\hline Components & $\begin{array}{l}\text { Liquid\% } \\
\text { to SBS }\end{array}$ & $\begin{array}{l}\text { Solid\% } \\
\text { to SBS }\end{array}$ & Descriptions \\
\hline $129-\mathrm{I}$ & 0.000 & 0.000 & $129-\mathrm{I}(1, \mathrm{~s}) \rightarrow 129-\mathrm{I}(\mathrm{g}) \mathrm{CF}=0.4167$, remaining go to glass \\
\hline $3-\mathrm{H}$ & 100.000 & 100.000 & Follows water, 100\% go to offgas \\
\hline $\mathrm{C} 12 \mathrm{H} 4 \mathrm{Cl6}$ & 0.000 & 0.000 & $\begin{array}{l}\mathrm{C} 12 \mathrm{H} 4 \mathrm{Cl6}(1, \mathrm{~s})+11.5 \mathrm{O} 2(\mathrm{~g})+\mathrm{H} 2 \mathrm{O}(\mathrm{l}) \rightarrow 12 \mathrm{CO} 2(\mathrm{~g})+6 \mathrm{HCl}(\mathrm{g}) \mathrm{CF}= \\
1.0\end{array}$ \\
\hline $\mathrm{C} 2 \mathrm{H} 3 \mathrm{~N}$ & 0.000 & 0.000 & $\mathrm{C} 2 \mathrm{H} 3 \mathrm{~N}(1, \mathrm{~s})+3.75 \mathrm{O} 2(\mathrm{~g}) \rightarrow 2 \mathrm{CO} 2(\mathrm{~g})+1.5 \mathrm{H} 2 \mathrm{O}(\mathrm{g})+\mathrm{NO} 2(\mathrm{~g}) \mathrm{CF}=$ \\
\hline
\end{tabular}




\begin{tabular}{|c|c|c|c|}
\hline Components & $\begin{array}{l}\text { Liquid\% } \\
\text { to SBS }\end{array}$ & $\begin{array}{l}\text { Solid\% } \\
\text { to SBS }\end{array}$ & Descriptions \\
\hline & & & 1.0 \\
\hline $\mathrm{C} 2 \mathrm{O} 4-2$ & 0.000 & 0.000 & $\mathrm{C} 2 \mathrm{O} 4-2(1, \mathrm{~s}) \rightarrow 2 \mathrm{CO} 2(\mathrm{~g}) \mathrm{CF}=1.0$ \\
\hline CHO2- & 0.000 & 0.000 & $\mathrm{CHO} 2-(1, \mathrm{~s})+0.25 \mathrm{O} 2(\mathrm{~g}) \rightarrow \mathrm{CO} 2(\mathrm{~g})+0.5 \mathrm{H} 2 \mathrm{O}(\mathrm{g}) \mathrm{CF}=1.0$ \\
\hline $\mathrm{Cl}-$ & 0.000 & 0.000 & $\begin{array}{l}2 \mathrm{Cl}-(1, \mathrm{~s})+2 \mathrm{Na}+(1, \mathrm{~s})+\mathrm{H} 2 \mathrm{O}(\mathrm{l}) \rightarrow \mathrm{Na} 2 \mathrm{O}(\mathrm{o})+2 \mathrm{HCl}(\mathrm{g}) \mathrm{CF}=0.5025, \\
\text { remaining go to glass }\end{array}$ \\
\hline $\mathrm{CN}-$ & 1.754 & 1.754 & $\begin{array}{l}2 \mathrm{CN}-(1, \mathrm{~s})+2 \mathrm{O} 2(\mathrm{~g}) \rightarrow 2 \mathrm{CO} 2(\mathrm{~g})+\mathrm{N} 2(\mathrm{~g}), \mathrm{CF}=1.0, \\
\text { partially split to offgas, remaining } 100 \% \text { destroyed }\end{array}$ \\
\hline $\mathrm{CO} 3-2$ & 0.000 & 0.000 & $\mathrm{CO} 3-2(1, \mathrm{~s}) \rightarrow \mathrm{CO} 2(\mathrm{~g})+0.5 \mathrm{O} 2(\mathrm{~g}) \mathrm{CF}=1.0$ \\
\hline F- & 0.000 & 0.000 & $\begin{array}{l}2 \mathrm{~F}-(1, \mathrm{~s})+2 \mathrm{Na}+(1, \mathrm{~s})+\mathrm{H} 2 \mathrm{O}(\mathrm{l}) \rightarrow \mathrm{Na} 2 \mathrm{O}(\mathrm{o})+2 \mathrm{HF}(\mathrm{g}) \mathrm{CF}=0.5319, \\
\text { remaining go to glass }\end{array}$ \\
\hline $\mathrm{H}+$ & 0.000 & 0.000 & $\mathrm{H}+(1, \mathrm{~s})+\mathrm{OH}(\mathrm{BOUND})(1, \mathrm{~s}) \rightarrow \mathrm{H} 2 \mathrm{O}(\mathrm{g}) \mathrm{CF}=1.0$ \\
\hline $\mathrm{H} 2 \mathrm{CO} 3$ & 0.000 & 0.000 & $\mathrm{H} 2 \mathrm{CO} 3(1, \mathrm{~s}) \rightarrow \mathrm{CO} 2(\mathrm{~g})+\mathrm{H} 2 \mathrm{O}(\mathrm{g}) \mathrm{CF}=1.0$ \\
\hline $\mathrm{H} 2 \mathrm{O}$ & 0.000 & 0.000 & $\mathrm{H} 2 \mathrm{O}(1, \mathrm{~s}) \rightarrow \mathrm{H} 2 \mathrm{O}(\mathrm{g}) \mathrm{CF}=1.0$ \\
\hline $\mathrm{H} 2 \mathrm{O} 2$ & 0.000 & 0.000 & $2 \mathrm{H} 2 \mathrm{O} 2(1, \mathrm{~s}) \rightarrow 2 \mathrm{H} 2 \mathrm{O}(\mathrm{g})+\mathrm{O} 2(\mathrm{~g}) \mathrm{CF}=1.0$ \\
\hline HCO3- & 0.000 & 0.000 & $2 \mathrm{HCO} 3-(1, \mathrm{~s}) \rightarrow 2 \mathrm{CO} 2(\mathrm{~g})+\mathrm{H} 2 \mathrm{O}(\mathrm{g})+0.5 \mathrm{O} 2(\mathrm{~g}) \mathrm{CF}=1.0$ \\
\hline NH3 & 0.000 & 0.000 & $2 \mathrm{NH} 3(1, \mathrm{~s})+3 \mathrm{O} 2(\mathrm{~g}) \rightarrow \mathrm{NO}(\mathrm{g})+\mathrm{NO} 2(\mathrm{~g})+3 \mathrm{H} 2 \mathrm{O}(\mathrm{g}) \mathrm{CF}=1.0$ \\
\hline NH4+ & 0.000 & 0.000 & $2 \mathrm{NH} 4+(1, \mathrm{~s})+3.5 \mathrm{O} 2(\mathrm{~g}) \rightarrow \mathrm{NO}(\mathrm{g})+\mathrm{NO} 2(\mathrm{~g})+4 \mathrm{H} 2 \mathrm{O}(\mathrm{g}) \mathrm{CF}=1.0$ \\
\hline NO2- & 0.000 & 0.000 & See Eq. 55 in the following discussions \\
\hline NO3- & 0.000 & 0.000 & See Eq. 54 in the following discussions \\
\hline $\mathrm{O}-2$ & 0.000 & 0.000 & $2 \mathrm{O}-2(1, \mathrm{~s}) \rightarrow \mathrm{O} 2(\mathrm{~g}) \mathrm{CF}=1.0$ \\
\hline OH(BOUND) & 0.000 & 0.000 & $2 \mathrm{OH}($ Bound $)(1, \mathrm{~s}) \rightarrow \mathrm{H} 2 \mathrm{O}(\mathrm{g})+0.5 \mathrm{O} 2(\mathrm{~g}) \mathrm{CF}=1.0$ \\
\hline OH- & 0.000 & 0.000 & $2 \mathrm{OH}-(\mathrm{l}, \mathrm{s}) \rightarrow \mathrm{H} 2 \mathrm{O}(\mathrm{g})+0.5 \mathrm{O} 2(\mathrm{~g}) \mathrm{CF}=1.0$ \\
\hline SO4-2 & 0.000 & 0.000 & $\begin{array}{l}\mathrm{SO} 4-2(1, \mathrm{~s}) \rightarrow \mathrm{SO} 2(\mathrm{~g})+\mathrm{O} 2(\mathrm{~g}), \mathrm{CF} \text { is dynamically determined, } \\
\text { remaining is oxidized and go to glass }\end{array}$ \\
\hline TOC & 0.000 & 0.000 & $\mathrm{TOC}(1, \mathrm{~s})+\mathrm{O} 2(\mathrm{~g}) \rightarrow \mathrm{CO} 2(\mathrm{~g}) \mathrm{CF}=1.0$ \\
\hline
\end{tabular}

Nitrate and nitrite decomposition reactions are shown as Eq. 54 and Eq. 55 where the stoichiometric coefficients are dependent upon the variable multiplier $\mathrm{M}$. These reactions provide the means for approximating the test data which found the SBS solution to be near neutral $(\mathrm{pH}=6.5$ to 7.5$)$ due to variations of ammonia gas production rate.

$$
\begin{aligned}
& \mathrm{r} 11 \times \mathrm{NO}_{3}{ }^{-}(1, \mathrm{~s})+\mathrm{r} 12 \times \mathrm{H}_{2} \mathrm{O}(\mathrm{l}) \rightarrow \mathrm{p} 11 \times \mathrm{N}_{2}(\mathrm{~g})+\mathrm{p} 12 \times \mathrm{NO}(\mathrm{g})+\mathrm{p} 13 \times \mathrm{NO}_{2}(\mathrm{~g})+\mathrm{p} 14 \times \mathrm{NH}_{3}(\mathrm{~g})+ \\
&(0.25+\mathrm{p} 15) \times \mathrm{O}_{2}(\mathrm{~g})
\end{aligned}
$$

$\mathrm{r} 21 \times \mathrm{NO}_{2}^{-}(1, \mathrm{~s})+\mathrm{r} 22 \times \mathrm{H}_{2} \mathrm{O}(\mathrm{l}) \rightarrow \mathrm{p} 21 \times \mathrm{N}_{2}(\mathrm{~g})+\mathrm{p} 22 \times \mathrm{NO}(\mathrm{g})+\mathrm{p} 23 \times \mathrm{NO}_{2}(\mathrm{~g})+\mathrm{p} 24 \times \mathrm{NH}_{3}(\mathrm{~g})+$ $(0.25+\mathrm{p} 25) \times \mathrm{O}_{2}(\mathrm{~g})$

where the stoichiometric coefficients are given as follows:

r11: 1

r12: $\quad 0.03623 \times \mathrm{M}$

p11: $0.2546-0.0121 \times(\mathrm{M}-1)$

p12: 0.4239

p13: 0.04277

p14: $0.024154 \times \mathrm{M}$

p15: $1.01339+0.0181 \times(\mathrm{M}-1)$ r21: $\quad 1$

r22: $\quad 0.03623 \times \mathrm{M}$

p21: $\quad 0.2546-0.0121 \times(\mathrm{M}-1)$

p22: $\quad 0.4239$

p23: $\quad 0.04277$

p24: $0.024154 \times \mathrm{M}$

p25: $\quad 0.5134+0.0181 \times(\mathrm{M}-1)$ 
$\mathrm{M}$ is a multiplier that can be used to determine the ammonia production rate in the next time step. As the $\mathrm{pH}$ values of the SBSs are very sensitive to the ammonia gas production in the melters, variable M values are applied based on the current $\mathrm{pH}$ value in the SBS. A lookup table is provided as follows:

\begin{tabular}{|l|l|}
\hline Current pH & M \\
\hline $\mathrm{pH}<=3.0$ & 1.05 \\
\hline $3.0<\mathrm{pH}<=4.0$ & 1.01 \\
\hline $4.0<\mathrm{pH}<=5.0$ & 1.005 \\
\hline $5.0<\mathrm{pH}<=6.0$ & 1.001 \\
\hline $6.0<\mathrm{pH}<=6.5$ & 1.0001 \\
\hline $6.5<\mathrm{pH}<=7.0$ & 1.00001 \\
\hline $7.0<\mathrm{pH}<=7.5$ & 0.99999 \\
\hline $7.5<\mathrm{pH}<=8.0$ & 0.9999 \\
\hline $8.0<\mathrm{pH}<=9.0$ & 0.999 \\
\hline $9.0<\mathrm{pH}<=10.0$ & 0.995 \\
\hline $10.0<\mathrm{pH}<=11.0$ & 0.99 \\
\hline $11.0<\mathrm{pH}$ & 0.95 \\
\hline
\end{tabular}

Ammonia production rate increases as $\mathrm{M}$ value increases. Relationships of $\mathrm{NH} 3$ production in the melter and $\mathrm{pH}$ in the SBS are discussed in Section 4.8.16.

The conversion factor for the SO4-2 decomposition reaction is also determined dynamically depending on the concentration of SO4-2 in the feed:

$\mathrm{C}_{\mathrm{SO} 3}=0.22369 /\left(\mathrm{M}_{\mathrm{Na} 2 \mathrm{O}} /\left(\mathrm{M}_{\mathrm{SO} 3}+20.321\right)\right)$

If $\mathrm{C}_{\mathrm{SO} 3} \leq 0.002941$ or $\mathrm{C}_{\mathrm{SO} 3} \geq 0.008$ then

$\mathrm{DF}_{\mathrm{SO} 4}=8000$

Else

$\mathrm{DF}_{\mathrm{SO} 4}=1 /\left(73.5 \times \mathrm{C}_{\mathrm{SO} 3}-0.216\right)$

$\mathrm{CF}_{\mathrm{SO} 4}=1 / \mathrm{DF}_{\mathrm{SO} 4}$

Where:

$\mathrm{C}_{\mathrm{SO} 3}=\mathrm{SO} 3$ if $100 \%$ of the SO4-2 are converted to glass (unitless)

$\mathrm{M}_{\mathrm{Na} 2 \mathrm{O}} \quad=\mathrm{Na} 2 \mathrm{O}$ equivalent of the melter feed sodium $(\mathrm{kgs})$

$\mathrm{M}_{\mathrm{SO} 3} \quad=\mathrm{SO} 3$ equivalent of the melter feed sulfate $(\mathrm{kgs})$

$\mathrm{DF}_{\mathrm{SO} 4} \quad=\mathrm{LAW}$ melter decontamination factor for sulfate (unitless)

$\mathrm{CF}_{\mathrm{SO} 4}=$ Conversion factor for the SO4-2 $(1, \mathrm{~s})$ decomposition reaction (fraction)

After splitting to the offgas system, the remaining solid and liquid arrays are combined in the liquid array and the following reactions occur in each melter, reactions take place in the order presented (note that some reactions have been shown in the table above).

\begin{tabular}{|l|l|}
\hline Organic Destruction Reactions & Conversion (Fraction) \\
\hline $14-\mathrm{C}(1, \mathrm{~s})+\mathrm{O} 2(\mathrm{~g}) \rightarrow \mathrm{CO} 2(\mathrm{~g})$ & $0.0(14-\mathrm{C}$ is $100 \%$ split $)$ \\
\hline $\mathrm{TOC}(1, \mathrm{~s})+\mathrm{O} 2(\mathrm{~g}) \rightarrow \mathrm{CO} 2(\mathrm{~g})$ & 1.0 \\
\hline
\end{tabular}




\begin{tabular}{|l|l|}
\hline Organic Destruction Reactions & Conversion (Fraction) \\
\hline $\mathrm{C} 12 \mathrm{H} 4 \mathrm{Cl} 6(\mathrm{l}, \mathrm{s})+11.5 \mathrm{O} 2(\mathrm{~g})+\mathrm{H} 2 \mathrm{O}(\mathrm{l}) \rightarrow 12 \mathrm{CO} 2(\mathrm{~g})+6 \mathrm{HCl}(\mathrm{g})$ & 1.0 \\
\hline $\mathrm{C} 2 \mathrm{H} 3 \mathrm{~N}(\mathrm{l}, \mathrm{s})+3.75 \mathrm{O} 2(\mathrm{~g}) \rightarrow 2 \mathrm{CO} 2(\mathrm{~g})+1.5 \mathrm{H} 2 \mathrm{O}(\mathrm{g})+\mathrm{NO} 2(\mathrm{~g})$ & 1.0 \\
\hline $\mathrm{C} 2 \mathrm{O} 4-2(1, \mathrm{~s}) \rightarrow 2 \mathrm{CO} 2(\mathrm{~g})$ & 1.0 \\
\hline $\mathrm{CHO} 2-(\mathrm{l}, \mathrm{s})+0.25 \mathrm{O} 2(\mathrm{~g}) \rightarrow \mathrm{CO} 2(\mathrm{~g})+0.5 \mathrm{H} 2 \mathrm{O}(\mathrm{g})$ & 1.0 \\
\hline $\mathrm{PPG}(\mathrm{l}, \mathrm{s})+240 \mathrm{O} 2(\mathrm{~g}) \rightarrow 180 \mathrm{CO} 2(\mathrm{~g})+181 \mathrm{H} 2 \mathrm{O}(\mathrm{g})$ & 1.0 \\
\hline $\mathrm{DPPG}(\mathrm{l}, \mathrm{s})+240 \mathrm{O} 2(\mathrm{~g}) \rightarrow 180 \mathrm{CO} 2(\mathrm{~g})+181 \mathrm{H} 2 \mathrm{O}(\mathrm{g})$ & 1.0 \\
\hline PDMS $(\mathrm{l}, \mathrm{s})+1892 \mathrm{O} 2(\mathrm{~g}) \rightarrow 946 \mathrm{CO} 2(\mathrm{~g})+1419 \mathrm{H} 2 \mathrm{O}(\mathrm{g})+472 \mathrm{SiO} 2(\mathrm{o})$ & 1.0 \\
\hline $\mathrm{DPDMS}(1, \mathrm{~s})+1892 \mathrm{O} 2(\mathrm{~g}) \rightarrow 946 \mathrm{CO} 2(\mathrm{~g})+1419 \mathrm{H} 2 \mathrm{O}(\mathrm{g})+472 \mathrm{SiO} 2(\mathrm{o})$ & 1.0 \\
\hline
\end{tabular}

\begin{tabular}{|c|c|}
\hline Miscellaneous Reactions & Conversion (Fraction) \\
\hline $2 \mathrm{Cl}-(1, \mathrm{~s})+2 \mathrm{Na}+(\mathrm{l}, \mathrm{s})+\mathrm{H} 2 \mathrm{O}(\mathrm{l}) \rightarrow \mathrm{Na} 2 \mathrm{O}(\mathrm{o})+2 \mathrm{HCl}(\mathrm{g})$ & 0.5025 \\
\hline $2 \mathrm{~F}-(1, \mathrm{~s})+2 \mathrm{Na}+(\mathrm{l}, \mathrm{s})+\mathrm{H} 2 \mathrm{O}(\mathrm{l}) \rightarrow \mathrm{Na} 2 \mathrm{O}(\mathrm{o})+2 \mathrm{HF}(\mathrm{g})$ & 0.5319 \\
\hline $129-\mathrm{I}(1, \mathrm{~s}) \rightarrow 129-\mathrm{I}(\mathrm{g})$ & 0.4167 \\
\hline $\mathrm{Hg}+2(1, \mathrm{~s}) \rightarrow \mathrm{Hg}(\mathrm{g})$ & 1.0 \\
\hline 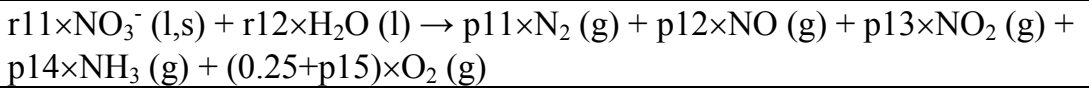 & dynamically calculated \\
\hline $\begin{array}{l}\mathrm{r} 21 \times \mathrm{NO}_{2}^{-}(\mathrm{l}, \mathrm{s})+\mathrm{r} 22 \times \mathrm{H}_{2} \mathrm{O}(\mathrm{l}) \rightarrow \mathrm{p} 21 \times \mathrm{N}_{2}(\mathrm{~g})+\mathrm{p} 22 \times \mathrm{NO}(\mathrm{g})+\mathrm{p} 23 \times \mathrm{NO}_{2}(\mathrm{~g})+ \\
\mathrm{p} 24 \times \mathrm{NH}_{3}(\mathrm{~g})+(0.25+\mathrm{p} 25) \times \mathrm{O}_{2}(\mathrm{~g})\end{array}$ & dynamically calculated \\
\hline $\mathrm{SO} 4-2(1, \mathrm{~s}) \rightarrow \mathrm{SO} 2(\mathrm{~g})+\mathrm{O} 2(\mathrm{~g})$ & $\begin{array}{l}\text { dynamically calculated, } \\
\text { remaining oxidized }\end{array}$ \\
\hline $\mathrm{C} 12 \mathrm{H} 22 \mathrm{O} 11(\mathrm{o})+8 \mathrm{NO} 3-(\mathrm{l}, \mathrm{s}) \rightarrow 6 \mathrm{C} 2 \mathrm{H} 3 \mathrm{~N}(\mathrm{~g})+2 \mathrm{H} 2 \mathrm{O}(\mathrm{g})+\mathrm{N} 2(\mathrm{~g})+16.5 \mathrm{O} 2(\mathrm{~g})$ & 0.0159 \\
\hline $\mathrm{C} 12 \mathrm{H} 22 \mathrm{O} 11(\mathrm{o})+6 \mathrm{HCl}(\mathrm{g})+0.5 \mathrm{O} 2(\mathrm{~g}) \rightarrow \mathrm{C} 12 \mathrm{H} 4 \mathrm{Cl}(\mathrm{g})+12 \mathrm{H} 2 \mathrm{O}(\mathrm{g})$ & $3.79 \mathrm{e}-10$ \\
\hline $\mathrm{C} 12 \mathrm{H} 22 \mathrm{O} 11(\mathrm{o})+8 \mathrm{NO} 3-(\mathrm{l}, \mathrm{s}) \rightarrow 12 \mathrm{CO} 2(\mathrm{~g})+11 \mathrm{H} 2 \mathrm{O}(\mathrm{g})+4 \mathrm{~N} 2(\mathrm{~g})$ & 0.6 \\
\hline $\mathrm{C} 12 \mathrm{H} 22 \mathrm{O} 11(\mathrm{o})+8 \mathrm{NO} 2(1, \mathrm{~s})+4 \mathrm{O} 2(\mathrm{~g}) \rightarrow 12 \mathrm{CO} 2(\mathrm{~g})+11 \mathrm{H} 2 \mathrm{O}(\mathrm{g})+4 \mathrm{~N} 2(\mathrm{~g})$ & 1.0 \\
\hline $\mathrm{C} 12 \mathrm{H} 22 \mathrm{O} 11(\mathrm{o})+12 \mathrm{O} 2(\mathrm{~g}) \rightarrow 12 \mathrm{CO} 2(\mathrm{~g})+11 \mathrm{H} 2 \mathrm{O}(\mathrm{g})$ & 1.0 \\
\hline $2 \mathrm{CN}-(\mathrm{l}, \mathrm{s})+2 \mathrm{O} 2(\mathrm{~g}) \rightarrow 2 \mathrm{CO} 2(\mathrm{~g})+\mathrm{N} 2(\mathrm{~g})$ & 1.0 \\
\hline $\mathrm{CO} 3-2(1, \mathrm{~s}) \rightarrow \mathrm{CO} 2(\mathrm{~g})+0.5 \mathrm{O} 2(\mathrm{~g})$ & 1.0 \\
\hline $\mathrm{H}+(1, \mathrm{~s})+\mathrm{OH}(\mathrm{BOUND})(1, \mathrm{~s}) \rightarrow \mathrm{H} 2 \mathrm{O}(\mathrm{g})$ & 1.0 \\
\hline $\mathrm{H} 2 \mathrm{O}(1, \mathrm{~s}) \rightarrow \mathrm{H} 2 \mathrm{O}(\mathrm{g})$ & 1.0 \\
\hline $2 \mathrm{H} 2 \mathrm{O} 2(\mathrm{l}, \mathrm{s}) \rightarrow 2 \mathrm{H} 2 \mathrm{O}(\mathrm{g})+\mathrm{O} 2(\mathrm{~g})$ & 1.0 \\
\hline $2 \mathrm{HCO} 3-(1, \mathrm{~s}) \rightarrow 2 \mathrm{CO} 2(\mathrm{~g})+\mathrm{H} 2 \mathrm{O}(\mathrm{g})+0.5 \mathrm{O} 2(\mathrm{~g})$ & 1.0 \\
\hline $\mathrm{H} 2 \mathrm{CO} 3(1, \mathrm{~s}) \rightarrow \mathrm{CO} 2(\mathrm{~g})+\mathrm{H} 2 \mathrm{O}(\mathrm{g})$ & 1.0 \\
\hline $2 \mathrm{NH} 3(1, \mathrm{~s})+3 \mathrm{O} 2(\mathrm{~g}) \rightarrow \mathrm{NO}(\mathrm{g})+\mathrm{NO} 2(\mathrm{~g})+3 \mathrm{H} 2 \mathrm{O}(\mathrm{g})$ & 1.0 \\
\hline $2 \mathrm{NH} 4+(\mathrm{l}, \mathrm{s})+3.5 \mathrm{O} 2(\mathrm{~g}) \rightarrow \mathrm{NO}(\mathrm{g})+\mathrm{NO} 2(\mathrm{~g})+4 \mathrm{H} 2 \mathrm{O}(\mathrm{g})$ & 1.0 \\
\hline $2 \mathrm{O}-2(\mathrm{l}, \mathrm{s}) \rightarrow \mathrm{O} 2(\mathrm{~g})$ & 1.0 \\
\hline \multicolumn{2}{|l|}{ Sum OH- and OH(BOUND) to OH- (i.e., combine both components into OH-) } \\
\hline $2 \mathrm{OH}-(1, \mathrm{~s}) \rightarrow \mathrm{H} 2 \mathrm{O}(\mathrm{g})+0.5 \mathrm{O} 2(\mathrm{~g})$ & 1.0 \\
\hline
\end{tabular}

\begin{tabular}{|l|l|}
\hline Oxidation Reactions & Conversion (Fraction) \\
\hline $2227-\mathrm{Ac}(1, \mathrm{~s})+1.5 \mathrm{O} 2(\mathrm{~g}) \rightarrow \mathrm{Ac} 2 \mathrm{O} 3(\mathrm{o})$ & 1.0 \\
\hline $2 \mathrm{Ag}+(1, \mathrm{~s})+0.5 \mathrm{O} 2(\mathrm{~g}) \rightarrow \mathrm{Ag} 2 \mathrm{O}(\mathrm{o})$ & 1.0 \\
\hline $2 \mathrm{Al}+3(1, \mathrm{~s})+1.5 \mathrm{O} 2(\mathrm{~g}) \rightarrow \mathrm{Al} 2 \mathrm{O} 3(\mathrm{o})$ & 1.0 \\
\hline $2 \mathrm{Al}(\mathrm{OH}) 4-(1, \mathrm{~s}) \rightarrow \mathrm{Al} 2 \mathrm{O} 3(\mathrm{o})+0.5 \mathrm{O} 2(\mathrm{~g})+4 \mathrm{H} 2 \mathrm{O}(\mathrm{g})$ & 1.0 \\
\hline $2 \mathrm{Al}(\mathrm{OH}) 3(1, \mathrm{~s}) \rightarrow \mathrm{Al} 2 \mathrm{O} 3(\mathrm{o})+3 \mathrm{H} 2 \mathrm{O}(\mathrm{g})$ & 1.0 \\
\hline
\end{tabular}

- Sum 241-Am and 243-Am to 241-Am

\begin{tabular}{|l|l}
\hline 2 241-Am $(1, \mathrm{~s})+1.5 \mathrm{O} 2(\mathrm{~g}) \rightarrow \mathrm{Am} 2 \mathrm{O} 3(\mathrm{o})$ & 1.0
\end{tabular}

$2 \mathrm{As}+5(\mathrm{l}, \mathrm{s})+2.5 \mathrm{O} 2(\mathrm{~g}) \rightarrow \mathrm{As} 2 \mathrm{O} 5(\mathrm{o})$

$2 \mathrm{~B}+3(1, \mathrm{~s})+1.5 \mathrm{O} 2(\mathrm{~g}) \rightarrow \mathrm{B} 2 \mathrm{O} 3(\mathrm{o})$

1.0

- $\quad$ Sum $\mathrm{Ba}+2$ and $137 \mathrm{~m}-\mathrm{Ba}$ to $\mathrm{Ba}+2$

$\mathrm{Ba}+2(1, \mathrm{~s})+0.5 \mathrm{O} 2(\mathrm{~g}) \rightarrow \mathrm{BaO}(\mathrm{o})$

$\mathrm{Be}+2(1, \mathrm{~s})+0.5 \mathrm{O} 2(\mathrm{~g}) \rightarrow \mathrm{BeO}(\mathrm{o})$

$2 \mathrm{Bi}+3(1, \mathrm{~s})+1.5 \mathrm{O} 2(\mathrm{~g}) \rightarrow \mathrm{Bi} 2 \mathrm{O} 3(\mathrm{o})$

$\mathrm{Ca}+2(1, \mathrm{~s})+0.5 \mathrm{O} 2(\mathrm{~g}) \rightarrow \mathrm{CaO}(\mathrm{o})$

- $\quad$ Sum $\mathrm{Cd}+2$ and $113 \mathrm{~m}-\mathrm{Cd}$ to $\mathrm{Cd}+2$ 
$\mathrm{Cd}+2(1, \mathrm{~s})+0.5 \mathrm{O} 2(\mathrm{~g}) \rightarrow \mathrm{CdO}(\mathrm{o})$

$4 \mathrm{Ce}+3(1, \mathrm{~s})+3 \mathrm{O} 2(\mathrm{~g}) \rightarrow 2 \mathrm{Ce} 2 \mathrm{O} 3$ (o)

$\mathrm{Cl}-(\mathrm{l}, \mathrm{s}) \rightarrow \mathrm{Cl}-(\mathrm{o})$

- Sum 242-Cm, 243-Cm, and 244-Cm to 242-Cm

\begin{tabular}{|l|l}
\hline $2242-\mathrm{Cm}(1, \mathrm{~s})+1.5 \mathrm{O} 2(\mathrm{~g}) \rightarrow \mathrm{Cm} 2 \mathrm{O} 3(\mathrm{o})$ & 1.0
\end{tabular}

- $\quad \mathrm{Sum} \mathrm{Co}+3$ and $60-\mathrm{Co}$ to $\mathrm{Co}+3$

$2 \mathrm{Co}+3(1, \mathrm{~s})+\mathrm{O} 2(\mathrm{~g}) \rightarrow 2 \mathrm{CoO}(\mathrm{o})$

$2 \mathrm{Cr}(\mathrm{TOTAL})(1, \mathrm{~s})+1.5 \mathrm{O} 2(\mathrm{~g}) \rightarrow \mathrm{Cr} 2 \mathrm{O} 3(\mathrm{o})$

$2 \mathrm{Cr}(\mathrm{OH}) 4-(1, \mathrm{~s}) \rightarrow \mathrm{Cr} 2 \mathrm{O} 3(\mathrm{o})+0.5 \mathrm{O} 2(\mathrm{~g})+4 \mathrm{H} 2 \mathrm{O}(\mathrm{g})$

- $\quad$ Sum Cs+, 134-Cs, and 137-Cs to $\mathrm{Cs}+$

$2 \mathrm{Cs}+(1, \mathrm{~s})+0.5 \mathrm{O} 2(\mathrm{~g}) \rightarrow \mathrm{Cs} 2 \mathrm{O}(\mathrm{o})$

$\mathrm{Cu}+2(1, \mathrm{~s})+0.5 \mathrm{O} 2(\mathrm{~g}) \rightarrow \mathrm{CuO}(\mathrm{o})$

\begin{tabular}{|l|}
\hline 1.0 \\
1.0 \\
1.0
\end{tabular}

- $\quad$ Sum 152-Eu, 154-Eu, and 155-Eu to 152-Eu

2 152-Eu $(1, \mathrm{~s})+0.5 \mathrm{O} 2(\mathrm{~g}) \rightarrow \mathrm{Eu} 2 \mathrm{O} 3(\mathrm{o})$

$\mathrm{F}-(1, \mathrm{~s}) \rightarrow \mathrm{F}-(\mathrm{o})$

$2 \mathrm{Fe}+3(1, \mathrm{~s})+1.5 \mathrm{O} 2(\mathrm{~g}) \rightarrow \mathrm{Fe} 2 \mathrm{O} 3(\mathrm{o})$

$\mathrm{Hg}+2(1, \mathrm{~s})+0.5 \mathrm{O} 2(\mathrm{~g}) \rightarrow \mathrm{HgO}(\mathrm{o})$

$129-\mathrm{I}(1, \mathrm{~s}) \rightarrow 129-\mathrm{I}(\mathrm{o})$

$2 \mathrm{~K}+(1, \mathrm{~s})+0.5 \mathrm{O} 2(\mathrm{~g}) \rightarrow \mathrm{K} 2 \mathrm{O}(\mathrm{o})$

$2 \mathrm{La}+3(1, \mathrm{~s})+1.5 \mathrm{O} 2(\mathrm{~g}) \rightarrow \mathrm{La} 2 \mathrm{O} 3(\mathrm{o})$

$2 \mathrm{Li}+(1, \mathrm{~s})+0.5 \mathrm{O} 2(\mathrm{~g}) \rightarrow \mathrm{Li} 2 \mathrm{O}(\mathrm{o})$

$\mathrm{Mg}+2(1, \mathrm{~s})+0.5 \mathrm{O} 2(\mathrm{~g}) \rightarrow \mathrm{MgO}(\mathrm{o})$

$2 \mathrm{MnO} 4-(1, \mathrm{~s}) \rightarrow 2 \mathrm{MnO}(\mathrm{o})+3 \mathrm{O} 2(\mathrm{~g})$

$2 \mathrm{Mn}+4(1, \mathrm{~s})+\mathrm{O} 2(\mathrm{~g}) \rightarrow 2 \mathrm{MnO}(\mathrm{o})$

$2 \mathrm{MnO} 2(1, \mathrm{~s}) \rightarrow 2 \mathrm{MnO}(\mathrm{o})+\mathrm{O} 2(\mathrm{~g})$

$2 \mathrm{Mnp}(1, \mathrm{~s})+1.5 \mathrm{O} 2(\mathrm{~g}) \rightarrow \mathrm{Mnp} 2 \mathrm{O} 3(\mathrm{o})$

$\mathrm{Mo}+6(\mathrm{l}, \mathrm{s})+1.5 \mathrm{O} 2(\mathrm{~g}) \rightarrow \mathrm{MoO} 3(\mathrm{o})$

$2 \mathrm{Na}+(1, \mathrm{~s})+0.5 \mathrm{O} 2(\mathrm{~g}) \rightarrow \mathrm{Na} 2 \mathrm{O}(\mathrm{o})$

$2 \mathrm{Nap}+(1, \mathrm{~s})+0.5 \mathrm{O} 2(\mathrm{~g}) \rightarrow \mathrm{Nap} 2 \mathrm{O}(\mathrm{o})$

$293 \mathrm{~m}-\mathrm{Nb}(\mathrm{l}, \mathrm{s})+2.5 \mathrm{O} 2(\mathrm{~g}) \rightarrow \mathrm{Nb} 2 \mathrm{O} 5$ (o)

$2 \mathrm{Nd}+3(1, \mathrm{~s})+1.5 \mathrm{O} 2(\mathrm{~g}) \rightarrow \mathrm{Nd} 2 \mathrm{O} 3(\mathrm{o})$

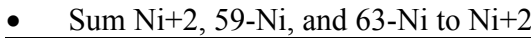

$\mathrm{Ni}+2(1, \mathrm{~s})+0.5 \mathrm{O} 2(\mathrm{~g}) \rightarrow \mathrm{NiO}(\mathrm{o})$

$237-\mathrm{Np}(1, \mathrm{~s})+\mathrm{O} 2(\mathrm{~g}) \rightarrow \mathrm{NpO} 2$ (o)

2 PO4-3 (1,s) $\rightarrow$ P2O5 (o) +1.5 O2 (g)

$2231-\mathrm{Pa}(1, \mathrm{~s})+1.5 \mathrm{O} 2(\mathrm{~g}) \rightarrow \mathrm{Pa} 2 \mathrm{O} 3(\mathrm{o})$

$\mathrm{Pb}+2(1, \mathrm{~s})+0.5 \mathrm{O} 2(\mathrm{~g}) \rightarrow \mathrm{PbO}(\mathrm{o})$

$\mathrm{Pd}+2(1, \mathrm{~s})+0.5 \mathrm{O} 2(\mathrm{~g}) \rightarrow \mathrm{PdO}(\mathrm{o})$

$2 \mathrm{Pr}+3(1, \mathrm{~s})+1.5 \mathrm{O} 2(\mathrm{~g}) \rightarrow \mathrm{Pr} 2 \mathrm{O} 3(\mathrm{o})$

- $\quad$ Sum $\mathrm{Pu}+4,238-\mathrm{Pu}, 239-\mathrm{Pu}, 240-\mathrm{Pu}, 241-\mathrm{Pu}$, and 242-Pu to $\mathrm{Pu}+4$

$\mathrm{Pu}+4(1, \mathrm{~s})+\mathrm{O} 2(\mathrm{~g}) \rightarrow \mathrm{PuO} 2(\mathrm{o})$

- $\quad$ Sum 226-Ra and 228-Ra to 226-Ra

$2226-\mathrm{Ra}(1, \mathrm{~s})+\mathrm{O} 2(\mathrm{~g}) \rightarrow 2 \mathrm{RaO}(\mathrm{o})$

$2 \mathrm{Rb}+(1, \mathrm{~s})+0.5 \mathrm{O} 2(\mathrm{~g}) \rightarrow \mathrm{Rb} 2 \mathrm{O}(\mathrm{o})$

$2 \mathrm{Rh}+3(\mathrm{l}, \mathrm{s})+1.5 \mathrm{O} 2(\mathrm{~g}) \rightarrow \mathrm{Rh} 2 \mathrm{O} 3(\mathrm{o})$

- $\quad$ Sum $\mathrm{Ru}+3$ and 106-Ru to $\mathrm{Ru}+3$

$\mathrm{Ru}+3(1, \mathrm{~s})+\mathrm{O} 2(\mathrm{~g}) \rightarrow \mathrm{RuO} 2(\mathrm{o})$

$\mathrm{SO} 4-2(1, \mathrm{~s}) \rightarrow \mathrm{SO} 3(\mathrm{o})+0.5 \mathrm{O} 2(\mathrm{~g})$

- $\quad \mathrm{Sum} \mathrm{Sb}+5$ and $125-\mathrm{Sb}$ to $\mathrm{Sb}+5$

$2 \mathrm{Sb}+5(1, \mathrm{~s})+1.5 \mathrm{O} 2(\mathrm{~g}) \rightarrow \mathrm{Sb} 2 \mathrm{O} 3(\mathrm{o})$

- $\quad$ Sum $\mathrm{Se}+6$ and 79-Se to $\mathrm{Se}+6$

$\mathrm{Se}+6(1, \mathrm{~s})+\mathrm{O} 2(\mathrm{~g}) \rightarrow \mathrm{SeO} 2(\mathrm{o})$

$\mathrm{Si}+4(1, \mathrm{~s})+\mathrm{O} 2(\mathrm{~g}) \rightarrow \mathrm{SiO} 2(\mathrm{o})$

2 151-Sm $(1, \mathrm{~s})+1.5 \mathrm{O} 2(\mathrm{~g}) \rightarrow \mathrm{Sm} 2 \mathrm{O} 3(\mathrm{o})$

$126-\mathrm{Sn}(1, \mathrm{~s})+\mathrm{O} 2(\mathrm{~g}) \rightarrow \mathrm{SnO} 2$ (o)

\begin{tabular}{|l}
1.0 \\
1.0 \\
1.0
\end{tabular}

1.0

1.0

\begin{tabular}{|l|}
\hline 1.0 \\
\hline 1.0 \\
\hline 1.0 \\
\hline $0.0(100 \%$ split $)$ \\
\hline 1.0 \\
\hline 1.0 \\
1.0 \\
1.0 \\
1.0 \\
1.0 \\
1.0 \\
1.0 \\
1.0 \\
1.0 \\
\hline 1.0 \\
1.0 \\
1.0 \\
\hline 1.0 \\
\hline
\end{tabular}

\begin{tabular}{|l|}
\hline 1.0 \\
1.0 \\
1.0 \\
1.0 \\
1.0 \\
1.0 \\
1.0 \\
\hline
\end{tabular}

1.0

1.0

1.0

1.0

\begin{tabular}{|l|l}
\hline & 1.0 \\
\hline & 1.0
\end{tabular}

\begin{tabular}{|l|l}
\hline (o) & 1.0 \\
\hline
\end{tabular}

\begin{tabular}{|l|l}
\hline & 1.0 \\
\hline & 1.0
\end{tabular}

\begin{tabular}{|l|}
\hline 1.0 \\
\hline 1.0 \\
\hline 1.0 \\
1.0
\end{tabular}


- $\quad \mathrm{Sum} \mathrm{Sr}+2$ and $90-\mathrm{Sr}$ to $\mathrm{Sr}+2$

\begin{tabular}{|c|c|}
\hline $\mathrm{Sr}+2(1, \mathrm{~s})+0.5 \mathrm{O} 2(\mathrm{~g}) \rightarrow \mathrm{SrO}(\mathrm{o})$ & 1.0 \\
\hline $2 \mathrm{Ta}+5(\mathrm{l}, \mathrm{s})+2.5 \mathrm{O} 2(\mathrm{~g}) \rightarrow \mathrm{Ta} 2 \mathrm{O} 5(\mathrm{o})$ & 1.0 \\
\hline \multicolumn{2}{|l|}{ - $\quad$ Sum Tc+7 and 99-Tc to Tc+7 } \\
\hline $\mathrm{Tc}+7(1, \mathrm{~s})+3.5 \mathrm{O} 2(\mathrm{~g}) \rightarrow \mathrm{Tc} 2 \mathrm{O} 7(\mathrm{o})$ & 1.0 \\
\hline $\mathrm{Te}+6(1, \mathrm{~s})+\mathrm{O} 2(\mathrm{~g}) \rightarrow \mathrm{TeO} 2(\mathrm{o})$ & 1.0 \\
\hline \multicolumn{2}{|l|}{ - $\quad$ Sum $\mathrm{Th}+4,229-\mathrm{Th}$, and 232-Th to $\mathrm{Th}+4$} \\
\hline $\mathrm{Th}+4(\mathrm{l}, \mathrm{s})+\mathrm{O} 2(\mathrm{~g}) \rightarrow \mathrm{ThO} 2(\mathrm{o})$ & 1.0 \\
\hline $\mathrm{Ti}+4(1, \mathrm{~s})+\mathrm{O} 2(\mathrm{~g}) \rightarrow \mathrm{TiO} 2(\mathrm{o})$ & 1.0 \\
\hline $4 \mathrm{~T} 1+3(1, \mathrm{~s})+\mathrm{O} 2(\mathrm{~g}) \rightarrow 2 \mathrm{~T} 12 \mathrm{O}(\mathrm{o})$ & 1.0 \\
\hline \multicolumn{2}{|c|}{ - $\quad$ Sum U(TOTAL), 232-U, 233-U, 234-U, 235-U, 236-U, and 238-U to U(TOTAL) } \\
\hline $2 \mathrm{U}(\mathrm{TOTAL})(1, \mathrm{~s})+3 \mathrm{O} 2(\mathrm{~g}) \rightarrow 2 \mathrm{UO} 3(\mathrm{o})$ & 1.0 \\
\hline $2 \mathrm{~V}+5(1, \mathrm{~s})+2.5 \mathrm{O} 2(\mathrm{~g}) \rightarrow \mathrm{V} 2 \mathrm{O} 5(\mathrm{o})$ & 1.0 \\
\hline $\mathrm{W}+6(\mathrm{l}, \mathrm{s})+1.5 \mathrm{O} 2(\mathrm{~g}) \rightarrow \mathrm{WO} 3(\mathrm{o})$ & 1.0 \\
\hline \multicolumn{2}{|l|}{ - $\quad$ Sum $\mathrm{Y}+3$ and $90-\mathrm{Y}$ to $\mathrm{Y}+3$} \\
\hline $2 \mathrm{Y}+3(1, \mathrm{~s})+1.5 \mathrm{O} 2(\mathrm{~g}) \rightarrow \mathrm{Y} 2 \mathrm{O} 3(\mathrm{o})$ & 1.0 \\
\hline $\mathrm{Zn}+2(1, \mathrm{~s})+0.5 \mathrm{O} 2(\mathrm{~g}) \rightarrow \mathrm{ZnO}(\mathrm{o})$ & 1.0 \\
\hline \multicolumn{2}{|l|}{ - $\quad$ Sum $\mathrm{Zr}+4$ and $93-\mathrm{Zr}$ to $\mathrm{Zr}+4$} \\
\hline $\mathrm{Zr}+4(1, \mathrm{~s})+\mathrm{O} 2(\mathrm{~g}) \rightarrow \mathrm{ZnO} 2(\mathrm{o})$ & 1.0 \\
\hline
\end{tabular}

A lower set volume (1,875 gallons) is applied to the LAW melters where the molten glasses above the set volume will be transferred to the ILAW containers. LMP-MLTR-00001 transfers molten glasses to ILAW1 while LMP-MLTR-00002 transfers molten glasses to ILAW2. The ILAW containers are 564 gallons in size. ILAW glass has a $2.58 \mathrm{~g} / \mathrm{cm}^{3}$ density. Container counts and glass compositions are tracked by the model. Gases from the melter are routed to a corresponding LAW Offgas Process system. LMP-MLTR-00001 transfers materials to LOP-SCB-00001 and LMP-MLTR-00002 transfers materials to LOP-SCB-00002.

Figures F-13 and F-14 provide graphical illustrations of the LMP/LOP systems where connections with upstream and downstream systems are shown. Table N-1 provides the totalizer files that are used to track the process streams and material flows into and out of the LMP/LOP.

\subsubsection{LAW Offgas Process (LOP) Systems}

\begin{tabular}{|l|l|}
\hline Vessel Tag \# & Equipment Description \\
\hline LOP-SCB-00001 & LAW Melter 1 Submerged Bed Scrubber \\
\hline LOP-SCB-00002 & LAW Melter 2 Submerged Bed Scrubber \\
\hline LOP-VSL-00001 & LAW Melter 1 SBS Condensate Vessel \\
\hline LOP-VSL-00002 & LAW Melter 2 SBS Condensate Vessel \\
\hline
\end{tabular}

The LAW offgas process includes two separate trains of LAW Melter Offgas Treatment Process systems and a LAW Secondary Offgas/Vessel Process system. The LAW SBSs receive offgas from a corresponding LAW melter. LOP-SCB-00001 receives materials from LMP-MLTR-00001 and LOP-SCB-00002 receives materials from LMP-MLTR-00002. Each of the LAW SBS vessels is associated with a SBS Condensate Vessel. For modeling simplicity, the condensate vessels have been merged into their associated SBSs, for example, working volumes have been combined as follows:

\section{LOP-SCB-00001/2}

Minimum Volume $=451+0$ gallons $=451$ gallons

Maximum Volume $=6,651+3,329$ gallons $=9,980$ gallons

Set Volume $=8,980$ gallons 
Maximum Accumulation Time $=48$ hours

Initially, the scrubbers are filled to the heel volume with demineralized water from a chem-add. Upon arriving at the SBS, the entrained liquid and solid from the melter are split to the WESP according to the split values given in Table D-13. The remaining liquid, solid, and gas materials undergo the following reactions (reactions take place in the order presented):

\begin{tabular}{|l|l|}
\hline Reactions & Conversion (Fraction) \\
\hline $\mathrm{H} 2 \mathrm{O}(\mathrm{g}) \rightarrow \mathrm{H} 2 \mathrm{O}(\mathrm{l})$ or $\mathrm{H} 2 \mathrm{O}(\mathrm{l}) \rightarrow \mathrm{H} 2 \mathrm{O}(\mathrm{g})$ & $\begin{array}{l}0.138 \text { mole fraction at } \\
\text { outlet }\end{array}$ \\
\hline $\mathrm{Hg}(\mathrm{g}) \rightarrow \mathrm{Hg}+2(\mathrm{l})$ & Eq. 60 \\
\hline $2 \mathrm{~B}+3(\mathrm{~s})+1.5 \mathrm{O} 2(\mathrm{~g})+3 \mathrm{H} 2 \mathrm{O}(\mathrm{l}) \rightarrow 2 \mathrm{H}+(\mathrm{l})+2 \mathrm{~B}+3(\mathrm{l})+4 \mathrm{O}-2(\mathrm{l})+2 \mathrm{H} 2 \mathrm{O}(\mathrm{l})$ & 1.0 \\
\hline $\mathrm{HCl}(\mathrm{g}) \rightarrow \mathrm{Cl}-(\mathrm{l})+\mathrm{H}+(\mathrm{l})$ & 0.8588 \\
\hline $\mathrm{HF}(\mathrm{g}) \rightarrow \mathrm{F}-(\mathrm{l})+\mathrm{H}+(\mathrm{l})$ & 0.8384 \\
\hline $3 \mathrm{NO} 2(\mathrm{~g})+\mathrm{H} 2 \mathrm{O}(\mathrm{l}) \rightarrow 2 \mathrm{NO} 3-(\mathrm{l})+2 \mathrm{H}+(\mathrm{l})+\mathrm{NO}(\mathrm{g})$ & 0.002 \\
\hline $4 \mathrm{NO}(\mathrm{g})+2 \mathrm{H} 2 \mathrm{O}(\mathrm{l})+3 \mathrm{O} 2(\mathrm{~g}) \rightarrow 4 \mathrm{NO} 3-(\mathrm{l})+4 \mathrm{H}+(\mathrm{l})$ & 0.0548 \\
\hline $\mathrm{P} 2 \mathrm{O} 5(\mathrm{~g})+3 \mathrm{H} 2 \mathrm{O}(\mathrm{l}) \rightarrow 0.5 \mathrm{PO} 4-3(\mathrm{l})+1.5 \mathrm{PO} 4-3(\mathrm{~s})+6 \mathrm{H}+(\mathrm{l})$ & 0.6 \\
\hline $\mathrm{SO} 2(\mathrm{~g})+\mathrm{H} 2 \mathrm{O}(\mathrm{l})+0.5 \mathrm{O} 2(\mathrm{~g}) \rightarrow \mathrm{SO} 4-2(\mathrm{l})+2 \mathrm{H}+(\mathrm{l})$ & 0.8721 \\
\hline $\mathrm{C} 2 \mathrm{H} 3 \mathrm{~N}(\mathrm{~g}) \rightarrow \mathrm{C} 2 \mathrm{H} 3 \mathrm{~N}(\mathrm{l})$ & 0.8 \\
\hline $\mathrm{C} 12 \mathrm{H} 4 \mathrm{Cl} 6(\mathrm{~g}) \rightarrow \mathrm{C} 12 \mathrm{H} 4 \mathrm{Cl} 6(\mathrm{l})$ & 0.8837 \\
\hline $129-\mathrm{I}(\mathrm{g}) \rightarrow 129-\mathrm{I}(\mathrm{l})$ & 0.7980 \\
\hline $\mathrm{Na}+(\mathrm{s})+\mathrm{Cl}-(\mathrm{s}) \rightarrow \mathrm{Na}+(\mathrm{l})+\mathrm{Cl}-(\mathrm{l})$ & $1.0[\mathrm{Cl}-](\mathrm{s})=0.0$ \\
\hline $\mathrm{Nap}+(\mathrm{s})+\mathrm{Cl}-(\mathrm{s}) \rightarrow \mathrm{Nap}+(\mathrm{l})+\mathrm{Cl}-(\mathrm{l})$ & $1.0[\mathrm{Cl}-](\mathrm{s})=0.0$ \\
\hline $\mathrm{Na}+(\mathrm{s})+\mathrm{F}-(\mathrm{s}) \rightarrow \mathrm{Na}+(\mathrm{l})+\mathrm{F}-(\mathrm{l})$ & $1.0[\mathrm{~F}-](\mathrm{s})=0.0$ \\
\hline $\mathrm{Nap}+(\mathrm{s})+\mathrm{F}-(\mathrm{s}) \rightarrow \mathrm{Nap}+(\mathrm{l})+\mathrm{F}-(\mathrm{l})$ & $1.0[\mathrm{~F}-](\mathrm{s})=0.0$ \\
\hline $4 \mathrm{Na}+(\mathrm{s})+\mathrm{O} 2(\mathrm{~g})+2 \mathrm{H} 2 \mathrm{O}(\mathrm{l}) \rightarrow 4 \mathrm{Na}+(\mathrm{l})+4 \mathrm{OH}-(\mathrm{l})$ & 1.0 \\
\hline $4 \mathrm{Nap}+(\mathrm{s})+\mathrm{O} 2(\mathrm{~g})+2 \mathrm{H} 2 \mathrm{O}(\mathrm{l}) \rightarrow 4 \mathrm{Nap}+(\mathrm{l})+4 \mathrm{OH}-(\mathrm{l})$ & 1.0 \\
\hline $4 \mathrm{~K}+(\mathrm{s})+\mathrm{O} 2(\mathrm{~g})+2 \mathrm{H} 2 \mathrm{O}(\mathrm{l}) \rightarrow 4 \mathrm{~K}+(\mathrm{l})+4 \mathrm{OH}-(\mathrm{l})$ & 1.0 \\
\hline $2 \mathrm{Ca}+2(\mathrm{~s})+\mathrm{O} 2(\mathrm{~g})+2 \mathrm{H} 2 \mathrm{O}(\mathrm{l}) \rightarrow 2 \mathrm{Ca}+2(\mathrm{l})+4 \mathrm{OH}-(\mathrm{l})$ & 0.3 \\
\hline $2 \mathrm{Sr}+2(\mathrm{~s})+\mathrm{O} 2(\mathrm{~g})+2 \mathrm{H} 2 \mathrm{O}(\mathrm{l}) \rightarrow 2 \mathrm{Sr}+2(\mathrm{l})+4 \mathrm{OH}-(\mathrm{l})$ & 0.5 \\
\hline $290-\mathrm{Sr}(\mathrm{s})+\mathrm{O} 2(\mathrm{~g})+2 \mathrm{H} 2 \mathrm{O}(\mathrm{l}) \rightarrow 290-\mathrm{Sr}(\mathrm{s})+4 \mathrm{OH}-(\mathrm{l})$ & 0.5 \\
\hline $4 \mathrm{Li}+(\mathrm{s})+\mathrm{O} 2(\mathrm{~g})+2 \mathrm{H} 2 \mathrm{O}(\mathrm{l}) \rightarrow 4 \mathrm{Li}+(\mathrm{l})+4 \mathrm{OH}-(\mathrm{l})$ & 1.0 \\
\hline $\mathrm{NH} 3(\mathrm{~g}) \rightarrow \mathrm{NH} 3(\mathrm{l})$ & 0.90 \\
\hline $\mathrm{NH} 3(\mathrm{l})+\mathrm{H} 2 \mathrm{O}(\mathrm{l}) \rightarrow \mathrm{NH} 4+(\mathrm{l})+\mathrm{OH}-(\mathrm{l})$ & $\mathrm{Eq} .68$ \\
\hline $\mathrm{CO} 2(\mathrm{~g})+\mathrm{H} 2 \mathrm{O}(\mathrm{l}) \rightarrow \mathrm{H} 2 \mathrm{CO} 3(\mathrm{l})$ & 0.001 \\
\hline $\mathrm{H} 2 \mathrm{CO} 3(\mathrm{l}) \rightarrow \mathrm{HCO} 3-(\mathrm{l})+\mathrm{H}+(\mathrm{l})$ & $\mathrm{Eq} .64$ \\
\hline $\mathrm{HCO} 3-(\mathrm{l}) \rightarrow \mathrm{CO} 3-2(\mathrm{l})+\mathrm{H}+(\mathrm{l})$ & $\mathrm{Eq.} 65$ \\
\hline $\mathrm{H}+(\mathrm{l})+\mathrm{OH}-(\mathrm{l}) \rightarrow \mathrm{H} 2 \mathrm{O}(\mathrm{l})$ & 1.0 \\
\hline
\end{tabular}

Conversion factor of the mercury reaction is dependent upon the mercury to chloride ratio in the LAW melter feed, for example,

$\begin{aligned} \mathrm{CF} & =0.833 & & \text { if } \operatorname{Hg}+2(1, \mathrm{~s}) / \mathrm{Cl}-(1, \mathrm{~s}) \leq 0.1 \\ & =0.559 & & \text { if } 0.1<\mathrm{Hg}+2(1, \mathrm{~s}) / \mathrm{Cl}-(1, \mathrm{~s}) \leq 0.5 \\ & =0.286 & & \text { if } \operatorname{Hg}+2(1, \mathrm{~s}) / \mathrm{Cl}-(1, \mathrm{~s})>0.5\end{aligned}$

The remaining gaseous mercury continues on as " $\mathrm{Hg}$ " to the downstream LOP/LVP equipment. The SBS split values for $\mathrm{Hg}+2(1, \mathrm{~s})$ are set to zero. 
Conversion factors for the $\mathrm{H}_{2} \mathrm{CO}_{3}(1)$ and $\mathrm{HCO}_{3}{ }^{-}$(1) reactions are calculated below:

$[$ Total $]=0.001 \times[$ gmoles of $\mathrm{CO} 2(\mathrm{~g})] /($ volume above setpoint in liters $)$

$[\mathrm{H} 2 \mathrm{CO} 3]-$ End $=0.1834 \times[$ Total $]$

$[$ HCO3- $]$-End $=0.8162 \times[$ Total $]$

$\mathrm{H} 2 \mathrm{CO} 3-\mathrm{CF}=([\mathrm{H} 2 \mathrm{CO} 3]$-Begin $-[\mathrm{H} 2 \mathrm{CO} 3]$-End $) /[\mathrm{H} 2 \mathrm{CO} 3]$-Begin

$\mathrm{HCO} 3-\mathrm{CF}=([\mathrm{HCO} 3-]-$ Begin $-[\mathrm{HCO} 3-]-$ End $) /[\mathrm{HCO} 3-]-$ Begin

The conversion factor for the $\mathrm{NH}_{4}^{+}$reaction is calculated using the equilibrium constant as

$[$ Total $]=0.9 \times[$ gmoles of NH3 $(\mathrm{g})] /($ volume above setpoint in liters $)$

$[\mathrm{NH} 3]-\mathrm{End}=5.682 \mathrm{e}-3 \times[$ Total $]$

$\mathrm{NH} 4-\mathrm{CF}=([\mathrm{NH} 3]-$ Begin $-[\mathrm{NH} 3]-$ End $) /[\mathrm{NH} 3]$-Begin

The production of $\mathrm{NH}_{3}(\mathrm{~g})$ from the melter is dependent upon the $\mathrm{pH}$ value at the SBS at the end of the above reactions. Initially, the multiplier $\mathrm{M}$ of the stoichiometric coefficients for Eq. 54 and Eq. 55 is set to 1.0. The $\mathrm{M}$ value is increased if $\mathrm{pH}$ at the $\mathrm{SBS}$ is below 7.0 and is decreased if $\mathrm{pH}$ is above 7.0. This provides the means for achieving a $\mathrm{pH} 7.0$ at the SBS by adjusting the production of $\mathrm{NH}_{3}$ at the melter. Due to the delay effect of the above modeling approach, the following chemical reactions are used to provide further adjustment.

if $\mathrm{pH}<7.0$ then

\begin{tabular}{|l|l|}
\hline $10 \mathrm{H}+(\mathrm{l})+\mathrm{NO} 3-(\mathrm{l}) \rightarrow \mathrm{NH} 4+(\mathrm{l})+3 \mathrm{H} 2 \mathrm{O}(\mathrm{l})$ & $\begin{array}{l}\text { until } \mathrm{H}+\text { or NO3- are } \\
\text { consumed }\end{array}$ \\
\hline $8 \mathrm{H}+(\mathrm{l})+\mathrm{NO} 2(\mathrm{l}) \rightarrow \mathrm{NH} 4+(\mathrm{l})+2 \mathrm{H} 2 \mathrm{O}(\mathrm{l})$ & $\begin{array}{l}\text { until } \mathrm{H}+\text { or NO2- are } \\
\text { consumed }\end{array}$ \\
\hline
\end{tabular}

if $\mathrm{pH}>7.0$ then

\begin{tabular}{|l|l|}
$\mathrm{NH} 4+(\mathrm{l})+2 \mathrm{H} 2 \mathrm{O} \rightarrow \mathrm{NO} 2-(\mathrm{l})+8 \mathrm{H}+$ & $\begin{array}{l}\text { until } \mathrm{pH}=7.0 \text { or NH4+ } \\
\text { is consumed }\end{array}$ \\
\hline
\end{tabular}

It shall be noted that the $\mathrm{pH}$ control measures above are a modeling technique used to approximate the experimental data, which indicated a near $\mathrm{pH}$ neutral environment in the SBS.

$\mathrm{H}_{2} \mathrm{O}$ is condensed or evaporated based on the mole fraction of $\mathrm{H}_{2} \mathrm{O}$ in the offgas stream exiting the LAW SBS. The goal is to have a mole fraction of $0.138 \mathrm{H}_{2} \mathrm{O}(\mathrm{g})$ in the offgas stream. When feed is unavailable to the melter, water vapor entering the SBS is dramatically reduced causing the water in SBS to evaporate. Under this circumstance, the SBS water chem-add will be utilized to replenish the scrubber column. Tritium $(3-\mathrm{H})$ and peroxide $\left(\mathrm{H}_{2} \mathrm{O}_{2}\right)$ follow water, e.g., splits are dynamically calculated in every time step based on the water condensation or evaporation situations.

The SBS volume is reset after the reactions. LOP-SCB-00001 transfers the offgas to LOP-WESP-00001 while LOP-SCB-00002 transfers to LOP-WESP-00002. A SBS will accumulate condensate until either 
the set volume or the 48-hour maximum accumulation time has been reached. The SBS discharges the condensate to RLD-VSL-00005 if the vessel is ready to receive, or continue to cumulate while waiting for RLD-VSL-00005 to become ready. A 1000-gallon headspace (e.g., $\mathrm{V}_{\max }-\mathrm{V}_{\text {set }}$ ) is reserved for each SBS to allow buffer for continuous operations.

\begin{tabular}{|l|l|}
\hline Vessel Tag \# & Equipment Description \\
\hline LOP-WESP-00001 & LAW Wet Electrostatic Precipitator \\
\hline LOP-WESP-00002 & LAW Wet Electrostatic Precipitator \\
\hline
\end{tabular}

Gases that exit the LAW SBS are routed to a corresponding LAW WESP. LOP-WESP-00001 receives the offgas from LOP-SCB-00001 and LOP-WESP-00002 receives the offgas from LOP-SCB-00002. Upon arriving at the WESP, the entrained liquid and solid from the SBS are split to LVP-HEPA00001AB, via LAW-Vessel-Vent, according to the split values given in Table D-14. Demin water is pumped to the WESP through a chem-add at a rate of $0.75 \mathrm{gpm}$ based on 15 metric tons of glass per day (MTG/day) melter operation. The rate is proportioned to the melter production rate if ramp up occurs. In addition, air is added to the WESP to ensure that the electrodes stay dry and clean. For modeling purposes, this air is combined with the LAW Vessel Vent air stream. The total air injected is $368 \mathrm{cfm}$ at $118.3^{\circ} \mathrm{F}$ and 14.7 psi.

The LAW WESP performs the following reactions:

\begin{tabular}{|l|l|}
\hline Reactions & Conversion (Fraction) \\
\hline $\mathrm{H} 2 \mathrm{O}(\mathrm{l}) \rightarrow \mathrm{H} 2 \mathrm{O}(\mathrm{g})$ or $\mathrm{H} 2 \mathrm{O}(\mathrm{g}) \rightarrow \mathrm{H} 2 \mathrm{O}(\mathrm{l})$ & $\begin{array}{l}0.163 \text { mole fraction at } \\
\text { outlet }\end{array}$ \\
\hline $\mathrm{HCl}(\mathrm{g}) \rightarrow \mathrm{Cl}-(\mathrm{l})+\mathrm{H}+(\mathrm{l})$ & 0.2308 \\
\hline $\mathrm{HF}(\mathrm{g}) \rightarrow \mathrm{F}-(\mathrm{l})+\mathrm{H}+(\mathrm{l})$ & 0.4764 \\
\hline $129-\mathrm{I}(\mathrm{g}) \rightarrow 129-\mathrm{I}(\mathrm{l})$ & 0.1071 \\
\hline $\mathrm{SO} 2(\mathrm{~g})+\mathrm{H} 2 \mathrm{O}(\mathrm{l})+0.5 \mathrm{O} 2(\mathrm{~g}) \rightarrow \mathrm{SO} 4-2(\mathrm{l})+2 \mathrm{H}+(\mathrm{l})$ & 0.9471 \\
\hline $\mathrm{P} 2 \mathrm{O} 5(\mathrm{~g})+3 \mathrm{H} 2 \mathrm{O}(\mathrm{l}) \rightarrow 6 \mathrm{H}+(\mathrm{l})+0.5 \mathrm{PO} 4-3(\mathrm{l})+1.5 \mathrm{PO} 4-3(\mathrm{~s})$ & 0.8 \\
\hline $2 \mathrm{NO}(\mathrm{g})+\mathrm{O} 2(\mathrm{~g}) \rightarrow 2 \mathrm{H} 2 \mathrm{O}+4 \mathrm{H}+(\mathrm{l})+2 \mathrm{NO} 3-(\mathrm{l})$ & 0.01 \\
\hline $\mathrm{C} 12 \mathrm{H} 4 \mathrm{Cl} 6(\mathrm{~g}) \rightarrow \mathrm{C} 12 \mathrm{H} 4 \mathrm{Cl} 6(\mathrm{l})$ & 0.0909 \\
\hline $2 \mathrm{~B}+3(\mathrm{~s})+1.5 \mathrm{O} 2(\mathrm{~g})+3 \mathrm{H} 2 \mathrm{O}(\mathrm{l}) \rightarrow 2 \mathrm{H}+(\mathrm{l})+2 \mathrm{~B}+3(\mathrm{l})+4 \mathrm{O}-2(\mathrm{l})+2 \mathrm{H} 2 \mathrm{O}(\mathrm{l})$ & 1.0 \\
\hline $\mathrm{H}+(\mathrm{l})+\mathrm{OH}-(\mathrm{l}) \rightarrow \mathrm{H} 2 \mathrm{O}(\mathrm{l})$ & 1.0 \\
\hline
\end{tabular}

$\mathrm{H}_{2} \mathrm{O}$ is evaporated or condensed based on the mole fraction of $\mathrm{H}_{2} \mathrm{O}$ in the offgas stream exiting the LAW WESP. The goal is to have a mole fraction of $0.163 \mathrm{H}_{2} \mathrm{O}(\mathrm{g})$ in the offgas stream. Tritium and peroxide follow water. The remaining gases exit the WESP and pass to the LAW HEPA while the liquid and solid flow to RLD-VSL-00004.

\begin{tabular}{|l|l|}
\hline Vessel Tag \# & Equipment Description \\
\hline LVP-HEPA-00001AB & LAW High Efficiency Particular Air Filter \\
\hline
\end{tabular}

Gases leaving the LAW WESP are combined with an air stream that models the LAW Vessel Vent and are routed to the LAW HEPA. A large percentage of the liquid and solid components entering the LAW HEPA are captured while few of the gas components are removed. The offgas leaving the HEPA is routed to the downstream Carbon Absorber. Percent removal by the HEPA is shown in Table D-15.

\begin{tabular}{|l|l|}
\hline Vessel Tag \# & Equipment Description \\
\hline LVP-ADBR-00001AB & LAW Carbon Absorber \\
\hline
\end{tabular}


The LAW Carbon Absorber captures $99.585 \%$ of mercury from the entrained liquid and solid. It also removes $99 \%$ of the 129 -I and $96.997 \%$ of the $\mathrm{HCl}$ and $\mathrm{HF}$ from the gas stream. The offgas leaving the absorber is routed to the downstream Catalytic Oxidizer Reducer. Percent removal by the carbon absorber is shown in Table D-16.

\begin{tabular}{|l|l|}
\hline Vessel Tag \# & Equipment Description \\
\hline LVP-SCR-00001-2 & Catalytic Oxidizer Reducer \\
\hline
\end{tabular}

The primary purpose of the Catalytic Oxidizer Reducer is to remove $\mathrm{NO}$ and $\mathrm{NO}_{2}$ from the offgas. $\mathrm{SO}_{2}$, $\mathrm{C}_{2} \mathrm{H}_{3} \mathrm{~N}$, and $\mathrm{C}_{12} \mathrm{H}_{4} \mathrm{Cl}_{6}$ are also reduced. Upon arriving at the Catalytic Oxidizer Reducer, entrained liquid and solid are removed according to the split values given in Table D-17. Pure anhydrous ammonia gas and dilution air are pumped in from the gas chem-adds named AMR-VSL-00001-2-NH3 and AMR-VSL00001-2-AIR, respectively. The Catalytic Oxidizer Reducer performs the following reactions:

\begin{tabular}{|l|l|}
\hline Reactions & Conversion (Fraction) \\
\hline $4 \mathrm{NO}(\mathrm{g})+4 \mathrm{NH} 3(\mathrm{~g})+\mathrm{O} 2(\mathrm{~g}) \rightarrow 4 \mathrm{~N} 2(\mathrm{~g})+6 \mathrm{H} 2 \mathrm{O}(\mathrm{g})$ & 0.98 \\
\hline $2 \mathrm{NO} 2(\mathrm{~g})+4 \mathrm{NH} 3(\mathrm{~g})+\mathrm{O} 2(\mathrm{~g}) \rightarrow 3 \mathrm{~N} 2(\mathrm{~g})+6 \mathrm{H} 2 \mathrm{O}(\mathrm{g})$ & 0.98 \\
\hline $\mathrm{SO} 2(\mathrm{~g})+0.5 \mathrm{O} 2(\mathrm{~g}) \rightarrow \mathrm{SO} 3(\mathrm{~g})$ & 0.95 \\
\hline $\mathrm{C} 2 \mathrm{H} 3 \mathrm{~N}(\mathrm{l}, \mathrm{g})+3.75 \mathrm{O} 2(\mathrm{~g}) \rightarrow 2 \mathrm{CO} 2(\mathrm{~g})+1.5 \mathrm{H} 2 \mathrm{O}(\mathrm{g})+\mathrm{NO} 2(\mathrm{~g})$ & 0.757 \\
\hline $\mathrm{C} 12 \mathrm{H} 4 \mathrm{Cl} 6(\mathrm{l}, \mathrm{g})+11.5 \mathrm{O} 2(\mathrm{~g})+\mathrm{H} 2 \mathrm{O}(\mathrm{g}) \rightarrow 12 \mathrm{CO} 2(\mathrm{~g})+6 \mathrm{HCl}(\mathrm{g})$ & 0.95 \\
\hline
\end{tabular}

The quantity of gas chem-add is dynamically determined to satisfy the stoichiometric requirements of the $\mathrm{NO}_{\mathrm{x}}$ reactions, the $\mathrm{NH}_{3}$ in the inlet to the Catalytic Oxidizer Reducer including the gas, liquid, and solid phases, and a 78 parts per million volume (ppmv) slip stream amount. The exiting gas is routed to the LAW Caustic Scrubber.

\begin{tabular}{|l|l|}
\hline Vessel Tag \# & Equipment Description \\
\hline LVP-SCB-00001 & Caustic Scrubber \\
\hline LVP-TK-00001 & Caustic Collection Vessel \\
\hline
\end{tabular}

The Caustic Scrubber is modeled as two parts. The column (LVP-SCB-00001) is modeled in the LAW Offgas Treatment System. The vessel (LVP-TK-00001) is modeled in the LAW Liquid Effluent workspace. Upon arriving at the Caustic Scrubber, the entrained liquid and solid from the Catalytic Oxidizer Reducer are split to the Stack according to the split values given Table D-18. Caustic solutions at $\mathrm{pH} 9$ or above are pumped in from LVP-TK-00001 at 60 gpm flowrate. Reactions take place as follows:

\begin{tabular}{|l|l|}
\hline Reactions & Conversion (Fraction) \\
\hline $\mathrm{H} 2 \mathrm{O}(\mathrm{l}) \rightarrow \mathrm{H} 2 \mathrm{O}(\mathrm{g})$ or $\mathrm{H} 2 \mathrm{O}(\mathrm{g}) \rightarrow \mathrm{H} 2 \mathrm{O}(\mathrm{l})$ & $\begin{array}{l}\text { o.189 mole fraction at } \\
\text { outlet }\end{array}$ \\
\hline $\mathrm{CO} 2(\mathrm{~g})+\mathrm{H} 2 \mathrm{O}(\mathrm{l}) \rightarrow \mathrm{H} 2 \mathrm{CO} 3(\mathrm{l})$ & 0.004 \\
\hline $\mathrm{H} 2 \mathrm{CO} 3(\mathrm{l}) \rightarrow \mathrm{HCO} 3-(\mathrm{l})+\mathrm{H}+(\mathrm{l})$ & Eq. 64 \\
\hline $\mathrm{HCO} 3-(\mathrm{l}) \rightarrow \mathrm{CO} 3-2(\mathrm{l})+\mathrm{H}+(\mathrm{l})$ & Eq. 65 \\
\hline $\mathrm{NH} 3(\mathrm{~g}) \rightarrow \mathrm{NH} 3(\mathrm{l})$ & 0.2857 \\
\hline $\mathrm{NH} 3(\mathrm{l})+\mathrm{H} 2 \mathrm{O}(\mathrm{l}) \rightarrow \mathrm{NH} 4+(\mathrm{l})+\mathrm{OH}-(\mathrm{l})$ & Eq. 68 \\
\hline $\mathrm{HCl}(\mathrm{g}) \rightarrow \mathrm{Cl}-(\mathrm{l})+\mathrm{H}+(\mathrm{l})$ & 0.99 \\
\hline $\mathrm{HF}(\mathrm{g}) \rightarrow \mathrm{F}-(\mathrm{l})+\mathrm{H}+(\mathrm{l})$ & 0.9874 \\
\hline $3 \mathrm{NO} 2(\mathrm{~g})+\mathrm{H} 2 \mathrm{O}(\mathrm{l}) \rightarrow 2 \mathrm{NO} 3-(\mathrm{l})+2 \mathrm{H}+(\mathrm{l})+\mathrm{NO}(\mathrm{g})$ & 0.2248 \\
\hline $4 \mathrm{NO}(\mathrm{g})+2 \mathrm{H} 2 \mathrm{O}(\mathrm{l})+3 \mathrm{O} 2(\mathrm{~g}) \rightarrow 4 \mathrm{NO} 3-(\mathrm{l})+4 \mathrm{H}+(\mathrm{l})$ & 0.2248 \\
\hline $\mathrm{P} 2 \mathrm{O} 5(\mathrm{~g})+3 \mathrm{H} 2 \mathrm{O}(\mathrm{l}) \rightarrow 0.5 \mathrm{PO} 4-3(\mathrm{l})+1.5 \mathrm{PO} 4-3(\mathrm{~s})+6 \mathrm{H}+(\mathrm{l})$ & 0.5 \\
\hline $\mathrm{SO} 2(\mathrm{~g})+\mathrm{H} 2 \mathrm{O}(\mathrm{l})+0.5 \mathrm{O} 2(\mathrm{~g}) \rightarrow \mathrm{SO} 4-2(\mathrm{l})+2 \mathrm{H}+(\mathrm{l})$ & 0.97 \\
\hline $\mathrm{SO} 3(\mathrm{~g})+\mathrm{H} 2 \mathrm{O}(\mathrm{l}) \rightarrow 2 \mathrm{H}+(\mathrm{l})+\mathrm{SO} 4-2(\mathrm{l})$ & 0.97 \\
\hline
\end{tabular}




\begin{tabular}{|l|l|}
\hline Reactions & Conversion (Fraction) \\
\hline $129-\mathrm{I}(\mathrm{g}) \rightarrow 129-\mathrm{I}(\mathrm{l})$ & 0.0 (reserved reaction) \\
\hline $\mathrm{H}+(\mathrm{l})+\mathrm{OH}-(\mathrm{l}) \rightarrow \mathrm{H} 2 \mathrm{O}(\mathrm{l})$ & 1.0 \\
\hline
\end{tabular}

$\mathrm{H}_{2} \mathrm{O}$ is evaporated or condensed based on the mole fraction of $\mathrm{H}_{2} \mathrm{O}$ in the offgas stream exiting the LAW Caustic Scrubber. The goal is to have a mole fraction of $0.189 \mathrm{H}_{2} \mathrm{O}(\mathrm{g})$ in the offgas stream. Gases are transferred to the Stack while liquid and solid are recycled to LVP-TK-00001.

LVP-TK-00001 is initially filled to halfway between its set volume (6,032 gallons) and its minimum volume (2,032 gallons) with $\mathrm{NapOH}$ to $\mathrm{pH}$ 9.0. The goal is to keep $\mathrm{pH}$ at constant at around 9.0. Within each time step, $\mathrm{H}^{+}$is produced from the Caustic Scrubber reactions that lower the $\mathrm{pH}$ value. If the $\mathrm{pH}$ in the vessel goes below 9.0, 20 gallons of 5M NapOH will be added (minimum pump capacity) which brings the $\mathrm{pH}$ to above 9.0. If the $\mathrm{SpG}$ goes above 1.1 as heavier components accumulate, demin water will be added at $22 \mathrm{gpm}$ flowrate. If the volume goes above its set volume, the vessel will be discharged to its minimum volume. If the volume goes below 4,032 gallons (water is lost in the caustic scrubber column due to evaporation) even if its $\mathrm{SpG}$ is below the 1.1 target volume, water will be added to replenish the vessel. The vessel will be discharged anyway if it has not been discharged for a month.

When the level in LVP-TK-00001 reaches the set volume, the contents are transferred to either RLD-VSL-00017A or RLD-VSL-00017B, depending on their availability. If RLD-VLS-00017A and RLD-VSL-00017B are not available, then LVP-TK-00001 continues to fill to maximum volume. If the maximum volume is reached and LVP-TK-00001 still does not have anywhere to transfer, the LAW melters will idle until LVP-TK-00001 can empty. LVP-TK-00001 still pumps to and receives from the LAW Caustic Scrubber Column while it is emptying.

Figure F-15 provides a graphical illustration of the LVP system where connections with upstream and downstream systems are shown. Table N-1 provides the totalizer files that are used to track the process streams and material flows into and out of the LVP.

\subsubsection{HLW Melter Feed Process (HFP) System}

\begin{tabular}{|l|l|}
\hline Vessel Tag \# & Equipment Description \\
\hline HFP-VSL-00001 & HLW Melter Feed Preparation Vessel \\
\hline HFP-VSL-00005 & HLW Melter Feed Preparation Vessel \\
\hline
\end{tabular}

The HLW Melter Feed Preparation Vessels (HLW MFPV) receive waste feed from HLP-VSL-00028 on a batch transfer basis and alternate with each other in operation. When a HLW MFPV becomes empty, a glass former analysis will be performed which determines the batch size and glass former recipe based on the chemical compositions in HLP-VSL-00028 so that the following conditions are met:

1) HFP-VSL-00001 and HFP-VSL-00005 will not exceed the maximum volume after waste, line flush, glass formers, dusting water, and rheology water are added

2) Rheology water target is met (550 grams oxide/L)

3) HLW glass quality specifications are met

The MFPV batch size is determined using the logic shown in Figure 15. The glass former recipe is determined using the HLW Glass Shell V2.0 presented in Figures 16 through 34. Details for the development of this HLW Glass Shell can be found in 24590-WTP-MCR-PET-09-0047 and further in the $B A R D$. A request is submitted to the HLW recipe queue for the Glass Former Reagent System. Glass 
formers can be prepared as soon as a recipe exists in the HLW recipe queue given that the GFR is available. LAW and HLW recipes compete for the use of GFR as there is only one in the plant (refer to Section 4.8.22 for GFR operations). HLW recipes have higher priorities than the LAW recipes to prevent HFP from backing up the ultrafiltration operations.

Glass former preparations can occur simultaneously with a waste batch transfer. When an HLW MFPV has reached its set volume, it receives a line flush and a pre- determined volume of rheology water. Then the vessel is held 12.79 hours for a pre-glass former sampling. The vessel will wait for the glass formers if they are not already ready. HFP-VSL-00001 receives glass formers from GFR-TK-00031 and HFPVSL-00005 receives glass formers from GFR-TK-00025. The MFPV will receive 48 gallons of demister flush water before the confirmation sample is taken. The vessel is held 9.59 hours for a confirmation sampling prior to discharge. HFP-VSL-00001 empties its contents to HFP-VSL-00002 and HFP-VSL00005 empties its contents to HFP-VSL-00006. After emptying, the HLW MFPV starts a new cycle. 


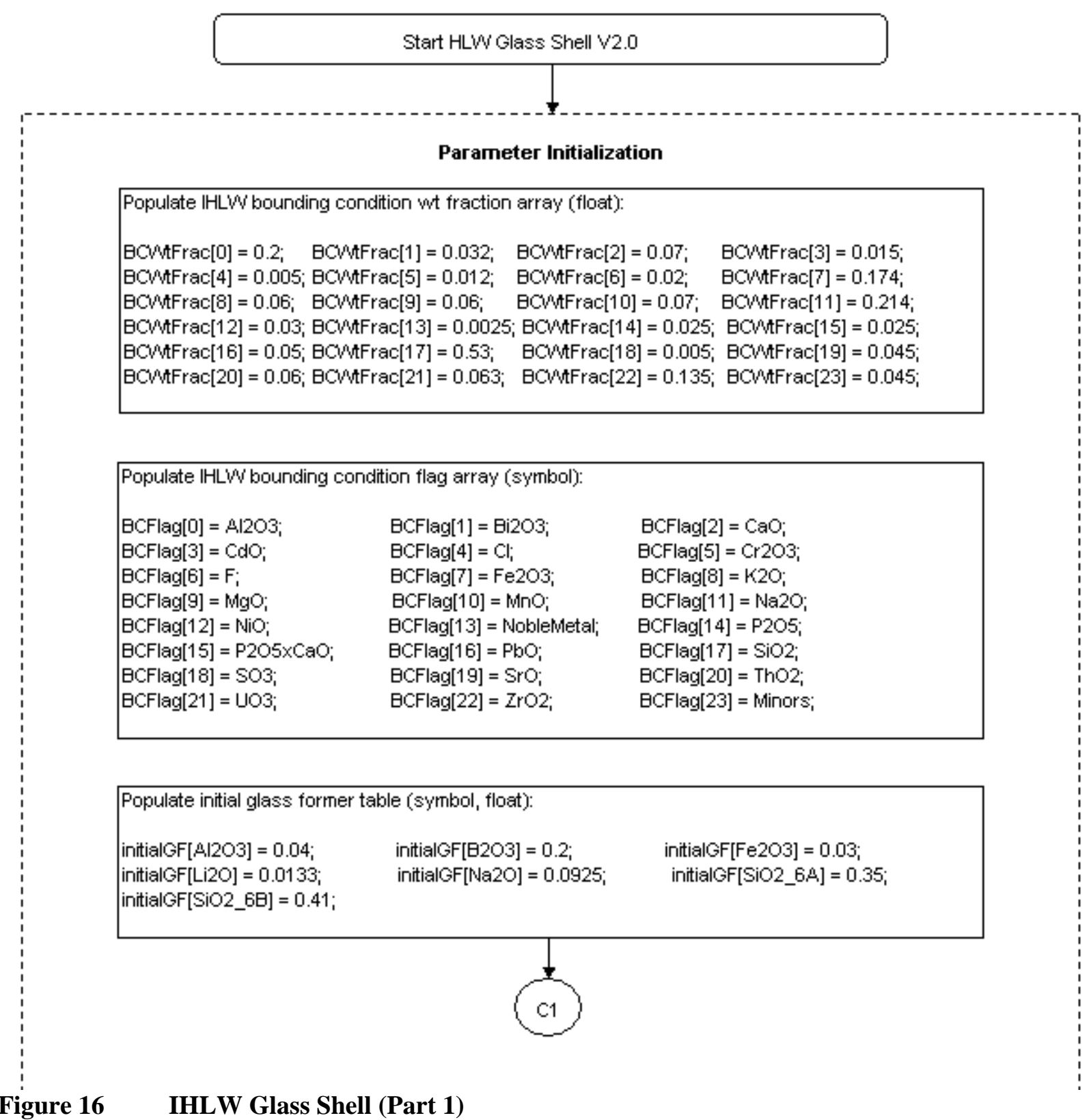

Figure 16 IHLW Glass Shell (Part 1) 


\begin{tabular}{|c|c|c|}
\hline \multicolumn{3}{|c|}{ Populate liquidus temperature coefficients: } \\
\hline $\begin{array}{l}\text { spinelCoef[0] = 2,835; } \\
\text { spinelCoef[3] = -410; } \\
\text { spinelCoef }[6]=11,916 ; \\
\text { spinelCoef }[9]=3,103 ; \\
\text { spinelCoef[12] = -201; } \\
\text { spinelCoef[15] = 425; }\end{array}$ & $\begin{array}{l}\text { spinelCoef[1] = 12,468; } \\
\text { spinelCoef[4] = 3,927; } \\
\text { spinelCoef[7] = 421; } \\
\text { spinelCoef }[10]=1,933 \\
\text { spinelCoef }[13]=-735 \\
\text { spinelCoef[16] = 736; }\end{array}$ & $\begin{array}{l}\text { spinelCoef[2] = 3,328; } \\
\text { spinelCoef[5] = 2,619; } \\
\text { spinelCoef[8] = 897; } \\
\text { spinelCoef[11] = 14,871; } \\
\text { spinelCoef[14] = -718; }\end{array}$ \\
\hline
\end{tabular}

Populate viscosity coefficients:

visCoef $[0]=10.609 ; \quad$ visCoef $[1]=-9.375$

visCoef $[4]=-12.344 ; \quad$ visCoef[5] $=-3.825$;

visCoef $[8]=-3.231$;

visCoef[12] = -23.144;

visCoef[16] $=-2.696$;

visCoef $[20]=17.517$;
visCoef $[9]=-6.887$;

visCoef $[13]=9.368$;

visCoef $[17]=7.140$;

visCoef $[21]=24.593$;
visCoef $[2]=-3.418$; visCoef $[6]=-4.970$; visCoef $[10]=-9.633$; visCoef[14] $=-4.351$; visCoef $[18]=-0.090$; visCoef[22] $=-8.135$;
visCoef $[3]=-6.933$;

visCoet $[7]=-39.025$;

visCoef[11 $=5.305$;

visCoef $[15]=2.151$;

visCoef[19] $=-26.957$;

visCoef[23] $=47.359$;

Figure 17 IHLW Glass Shell (Part 2) 


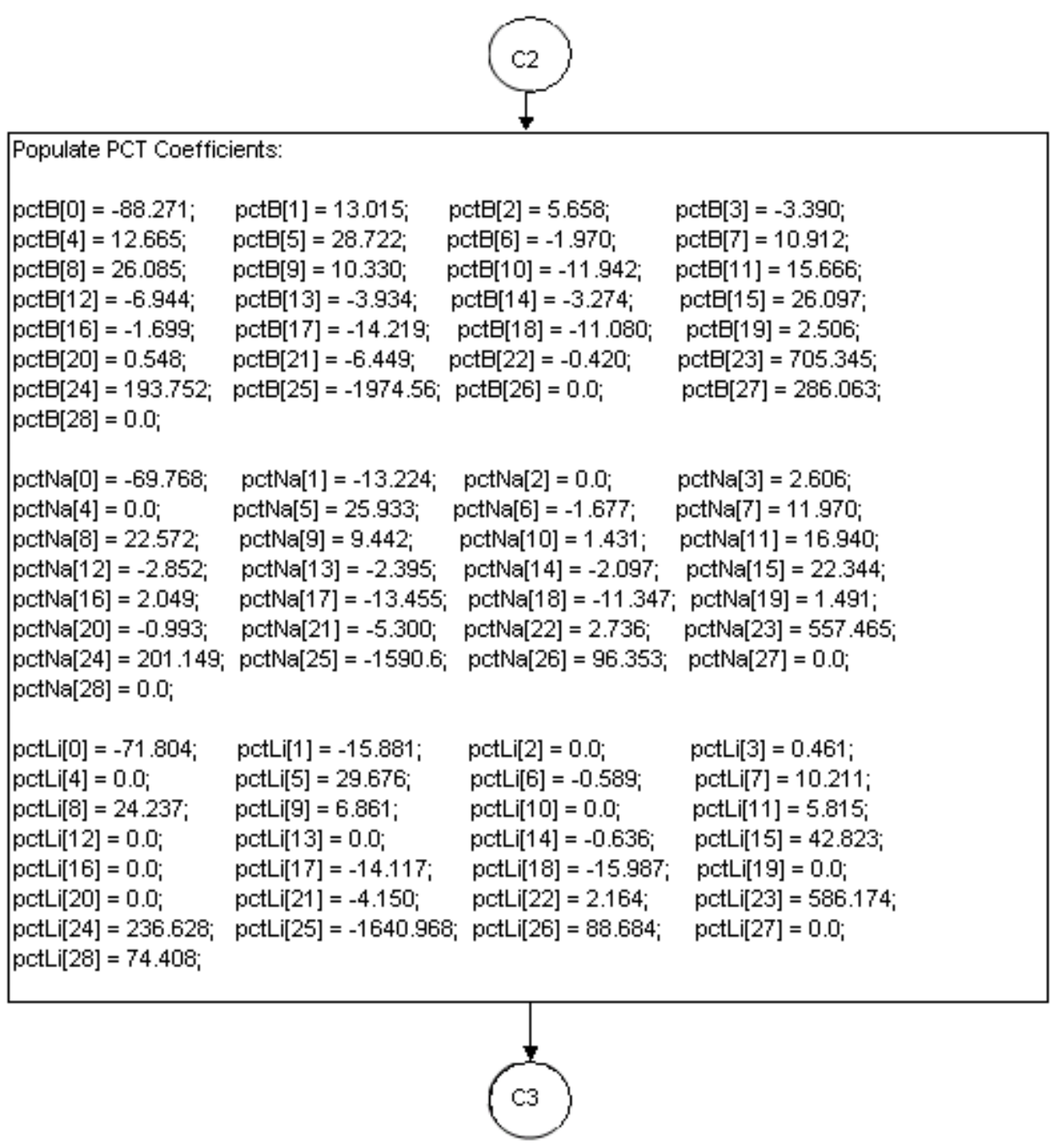

Figure 18 IHLW Glass Shell (Part 3) 


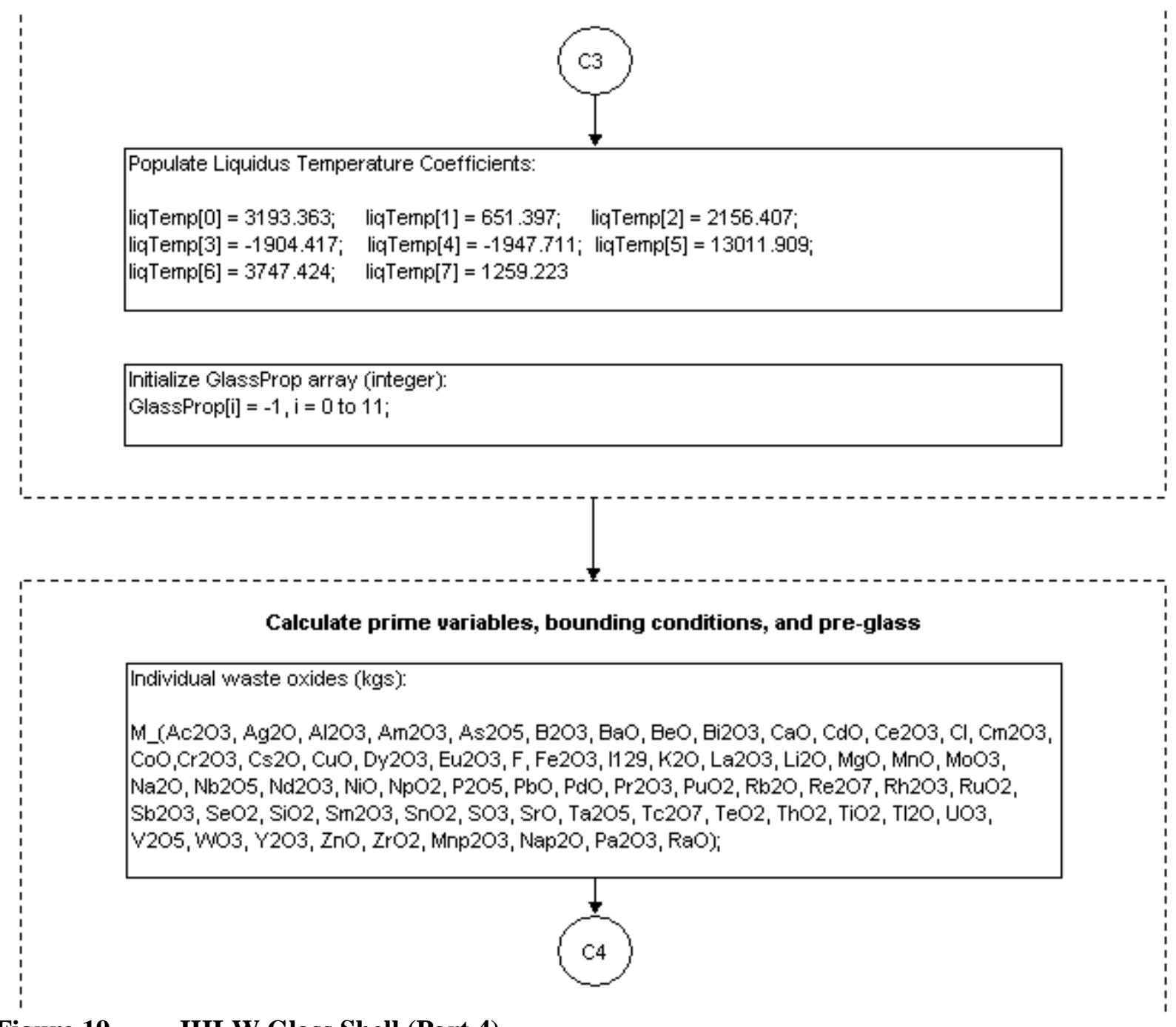

Figure 19 IHLW Glass Shell (Part 4) 


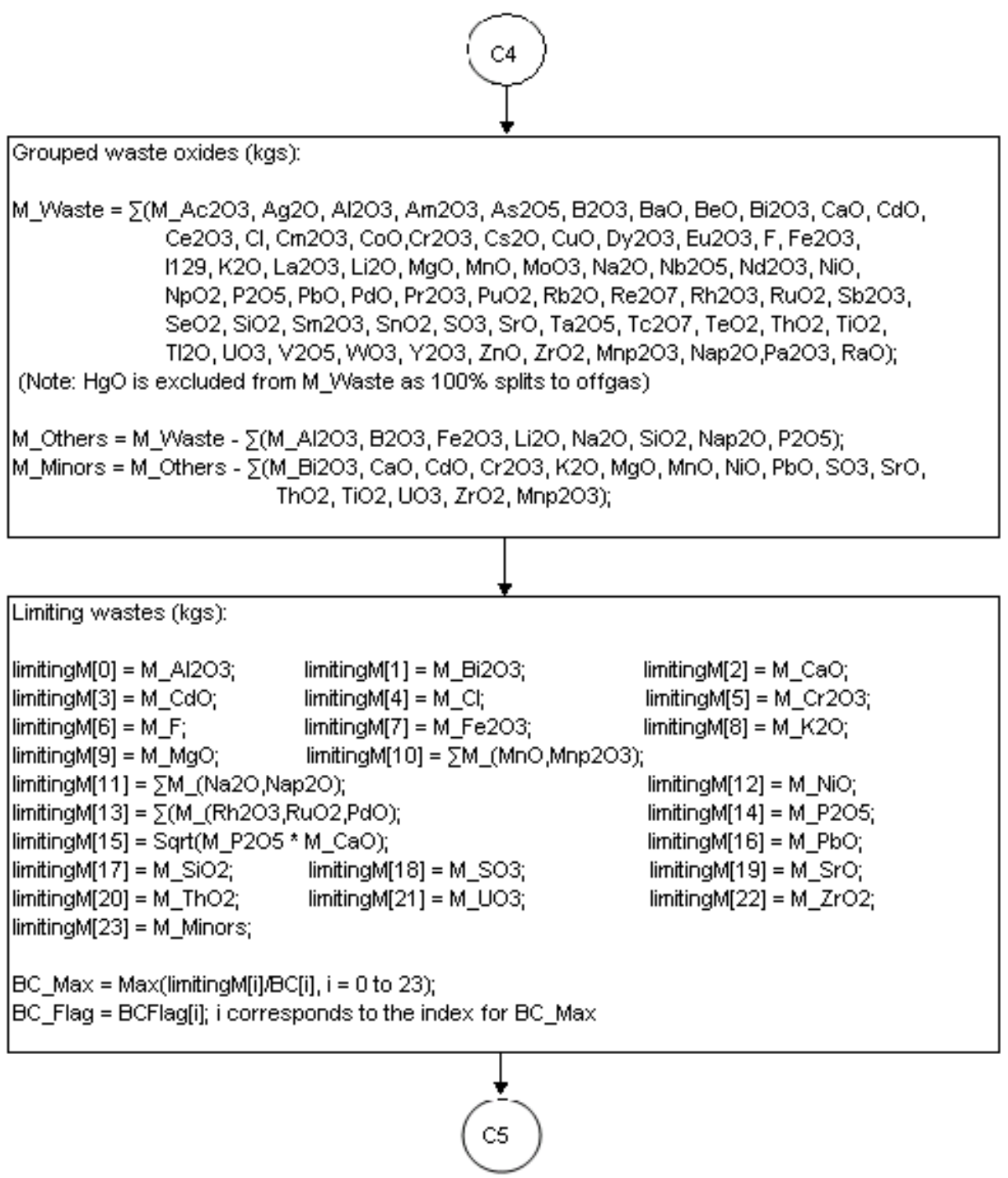

Figure 20 IHLW Glass Shell (Part 5) 


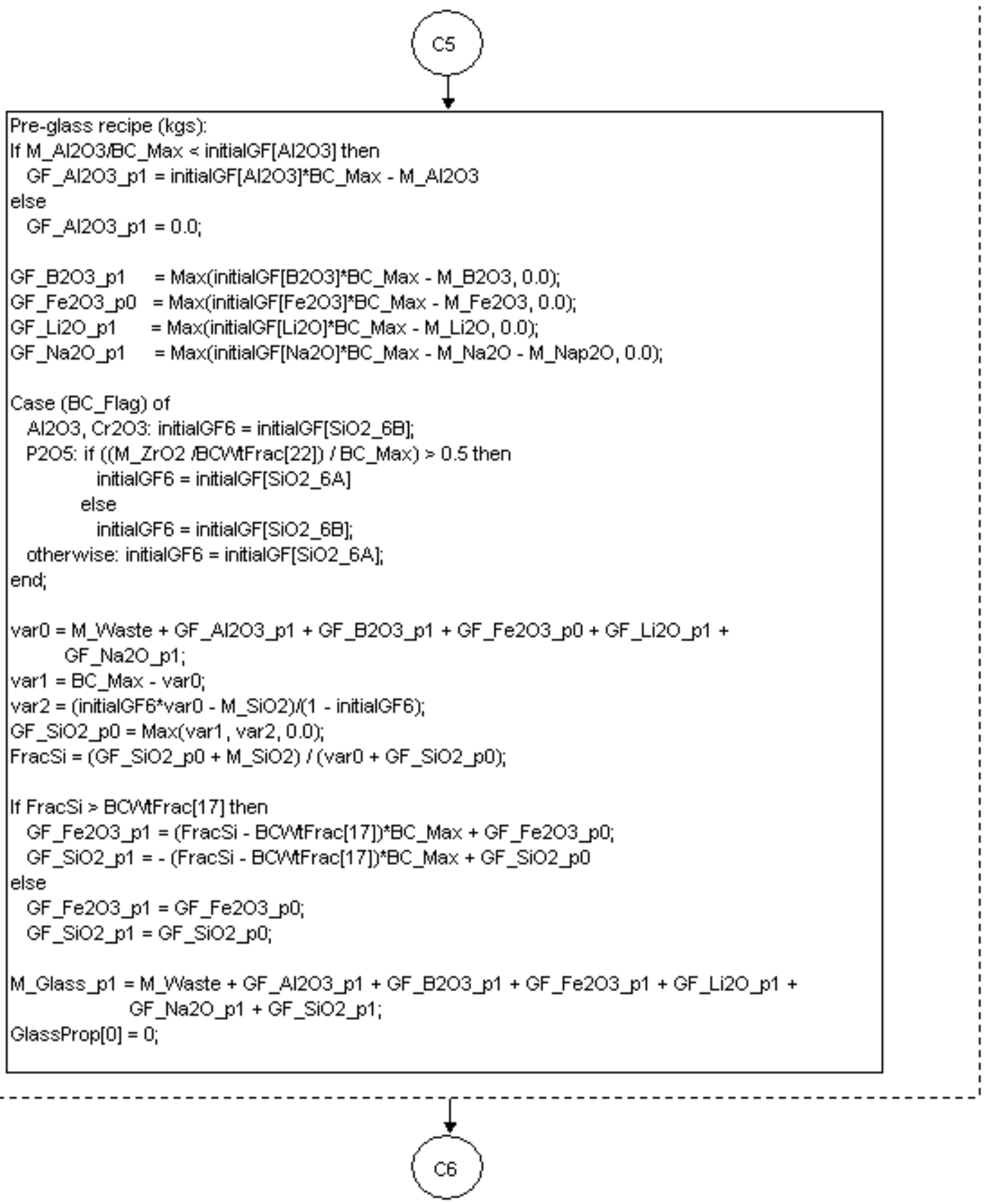

\section{Figure 21 IHLW Glass Shell (Part 6)}




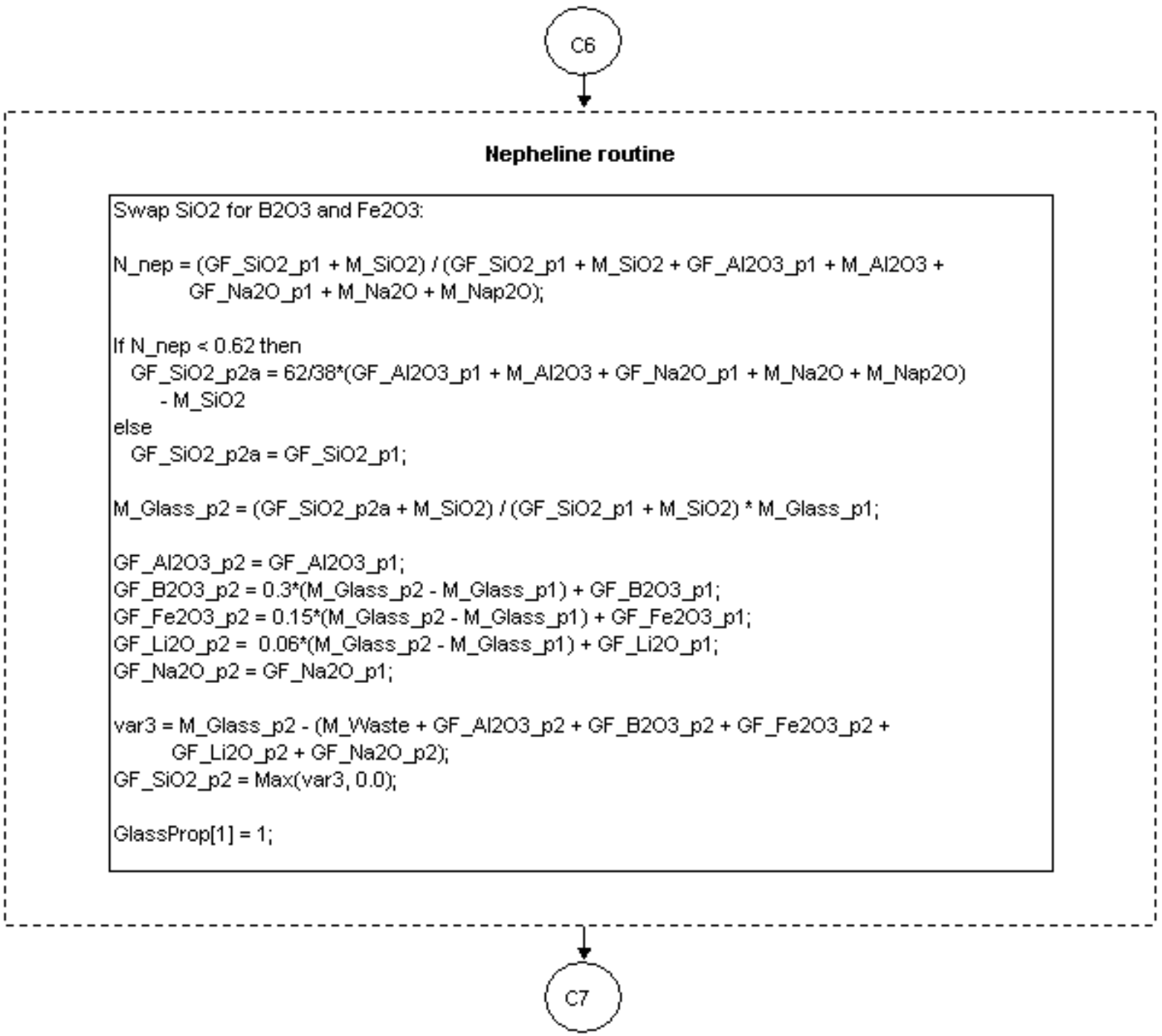

Figure 22 IHLW Glass Shell (Part 7) 


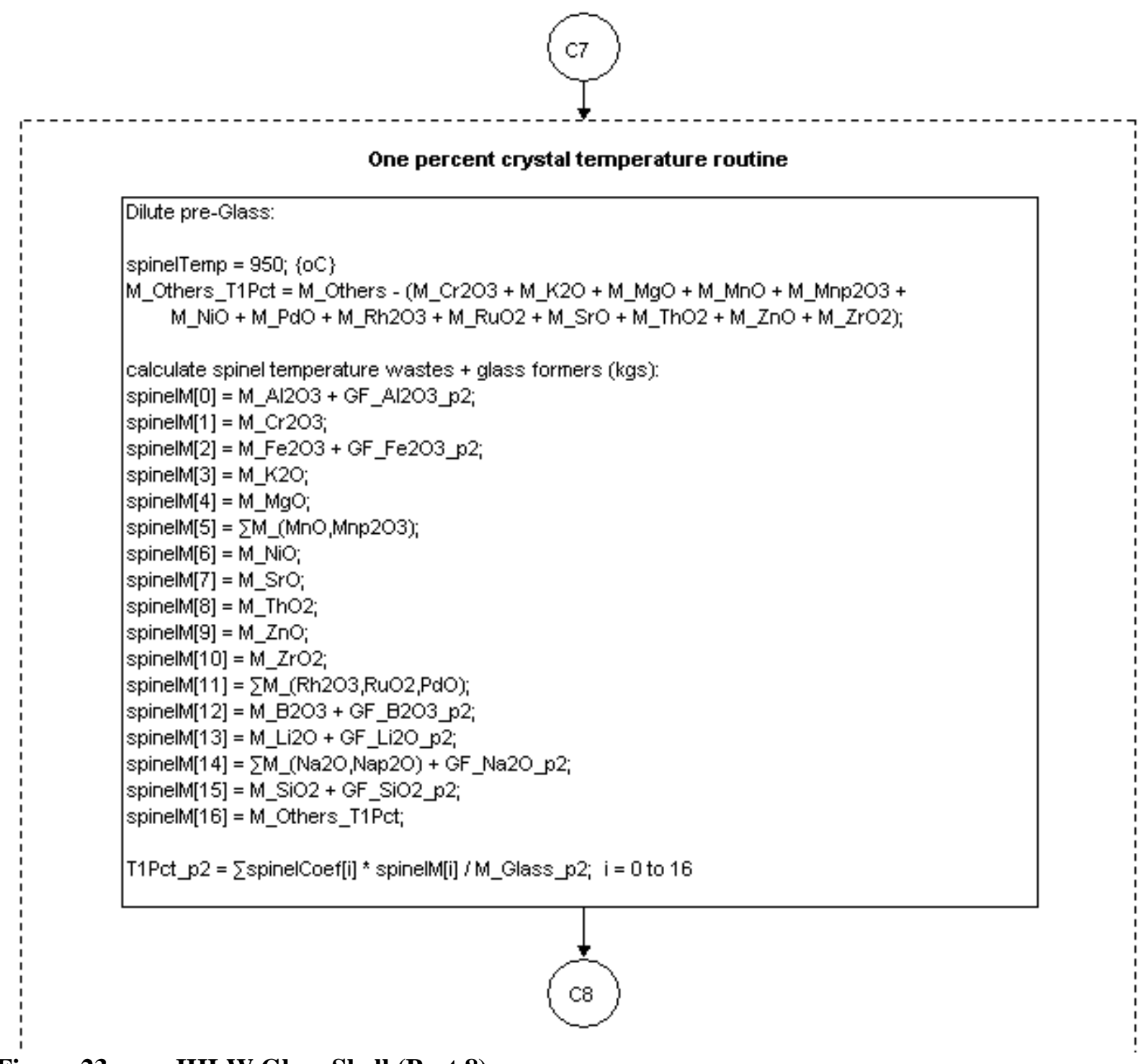

Figure 23 IHLW Glass Shell (Part 8) 


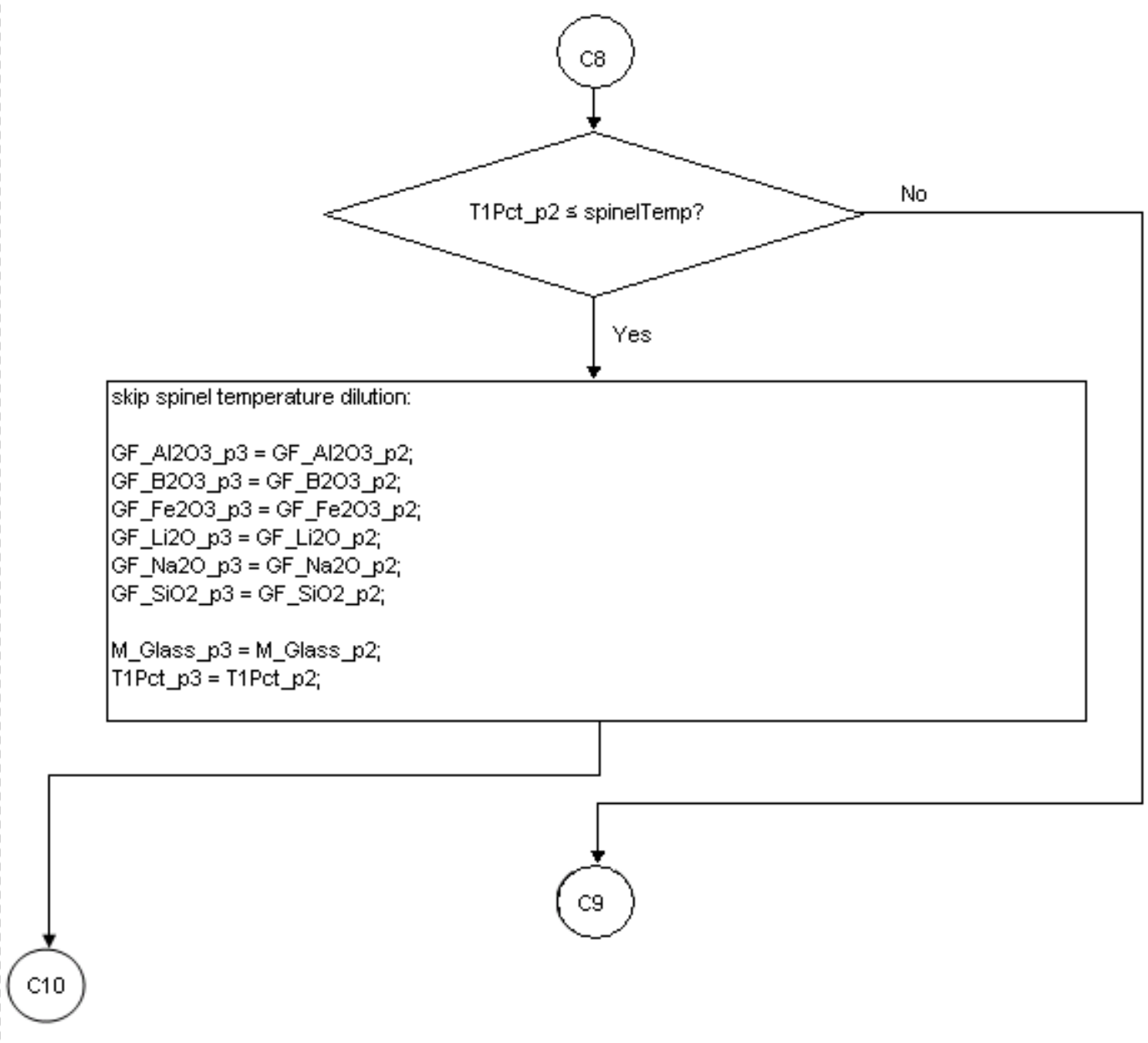

Figure 24 IHLW Glass Shell (Part 9) 


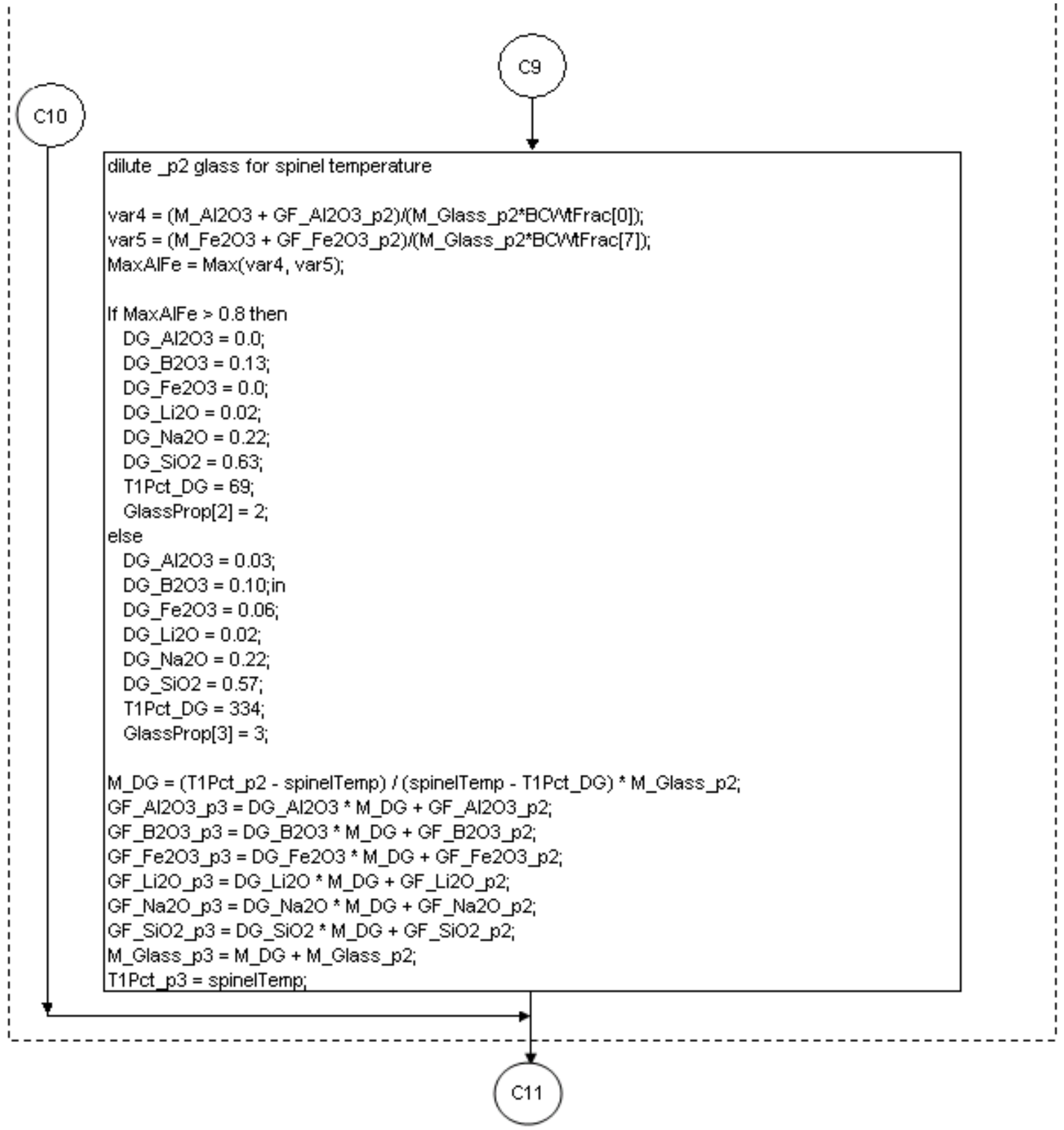

Figure 25 IHLW Glass Shell (Part 10) 


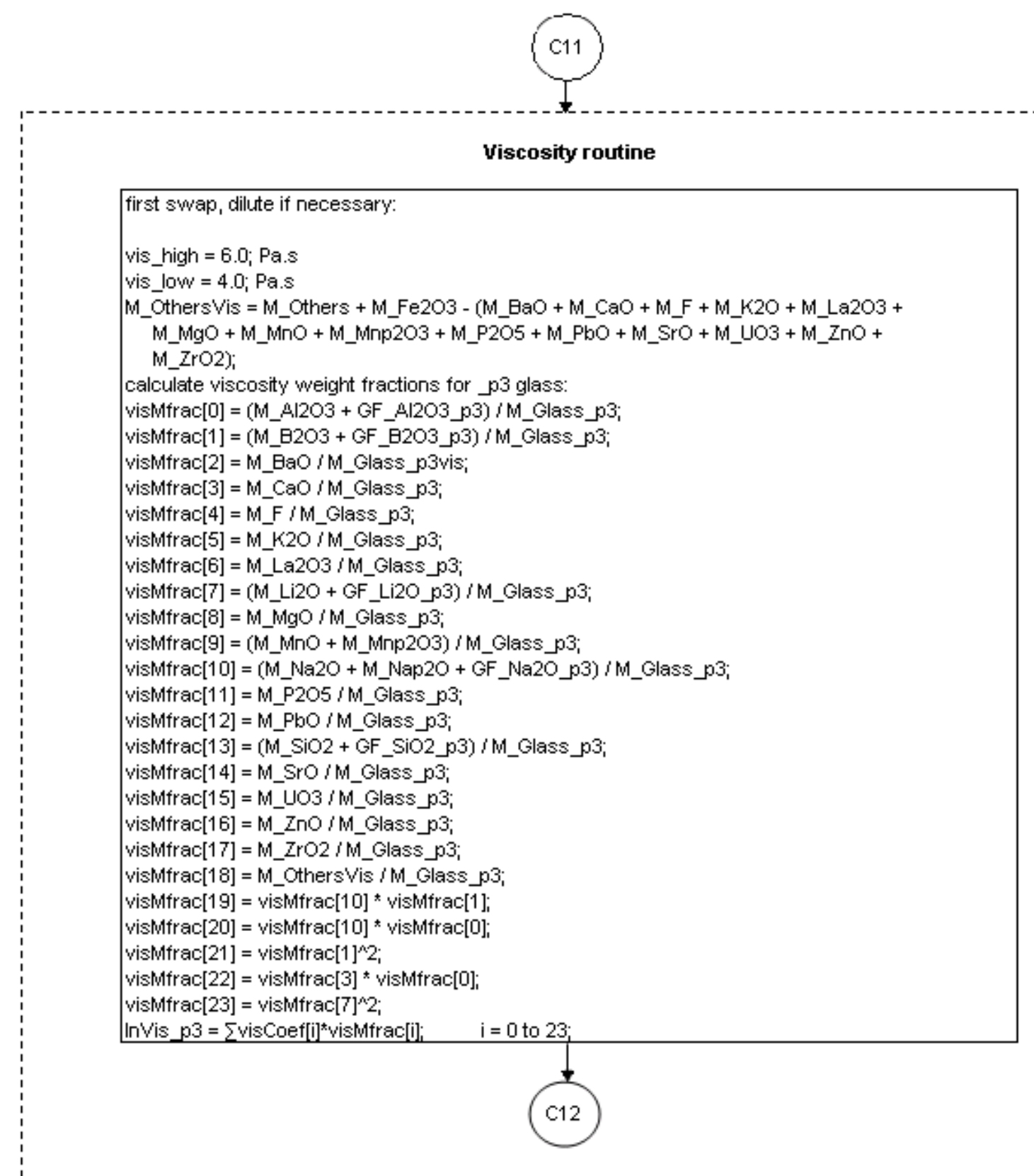

Figure 26 IHLW Glass Shell (Part 11) 


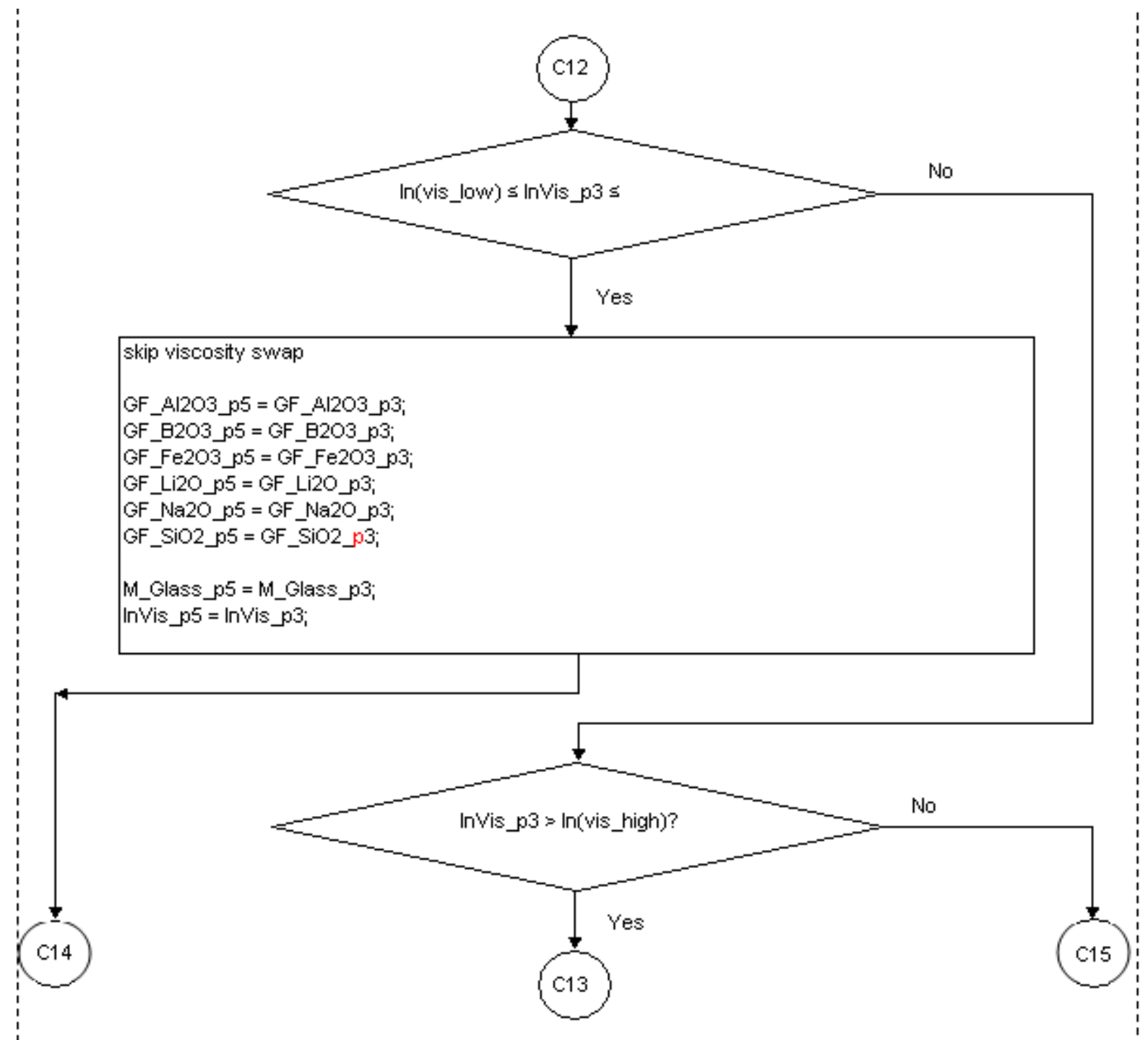

Figure 27 IHLW Glass Shell (Part 12) 


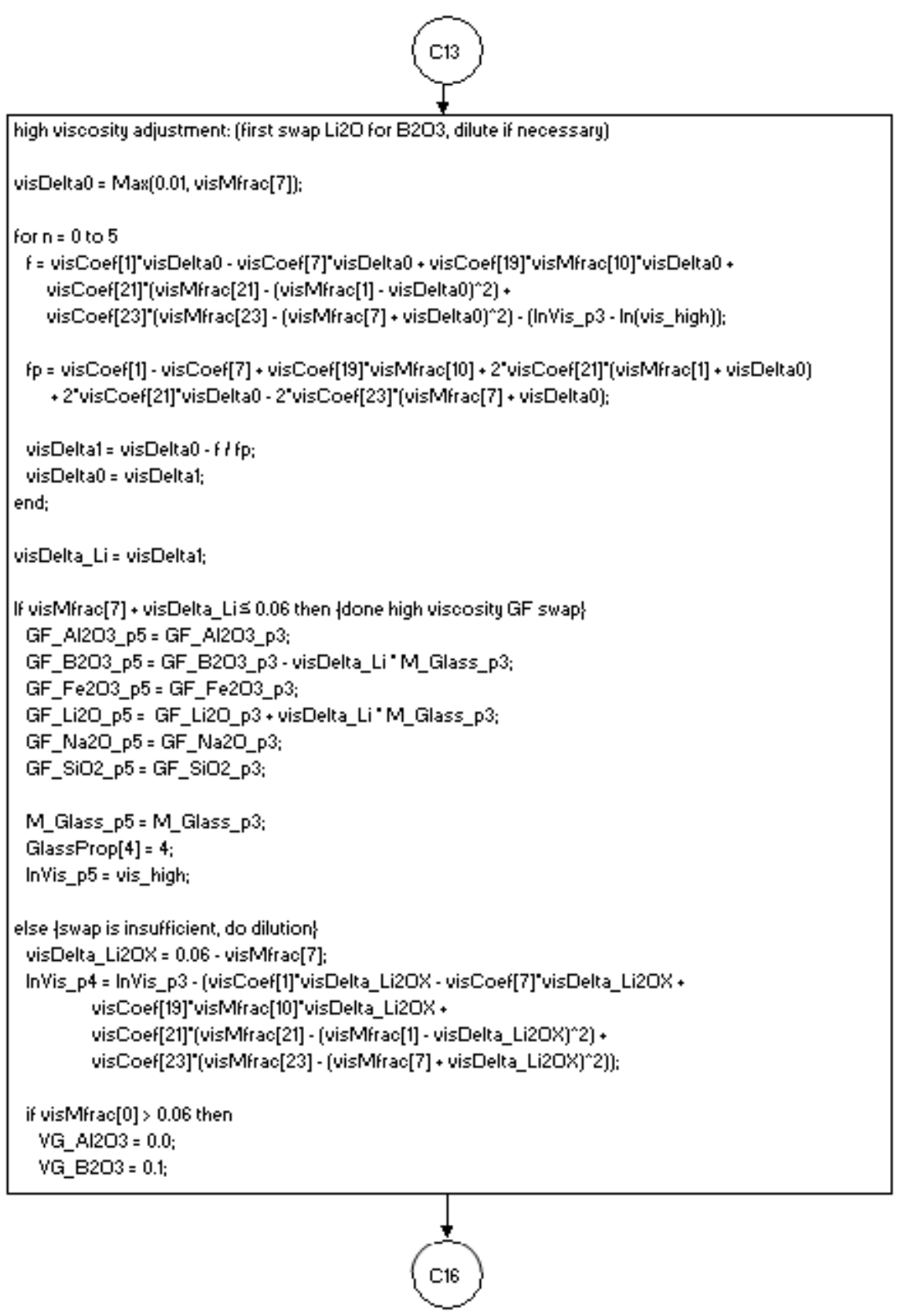

Figure 28 IHLW Glass Shell (Part 13) 


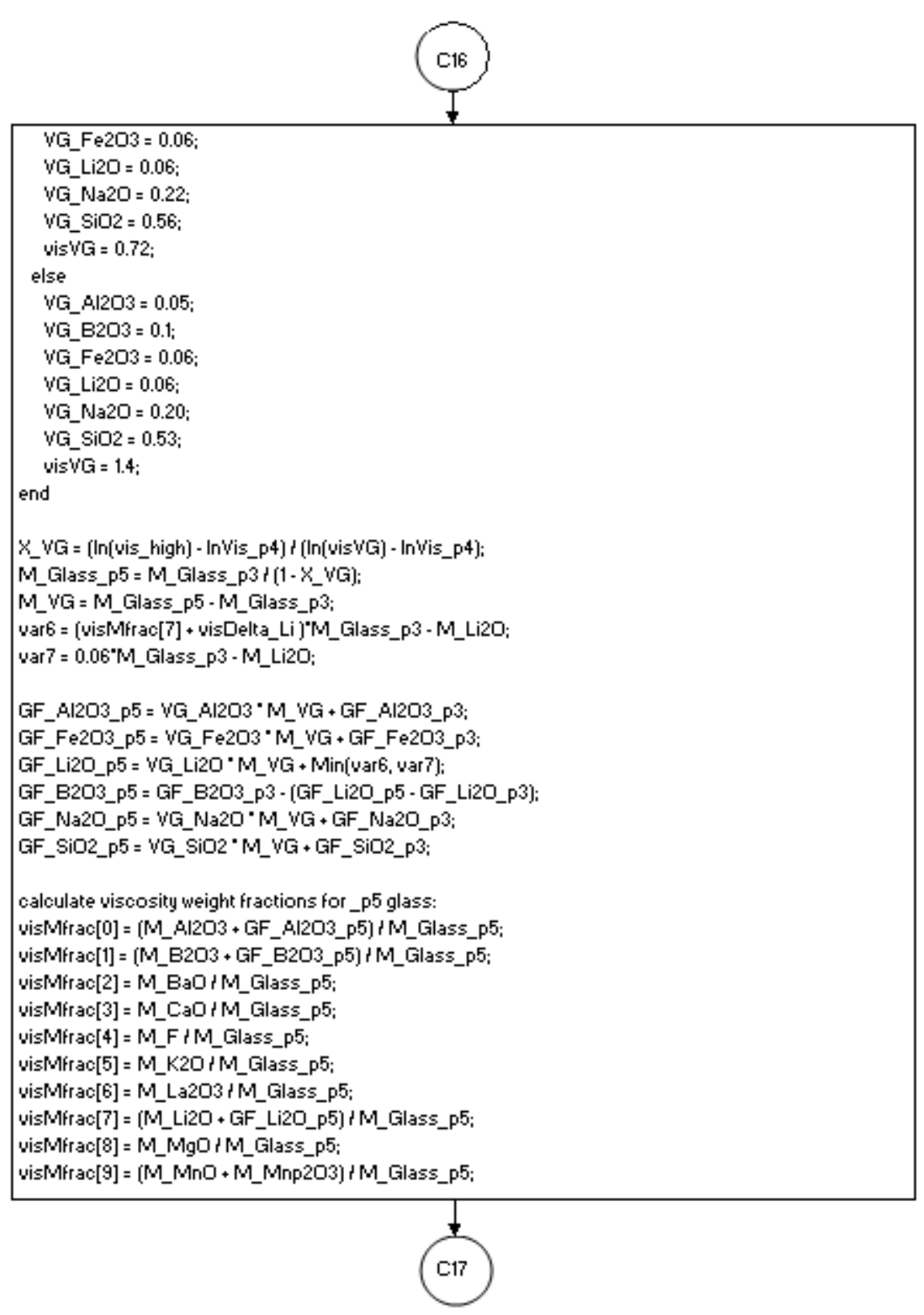

Figure 29 IHLW Glass Shell (Part 14) 


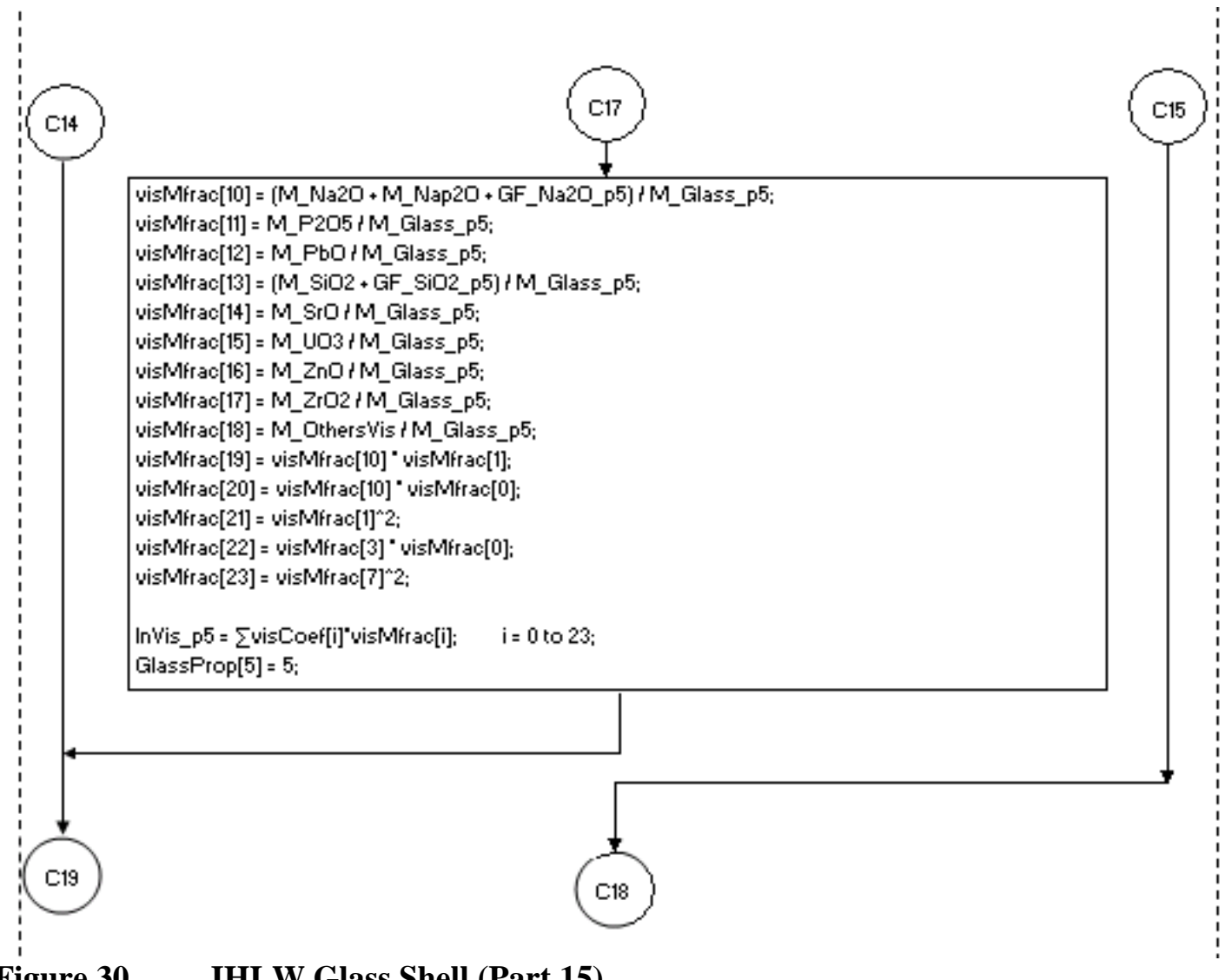

Figure $30 \quad$ IHLW Glass Shell (Part 15) 


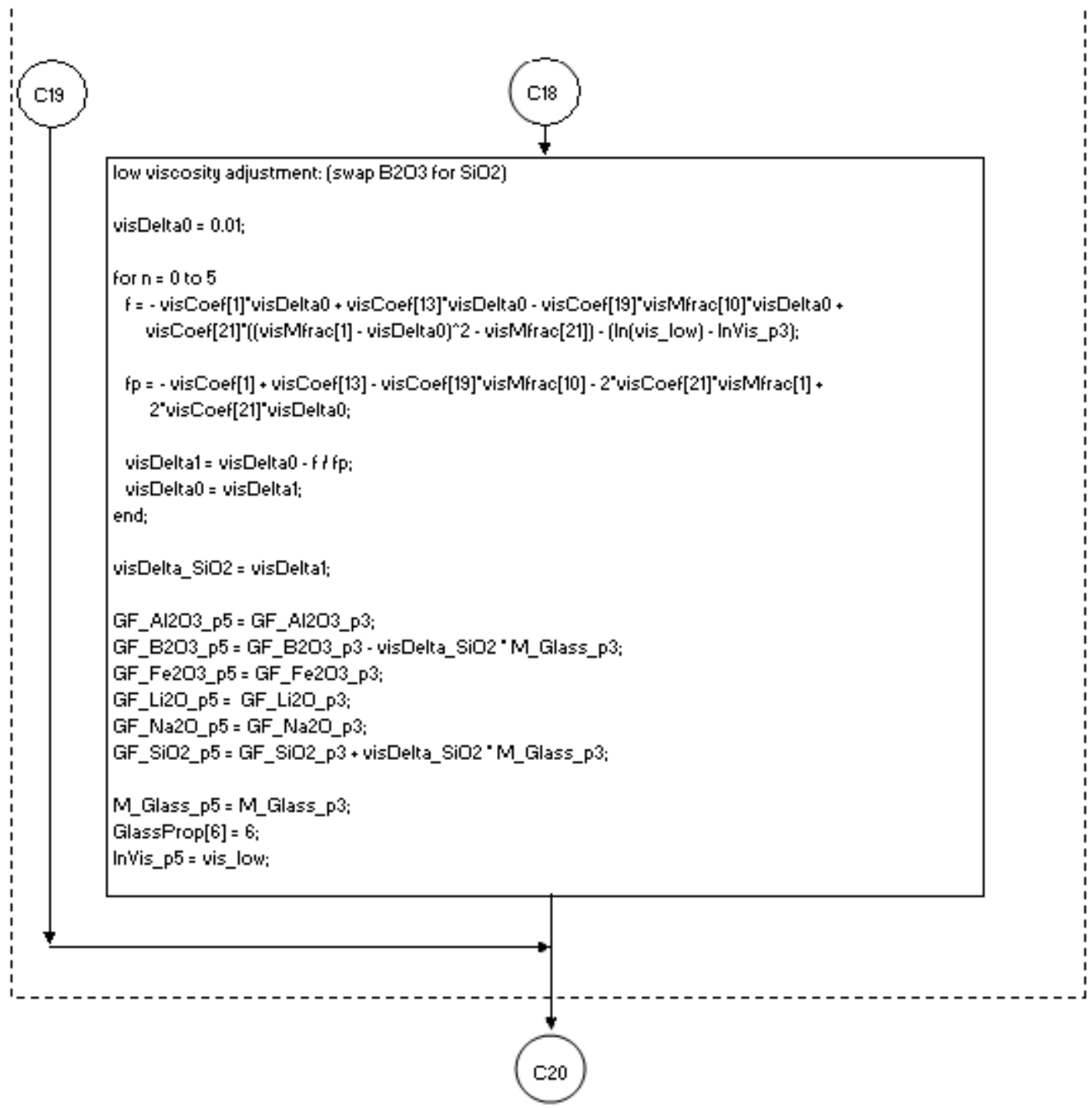

Figure $31 \quad$ IHLW Glass Shell (Part 16) 
24590-WTP-MDD-PR-01-002, Rev 12 Dynamic (G2) Model Design Document

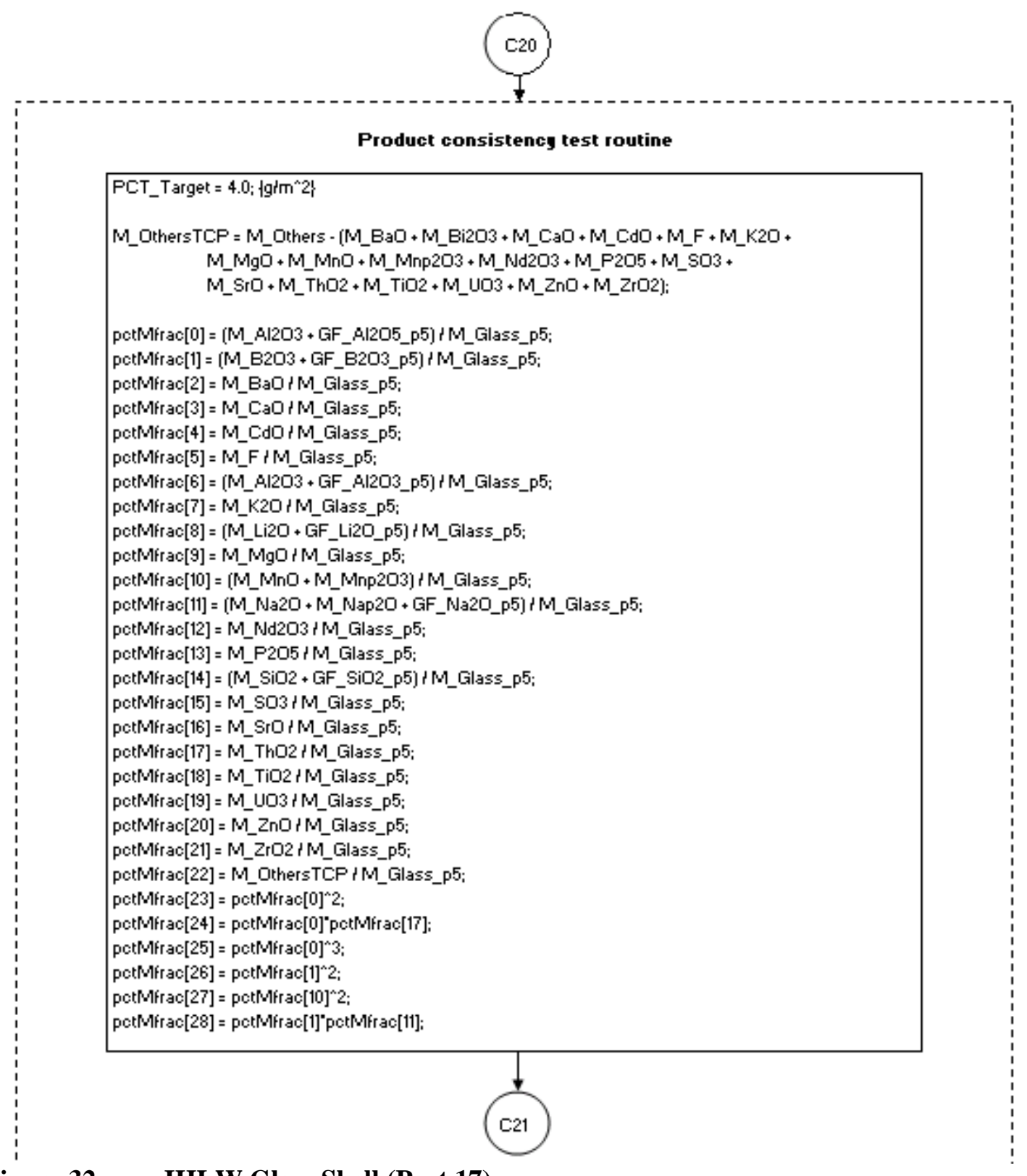

Figure 32 IHLW Glass Shell (Part 17) 


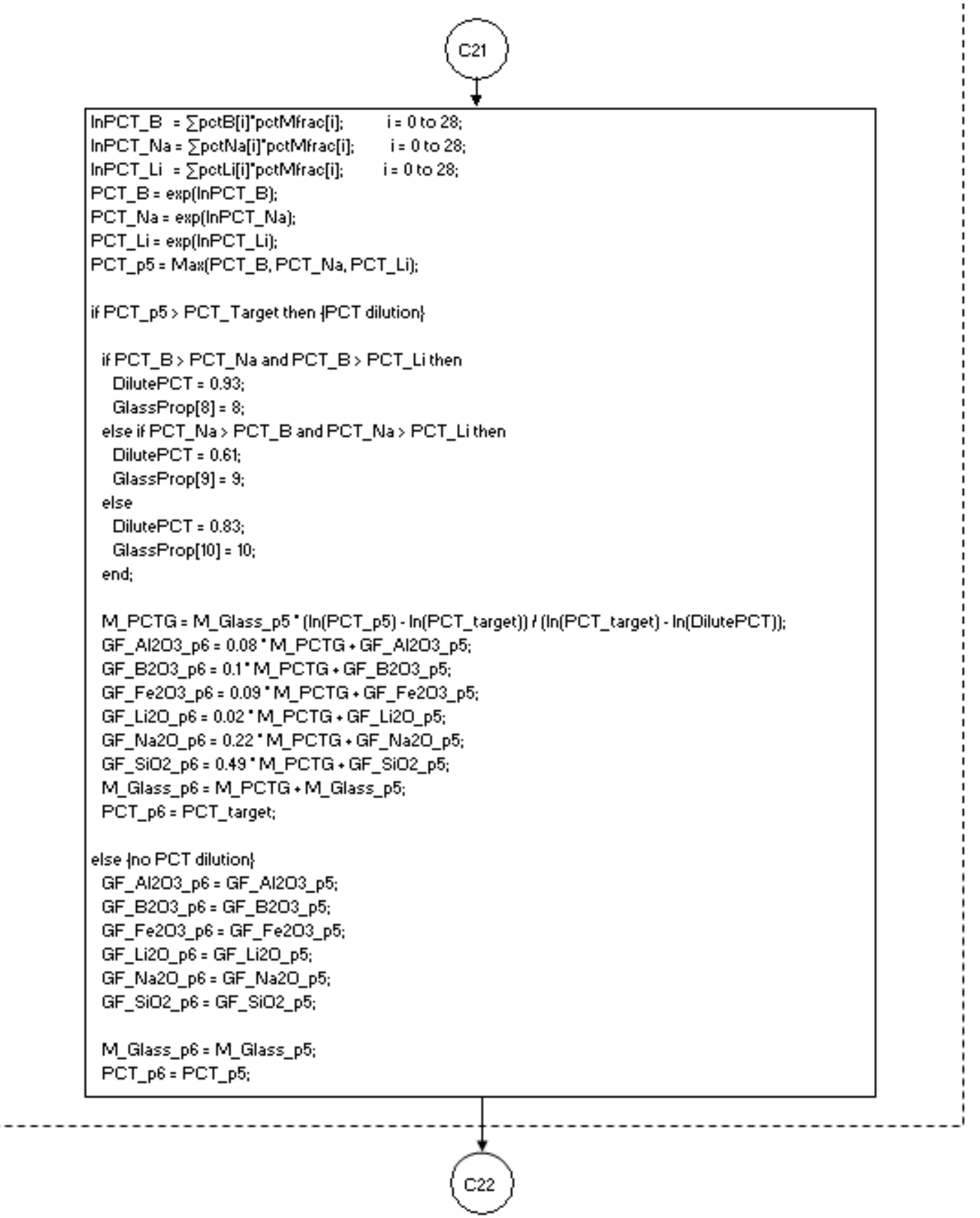

Figure 33 IHLW Glass Shell (Part 18) 


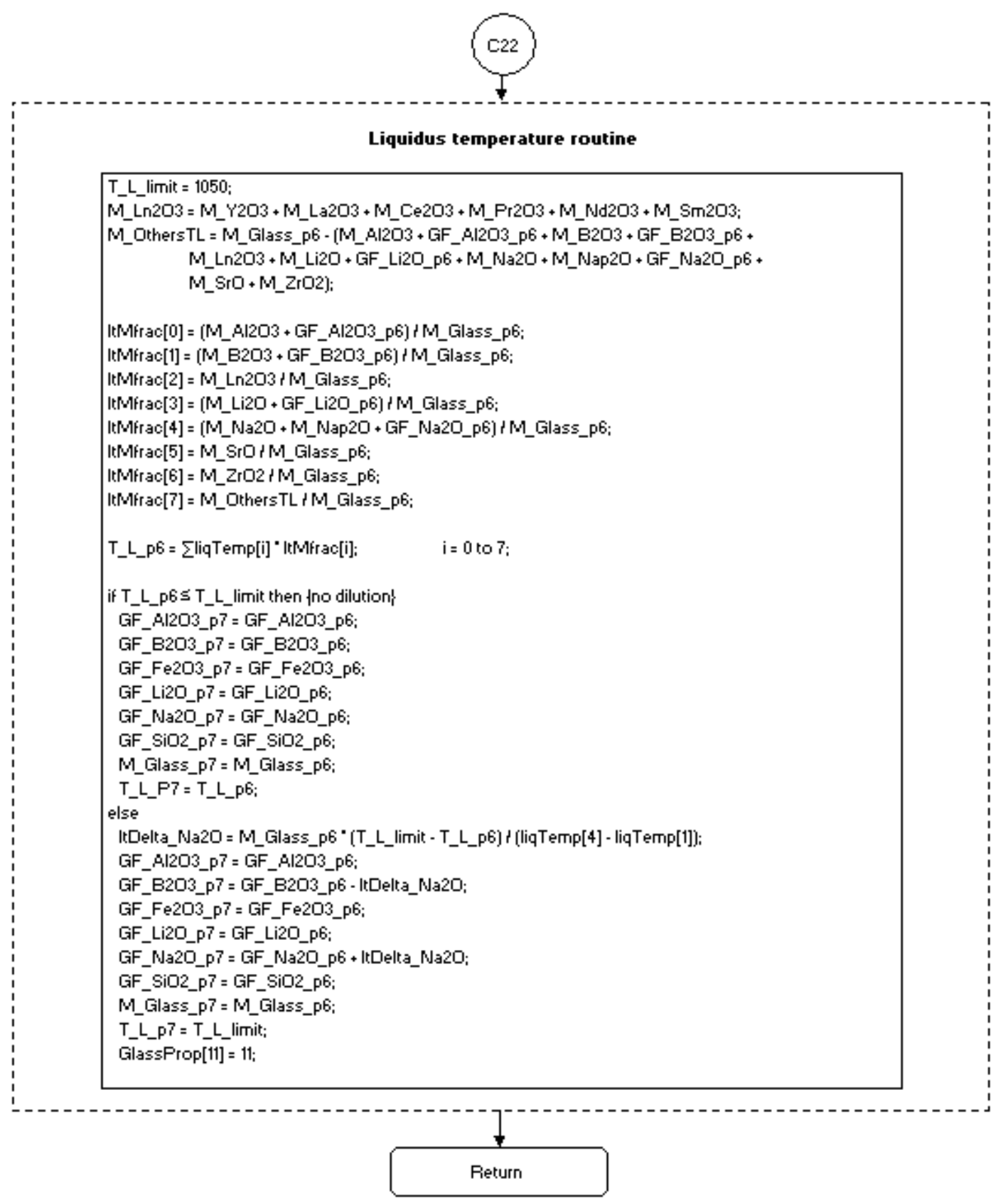

Figure 34 IHLW Glass Shell (Part 19) 
The glass former recipe, including the quantities of borax, boric acid, kyanite, lithium carbonate, olivine, rutile, soda ash, sugar, wollastonite, zinc oxide, and zircon sand, are returned from the Immobilized HighLevel Waste (IHLW) Glass Shell.

\begin{tabular}{|l|l|}
\hline Vessel Tag \# & Equipment Description \\
\hline HFP-VSL-00002 & HLW Melter 1 Feed Vessel \\
\hline HFP-VSL-00006 & HLW Melter 2 Feed Vessel \\
\hline
\end{tabular}

The HLW MFVs receive feed from the corresponding HLW MFPVs. HFP-VSL-00002 transfers material only to HMP-MLTR-00001, and HFP-VSL-00006 transfers material only to HMP-MLTR-00002. The objective of the MFVs is to feed enough waste to the melters to maintain a set target production rate for MTG/day. Because the composition of the waste batches are variable, the transfer rate required to maintain the target throughput in the melters is also variable. A more diluted feed will have a higher transfer rate so that the target melter production rate can be maintained. When an HLW MFV reaches its lower setpoint (1,563 gallons) it becomes available to receive feed again while continuing to empty to its minimum volume (1,543 gallons). This prevents the HLW melter from becoming idle. The only time that an HLW MFV will stop pumping to the melter is when there is no feed available from the HLW MFPV and the HLW MFV is at its minimum volume.

The HLW melter feed pumps will be shut down temporarily when the number of canisters on the canister decontamination system cooling rack reach the 24 capacity limit and/or when RLD-VSL-00007 is filled but unable to discharge. The melter feed pumps resume as soon as the restricting conditions are relieved.

Figure F-18 provides the graphical illustration of the HFP system where connections with upstream and downstream systems are shown. Table N-1 provides the totalizer files that are used to track the process streams and material flows into and out of the HFP.

\subsubsection{HLW Melter Process (HMP) System}

\begin{tabular}{|l|l|}
\hline Vessel Tag \# & Equipment Description \\
\hline HMP-MLTR-00001 & HLW Melter 1 \\
\hline HMP-MLTR-00002 & HLW Melter 2 \\
\hline
\end{tabular}

HLW Melters (HMP-MLTR-00001 and HMP-MLTR-00002) are used to transform the HLW into glass. Liquid and solid wastes, oxide glass formers and impurities, and air are delivered to the melters. The inflow of wastes and glass formers depends on the availability of feed; however, the inflow of air is continuous once started. The air stream is made up of: 1) air inleakage, 2) bubbler air, 3) film cooler air, and 4) pressure control air. The combined air flowrate and the associated temperature and pressure are shown in Table B-19.

When feed enters the melter, a split is applied to the non-gas components including the liquid and solid wastes, and oxide glass formers and associated mineral impurities. The liquid split values apply to the liquid components that come into the melter while the solid split values apply to the combined solids of solid wastes, oxide glass formers, and impurities. The predefined split values are shown in Table D-19. Exceptions are applied to the following components of which melter reactions or a combination of melter reactions and splits are more appropriate: 


\begin{tabular}{|c|c|c|c|}
\hline Components & $\begin{array}{l}\text { Liquid } \\
\% \text { to } \\
\text { SBS }\end{array}$ & $\begin{array}{l}\text { Solid\% } \\
\text { to SBS }\end{array}$ & Descriptions \\
\hline $129-\mathrm{I}$ & 0.000 & 0.000 & $129-\mathrm{I}(\mathrm{l}, \mathrm{s}) \rightarrow 129-\mathrm{I}(\mathrm{g}) \mathrm{CF}=0.7692$, remaining go to glass \\
\hline $3-\mathrm{H}$ & 100.000 & 100.000 & Follows water, $100 \%$ go to offgas \\
\hline $\mathrm{C} 12 \mathrm{H} 4 \mathrm{Cl} 6$ & 0.000 & 0.000 & $\mathrm{C} 12 \mathrm{H} 4 \mathrm{Cl} 6(\mathrm{l}, \mathrm{s})+11.5 \mathrm{O} 2(\mathrm{~g})+\mathrm{H} 2 \mathrm{O}(\mathrm{l}) \rightarrow 12 \mathrm{CO} 2(\mathrm{~g})+6 \mathrm{HCl}(\mathrm{g}) \mathrm{CF}=1.0$ \\
\hline $\mathrm{C} 2 \mathrm{H} 3 \mathrm{~N}$ & 0.000 & 0.000 & $\mathrm{C} 2 \mathrm{H} 3 \mathrm{~N}(\mathrm{l}, \mathrm{s})+3.75 \mathrm{O} 2(\mathrm{~g}) \rightarrow 2 \mathrm{CO} 2(\mathrm{~g})+1.5 \mathrm{H} 2 \mathrm{O}(\mathrm{g})+\mathrm{NO} 2(\mathrm{~g}) \mathrm{CF}=1.0$ \\
\hline $\mathrm{C} 2 \mathrm{O} 4-2$ & 0.000 & 0.000 & $\mathrm{C} 2 \mathrm{O} 4-2(1, \mathrm{~s}) \rightarrow 2 \mathrm{CO} 2(\mathrm{~g}) \mathrm{CF}=1.0$ \\
\hline CHO2- & 0.000 & 0.000 & $\mathrm{CHO} 2-(1, \mathrm{~s})+0.25 \mathrm{O} 2(\mathrm{~g}) \rightarrow \mathrm{CO} 2(\mathrm{~g})+0.5 \mathrm{H} 2 \mathrm{O}(\mathrm{g}) \mathrm{CF}=1.0$ \\
\hline $\mathrm{Cl}-$ & 0.000 & 0.000 & $\begin{array}{l}2 \mathrm{Cl}-(\mathrm{l}, \mathrm{s})+2 \mathrm{Na}+(1, \mathrm{~s})+\mathrm{H} 2 \mathrm{O}(\mathrm{l}) \rightarrow \mathrm{Na} 2 \mathrm{O}(\mathrm{o})+2 \mathrm{HCl}(\mathrm{g}) \mathrm{CF}=0.7353, \\
\text { remaining go to glass }\end{array}$ \\
\hline $\mathrm{CN}-$ & 1.695 & 1.695 & $\begin{array}{l}2 \mathrm{CN}-(1, \mathrm{~s})+2 \mathrm{O} 2(\mathrm{~g}) \rightarrow 2 \mathrm{CO} 2(\mathrm{~g})+\mathrm{N} 2(\mathrm{~g}), \mathrm{CF}=1.0, \\
\text { partially split to offgas, remaining } 100 \% \text { destroyed }\end{array}$ \\
\hline $\mathrm{CO} 3-2$ & 0.000 & 0.000 & $\mathrm{CO} 3-2(1, \mathrm{~s}) \rightarrow \mathrm{CO} 2(\mathrm{~g})+0.5 \mathrm{O} 2(\mathrm{~g}) \mathrm{CF}=1.0$ \\
\hline F- & 0.000 & 0.000 & $\begin{array}{l}2 \mathrm{~F}-(1, \mathrm{~s})+2 \mathrm{Na}+(\mathrm{l}, \mathrm{s})+\mathrm{H} 2 \mathrm{O}(\mathrm{l}) \rightarrow \mathrm{Na} 2 \mathrm{O}(\mathrm{o})+2 \mathrm{HF}(\mathrm{g}) \mathrm{CF}=0.8065, \\
\text { remaining go to glass }\end{array}$ \\
\hline $\mathrm{H}+$ & 0.000 & 0.000 & $\mathrm{H}+(1, \mathrm{~s})+\mathrm{OH}(\mathrm{BOUND})(1, \mathrm{~s}) \rightarrow \mathrm{H} 2 \mathrm{O}(\mathrm{g}) \mathrm{CF}=1.0$ \\
\hline $\mathrm{H} 2 \mathrm{CO} 3$ & 0.000 & 0.000 & $\mathrm{H} 2 \mathrm{CO} 3(1, \mathrm{~s}) \rightarrow \mathrm{CO} 2(\mathrm{~g})+\mathrm{H} 2 \mathrm{O}(\mathrm{g}) \mathrm{CF}=1.0$ \\
\hline $\mathrm{H} 2 \mathrm{O}$ & 0.000 & 0.000 & $\mathrm{H} 2 \mathrm{O}(\mathrm{l}, \mathrm{s}) \rightarrow \mathrm{H} 2 \mathrm{O}(\mathrm{g}) \mathrm{CF}=1.0$ \\
\hline $\mathrm{H} 2 \mathrm{O} 2$ & 0.000 & 0.000 & $2 \mathrm{H} 2 \mathrm{O} 2(1, \mathrm{~s}) \rightarrow 2 \mathrm{H} 2 \mathrm{O}(\mathrm{g})+\mathrm{O} 2(\mathrm{~g}) \mathrm{CF}=1.0$ \\
\hline HCO3- & 0.000 & 0.000 & $2 \mathrm{HCO} 3-(1, \mathrm{~s}) \rightarrow 2 \mathrm{CO} 2(\mathrm{~g})+\mathrm{H} 2 \mathrm{O}(\mathrm{g})+0.5 \mathrm{O} 2(\mathrm{~g}) \mathrm{CF}=1.0$ \\
\hline NH3 & 0.000 & 0.000 & $2 \mathrm{NH} 3(1, \mathrm{~s})+3 \mathrm{O} 2(\mathrm{~g}) \rightarrow \mathrm{NO}(\mathrm{g})+\mathrm{NO} 2(\mathrm{~g})+3 \mathrm{H} 2 \mathrm{O}(\mathrm{g}) \mathrm{CF}=1.0$ \\
\hline NH4+ & 0.000 & 0.000 & $2 \mathrm{NH} 4+(1, \mathrm{~s})+3.5 \mathrm{O} 2(\mathrm{~g}) \rightarrow \mathrm{NO}(\mathrm{g})+\mathrm{NO} 2(\mathrm{~g})+4 \mathrm{H} 2 \mathrm{O}(\mathrm{g}) \mathrm{CF} 1.0$ \\
\hline $\mathrm{NO} 2-$ & 0.000 & 0.000 & Eq. 55 \\
\hline NO3- & 0.000 & 0.000 & Eq. 54 \\
\hline $\mathrm{O}-2$ & 0.000 & 0.000 & $2 \mathrm{O}-2(\mathrm{l}, \mathrm{s}) \rightarrow \mathrm{O} 2(\mathrm{~g}) \mathrm{CF}=1.0$ \\
\hline OH(BOUND) & 0.000 & 0.000 & $2 \mathrm{OH}($ Bound $)(1, \mathrm{~s}) \rightarrow \mathrm{H} 2 \mathrm{O}(\mathrm{g})+0.5 \mathrm{O} 2(\mathrm{~g}) \mathrm{CF}=1.0$ \\
\hline $\mathrm{OH}-$ & 0.000 & 0.000 & $2 \mathrm{OH}-(\mathrm{l}, \mathrm{s}) \rightarrow \mathrm{H} 2 \mathrm{O}(\mathrm{g})+0.5 \mathrm{O} 2(\mathrm{~g}) \mathrm{CF}=1.0$ \\
\hline SO4-2 & 0.000 & 0.000 & $\begin{array}{l}\mathrm{SO} 4-2(1, \mathrm{~s}) \rightarrow \mathrm{SO} 2(\mathrm{~g})+\mathrm{O} 2(\mathrm{~g}), \mathrm{CF}=0.5814, \\
\text { remaining is oxidized and go to glass }\end{array}$ \\
\hline TOC & 0.000 & 0.000 & $\mathrm{TOC}(\mathrm{l}, \mathrm{s})+\mathrm{O} 2(\mathrm{~g}) \rightarrow \mathrm{CO} 2(\mathrm{~g}) \mathrm{CF}=1.0$ \\
\hline
\end{tabular}

Nitrate and nitrite decomposition reactions are shown as Eq. 54 and Eq. 55 where the stoichiometric coefficients are dependent upon the variable multiplier M. These reactions provide the means for approximating the experimental results which found the SBS solution to be near neutral ( $\mathrm{pH}=6.5$ to 7.5) due to variations of ammonia gas production rate. Refer to Section 4.8 .15 for detailed discussions on nitrate and nitrite decomposition reactions.

The splits of sulfate include SO4-2 (1,s) to the offgas, SO2 (g) to the offgas, and SO3 (o) to the glass. The overall split to the offgas is $33.3 \%$ of which $6.25 \%$ is SO4-2 $(1, \mathrm{~s})$ and $93.75 \%$ is SO2 $(\mathrm{g})$. This is equivalent to: a) $2.08125 \%$ of the melter feed sulfate being split to the SBS as SO4-2 $(1, \mathrm{~s})$, b) $31.8823 \%$ of the melter feed sulfate being converted to SO2 (g) that goes to the SBS, and c) $66.7 \%$ of the melter feed sulfate is oxidized to $\mathrm{SO} 3$ (o) that goes to the glass.

After splitting to the offgas system, the remaining solid and liquid arrays are combined in the liquid array and the following reactions occur in each melter, reactions take place in the order presented (note that some reactions have been shown in the table above). 


\begin{tabular}{|l|l|}
\hline Organic Destruction Reactions & Conversion (Fraction) \\
\hline $14-\mathrm{C}(1, \mathrm{~s})+\mathrm{O} 2(\mathrm{~g}) \rightarrow \mathrm{CO} 2(\mathrm{~g})$ & $0.0(14-\mathrm{C}$ is $100 \%$ split $)$ \\
\hline $\mathrm{TOC}(1, \mathrm{~s})+\mathrm{O} 2(\mathrm{~g}) \rightarrow \mathrm{CO} 2(\mathrm{~g})$ & 1.0 \\
\hline $\mathrm{C} 12 \mathrm{H} 4 \mathrm{Cl} 6(1, \mathrm{~s})+11.5 \mathrm{O} 2(\mathrm{~g})+\mathrm{H} 2 \mathrm{O}(\mathrm{l}) \rightarrow 12 \mathrm{CO} 2(\mathrm{~g})+6 \mathrm{HCl}(\mathrm{g})$ & 1.0 \\
\hline $\mathrm{C} 2 \mathrm{H} 3 \mathrm{~N}(\mathrm{l}, \mathrm{s})+3.75 \mathrm{O} 2(\mathrm{~g}) \rightarrow 2 \mathrm{CO} 2(\mathrm{~g})+1.5 \mathrm{H} 2 \mathrm{O}(\mathrm{g})+\mathrm{NO} 2(\mathrm{~g})$ & 1.0 \\
\hline $\mathrm{C} 2 \mathrm{O} 4-2(\mathrm{l}, \mathrm{s}) \rightarrow 2 \mathrm{CO} 2(\mathrm{~g})$ & 1.0 \\
\hline $\mathrm{CHO} 2-(\mathrm{l}, \mathrm{s})+0.25 \mathrm{O} 2(\mathrm{~g}) \rightarrow \mathrm{CO} 2(\mathrm{~g})+0.5 \mathrm{H} 2 \mathrm{O}(\mathrm{g})$ & 1.0 \\
\hline PPG $(\mathrm{l}, \mathrm{s})+240 \mathrm{O} 2(\mathrm{~g}) \rightarrow 180 \mathrm{CO} 2(\mathrm{~g})+181 \mathrm{H} 2 \mathrm{O}(\mathrm{g})$ & 1.0 \\
\hline $\mathrm{DPPG}(1, \mathrm{~s})+240 \mathrm{O} 2(\mathrm{~g}) \rightarrow 180 \mathrm{CO} 2(\mathrm{~g})+181 \mathrm{H} 2 \mathrm{O}(\mathrm{g})$ & 1.0 \\
\hline PDMS $(\mathrm{l}, \mathrm{s})+1892 \mathrm{O} 2(\mathrm{~g}) \rightarrow 946 \mathrm{CO} 2(\mathrm{~g})+1419 \mathrm{H} 2 \mathrm{O}(\mathrm{g})+472 \mathrm{SiO} 2(\mathrm{o})$ & 1.0 \\
\hline $\mathrm{DPDMS}(\mathrm{l}, \mathrm{s})+1892 \mathrm{O} 2(\mathrm{~g}) \rightarrow 946 \mathrm{CO} 2(\mathrm{~g})+1419 \mathrm{H} 2 \mathrm{O}(\mathrm{g})+472 \mathrm{SiO} 2(\mathrm{o})$ & 1.0 \\
\hline
\end{tabular}

\begin{tabular}{|c|c|}
\hline Miscellaneous Reactions & Conversion (Fraction) \\
\hline $2 \mathrm{Cl}-(1, \mathrm{~s})+2 \mathrm{Na}+(1, \mathrm{~s})+\mathrm{H} 2 \mathrm{O}(\mathrm{l}) \rightarrow \mathrm{Na} 2 \mathrm{O}(\mathrm{o})+2 \mathrm{HCl}(\mathrm{g})$ & 0.7353 \\
\hline $2 \mathrm{~F}-(1, \mathrm{~s})+2 \mathrm{Na}+(1, \mathrm{~s})+\mathrm{H} 2 \mathrm{O}(\mathrm{l}) \rightarrow \mathrm{Na} 2 \mathrm{O}(\mathrm{o})+2 \mathrm{HF}(\mathrm{g})$ & 0.8065 \\
\hline $129-\mathrm{I}(1, \mathrm{~s}) \rightarrow 129-\mathrm{I}(\mathrm{g})$ & 0.7692 \\
\hline $\mathrm{Hg}+2(1, \mathrm{~s}) \rightarrow \mathrm{Hg}(\mathrm{g})$ & 1.0 \\
\hline $\begin{array}{l}\mathrm{r} 11 \times \mathrm{NO}_{3}{ }^{-}(1, \mathrm{~s})+\mathrm{r} 12 \times \mathrm{H}_{2} \mathrm{O}(\mathrm{l}) \rightarrow \mathrm{p} 11 \times \mathrm{N}_{2}(\mathrm{~g})+\mathrm{p} 12 \times \mathrm{NO}(\mathrm{g})+\mathrm{p} 13 \times \mathrm{NO}_{2}(\mathrm{~g})+ \\
\mathrm{p} 14 \times \mathrm{NH}_{3}(\mathrm{~g})+(0.25+\mathrm{p} 15) \times \mathrm{O}_{2}(\mathrm{~g})\end{array}$ & dynamically calculated \\
\hline $\begin{array}{l}\mathrm{r} 21 \times \mathrm{NO}_{2}^{-}(\mathrm{l}, \mathrm{s})+\mathrm{r} 22 \times \mathrm{H}_{2} \mathrm{O}(\mathrm{l}) \rightarrow \mathrm{p} 21 \times \mathrm{N}_{2}(\mathrm{~g})+\mathrm{p} 22 \times \mathrm{NO}(\mathrm{g})+\mathrm{p} 23 \times \mathrm{NO}_{2}(\mathrm{~g})+ \\
\mathrm{p} 24 \times \mathrm{NH}_{3}(\mathrm{~g})+(0.25+\mathrm{p} 25) \times \mathrm{O}_{2}(\mathrm{~g})\end{array}$ & dynamically calculated \\
\hline $\mathrm{SO} 4-2(1, \mathrm{~s}) \rightarrow \mathrm{SO} 2(\mathrm{~g})+\mathrm{O} 2(\mathrm{~g})$ & 0.318823 \\
\hline $\mathrm{C} 12 \mathrm{H} 22 \mathrm{O} 11(\mathrm{o})+8 \mathrm{NO} 3-(1, \mathrm{~s}) \rightarrow 6 \mathrm{C} 2 \mathrm{H} 3 \mathrm{~N}(\mathrm{~g})+2 \mathrm{H} 2 \mathrm{O}(\mathrm{g})+\mathrm{N} 2(\mathrm{~g})+16.5 \mathrm{O} 2(\mathrm{~g})$ & 0.0159 \\
\hline $\mathrm{C} 12 \mathrm{H} 22 \mathrm{O} 11(\mathrm{o})+6 \mathrm{HCl}(\mathrm{g})+0.5 \mathrm{O} 2(\mathrm{~g}) \rightarrow \mathrm{C} 12 \mathrm{H} 4 \mathrm{Cl} 6(\mathrm{~g})+12 \mathrm{H} 2 \mathrm{O}(\mathrm{g})$ & $3.79 \mathrm{e}-10$ \\
\hline $\mathrm{C} 12 \mathrm{H} 22 \mathrm{O} 11(\mathrm{o})+8 \mathrm{NO} 3-(\mathrm{l}, \mathrm{s}) \rightarrow 12 \mathrm{CO} 2(\mathrm{~g})+11 \mathrm{H} 2 \mathrm{O}(\mathrm{g})+4 \mathrm{~N} 2(\mathrm{~g})$ & 0.6 \\
\hline $\mathrm{C} 12 \mathrm{H} 22 \mathrm{O} 11(\mathrm{o})+8 \mathrm{NO} 2(\mathrm{l}, \mathrm{s})+4 \mathrm{O} 2(\mathrm{~g}) \rightarrow 12 \mathrm{CO} 2(\mathrm{~g})+11 \mathrm{H} 2 \mathrm{O}(\mathrm{g})+4 \mathrm{~N} 2(\mathrm{~g})$ & 1.0 \\
\hline $\mathrm{C} 12 \mathrm{H} 22 \mathrm{O} 11(\mathrm{o})+12 \mathrm{O} 2(\mathrm{~g}) \rightarrow 12 \mathrm{CO} 2(\mathrm{~g})+11 \mathrm{H} 2 \mathrm{O}(\mathrm{g})$ & 1.0 \\
\hline $2 \mathrm{CN}-(1, \mathrm{~s})+2 \mathrm{O} 2(\mathrm{~g}) \rightarrow 2 \mathrm{CO} 2(\mathrm{~g})+\mathrm{N} 2(\mathrm{~g})$ & 1.0 \\
\hline $\mathrm{CO} 3-2(1, \mathrm{~s}) \rightarrow \mathrm{CO} 2(\mathrm{~g})+0.5 \mathrm{O} 2(\mathrm{~g})$ & 1.0 \\
\hline $\mathrm{H}+(1, \mathrm{~s})+\mathrm{OH}(\mathrm{BOUND})(1, \mathrm{~s}) \rightarrow \mathrm{H} 2 \mathrm{O}(\mathrm{g})$ & 1.0 \\
\hline $\mathrm{H} 2 \mathrm{O}(1, \mathrm{~s}) \rightarrow \mathrm{H} 2 \mathrm{O}(\mathrm{g})$ & 1.0 \\
\hline $2 \mathrm{H} 2 \mathrm{O} 2(\mathrm{l}, \mathrm{s}) \rightarrow 2 \mathrm{H} 2 \mathrm{O}(\mathrm{g})+\mathrm{O} 2(\mathrm{~g})$ & 1.0 \\
\hline $2 \mathrm{HCO} 3-(1, \mathrm{~s}) \rightarrow 2 \mathrm{CO} 2(\mathrm{~g})+\mathrm{H} 2 \mathrm{O}(\mathrm{g})+0.5 \mathrm{O} 2(\mathrm{~g})$ & 1.0 \\
\hline $\mathrm{H} 2 \mathrm{CO} 3(1, \mathrm{~s}) \rightarrow \mathrm{CO} 2(\mathrm{~g})+\mathrm{H} 2 \mathrm{O}(\mathrm{g})$ & 1.0 \\
\hline $2 \mathrm{NH} 3(\mathrm{l}, \mathrm{s})+3 \mathrm{O} 2(\mathrm{~g}) \rightarrow \mathrm{NO}(\mathrm{g})+\mathrm{NO} 2(\mathrm{~g})+3 \mathrm{H} 2 \mathrm{O}(\mathrm{g})$ & 1.0 \\
\hline $2 \mathrm{NH} 4+(\mathrm{l}, \mathrm{s})+3.5 \mathrm{O} 2(\mathrm{~g}) \rightarrow \mathrm{NO}(\mathrm{g})+\mathrm{NO} 2(\mathrm{~g})+4 \mathrm{H} 2 \mathrm{O}(\mathrm{g})$ & 1.0 \\
\hline $2 \mathrm{O}-2(1, \mathrm{~s}) \rightarrow \mathrm{O} 2(\mathrm{~g})$ & 1.0 \\
\hline \multicolumn{2}{|l|}{ Sum OH- and $\mathrm{OH}(\mathrm{BOUND})$ to OH- (i.e., combine both components into OH-) } \\
\hline $2 \mathrm{OH}-(1, \mathrm{~s}) \rightarrow \mathrm{H} 2 \mathrm{O}(\mathrm{g})+0.5 \mathrm{O} 2(\mathrm{~g})$ & 1.0 \\
\hline
\end{tabular}

\begin{tabular}{|l|l|}
\hline Oxidation Reactions & Conversion (Fraction) \\
\hline $2227-\mathrm{Ac}(1, \mathrm{~s})+1.5 \mathrm{O} 2(\mathrm{~g}) \rightarrow \mathrm{Ac} 2 \mathrm{O} 3(\mathrm{o})$ & 1.0 \\
\hline $2 \mathrm{Ag}+(1, \mathrm{~s})+0.5 \mathrm{O} 2(\mathrm{~g}) \rightarrow \mathrm{Ag} 2 \mathrm{O}(\mathrm{o})$ & 1.0 \\
\hline $2 \mathrm{Al}+3(1, \mathrm{~s})+1.5 \mathrm{O} 2(\mathrm{~g}) \rightarrow \mathrm{Al} 2 \mathrm{O} 3(\mathrm{o})$ & 1.0 \\
\hline $2 \mathrm{Al}(\mathrm{OH}) 4-(\mathrm{l}, \mathrm{s}) \rightarrow \mathrm{Al} 2 \mathrm{O} 3(\mathrm{o})+0.5 \mathrm{O} 2(\mathrm{~g})+4 \mathrm{H} 2 \mathrm{O}(\mathrm{g})$ & 1.0 \\
\hline $2 \mathrm{Al}(\mathrm{OH}) 3(\mathrm{l}, \mathrm{s}) \rightarrow \mathrm{Al} 2 \mathrm{O} 3(\mathrm{o})+3 \mathrm{H} 2 \mathrm{O}(\mathrm{g})$ & 1.0 \\
\hline$\quad \mathrm{Sum} 241-\mathrm{Am}$ and $243-\mathrm{Am}$ to $241-\mathrm{Am}$ & 1.0 \\
\hline $2241-\mathrm{Am}(1, \mathrm{~s})+1.5 \mathrm{O} 2(\mathrm{~g}) \rightarrow \mathrm{Am} 2 \mathrm{O} 3(\mathrm{o})$ & 1.0 \\
\hline $2 \mathrm{As}+5(1, \mathrm{~s})+2.5 \mathrm{O} 2(\mathrm{~g}) \rightarrow \mathrm{As} 2 \mathrm{O} 5(\mathrm{o})$ & 1.0 \\
\hline $2 \mathrm{~B}+3(1, \mathrm{~s})+1.5 \mathrm{O} 2(\mathrm{~g}) \rightarrow \mathrm{B} 2 \mathrm{O} 3(\mathrm{o})$ & \\
\hline$\quad \mathrm{Sum} \mathrm{Ba}+2$ and $137 \mathrm{~m}-\mathrm{Ba}$ to Ba+2 & 1.0 \\
\hline $\mathrm{Ba}+2(1, \mathrm{~s})+0.5 \mathrm{O} 2(\mathrm{~g}) \rightarrow \mathrm{BaO}(\mathrm{o})$ & 1.0 \\
\hline $\mathrm{Be}+2(1, \mathrm{~s})+0.5 \mathrm{O} 2(\mathrm{~g}) \rightarrow \mathrm{BeO}(\mathrm{o})$ & 1.0 \\
\hline $2 \mathrm{Bi}+3(1, \mathrm{~s})+1.5 \mathrm{O} 2(\mathrm{~g}) \rightarrow \mathrm{Bi} 2 \mathrm{O} 3(\mathrm{o})$ & \\
\hline
\end{tabular}


$\mathrm{Ca}+2(1, \mathrm{~s})+0.5 \mathrm{O} 2(\mathrm{~g}) \rightarrow \mathrm{CaO}(\mathrm{o})$ 1.0

- $\quad$ Sum $\mathrm{Cd}+2$ and $113 \mathrm{~m}-\mathrm{Cd}$ to $\mathrm{Cd}+2$

$\mathrm{Cd}+2(1, \mathrm{~s})+0.5 \mathrm{O} 2(\mathrm{~g}) \rightarrow \mathrm{CdO}(\mathrm{o})$

$4 \mathrm{Ce}+3(1, \mathrm{~s})+3 \mathrm{O} 2(\mathrm{~g}) \rightarrow 2 \mathrm{Ce} 2 \mathrm{O} 3$ (o)

$\mathrm{Cl}-(1, \mathrm{~s}) \rightarrow \mathrm{Cl}-(\mathrm{o})$

- $\quad$ Sum 242-Cm, 243-Cm, and 244-Cm to 242-Cm

2 242-Cm (1,s) + $1.5 \mathrm{O} 2$ (g) $\rightarrow \mathrm{Cm} 2 \mathrm{O} 3$ (o)

- $\quad$ Sum $\mathrm{Co}+3$ and $60-\mathrm{Co}$ to $\mathrm{Co}+3$

$2 \mathrm{Co}+3(1, \mathrm{~s})+\mathrm{O} 2(\mathrm{~g}) \rightarrow 2 \mathrm{CoO}(\mathrm{o})$

2 Cr(TOTAL) $(1, \mathrm{~s})+1.5 \mathrm{O} 2(\mathrm{~g}) \rightarrow \mathrm{Cr} 2 \mathrm{O} 3(\mathrm{o})$

$2 \mathrm{Cr}(\mathrm{OH}) 4-(1, \mathrm{~s}) \rightarrow \mathrm{Cr} 2 \mathrm{O} 3(\mathrm{o})+0.5 \mathrm{O} 2(\mathrm{~g})+4 \mathrm{H} 2 \mathrm{O}(\mathrm{g})$

1.0

1.0

1.0

1.0

1.0

1.0

1.0

- $\quad$ Sum $\mathrm{Cs}^{+}, 134-\mathrm{Cs}$, and $137-\mathrm{Cs}$ to $\mathrm{Cs}^{+}$

$2 \mathrm{Cs}+(1, \mathrm{~s})+0.5 \mathrm{O} 2(\mathrm{~g}) \rightarrow \mathrm{Cs} 2 \mathrm{O}(\mathrm{o})$

$\mathrm{Cu}+2(1, \mathrm{~s})+0.5 \mathrm{O} 2(\mathrm{~g}) \rightarrow \mathrm{CuO}(\mathrm{o})$

1.0

1.0

- $\quad$ Sum 152-Eu, 154-Eu, and 155-Eu to 152-Eu

$2152-\mathrm{Eu}(1, \mathrm{~s})+0.5 \mathrm{O} 2(\mathrm{~g}) \rightarrow \mathrm{Eu} 2 \mathrm{O} 3(\mathrm{o})$

$\mathrm{F}-(1, \mathrm{~s}) \rightarrow \mathrm{F}-(\mathrm{o})$

$2 \mathrm{Fe}+3(1, \mathrm{~s})+1.5 \mathrm{O} 2(\mathrm{~g}) \rightarrow \mathrm{Fe} 2 \mathrm{O} 3(\mathrm{o})$

$\mathrm{Hg}+2(\mathrm{l}, \mathrm{s})+0.5 \mathrm{O} 2(\mathrm{~g}) \rightarrow \mathrm{HgO}(\mathrm{o})$

$129-\mathrm{I}(1, \mathrm{~s}) \rightarrow 129-\mathrm{I}(\mathrm{o})$

$2 \mathrm{~K}+(1, \mathrm{~s})+0.5 \mathrm{O} 2(\mathrm{~g}) \rightarrow \mathrm{K} 2 \mathrm{O}(\mathrm{o})$

$2 \mathrm{La}+3(\mathrm{l}, \mathrm{s})+1.5 \mathrm{O} 2(\mathrm{~g}) \rightarrow \mathrm{La} 2 \mathrm{O} 3(\mathrm{o})$

$2 \mathrm{Li}+(1, \mathrm{~s})+0.5 \mathrm{O} 2(\mathrm{~g}) \rightarrow \mathrm{Li} 2 \mathrm{O}(\mathrm{o})$

$\mathrm{Mg}+2(1, \mathrm{~s})+0.5 \mathrm{O} 2(\mathrm{~g}) \rightarrow \mathrm{MgO}(\mathrm{o})$

$2 \mathrm{MnO} 4-(1, \mathrm{~s}) \rightarrow 2 \mathrm{MnO}(\mathrm{o})+3 \mathrm{O} 2(\mathrm{~g})$

$2 \mathrm{Mn}+4(1, \mathrm{~s})+\mathrm{O} 2(\mathrm{~g}) \rightarrow 2 \mathrm{MnO}(\mathrm{o})$

$2 \mathrm{MnO} 2(\mathrm{l}, \mathrm{s}) \rightarrow 2 \mathrm{MnO}(\mathrm{o})+\mathrm{O} 2(\mathrm{~g})$

$2 \mathrm{Mnp}(1, \mathrm{~s})+1.5 \mathrm{O} 2(\mathrm{~g}) \rightarrow \mathrm{Mnp} 2 \mathrm{O} 3(\mathrm{o})$

$\mathrm{Mo}+6(1, \mathrm{~s})+1.5 \mathrm{O} 2(\mathrm{~g}) \rightarrow \mathrm{MoO} 3(\mathrm{o})$

$2 \mathrm{Na}+(1, \mathrm{~s})+0.5 \mathrm{O} 2(\mathrm{~g}) \rightarrow \mathrm{Na} 2 \mathrm{O}(\mathrm{o})$

$2 \mathrm{Nap}+(\mathrm{l}, \mathrm{s})+0.5 \mathrm{O} 2(\mathrm{~g}) \rightarrow \mathrm{Nap} 2 \mathrm{O}(\mathrm{o})$

$293 \mathrm{~m}-\mathrm{Nb}(\mathrm{l}, \mathrm{s})+2.5 \mathrm{O} 2(\mathrm{~g}) \rightarrow \mathrm{Nb} 2 \mathrm{O} 5(\mathrm{o})$

$2 \mathrm{Nd}+3(1, \mathrm{~s})+1.5 \mathrm{O} 2(\mathrm{~g}) \rightarrow \mathrm{Nd} 2 \mathrm{O} 3(\mathrm{o})$

- $\quad \mathrm{Sum} \mathrm{Ni}+2,59-\mathrm{Ni}$, and $63-\mathrm{Ni}$ to $\mathrm{Ni}+2$

$\mathrm{Ni}+2(1, \mathrm{~s})+0.5 \mathrm{O} 2(\mathrm{~g}) \rightarrow \mathrm{NiO}(\mathrm{o})$

$237-\mathrm{Np}(1, \mathrm{~s})+\mathrm{O} 2(\mathrm{~g}) \rightarrow \mathrm{NpO} 2(\mathrm{o})$

2 PO4-3 (1,s) $\rightarrow$ P2O5 (o) + $1.5 \mathrm{O} 2(\mathrm{~g})$

$2231-\mathrm{Pa}(1, \mathrm{~s})+1.5 \mathrm{O} 2(\mathrm{~g}) \rightarrow \mathrm{Pa} 2 \mathrm{O} 3(\mathrm{o})$

$\mathrm{Pb}+2(\mathrm{l}, \mathrm{s})+0.5 \mathrm{O} 2(\mathrm{~g}) \rightarrow \mathrm{PbO}(\mathrm{o})$

$\mathrm{Pd}+2(1, \mathrm{~s})+0.5 \mathrm{O} 2(\mathrm{~g}) \rightarrow \mathrm{PdO}(\mathrm{o})$

$2 \mathrm{Pr}+3(1, \mathrm{~s})+1.5 \mathrm{O} 2(\mathrm{~g}) \rightarrow \mathrm{Pr} 2 \mathrm{O} 3(\mathrm{o})$

- $\quad \mathrm{Sum} \mathrm{Pu}+4,238-\mathrm{Pu}, 239-\mathrm{Pu}, 240-\mathrm{Pu}, 241-\mathrm{Pu}$, and 242-Pu to $\mathrm{Pu}+4$

$\mathrm{Pu}+4(1, \mathrm{~s})+\mathrm{O} 2(\mathrm{~g}) \rightarrow \mathrm{PuO} 2(\mathrm{o})$

- $\quad$ Sum 226-Ra and 228-Ra to 226-Ra

$2226-\mathrm{Ra}(1, \mathrm{~s})+\mathrm{O} 2(\mathrm{~g}) \rightarrow 2 \mathrm{RaO}(\mathrm{o})$

$2 \mathrm{Rb}+(1, \mathrm{~s})+0.5 \mathrm{O} 2(\mathrm{~g}) \rightarrow \mathrm{Rb} 2 \mathrm{O}(\mathrm{o})$

$2 \mathrm{Rh}+3(1, \mathrm{~s})+1.5 \mathrm{O} 2(\mathrm{~g}) \rightarrow \mathrm{Rh} 2 \mathrm{O} 3(\mathrm{o})$

- Sum $\mathrm{Ru}+3$ and $106-\mathrm{Ru}$ to $\mathrm{Ru}+3$

$\mathrm{Ru}+3(1, \mathrm{~s})+\mathrm{O} 2(\mathrm{~g}) \rightarrow \mathrm{RuO} 2(\mathrm{o})$

$\mathrm{SO} 4-2(1, \mathrm{~s}) \rightarrow \mathrm{SO} 3(\mathrm{o})+0.5 \mathrm{O} 2(\mathrm{~g})$

- $\quad \mathrm{Sum} \mathrm{Sb}+5$ and $125-\mathrm{Sb}$ to $\mathrm{Sb}+5$

$2 \mathrm{Sb}+5(1, \mathrm{~s})+1.5 \mathrm{O} 2(\mathrm{~g}) \rightarrow \mathrm{Sb} 2 \mathrm{O} 3(\mathrm{o})$

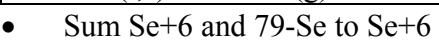

$\mathrm{Se}+6(1, \mathrm{~s})+\mathrm{O} 2(\mathrm{~g}) \rightarrow \mathrm{SeO} 2(\mathrm{o})$

$\mathrm{Si}+4(1, \mathrm{~s})+\mathrm{O} 2(\mathrm{~g}) \rightarrow \mathrm{SiO} 2(\mathrm{o})$
1.0

1.0

1.0

$0.0(100 \%$ split $)$

1.0

1.0

1.0

1.0

1.0

1.0

1.0

1.0

1.0

1.0

1.0

1.0

1.0

\begin{tabular}{|l|}
\hline 1.0 \\
\hline 1.0 \\
\hline 1.0 \\
1.0 \\
1.0 \\
\hline 1.0 \\
1.0 \\
\hline
\end{tabular}

1.0

1.0

1.0

1.0

1.0

1.0

1.0

1.0

1.0 


\begin{tabular}{|c|c|}
\hline $2151-\mathrm{Sm}(1, \mathrm{~s})+1.5 \mathrm{O} 2(\mathrm{~g}) \rightarrow \mathrm{Sm} 2 \mathrm{O} 3(\mathrm{o})$ & 1.0 \\
\hline $126-\mathrm{Sn}(1, \mathrm{~s})+\mathrm{O} 2(\mathrm{~g}) \rightarrow \mathrm{SnO} 2(\mathrm{o})$ & 1.0 \\
\hline \multicolumn{2}{|l|}{ - $\quad$ Sum $\mathrm{Sr}+2$ and $90-\mathrm{Sr}$ to $\mathrm{Sr}+2$} \\
\hline $\mathrm{Sr}+2(1, \mathrm{~s})+0.5 \mathrm{O} 2(\mathrm{~g}) \rightarrow \mathrm{SrO}(\mathrm{o})$ & 1.0 \\
\hline $2 \mathrm{Ta}+5(1, \mathrm{~s})+2.5 \mathrm{O} 2(\mathrm{~g}) \rightarrow \mathrm{Ta} 2 \mathrm{O} 5(\mathrm{o})$ & 1.0 \\
\hline \multicolumn{2}{|l|}{ - $\quad$ Sum $\mathrm{Tc}+7$ and $99-\mathrm{Tc}$ to $\mathrm{Tc}+7$} \\
\hline $\mathrm{Tc}+7(1, \mathrm{~s})+3.5 \mathrm{O} 2(\mathrm{~g}) \rightarrow \mathrm{Tc} 2 \mathrm{O} 7(\mathrm{o})$ & 1.0 \\
\hline $\mathrm{Te}+6(\mathrm{l}, \mathrm{s})+\mathrm{O} 2(\mathrm{~g}) \rightarrow \mathrm{TeO} 2(\mathrm{o})$ & 1.0 \\
\hline \multicolumn{2}{|l|}{ - $\quad$ Sum $\mathrm{Th}+4,229-\mathrm{Th}$, and 232-Th to $\mathrm{Th}+4$} \\
\hline $\mathrm{Th}+4(\mathrm{l}, \mathrm{s})+\mathrm{O} 2(\mathrm{~g}) \rightarrow \mathrm{ThO} 2(\mathrm{o})$ & 1.0 \\
\hline $\mathrm{Ti}+4(1, \mathrm{~s})+\mathrm{O} 2(\mathrm{~g}) \rightarrow \mathrm{TiO} 2(\mathrm{o})$ & 1.0 \\
\hline $4 \mathrm{Tl}+3(\mathrm{l}, \mathrm{s})+\mathrm{O} 2(\mathrm{~g}) \rightarrow 2 \mathrm{~T} 12 \mathrm{O}(\mathrm{o})$ & 1.0 \\
\hline \multicolumn{2}{|c|}{ - $\quad$ Sum U(TOTAL), 232-U, 233-U, 234-U, 235-U, 236-U, and 238-U to U(TOTAL) } \\
\hline $2 \mathrm{U}(\mathrm{TOTAL})(1, \mathrm{~s})+3 \mathrm{O} 2(\mathrm{~g}) \rightarrow 2 \mathrm{UO} 3(\mathrm{o})$ & 1.0 \\
\hline $2 \mathrm{~V}+5(1, \mathrm{~s})+2.5 \mathrm{O} 2(\mathrm{~g}) \rightarrow \mathrm{V} 2 \mathrm{O} 5(\mathrm{o})$ & 1.0 \\
\hline $\mathrm{W}+6(1, \mathrm{~s})+1.5 \mathrm{O} 2(\mathrm{~g}) \rightarrow \mathrm{WO} 3(\mathrm{o})$ & 1.0 \\
\hline \multicolumn{2}{|l|}{ - $\quad$ Sum $\mathrm{Y}+3$ and $90-\mathrm{Y}$ to $\mathrm{Y}+3$} \\
\hline $2 \mathrm{Y}+3(1, \mathrm{~s})+1.5 \mathrm{O} 2(\mathrm{~g}) \rightarrow \mathrm{Y} 2 \mathrm{O} 3(\mathrm{o})$ & 1.0 \\
\hline $\mathrm{Zn}+2(\mathrm{l}, \mathrm{s})+0.5 \mathrm{O} 2(\mathrm{~g}) \rightarrow \mathrm{ZnO}(\mathrm{o})$ & 1.0 \\
\hline \multicolumn{2}{|l|}{ - $\quad$ Sum $\mathrm{Zr}+4$ and $93-\mathrm{Zr}$ to $\mathrm{Zr}+4$} \\
\hline $\mathrm{Zr}+4(1, \mathrm{~s})+\mathrm{O} 2(\mathrm{~g}) \rightarrow \mathrm{ZnO} 2(\mathrm{o})$ & 1.0 \\
\hline
\end{tabular}

A lower set volume (1,067 gallons) is applied to the HLW melters where the molten glass above the set volume is transferred to the IHLW canisters. HMP-MLTR-00001 transfers molten glass to IHLW1 while HMP-MLTR-00002 transfers molten glass to IHLW2. The IHLW canisters are 300 gallons in size. IHLW glass has a $2.66 \mathrm{~g} / \mathrm{cm}^{3}$ density. Canister counts and glass compositions are tracked by the model. Gases from the melter are routed to a corresponding HLW Offgas Process system. HMP-MLTR-00001 transfers materials to HOP-SCB-00001 and HMP-MLTR-00002 transfers materials to HOP-SCB-00002.

Figures F-19 and F-20 provide the graphical illustration of the HMP systems where connections with upstream and downstream systems are shown. Table N-1 provides the totalizer files that are used to track the process streams and material flows into and out of the HMP.

\subsubsection{HLW Melter Offgas Treatment Process (HOP) System}

\begin{tabular}{|l|l|}
\hline Vessel Tag \# & Equipment Description \\
\hline HOP-SCB-00001 & HLW Melter 1 Submerged Bed Scrubber \\
\hline HOP-SCB-00002 & HLW Melter 2 Submerged Bed Scrubber \\
\hline HOP-VSL-00903 & HLW SBS Condensate Receiver Vessel \\
\hline HOP-VSL-00904 & HLW SBS Condensate Receiver Vessel \\
\hline
\end{tabular}

The HLW SBSs receive offgas from the HLW melters. HOP-SCB-00001 receives material from HMP-MLTR-00001 and HOP-SCB-00002 receives material from HMP-MLTR-00002. Each of the HLW SBSs are associated with a SBS Condensate Vessel. For modeling simplicity, the condensate vessels have been merged into their associated SBSs, for example, working volumes have been combined as follows:

\section{HOP-SCB-00001/2}

Minimum Volume $=1,758+0$ gallons $=1,758$ gallons

Maximum Volume $=5,808+3,329$ gallons $=9,137$ gallons

Set Volume $=8,137$ gallons 
Maximum Accumulation Time $=24$ hours

Initially, the scrubbers are filled to their set volume with demineralized water from a chem-add. Upon arriving at the SBS, entrained liquid and solid from the melter are split to the WESP according to the split values given in Table D-20. The remaining liquid and solid materials undergo the following reactions (reactions take place in the order presented):

\begin{tabular}{|l|l|}
\hline Reactions & Conversion (Fraction) \\
\hline $\mathrm{H} 2 \mathrm{O}(\mathrm{g}) \rightarrow \mathrm{H} 2 \mathrm{O}(\mathrm{l})$ or $\mathrm{H} 2 \mathrm{O}(\mathrm{l}) \rightarrow \mathrm{H} 2 \mathrm{O}(\mathrm{g})$ & $\begin{array}{l}0.138 \text { mole fraction at } \\
\text { outlet }\end{array}$ \\
\hline $\mathrm{Hg}(\mathrm{g}) \rightarrow \mathrm{Hg}+2(\mathrm{l})$ & Eq. 69 \\
\hline $2 \mathrm{~B}+3(\mathrm{~s})+1.5 \mathrm{O} 2(\mathrm{~g})+3 \mathrm{H} 2 \mathrm{O}(\mathrm{l}) \rightarrow 2 \mathrm{H}+(\mathrm{l})+2 \mathrm{~B}+3(\mathrm{l})+4 \mathrm{O}-2(\mathrm{l})+2 \mathrm{H} 2 \mathrm{O}(\mathrm{l})$ & 1.0 \\
\hline $\mathrm{HCl}(\mathrm{g}) \rightarrow \mathrm{Cl}-(\mathrm{l})+\mathrm{H}+(\mathrm{l})$ & 0.7561 \\
\hline $\mathrm{HF}(\mathrm{g}) \rightarrow \mathrm{F}-(\mathrm{l})+\mathrm{H}+(\mathrm{l})$ & 0.9825 \\
\hline $3 \mathrm{NO} 2(\mathrm{~g})+\mathrm{H} 2 \mathrm{O}(\mathrm{l}) \rightarrow 2 \mathrm{NO} 3-(\mathrm{l})+2 \mathrm{H}+(\mathrm{l})+\mathrm{NO}(\mathrm{g})$ & 0.002 \\
\hline $4 \mathrm{NO}(\mathrm{g})+2 \mathrm{H} 2 \mathrm{O}(\mathrm{l})+3 \mathrm{O} 2(\mathrm{~g}) \rightarrow 4 \mathrm{NO} 3-(\mathrm{l})+4 \mathrm{H}+(\mathrm{l})$ & 0.0548 \\
\hline $\mathrm{P} 2 \mathrm{O} 5(\mathrm{~g})+3 \mathrm{H} 2 \mathrm{O}(\mathrm{l}) \rightarrow 0.5 \mathrm{PO} 4-3(\mathrm{l})+1.5 \mathrm{PO} 4-3(\mathrm{~s})+6 \mathrm{H}+(\mathrm{l})$ & 0.9451 \\
\hline $\mathrm{SO} 2(\mathrm{~g})+\mathrm{H} 2 \mathrm{O}(\mathrm{l})+0.5 \mathrm{O} 2(\mathrm{~g}) \rightarrow \mathrm{SO} 4-2(\mathrm{l})+2 \mathrm{H}+(\mathrm{l})$ & 0.8534 \\
\hline $\mathrm{C} 2 \mathrm{H} 3 \mathrm{~N}(\mathrm{~g}) \rightarrow \mathrm{C} 2 \mathrm{H} 3 \mathrm{~N}(\mathrm{l})$ & 0.8 \\
\hline $\mathrm{C} 12 \mathrm{H} 4 \mathrm{Cl} 6(\mathrm{~g}) \rightarrow \mathrm{C} 12 \mathrm{H} 4 \mathrm{Cl} 6(\mathrm{l})$ & 0.8837 \\
\hline $129-\mathrm{I}(\mathrm{g}) \rightarrow 129-\mathrm{I}(\mathrm{l})$ & 0.6875 \\
\hline $\mathrm{Na}+(\mathrm{s})+\mathrm{Cl}-(\mathrm{s}) \rightarrow \mathrm{Na}+(\mathrm{l})+\mathrm{Cl}-(\mathrm{l})$ & $1.0[\mathrm{Cl}-](\mathrm{s})=0.0$ \\
\hline $\mathrm{Nap}+(\mathrm{s})+\mathrm{Cl}-(\mathrm{s}) \rightarrow \mathrm{Nap}+(\mathrm{l})+\mathrm{Cl}-(\mathrm{l})$ & $1.0[\mathrm{Cl}-](\mathrm{s})=0.0$ \\
\hline $\mathrm{Na}+(\mathrm{s})+\mathrm{F}-(\mathrm{s}) \rightarrow \mathrm{Na}+(\mathrm{l})+\mathrm{F}-(\mathrm{l})$ & $1.0[\mathrm{~F}-](\mathrm{s})=0.0$ \\
\hline $\mathrm{Nap}+(\mathrm{s})+\mathrm{F}-(\mathrm{s}) \rightarrow \mathrm{Nap}+(\mathrm{l})+\mathrm{F}-(\mathrm{l})$ & $1.0[\mathrm{~F}-](\mathrm{s})=0.0$ \\
\hline $4 \mathrm{Na}+(\mathrm{s})+\mathrm{O} 2(\mathrm{~g})+2 \mathrm{H} 2 \mathrm{O}(\mathrm{l}) \rightarrow 4 \mathrm{Na}+(\mathrm{l})+4 \mathrm{OH}-(\mathrm{l})$ & 0.9 \\
\hline $4 \mathrm{Nap}+(\mathrm{s})+\mathrm{O} 2(\mathrm{~g})+2 \mathrm{H} 2 \mathrm{O}(\mathrm{l}) \rightarrow 4 \mathrm{Nap}+(\mathrm{l})+4 \mathrm{OH}-(\mathrm{l})$ & 0.9 \\
\hline $4 \mathrm{~K}+(\mathrm{s})+\mathrm{O} 2(\mathrm{~g})+2 \mathrm{H} 2 \mathrm{O}(\mathrm{l}) \rightarrow 4 \mathrm{~K}+(\mathrm{l})+4 \mathrm{OH}-(\mathrm{l})$ & 0.95 \\
\hline $2 \mathrm{Ca}+2(\mathrm{~s})+\mathrm{O} 2(\mathrm{~g})+2 \mathrm{H} 2 \mathrm{O}(\mathrm{l}) \rightarrow 2 \mathrm{Ca}+2(\mathrm{l})+4 \mathrm{OH}-(\mathrm{l})$ & 0.75 \\
\hline $2 \mathrm{Sr}+2(\mathrm{~s})+\mathrm{O} 2(\mathrm{~g})+2 \mathrm{H} 2 \mathrm{O}(\mathrm{l}) \rightarrow 2 \mathrm{Sr}+2(\mathrm{l})+4 \mathrm{OH}-(\mathrm{l})$ & 0.65 \\
\hline $290-\mathrm{Sr}(\mathrm{s})+\mathrm{O} 2(\mathrm{~g})+2 \mathrm{H} 2 \mathrm{O}(\mathrm{l}) \rightarrow 290-\mathrm{Sr}(\mathrm{s})+4$ OH- $(\mathrm{l})$ & 0.65 \\
\hline $4 \mathrm{Li}+(\mathrm{s})+\mathrm{O} 2(\mathrm{~g})+2 \mathrm{H} 2 \mathrm{O}(\mathrm{l}) \rightarrow 4 \mathrm{Li}+(\mathrm{l})+4 \mathrm{OH}-(\mathrm{l})$ & 0.95 \\
\hline $\mathrm{NH} 3(\mathrm{~g}) \rightarrow \mathrm{NH} 3(\mathrm{l})$ & 0.90 \\
\hline $\mathrm{NH} 3(\mathrm{l})+\mathrm{H} 2 \mathrm{O}(\mathrm{l}) \rightarrow \mathrm{NH} 4+(\mathrm{l})+\mathrm{OH}-(\mathrm{l})$ & $\mathrm{Eq} .68$ \\
\hline $\mathrm{CO} 2(\mathrm{~g})+\mathrm{H} 2 \mathrm{O}(\mathrm{l}) \rightarrow \mathrm{H} 2 \mathrm{CO} 3(\mathrm{l})$ & 0.001 \\
\hline $\mathrm{H} 2 \mathrm{CO} 3(\mathrm{l}) \rightarrow \mathrm{HCO} 3-(\mathrm{l})+\mathrm{H}+(\mathrm{l})$ & $\mathrm{Eq} .64$ \\
\hline $\mathrm{HCO} 3-(\mathrm{l}) \rightarrow \mathrm{CO}-2(\mathrm{l})+\mathrm{H}+(\mathrm{l})$ & Eq. 65 \\
\hline $\mathrm{H}+(\mathrm{l})+\mathrm{OH}-(\mathrm{l}) \rightarrow \mathrm{H} 2 \mathrm{O}(\mathrm{l})$ & 1.0 \\
\hline & \\
\hline
\end{tabular}

Conversion factor of the mercury reaction is dependent upon the mercury to chloride ratio in the HLW melter feed, for example,
$\mathrm{CF}=0.833$
$=0.559$
if $\operatorname{Hg}+2(1, \mathrm{~s}) / \mathrm{Cl}-(1, \mathrm{~s}) \leq 0.1$
$=0.286$
if $0.1<\mathrm{Hg}+2(1, \mathrm{~s}) / \mathrm{Cl}-(1, \mathrm{~s}) \leq 0.5$
if $\mathrm{Hg}+2(1, \mathrm{~s}) / \mathrm{Cl}-(1, \mathrm{~s})>0.5$

The remaining gaseous mercury continues on as "Hg" to HOP-WESP-00001/2. The SBS split values for $\mathrm{Hg}+2(1, \mathrm{~s})$ are set to zero.

The production of $\mathrm{NH}_{3}(\mathrm{~g})$ from the melter is dependent upon the $\mathrm{pH}$ value at the SBS at the end of the above reactions. Initially, the multiplier $\mathrm{M}$ of the stoichiometric coefficients for Eq. 54 and Eq. 55 is set to 1.0. The $\mathrm{M}$ value is increased if $\mathrm{pH}$ at the $\mathrm{SBS}$ is below 7.0 and is decreased if $\mathrm{pH}$ is above 7.0. This provides the means for achieving a $\mathrm{pH} 7.0$ at the SBS by adjusting the production of $\mathrm{NH}_{3}$ at the melter. 
Due to the delay effect of the above modeling approach, the following chemical reactions are used to provide further adjustment.

if $\mathrm{pH}<7.0$ then

\begin{tabular}{|l|l|}
\hline $10 \mathrm{H}+(\mathrm{l})+\mathrm{NO} 3-(\mathrm{l}) \rightarrow \mathrm{NH} 4+(\mathrm{l})+3 \mathrm{H} 2 \mathrm{O}(\mathrm{l})$ & $\begin{array}{l}\text { until } \mathrm{H}+\text { or NO3- are } \\
\text { consumed }\end{array}$ \\
\hline $8 \mathrm{H}+(\mathrm{l})+\mathrm{NO} 2(\mathrm{l}) \rightarrow \mathrm{NH} 4+(\mathrm{l})+2 \mathrm{H} 2 \mathrm{O}(\mathrm{l})$ & $\begin{array}{l}\text { until } \mathrm{H}+\text { or NO2- are } \\
\text { consumed }\end{array}$ \\
\hline
\end{tabular}

if $\mathrm{pH}>7.0$ then

$\mathrm{NH} 4+(\mathrm{l})+2 \mathrm{H} 2 \mathrm{O} \rightarrow \mathrm{NO} 2-(\mathrm{l})+8 \mathrm{H}+$

until $\mathrm{pH}=7.0$ or $\mathrm{NH} 4+$

is consumed

It shall be noted that the $\mathrm{pH}$ control measures above are a modeling technique to approximate the experimental data, which indicated a near $\mathrm{pH}$ neutral environment in the SBS.

Water is condensed or evaporated based on the mole fraction of $\mathrm{H}_{2} \mathrm{O}$ in the offgas stream exiting the HLW SBS. The goal is to have a mole fraction of $0.123 \mathrm{H}_{2} \mathrm{O}(\mathrm{g})$ in the offgas stream. When feed is unavailable to the melter, water vapor entering the SBS is dramatically reduced causing the water in SBS to evaporate. Under this circumstance, the SBS water chem-add will be utilized to replenish the scrubber column. Tritium (3-H) and peroxide $\left(\mathrm{H}_{2} \mathrm{O}_{2}\right)$ follow water, e.g., splits are dynamically calculated in every time step in accordance with water condensation/evaporation.

The SBS volume is recalculated after the reactions. HOP-SCB-00001 transfers the offgas to HOP-WESP-00001 while HOP-SCB-00002 to HOP-WESP-00002. A SBS will accumulate condensate until either the set volume or the 24-hour maximum accumulation time has been reached. The SBS discharges the condensate to RLD-VSL-00007 if the vessel is ready to receive, or continue to accumulate while waiting for RLD-VSL-00007 to become ready. A 1000-gallon headspace (e.g., $\mathrm{V}_{\max }-\mathrm{V}_{\text {set }}$ ) is reserved for each SBS to allow buffer for continuous operations.

\begin{tabular}{|l|l|}
\hline Vessel Tag \# & Equipment Description \\
\hline HOP-WESP-00001 & HLW Wet Electrostatic Precipitator \\
\hline HOP-WESP-00002 & HLW Wet Electrostatic Precipitator \\
\hline
\end{tabular}

Gases that exit each HLW SBS are routed to a corresponding HLW WESP. HOP-WESP-00001 receives the offgas from HOP-SCB-00001 and HOP-WESP-00002 receives the offgas from HOP-SCB-00002. Upon arriving at the WESP, entrained liquid and solid from the SBS are split to the HEME according to the split values given in Table D-21. Demin water is initially pumped to the WESP through a chem-add at a rate of $0.42 \mathrm{gpm}$ which is determined base on a $3 \mathrm{MTG} /$ day IHLW glass production. The demin water rate is proportioned to the melter production rate when HLW ramps up to $3.75 \mathrm{MTG}$ /day in 2023 . In addition, air is added to the WESP to maintain the electrodes are dry and clean. For modeling purpose, this air is combined with the HLW Vessel Vent air stream. The total air injected is $131.5 \mathrm{cfm}$ at $95^{\circ} \mathrm{F}$ and 14.7 psi.

The HLW WESP then performs the following reactions:

\begin{tabular}{|l|l|}
\hline Reactions & Conversion (Fraction) \\
\hline $\mathrm{H} 2 \mathrm{O}(\mathrm{l}) \rightarrow \mathrm{H} 2 \mathrm{O}(\mathrm{g})$ or $\mathrm{H} 2 \mathrm{O}(\mathrm{g}) \rightarrow \mathrm{H} 2 \mathrm{O}(\mathrm{l})$ & $\begin{array}{l}0.162 \text { mole fraction at } \\
\text { outlet }\end{array}$ \\
\hline $\mathrm{HCl}(\mathrm{g}) \rightarrow \mathrm{Cl}-(\mathrm{l})+\mathrm{H}+(\mathrm{l})$ & 0.8947 \\
\hline
\end{tabular}




\begin{tabular}{|l|l|}
\hline Reactions & Conversion (Fraction) \\
\hline $\mathrm{HF}(\mathrm{g}) \rightarrow \mathrm{F}-(\mathrm{l})+\mathrm{H}+(\mathrm{l})$ & 0.5000 \\
\hline $129-\mathrm{I}(\mathrm{g}) \rightarrow 129-\mathrm{I}(\mathrm{l})$ & 0.4118 \\
\hline $\mathrm{SO} 2(\mathrm{~g})+\mathrm{H} 2 \mathrm{O}(\mathrm{l})+0.5 \mathrm{O} 2(\mathrm{~g}) \rightarrow \mathrm{SO} 4-2(\mathrm{l})+2 \mathrm{H}+(\mathrm{l})$ & 0.1667 \\
\hline $\mathrm{P} 2 \mathrm{O} 5(\mathrm{~g})+3 \mathrm{H} 2 \mathrm{O}(\mathrm{l}) \rightarrow 6 \mathrm{H}+(\mathrm{l})+0.5 \mathrm{PO} 4-3(\mathrm{l})+1.5 \mathrm{PO} 4-3(\mathrm{~s})$ & 0.8 \\
\hline $2 \mathrm{NO}(\mathrm{g})+\mathrm{O} 2(\mathrm{~g}) \rightarrow 2 \mathrm{H} 2 \mathrm{O}+4 \mathrm{H}+(\mathrm{l})+2 \mathrm{NO} 3-(\mathrm{l})$ & 0.01 \\
\hline $\mathrm{C} 12 \mathrm{H} 4 \mathrm{Cl} 6(\mathrm{~g}) \rightarrow \mathrm{C} 12 \mathrm{H} 4 \mathrm{Cl} 6(\mathrm{l})$ & 0.0909 \\
\hline $2 \mathrm{~B}+3(\mathrm{~s})+1.5 \mathrm{O} 2(\mathrm{~g})+3 \mathrm{H} 2 \mathrm{O}(\mathrm{l}) \rightarrow 2 \mathrm{H}+(\mathrm{l})+2 \mathrm{~B}+3(\mathrm{l})+4 \mathrm{O}-2(\mathrm{l})+2 \mathrm{H} 2 \mathrm{O}(\mathrm{l})$ & 1.0 \\
\hline $\mathrm{H}+(\mathrm{l})+\mathrm{OH}-(\mathrm{l}) \rightarrow \mathrm{H} 2 \mathrm{O}(\mathrm{l})$ & 1.0 \\
\hline
\end{tabular}

$\mathrm{H}_{2} \mathrm{O}$ is evaporated or condensed based on the mole fraction of $\mathrm{H}_{2} \mathrm{O}$ in the offgas stream exiting the HLW WESP. The goal is to have a mole fraction of $0.162 \mathrm{H}_{2} \mathrm{O}(\mathrm{g})$ in the offgas stream. The gases exit the WESP and pass to the corresponding HLW HEME while the remaining flows to the corresponding HLW SBS Condensate Vessel. HOP-WESP-00001 transfers offgas to HOP-HEME-00001 and transfers condensate to HOP-VSL-00903. HOP-WESP-00002 transfers offgas to HOP-HEME-00002 and transfers condensate to HOP-VSL-00904.

\begin{tabular}{|l|l|}
\hline Vessel Tag \# & Equipment Description \\
\hline HOP-HEME-00001 & High Efficiency Mist Eliminator \\
\hline HOP-HEME-00002 & High Efficiency Mist Eliminator \\
\hline
\end{tabular}

Gases that leave the HLW WESP are combined with an air stream that models the HLW Vessel Vent and is routed to the corresponding HLW HEME. HOP-HEME-00001 receives offgas from HOP-WESP-00001 and HOP-HEME-00002 receives offgas from HOP-WESP-00002. Upon arriving at the HEME, entrained liquid and solid are split to the HLW HEPA according to the split values given in Table D-22. Water is transferred to each HLW HEME through a chem-add at a rate of 0.15 gpm per 3.0 MTG/day melter operations initially. The flowrate is proportioned to the melter production rate during the ramp up in 2023. The HEME then performs the following reactions:

\begin{tabular}{|l|l|}
\hline Reactions & Conversion (Fraction) \\
\hline $\mathrm{H} 2 \mathrm{O}(\mathrm{l}) \rightarrow \mathrm{H} 2 \mathrm{O}(\mathrm{g})$ or $\mathrm{H} 2 \mathrm{O}(\mathrm{g}) \rightarrow \mathrm{H} 2 \mathrm{O}(\mathrm{l})$ & $\begin{array}{l}0.118 \text { mole fraction at } \\
\text { outlet }\end{array}$ \\
\hline $\mathrm{Hg}(\mathrm{g}) \rightarrow \mathrm{Hg}+2(\mathrm{l})$ & Eq. 70 \\
\hline $\mathrm{HCl}(\mathrm{g}) \rightarrow \mathrm{Cl}-(\mathrm{l})+\mathrm{H}+(\mathrm{l})$ & 0.8684 \\
\hline $\mathrm{HF}(\mathrm{g}) \rightarrow \mathrm{F}-(\mathrm{l})+\mathrm{H}+(\mathrm{l})$ & 0.8684 \\
\hline $129-\mathrm{I}(\mathrm{g}) \rightarrow 129-\mathrm{I}(\mathrm{l})$ & 0.0 \\
\hline $\mathrm{SO} 2(\mathrm{~g})+\mathrm{H} 2 \mathrm{O}(\mathrm{l})+0.5 \mathrm{O} 2(\mathrm{~g}) \rightarrow \mathrm{SO} 4-2(\mathrm{l})+2 \mathrm{H}+(\mathrm{l})$ & 0.9845 \\
\hline $\mathrm{P} 2 \mathrm{O} 5(\mathrm{~g})+3 \mathrm{H} 2 \mathrm{O}(\mathrm{l}) \rightarrow 6 \mathrm{H}+(\mathrm{l})+0.5 \mathrm{PO} 4-3(\mathrm{l})+1.5 \mathrm{PO} 4-3(\mathrm{~s})$ & 0.99 \\
\hline $\mathrm{C} 12 \mathrm{H} 4 \mathrm{Cl} 6(\mathrm{~g}) \rightarrow \mathrm{C} 12 \mathrm{H} 4 \mathrm{Cl} 6(\mathrm{l})$ & 0.997959 \\
\hline $2 \mathrm{~B}+3(\mathrm{~s})+1.5 \mathrm{O} 2(\mathrm{~g})+3 \mathrm{H} 2 \mathrm{O}(\mathrm{l}) \rightarrow 2 \mathrm{H}+(\mathrm{l})+2 \mathrm{~B}+3(\mathrm{l})+4 \mathrm{O}-2(\mathrm{l})+2 \mathrm{H} 2 \mathrm{O}(\mathrm{l})$ & 1.0 \\
\hline $\mathrm{H}+(\mathrm{l})+\mathrm{OH}-(\mathrm{l}) \rightarrow \mathrm{H} 2 \mathrm{O}(\mathrm{l})$ & 1.0 \\
\hline
\end{tabular}

Conversion factor of the mercury reaction is dependent upon the mercury to chloride ratio in the HLW melter feed, for example,

$$
\begin{aligned}
\mathrm{CF} & =0.714 \\
& =0.357 \\
& =0.0
\end{aligned}
$$

if $\operatorname{Hg}+2(1, \mathrm{~s}) / \mathrm{Cl}-(1, \mathrm{~s}) \leq 0.1$ if $0.1<\mathrm{Hg}+2(1, \mathrm{~s}) / \mathrm{Cl}-(1, \mathrm{~s}) \leq 0.5$ if $\mathrm{Hg}+2(1, \mathrm{~s}) / \mathrm{Cl}-(1, \mathrm{~s})>0.5$
(Eq. 70) 
The remaining gaseous mercury continues on as " $\mathrm{Hg}$ " to the downstream HOP equipment. The HEME split values for $\mathrm{Hg}+2(1, \mathrm{~s})$ are set to zero.

$\mathrm{H}_{2} \mathrm{O}$ is condensed or evaporated based on the mole fraction of $\mathrm{H}_{2} \mathrm{O}$ in the offgas stream exiting the HLW HEME. The goal is to have a mole fraction of $0.118 \mathrm{H}_{2} \mathrm{O}(\mathrm{g})$ in the offgas stream. The remaining material is captured based on splits shown in Table D-22 and transferred to the SBS Condensate Vessel. The remaining gases exit the HEME and pass to the corresponding HLW HEPA. HOP-HEME-00001 transfers offgas to HOP-HEPA-00001-2-A/B and transfers condensate to HOP-VSL-00903. HOP-HEME-00002 transfers offgas to HOP-HEPA-00007-8-A/B and transfers condensate to HOP-VSL-00904.

\begin{tabular}{|l|l|}
\hline Vessel Tag \# & Equipment Description \\
\hline HOP-HEPA-00001-2-A/B & HLW High Efficiency Particular Air Filter \\
\hline HOP-HEPA-00007-8-A/B & HLW High Efficiency Particular Air Filter \\
\hline
\end{tabular}

A large percentage of the entrained liquid and solid components entering the HLW carbon absorbers is captured. Large percentages of the gas components are also removed. The offgas leaving the HEPA is routed to the downstream carbon absorber. Percent removals by the HEPAs are shown in Table D-23.

\begin{tabular}{|l|l|}
\hline Vessel Tag \# & Equipment Description \\
\hline HOP-ADBR-00001A/B & HLW Carbon Absorber \\
\hline HOP-ADBR-00002A/B & HLW Carbon Absorber \\
\hline
\end{tabular}

The HLW Carbon Absorber captures $99.9 \%$ of mercury from the entrained liquid and solid. It also removes $90 \%$ of the PCB from the gas stream. The offgas leaving the absorber is routed to the downstream Silver Mordenite Absorption Column. Percent removal by the carbon absorber is shown in Table D-24.

\begin{tabular}{|l|l|}
\hline Vessel Tag \# & Equipment Description \\
\hline HOP-ABS-00002 & Silver Mordenite Absorption Column \\
\hline HOP-ABS-00003 & Silver Mordenite Absorption Column \\
\hline
\end{tabular}

Gases enter the Silver Mordenite Absorption Columns from the corresponding HLW carbon absorbers and material is captured based on splits shown in Table D-25. Up to $99.9 \%$ of the 129-I, HCl, and HF gases are removed by the Silver Mordenite Absorption Column. The remaining gases are routed to the

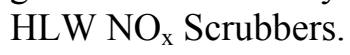

\begin{tabular}{|l|l|}
\hline Vessel Tag \# & Equipment Description \\
\hline HOP-SCR-00001 & HLW NOx Scrubber \\
\hline HOP-SCR-00002 & HLW NOx Scrubber \\
\hline
\end{tabular}

Gases leaving the HLW Silver Mordenite Absorption Columns are routed to the corresponding $\mathrm{HLW} \mathrm{NO}_{\mathrm{x}}$ Scrubber. HOP-SCR-00001 receives offgas from HOP-ADBR-00001A/B and HOP-SCR-00002 receives offgas from HOP-ADBR-00002A/B. The primary purpose of the HLW $\mathrm{NO}_{\mathrm{x}}$ Scrubber is to remove NO and $\mathrm{NO}_{2}$ from the offgas. Pure anhydrous ammonia gas and dilution air are added through chem-adds based on the amount of $\mathrm{NO}, \mathrm{NO}_{2}$, and $\mathrm{NH}_{3}$ entering the $\mathrm{HLW} \mathrm{NO}$ Scrubber, and a 10 parts per million by volume (ppmv) $\mathrm{NH}_{3}$ concentration in the outlet stream. After the $\mathrm{NH}_{3}$ has entered, the following reactions occur: 


\begin{tabular}{|l|l|}
\hline Reactions & Conversion (Fraction) \\
\hline $\mathrm{NH} 3(\mathrm{l}) \rightarrow \mathrm{NH} 3(\mathrm{~g})$ & 1.0 \\
\hline $4 \mathrm{NO}(\mathrm{g})+4 \mathrm{NH} 3(\mathrm{~g})+\mathrm{O} 2(\mathrm{~g}) \rightarrow 4 \mathrm{~N} 2(\mathrm{~g})+6 \mathrm{H} 2 \mathrm{O}(\mathrm{g})$ & 0.95 \\
\hline $2 \mathrm{NO} 2(\mathrm{~g})+4 \mathrm{NH} 3(\mathrm{~g})+\mathrm{O} 2(\mathrm{~g}) \rightarrow 3 \mathrm{~N} 2(\mathrm{~g})+6 \mathrm{H} 2 \mathrm{O}(\mathrm{g})$ & 0.95 \\
\hline $\mathrm{SO} 2(\mathrm{~g})+0.5 \mathrm{O} 2(\mathrm{~g}) \rightarrow \mathrm{SO} 3(\mathrm{~g})$ & 0.95 \\
\hline $\mathrm{C} 2 \mathrm{H} 3 \mathrm{~N}(\mathrm{l}, \mathrm{g})+3.75 \mathrm{O} 2(\mathrm{~g}) \rightarrow 2 \mathrm{CO} 2+1.5 \mathrm{H} 2 \mathrm{O}(\mathrm{g})+\mathrm{NO} 2(\mathrm{~g})$ & 0.757 \\
\hline $\mathrm{C} 12 \mathrm{H} 4 \mathrm{Cl} 6(1, \mathrm{~g})+11.5 \mathrm{O} 2(\mathrm{~g})+\mathrm{H} 2 \mathrm{O}(\mathrm{g}) \rightarrow 12 \mathrm{CO} 2(\mathrm{~g})+6 \mathrm{HCl}(\mathrm{g})$ & 0.95 \\
\hline
\end{tabular}

The HLW $\mathrm{NO}_{x}$ Scrubber then captures components based on splits shown in Table D-26. The HLW $\mathrm{NO}_{x}$ Scrubbers are the final units in HOP before entering the stack. The remaining gases exit out of the HLW $\mathrm{NO}_{\mathrm{x}}$ Scrubbers and through HLW Offgas Stack into the atmosphere.

\begin{tabular}{|l|l|}
\hline Vessel Tag \# & Equipment Description \\
\hline PJV-HEPA-00004-5 & PJV High Efficiency Particular Air Filter \\
\hline
\end{tabular}

The PJV HEPA receives Pulse Jet Ventilation air from HOP-SCB-00001/2 and RLD-VSL-00007. Entrained liquid and solid are removed in accordance with splits shown in Table D-27. Currently, the G2 model simulates dynamic air usage for clean air only (refer to Appendix $G$ for flow rates). Air exiting the PJV HEPA is routed to the HLW PJV Stack.

Figures F-19 and F-20 provide the graphical illustration of the HOP systems where connections with upstream and downstream systems are shown. Table N-1 provides the totalizer files that are used to track the process streams and material flows into and out of the HOP.

\subsubsection{HLW Canister Decontamination Handling (HDH) System}

\begin{tabular}{|l|l|}
\hline Vessel Tag \# & Equipment Description \\
\hline HDH-VSL-00001 & Canister Rinse Bogie Decon Vessel \\
\hline HDH-VSL-00002 & Canister Decontamination Vessel \\
\hline HDH-VSL-00004 & Canister Decontamination Vessel \\
\hline HDH-VSL-00003 & Waste Neutralization Vessel \\
\hline
\end{tabular}

The sequence of HLW canister decontamination is described as follows:

1) The bogie vessel (HDH-VSL-00001) waits until the HLW canister cooling rack has one or more canisters (the rack can hold up to 24 canisters). A canister must wait 24 hours for cooling and welding before it can be decontaminated.

2) A canister is moved to the bogie and waits 26 minutes.

3) The canister is rinsed with 180 -gallon water at $90 \mathrm{gpm}$; the rinse water is drained to the waste neutralization vessel (HDH-VSL-00003) at 80 gpm concurrently. Volume changes in the bogie vessel will not be shown due to the resolution of time interval.

4) The canister is dried for 29 minutes.

5) The canister is rinsed a second time with 180 -gallon water at 90 gpm, the rinse water is drained to HDH-VSL-00003 at 80 gpm concurrently. Volume changes in the bogie vessel will not be shown.

6) The canister is dried for 29 minutes.

7) The dry canister is moved to a decontamination vessel (HDH-VSL-00002 or HDH-VSL-00004). The turnaround time for the bogie vessel to become available for the next canister wash is 10 minutes 
from the end of drying, the time for the decontamination vessel to start decon is 62 minutes from the end of drying. HDH-VSL-00002 and HDH-VSL-00004 are used in an alternating fashion.

8) HDH-VSL-00002 or HDH-VSL-00004 receives 20 gallons of cerium nitrate [0.5M Ce(NO3)3] at 3.2 gpm and 194 gallons of nitric acid (1M HNO3) at $32 \mathrm{gpm}$.

9) HDH-VSL-00003 receives 122 gallons of steam condensate (1,020 lbs) at $0.27 \mathrm{gpm}$.

10) HDH-VSL-00002 or HDH-VSL-00004 waits 540 minutes (90 minutes heating, 360 minutes maintaining, 90 minutes cooling).

11) Decon solution in HDH-VSL-00002 or HDH-VSL-00004 is drained to HDH-VSL-00003 at 48 gpm.

12) Add 5.6 gallons of steam condensate to HDH-VSL-00003 at $1 \mathrm{gpm}$.

13) HDH-VSL-00002 or HDH-00004 waits 28 minutes.

14) HDH-VSL-00002 or HDH-00004 receives 201 gallons of nitric acid (1M HNO3) at 32 gpm.

15) Nitric acid in HDH-VSL-00002 or HDH-00004 is drained to HDH-VSL-00003 at 48 gpm.

16) Add 5.6 gallons of steam condensate to HDH-VSL-00003 at 1 gpm.

17) HDH-VSL-00002 or HDH-VSL-00004 waits 21 minutes.

18) HDH-VSL-00002 or HDH-VSL-00004 receives 200 gallons of demin water at $33 \mathrm{gpm}$.

19) Demin water is drained to HDH-VSL-00003 at $48 \mathrm{gpm}$.

20) HDH-VSL-00002 or HDH-VSL-00004 waits 31 minutes (drying).

21) Add 5.6 gallons of steam condensate to HDH-VSL-00003 at $1 \mathrm{gpm}$.

22) $\mathrm{HDH}-V S L-00003$ receives 0.5 gallons of hydrogen peroxide $(1 \mathrm{M} \mathrm{H} 2 \mathrm{O} 2)$ at $0.033 \mathrm{gpm}$.

23) HDH-VSL-00003 receives 83 gallons of caustic $(5 \mathrm{M} \mathrm{NaOH})$ at $20.75 \mathrm{gpm}$.

24) The canister is moved to the HLW Canister Decon Swabbing Station and waits 235 minutes.

Repeat Steps 1 through 24 for a second HLW canister. Discharge HDH-VSL-00003 to RLD-VSL-00007 after two canisters have been decontaminated. If the canisters on the cooling rack reach the capacity limit, the HLW melter feed pumps will be shut down temporarily.

\subsubsection{Plant Wash and Disposal (PWD) System}

\begin{tabular}{|l|l|}
\hline Vessel Tag \# & Equipment Description \\
\hline PWD-VSL-00015 & Acidic/Alkaline Effluent Vessel \\
\hline PWD-VSL-00016 & Acidic/Alkaline Effluent Vessel \\
\hline
\end{tabular}

PWD-VSL-00015 and PWD-VSL-00016 operate in alternate mode and receive material from the following vessels:

\begin{tabular}{|l|l|}
\hline Vessel Tag \# & Equipment Description \\
\hline UFP-VSL-00002A & Ultrafiltration Feed Vessel \\
\hline UFP-VSL-00002B & Ultrafiltration Feed Vessel \\
\hline CXP-IXC-00001 & Cesium Ion Exchange Column \\
\hline CXP-IXC-00002 & Cesium Ion Exchange Column \\
\hline CXP-IXC-00003 & Cesium Ion Exchange Column \\
\hline
\end{tabular}




\begin{tabular}{|l|l|}
\hline CXP-IXC-00004 & Cesium Ion Exchange Column \\
\hline CNP-DISTC-00001 & Cesium Evaporator Nitric Acid Rectifier \\
\hline
\end{tabular}

Each vessel can only receive material from one source at a time. However, both vessels can be receiving material at the same time if receiving from different sources. If both vessels are available at the time of request, the vessel that is the fullest will be selected. PWD-VSL-00015 and PWD-VSL-00016 will receive until the set volume ( 82,148 gallons) is reached. A 5,000-gallon headspace is reversed in each vessel for potential $\mathrm{pH}$ adjustment operations. If the $\mathrm{pH}$ is less than 12.0 , a calculated volume of $19 \mathrm{M}$ $\mathrm{NapOH}$ is added through a chem-add to the vessel until the $\mathrm{pH}$ reaches 12.0. The vessel then becomes available to send material to one of the two Waste Feed Evaporator Feed Vessels (FEP-VSL-00017A or FEP-VSL-00017B), depending on their availability.

\begin{tabular}{|l|l|}
\hline Vessel Tag \# & Equipment Description \\
\hline PWD-VSL-00033 & Ultimate Overflow Vessel \\
\hline
\end{tabular}

The Ultimate Overflow Vessel receives line flushes and drains from pretreatment and ultrafiltration recirculation loop flush. It also receives inhibited water from post-transfer line flushes from the Tank Farm. A contingent volume is set between the lower set point (10,386 gallons) and the upper set point (14,386 gallons). The vessel can only receive when its volume is below the lower set point. The vessel can receive and discharge when its volume is within the contingency zone. PWD-VSL-00033 can only be discharged when its volume is above the upper set point. When the vessel has met its discharge conditions, it empties its contents into PWD-VSL-00044.

\begin{tabular}{|l|l|}
\hline Vessel Tag \# & Equipment Description \\
\hline PWD-VSL-00043 & HLW Effluent Transfer Vessel \\
\hline
\end{tabular}

PWD-VSL-00043 receives neutralized HLW recycle from RLD-VSL-00007, demister solution from PJVVSL-00001, and inhibited water for post-transfer line flushes from the Tank Farm. A contingent volume is set between the lower set point (10,301 gallons) and the upper set point (14,301 gallons). The vessel can only receive when its volume is below the lower set point. The vessel can receive and discharge when its volume is within the contingency zone. PWD-VSL-00033 can only be discharged when its volume is above the upper set point. When the vessel has met its discharge conditions, it empties its contents into PWD-VSL-00044.

\begin{tabular}{|l|l|}
\hline Vessel Tag \# & Equipment Description \\
\hline PWD-VSL-00044 & Plant Wash Vessel \\
\hline
\end{tabular}

The Plant Wash Vessel receives material from PWD-VSL-00033, PWD-VSL-00043, PVP-SCB-00002, and PVP-VSL-00001. When the vessel has reached its setpoint level, the vessel performs the following reactions:

\begin{tabular}{|l|l|}
\hline Reaction & $\begin{array}{l}\text { Conversion } \\
\text { (Fraction) }\end{array}$ \\
\hline $\mathrm{H} 2 \mathrm{CO} 3(\mathrm{l}) \rightarrow \mathrm{CO} 3-2(\mathrm{l})+2 \mathrm{H}+(\mathrm{l})$ & 1.0 \\
\hline $\mathrm{HCO} 3-(\mathrm{l}) \rightarrow \mathrm{CO} 3-2(\mathrm{l})+\mathrm{H}+(\mathrm{l})$ & 1.0 \\
\hline $\mathrm{NH} 4+(\mathrm{l}) \rightarrow \mathrm{NH} 3(\mathrm{l})+\mathrm{H}+(\mathrm{l})$ & 1.0 \\
\hline $\mathrm{Hg}+2(\mathrm{l})+2 \mathrm{OH}-(\mathrm{l}) \rightarrow \mathrm{Hg}+2(\mathrm{~s})+\mathrm{O}-2(\mathrm{~s})+\mathrm{H} 2 \mathrm{O}($ Bound $)(\mathrm{s})$ & 1.0 \\
\hline $\mathrm{H}+(\mathrm{l})+\mathrm{OH}-(\mathrm{l}) \rightarrow \mathrm{H} 2 \mathrm{O}(\mathrm{l})$ & 1.0 \\
\hline
\end{tabular}


PWD-VSL-00044 will receive until the set volume (68,644 gallons) is reached. A 5,000-gallon headspace is reversed in the vessel for potential $\mathrm{pH}$ adjustment operation. If the $\mathrm{pH}$ is less than 12.0, a calculated volume of $19 \mathrm{M} \mathrm{NapOH}$ is added through a chem-add to the vessel until the $\mathrm{pH}$ reaches 12.0. The vessel then becomes available to send material to one of the two Waste Feed Evaporator Feed Vessels (FEP-VSL-00017A or FEP-VSL-00017B), depending on their availability.

Figure F-7 provides a graphical illustration of the PWD system where connections with upstream and downstream systems are shown. Table N-1 provides the totalizer files that are used to track the process streams and material flows into and out of the PWD.

\subsubsection{Glass Formers Reagent (GFR) System}

Glass formers are minerals that are added to waste batches that, when melted, produce glass. Each batch of waste is evaluated based on composition and a recipe of glass formers is generated that will produce an optimized volume of glass in the melters. with the appropriate waste loadings. The names and chemical formulas of the glass formers are shown in Table 16.

Table $16 \quad$ Glass Former Minerals

\begin{tabular}{|l|l|}
\hline Names & Chemical Formulas \\
\hline Borax & $\mathrm{Na}_{2} \mathrm{~B}_{4} \mathrm{O}_{7} 10 \mathrm{H}_{2} \mathrm{O}$ \\
\hline Boric Acid & $\mathrm{H}_{3} \mathrm{BO}_{3}$ \\
\hline Iron Oxide & $\mathrm{Fe}_{2} \mathrm{O}_{3}$ \\
\hline Kyanite & $\mathrm{Al}_{2} \mathrm{SiO}_{5}$ \\
\hline Lithium Carbonate & $\mathrm{Li}_{2} \mathrm{CO}_{3}$ \\
\hline Olivine & $\mathrm{Mg}_{2} \mathrm{SiO}_{4} \mathrm{Fe}_{2} \mathrm{SiO}_{4}$ \\
\hline Rutile & $\mathrm{TiO}_{2}$ \\
\hline Silica & $\mathrm{SiO}_{2}$ \\
\hline Soda Ash & $\mathrm{Na}_{2} \mathrm{CO}_{3}$ \\
\hline Sugar & $\mathrm{C}_{12} \mathrm{H}_{2} \mathrm{O}_{11}$ \\
\hline Wollastonite & $\mathrm{CaSiO}$ \\
\hline Zinc Oxide & $\mathrm{ZnO}_{3}$ \\
\hline Zircon Sand & $\mathrm{ZrSiO}_{4}$ \\
\hline
\end{tabular}

Glass former quantities in the oxide form are calculated using the LAW and HLW glass models discussed in Sections 4.8.13 and 4.8.17, respectively, and in the BARD. However, minerals equivalent to the calculated oxide glass formers are actually transferred to the MFPVs. These minerals contain the glass former oxides, impurities, and water moisture. Table 17 shows the makeup of the mineral glass formers.

For most glass formers, the moisture contents are partitioned into detachable water and non-detachable water in a 50:50 ratio. The $\mathrm{H} 2 \mathrm{O}$ (Bound) in Table 16 has combined the non-detachable portion of moisture with $\mathrm{H} 2 \mathrm{O}$ (Bound) attached to the minerals. The $\mathrm{H} 2 \mathrm{O}$ (Bound) acts like a solid and will contribute to weight percent solid calculations and solid volume calculations. Upon arriving at an MFPV, the detachable portion of moisture will be removed from the oxide array and added to the liquid array that contributes to the rheology water calculations and liquid volume calculations. The $\mathrm{H} 2 \mathrm{O}$ (Bound) that remains in the oxide array is vaporized in the melter. 


\section{Table 17 Makeup of Mineral Glass Formers}

\begin{tabular}{|c|c|c|c|c|c|c|c|c|c|c|c|c|c|}
\hline $\begin{array}{l}\text { Oxide } \\
\text { Array }\end{array}$ & Borax & $\begin{array}{r}\text { Boric } \\
\text { Acid } \\
\end{array}$ & $\begin{array}{r}\text { Iron } \\
\text { Oxide }\end{array}$ & Kyanite & \begin{tabular}{|r|}
$\begin{array}{r}\text { Lithium } \\
\text { Carbonate }\end{array}$ \\
\end{tabular} & Olivine & Rutile & Silica & Soda Ash & Sugar & Wollastonite & $\begin{array}{r}\text { Zinc } \\
\text { Oxide } \\
\end{array}$ & $\begin{array}{r}\text { Zircon } \\
\text { Sand } \\
\end{array}$ \\
\hline $\mathbf{A c}_{2} \mathbf{O}_{3}$ & 0 & 0 & 0 & 0 & 0 & 0 & 0 & 0 & 0 & 0 & 0 & 0 & 0 \\
\hline $\mathrm{Ag}_{2} \mathrm{O}$ & 0 & 0 & 0 & 0 & 0 & 0 & 0 & 0 & 0 & 0 & 0 & 0 & 0 \\
\hline $\mathrm{Al}_{2} \mathrm{O}_{3}$ & 0 & 0 & 15,000 & 570,000 & 0 & 1,900 & 5,000 & 1,350 & 0 & 0 & 2,000 & 0 & 2,500 \\
\hline $\mathrm{Am}_{2} \mathrm{O}_{3}$ & 0 & 0 & 0 & 0 & 0 & 0 & 0 & 0 & 0 & 0 & 0 & 0 & 0 \\
\hline $\mathbf{A s}_{2} \mathbf{O}_{5}$ & 0 & 0 & 0 & 0 & 0 & 0 & 0 & 0 & 0 & 0 & 0 & 0 & 0 \\
\hline $\mathbf{B}_{2} \mathbf{O}_{3}$ & 375,000 & 565,300 & 0 & 0 & 0 & 0 & 0 & 0 & 0 & 0 & 0 & 0 & 0 \\
\hline BaO & 0 & 0 & 0 & 0 & 0 & 0 & 0 & 0 & 0 & 0 & 0 & 0 & 0 \\
\hline $\mathrm{BeO}$ & 0 & 0 & 0 & 0 & 0 & 0 & 0 & 0 & 0 & 0 & 0 & 0 & 0 \\
\hline $\mathbf{B i}_{2} \mathbf{O}_{3}$ & 0 & 0 & 0 & 0 & 0 & 0 & 0 & 0 & 0 & 0 & 0 & 0 & 0 \\
\hline $\mathrm{CaO}$ & 0 & 0 & 400 & 400 & 14 & 200 & 0 & 80 & 49 & 0 & 475,000 & 0 & 0 \\
\hline CdO & 0 & 0 & 0 & 0 & 0 & 0 & 0 & 0 & 0 & 0 & 0 & 50 & 0 \\
\hline $\mathrm{CeO}_{2}$ & 0 & 0 & 0 & 0 & 0 & 0 & 0 & 0 & 0 & 0 & 0 & 0 & 0 \\
\hline $\mathrm{Cl}$ & 700 & 18 & 0 & 0 & 100 & 0 & 0 & 0 & 182 & 0 & 0 & 0 & 0 \\
\hline $\mathrm{Cm}_{2} \mathrm{O}_{3}$ & 0 & 0 & 0 & 0 & 0 & 0 & 0 & 0 & 0 & 0 & 0 & 0 & 0 \\
\hline $\mathrm{Co}_{2} \mathrm{O}_{3}$ & 0 & 0 & 0 & 0 & 0 & 0 & 0 & 0 & 0 & 0 & 0 & 0 & 0 \\
\hline $\mathrm{Cr}_{2} \mathrm{O}_{3}$ & 0 & 0 & 0 & 0 & 0 & 1,300 & 1,600 & 0 & 0 & 0 & 0 & 0 & 0 \\
\hline $\mathrm{Cs}_{2} \mathrm{O}$ & 0 & 0 & 0 & 0 & 0 & 0 & 0 & 0 & 0 & 0 & 0 & 0 & 0 \\
\hline $\mathrm{CuO}$ & 0 & 0 & 0 & 0 & 0 & 0 & 0 & 0 & 0 & 0 & 0 & 0 & 0 \\
\hline $\mathrm{Dy}_{2} \mathrm{O}_{3}$ & 0 & 0 & 0 & 0 & 0 & 0 & 0 & 0 & 0 & 0 & 0 & 0 & 0 \\
\hline $\mathrm{Eu}_{2} \mathrm{O}_{3}$ & 0 & 0 & 0 & 0 & 0 & 0 & 0 & 0 & 0 & 0 & 0 & 0 & 0 \\
\hline $\mathbf{F}$ & 0 & 0 & 0 & 0 & 0 & 0 & 0 & 0 & 0 & 0 & 0 & 0 & 0 \\
\hline $\mathrm{Fe}_{2} \mathrm{O}_{3}$ & 43 & 9 & 965,373 & 7,100 & 400 & 76,800 & 7,000 & 160 & 3 & 0 & 4,000 & 10 & 750 \\
\hline HgO & 0 & 0 & 0 & 0 & 0 & 0 & 0 & 0 & 0 & 0 & 0 & 0 & 0 \\
\hline $\mathbf{K}_{2} \mathbf{O}$ & 0 & 0 & 0 & 700 & 0 & 100 & 0 & 170 & 0 & 0 & 0 & 0 & 0 \\
\hline $\mathrm{La}_{2} \mathrm{O}_{3}$ & 0 & 0 & 0 & 0 & 0 & 0 & 0 & 0 & 0 & 0 & 0 & 0 & 0 \\
\hline $\mathbf{L i}_{2} \mathbf{O}$ & 0 & 0 & 0 & 0 & 402,000 & 0 & 0 & 0 & 0 & 0 & 0 & 0 & 0 \\
\hline
\end{tabular}


24590-WTP-MDD-PR-01-002, Rev 12

Dynamic (G2) Model Design Document

\begin{tabular}{|c|c|c|c|c|c|c|c|c|c|c|c|c|c|}
\hline $\begin{array}{l}\text { Oxide } \\
\text { Array }\end{array}$ & Borax & $\begin{array}{r}\text { Boric } \\
\text { Acid } \\
\end{array}$ & $\begin{array}{r}\text { Iron } \\
\text { Oxide }\end{array}$ & Kyanite & $\begin{array}{r}\text { Lithium } \\
\text { Carbonate }\end{array}$ & Olivine & Rutile & Silica & Soda Ash & Sugar & Wollastonite & $\begin{array}{r}\text { Zinc } \\
\text { Oxide }\end{array}$ & $\begin{array}{r}\text { Zircon } \\
\text { Sand } \\
\end{array}$ \\
\hline MgO & 0 & 0 & 1,000 & 400 & 995 & 480,100 & 0 & 80 & 41 & 0 & 1,000 & 0 & 0 \\
\hline $\mathrm{MnO}_{2}$ & 0 & 0 & 0 & 0 & 0 & 0 & 0 & 0 & 0 & 0 & 0 & 0 & 0 \\
\hline $\mathrm{MoO}_{3}$ & 0 & 0 & 0 & 0 & 0 & 0 & 0 & 0 & 0 & 0 & 0 & 0 & 0 \\
\hline $\mathrm{Na}_{2} \mathrm{O}$ & 0 & 0 & 0 & 0 & 0 & 0 & 0 & 0 & 0 & 0 & 0 & 0 & 0 \\
\hline $\mathrm{Nb}_{2} \mathbf{O}_{5}$ & 0 & 0 & 0 & 0 & 0 & 0 & 4,000 & 0 & 0 & 0 & 0 & 0 & 0 \\
\hline $\mathrm{Nd}_{2} \mathrm{O}_{3}$ & 0 & 0 & 0 & 0 & 0 & 0 & 0 & 0 & 0 & 0 & 0 & 0 & 0 \\
\hline $\mathrm{NiO}$ & 0 & 0 & 0 & 0 & 0 & 3,700 & 0 & 0 & 0 & 0 & 0 & 0 & 0 \\
\hline $\mathrm{NpO}_{2}$ & 0 & 0 & 0 & 0 & 0 & 0 & 0 & 0 & 0 & 0 & 0 & 0 & 0 \\
\hline $\mathbf{P}_{2} \mathbf{O}_{5}$ & 0 & 0 & 2,635 & 0 & 0 & 0 & 458 & 0 & 0 & 0 & 0 & 0 & 0 \\
\hline PbO & 0 & 0 & 0 & 0 & 0 & 0 & 0 & 0 & 0 & 0 & 0 & 10 & 0 \\
\hline PdO & 0 & 0 & 0 & 0 & 0 & 0 & 0 & 0 & 0 & 0 & 0 & 0 & 0 \\
\hline $\mathbf{P r}_{2} \mathbf{O}_{3}$ & 0 & 0 & 0 & 0 & 0 & 0 & 0 & 0 & 0 & 0 & 0 & 0 & 0 \\
\hline $\mathrm{PuO}_{2}$ & 0 & 0 & 0 & 0 & 0 & 0 & 0 & 0 & 0 & 0 & 0 & 0 & 0 \\
\hline $\mathbf{R} \mathbf{b}_{2} \mathbf{O}$ & 0 & 0 & 0 & 0 & 0 & 0 & 0 & 0 & 0 & 0 & 0 & 0 & 0 \\
\hline $\mathbf{R e}_{2} \mathbf{O}_{3}$ & 0 & 0 & 0 & 0 & 0 & 0 & 0 & 0 & 0 & 0 & 0 & 0 & 0 \\
\hline $\mathbf{R h}_{2} \mathbf{O}_{3}$ & 0 & 0 & 0 & 0 & 0 & 0 & 0 & 0 & 0 & 0 & 0 & 0 & 0 \\
\hline $\mathrm{Ru}_{2} \mathrm{O}_{3}$ & 0 & 0 & 0 & 0 & 0 & 0 & 0 & 0 & 0 & 0 & 0 & 0 & 0 \\
\hline $\mathrm{Sb}_{2} \mathrm{O}_{3}$ & 0 & 0 & 0 & 0 & 0 & 0 & 0 & 0 & 0 & 0 & 0 & 0 & 0 \\
\hline $\mathrm{SeO}_{2}$ & 0 & 0 & 0 & 0 & 0 & 0 & 0 & 0 & 0 & 0 & 0 & 0 & 0 \\
\hline $\mathrm{SiO}_{2}$ & 0 & 0 & 13,500 & 410,500 & 0 & 435,700 & 22,000 & 998,060 & 0 & 0 & 516,687 & 0 & 340,250 \\
\hline $\mathrm{Sm}_{2} \mathrm{O}_{3}$ & 0 & 0 & 0 & 0 & 0 & 0 & 0 & 0 & 0 & 0 & 0 & 0 & 0 \\
\hline $\mathrm{SnO}_{2}$ & 0 & 0 & 0 & 0 & 0 & 0 & 0 & 0 & 0 & 0 & 0 & 0 & 0 \\
\hline $\mathrm{SO}_{3}$ & 500 & 292 & 799 & 0 & 33 & 0 & 250 & 0 & 56 & 0 & 0 & 0 & 0 \\
\hline SrO & 0 & 0 & 0 & 0 & 0 & 0 & 0 & 0 & 0 & 0 & 0 & 0 & 0 \\
\hline $\mathbf{T a}_{2} \mathbf{O}_{5}$ & 0 & 0 & 0 & 0 & 0 & 0 & 0 & 0 & 0 & 0 & 0 & 0 & 0 \\
\hline $\mathbf{T c}_{2} \mathbf{O}_{7}$ & 0 & 0 & 0 & 0 & 0 & 0 & 0 & 0 & 0 & 0 & 0 & 0 & 0 \\
\hline $\mathrm{TeO}_{2}$ & 0 & 0 & 0 & 0 & 0 & 0 & 0 & 0 & 0 & 0 & 0 & 0 & 0 \\
\hline $\mathrm{ThO}_{2}$ & 0 & 0 & 0 & 0 & 0 & 0 & 0 & 0 & 0 & 0 & 0 & 0 & 450 \\
\hline
\end{tabular}


24590-WTP-MDD-PR-01-002, Rev 12

Dynamic (G2) Model Design Document

\begin{tabular}{|c|c|c|c|c|c|c|c|c|c|c|c|c|c|}
\hline $\begin{array}{l}\text { Oxide } \\
\text { Array }\end{array}$ & Borax & $\begin{array}{r}\text { Boric } \\
\text { Acid } \\
\end{array}$ & $\begin{array}{r}\text { Iron } \\
\text { Oxide }\end{array}$ & Kyanite & $\begin{array}{r}\text { Lithium } \\
\text { Carbonate }\end{array}$ & Olivine & Rutile & Silica & Soda Ash & Sugar & Wollastonite & $\begin{array}{r}\text { Zinc } \\
\text { Oxide }\end{array}$ & $\begin{array}{r}\text { Zircon } \\
\text { Sand } \\
\end{array}$ \\
\hline $\mathrm{TiO}_{2}$ & 0 & 0 & 0 & 10,500 & 0 & 0 & 936,192 & 80 & 0 & 0 & 200 & 0 & 1,050 \\
\hline $\mathrm{TI}_{2} \mathrm{O}_{3}$ & 0 & 0 & 0 & 0 & 0 & 0 & 0 & 0 & 0 & 0 & 0 & 0 & 0 \\
\hline $\mathbf{U}_{3} \mathbf{O}_{8}$ & 0 & 0 & 0 & 0 & 0 & 0 & 0 & 0 & 0 & 0 & 0 & 0 & 0 \\
\hline $\mathbf{V}_{2} \mathbf{O}_{5}$ & 0 & 0 & 0 & 0 & 0 & 0 & 4,500 & 0 & 0 & 0 & 0 & 0 & 0 \\
\hline $\mathrm{WO}_{3}$ & 0 & 0 & 0 & 0 & 0 & 0 & 0 & 0 & 0 & 0 & 0 & 0 & 0 \\
\hline $\mathbf{Y}_{2} \mathbf{O}_{3}$ & 0 & 0 & 0 & 0 & 0 & 0 & 0 & 0 & 0 & 0 & 0 & 0 & 0 \\
\hline $\mathrm{ZnO}$ & 0 & 0 & 0 & 0 & 0 & 0 & 0 & 0 & 0 & 0 & 0 & 999,730 & 0 \\
\hline $\mathrm{ZrO}_{2}$ & 0 & 0 & 0 & 0 & 0 & 0 & 19,000 & 0 & 0 & 0 & 0 & 0 & 589,500 \\
\hline $\mathrm{CO}_{2}$ & 0 & 0 & 0 & 0 & 596,454 & 0 & 0 & 0 & 414,300 & 0 & 0 & 0 & 0 \\
\hline $\mathrm{H}_{2} \mathbf{O}(\mathbf{b})$ & 486,757 & 464,382 & 30,000 & 30,000 & 30,000 & 30,000 & 30,000 & 30,000 & 30,200 & 30,000 & 30,000 & 30,000 & 30,000 \\
\hline $\mathrm{H}_{2} \mathrm{O}$ & 30,000 & 30,000 & 30,000 & 30,000 & 30,000 & 30,000 & 30,000 & 30,000 & 30,000 & 30,000 & 30,000 & 30,000 & 30,000 \\
\hline $\mathrm{HfO}_{2}$ & 0 & 0 & 0 & 0 & 0 & 0 & 0 & 0 & 0 & 0 & 0 & 0 & 65,500 \\
\hline $\mathbf{M n p}_{2} \mathrm{O}_{3}$ & 0 & 0 & 1,293 & 0 & 0 & 0 & 0 & 0 & 0 & 0 & 1,113 & 0 & 0 \\
\hline $\mathrm{Nap}_{2} \mathrm{O}$ & 167,000 & 0 & 0 & 400 & 4 & 200 & 0 & 20 & 585,169 & 0 & 0 & 200 & 0 \\
\hline 129-I & 0 & 0 & 0 & 0 & 0 & 0 & 0 & 0 & 0 & 0 & 0 & 0 & 0 \\
\hline Pa2O3 & 0 & 0 & 0 & 0 & 0 & 0 & 0 & 0 & 0 & 0 & 0 & 0 & 0 \\
\hline RaO & 0 & 0 & 0 & 0 & 0 & 0 & 0 & 0 & 0 & 0 & 0 & 0 & 0 \\
\hline $\begin{array}{l}\text { C12H22O } \\
11\end{array}$ & 0 & 0 & 0 & 0 & 0 & 0 & 0 & 0 & 0 & $1,000,000$ & 0 & 0 & 0 \\
\hline Ox5 & 0 & 0 & 0 & 0 & 0 & 0 & 0 & 0 & 0 & 0 & 0 & 0 & 0 \\
\hline Total $^{(1)}$ & $1,060,000$ & $1,060,000$ & $1,060,000$ & $1,060,000$ & $1,060,000$ & $1,060,000$ & $1,060,000$ & $1,060,000$ & $1,060,000$ & $1,060,000$ & $1,060,000$ & $1,060,000$ & $1,060,000$ \\
\hline
\end{tabular}

Note:

${ }^{(1)}$ Unit is in parts per million (ppm) excluding the detachable and non-detachable moisture. 
The glass former reagent system modeled includes mineral stockpiles, glass former trucks, storage silos, weigh hoppers, transporters, blending silos, mixers, and dusting water as shown in Figure F-23. A glass former equipment class is created with attributes commonly used for logical operations and mass balance calculations. A number of sub-classes are also created to accommodate the required equipment. The equipment class hierarchy is illustrated as follows (see Figure 1 for a complete WTP equipment class hierarchy):

- Glass Former Equipment

- Mineral Chem. Add

- Glass Former Truck

- Silo

- Transporter

- Glass Former Mixer

Mineral Chem. Adds are used to represent the glass former stockpiles shown in Table 18 where the names and bulk densities of the minerals are specified. It is assumed that glass former minerals are available in unlimited quantities at any time as needed.

Table 18 Names and Bulk Densities of Glass Former Stockpiles

\begin{tabular}{|l|c|}
\hline Stockpile Name & Bulk Density $\left(\mathbf{l b s} / \mathbf{f t}^{\mathbf{3}}\right)$ \\
\hline Silica & 49.6 \\
\hline Wollastonite & 46.4 \\
\hline Kyanite & 56.1 \\
\hline Boric Acid & 54.6 \\
\hline Sugar & 52.6 \\
\hline Lithium Carbonate & 53.1 \\
\hline Zinc Oxide & 33.8 \\
\hline Soda Ash & 64.2 \\
\hline Borax & 48.0 \\
\hline Olivine & 89.3 \\
\hline Rutile & 91.0 \\
\hline Zircon Sand & 96.0 \\
\hline Iron Oxide & 88.9 \\
\hline
\end{tabular}

Trucks are used to transport glass formers from stockpiles to storage silos. A convoy of trucks can be used for loading and hauling but only two can be unloading at the same time. It is outside the scope of this model to simulate the loading and hauling of the glass formers from the stockpiles and therefore it is assumed that a truckload of minerals is available at the truck lane entrance for unloading at any time. Because only two trucks can be unloading at the same time, a truck lane is dedicated for Systems 100, 200, and 500 while another truck lane is dedicated for Systems 300 and 400 (see Figure F-23 for details).

After a delivery order is received, a truck with the requested minerals arrives at the truck lane entrance; moves to the requesting silo; docks to the silo's truck unload line; and starts unloading. These steps are assumed to take 30-minutes. Then the mass is transferred to the silo from the truck at a rate of 500 $\mathrm{lbs} / \mathrm{min}$. A truck has a fixed capacity of $40,000 \mathrm{lbs}$ although its holding volume varies depending on the bulk density of minerals. The truck undocks from the silo and leaves after it is completely emptied. These steps are assumed to take 30-minutes. The next truck can enter the truck lane after the previous one exits. 
Some attributes of the truck class are shown in Table 19 where descriptions for these attributes are also provided.

Table 19 Attributes of the Truck Class

\begin{tabular}{|l|l|}
\hline Attributes & Description \\
\hline Trip No. & Keeps track of the total number of trips. \\
\hline Mineral Type & $\begin{array}{l}\text { Varies, is used in combination with trip no. so that glass former } \\
\text { purchasing orders vs. time information can be collected. }\end{array}$ \\
\hline Capacity $(\mathrm{lbs})$ & $40,000 \mathrm{lbs}$ per load, volume $\left(\mathrm{ft}^{3}\right)=$ capacity / bulk density \\
\hline Current Weight $(\mathrm{lbs})$ & Equals to $($ capacity - flow rate $\times$ time step $) \geq 0.0$ \\
\hline Flow Rate $(\mathrm{lbs} / \mathrm{min})$ & 500 \\
\hline Solid Density $\left(\mathrm{lbs} / \mathrm{ft}^{3}\right)$ & Varies depending on mineral types \\
\hline
\end{tabular}

Storage silos are used to receive, store, and deliver glass formers. The thirteen storage silos are grouped in five systems. Silos of the same system share a weigh hopper and a transporter. A silo will be restocked once more than a truckload of minerals $(40,000 \mathrm{lbs})$ has been consumed. Silos will be restocked in the order in which they are requested. If requests are received from more than one silo at the same time, priority is given to the one that has a lower percent of remaining volume (emptier). A silo can receive and deliver glass formers at the same time. All the storage silos are stocked initially before the plant startup. Table 20 shows the attributes of the silo class and Table 21 summarizes the attribute values of the storage silos.

Table $20 \quad$ Attributes of the Silo Class

\begin{tabular}{|l|l|}
\hline Attributes & Description \\
\hline Minimum Volume $\left(\mathrm{ft}^{3}\right)$ & Heel volume, equals to 0.0 \\
\hline Current Solid Volume $\left(\mathrm{ft}^{3}\right)$ & Remaining volume in silo \\
\hline Set Volume $\left(\mathrm{ft}^{3}\right)$ & $\begin{array}{l}\text { Equals to (max vol. - 40,000 / solid density), rounded lower to } \\
\text { prevent silo overfill. Triggers a purchasing order }\end{array}$ \\
\hline Maximum Volume $\left(\mathrm{ft}^{3}\right)$ & Equals to capacity \\
\hline Flow Rate $\left(\mathrm{ft}^{3} / \mathrm{min}\right)$ & Varies depending on silos \\
\hline Solid Density $\left(\mathrm{lbs} / \mathrm{ft}^{3}\right)$ & Varies depending on mineral types \\
\hline
\end{tabular}

Table 21 Attribute Values of the Storage Silos

\begin{tabular}{|c|c|c|c|c|c|}
\hline Storage Silo & $\begin{array}{l}\text { Alternate } \\
\text { Name }\end{array}$ & $\begin{array}{l}\text { Capacity } \\
\left(\mathrm{ft}^{3}\right)\end{array}$ & $\begin{array}{l}\text { Flow Rate } \\
\left(\mathbf{f t}^{3} / \mathrm{min}\right)\end{array}$ & $\begin{array}{l}\text { Density } \\
\left(\mathbf{l b s} / \mathrm{ft}^{3}\right)\end{array}$ & $\begin{array}{l}\text { Set Volume } \\
\left(\mathbf{f t}^{3}\right)\end{array}$ \\
\hline GFR-TK-00001 & Silica & 8,500 & 5.7 & 49.6 & 7,650 \\
\hline GFR-TK-00010 & Wollastonite & 3,000 & 9 & 46.4 & 2,100 \\
\hline GFR-TK-00008 & Kyanite & 2,175 & 5.88 & 56.1 & 1,450 \\
\hline GFR-TK-00007 & Boric Acid & 3,000 & 10.2 & 54.6 & 2,250 \\
\hline GFR-TK-00011 & Sugar & 1,800 & 7.2 & 52.6 & 1,000 \\
\hline GFR-TK-00006 & $\begin{array}{l}\text { Lithium } \\
\text { Carbonate }\end{array}$ & 2,500 & 6 & 53.1 & 1,700 \\
\hline GFR-TK-00002 & Zinc Oxide & 2,500 & 2.88 & 33.8 & 1,300 \\
\hline GFR-TK-00012 & Soda Ash & 1,500 & 7.62 & 64.2 & 850 \\
\hline GFR-TK-00028 & Borax & 2,150 & 9.18 & 48.0 & 1,300 \\
\hline GFR-TK-00009 & Olivine & 1,000 & 3 & 89.3 & 550 \\
\hline GFR-TK-00003 & Rutile & 1,000 & 0.78 & 91.0 & 550 \\
\hline GFR-TK-00005 & Zircon Sand & 1,000 & 4.68 & 96.0 & 550 \\
\hline GFR-TK-00004 & Iron Oxide & 1,000 & 0.72 & 88.9 & 550 \\
\hline
\end{tabular}


A glass former recipe must be determined for each batch of LAW and HLW wastes to the Melter Feed Preparation Vessels. Glass former recipe determinations are discussed in Section 4.8.13 for LAW and Section 4.8.17 for HLW. After a MFPV receives an entire waste batch, the vessel must wait if the glass formers have not already been prepared.

Glass former requests are processed in the order they are received as the storage silos, weigh hoppers, transporters, and blending silos are shared. However, higher priority will be given to the HLW facilities if requests are received at the same time.

Systems 100 through 500 will operate in parallel, for example, the five weigh hoppers and their associated transporters can receive feeds from storage silos and discharge to the blending silo at the same time. Because they share the same weigh hopper and transporter, silos of the same system (i.e., Systems 200, 300, 400, and 500) will operate in sequence in the order from left to right. Multiple batches might be needed if the required glass former quantity exceeds the capacity of the weigh hopper/transporter. If a weigh hopper is partially filled with a glass former, it will continue to be filled with other required glass formers. Density of the mixture is calculated using the equation below:

$\rho_{\mathrm{m}}=\left(\mathrm{V}_{1} \times \rho_{1}+\mathrm{V}_{2} \times \rho_{2}+\ldots \mathrm{V}_{\mathrm{i}} \times \rho_{\mathrm{i}}\right) /\left(\mathrm{V}_{1}+\mathrm{V}_{2}+\ldots .+\mathrm{V}_{\mathrm{i}}\right)$

Where:

$\mathrm{V} \quad=$ Volume of a glass former mineral $\left(\mathrm{ft}^{3}\right) ;$

$\rho \quad=$ Density of a glass former mineral $\left(\mathrm{lbs} / \mathrm{ft}^{3}\right)$;

Similar to the weigh hoppers, bulk density of the transporters varies depending on the mineral types and mixtures.

Weigh hoppers are derived from the silo equipment class. Transporters also share the same class attributes as silos. Attribute values of the weigh hoppers and their associated transporters are shown in Table 22 and Table 23.

Table 22 Attribute Values of the Weigh Hoppers

\begin{tabular}{|c|c|c|c|c|}
\hline Weigh Hopper & Alternate Name & $\begin{array}{l}\text { Capacity } \\
\left(\mathbf{f t}^{3}\right)\end{array}$ & $\begin{array}{l}\text { Flow Rate } \\
\left(\mathrm{ft}^{3} / \mathrm{min}\right)\end{array}$ & $\begin{array}{l}\text { Density } \\
\left.(\mathbf{l b}) / \mathrm{ft}^{3}\right)\end{array}$ \\
\hline GFR-TK-00013 & System 100 Weigh Hopper & 10 & 163.8 & 49.6 \\
\hline GFR-TK-00016 & System 200 Weigh Hopper & 10 & 129 & Varies \\
\hline GFR-TK-00015 & System 300 Weigh Hopper & 10 & 51.6 & Varies \\
\hline GFR-TK-00018 & System 400 Weigh Hopper & 10 & 136.8 & Varies \\
\hline GFR-TK-00014 & System 500 Weigh Hopper & 3 & 61.2 & Varies \\
\hline
\end{tabular}

Note:

(a) Bulk density varies as the weigh hoppers are used to handle different minerals and their mixtures. 
Table 23 Attribute Values of the Transporters

\begin{tabular}{|c|c|c|c|c|}
\hline Transporter & Alternate Name & $\begin{array}{l}\text { Capacity } \\
\left(\mathbf{f t}^{3}\right)\end{array}$ & $\begin{array}{l}\text { Flow Rate } \\
\left(\mathrm{ft}^{3} / \mathrm{min}\right)\end{array}$ & $\begin{array}{l}\text { Density } \\
\left(\text { lbs/ft }{ }^{3}\right)\end{array}$ \\
\hline GFR-VSL-00002 & System 100 Transporter & 10 & 2.5 & 49.6 \\
\hline GFR-VSL-00003 & System 200 Transporter & 10 & 2.9 & Varies \\
\hline GFR-VSL-00004 & System 300 Transporter & 10 & 2.5 & Varies \\
\hline GFR-VSL-00005 & System 400 Transporter & 10 & 2.5 & Varies \\
\hline GFR-VSL-00006 & System 500 Transporter & 3 & 0.7 & Varies \\
\hline
\end{tabular}

Two blending silos are used to receive and blend the different minerals from the storage silos and their associated weigh hoppers and transporters. GFR-TK-00019 is dedicated for LAW glass formers while GFR-TK-00021 is dedicated for HLW glass formers. A blending silo can receive glass formers from any number of transporters simultaneously. If the volume of a glass former recipe is greater than the capacity of the blending silo, two batch transfers will be made into and out of the blending silo, the following mixer, then to the requesting MFPV. These two batches will come proportionally from the associated storage silos. Blending starts after the entire contents of the glass former recipe are received. Blending will take 15 minutes to complete.

Blending silos are derived from the silo equipment class and therefore posses the same attributes. Table 24 summarizes the attribute values of the LAW and HLW blending silos.

Table $24 \quad$ Attribute Values of the Blending Silos

\begin{tabular}{|c|c|c|c|c|}
\hline $\begin{array}{l}\text { Blending } \\
\text { Hopper }\end{array}$ & Alternate Name & $\begin{array}{l}\text { Capacity } \\
\left(\mathbf{f t}^{3}\right)\end{array}$ & $\begin{array}{l}\text { Flow Rate } \\
\left(\mathrm{ft}^{3} / \mathrm{min}\right)\end{array}$ & $\begin{array}{l}\text { Density } \\
\left(\mathbf{l b s} / \mathbf{f t}^{3}\right)\end{array}$ \\
\hline GFR-TK-00019 & LAW Blending Silo & 470 & 252.7 & Variable \\
\hline GFR-TK-00021 & HLW Blending Silo & 320 & 205.8 & Variable \\
\hline
\end{tabular}

Blended glass formers are delivered to the associated transporters which then transfer the contents to the designated glass former mixers. Transporter GFR-VSL-00007 is associated upstream with the LAW blending silo and downstream with the LAW glass former mixers. Transporter GFR-VSL-00008 is associated upstream with the HLW blending silo and downstream with the HLW glass former mixers. Attribute values of the LAW/HLW transporters are summarized in Table 25.

Table 25 Attribute Values of the LAW/HLW Transporters

\begin{tabular}{|c|c|c|c|c|}
\hline Transporter & Alternate Name & $\begin{array}{l}\text { Capacity } \\
\left(\mathbf{f t}^{3}\right)\end{array}$ & $\begin{array}{l}\text { Flow Rate } \\
\left(\mathbf{f t}^{3} / \mathrm{min}\right)\end{array}$ & $\begin{array}{l}\text { Density } \\
\left(\mathbf{l b s} / \mathbf{f t}^{3}\right)\end{array}$ \\
\hline GFR-VSL-00007 & LAW Transporter & 80 & 7.8 & Variable \\
\hline GFR-VSL-00008 & HLW Transporter & 80 & 5.3 & Variable \\
\hline
\end{tabular}

Four glass former mixers are used to receive, mix, and deliver the finished glass former contents to the dedicated MFPVs. After the entire contents of the LAW/HLW transporters are received, dusting water is added to the mixer, i.e., $4 \mathrm{wt} \%$ for LAW and $5 \mathrm{wt} \%$ for HLW and mixed. The mixing process takes 20 minutes. Each mixer is dedicated to a MFPV, for example, GFR-TK-00022 to LFP-VSL-00003, GFRTK-00023 to LFP-VSL-00001, GFR-TK-00025 to HFP-VSL-00005, and GFR-TK-00031 to HFP-VSL00001, respectively. Once again, it is assumed that the glass former mixers have the capacities to hold the ingredients of any glass former recipes, and if not, the mixers will allow topping off their maximum volumes. Attribute values of the mixers are summarized in Table 26. 
Table 26 Attribute Values of the LAW/HLW Glass Former Mixers

\begin{tabular}{|c|c|c|c|c|}
\hline Transporter & Alternate Name & $\begin{array}{l}\text { Capacity } \\
\left(\mathbf{f t}^{3}\right)\end{array}$ & $\begin{array}{l}\text { Flow Rate } \\
\left(\mathrm{ft}^{3} / \mathrm{min}\right)\end{array}$ & $\begin{array}{l}\text { Density } \\
\left(\text { lbs/ft }{ }^{3}\right)\end{array}$ \\
\hline GFR-TK-00022 & LFP-VSL-00003 Mixer & 470 & 6.5 & Variable \\
\hline GFR-TK-00023 & LFP-VSL-00001 Mixer & 470 & 6.5 & Variable \\
\hline GFR-TK-00025 & HFP-VSL-00005 Mixer & 320 & 4.5 & Variable \\
\hline GFR-TK-00031 & HFP-VSL-00001 Mixer & 320 & 4.5 & Variable \\
\hline
\end{tabular}

Figure F-23 provides a graphical illustration of the GFR system where connections with upstream and downstream systems are shown. Table N-1 provides the totalizer files that are used to track the process streams and material flows into and out of the GFR.

\subsection{Workspace Description}

The model is divided into workspaces. Each workspace can model up to two systems. Table 27 provides a list showing which systems are modeled in each workspace. Screenshots of the workspaces are shown in Appendix F.

Table 27 Workspace Description

\begin{tabular}{|l|l|c|}
\hline \multicolumn{1}{|c|}{ RPP-WTP System } & \multicolumn{1}{c|}{ G2 Model Workspace } & Figure No \\
\hline FRP - Waste Feed Receipt Process System & Waste Feed Receipt & F-3 \\
\hline FEP - Waste Feed Evaporation Process System & Waste Feed Evaporation & F-5 \\
\hline UFP - Ultrafiltration Process System & Ultrafiltration & F-17 \\
\hline $\begin{array}{l}\text { HLP - HLW Lag Storage and Feed Blending Process } \\
\text { System }\end{array}$ & HLW Lag Storage and Feed Blending & F-8 \\
\hline CXP - Cesium Ion Exchange Process System & Cesium Ion Exchange & F-10 \\
\hline CNP - Cesium Nitric Acid Recovery Process System & Cesium Nitric Acid Recovery & F-9 \\
\hline CRP - Cesium Resin Addition Process System & Resin Dewatering Process & F-9 \\
\hline $\begin{array}{l}\text { RDP - Spent Resin Collection and Dewatering Process } \\
\text { System }\end{array}$ & Spent Resin Collection and Dewatering & F-11 \\
\hline TLP - Treated LAW Evaporation Process System & $\begin{array}{l}\text { Treated LAW Evaporation and } \\
\text { Concentrate Storage }\end{array}$ & F-11 \\
\hline TCP - Treated LAW Concentrate Storage Process System & $\begin{array}{l}\text { Treated LAW Evaporation and } \\
\text { Concentrate Storage }\end{array}$ & F-6 \\
\hline PVP - Pretreatment Vessel Vent Process System & Pretreatment Vessel Vent Process & F-7 \\
\hline PWD - Plant Wash and Disposal System & $\begin{array}{l}\text { Plant Wash and Disposal (Recycle)/ } \\
\text { PWD Effluent Collection }\end{array}$ & Throughout \\
\hline Pretreatment Process Reagent Handling System & Modeled by Chem-Adds & F-12 \\
\hline $\begin{array}{l}\text { LCP - LAW Concentrate Receipt Process System } \\
\text { LFP - LAW Melter Feed Process System }\end{array}$ & LAW Melter Feed & $\begin{array}{l}\text { F-13 } \\
\text { L-14 }\end{array}$ \\
\hline $\begin{array}{l}\text { LMP - LAW Melter Process System } \\
\text { LOP - LAW Primary Offgas Process System }\end{array}$ & LAW Melters & F-15 \\
\hline LVP - LAW Secondary Offgas/Vessel Vent Process System & LAW Offgas Treatment System & Not \\
\hline $\begin{array}{l}\text { LAW-RLD - LAW Radioactive Liquid Waste Disposal } \\
\text { System } \\
\text { LAW-NLD - LAW Non-Radioactive Liquid Waste Disposal } \\
\text { System }\end{array}$ & $\begin{array}{l}\text { RLD Process Condensate Collection/ } \\
\text { LAW Liquid Effluent }\end{array}$ & $\begin{array}{l}\text { F-16 } \\
\text { GFR - Glass Formers Reagent System }\end{array}$ \\
\hline \begin{tabular}{l} 
LFH - LAW Container Finishing Handling System \\
\hline
\end{tabular} & Glass Formers & Not modeled by G2 model \\
\hline
\end{tabular}




\begin{tabular}{|l|l|c|}
\hline \multicolumn{1}{|c|}{ RPP-WTP System } & \multicolumn{1}{c|}{ G2 Model Workspace } & Figure No \\
\hline LAW Reagent Handling Systems & Modeled by Chem-Adds & Throughout \\
\hline HFP - HLW Melter Feed Process System & HLW Melter Feed and Melter & F-18 \\
\hline HMP - HLW Melter Process System & HLW Melter Feed and Melter & F-19 \\
& & F-20 \\
\hline HOP - HLW Melter Offgas Treatment Process System & HLW Offgas Treatment & F-19 \\
& & F-20 \\
\hline PJV - Pulse Jet Ventilation & Pretreatment Vessel Vent Process & F-6 \\
\hline HLW-RLD - HLW Radioactive Liquid Waste Disposal & RLD Process Condensate Collection/ & F-22 \\
$\begin{array}{l}\text { System } \\
\text { HLW-NLD - HLW Non-Radioactive Liquid Waste Disposal } \\
\text { System }\end{array}$ & HLW Liquid Effluent & \\
\hline HDH - HLW Canister Decontamination Handling System & HLW Canister Decon & \\
\hline HLW Reagent Handling Systems & Modeled by Chem-Adds & F-21 \\
\hline
\end{tabular}

An exception to the information in Table 27 is the Tank Farms HLW Batch Receipt Vessel HLP-VSL-00022 residing in the workspace Waste Feed Receipt (FRP).

\section{Model Construction}

The G2 model is constructed of many knowledge base files. The KBs contain objects, procedures (codes), and graphical interfaces.

\subsection{Knowledge Base}

G2 applications are stored in American Standard Code for Information Interchange (ASCII) files with a . $\mathrm{kb}$ extension. These files are referred to as $\mathrm{KBs}$, and contain all the information the application needs to run. G2 applications can have a single file or many files. Elements of KBs come in several different forms in the G2 model, including the following:

- Object - An object is a piece of information that contains all related knowledge in one location. In object-oriented terms, an object's data is called its attributes, and an object's operations include rules, methods, and procedures (e.g., a tank object contains all the data that defines the tank and all the operations that the tank can perform).

- Definitions - Definitions describe the common features of objects (e.g., all the tanks have volumes, all pipes have flowrates, and all pumps have pump procedures).

- Operations - Operations are the codes that perform the computation using rules, methods, and procedures.

- Graphical user interface - GUIs enable the user to interact with the application.

A workspace is a two-dimensional area upon which objects and definitions are placed and the GUI is built. There are top-level workspaces and sub-workspaces. The user can move a workspace by dragging it to a desired place within Opened G2-Windows. The user can manipulate workspaces by using the KB Workspace menu, (e.g., to hide, print, lift to top, and drop to bottom). The user can single-right-click the background area with a workspace to obtain the KB Workspace menu. The user can use the lift to top command to make a workspace entirely visible or use the drop to bottom command to bring up a workspace which is covered by the active workspace. A workspace can be removed from G2-Window by using the hide command. 
The programming code used in G2 is object oriented. G2 uses it own type of coding language, which is similar to $\mathrm{C \#}$. The procedures are located within each workspace. A procedure begins with a statement of its title and function. Next, the variables used within the program are listed and the operating logic or control logic of the procedure is given such as other procedures being called, if/then statements, pumping orders, and data reporting functions. The procedures are written in G2 language. G2 language is strongly object oriented and is most similar to Pascal.

\subsection{Computation Environment}

See System Design for Process Modeling (24590-WTP-PL-TE-01-008) for the modeling environment.

\subsection{Inputs and Outputs}

\subsubsection{Input Data}

Because of the object-oriented nature of the model, input data such as those discussed throughout this document are embedded as attributes of the WTP equipment objects. These primarily include tank volumes, flowrates, sample times, splits, and chem-add compositions. Chemical reactions are also programmed in the reaction procedures that are the methods of the equipment objects.

\subsubsection{Output Data}

Output data from the G2 model is stored using Oracle, Version 8i. MatLab is used to extract data from Oracle then convert the data to a form that can be used in Excel spreadsheets. The spreadsheets present the data in a readable fashion for the user. The data can be written at various time intervals of every 6 simulated minutes or greater. Vessel compositions are gathered through the use of a snapshot composition which captures the contents of vessels at specified events, and through totalizing data, which acts as a summation tool for storing the component mass transferred from one piece of equipment to another over a set time interval. The duration over which the totalizer information is packaged together is specified during data processing. The typical duration is set to 30 days.

The Dynamic (G2) Flowsheet Version 7.0 is designed to provide the general data output shown in Table 28.

Table 28 General Data Output

\begin{tabular}{|l|l|}
\hline General Data Output & Description \\
\hline FEP and TLP evaporator concentrate data & $\begin{array}{l}\text { SpG/Na molarity vs. time plots for FEP/TLP evaporator bottoms } \\
\text { in Joint Photographic Experts Group (JPEG) format }\end{array}$ \\
\hline Final screenshot & $\begin{array}{l}\text { Final states of a model run on the GUI are captured by a } \\
\text { screenshot in Bitmap (BMP) format. Critical information on the } \\
\text { captured GUI include WTP mission duration, IHLW canister } \\
\text { and ILAW container counts, and volume of treated LAW sent to } \\
\text { the Supplemental LAW Facility }\end{array}$ \\
\hline Glass property files & $\begin{array}{l}\text { IHLW glass heat load, ILAW glass Cs, Sr, Tc, and TRU } \\
\text { concentrations vs. time plots (JPEG) }\end{array}$ \\
\hline Heel cleaning and dilution files & $\begin{array}{l}\text { Details of the heal cleaning/dilution operations in Comma } \\
\text { Separated Value (CSV) format }\end{array}$ \\
\hline HLP-VSL-00028 conditions & $\begin{array}{l}\text { Volumes and wt\% solids of HLP-VSL-00028 after each } \\
\text { sampling event (CSV) }\end{array}$ \\
\hline HLW glass property data & Details of the IHLW glass formulation in Text (TXT) format \\
\hline
\end{tabular}




\begin{tabular}{|l|l|}
\hline General Data Output & Description \\
\hline Hydrogen generation rate data & HGR vs. time plots for vessels of interest (JPEG) \\
\hline LAW glass property data & Details of the ILAW glass formulation (TXT) \\
\hline Leach caustic addition & $\begin{array}{l}\text { Over process parameters related to caustic leach (CSV) } \\
\text { Diagrams include AFA, Al, C2O4, Cr, Cs137, total mass, total } \\
\text { volume, Fe, H2O, Hg, I129, Na, PO4, radionuclides, Sr90, and } \\
\text { Tc99 (XLS) }\end{array}$ \\
\hline Mass balance diagrams & $\begin{array}{l}\text { Key process parameters used for flowsheet performance } \\
\text { assessment (CSV) }\end{array}$ \\
\hline Run summary data & Instantaneous composition data at locations of interest (CSV). \\
\hline Snapshot composition & $\begin{array}{l}\text { Oxalate, phosphate, and aluminum phase equilibrium data at } \\
\text { Pretreatment vessels of interest (CSV) }\end{array}$ \\
\hline Solubility data files & $\begin{array}{l}\text { Additions of dilution water and aluminum solubility caustic to } \\
\text { Supertank (CSV) }\end{array}$ \\
\hline Supertank dilution conditions & Tank Farm batch waste characteristics (CSV) \\
\hline Tank Farm batch delivery data & $\begin{array}{l}\text { Cumulated mass for the liquid, solid, oxide, and gas components } \\
\text { through every process stream in the model (CSV) }\end{array}$ \\
\hline Totalizer data for process streams & $\begin{array}{l}\text { Key process parameters at different stages of a UFP2 cycle, such } \\
\text { as end of solids concentration, end of caustic leach, end of } \\
\text { recon, end of wash forward, end of wash recycle, end of solid } \\
\text { discharge, and start of a new cycle (CSV) }\end{array}$ \\
\hline UFP2 cycle times & $\begin{array}{l}\text { Volumes for wash forward, wash recycle, and oxidative wash } \\
\text { (CSV) }\end{array}$ \\
\hline UFP2 washing data & $\begin{array}{l}\text { Data output options include CSV files and JPEG images at } \\
\text { selected time interval: 6 minutes, 30 minutes, 60 minutes, and } \\
\text { daily. }\end{array}$ \\
\hline $\begin{array}{l}\text { Volume and Sodium molarity vs. time plots form } \\
\text { key process vessels }\end{array}$ & $\begin{array}{l}\text { Data output options include CSV files and JPEG images at } \\
\text { daily. }\end{array}$ \\
\hline $\begin{array}{l}\text { Volume and weight percent solids vs. time plater } \\
\text { for key process vessels }\end{array}$ & $\begin{array}{l}\text { Cumulated glass output in volume and number of canisters } \\
\text { through the melters (LAW and HLW) are provided in CSV files } \\
\text { and JPEG images. }\end{array}$ \\
\hline Volume history data for key process vessels \\
\hline Volume history plots for LAW and HLW glass \\
$\begin{array}{l}\text { Initial and final volumes of chem-adds for the model runs are } \\
\text { saved in CSV files. }\end{array}$ \\
\hline
\end{tabular}

A model run can provide data from none of the above to all of the above depending on model run scenarios. In addition to the general data output, supplemental data outputs (SDO) can also be gathered as needed.

\subsection{G2 Mass Balance Calculator}

The Microsoft Visual Studio 2005 software is used for the development of automation tools for pre-/post$\mathrm{G} 2$ run data manipulation/processing. The Visual C\# programming language is selected because of its features in providing graphical user interface, easy interaction with Excel spreadsheet, and modularization. The Visual C\# application is named "G2 Mass Balance Calculator" or G2MBC for short. G2MBC consists of three modules: 1) Feed Vector Conversion, 2) Mass Balance Diagrams, and 3) Totalizer Value Extraction. The modules are developed using three Windows forms which are then merged into a Multiple Document Interface (MDI) named "G2MBC_GUI". G2MBC_GUI provides a pull-down menu as shown in Figure 35 where each module application can be launched independently. 
The automation tools are intended for limited use, e.g., only trained modelers with Visual Studio installed, therefore, executables will not be produced for wide distribution. Functional structures of the three modules are presented in Table 29 while methods for the module development are presented in Appendices I and J. All modules have been verified and validated as part of the G2 Version 7.0 release. 


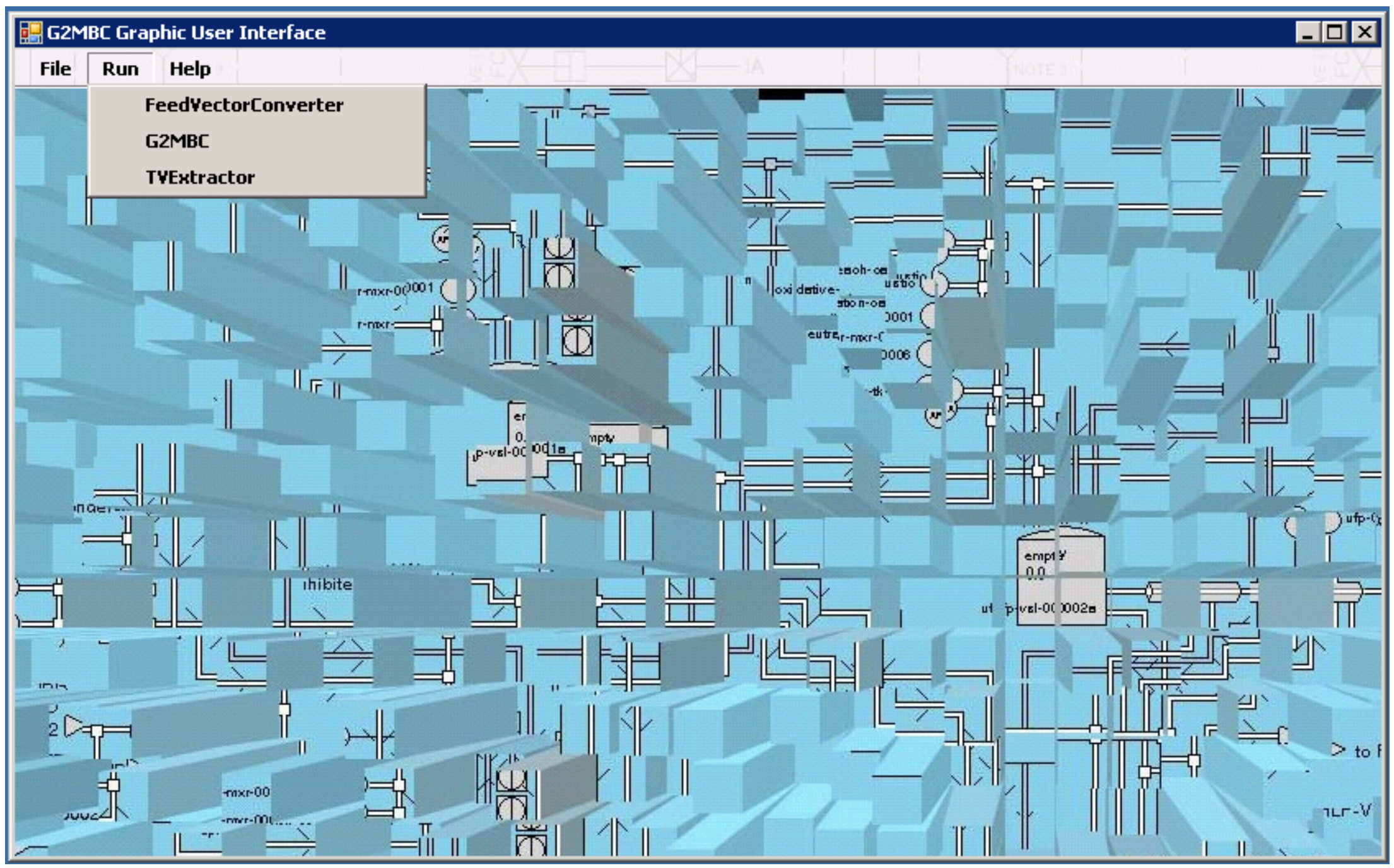

Figure 35

G2MBC GUI 
Table 29 G2MBC Programming Structures

\begin{tabular}{|c|c|c|}
\hline Module & Routines/Subroutines & Functional Descriptions \\
\hline \multirow[t]{2}{*}{$\begin{array}{l}\text { Feed Vector } \\
\text { Conversion }\end{array}$} & $\operatorname{main}()$ & $\begin{array}{l}\text { Specify input/output file names and directories, } \\
\text { Input, } \\
\text { Call subroutines, } \\
\text { Output }\end{array}$ \\
\hline & showFVCinfo() & $\begin{array}{l}\text { Display module development status (Note: this feature is } \\
\text { disabled as methods for feed vector charge balance are } \\
\text { still maturing and conversions are performed by } \\
\text { independent Flowsheet Engineer) }\end{array}$ \\
\hline \multirow[t]{4}{*}{$\begin{array}{l}\text { Mass Balance } \\
\text { Diagrams }\end{array}$} & $\operatorname{main}()$ & $\begin{array}{l}\text { Specify input/output file names and directories, } \\
\text { Input, } \\
\text { Call runCalculator(), } \\
\text { Output }\end{array}$ \\
\hline & runCalculator() & $\begin{array}{l}\text { Call calculateEntireFlowsheetMassBalance(), } \\
\text { Call calculateCumulativeTotalMass(), } \\
\text { Call calculateCumulativeTotalVolume(), } \\
\text { Call calculateCumulativeRadioactivity(), } \\
\text { Call calculateAluminum(), } \\
\text { Call calculateChromium(), } \\
\text { Call calculateIron(), } \\
\text { Call calculateSodium(), } \\
\text { Call calculateOxalate(), } \\
\text { Call calculateCesium(), } \\
\text { Call calculateStrontium(), } \\
\text { Call calculateIodine(), } \\
\text { Call calculateTechnetium(), } \\
\text { Call calculateMercury(), } \\
\text { Call calculateWater(), } \\
\text { Call calculateAFA(), } \\
\text { Call calculatePhosphate() }\end{array}$ \\
\hline & calculateEntireFlowsheetMassBalance() & $\begin{array}{l}\text { Calculate mass balance for all the streams in Figure A-1 } \\
\text { for every liquid, solid, oxide, and gas components, } \\
\text { Call calculateOneFile() repeatedly }\end{array}$ \\
\hline & calculateOneFile() & Calculate mass balance for a single totalizer file \\
\hline
\end{tabular}




\begin{tabular}{|c|c|c|}
\hline Module & Routines/Subroutines & Functional Descriptions \\
\hline & calculateCumulativeTotalMass & $\begin{array}{l}\text { Calculate cumulative total mass (liq. sol., ox, and g), } \\
\text { Post cumulative total mass on flowsheet diagrams }\end{array}$ \\
\hline & calculateCumulativeTotalVolume() & $\begin{array}{l}\text { Calculate cumulative total volume (liq. and sol.), } \\
\text { Post cumulative total volume on flowsheet diagrams }\end{array}$ \\
\hline & calculateCumulativeRadioactivity() & $\begin{array}{l}\text { Calculate cumulative radioactivity, } \\
\text { Post cumulative radioactivity on flowsheet diagrams }\end{array}$ \\
\hline & calculateAluminum() & $\begin{array}{l}\text { Calculate cumulative aluminum mass, } \\
\text { Post cumulative aluminum mass of flowsheet diagrams }\end{array}$ \\
\hline & calculateChromium() & $\begin{array}{l}\text { Calculate cumulative chromium mass, } \\
\text { Post cumulative chromium mass of flowsheet diagrams }\end{array}$ \\
\hline & calculateIron() & $\begin{array}{l}\text { Calculate cumulative iron mass, } \\
\text { Post cumulative iron mass of flowsheet diagrams }\end{array}$ \\
\hline & calculateSodium() & $\begin{array}{l}\text { Calculate cumulative sodium mass, } \\
\text { Post cumulative sodium mass of flowsheet diagrams }\end{array}$ \\
\hline & calculateOxalate() & $\begin{array}{l}\text { Calculate cumulative oxalate mass, } \\
\text { Post cumulative oxalate mass of flowsheet diagrams }\end{array}$ \\
\hline & calculateCesium() & $\begin{array}{l}\text { Calculate cumulative cesium mass, } \\
\text { Post cumulative cesium mass of flowsheet diagrams }\end{array}$ \\
\hline & calculateStrontium() & $\begin{array}{l}\text { Calculate cumulative strontium mass, } \\
\text { Post cumulative strontium mass of flowsheet diagrams }\end{array}$ \\
\hline & calculateIodine() & $\begin{array}{l}\text { Calculate cumulative iodine mass, } \\
\text { Post cumulative iodine mass of flowsheet diagrams }\end{array}$ \\
\hline & calculateTechnetium() & $\begin{array}{l}\text { Calculate cumulative technetium mass, } \\
\text { Post cumulative technetium mass of flowsheet diagrams }\end{array}$ \\
\hline & calculateMercury() & $\begin{array}{l}\text { Calculate cumulative mercury mass, } \\
\text { Post cumulative mercury mass of flowsheet diagrams }\end{array}$ \\
\hline & calculateWater() & $\begin{array}{l}\text { Calculate cumulative water mass, } \\
\text { Post cumulative water mass of flowsheet diagrams }\end{array}$ \\
\hline & calculateAFA() & $\begin{array}{l}\text { Calculate cumulative AFA mass, } \\
\text { Post cumulative AFA mass of flowsheet diagrams }\end{array}$ \\
\hline & calculatePhosphate() & $\begin{array}{l}\text { Calculate cumulative phosphate mass, } \\
\text { Post cumulative phosphate mass of flowsheet diagrams }\end{array}$ \\
\hline
\end{tabular}




\begin{tabular}{|c|c|c|}
\hline Module & Routines/Subroutines & Functional Descriptions \\
\hline \multirow[t]{3}{*}{$\begin{array}{l}\text { Totalizer Value } \\
\text { Extraction }\end{array}$} & $\operatorname{main}()$ & $\begin{array}{l}\text { Specify input/output file names and directories, } \\
\text { Input, } \\
\text { Call extractEntireStreamMakeup(), } \\
\text { Output }\end{array}$ \\
\hline & extractEntireStreamMakeup() & Call extractOneFile() repeatedly \\
\hline & extractOneFile() & $\begin{array}{l}\text { Extract monthly data for liquid and solid volumes, } \\
\text { Calculate monthly total volume }\end{array}$ \\
\hline
\end{tabular}




\section{References}

\section{Project Documents}

24590-WTP-GPP-PT-013, Rev 7, Lifecycle Control of Process Model.

24590-WTP-ICD-MG-01-019, Rev 4, ICD 19 - Interface Control Document for Waste Feed

24590-WTP-M4C-V37T-00009, Rev. 0, Phosphate Solubility Algorithm

24590-WTP-MCR-PET-09-0047, Rev 1, Incorporate HLW Glass Shell V2.0 into Dynamic Flowsheet G2

24590-WTP-MCR-PET-09-0048, Rev 1, Caustic Leaching Requirements and Optimum Aluminum Leaching Routine to Use with HLW Glass Shell V2.0

24590-WTP-MCR-PET-09-0049, Rev 1, Oxidative Leaching Smart Logic to Use with HLW Glass Shell V2.0

24590-WTP-PL-ENG-06-0008, Rev 0, Hanford Waste Treatment and Immobilization Plant (WTP) Project Response Plan for Resolution of Issues Identified by the Comprehensive Review of the WTP Flowsheet and Throughput

24590-WTP-PL-PR-04-0001, Rev 2, Integrated Sampling and Analysis Requirements Document (ISARD) 24590-WTP-PL-TE-01-008, Rev 17, System Design for Process Modeling

24590-WTP-RPT-M-06-002, Rev 0, Hydrogen in Piping and Ancillary Vessels - Plant Availability Assessment.

24590-WTP-RPT-PE-11-008, Rev 0, TPA Milestone M-062-49 - Demonstration of the WTP Design Capacity

24590-WTP-RPT-PET-10-020, Rev 0, 2010 WTP Tank Utilization Assessment

24590-WTP-RPT-PR-01-011, Rev 0, Mercury Pathway and Treatment Assessment for the WTP.

24590-WTP-RPT-PT-02-005, Rev 6, Flowsheet Bases, Assumptions, and Requirements.

24590-WTP-TP-PE-11-001, Rev 0, Dynamic (G2) Flowsheet Version 7.0 Test Plan.

24590-WTP-VV-PE-11-001, Rev 0, Dynamic (G2) Flowsheet Version 7.0 Verification and Validation Report.

CCN 160514, Gibbsite Solubility Models, Supersedes CCN 137192.

CCN 160518, Sodium Oxalate Solubility Model for the Dynamic Flowsheet Rev 1. Supersedes CCN 15320 
CCN 221451, Conversion of CCN 150372 Aluminum Solubility Equations (3) to a Reversible Form

\section{Other Documents}

DOE. 2000. DOE Contract DE-AC27-01RV14136. US Department of Energy, Richland, WA, as amended. 
24590-WTP-MDD-PR-01-002, Rev 12

Dynamic (G2) Model Design Document

\section{Appendix A}

\section{Component List}




\section{Appendix A \\ Component List}

Component lists are used to perform material balance calculations such as storage, material flow, separation, and reaction. These include the liquid, solid, gas, and oxide components. Each list consists of a pre-defined number of constituents that can exist in the WTP equipment object.

Table A-1 Liquid/Solid Components

\begin{tabular}{|c|c|c|c|}
\hline \begin{tabular}{|l|} 
Liquid/Solid \\
Components
\end{tabular} & $\begin{array}{l}\text { Component } \\
\text { Name }\end{array}$ & $\begin{array}{c}\text { Molecular Weight } \\
\text { (kg/kg-mole) }\end{array}$ & $\begin{array}{c}\text { Specific Activity } \\
\text { (Ci/gram) }\end{array}$ \\
\hline 106-Ru & Ruthenium-106 & 105.907 & $3.307 \mathrm{E}+03$ \\
\hline $113 \mathrm{~m}-\mathrm{Cd}$ & Cadmium-113 & 112.904 & $2.244 \mathrm{E}+02$ \\
\hline $125-\mathrm{Sb}$ & Antimony-125 & 124.905 & $1.037 \mathrm{E}+03$ \\
\hline 126-Sn & Tin-126 & 125.908 & $1.135 \mathrm{E}-02$ \\
\hline 129-I & Iodine-129 & 128.905 & $1.765 \mathrm{E}-04$ \\
\hline 134-Cs & Cesium-134 & 133.907 & $1.292 \mathrm{E}+03$ \\
\hline 137-Cs & Cesium-137 & 136.907 & $8.678 \mathrm{E}+01$ \\
\hline $137 \mathrm{~m}-\mathrm{Ba}$ & Barium-137 & 136.906 & $5.380 \mathrm{E}+08$ \\
\hline 14-C & Carbon-14 & 14.003 & $4.468 \mathrm{E}+00$ \\
\hline 151-Sm & Samarium-151 & 150.920 & $2.631 \mathrm{E}+01$ \\
\hline $152-\mathrm{Eu}$ & Europium-152 & 151.922 & $1.737 \mathrm{E}+02$ \\
\hline 154-Eu & Europium-154 & 153.923 & $2.702 \mathrm{E}+02$ \\
\hline 155-Eu & Europium-155 & 154.923 & $4.856 \mathrm{E}+02$ \\
\hline 226-Ra & Radium-226 & 226.025 & 9.893E-01 \\
\hline $227-A c$ & Actinium-227 & 227.028 & $7.233 \mathrm{E}+01$ \\
\hline 228-Ra & Radium-228 & 228.031 & $2.722 \mathrm{E}+02$ \\
\hline 229-Th & Thorium-229 & 229.032 & $2.139 \mathrm{E}-01$ \\
\hline $231-\mathrm{Pa}$ & Protactinium-231 & 231.036 & $4.718 \mathrm{E}-02$ \\
\hline 232-Th & Thorium-232 & 232.038 & $1.100 \mathrm{E}-07$ \\
\hline $232-\mathrm{U}$ & Uranium-232 & 232.037 & $2.208 \mathrm{E}+01$ \\
\hline $233-\mathrm{U}$ & Uranium-233 & 233.040 & $9.638 \mathrm{E}-03$ \\
\hline $234-\mathrm{U}$ & Uranium-234 & 234.041 & $6.210 \mathrm{E}-03$ \\
\hline $235-\mathrm{U}$ & Uranium-235 & 235.044 & $2.160 \mathrm{E}-06$ \\
\hline $236-U$ & Uranium-236 & 236.046 & $6.468 \mathrm{E}-05$ \\
\hline 237-Np & Neptunium-237 & 237.048 & 7.049E-04 \\
\hline $238-\mathrm{Pu}$ & Plutonium-238 & 238.050 & $1.713 \mathrm{E}+01$ \\
\hline $238-\mathrm{U}$ & Uranium-238 & 238.051 & $3.360 \mathrm{E}-07$ \\
\hline 239-Pu & Plutonium-239 & 239.052 & $6.207 \mathrm{E}-02$ \\
\hline $240-\mathrm{Pu}$ & Plutonium-240 & 240.054 & $2.271 \mathrm{E}-01$ \\
\hline 241-Am & Americium-241 & 241.057 & $3.428 \mathrm{E}+00$ \\
\hline $241-\mathrm{Pu}$ & Plutonium-241 & 241.057 & $1.030 \mathrm{E}+02$ \\
\hline $242-\mathrm{Cm}$ & Curium-242 & 242.059 & $3.315 \mathrm{E}+03$ \\
\hline $242-\mathrm{Pu}$ & Plutonium-242 & 242.059 & 3.939E-03 \\
\hline 243-Am & Americium-243 & 243.061 & $1.996 \mathrm{E}-01$ \\
\hline $243-\mathrm{Cm}$ & Curium-243 & 243.061 & $5.056 \mathrm{E}+01$ \\
\hline 244-Cm & Curium-244 & 244.063 & $8.095 \mathrm{E}+01$ \\
\hline $3-\mathrm{H}$ & Tritium & 3.016 & $9.673 \mathrm{E}+03$ \\
\hline $59-\mathrm{Ni}$ & Nickel-59 & 58.934 & 7.973E-02 \\
\hline
\end{tabular}


24590-WTP-MDD-PR-01-002, Rev 12 Dynamic (G2) Model Design Document

\begin{tabular}{|c|c|c|c|}
\hline $\begin{array}{l}\text { Liquid/Solid } \\
\text { Components }\end{array}$ & \begin{tabular}{|l|} 
Component \\
Name
\end{tabular} & $\begin{array}{c}\begin{array}{c}\text { Molecular Weight } \\
\text { (kg/kg-mole) }\end{array} \\
\end{array}$ & $\begin{array}{c}\text { Specific Activity } \\
\text { (Ci/gram) }\end{array}$ \\
\hline 60-Co & Cobalt-60 & 59.934 & $1.130 \mathrm{E}+03$ \\
\hline 63-Ni & Nickel-63 & 62.930 & $5.675 \mathrm{E}+01$ \\
\hline 79-Se & Selenium-79 & 78.918 & $6.962 \mathrm{E}-03$ \\
\hline $90-\mathrm{Sr}$ & Strontium-90 & 89.908 & $1.380 \mathrm{E}+02$ \\
\hline $90-\mathrm{Y}$ & Yttrium-90 & 89.907 & $5.434 \mathrm{E}+05$ \\
\hline $93-\mathrm{Zr}$ & Zirconium-93 & 92.906 & $2.563 \mathrm{E}-03$ \\
\hline $93 \mathrm{~m}-\mathrm{Nb}$ & Niobium-93 & 92.906 & $2.388 \mathrm{E}+02$ \\
\hline 99-Tc & Technetium-99 & 98.906 & $1.695 \mathrm{E}-02$ \\
\hline $\mathrm{Ag}+$ & Silver Ion & 107.868 & $\mathrm{n} / \mathrm{a}$ \\
\hline $\mathrm{Al}(\mathrm{OH}) 4-$ & Aluminum Hydroxide & 95.011 & $\mathrm{n} / \mathrm{a}$ \\
\hline $\mathrm{Al}+3$ & Aluminum Ion & 26.982 & $\mathrm{n} / \mathrm{a}$ \\
\hline As+5 & Arsenic Ion & 74.922 & $\mathrm{n} / \mathrm{a}$ \\
\hline $\mathrm{B}+3$ & Boron Ion & 10.810 & $\mathrm{n} / \mathrm{a}$ \\
\hline $\mathrm{Ba}+2$ & Barium Ion & 137.340 & $\mathrm{n} / \mathrm{a}$ \\
\hline $\mathrm{Be}+2$ & Beryllium Ion & 9.012 & $\mathrm{n} / \mathrm{a}$ \\
\hline $\mathrm{Bi}+3$ & Bismuth Ion & 208.981 & $\mathrm{n} / \mathrm{a}$ \\
\hline C10H12N2O8-4 & EDTA & 288.213 & $\mathrm{n} / \mathrm{a}$ \\
\hline $\mathrm{C} 10 \mathrm{H} 15 \mathrm{~N} 2 \mathrm{O} 7-3$ & N-Hydroxyethylethylenediaminetriacetate & 275.238 & $\mathrm{n} / \mathrm{a}$ \\
\hline $\mathrm{C} 12 \mathrm{H} 27 \mathrm{O} 4 \mathrm{P}$ & Phosphoric Acid Tributyl Ester & 266.317 & $\mathrm{n} / \mathrm{a}$ \\
\hline $\mathrm{C} 13 \mathrm{H} 28$ & Tridecane & 184.364 & $\mathrm{n} / \mathrm{a}$ \\
\hline $\mathrm{C} 2 \mathrm{Cl} 4$ & Tetrachloroethene & 165.834 & $\mathrm{n} / \mathrm{a}$ \\
\hline $\mathrm{C} 2 \mathrm{H} 3 \mathrm{O} 2-$ & Acetate Ion & 59.044 & $\mathrm{n} / \mathrm{a}$ \\
\hline C2H3O3- & Hydroxyacetate Ion & 75.044 & $\mathrm{n} / \mathrm{a}$ \\
\hline $\mathrm{C} 2 \mathrm{HCl} 3$ & Trichloroethylene & 131.389 & $\mathrm{n} / \mathrm{a}$ \\
\hline $\mathrm{C} 2 \mathrm{O} 4-2$ & Oxalate & 88.020 & $\mathrm{n} / \mathrm{a}$ \\
\hline $\mathrm{C} 3 \mathrm{H} 6 \mathrm{O}$ & Acetone & 58.080 & $\mathrm{n} / \mathrm{a}$ \\
\hline $\mathrm{C} 4 \mathrm{H} 10 \mathrm{O}$ & Ether & 74.122 & $\mathrm{n} / \mathrm{a}$ \\
\hline $\mathrm{C} 4 \mathrm{H} 8 \mathrm{O}$ & Methyl Ethyl Ketone & 72.107 & $\mathrm{n} / \mathrm{a}$ \\
\hline $\mathrm{C} 5 \mathrm{H} 5 \mathrm{~N}$ & Pyridine & 79.101 & $\mathrm{n} / \mathrm{a}$ \\
\hline C6H5NO2 & Nito-Benzene & 123.111 & $\mathrm{n} / \mathrm{a}$ \\
\hline C6H5O7-3 & Citrate Ion & 189.101 & $\mathrm{n} / \mathrm{a}$ \\
\hline C6H6 & Benzene & 78.113 & $\mathrm{n} / \mathrm{a}$ \\
\hline C7H6N2O4 & Dinitrotoluene & 182.135 & $\mathrm{n} / \mathrm{a}$ \\
\hline $\mathrm{C} 7 \mathrm{H} 8 \mathrm{O}$ & Anisole & 108.140 & $\mathrm{n} / \mathrm{a}$ \\
\hline $\mathrm{Ca}+2$ & Calcium Ion & 40.080 & $\mathrm{n} / \mathrm{a}$ \\
\hline $\mathrm{Cd}+2$ & Cadmium Ion & 112.400 & $\mathrm{n} / \mathrm{a}$ \\
\hline $\mathrm{Ce}+3$ & Cerium Ion & 140.120 & $\mathrm{n} / \mathrm{a}$ \\
\hline $\mathrm{CH} 2 \mathrm{Cl} 2$ & Dichloromethane & 84.933 & $\mathrm{n} / \mathrm{a}$ \\
\hline $\mathrm{CHCl} 3$ & Trichloromethane & 119.378 & $\mathrm{n} / \mathrm{a}$ \\
\hline CHO2- & Formate Ion & 45.018 & $\mathrm{n} / \mathrm{a}$ \\
\hline $\mathrm{Cl}-$ & Chloride Ion & 35.453 & $\mathrm{n} / \mathrm{a}$ \\
\hline $\mathrm{CN}-$ & Cyanide Ion & 26.018 & $\mathrm{n} / \mathrm{a}$ \\
\hline $\mathrm{Co}+3$ & Cobalt Ion & 58.933 & $\mathrm{n} / \mathrm{a}$ \\
\hline $\mathrm{CO} 3-2$ & Carbonate Ion & 60.009 & $\mathrm{n} / \mathrm{a}$ \\
\hline $\mathrm{Cr}(\mathrm{OH}) 4-$ & Chromium Hydroxide & 120.025 & $\mathrm{n} / \mathrm{a}$ \\
\hline $\mathrm{Cr}(\mathrm{TOTAL})$ & Chromium & 51.996 & $\mathrm{n} / \mathrm{a}$ \\
\hline Cs+ & Cesium ion & 137.000 & $\mathrm{n} / \mathrm{a}$ \\
\hline
\end{tabular}


24590-WTP-MDD-PR-01-002, Rev 12 Dynamic (G2) Model Design Document

\begin{tabular}{|c|c|c|c|}
\hline \begin{tabular}{|l|} 
Liquid/Solid \\
Components \\
\end{tabular} & \begin{tabular}{|l|} 
Component \\
Name
\end{tabular} & $\begin{array}{c}\begin{array}{c}\text { Molecular Weight } \\
\text { (kg/kg-mole) }\end{array} \\
\end{array}$ & $\begin{array}{c}\text { Specific Activity } \\
\text { (Ci/gram) }\end{array}$ \\
\hline $\mathrm{Cu}+2$ & Copper Ion & 63.546 & $\mathrm{n} / \mathrm{a}$ \\
\hline F- & Fluoride Ion & 18.998 & $\mathrm{n} / \mathrm{a}$ \\
\hline $\mathrm{Fe}+3$ & Iron Ion & 55.847 & $\mathrm{n} / \mathrm{a}$ \\
\hline $\mathrm{H}+$ & Hydrogen Ion & 1.008 & $\mathrm{n} / \mathrm{a}$ \\
\hline $\mathrm{H} 2 \mathrm{O}$ & Water & 18.015 & $\mathrm{n} / \mathrm{a}$ \\
\hline $\mathrm{H} 2 \mathrm{O} 2$ & Hydrogen Peroxide & 34.015 & $\mathrm{n} / \mathrm{a}$ \\
\hline $\mathrm{Hg}+2$ & Mercury Ion & 200.590 & $\mathrm{n} / \mathrm{a}$ \\
\hline $\mathrm{K}+$ & Potassium Ion & 39.090 & $\mathrm{n} / \mathrm{a}$ \\
\hline $\mathrm{La}+3$ & Lanthanum Ion & 138.905 & $\mathrm{n} / \mathrm{a}$ \\
\hline $\mathrm{Li}+$ & Lithium Ion & 6.940 & $\mathrm{n} / \mathrm{a}$ \\
\hline $\mathrm{Mg}+2$ & Magnesium Ion & 24.305 & $\mathrm{n} / \mathrm{a}$ \\
\hline $\mathrm{Mn}+4$ & Manganese Ion & 54.938 & $\mathrm{n} / \mathrm{a}$ \\
\hline \begin{tabular}{|l|}
$\mathrm{MnO} 2$ \\
\end{tabular} & Manganese Oxide & 86.937 & $\mathrm{n} / \mathrm{a}$ \\
\hline MnO4- & Permanganate Ion & 118.936 & $\mathrm{n} / \mathrm{a}$ \\
\hline $\mathrm{Mo}+6$ & Molybdenum Ion & 95.940 & $\mathrm{n} / \mathrm{a}$ \\
\hline $\mathrm{Na}+$ & Sodium Ion & 22.990 & $\mathrm{n} / \mathrm{a}$ \\
\hline $\mathrm{Nd}+3$ & Neodymium Ion & 144.240 & $\mathrm{n} / \mathrm{a}$ \\
\hline NH3 & Ammonia & 17.030 & $\mathrm{n} / \mathrm{a}$ \\
\hline $\mathrm{Ni}+2$ & Nickel Ion & 58.710 & $\mathrm{n} / \mathrm{a}$ \\
\hline NO2- & Nitrite & 46.005 & $\mathrm{n} / \mathrm{a}$ \\
\hline NO3- & Nitrate & 62.005 & $\mathrm{n} / \mathrm{a}$ \\
\hline OH(BOUND) & Bound Hydroxide & 17.007 & $\mathrm{n} / \mathrm{a}$ \\
\hline $\mathrm{OH}-$ & Hydroxide & 17.007 & $\mathrm{n} / \mathrm{a}$ \\
\hline $\mathrm{Pb}+2$ & Lead Ion & 207.200 & $\mathrm{n} / \mathrm{a}$ \\
\hline $\mathrm{Pd}+2$ & Palladium Ion & 106.400 & $\mathrm{n} / \mathrm{a}$ \\
\hline PO4-3 & Phosphate Ion & 94.971 & $\mathrm{n} / \mathrm{a}$ \\
\hline $\mathrm{Pr}+3$ & Praseodymium Ion & 140.908 & $\mathrm{n} / \mathrm{a}$ \\
\hline $\mathrm{Pu}+4$ & Plutonium Ion & 239.000 & $\mathrm{n} / \mathrm{a}$ \\
\hline $\mathrm{Rb}+$ & Rubidium Ion & 85.467 & $\mathrm{n} / \mathrm{a}$ \\
\hline $\mathrm{Rh}+3$ & Rhodium Ion & 102.906 & $\mathrm{n} / \mathrm{a}$ \\
\hline $\mathrm{Ru}+3$ & Ruthenium Ion & 101.070 & $\mathrm{n} / \mathrm{a}$ \\
\hline $\mathrm{Sb}+5$ & Antimony Ion & 121.750 & $\mathrm{n} / \mathrm{a}$ \\
\hline $\mathrm{Se}+6$ & Selenium Ion & 78.960 & $\mathrm{n} / \mathrm{a}$ \\
\hline $\mathrm{Si}+4$ & Silicon Ion & 28.086 & $\mathrm{n} / \mathrm{a}$ \\
\hline SO4-2 & Sulfate Ion & 96.058 & $\mathrm{n} / \mathrm{a}$ \\
\hline $\mathrm{Sr}+2$ & Strontium Ion & 90.000 & $\mathrm{n} / \mathrm{a}$ \\
\hline $\mathrm{Ta}+5$ & Tantalum Ion & 180.947 & $\mathrm{n} / \mathrm{a}$ \\
\hline $\mathrm{Tc}+7$ & Technetium Ion & 98.906 & $\mathrm{n} / \mathrm{a}$ \\
\hline $\mathrm{Te}+6$ & Tellurium Ion & 127.600 & $\mathrm{n} / \mathrm{a}$ \\
\hline $\mathrm{Th}+4$ & Thorium Ion & 232.038 & $\mathrm{n} / \mathrm{a}$ \\
\hline $\mathrm{Ti}+4$ & Titanium Ion & 47.900 & $\mathrm{n} / \mathrm{a}$ \\
\hline $\mathrm{Tl}+3$ & Thallium Ion & 204.370 & $\mathrm{n} / \mathrm{a}$ \\
\hline TOC & Total Organic Carbon & 12.011 & $\mathrm{n} / \mathrm{a}$ \\
\hline U(TOTAL) & Uranium & 238.029 & $\mathrm{n} / \mathrm{a}$ \\
\hline $\mathrm{V}+5$ & Vanadium Ion & 50.941 & $\mathrm{n} / \mathrm{a}$ \\
\hline $\mathrm{W}+6$ & Tungsten Ion & 183.850 & $\mathrm{n} / \mathrm{a}$ \\
\hline $\mathrm{Y}+3$ & Yttrium Ion & 88.906 & $\mathrm{n} / \mathrm{a}$ \\
\hline
\end{tabular}


24590-WTP-MDD-PR-01-002, Rev 12 Dynamic (G2) Model Design Document

\begin{tabular}{|c|c|c|c|}
\hline \begin{tabular}{|l|} 
Liquid/Solid \\
Components \\
\end{tabular} & \begin{tabular}{|l|} 
Component \\
Name
\end{tabular} & $\begin{array}{c}\text { Molecular Weight } \\
(\mathrm{kg} / \mathrm{kg}-\mathrm{mole})\end{array}$ & $\begin{array}{c}\text { Specific Activity } \\
\text { (Ci/gram) }\end{array}$ \\
\hline $\mathrm{Zn}+2$ & Zinc Ion & 65.380 & $\mathrm{n} / \mathrm{a}$ \\
\hline $\mathrm{Zr}+4$ & Zirconium Ion & 91.220 & $\mathrm{n} / \mathrm{a}$ \\
\hline $\mathrm{C} 10 \mathrm{H} 8$ & Naphthalene & 128.175 & $\mathrm{n} / \mathrm{a}$ \\
\hline $\mathrm{C} 12 \mathrm{H} 4 \mathrm{Cl} 6$ & PCBs & 360.884 & $\mathrm{n} / \mathrm{a}$ \\
\hline $\mathrm{C} 12 \mathrm{H} 8$ & Acenaphthylene & 152.198 & $\mathrm{n} / \mathrm{a}$ \\
\hline $\mathrm{C} 2 \mathrm{H} 3 \mathrm{~N}$ & Acetonitrile & 41.053 & $\mathrm{n} / \mathrm{a}$ \\
\hline $\mathrm{C} 2 \mathrm{H} 6 \mathrm{SiO}$ & Dimethylsiloxane & 74.156 & $\mathrm{n} / \mathrm{a}$ \\
\hline $\mathrm{C} 2 \mathrm{H} 8 \mathrm{~N} 2$ & 1,1-Dimethylhdrazine & 60.099 & $\mathrm{n} / \mathrm{a}$ \\
\hline $\mathrm{H} 2 \mathrm{O}(\mathrm{BOUND})$ & Bound Water & 18.015 & $\mathrm{n} / \mathrm{a}$ \\
\hline C4H7NO4 & Iminodiacetic Acid & 133.105 & $\mathrm{n} / \mathrm{a}$ \\
\hline C6H6O & Phenol & 94.114 & $\mathrm{n} / \mathrm{a}$ \\
\hline $\mathrm{C} 7 \mathrm{H} 8$ & Toluene & 92.142 & $\mathrm{n} / \mathrm{a}$ \\
\hline $\mathrm{C} 8 \mathrm{H} 8 \mathrm{O}$ & Acetophenone & 120.152 & $\mathrm{n} / \mathrm{a}$ \\
\hline $\mathrm{CCl} 4$ & Carbon Tetrachloride & 153.823 & $\mathrm{n} / \mathrm{a}$ \\
\hline $\mathrm{H} 2 \mathrm{CO} 3$ & Carbonic Acid & 62.025 & $\mathrm{n} / \mathrm{a}$ \\
\hline HCO3- & Hydrogen Carbonate Ion & 61.017 & $\mathrm{n} / \mathrm{a}$ \\
\hline Mnp & Process Manganese Ion & 54.938 & $\mathrm{n} / \mathrm{a}$ \\
\hline Nap+ & Process Sodium Ion & 22.990 & $\mathrm{n} / \mathrm{a}$ \\
\hline NH4+ & Ammonium Ion & 18.039 & $\mathrm{n} / \mathrm{a}$ \\
\hline $\mathrm{O}-2$ & Oxide Ion & 15.999 & $\mathrm{n} / \mathrm{a}$ \\
\hline $\mathrm{Al}(\mathrm{OH}) 3$ & Gibbsite & 78.004 & $\mathrm{n} / \mathrm{a}$ \\
\hline PO4-3(HARD) & Hard to Dissolve Phosphate & 94.971 & $\mathrm{n} / \mathrm{a}$ \\
\hline PPG & Polypropyleneglycol & 3,503 & $\mathrm{n} / \mathrm{a}$ \\
\hline DPPG & Decomposed Polypropyleneglycol & 3,503 & $\mathrm{n} / \mathrm{a}$ \\
\hline PDMS & Polydimethoylsiloxane & 35,015 & $\mathrm{n} / \mathrm{a}$ \\
\hline DPDMS & Decomposed Polydimethoylsiloxane & 35,015 & $\mathrm{n} / \mathrm{a}$ \\
\hline NO3-D & Nitrate (Charge Balance) & 62.005 & $\mathrm{n} / \mathrm{a}$ \\
\hline $\mathrm{AlOOH}$ & Boehmite & 59.988 & $\mathrm{n} / \mathrm{a}$ \\
\hline LS9 & Liquid-Solid-9 & 0.000 & $\mathrm{n} / \mathrm{a}$ \\
\hline LS10 & Liquid-Solid-10 & 0.000 & $\mathrm{n} / \mathrm{a}$ \\
\hline
\end{tabular}

\section{Table A-2 Gas Components}

\begin{tabular}{|l|l|c|}
\hline Gas Components & Component Name & Molecular Weight \\
\hline $\mathrm{CO} 2$ & Carbon Dioxide & 44.010 \\
\hline $\mathrm{H} 2 \mathrm{O}$ & Water & 18.015 \\
\hline $\mathrm{HCl}$ & Hydrogen Chloride & 36.461 \\
\hline $\mathrm{HF}$ & Hydrogen Fluoride & 20.006 \\
\hline $\mathrm{N} 2$ & Nitrogen & 28.013 \\
\hline $\mathrm{NH} 3$ & Ammonia & 17.030 \\
\hline $\mathrm{NO}$ & Nitrogen Oxide & 30.006 \\
\hline $\mathrm{NO} 2$ & Nitrogen Dioxide & 46.005 \\
\hline $\mathrm{O} 2$ & Oxygen & 31.999 \\
\hline $\mathrm{P} 2 \mathrm{O} 5$ & Phosphate & 141.945 \\
\hline $\mathrm{SO} 2$ & Sulfur Dioxide & 64.059 \\
\hline $129-\mathrm{I}$ & Iodine-129 & 128.905 \\
\hline $\mathrm{SO} 3$ & Sulfur Trioxide & 80.062 \\
\hline $\mathrm{C} 12 \mathrm{H} 4 \mathrm{Cl} 6$ & PCBs & 360.852 \\
\hline
\end{tabular}


Gas Components

$\mathrm{C} 2 \mathrm{H} 3 \mathrm{~N}$

$\mathrm{Hg}$
Component Name

Acetonitrile

Mercury
Molecular Weight

41.054

200.59

\section{Table A-3 Oxide Components}

\begin{tabular}{|c|c|c|}
\hline Oxide Components & Component Name & Molecular Weight \\
\hline $\mathrm{Ac} 2 \mathrm{O} 3$ & Actinium Oxide & 501.998 \\
\hline $\mathrm{Ag} 2 \mathrm{O}$ & Silver Oxide & 231.735 \\
\hline $\mathrm{Al} 2 \mathrm{O} 3$ & Aluminum Oxide & 101.961 \\
\hline $\mathrm{Am} 2 \mathrm{O} 3$ & Americium Oxide & 533.998 \\
\hline As2O5 & Arsenic Oxide & 229.840 \\
\hline $\mathrm{B} 2 \mathrm{O} 3$ & Boron Oxide & 69.618 \\
\hline $\mathrm{BaO}$ & Barium Oxide & 153.339 \\
\hline $\mathrm{BeO}$ & Beryllium Oxide & 25.012 \\
\hline Bi2O3 & Bismuth Oxide & 465.960 \\
\hline $\mathrm{CaO}$ & Calcium Oxide & 56.079 \\
\hline $\mathrm{CdO}$ & Cadmium Oxide & 128.399 \\
\hline $\mathrm{Ce} 2 \mathrm{O} 3$ & Cerium Oxide & 328.2382 \\
\hline $\mathrm{Cl}-$ & Chloride ion & 35.453 \\
\hline $\mathrm{Cm} 2 \mathrm{O} 3$ & Curium Oxide & 541.998 \\
\hline $\mathrm{CoO}$ & Cobalt Oxide & 74.9326 \\
\hline Cr2O3 & Chromium Oxide & 151.990 \\
\hline $\mathrm{Cs} 2 \mathrm{O}$ & Cesium Oxide & 289.999 \\
\hline $\mathrm{CuO}$ & Copper Oxide & 79.545 \\
\hline Dy2O3 & Dysprosium Oxide & 372.998 \\
\hline Eu2O3 & Europium Oxide & 351.918 \\
\hline F- & Fluoride ion & 18.998 \\
\hline $\mathrm{Fe} 2 \mathrm{O} 3$ & Iron Oxide & 159.692 \\
\hline $\mathrm{HgO}$ & Mercury Oxide & 216.589 \\
\hline $\mathrm{K} 2 \mathrm{O}$ & Potassium Oxide & 94.179 \\
\hline La2O3 & Lanthanum Oxide & 325.809 \\
\hline $\mathrm{Li} 2 \mathrm{O}$ & Lithium Oxide & 29.879 \\
\hline $\mathrm{MgO}$ & Magnesium Oxide & 40.304 \\
\hline $\mathrm{MnO}$ & Manganese Oxide & 70.9374 \\
\hline $\mathrm{MoO} 3$ & Molybdenum Oxide & 143.938 \\
\hline $\mathrm{Na} 2 \mathrm{O}$ & Sodium Oxide & 61.979 \\
\hline $\mathrm{Nb} 2 \mathrm{O} 5$ & Niobium Oxide & 265.810 \\
\hline $\mathrm{Nd} 2 \mathrm{O} 3$ & Neodymium Oxide & 336.478 \\
\hline $\mathrm{NiO}$ & Nickel Oxide & 74.709 \\
\hline $\mathrm{NpO} 2$ & Neptunium Oxide & 269.047 \\
\hline $\mathrm{P} 2 \mathrm{O} 5$ & Phosphate & 141.945 \\
\hline $\mathrm{PbO}$ & Lead Oxide & 223.199 \\
\hline $\mathrm{PdO}$ & Palladium Oxide & 122.399 \\
\hline Pr2O3 & Praseodymium Oxide & 329.814 \\
\hline $\mathrm{PuO} 2$ & Plutonium Oxide & 270.999 \\
\hline $\mathrm{Rb} 2 \mathrm{O}$ & Rubidium Oxide & 186.933 \\
\hline $\operatorname{Re} 2 \mathrm{O}^{(\mathrm{a})}$ & Rhenium Oxide & 484.3958 \\
\hline $\mathrm{Rh} 2 \mathrm{O} 3$ & Rhodium Oxide & 253.809 \\
\hline $\mathrm{RuO} 2$ & Ruthenium Oxide & 133.0688 \\
\hline
\end{tabular}




\begin{tabular}{|l|l|c|}
\hline Oxide Components & Component Name & Molecular Weight \\
\hline $\mathrm{Sb} 2 \mathrm{O} 3$ & Antimony Oxide & 291.498 \\
\hline $\mathrm{SeO} 2$ & Selenium Oxide & 110.959 \\
\hline $\mathrm{SiO} 2$ & Silicon Dioxide & 60.085 \\
\hline $\mathrm{Sm} 2 \mathrm{O} 3$ & Samarium Oxide & 348.798 \\
\hline $\mathrm{SnO} 2$ & Tin Oxide & 150.689 \\
\hline $\mathrm{SO} 3$ & Sulfur Trioxide & 80.058 \\
\hline $\mathrm{SrO}$ & Strontium Oxide & 105.999 \\
\hline $\mathrm{Ta} 2 \mathrm{O} 5$ & Tantalum Oxide & 441.891 \\
\hline $\mathrm{Tc2O} 7$ & Technetium Oxide & 309.808 \\
\hline $\mathrm{TeO} 2$ & Tellurium Oxide & 159.599 \\
\hline $\mathrm{ThO} 2$ & Thorium Oxide & 264.037 \\
\hline $\mathrm{TiO} 2$ & Titanium Oxide & 79.899 \\
\hline $\mathrm{T} 2 \mathrm{O}$ & Thallium Oxide & 424.7394 \\
\hline $\mathrm{UO} 3$ & Uranium Oxide & 286.0282 \\
\hline $\mathrm{V} 2 \mathrm{O} 5$ & Vanadium Oxide & 181.879 \\
\hline $\mathrm{WO} 3$ & Tungsten Oxide & 231.848 \\
\hline $\mathrm{Y} 2 \mathrm{O} 3$ & Yttrium Oxide & 225.810 \\
\hline $\mathrm{ZnO}$ & Zinc Oxide & 81.379 \\
\hline $\mathrm{ZrO} 2$ & Zirconium Oxide & 123.219 \\
\hline $\mathrm{CO} 2$ & Carbon Dioxide & 44.010 \\
\hline $\mathrm{H} 2 \mathrm{O}(\mathrm{Bound})$ & Bound Water & 18.015 \\
\hline $\mathrm{H} 2 \mathrm{O}$ & Water & 18.015 \\
\hline $\mathrm{HfO} 2$ & Hafnium Oxide & 210.489 \\
\hline $\mathrm{Mnp2O3}$ & Process Manganese Oxide & 157.875 \\
\hline $\mathrm{Nap2O}$ & Process Sodium Oxide & 61.979 \\
\hline $129-\mathrm{I}$ & Iodine-129 & 128.905 \\
\hline $\mathrm{Pa} 2 \mathrm{O} 3$ & Protactinium Oxide & 510.000 \\
\hline $\mathrm{RaO}$ & Radium Oxide & 242.000 \\
\hline $\mathrm{C} 12 \mathrm{H} 22 \mathrm{O} 11$ & Sugar & 342.299 \\
\hline $\mathrm{Ox} 5$ & Oxide-5 & 0.000 \\
\hline & & \\
\hline & & \\
\hline
\end{tabular}

(a) Rhenium oxide is tracked but not used in the model as the TFC feed vector does not provide the quantities of rhenium. 
24590-WTP-MDD-PR-01-002, Rev 12

Dynamic (G2) Model Design Document

\section{Appendix B}

\section{Chemical Adds}




\section{Appendix B Chemical Adds}

Chemical reagents including chemicals, minerals, and air are used in various systems and unit operations. Utilization of these chemical reagents is discussed below in a system-by-system manner. Process water and process condensate for line flushes are excluded in these discussions but can be found in Sections 4.7.13 and 4.8. It shall be noted in the following that $\mathrm{Na}+$ represents the waste sodium from the Tank Farm and includes the sodium for caustic leach and aluminum solubility applications, Nap+ represents the process sodium such as the chemical reagents utilized in other parts of the WTP, and Mnp represents the process manganese. All of the caustic soda contains $2 \mathrm{wt} \%$ of $\mathrm{NapCl}$ or $\mathrm{NaCl}$ as appropriate.

To quickly evaluate the utilization of the chemical reagents, the model can output the initial and final volumes of these chemical reagents for any scenario runs. Table B-23 provides the initial volumes of the chemical reagents in Version 7.0 of the Dynamic (G2) Model.

\section{WASTE FEED RECEIPT PROCESS SYSTEM}

Table B-1 Chemical Reagents for FRP/HLP-VSL-00022

\begin{tabular}{|l|l|l|l|}
\hline Chem Add & Composition & Flowrate & Description \\
\hline INHIBITED-WATER & $0.1 \mathrm{M} \mathrm{NapOH}$ & $73 \mathrm{gpm}$ & Pre- and post- Tank Farm batch transfer \\
& $0.011 \mathrm{M} \mathrm{NapNO}_{2}$ & & line flush \\
\hline
\end{tabular}

\section{WASTE FEED EVAPORATION PROCESS SYSTEM}

Table B-2 Chemical Reagents for FEP

\begin{tabular}{|c|c|c|c|}
\hline Chem Add & Composition & Flowrate & Description \\
\hline Antifoam Agent & $\begin{array}{l}0.034 \mathrm{M} \text { PPG } \\
0.003 \mathrm{M} \text { PDMS }\end{array}$ & $50 \mathrm{gpm}$ & $\begin{array}{l}\text { Mitigates foaming in process vessels, } \\
\text { Target concentrations: } \\
\text { PPG }=250 \mathrm{ppm} \\
\text { PDMS }=250 \mathrm{ppm}\end{array}$ \\
\hline FEP-DMST-00001A-AIR & $\begin{array}{l}\text { Air }\left(0.0012 \mathrm{wt}^{\%} \%\right. \\
\mathrm{H}_{2} \mathrm{O}, 76.7 \mathrm{wt}^{0} \% \mathrm{~N}_{2} \\
\left.23.3 \mathrm{wt}^{0} \% \mathrm{O}_{2}\right)\end{array}$ & $\begin{array}{l}5 \mathrm{ft}^{3} / \mathrm{min} \\
\mathrm{T}=70^{\circ} \mathrm{F}, \mathrm{P}= \\
14.7 \mathrm{psi}\end{array}$ & Pump when FEP-SEP-00001A boils off \\
\hline FEP-DMST-00001B-AIR & $\begin{array}{l}\text { Air }\left(0.0012 w^{0} \%\right. \\
\mathrm{H}_{2} \mathrm{O}, 76.7 \mathrm{wt}^{0} \% \mathrm{~N}_{2} \\
\left.23.3 \mathrm{wt}^{0} \% \mathrm{O}_{2}\right)\end{array}$ & $\begin{array}{l}5 \mathrm{ft}^{3} / \mathrm{min} \\
\mathrm{T}=70^{\circ} \mathrm{F}, \mathrm{P}= \\
14.7 \mathrm{psi}\end{array}$ & Pump when FEP-SEP-00001B boils off \\
\hline $\begin{array}{l}\text { FEPA-INTER-AFTER- } \\
\text { CONDENSER-WATER }\end{array}$ & $\mathrm{H}_{2} \mathrm{O}$ & $0.96 \mathrm{gpm}$ & Pump when FEP-SEP-00001A boils off \\
\hline $\begin{array}{l}\text { FEPB-INTER-AFTER- } \\
\text { CONDENSER-WATER }\end{array}$ & $\mathrm{H}_{2} \mathrm{O}$ & $0.96 \mathrm{gpm}$ & Pump when FEP-SEP-00001B boils off \\
\hline
\end{tabular}

\section{ULTRAFILTRATION PROCESS SYSTEM}

Table B-3 Reserved Chemical Reagent Capabilities for Envelope C Feeds

\begin{tabular}{|l|l|l|l|}
\hline Chem Add & Composition & Flowrate & Description \\
\hline STR-MXR-00001 & $1 \mathrm{M} \mathrm{Sr}\left(\mathrm{NO}_{3}\right)_{2}$ & $\mathrm{n} / \mathrm{a}$ & $\begin{array}{l}\text { WTP legacy design capability (not used } \\
\text { in the model) }\end{array}$ \\
\hline
\end{tabular}




\begin{tabular}{|l|l|l|l|}
\hline Chem Add & Composition & Flowrate & Description \\
\hline SPR-MXR-00001 & $1 \mathrm{M} \mathrm{Nap}\left(\mathrm{MnpO}_{4}\right)$ & $\mathrm{n} / \mathrm{a}$ & $\begin{array}{l}\text { WTP legacy design capability (not used } \\
\text { in the model) }\end{array}$ \\
\hline
\end{tabular}

Table B-4 Chemical Reagents for UFP-VSL-00001A/B

\begin{tabular}{|l|l|l|l|}
\hline Chem Add & Composition & Flowrate & Description \\
\hline Antifoam Agent & $\begin{array}{l}0.034 \mathrm{M} \text { PPG } \\
0.003 \mathrm{M} \text { PDMS }\end{array}$ & $50 \mathrm{gpm}$ & $\begin{array}{l}\text { Mitigates foaming in process vessels, } \\
\text { Target concentrations: } \\
\text { PPG }=175 \mathrm{ppm} \\
\text { PDMS }=175 \mathrm{ppm}\end{array}$ \\
\hline STEAM & $\mathrm{H}_{2} \mathrm{O}$ & variable gpm & $\begin{array}{l}\text { Utilized for heating, } \\
\text { volume = batch size dependent }\end{array}$ \\
\hline DIW-TK-00001 & $\mathrm{H}_{2} \mathrm{O}$ & $73 \mathrm{gpm}$ & Reserved capability for sodium dilution \\
\hline SHR-TK-00006 & $19 \mathrm{M} \mathrm{NaOH}$ & $50 \mathrm{gpm}$ & Reserved capability for caustic leaching \\
\hline
\end{tabular}

Table B-5 Chemical Reagents for UFP-VSL-00002A/B

\begin{tabular}{|c|c|c|c|}
\hline Chem Add & Composition & Flowrate & Description \\
\hline Antifoam Agent & $\begin{array}{l}0.034 \mathrm{M} \text { PPG } \\
0.003 \mathrm{M} \text { PDMS }\end{array}$ & $50 \mathrm{gpm}$ & $\begin{array}{l}\text { Mitigate foaming in process vessels, } \\
\text { Target concentrations: } \\
\text { PPG }=175 \mathrm{ppm} \\
\text { PDMS }=175 \mathrm{ppm}\end{array}$ \\
\hline SHR-TK-00006 & $19 \mathrm{M} \mathrm{NaOH}$ & $50 \mathrm{gpm}$ & $\begin{array}{l}\text { Utilized for caustic leaching and } \\
\text { aluminum solubility, } \\
\text { volume = batch size and waste } \\
\text { composition dependent }\end{array}$ \\
\hline STEAM & $\mathrm{H}_{2} \mathrm{O}$ & variable gpm & $\begin{array}{l}\text { Utilized for heating and reaction } \\
\text { temperature maintaining, } \\
\text { volume = batch size dependent }\end{array}$ \\
\hline $\begin{array}{l}\text { OXIDATIVE-LEACH- } \\
\text { CAUSTIC }\end{array}$ & 2M NapOH & $87.5 \mathrm{gpm}$ & $\begin{array}{l}\text { Used for oxidative leach, } \\
\text { volume }=\text { batch size and composition } \\
\text { dependent, endpoint }=0.2 \mathrm{M}[\mathrm{OH}-]\end{array}$ \\
\hline SPR-MXR-00001 & $1 \mathrm{M} \operatorname{Nap}\left(\mathrm{MnpO}_{4}\right)$ & $22 \mathrm{gpm}$ & $\begin{array}{l}\text { Used for oxidative leach, } \\
\text { volume }=\text { calculated, endpoint }=(\mathrm{MnO} 4- \\
\text { to solid chromium mole ratio equal to } \\
1.1)\end{array}$ \\
\hline NAR-TK-00006 & $2 \mathrm{M} \mathrm{HNO}_{3}$ & $87.5 \mathrm{gpm}$ & $\begin{array}{l}\text { Used for acid cleaning, } \\
\text { volume }=17,076 \mathrm{gal}\end{array}$ \\
\hline $\begin{array}{l}\text { NEUTRALIZATION- } \\
\text { CAUSTIC }\end{array}$ & 19M NapOH & $87.5 \mathrm{gpm}$ & $\begin{array}{l}\text { Used for neutralizing acids from filter } \\
\text { cleaning, volume }=50 \text { gal }\end{array}$ \\
\hline DIW-TK-00001 & $\mathrm{H}_{2} \mathrm{O}$ & $73 \mathrm{gpm}$ & $\begin{array}{l}\text { Secondary source for washing. } \\
\text { Primary source is process condensate } \\
\text { from RLD-TK-00006A }\end{array}$ \\
\hline INHIBITED-WATER & $\begin{array}{l}0.1 \mathrm{M} \mathrm{NapOH} \\
0.011 \mathrm{M} \mathrm{NapNO}_{2} \\
\end{array}$ & $73 \mathrm{gpm}$ & $\begin{array}{l}\text { Power flush, UFP1 to UFP2 transfer line } \\
\text { flush }\end{array}$ \\
\hline UFP-02A-P2-NAOH & $0.1 \mathrm{M} \mathrm{NapOH}$ & $87.5 \mathrm{gpm}$ & $\begin{array}{l}\text { Post isolation valve pipe }=1,576 \text { gal } \\
\text { initially filled before startup }\end{array}$ \\
\hline
\end{tabular}


Table B-6 Chemical Reagents for Super Tank

\begin{tabular}{|l|l|l|l|}
\hline Chem Add & Composition & Flowrate & Description \\
\hline SHR-TK-00006 & $19 \mathrm{M} \mathrm{NaOH}$ & $50 \mathrm{gpm}$ & $\begin{array}{l}\text { Used to prevent aluminate -> gibbsite } \\
\text { reaction, volume }=\text { waste composition } \\
\text { dependent }\end{array}$ \\
\hline DIW-TK-00001 & $\mathrm{H}_{2} \mathrm{O}$ & $73 \mathrm{gpm}$ & $\begin{array}{l}\text { Secondary source for sodium dilution, } \\
\text { dilution endpoint }=8 \mathrm{M}\left[\mathrm{Na}^{+}\right] . \\
\text {Primary source is process condensate } \\
\text { from RLD-TK-00006A }\end{array}$ \\
\hline Steam & $\mathrm{H}_{2} \mathrm{O}$ & variable gpm & Heating \\
\hline
\end{tabular}

\section{CESIUM REMOVAL USING ION EXCHANGE PROCESS SYSTEM}

Table B-7 Chemical Reagents for CXP

\begin{tabular}{|l|l|l|l|}
\hline Chem Add & Composition & Flowrate & Description \\
\hline $\begin{array}{l}\text { SHR-TK-00005 to } \\
\text { CXP-VSL-00005 }\end{array}$ & $0.1 \mathrm{M} \mathrm{NapOH}$ & $5 \mathrm{gpm}$ & $\begin{array}{l}\text { Replenish CXP-VSL-00005 for } \\
\text { displacement }\end{array}$ \\
\hline DIW-IXC-RINSE-1 & $\mathrm{H}_{2} \mathrm{O}$ & $30 \mathrm{gpm}$ & Pre-elution rinse, volume $=2 \mathrm{cv}$ \\
\hline $\begin{array}{l}\text { NAR-TK-00006 through } \\
\text { CNP-VSL-00004 }\end{array}$ & $0.45 \mathrm{M} \mathrm{HNO}{ }_{3}$ & $\begin{array}{l}10 \mathrm{gpm} \\
30 \mathrm{gpm}\end{array}$ & $\begin{array}{l}\text { Elution, volume }=15 \mathrm{cv} \\
\text { Resin outlet flush, volume }=21 \mathrm{gal}\end{array}$ \\
\hline DIW-IXC-RINSE-2 & $\mathrm{H}_{2} \mathrm{O}$ & $10 \mathrm{gpm}$ & Post-elution rinse, volume $=2.5 \mathrm{cv}$ \\
\hline $\begin{array}{l}\text { SHR-TK-00005 to } \\
\text { CXP-IXC-00001/2/3/4 }\end{array}$ & $0.5 \mathrm{M} \mathrm{NapOH}$ & $27.8 \mathrm{gpm}$ & Regeneration, volume $=4.292 \mathrm{cv}$ \\
\hline IX-Caustic & $0.5 \mathrm{M} \mathrm{NapOH}$ & $30 \mathrm{gpm}$ & Resin outlet flush, volume $=21 \mathrm{gal}$ \\
\hline IX-DI-Water & $\mathrm{H}_{2} \mathrm{O}$ & $30 \mathrm{gpm}$ & Resin outlet flush, volume $=21 \mathrm{gal}$ \\
\hline $\begin{array}{l}\text { SHR-TK-00005 to } \\
\text { CXP-FILT-00001 }\end{array}$ & $0.1 \mathrm{M} \mathrm{NapOH}$ & $5 \mathrm{gpm}$ & Guard filter backflush, volume $=80 \mathrm{gal}$ \\
\hline
\end{tabular}

$1 \mathrm{cv}=600$ gallons

\section{CESIUM NITRIC ACID RECOVERY PROCESS SYSTEM}

Table B-8 Chemical Reagents for CNP

\begin{tabular}{|l|l|l|l|}
\hline Chem Add & Composition & Flowrate & Description \\
\hline NAR-TK-00006 & $0.45 \mathrm{M} \mathrm{HNO}_{3}$ & $87.5 \mathrm{gpm}$ & $\begin{array}{l}\text { Replenish CNP-VSL-00004 for IX } \\
\text { elusion and resin outlet flush }\end{array}$ \\
\hline NAR-TK-00005 & $5 \mathrm{M} \mathrm{HNO}_{3}$ & $10 \mathrm{gpm}$ & $\begin{array}{l}\text { Volume }=2,128 \text { gallons initially before } \\
\text { startup and after each evaporator } \\
\text { concentrate discharge }\end{array}$ \\
\hline
\end{tabular}




\section{CESIUM FRESH RESIN ADDITION/SPENT RESIN COLLECTION AND DEWATERING PROCESS SYSTEMS}

Table B-9 Chemical Reagents for CRP/RDP

\begin{tabular}{|l|l|l|l|}
\hline Chem Add & Composition & Flowrate & Description \\
\hline DIW-TK-00004 & $\mathrm{H}_{2} \mathrm{O}$ & $20 \mathrm{gpm}$ & $\begin{array}{l}\text { Fresh resin preparation, } \\
\text { volume }=180 \text { gal }\end{array}$ \\
\hline NAR-MXR-00003 & $0.5 \mathrm{M} \mathrm{HNO}_{3}$ & $40 \mathrm{gpm}$ & $\begin{array}{l}\text { WTP legacy design capability (not used } \\
\text { in the model) }\end{array}$ \\
\hline SHR-TK-00004 & $2 \mathrm{M} \mathrm{NapOH}$ & $20 \mathrm{gpm}$ & $\begin{array}{l}\text { Fresh resin preparation, } \\
\text { volume }=750 \text { gal }\end{array}$ \\
\hline RF-RESIN & $\mathrm{R}-\mathrm{H}$ & $600 \mathrm{gal} / \mathrm{batch}$ & $\begin{array}{l}\text { Hydrogen ion attached to fresh RF resin, } \\
\mathrm{H}^{+}=4.63 \mathrm{~kg} \text {-mole }\end{array}$ \\
\hline $\begin{array}{l}\text { RDP-VSL-00002A- } \\
\text { CAUSTIC }\end{array}$ & $0.25 \mathrm{M} \mathrm{NapOH}$ & $140 \mathrm{gpm}$ & $\begin{array}{l}\text { Spent resin flush solution, initially } \\
\text { before startup, volume }=7,500 \text { gallons }\end{array}$ \\
\hline
\end{tabular}

\section{TREATED LAW EVAPORATION/CONCENTRATE STORAGE PROCESS SYSTEMS}

Table B-10 Chemical Reagents for TLP/TCP

\begin{tabular}{|l|l|l|l|}
\hline Chem Add & Composition & Flowrate & Description \\
\hline Antifoam Agent & $\begin{array}{l}0.034 \mathrm{M} \text { PPG } \\
0.003 \mathrm{M} \text { PDMS }\end{array}$ & $50 \mathrm{gpm}$ & $\begin{array}{l}\text { Mitigates foaming in process vessels, } \\
\text { Target concentrations: } \\
\text { PPG }=250 \mathrm{ppm} \\
\text { PDMS }=250 \mathrm{ppm}\end{array}$ \\
\hline SHR-TK-00007 & $5 \mathrm{M} \mathrm{NapOH}$ & $30 \mathrm{gpm}$ & $\begin{array}{l}\text { Used for } \mathrm{pH} \text { adjustment, target } \mathrm{pH}=12, \\
\text { Volume }=\text { variable }\end{array}$ \\
\hline TLP-DMST-00001-AIR & $\begin{array}{l}\text { Air }(0.0012 \mathrm{wt} \% \\
\mathrm{H}_{2} \mathrm{O}, 76.7 \mathrm{wt}_{0} \% \mathrm{~N}_{2},\end{array}$ & $\begin{array}{l}5 \mathrm{ft}^{3} / \mathrm{min} \\
\mathrm{T}=70^{\circ} \mathrm{F}, \mathrm{P}= \\
14.7 \mathrm{psi}\end{array}$ & Pump when evaporator boils off \\
\hline $\begin{array}{l}\left.23.3 \mathrm{wt} \% \mathrm{O}_{2}\right) \\
\text { TLP-INTER-AFTER-CON }\end{array}$ & $\mathrm{H}_{2} \mathrm{O}$ & $0.96 \mathrm{gpm}$ & Pump when evaporator boils off \\
DENSER-WATER & & & \\
\hline
\end{tabular}

\section{PRETREATMENT VESSEL VENT PROCESS SYSTEM}

Table B-11 Chemical Reagents for PVP/PJV

\begin{tabular}{|c|c|c|c|}
\hline Chem Add & Composition & Flowrate & Description \\
\hline AIR-VENT-LUMP-SUM & $\begin{array}{l}\text { Air }(0.0012 \text { wt } \% \\
\mathrm{H}_{2} \mathrm{O}, 76.7 \text { wt } \% \mathrm{~N}_{2}, \\
\left.23.3 \text { wt } \% \mathrm{O}_{2}\right)\end{array}$ & $\begin{array}{l}1513 \mathrm{ft}^{3} / \mathrm{min} \\
\mathrm{T}=77^{\circ} \mathrm{F}, \mathrm{P}= \\
14.7 \mathrm{psi}\end{array}$ & Continuous \\
\hline $\begin{array}{l}\text { UFV-HLP-3HR- } \\
\text { ROTATION }\end{array}$ & $\begin{array}{l}\text { Air }(0.0012 \text { wt } \% \\
\mathrm{H}_{2} \mathrm{O}, 76.7 \text { wt } \% \mathrm{~N}_{2} \\
\left.23.3 \text { wt } \% \mathrm{O}_{2}\right)\end{array}$ & $\begin{array}{l}\text { varies (initial } \\
\text { value }=2001.9 \\
\mathrm{ft}^{3} / \mathrm{min} \text { ) } \\
\mathrm{T}=77^{\circ} \mathrm{F}, \mathrm{P}= \\
14.7 \mathrm{psi}\end{array}$ & $\begin{array}{l}\text { Rotational flowrate is used from hour } 1 \\
\text { to hour } 2 \text { to hour } 3\end{array}$ \\
\hline SHR-TK-00007 & $0.01 \mathrm{M} \mathrm{NapOH}$ & $70 \mathrm{gpm}$ & $\begin{array}{l}\text { Fill to set volume after PVP-SCB-00002 } \\
\text { is discharged }\end{array}$ \\
\hline $\begin{array}{l}\text { DIW-TK-00004 to PJV- } \\
\text { VSL-00001 }\end{array}$ & $\mathrm{n} / \mathrm{a}$ & $\mathrm{n} / \mathrm{a}$ & Chemical addition not modeled \\
\hline
\end{tabular}




\begin{tabular}{|l|l|l|l|}
\hline Chem Add & Composition & Flowrate & Description \\
\hline $\begin{array}{l}\text { DIW-TK-00004 to PVP- } \\
\text { HEME-00001 }\end{array}$ & $\mathrm{H}_{2} \mathrm{O}$ & $0.133 \mathrm{gpm}$ & Continuous \\
\hline
\end{tabular}

\section{RADIOACTIVE LIQUID DISPOSAL SYSTEM - Pretreatment}

None

\section{HLW LAG STORAGE AND FEED BLENDING PROCESS SYSTEM}

Table B-12 Chemical Reagents for HLP

\begin{tabular}{|l|l|l|l|}
\hline Chem Add & Composition & Flowrate & Description \\
\hline Antifoam Agent & $\begin{array}{l}0.034 \mathrm{M} \text { PPG } \\
0.003 \mathrm{M} \text { PDMS }\end{array}$ & $50 \mathrm{gpm}$ & $\begin{array}{l}\text { Mitigates foaming in process vessels, } \\
\text { Target concentrations: } \\
\text { PPG }=175 \mathrm{ppm} \\
\text { PDMS }=175 \mathrm{ppm}\end{array}$ \\
\hline INHIBITED-WATER & $\begin{array}{l}0.1 \mathrm{M} \mathrm{NapOH} \\
0.011 \mathrm{M} \mathrm{NapNO}{ }_{2}\end{array}$ & $73 \mathrm{gpm}$ & Line flush after solid discharge \\
\hline HLP14 & $19 \mathrm{M} \mathrm{NapOH}$ & $50 \mathrm{gpm}$ & $\begin{array}{l}\text { Used for neutralizing CNP evaporator } \\
\text { bottoms, volume }=\left(\mathrm{OH}^{-} \text {to } \mathrm{H}^{+} \mathrm{mole} \mathrm{ratio}\right.\end{array}$ \\
\hline
\end{tabular}

\section{PLANT WASH AND DISPOSAL SYSTEM}

Table B-13 Chemical Reagents for PWD

\begin{tabular}{|l|l|l|l|}
\hline Chem Add & Composition & Flowrate & Description \\
\hline INHIBITED-WATER & $\begin{array}{l}0.1 \mathrm{M} \mathrm{NapOH} \\
0.011 \mathrm{M} \mathrm{NapNO}\end{array}$ & $73 \mathrm{gpm}$ & Line flush from Tank Farm \\
\hline $\begin{array}{l}\text { SHR-TK-00006 to PWD- } \\
\text { VSL-00015/00016/00044 }\end{array}$ & $19 \mathrm{M} \mathrm{NapOH}$ & $26.4 \mathrm{gpm}$ & Volume = variable until pH $>=12$ \\
\hline
\end{tabular}

\section{LAW CONCENTRATE/FEED PROCESS SYSTEMS (LCP/LFP)}

Table B-14 Chemical Reagents for LCP/LFP

\begin{tabular}{|l|l|l|l|}
\hline Chem Add & Composition & Flowrate & Description \\
\hline RHEOLOGY-WATER & $\mathrm{H}_{2} \mathrm{O}$ & $100 \mathrm{gpm}$ & $\begin{array}{l}\text { Volume }=\text { variable until } 36 \mathrm{wt}_{0} \mathrm{H}_{2} \mathrm{O} \text { in } \\
\text { the LAW MFPVs }\end{array}$ \\
\hline
\end{tabular}




\section{LAW MELTER PROCESS AND LAW PRIMARY OFFGAS PROCESS SYSTEMS (LMP/LOP)}

Table B-15 Chemical Reagents for LMP/LOP

\begin{tabular}{|l|l|l|l|}
\hline Chem Add & Composition & Flowrate & Description \\
\hline LMP-MLTR-00001-AIR & Air $(0.0012 \mathrm{wt} \%$ & $\begin{array}{l}946 \mathrm{ft}^{3} / \mathrm{min} \\
\mathrm{T}=68^{\circ} \mathrm{F}, \mathrm{P}= \\
14.7 \mathrm{psi}_{2} \mathrm{O}, 76.7 \mathrm{wt} \% \mathrm{~N}_{2}, \\
\left.23.3 \mathrm{wt} \% \mathrm{O}_{2}\right)\end{array}$ & Continuously after melter starts, \\
\hline LMP-MLTR-00002-AIR & $\begin{array}{l}\text { Air }(0.0012 \mathrm{wt} \% \\
\mathrm{H}_{2} \mathrm{O}, 76.7 \mathrm{wt} \% \mathrm{~N}_{2}, \\
\left.23.3 \mathrm{wt} \% \mathrm{O}_{2}\right)\end{array}$ & $\begin{array}{l}946 \mathrm{ft}^{3} / \mathrm{min} \\
\mathrm{T}=68^{\circ} \mathrm{F}, \mathrm{P}= \\
14.7 \mathrm{psi}\end{array}$ & Continuously after melter starts, \\
\hline $\begin{array}{l}\text { LMP-MLTR-00001- } \\
\text { WATER }\end{array}$ & $\mathrm{H}_{2} \mathrm{O}$ & $0.007 \mathrm{gpm}$ & $\begin{array}{l}\text { Film cooler water rinse, continuously } \\
\text { after melter starts }\end{array}$ \\
\hline $\begin{array}{l}\text { LMP-MLTR-00002- } \\
\text { WATER }\end{array}$ & $\mathrm{H}_{2} \mathrm{O}$ & $0.007 \mathrm{gpm}$ & $\begin{array}{l}\text { Film cooler water rinse, continuously } \\
\text { after melter starts }\end{array}$ \\
\hline LOP-SCB-00001-WATER & $\mathrm{H}_{2} \mathrm{O}$ & $30 \mathrm{gpm}$ & $\begin{array}{l}\text { Volume }=451 \text { gal (SBS Condensate } \\
\text { Vessel) initially before startup }\end{array}$ \\
\hline LOP-SCB-00002-WATER & $\mathrm{H}_{2} \mathrm{O}$ & $30 \mathrm{gpm}$ & $\begin{array}{l}\text { Volume }=451 \text { gal (SBS Condensate } \\
\text { Vessel) initially before startup }\end{array}$ \\
\hline LOP09 & $\mathrm{H}_{2} \mathrm{O}$ & $0.75 \mathrm{gpm}$ & Continuous \\
\hline
\end{tabular}

\section{LAW SECONDARY OFFGAS PROCESS SYSTEM (LVP)}

Table B-16 Chemical Reagents for LVP

\begin{tabular}{|c|c|c|c|}
\hline Chem Add & Composition & Flowrate & Description \\
\hline $\begin{array}{l}\text { LAW- } \\
\text { VESSEL-VENT-AIR }\end{array}$ & $\begin{array}{l}74.7 \text { mole } \% \mathrm{~N}_{2} \text {, } \\
19.9 \text { mole } \% \mathrm{O}_{2} \text {, and } \\
5.4 \text { mole } \% \mathrm{H}_{2} \mathrm{O}\end{array}$ & $\begin{array}{l}368 \mathrm{ft}^{3} / \mathrm{min} \\
\mathrm{T}=118.3^{\circ} \mathrm{F} \\
\mathrm{P}=14.7 \mathrm{psi}\end{array}$ & Continuous, \\
\hline AMR-VSL-00001-2-NH3 & $\mathrm{NH}_{3}$ gas & $\begin{array}{l}0.5 \mathrm{ft}^{3} / \mathrm{min} \\
\mathrm{T}=100^{\circ} \mathrm{F} \\
\mathrm{P}=100 \mathrm{psi}\end{array}$ & $\begin{array}{l}\text { Variable quantity depending on the } \\
\text { kg-moles of } \mathrm{NO}, \mathrm{NO}_{2} \text {, and } \mathrm{NH}_{3} \text { in the } \\
\text { offgas stream. }\end{array}$ \\
\hline AMR-VSL-00001-2-AIR & $\begin{array}{l}74.7 \text { mole } \% \mathrm{~N}_{2}, \\
19.9 \text { mole } \% \mathrm{O}_{2} \text {, and } \\
5.4 \text { mole } \% \mathrm{H}_{2} \mathrm{O}\end{array}$ & $\begin{array}{l}700 \mathrm{ft}^{3} / \mathrm{min} \\
\mathrm{T}=100^{\circ} \mathrm{F} \\
\mathrm{P}=100 \mathrm{psi}\end{array}$ & $\begin{array}{l}\text { Variable quantity depending on the } \\
\text { kg-moles of } \mathrm{NO}, \mathrm{NO}_{2} \text {, and } \mathrm{NH}_{3} \text { in the } \\
\text { offgas stream. }\end{array}$ \\
\hline
\end{tabular}

\section{LAW RADIOACTIVE LIQUID DISPOSAL}

Table B-17 Chemical Reagents for LAW RLD

\begin{tabular}{|l|l|l|l|}
\hline Chem Add & Composition & Flowrate & Description \\
\hline LVP-VSL-00001-NAOH & $5 \mathrm{M} \mathrm{NaOH}$ & $20 \mathrm{gpm}$ & $\begin{array}{l}\text { Filled to half way between set volume } \\
\text { (6,032 gal) and minimum volume }(2,032 \\
\text { gal) with } \mathrm{NaOH} \text { and } \mathrm{H}_{2} \mathrm{O} \text { to } \mathrm{pH}=9.0 \\
\text { initially, 20 gallons are added } \\
\text { incrementally after to keep } \mathrm{pH}>=9.0\end{array}$ \\
\hline LVP-VSL-00001-WATER & $\mathrm{H}_{2} \mathrm{O}$ & $22 \mathrm{gpm}$ & $\begin{array}{l}\text { Filled to half way between set volume } \\
\text { (6,032 gal) and minimum volume }(2,032 \\
\text { gal) with NaOH and } \mathrm{H}_{2} \mathrm{O} \text { to } \mathrm{pH}=9.0 \\
\text { initially, enough volume will added to } \\
\text { keep SpG }<1.1\end{array}$ \\
\hline
\end{tabular}




\section{HLW FEED PROCESS SYSTEMS}

Table B-18 Chemical Reagents for HFP

\begin{tabular}{|l|l|l|l|}
\hline Chem Add & Composition & Flowrate & Description \\
\hline RHEOLOGY-HLW & $\mathrm{H}_{2} \mathrm{O}$ & $100 \mathrm{gpm}$ & $\begin{array}{l}\text { Volume = variable, target oxide } \\
\text { concentration }=550 \mathrm{~g} / \mathrm{liter} \text { in the HLW } \\
\text { MFPVs }\end{array}$ \\
\hline
\end{tabular}

\section{HLW MELTER PROCESS/HLW PRIMARY OFFGAS PROCESS SYSTEMS}

Table B-19 Chemical Reagents for HMP/HOP

\begin{tabular}{|c|c|c|c|}
\hline Chem Add & Composition & Flowrate & Description \\
\hline HMP-MLTR-00001-AIR & $\begin{array}{l}\text { Combined wet and } \\
\text { dry air }(0.78 \text { mole } \% \\
\mathrm{H}_{2} \mathrm{O}, 78.4{\text { mole } \% \mathrm{~N}_{2}}_{2} \\
20.8{\left.\text { mole } \% \mathrm{O}_{2}\right)}\end{array}$ & $\begin{array}{l}711.5 \mathrm{ft}^{3} / \mathrm{min} \\
\mathrm{T}=68^{\circ} \mathrm{F} \\
\mathrm{P}=14.696 \mathrm{psi}\end{array}$ & $\begin{array}{l}\text { Air to HLW melter, } \\
\text { continuous }\end{array}$ \\
\hline HMP-MLTR-00002-AIR & $\begin{array}{l}\text { Combined wet and } \\
\text { dry air }(0.78 \text { mole } \% \\
\mathrm{H}_{2} \mathrm{O}, 78.4{\text { mole } \% \mathrm{~N}_{2}}_{2} \\
\left.20.8 \text { mole } \% \mathrm{O}_{2}\right)\end{array}$ & $\begin{array}{l}711.5 \mathrm{ft}^{3} / \mathrm{min} \\
\mathrm{T}=68^{\circ} \mathrm{F} \\
\mathrm{P}=14.696 \mathrm{psi}\end{array}$ & $\begin{array}{l}\text { Air to HLW melter, } \\
\text { continuous }\end{array}$ \\
\hline HOP08-1 & $\mathrm{H}_{2} \mathrm{O}$ & $30 \mathrm{gpm}$ & $\begin{array}{l}\text { Volume }=1,758 \text { gal to SBS Condensate } \\
\text { vessel initially before startup. Added to } \\
\text { SBS at minimum volume as needed if } \\
\text { volume goes below } 1,758 \text { gal due to } \\
\text { evaporation }\end{array}$ \\
\hline HOP08-2 & $\mathrm{H}_{2} \mathrm{O}$ & $30 \mathrm{gpm}$ & $\begin{array}{l}\text { Volume }=1,758 \text { gal to SBS Condensate } \\
\text { vessel initially before startup. Added to } \\
\text { SBS at minimum volume as needed if } \\
\text { volume goes below } 1,758 \text { gal due to } \\
\text { evaporation }\end{array}$ \\
\hline $\begin{array}{l}\text { DIW-TK-00002 to HOP- } \\
\text { WESP-00001/00002 }\end{array}$ & $\mathrm{H}_{2} \mathrm{O}$ & $0.42 \mathrm{gpm}$ & Continuous \\
\hline $\begin{array}{l}\text { HLW-VESSEL-VENT-1- } \\
\text { AIR }\end{array}$ & $\begin{array}{l}79 \text { mole } \% \mathrm{~N}_{2}, \\
21 \mathrm{~mole}^{2} \mathrm{O}_{2} \text {, and } \\
0.002 \mathrm{~mole} \% \mathrm{H}_{2} \mathrm{O}\end{array}$ & $131.5 \mathrm{ft}^{3} / \mathrm{min}$ & $\begin{array}{l}\text { Continuous } \\
\mathrm{T}=95^{\circ} \mathrm{F} \\
\mathrm{P}=14.7 \mathrm{psi}\end{array}$ \\
\hline $\begin{array}{l}\text { HLW-VESSEL-VENT-2- } \\
\text { AIR }\end{array}$ & $\begin{array}{l}79 \text { mole } \% \mathrm{~N}_{2}, \\
21 \text { mole } \% \mathrm{O}_{2} \text {, and } \\
0.002 \text { mole } \% \mathrm{H}_{2} \mathrm{O}\end{array}$ & $131.5 \mathrm{ft}^{3} / \mathrm{min}$ & $\begin{array}{l}\text { Continuous } \\
\mathrm{T}=95^{\circ} \mathrm{F} \\
\mathrm{P}=14.7 \mathrm{psi}\end{array}$ \\
\hline $\begin{array}{l}\text { DIW-TK-00002 to HOP- } \\
\text { HEME-00001/00002 }\end{array}$ & $\mathrm{H}_{2} \mathrm{O}$ & $0.15 \mathrm{gpm}$ & Continuous \\
\hline HOP-SCR-00001-NH3 & $\mathrm{NH}_{3}$ gas & $\begin{array}{l}1 \mathrm{ft}^{3} / \mathrm{min} \\
\mathrm{T}=70^{\circ} \mathrm{F} \\
\mathrm{P}=100 \mathrm{psi}\end{array}$ & $\begin{array}{l}\text { Variable quantity depending on the } \\
\text { kg-moles of } \mathrm{NO}, \mathrm{NO}_{2} \text {, and } \mathrm{NH}_{3} \text { in the } \\
\text { offgas stream. }\end{array}$ \\
\hline HOP-SCR-00001-AIR & $\begin{array}{l}74.7 \text { mole } \% \mathrm{~N}_{2}, \\
19.9 \text { mole } \% \mathrm{O}_{2} \text {, and } \\
5.4 \text { mole } \% \mathrm{H}_{2} \mathrm{O}\end{array}$ & $\begin{array}{l}200 \mathrm{ft}^{3} / \mathrm{min} \\
\mathrm{T}=70^{\circ} \mathrm{F} \\
\mathrm{P}=100 \mathrm{psi}\end{array}$ & $\begin{array}{l}\text { Variable quantity depending on the } \\
\text { kg-moles of } \mathrm{NO}, \mathrm{NO}_{2} \text {, and } \mathrm{NH} 3 \text { in the } \\
\text { offgas stream. }\end{array}$ \\
\hline HOP-SCR-00002-NH3 & $\mathrm{NH}_{3}$ gas & $\begin{array}{l}1 \mathrm{ft}^{3} / \mathrm{min} \\
\mathrm{T}=70^{\circ} \mathrm{F} \\
\mathrm{P}=100 \mathrm{psi}\end{array}$ & $\begin{array}{l}\text { Variable quantity depending on the } \\
\text { kg-moles of } \mathrm{NO}, \mathrm{NO}_{2} \text {, and } \mathrm{NH}_{3} \text { in the } \\
\text { offgas stream. }\end{array}$ \\
\hline
\end{tabular}




\begin{tabular}{|l|l|l|l|}
\hline Chem Add & Composition & Flowrate & Description \\
\hline HOP-SCR-00002-AIR & 74.7 mole $\% \mathrm{~N}_{2}$, & $200 \mathrm{ft}^{3} / \mathrm{min}$, & Variable quantity depending on the \\
& 19.9 mole $\% \mathrm{O}_{2}$, and & $\mathrm{T}=70 \% \mathrm{~F}$, & kg-moles of $\mathrm{NO}_{2} \mathrm{NO}_{2}$, and $\mathrm{NH}_{3}$ in the \\
& 5.4 mole $\% \mathrm{H}_{2} \mathrm{O}$ & $\mathrm{P}=100 \mathrm{psi}$ & offgas stream. \\
\hline
\end{tabular}

\section{HLW CANISTER DECONTAMINATION HANDLING SYSTEM}

Table B-20 Chemical Reagents for HDH

\begin{tabular}{|c|c|c|c|}
\hline Chem Add & Composition & Flowrate & Description \\
\hline $\begin{array}{l}\text { HDH-VSL-00001- } \\
\text { WATER }\end{array}$ & $\mathrm{H}_{2} \mathrm{O}$ & $90 \mathrm{gpm}$ & $\begin{array}{l}\text { Rinse } 1, \text { volume }=180 \mathrm{gal} \\
\text { Rinse } 2, \text { volume }=180 \mathrm{gal}\end{array}$ \\
\hline NAR-TK-00001 $1^{(\mathrm{a})}$ & $1.001 \mathrm{M} \mathrm{HNO}_{3}$ & $32 \mathrm{gpm}$ & $\begin{array}{l}\text { Decon volume }=194 \text { gal } \\
\text { Acid rinse volume }=201 \mathrm{gal}\end{array}$ \\
\hline HDH-TK-00002 (a) & $0.5 \mathrm{M} \mathrm{Ce}\left(\mathrm{NO}_{3}\right)_{3}$ & $3.2 \mathrm{gpm}$ & Decon, volume $=20$ gal \\
\hline DIW-TK-00002 (a) & $\mathrm{H}_{2} \mathrm{O}$ & $33 \mathrm{gpm}$ & Canister spray volume $=200 \mathrm{gal}$ \\
\hline SHR-TK-00002 & $5 \mathrm{M} \mathrm{NaOH}$ & 2 gpm & Neutralization volume $=83$ gal \\
\hline HPS & $\mathrm{H}_{2} \mathrm{O}$ & $\begin{array}{l}\text { (initially set to } \\
0.0 \mathrm{gpm} \text { ) } \\
0.27 \mathrm{gpm} \\
1 \mathrm{gpm} \\
1 \mathrm{gpm} \\
1 \mathrm{gpm}\end{array}$ & $\begin{array}{l}1 \mathrm{st} \text { use volume }=122 \mathrm{gal} \\
2 \mathrm{nd} \text { use volume }=5.6 \mathrm{gal} \\
3 \mathrm{rd} \text { use volume }=5.6 \mathrm{gal} \\
4 \text { th use volume }=5.6 \mathrm{gal}\end{array}$ \\
\hline HDH-VSL-00003-FLUSH & $\mathrm{H}_{2} \mathrm{O}$ & $31 \mathrm{gpm}$ & Not used \\
\hline HDH-TK-00003 & $1 \mathrm{M} \mathrm{H}_{2} \mathrm{O}_{2}$ & $0.08 \mathrm{gpm}$ & $\mathrm{Ce}^{+4}$ neutralization, volume $=0.5$ gal \\
\hline
\end{tabular}

(a) Chem add supplied to HDH-VSL-00002 and HDH-VSL-00004

\section{HLW RADIOACTIVE LIQUID DISPOSAL PROCESS SYSTEM}

Table B-21 Chemical Reagents for HLW RLD

\begin{tabular}{|l|l|l|l|}
\hline Chem Add & Composition & Flowrate & Description \\
\hline SHR-TK-00002 & $5 \mathrm{M} \mathrm{NapOH}$ & $20 \mathrm{gpm}$ & Volume $=$ variable until $\mathrm{pH}>=8$ \\
\hline
\end{tabular}

\section{GLASS FORMER FACILITY}

Table B-22 Glass Formers

\begin{tabular}{|l|l|l|l|}
\hline Chem Add & $\begin{array}{l}\text { Primary } \\
\text { Composition }\end{array}$ & Flowrate & Description \\
\hline BORAX & $\mathrm{B}_{2} \mathrm{O}_{3}$ & instant & Stockpile, contains mineral impurities \\
\hline BORIC-ACID & $\mathrm{B}_{2} \mathrm{O}_{3}$ & instant & Stockpile, contains mineral impurities \\
\hline IRON-OXIDE & $\mathrm{Fe}_{2} \mathrm{O}_{3}$ & instant & Stockpile, contains mineral impurities \\
\hline KYANITE & $\mathrm{Al}_{2} \mathrm{O}_{3}$ & instant & Stockpile, contains mineral impurities \\
\hline LITHIUM-CARBONATE & $\mathrm{Li}_{2} \mathrm{O}$ & instant & Stockpile, contains mineral impurities \\
\hline OLIVINE & $\mathrm{MgO}_{2}$ & instant & Stockpile, contains mineral impurities \\
\hline RUTILE & $\mathrm{TiO}_{2}$ & instant & Stockpile, contains mineral impurities \\
\hline
\end{tabular}




\begin{tabular}{|l|l|l|l|}
\hline Chem Add & $\begin{array}{l}\text { Primary } \\
\text { Composition }\end{array}$ & Flowrate & Description \\
\hline SILICA & $\mathrm{SiO}_{2}$ & instant & Stockpile, contains mineral impurities \\
\hline SODA-ASH & $\mathrm{Nap}_{2} \mathrm{O}$ & instant & Stockpile, contains mineral impurities \\
\hline SUGAR & $\mathrm{C}_{12} \mathrm{H}_{22} \mathrm{O}_{11}$ & instant & Stockpile, contains mineral impurities \\
\hline WOLLASTONITE & $\mathrm{CaO}$ & instant & Stockpile, contains mineral impurities \\
\hline ZINC-OXIDE & $\mathrm{ZnO}$ & instant & Stockpile, contains mineral impurities \\
\hline ZIRCON-SAND & $\mathrm{ZrSiO}_{4}$ & instant & Stockpile, contains mineral impurities \\
\hline DUSTING-WATER & $\mathrm{H}_{2} \mathrm{O}$ & $100 \mathrm{gpm}$ & Glass former mixer dusting \\
\hline
\end{tabular}

\section{CHEMICAL REAGENT INITIAL VOLUMES}

\section{Table B-23 Chemical Reagent Initial Volumes}

Liquid Reagents

\begin{tabular}{|l|l|r|}
\hline Chem Add Name & ChemAdd Alternate Name & Volume (gal) \\
\hline $\begin{array}{l}\text { FEPB-INTER-AFTER- } \\
\text { CONDENSER-WATER }\end{array}$ & FEPB-INTER-AFTER-CONDENSER-WATER & \\
\hline $\begin{array}{l}\text { FEPA-INTER-AFTER- } \\
\text { CONDENSER-WATER }\end{array}$ & FEPA-INTER-AFTER-CONDENSER-WATER & $9.99890 \mathrm{E}+07$ \\
\hline HDH-VSL-00003-FLUSH & & $9.99890 \mathrm{E}+07$ \\
\hline DIW-TK-00002 & V33002-STEAM-EJECTOR & $9.99890 \mathrm{E}+07$ \\
\hline HDH-TK-00002 & H2O-CHEM-ADD-1 & $9.99890 \mathrm{E}+07$ \\
\hline NAR-TK-00001 & DECON-CE4-CHEM-ADD-1 & $9.50573 \mathrm{E}+07$ \\
\hline HDH-TK-00003 & DECON-NITRIC-ACID-CHEM-ADD-1 & $9.78791 \mathrm{E}+07$ \\
\hline HPS & HYDROGEN-PEROXIDE & $9.89732 \mathrm{E}+07$ \\
\hline SHR-TK-00002 & V33002-STEAM-CONDENSATE & $9.99890 \mathrm{E}+07$ \\
\hline DIW-TK-00002 & V33002-NAOH-ADD & $9.43030 \mathrm{E}+07$ \\
\hline HDH-TK-00002 & H2O-CHEM-ADD & $9.99890 \mathrm{E}+07$ \\
\hline HDH-VSL-00001-WATER & DECON-CE4-CHEM-ADD & $9.50573 \mathrm{E}+07$ \\
\hline NAR-TK-00001 & BOGIE-RINSE-H2O & $9.99890 \mathrm{E}+07$ \\
\hline DIW-TK-00002 & DECON-NITRIC-ACID-CHEM-ADD & $9.78791 \mathrm{E}+07$ \\
\hline HOP08-2 & HEME-WATER-2 & $9.99890 \mathrm{E}+08$ \\
\hline DIW-TK-00002 & SBS-1-WATER-2 & $9.99890 \mathrm{E}+07$ \\
\hline DIW-TK-00002 & WESP-LIQUID-2 & $9.99890 \mathrm{E}+08$ \\
\hline HOP08-1 & HEME-WATER-1 & $9.99890 \mathrm{E}+08$ \\
\hline DIW-TK-00002 & SBS-1-WATER-1 & $9.99890 \mathrm{E}+07$ \\
\hline LMP-MLTR-00001-WATER & WESP-LIQUID-1 & $9.99890 \mathrm{E}+08$ \\
\hline LOP-SCB-00001-WATER & LAW-MELTER-WATER-1 & $9.99890 \mathrm{E}+07$ \\
\hline LOP09 & LAW-SBS-CHEM-ADD-1 & $9.99890 \mathrm{E}+07$ \\
\hline RHEOLOGY-HLW & LAW-WESP-CHEM-ADD-1 & $9.99890 \mathrm{E}+08$ \\
\hline LMP-MLTR-00002-WATER & RHEOLOGY-HLW & $9.99890 \mathrm{E}+07$ \\
\hline LOP-SCB-00002-WATER & LAW-MELTER-WATER-2 & $9.99890 \mathrm{E}+07$ \\
\hline LOP09 & LAW-SBS-CHEM-ADD-2 & $9.99890 \mathrm{E}+07$ \\
\hline LVP-VSL-00001-WATER & LAW-WESP-CHEM-ADD-2 & $9.99890 \mathrm{E}+08$ \\
\hline LVP-VSL-00001-NAOH & WATER-V22001 & $9.99890 \mathrm{E}+08$ \\
\hline SHR-TK-00007 & NAOH-V22001 & $9.43030 \mathrm{E}+07$ \\
\hline DIW-TK-00004 & PVP-CAUSTIC-SCRUBBER-CHEM-ADD & $1.00393 \mathrm{E}+08$ \\
\hline DIW-TK-00004 & PJV-DMST-WATER & $1.00321 \mathrm{E}+08$ \\
\hline & PVP-HEME-WATER & $9.99890 \mathrm{E}+07$ \\
\hline
\end{tabular}




\begin{tabular}{|c|c|c|}
\hline Chem Add Name & ChemAdd Alternate Name & Volume (gal) \\
\hline SHR-TK-00005 & FILTER-BACKFLUSH-CAUSTIC & $1.00001 \mathrm{E}+07$ \\
\hline SHR-TK-00001 & IX-CAUSTIC & $1.00024 \mathrm{E}+08$ \\
\hline IX-DI-WATER & IX-DI-WATER & $9.99890 \mathrm{E}+07$ \\
\hline SHR-TK-00005 & CAUSTIC-V13008 & $1.00001 \mathrm{E}+07$ \\
\hline SHR-TK-00005 & $\mathrm{C} 5-\mathrm{C} 13003$ & $1.00024 \mathrm{E}+08$ \\
\hline DIW-IXC-RINSE-2 & $\mathrm{C} 4-\mathrm{C} 13003$ & $9.99890 \mathrm{E}+07$ \\
\hline DIW-IXC-RINSE-1 & $\mathrm{C} 2-\mathrm{C} 13003$ & $9.99890 \mathrm{E}+07$ \\
\hline SHR-TK-00005 & $\mathrm{C} 5-\mathrm{C} 13004$ & $1.00024 \mathrm{E}+08$ \\
\hline DIW-IXC-RINSE-2 & C4-C13004 & $9.99890 \mathrm{E}+07$ \\
\hline DIW-IXC-RINSE-1 & $\mathrm{C} 2-\mathrm{C} 13004$ & $9.99890 \mathrm{E}+07$ \\
\hline SHR-TK-00005 & $\mathrm{C} 5-\mathrm{C} 13001$ & $1.00024 \mathrm{E}+08$ \\
\hline DIW-IXC-RINSE-2 & $\mathrm{C} 4-\mathrm{C} 13001$ & $9.99890 \mathrm{E}+07$ \\
\hline DIW-IXC-RINSE-1 & $\mathrm{C} 2-\mathrm{C} 13001$ & $9.99890 \mathrm{E}+07$ \\
\hline SHR-TK-00005 & $\mathrm{C} 5-\mathrm{C} 13002$ & $1.00024 \mathrm{E}+08$ \\
\hline DIW-IXC-RINSE-2 & $\mathrm{C} 4-\mathrm{C} 13002$ & $9.99890 \mathrm{E}+07$ \\
\hline DIW-IXC-RINSE-1 & $\mathrm{C} 2-\mathrm{C} 13002$ & $9.99890 \mathrm{E}+07$ \\
\hline SHR-TK-00002 & V35002-NAOH-ADD & $9.43030 \mathrm{E}+07$ \\
\hline SHR-TK-00006 & PWD-NEUTRALIZATION-CAUSTIC & $9.06675 \mathrm{E}+07$ \\
\hline NAR-TK-00005 & ACID-CHARGE & $9.23799 \mathrm{E}+07$ \\
\hline NAR-TK-00006 & V13028-CHEM-ADD & $9.90054 \mathrm{E}+07$ \\
\hline HLP14 & HLP14 & $9.06675 \mathrm{E}+07$ \\
\hline RHEOLOGY-WATER & RHEOLOGY-WATER & $9.99890 \mathrm{E}+07$ \\
\hline DUSTING-WATER & DUSTING-WATER & $9.99890 \mathrm{E}+07$ \\
\hline ANTIFOAM-AGENT & AFA & $1.03036 \mathrm{E}+07$ \\
\hline INHIBITED-WATER & INHIBITED-WATER & $2.08252 \mathrm{E}+08$ \\
\hline DIW-TK-00001 & DEMIN-WATER-CHEM-ADD & $9.99890 \mathrm{E}+07$ \\
\hline OXIDATIVE-LEACH-CAUSTIC & OXIDATIVE-LEACH-CAUSTIC & $9.72108 \mathrm{E}+07$ \\
\hline NEUTRALIZATION-CAUSTIC & NEUTRALIZATION-CAUSTIC & $9.06675 \mathrm{E}+07$ \\
\hline SHR-TK-00006 & UFP-CAUSTIC & $9.06674 \mathrm{E}+07$ \\
\hline SPR-MXR-00001 & SPR-MXR-00001 & $9.55658 \mathrm{E}+07$ \\
\hline UFP-02-P2-NAOH & NAOH-UFP-02B-P2 & $1.00001 \mathrm{E}+04$ \\
\hline UFP-02-P2-NAOH & NAOH-UFP-02A-P2 & $1.00001 \mathrm{E}+04$ \\
\hline STEAM & UFP-STEAM & $9.99890 \mathrm{E}+07$ \\
\hline STR-MXR-00001 & SRNITRATE-V12010B & $9.39463 \mathrm{E}+07$ \\
\hline SPR-MXR-00001 & NAMNO4-V12010B & $9.55663 \mathrm{E}+07$ \\
\hline STR-MXR-00001 & SRNITRATE-V12010A & $9.39463 \mathrm{E}+07$ \\
\hline SPR-MXR-00001 & NAMNO4-V12010A & $9.55663 \mathrm{E}+07$ \\
\hline NAR-TK-00006 & NAR-TK-00006 & $9.59799 \mathrm{E}+07$ \\
\hline SHR-TK-00007 & V45009B-CHEM-ADD & $9.43030 \mathrm{E}+07$ \\
\hline SHR-TK-00007 & V45009A-CHEM-ADD & $9.43030 \mathrm{E}+07$ \\
\hline $\begin{array}{l}\text { TLP-INTER-AFTER-CONDENSER- } \\
\text { WATER }\end{array}$ & TLP-INTER-AFTER-CONDENSER-WATER & $9.99890 \mathrm{E}+07$ \\
\hline DIW-TK-00004 & FRESH-RESIN-WATER & $9.99890 \mathrm{E}+07$ \\
\hline SHR-TK-00004 & FRESH-RESIN-CAUSTIC & $9.72108 \mathrm{E}+07$ \\
\hline RDP-VSL-00002A-CAUSTIC & CAUSTIC-RDP1 & $9.99361 \mathrm{E}+07$ \\
\hline
\end{tabular}


Gas Reagents

\begin{tabular}{|l|l|r|}
\hline Chem Add Name & ChemAdd Alternate Name & $\begin{array}{r}\text { Volume } \\
\text { (cubic feet) }\end{array}$ \\
\hline FEP-DMST-00001B-AIR & $\begin{array}{l}\text { V11002B-CONDENSER-COLLECTION-VENT- } \\
\text { GAS }\end{array}$ & $9.99999 \mathrm{E}+09$ \\
\hline FEP-DMST-00001A-AIR & $\begin{array}{l}\text { V11002A-CONDENSER-COLLECTION-VENT- } \\
\text { GAS }\end{array}$ & $9.99999 \mathrm{E}+09$ \\
\hline RFD-AIR & RFD-AIR & $1.00000 \mathrm{E}+15$ \\
\hline PSA & PJM-AIR & $1.00000 \mathrm{E}+15$ \\
\hline HOP-SCR-00002-AIR & HOP-SCR-00002-AIR & $1.47000 \mathrm{E}+14$ \\
\hline HOP-SCR-00002-NH3 & HOP-SCR-00002-NH3 & $1.47022 \mathrm{E}+10$ \\
\hline HLW-VESSEL-VENT-2-AIR & VESSEL-VENT-AIR-2 & $1.00000 \mathrm{E}+15$ \\
\hline HMP-MLTR-00002-AIR & HLW-MELTER-AIR-2 & $9.96589 \mathrm{E}+14$ \\
\hline HOP-SCR-00001-AIR & HOP-SCR-00001-AIR & $1.47000 \mathrm{E}+14$ \\
\hline HLW-VESSEL-VENT-1-AIR & VESSEL-VENT-AIR-1 & $1.00000 \mathrm{E}+15$ \\
\hline HOP-SCR-00001-NH3 & HOP-SCR-00001-NH3 & $1.47022 \mathrm{E}+10$ \\
\hline HMP-MLTR-00001-AIR & HLW-MELTER-AIR-1 & $9.96589 \mathrm{E}+14$ \\
\hline LMP-MLTR-00001-AIR & LAW-MELTER-AIR-1 & $9.96492 \mathrm{E}+14$ \\
\hline AMR-VSL-00001-2-AIR & AMR-VSL-00001-2-AIR & $1.32781 \mathrm{E}+11$ \\
\hline LAW-VESSEL-VENT-AIR & VESSEL-VENT-AIR & $9.32819 \mathrm{E}+11$ \\
\hline AMR-VSL-00001-2-NH3 & AMR-VSL-00001-2-NH3 & $1.99171 \mathrm{E}+09$ \\
\hline LMP-MLTR-00002-AIR & LAW-MELTER-AIR-2 & $9.96492 \mathrm{E}+14$ \\
\hline UFV-HLP-3HR-ROTATION & UFV-HLP-3HR-ROTATION & $1.00000 \mathrm{E}+15$ \\
\hline AIR-VENT-LUMP-SUM & AIR-VENT-LUMP-SUM & $1.00000 \mathrm{E}+15$ \\
\hline TLP-DMST-00001-AIR & V41011-CONDENSER-COLLECTION-VENT- & $9.99999 \mathrm{E}+09$ \\
\hline & GAS & \\
\hline
\end{tabular}

Mineral Reagents

\begin{tabular}{|l|l|r|}
\hline Chem Add Name & ChemAdd Alternate Name & Volume (gal) \\
\hline H3BO3 & BORIC-ACID & $4.28019 \mathrm{E}+06$ \\
\hline ZNO & ZINC-OXIDE & $6.91128 \mathrm{E}+06$ \\
\hline NA2B4O710H2O & BORAX & $4.86857 \mathrm{E}+06$ \\
\hline LI2CO3 & LITHIUM-CARBONATE & $4.39990 \mathrm{E}+06$ \\
\hline NA2CO3 & SODA-ASH & $3.63993 \mathrm{E}+06$ \\
\hline AL2SIO5 & KYANITE & $4.16541 \mathrm{E}+06$ \\
\hline CASIO3 & WOLLASTONITE & $5.03625 \mathrm{E}+06$ \\
\hline FE2O3 & IRON-OXIDE & $2.62857 \mathrm{E}+06$ \\
\hline ZRSIO4 & ZIRCON-SAND & $2.89238 \mathrm{E}+06$ \\
\hline SIO2 & SILICA & $4.71121 \mathrm{E}+07$ \\
\hline TIO2 & RUTILE & $2.56853 \mathrm{E}+06$ \\
\hline MG2SIO4FE2SIO4 & OLIVINE & $2.61664 \mathrm{E}+06$ \\
\hline C12H22O11 & SUGAR & $4.44212 \mathrm{E}+06$ \\
\hline
\end{tabular}




\section{Appendix C}

\section{Sample Identification and Logic}




\section{Appendix C Sample Identification and Logic}

Instantaneous chemical compositions at specified ISARD sample locations and data reconciliation locations can be collected as requested. The equipment names, sample identification, and sampling logic are described in the tables below, and are listed in order by lab or sample identification.

Table C-1 Sample Identification and Logic for ISARD

\begin{tabular}{|l|l|l|l|l|}
\hline $\begin{array}{l}\text { Snapshot } \\
\text { Filename }\end{array}$ & $\begin{array}{l}\text { ISARD } \\
\text { Sample Point } \\
\text { ID }\end{array}$ & $\begin{array}{l}\text { Lab OR } \\
\text { Sample ID }\end{array}$ & Vessel & Sample Trigger \\
\hline CXP1 & PT28 & 38 & CXP-VSL-00026A & $\begin{array}{l}\text { When vessel volume reaches above upper } \\
\text { setpoint }\end{array}$ \\
\hline CXP2 & PT28 & 38 & CXP-VSL-00026B & $\begin{array}{l}\text { When vessel volume reaches above upper } \\
\text { setpoint }\end{array}$ \\
\hline CXP3 & PT28 & 38 & CXP-VSL-00026C & $\begin{array}{l}\text { When vessel volume reaches above upper } \\
\text { setpoint }\end{array}$ \\
\hline FRP1 & PT2 & 9 & FRP-VSL-00002A & $\begin{array}{l}\text { When vessel is full or Tank Farm transfer is } \\
\text { complete }\end{array}$ \\
\hline FRP2 & PT2 & 9 & FRP-VSL-00002B & $\begin{array}{l}\text { When vessel is full or Tank Farm transfer is } \\
\text { complete }\end{array}$ \\
\hline FRP3 & PT2 & 9 & FRP-VSL-00002C & $\begin{array}{l}\text { When vessel is full or Tank Farm transfer is } \\
\text { complete }\end{array}$ \\
\hline FRP4 & PT2 & 9 & FRP-VSL-00002D & $\begin{array}{l}\text { When vessel is full or Tank Farm transfer is } \\
\text { complete }\end{array}$ \\
\hline HCP1 & HLW1 & 67 & HFP-VSL-00001 & Before glass former addition \\
\hline HCP2 & HLW1 & 67 & HFP-VSL-00005 & Before glass former addition \\
\hline HFP1 & HLW2 & 71 & HFP-VSL-00001 & $\begin{array}{l}\text { After glass former addition and prior to } \\
\text { discharge }\end{array}$ \\
\hline HFP2 & HLW2 & 71 & HFP-VSL-00005 & $\begin{array}{l}\text { After glass former addition and prior to } \\
\text { discharge }\end{array}$ \\
\hline HLP1 & PT17 & 24 & HLP-VSL-00022 & $\begin{array}{l}\text { When vessel is full or a Tank Farm transfer } \\
\text { is complete }\end{array}$ \\
\hline HLP4 & PT13 & 19 & HLP-VSL-00028 & $\begin{array}{l}\text { When vessel volume reaches above upper } \\
\text { setpoint }\end{array}$ \\
\hline LCP1 & LAW1 & 48 & RCP-VSL-00001 & $\begin{array}{l}\text { When vessel volume reaches above upper } \\
\text { setpoint }\end{array}$ \\
\hline RCP2 & LAW1 & 48 & $\begin{array}{l}\text { When vessel volume reaches above upper } \\
\text { setpoint }\end{array}$ \\
\hline RLD2 & PT35 & 43 & $\begin{array}{l}\text { When vessel volume reaches above upper } \\
\text { setpoint }\end{array}$ \\
\hline
\end{tabular}


Table C-2 Sample Identification and Logic

\begin{tabular}{|c|c|c|c|c|}
\hline $\begin{array}{l}\text { Snapshot } \\
\text { Filename }\end{array}$ & Sample ID & $\begin{array}{l}\text { Lab OR } \\
\text { Sample ID }\end{array}$ & Vessel & Sample Trigger \\
\hline CNP1 & PT10 & 16 & CNP-VSL-00004 & $\begin{array}{l}\text { Once a month when vessel volume is above } \\
\text { lower setpoint }\end{array}$ \\
\hline CNP2 & PT38 & 46 & CNP-EVAP-00001 & $\begin{array}{l}\text { When target Na molarity is achieved and } \\
\text { prior to discharge }\end{array}$ \\
\hline FEP1 & FEP & 102 & FEP-VSL-00017A & Prior to discharge \\
\hline FEP2 & FEP & 102 & FEP-VSL-00017B & Prior to discharge \\
\hline HDH1 & HLW6 & 74 & HDH-VSL-00003 & $\begin{array}{l}\text { After HLW canister decontamination and } \\
\text { prior to discharge }\end{array}$ \\
\hline HLP2 & PT16F & 22 & HLP-VSL-00027A & Prior to discharge \\
\hline HLP3 & PT16F & 22 & HLP-VSL-00027B & Prior to discharge \\
\hline HOP1 & HOP1 & 111 & HOP-VSL-00903 & Prior to discharge \\
\hline HOP2 & HOP2 & 111 & HOP-VSL-00904 & Prior to discharge \\
\hline HOP3 & HOP3 & 112 & HOP-WESP-00001 & Every 3 days $\&$ after WESP reactions \\
\hline HOP4 & HOP4 & 112 & HOP-WESP-00002 & Every 3 days \& after WESP reactions \\
\hline LFP1 & LAW6 & 108 & LFP-VSL-00001 & $\begin{array}{l}\text { After glass former addition and prior to } \\
\text { discharge }\end{array}$ \\
\hline LFP2 & LAW6 & 108 & LFP-VSL-00003 & $\begin{array}{l}\text { After glass former addition and prior to } \\
\text { discharge }\end{array}$ \\
\hline LFP3 & LFP3 & 109 & LFP-VSL-00002 & After receiving a full batch from MFPV \\
\hline LFP4 & LFP4 & 109 & LFP-VSL-00004 & After receiving a full batch from MFPV \\
\hline LOP1 & LOP1 & 110 & LOP-VSL-00001 & Prior to discharge \\
\hline LOP2 & LOP2 & 110 & LOP-VSL-00002 & Prior to discharge \\
\hline OF100 & OF100 & 100 & $\begin{array}{l}\text { SUPPLEMENTAL- } \\
\text { LAW-TREATED }\end{array}$ & Quarterly \\
\hline OF101 & OF101 & 101 & $\begin{array}{l}\text { SUPPLEMENTAL- } \\
\text { LAW-RECYCLE }\end{array}$ & $\begin{array}{l}\text { Quarterly (Not used, LAW SBS recycle to } \\
\text { Supplemental LAW Facility not in baseline) }\end{array}$ \\
\hline PVP1 & PT7 & 13 & PVP-SCB-00002 & Daily \\
\hline PWD1 & PT14 & 20 & PWD-VSL-00015 & $\begin{array}{l}\text { When vessel is above upper setpoint and } \\
\text { prior to discharge }\end{array}$ \\
\hline PWD2 & PT14 & 20 & PWD-VSL-00016 & $\begin{array}{l}\text { When vessel is above upper setpoint and } \\
\text { prior to discharge }\end{array}$ \\
\hline PWD3 & PT12A & 17 & PWD-VSL-00044 & $\begin{array}{l}\text { When vessel is above upper setpoint and } \\
\text { prior to discharge }\end{array}$ \\
\hline RLD3 & PT27 & 37 & RLD-TK-00006B & $\begin{array}{l}\text { When vessel volume reaches above upper } \\
\text { setpoint (1st } 10 \text { batches, then every 10th } \\
\text { batch) }\end{array}$ \\
\hline RLD4 & LAW8 & 54 & RLD-VSL-00004 & $\begin{array}{l}\text { When vessel volume reaches above upper } \\
\text { setpoint }\end{array}$ \\
\hline RLD5 & LAW10 & 56 & RLD-VSL-00005 & $\begin{array}{l}\text { When vessel volume reaches above upper } \\
\text { setpoint }\end{array}$ \\
\hline RLD6 & HLW4B & 73 & RLD-VSL-00007 & $\begin{array}{l}\text { When vessel volume reaches above upper } \\
\text { setpoint }\end{array}$ \\
\hline TCP1 & TCP1 & 107 & TCP-VSL-00001 & Sample when LAW CRV is sampled \\
\hline TLP1 & PT34 & 42 & TLP-VSL-00009A & $\begin{array}{l}\text { When vessel volume reaches above upper } \\
\text { setpoint }\end{array}$ \\
\hline TLP2 & PT34 & 42 & TLP-VSL-00009B & $\begin{array}{l}\text { When vessel volume reaches above upper } \\
\text { setpoint }\end{array}$ \\
\hline
\end{tabular}




\begin{tabular}{|l|l|l|l|l|}
\hline $\begin{array}{l}\text { Snapshot } \\
\text { Filename }\end{array}$ & Sample ID & $\begin{array}{l}\text { Lab OR } \\
\text { Sample ID }\end{array}$ & Vessel & Sample Trigger \\
\hline TLP3 & TLP3 & 106 & TLP-SEP-00001 & $\begin{array}{l}\text { Sample evaporator bottom when LAW CRV } \\
\text { is sampled }\end{array}$ \\
\hline UFP1 & PT5 & 11 & UFP-VSL-00002A & $\begin{array}{l}\text { After oxidative leach and before oxidative } \\
\text { wash }\end{array}$ \\
\hline UFP2 & PT5 & 11 & UFP-VSL-00002B & $\begin{array}{l}\text { After oxidative leach and before oxidative } \\
\text { wash }\end{array}$ \\
\hline UFP3 & PT19 & 28 & UFP-VSL-00002A & After solid concentration and before wash \\
\hline UFP4 & PT19 & 28 & UFP-VSL-00002B & After solid concentration and before wash \\
\hline UFP10 & UFP0 & 104 & UFP-VSL-00001A & After leaching and prior to discharge \\
\hline UFP11 & UFP0 & 104 & UFP-VSL-00001B & After leaching and prior to discharge \\
\hline
\end{tabular}


24590-WTP-MDD-PR-01-002, Rev 12

Dynamic (G2) Model Design Document

\section{Appendix D}

\section{Component Splits}




\section{Appendix D Component Splits}

The primary method for separation of mass is achieved by using splits in the G2 mode, e.g., upon arriving at a separation unit operation an influent is split into two output streams based on the split factors assigned. Splits are primarily applied to liquid and solid components, but are also applied to gas components. Special cases exist such that the separation is handled by reactions or by the combination of reactions and splits. This is especially true for the melters and offgas equipment where the destruction and formation of compounds occur.

The following tables present the split values applied to various WTP equipment where one table is shown if multiple trains of the same equipment exist in a unit operation.

\section{Table D-1 FEP-SEP-00001 Splits}

$\begin{array}{lr}\begin{array}{l}\text { FEP-SEP-00001A/B } \\ \text { Components }\end{array} & \begin{array}{r}\text { Liquid/Solid Fractions } \\ \text { to FEP-DMST-00001A/B }\end{array} \\ 113 \mathrm{~m}-\mathrm{Cd} & 0.0000452 \\ 125-\mathrm{Sb} & 0.00025 \\ 126-\mathrm{Sn} & 0.00025 \\ 129-\mathrm{I} & 0.002 \\ 134-\mathrm{Cs} & 3.57 \mathrm{E}-08 \\ 137-\mathrm{Cs} & 3.57 \mathrm{E}-08 \\ 137 \mathrm{~m}-\mathrm{Ba} & 3.57 \mathrm{E}-08 \\ 14-\mathrm{C} & 0.00099 \\ 151-\mathrm{Sm} & 2.67 \mathrm{E}-07 \\ 152-\mathrm{Eu} & 7.09 \mathrm{E}-07 \\ 154-\mathrm{Eu} & 7.09 \mathrm{E}-07 \\ 155-\mathrm{Eu} & 7.09 \mathrm{E}-07 \\ 229-\mathrm{Th} & 0.00099 \\ 232-\mathrm{Th} & 0.00099 \\ 232-\mathrm{U} & 0.00099 \\ 233-\mathrm{U} & 0.00099 \\ 234-\mathrm{U} & 0.00099 \\ 235-\mathrm{U} & 0.00099 \\ 236-\mathrm{U} & 0.00099 \\ 237-\mathrm{Np} & 0.00099 \\ 238-\mathrm{Pu} & 2.67 \mathrm{E}-07 \\ 238-\mathrm{U} & 0.00099 \\ 239-\mathrm{Pu} & 2.67 \mathrm{E}-07 \\ 240-\mathrm{Pu} & 2.67 \mathrm{E}-07 \\ 241-\mathrm{Am} & 7.09 \mathrm{E}-07 \\ 241-\mathrm{Pu} & 2.67 \mathrm{E}-07 \\ 242-\mathrm{Cm} & 0.00099 \\ 242-\mathrm{Pu} & 2.67 \mathrm{E}-07 \\ 243-\mathrm{Am} & 7.09 \mathrm{E}-07 \\ 243-\mathrm{Cm} & 0.00099 \\ 244-\mathrm{Cm} & 0.00099 \\ 3-\mathrm{H} & 0.599 \\ & \\ & \end{array}$




\begin{tabular}{|c|c|}
\hline $\begin{array}{l}\text { FEP-SEP-00001A/B } \\
\text { Components }\end{array}$ & $\begin{array}{l}\text { Liquid/Solid Fractions } \\
\text { to FEP-DMST-00001A/B }\end{array}$ \\
\hline $59-\mathrm{Ni}$ & 0.0000339 \\
\hline 60-Co & 0.00099 \\
\hline $63-\mathrm{Ni}$ & 0.0000339 \\
\hline $90-\mathrm{Sr}$ & $4.31 \mathrm{E}-06$ \\
\hline $90-\mathrm{Y}$ & $4.31 \mathrm{E}-06$ \\
\hline $93-\mathrm{Zr}$ & 0.00099 \\
\hline 99-Тc & 0.00099 \\
\hline $\mathrm{Ag}+$ & 0.00099 \\
\hline $\mathrm{Al}(\mathrm{OH}) 4-$ & 0.000658 \\
\hline $\mathrm{Al}+3$ & 0.000658 \\
\hline $\mathrm{As}+5$ & 0.00099 \\
\hline $\mathrm{B}+3$ & 0.00099 \\
\hline $\mathrm{Ba}+2$ & 0.00099 \\
\hline $\mathrm{Bi}+3$ & 0.00025 \\
\hline $\mathrm{C} 10 \mathrm{H} 12 \mathrm{~N} 2 \mathrm{O} 8-4$ & 0.001 \\
\hline $\mathrm{C} 10 \mathrm{H} 15 \mathrm{~N} 2 \mathrm{O} 7-3$ & 0.001 \\
\hline $\mathrm{C} 12 \mathrm{H} 27 \mathrm{O} 4 \mathrm{P}$ & 0.001 \\
\hline $\mathrm{C} 2 \mathrm{O} 4-2$ & 0.001 \\
\hline C6H5O7-3 & 0.09346 \\
\hline $\mathrm{Ca}+2$ & 0.001 \\
\hline $\mathrm{Cd}+2$ & 0.0000452 \\
\hline $\mathrm{Ce}+3$ & 0.00099 \\
\hline Cl- & 0.002 \\
\hline $\mathrm{Co}+3$ & 0.00099 \\
\hline $\mathrm{CO} 3-2$ & 0.00099 \\
\hline $\mathrm{Cr}(\mathrm{OH}) 4-$ & 0.0000282 \\
\hline Cr(TOTAL) & 0.0000282 \\
\hline Cs + & $3.57 \mathrm{E}-08$ \\
\hline $\mathrm{Cu}+2$ & 0.00099 \\
\hline F- & 0.0000411 \\
\hline $\mathrm{Fe}+3$ & 0.00099 \\
\hline $\mathrm{H}+$ & 0.1 \\
\hline $\mathrm{Hg}+2$ & 0.00099 \\
\hline $\mathrm{K}+$ & 0.000282 \\
\hline $\mathrm{La}+3$ & 0.00099 \\
\hline $\mathrm{Li}+$ & 0.000033 \\
\hline $\mathrm{Mg}+2$ & 0.00099 \\
\hline $\mathrm{Mn}+4$ & 0.00099 \\
\hline MnO4- & 0.00099 \\
\hline $\mathrm{Na}+$ & 0.000033 \\
\hline NH3 & 0.9 \\
\hline $\mathrm{Ni}+2$ & 0.0000339 \\
\hline NO2- & 0.000412 \\
\hline NO3- & 0.002 \\
\hline OH(BOUND) & 0.00099 \\
\hline $\mathrm{OH}-$ & 0.00099 \\
\hline $\mathrm{Pb}+2$ & 0.00025 \\
\hline $\mathrm{Pd}+2$ & 0.0000339 \\
\hline
\end{tabular}




$\begin{array}{lr}\begin{array}{l}\text { FEP-SEP-00001A/B } \\ \text { Components }\end{array} & \begin{array}{r}\text { Liquid/Solid Fractions } \\ \text { to FEP-DMST-00001A/B }\end{array} \\ \mathrm{PO} 4-3 & 0.00099 \\ \mathrm{Pu}+4 & 2.67 \mathrm{E}-07 \\ \mathrm{Sb}+5 & 0.00025 \\ \mathrm{Si}+4 & 0.00099 \\ \mathrm{SO} 4-2 & 0.000704 \\ \mathrm{Sr}+2 & 4.31 \mathrm{E}-06 \\ \mathrm{Tc}+7 & 0.00099 \\ \mathrm{Th}+4 & 0.00099 \\ \mathrm{Ti}+4 & 0.00099 \\ \mathrm{U}(\mathrm{TOTAL}) & 0.00099 \\ \mathrm{Zn}+2 & 0.00099 \\ \mathrm{Zr}+4 & 0.00099 \\ \mathrm{C} 12 \mathrm{H} 4 \mathrm{Cl6} & 0.0004 \\ \mathrm{C} 2 \mathrm{H} 3 \mathrm{~N} & 0.969 \\ \mathrm{Mnp} & 0.00099 \\ \mathrm{Nap}+ & 0.000033 \\ \mathrm{Al}(\mathrm{OH}) 3 & 0.000658 \\ \text { PO4-3(HARD) } & 0.00099 \\ \text { PPG } & 1.00 \mathrm{E}-08 \\ \mathrm{DPPG} & 1.00 \mathrm{E}-08 \\ \text { PDMS } & 1.00 \mathrm{E}-08 \\ \mathrm{DPDMS} & 1.00 \mathrm{E}-08 \\ \mathrm{AlOOH} & 0.000658\end{array}$

Table D-2 FEP-DMST-00001 Splits

$\begin{array}{lr}\begin{array}{l}\text { FEP-DMST-00001A/B } \\ \text { Components }\end{array} & \begin{array}{r}\text { Liquid/Solid Fractions } \\ \text { to PVP Vessel Vent }\end{array} \\ 113 \mathrm{~m}-\mathrm{Cd} & 0.002 \\ 125-\mathrm{Sb} & 0.0000358 \\ 126-\mathrm{Sn} & 0.0000358 \\ 129-\mathrm{I} & 0.001 \\ 134-\mathrm{Cs} & 0.0000427 \\ 137-\mathrm{Cs} & 0.0000427 \\ 137 \mathrm{~m}-\mathrm{Ba} & 0.0000427 \\ 14-\mathrm{C} & 0.002 \\ 151-\mathrm{Sm} & 0.00032 \\ 152-\mathrm{Eu} & 0.00032 \\ 154-\mathrm{Eu} & 0.00032 \\ 155-\mathrm{Eu} & 0.00032 \\ 229-\mathrm{Th} & 0.002 \\ 232-\mathrm{Th} & 0.002 \\ 232-\mathrm{U} & 0.002 \\ 233-\mathrm{U} & 0.002 \\ 234-\mathrm{U} & 0.002 \\ 235-\mathrm{U} & 0.002 \\ 236-\mathrm{U} & 0.002 \\ 237-\mathrm{Np} & 0.002\end{array}$




\begin{tabular}{|c|c|}
\hline $\begin{array}{l}\text { FEP-DMST-00001A/B } \\
\text { Components }\end{array}$ & $\begin{array}{l}\text { Liquid/Solid Fractions } \\
\text { to PVP Vessel Vent }\end{array}$ \\
\hline $238-\mathrm{Pu}$ & 0.00032 \\
\hline $238-U$ & 0.002 \\
\hline $239-\mathrm{Pu}$ & 0.00032 \\
\hline $240-\mathrm{Pu}$ & 0.00032 \\
\hline 241-Am & 0.00032 \\
\hline 241-Pu & 0.00032 \\
\hline $242-\mathrm{Cm}$ & 0.002 \\
\hline $242-\mathrm{Pu}$ & 0.00032 \\
\hline 243-Am & 0.00032 \\
\hline 243-Cm & 0.002 \\
\hline 244-Cm & 0.002 \\
\hline $3-\mathrm{H}$ & 0.014 \\
\hline $59-\mathrm{Ni}$ & 0.002 \\
\hline 60-Co & 0.002 \\
\hline $63-\mathrm{Ni}$ & 0.002 \\
\hline $90-\mathrm{Sr}$ & 0.0000855 \\
\hline $90-Y$ & 0.0000855 \\
\hline $93-\mathrm{Zr}$ & 0.002 \\
\hline 99-Tc & 0.002 \\
\hline $\mathrm{Ag}^{+}$ & 0.002 \\
\hline $\mathrm{Al}(\mathrm{OH}) 4-$ & 0.002 \\
\hline $\mathrm{Al}+3$ & 0.002 \\
\hline $\mathrm{As}+5$ & 0.002 \\
\hline $\mathrm{B}+3$ & 0.002 \\
\hline $\mathrm{Ba}+2$ & 0.002 \\
\hline $\mathrm{Bi}+3$ & 0.0000358 \\
\hline $\mathrm{C} 10 \mathrm{H} 12 \mathrm{~N} 2 \mathrm{O} 8-4$ & 0.05814 \\
\hline $\mathrm{C} 10 \mathrm{H} 15 \mathrm{~N} 2 \mathrm{O} 7-3$ & 0.05814 \\
\hline $\mathrm{C} 12 \mathrm{H} 27 \mathrm{O} 4 \mathrm{P}$ & 0.05814 \\
\hline C2O4-2 & 0.05814 \\
\hline $\mathrm{C} 6 \mathrm{H} 5 \mathrm{O} 7-3$ & $1.53 \mathrm{E}-06$ \\
\hline $\mathrm{Ca}+2$ & 0.058 \\
\hline $\mathrm{Cd}+2$ & 0.002 \\
\hline $\mathrm{Ce}+3$ & 0.002 \\
\hline $\mathrm{Cl}-$ & 0.0001299 \\
\hline $\mathrm{Co}+3$ & 0.002 \\
\hline $\mathrm{CO} 3-2$ & 0.002 \\
\hline $\mathrm{Cr}(\mathrm{OH}) 4-$ & 0.000188 \\
\hline Cr(TOTAL) & 0.000188 \\
\hline $\mathrm{Cs}+$ & 0.0000427 \\
\hline $\mathrm{Cu}+2$ & 0.002 \\
\hline F- & 0.00053 \\
\hline $\mathrm{Fe}+3$ & 0.002 \\
\hline $\mathrm{H}+$ & 0.01 \\
\hline $\mathrm{Hg}+2$ & 0.002 \\
\hline $\mathrm{K}+$ & 0.002 \\
\hline $\mathrm{La}+3$ & 0.002 \\
\hline
\end{tabular}




$\begin{array}{lr}\text { FEP-DMST-00001A/B } & \begin{array}{r}\text { Liquid/Solid Fractions } \\ \text { Components }\end{array} \\ \mathrm{Li}+ & 0.002 \\ \mathrm{Mg}+2 & 0.035 \\ \mathrm{Mn}+4 & 0.002 \\ \mathrm{MnO} 4- & 0.002 \\ \mathrm{Na}+ & 0.002 \\ \mathrm{NH} 3 & 0.081 \\ \mathrm{Ni}+2 & 0.002 \\ \mathrm{NO} 2- & 0.0000962 \\ \mathrm{NO} 3- & 0.0000962 \\ \mathrm{OH}(\mathrm{BOUND}) & 0.002 \\ \mathrm{OH}- & 0.002 \\ \mathrm{~Pb}+2 & 0.0000358 \\ \mathrm{Pd}+2 & 0.002 \\ \mathrm{PO} 4-3 & 0.002 \\ \mathrm{Pu}+4 & 0.00032 \\ \mathrm{Sb}+5 & 0.0000358 \\ \mathrm{Si}+4 & 0.004 \\ \mathrm{SO} 4-2 & 0.0002375 \\ \mathrm{Sr}+2 & 0.0000855 \\ \mathrm{Tc}+7 & 0.002 \\ \mathrm{Th}+4 & 0.002 \\ \mathrm{Ti}+4 & 0.002 \\ \mathrm{U}(\mathrm{TOTAL}) & 0.002 \\ \mathrm{Zn}+2 & 0.002 \\ \mathrm{Zr}+4 & 0.002 \\ \mathrm{C} 12 \mathrm{H} 4 \mathrm{Cl} 6 & 0.0004 \\ \mathrm{C} 2 \mathrm{H} 3 \mathrm{~N} & 0.8314 \\ \mathrm{Mnp} & 0.002 \\ \mathrm{Nap}+ & 0.002 \\ \mathrm{Al}(\mathrm{OH}) 3 & 0.002 \\ \mathrm{AlOOH} & 0.002 \\ & \\ \mathrm{~N}+4\end{array}$

Table D-3 PVP-SCB-00002 Splits

$\begin{array}{lrr}\begin{array}{l}\text { PVP-SCB-00002 } \\ \text { Components }\end{array} & \begin{array}{r}\text { Liquid \% } \\ \text { to PVP-HEME-00001 }\end{array} & \begin{array}{r}\text { Solid \% } \\ \text { to PVP-HEME-00001 }\end{array} \\ 125-\mathrm{Sb} & 30.03003003 & 30.03003003 \\ 126-\mathrm{Sn} & 30.03003003 & 30.03003003 \\ 129-\mathrm{I} & 30.03003003 & 30.03003003 \\ 134-\mathrm{Cs} & 30.03003003 & 30.03003003 \\ 137-\mathrm{Cs} & 30.03 & 30.03 \\ 137 \mathrm{~m}-\mathrm{Ba} & 30.03 & 30.03 \\ 14-\mathrm{C} & 100 & 100 \\ 151-\mathrm{Sm} & 30.03003003 & 30.03003003 \\ 152-\mathrm{Eu} & 30.03003003 & 30.03003003 \\ 154-\mathrm{Eu} & 30.03003003 & 30.03003003 \\ 155-\mathrm{Eu} & 30.03003003 & 30.03003003 \\ 232-\mathrm{Th} & 30.03003003 & 30.03003003\end{array}$


PVP-SCB-00002

Components

233-U

234-U

235-U

236-U

237-Np

$238-\mathrm{Pu}$

238-U

239-Pu

240-Pu

241-Am

241-Pu

243-Cm

244-Cm

3-H

60-Co

63-Ni

$90-\mathrm{Sr}$

90-Y

99-Tc

$\mathrm{Ag}^{+}$

$\mathrm{Al}(\mathrm{OH}) 3$

$\mathrm{Al}(\mathrm{OH}) 4-$

$\mathrm{Al}+3$

$\mathrm{AlOOH}$

$\mathrm{As}+5$

$\mathrm{B}+3$

$\mathrm{Ba}+2$

$\mathrm{Bi}+3$

$\mathrm{C} 12 \mathrm{H} 4 \mathrm{Cl} 6$

$\mathrm{C} 2 \mathrm{H} 3 \mathrm{~N}$

C2O4-2

$\mathrm{Ca}+2$

$\mathrm{Cd}+2$

$\mathrm{Ce}+3$

Cl-

CO3-2

Cr(TOTAL)

$\mathrm{Cu}+2$

F-

$\mathrm{Fe}+3$

$\mathrm{Hg}+2$

$\mathrm{K}+$

$\mathrm{La}+3$

$\mathrm{Li}+$

$\mathrm{Mg}+2$

$\mathrm{Mn}+4$

Mnp
Liquid \%

to PVP-HEME-00001

30.03003003

30.03003003

30.03003003

30.03003003

30.03003003

30.03003003

30.03003003

30.03003003

30.03003003

30.03003003

30.03003003

30.03003003

30.03003003

100

30.03003003

30.03003003

30.03

30.03

30.03003003

30.03003003

30.03

30.03003003

30.03003003

30.03

30.03003003

30.03003003

30.03003003

30.03003003

33.3

100

33.33333337

30.03003003

30.03003003

30.03003003

30.03003003

33.33333337

30.03003003

30.03003003

30.03003003

30.03003003

100

30.03003003

30.03003003

30.03003003

30.03003003

30.03003003

30.03
Solid \%

to PVP-HEME-00001

30.03003003

30.03003003

30.03003003

30.03003003

30.03003003

30.03003003

30.03003003

30.03003003

30.03003003

30.03003003

30.03003003

30.03003003

30.03003003

100

30.03003003

30.03003003

30.03

30.03

30.03003003

30.03003003

30.03

30.03003003

30.03003003

30.03

30.03003003

30.03003003

30.03003003

30.03003003

33.3

100

33.33333337

30.03003003

30.03003003

30.03003003

30.03003003

33.33333337

30.03003003

30.03003003

30.03003003

30.03003003

100

30.03003003

30.03003003

30.03003003

30.03003003

30.03003003

30.03 


PVP-SCB-00002
Components
$\mathrm{Na}+$
$\mathrm{Nap}+$
$\mathrm{NH} 3$
$\mathrm{Ni}+2$
$\mathrm{NO} 2-$
$\mathrm{NO} 3-$
$\mathrm{OH}(\mathrm{BOUND})$
$\mathrm{OH}-$
$\mathrm{Pb}+2$
$\mathrm{Pd}+2$
$\mathrm{PO} 4-3$
$\mathrm{PO} 4-3$ (HARD)
$\mathrm{Si}+4$
$\mathrm{SO} 4-2$
$\mathrm{Sr}+2$
$\mathrm{Ti}+4$
$\mathrm{Zn}+2$
$\mathrm{Zr}+4$

$\begin{array}{rr}\begin{array}{r}\text { Liquid \% } \\ \text { to PVP-HEME-00001 }\end{array} & \begin{array}{r}\text { Solid \% } \\ \text { to PVP-HEME-00001 }\end{array} \\ 30.03003003 & 30.03003003 \\ 30.03 & 30.03 \\ 33.33333337 & 33.33333337 \\ 30.03003003 & 30.03003003 \\ 30.03003003 & 30.03003003 \\ 30.03003003 & 30.03003003 \\ 30.03003003 & 30.03003003 \\ 30.03003003 & 30.03003003 \\ 30.03003003 & 30.03003003 \\ 30.03003003 & 30.03003003 \\ 30.03003003 & 30.03003003 \\ 30.03 & 30.03 \\ 30.03003003 & 30.03003003 \\ 30.03003003 & 30.03003003 \\ 30.03003003 & 30.03003003 \\ 30.03003003 & 30.03003003 \\ 30.03003003 & 30.03003003 \\ 30.03003003 & 30.03003003\end{array}$

Table D-4 PVP-HEME-00001 Splits

$\begin{array}{lrr}\text { PVP-HEME-00001 } & \begin{array}{r}\text { Liquid \% } \\ \text { Components }\end{array} & \begin{array}{c}\text { Solid \% } \\ \text { to PVP-VSL-00001 }\end{array} \\ \text { 125-Sb } & 99.5 & 99.5 \\ 126-\mathrm{Sn} & 99.5 & 99.5 \\ 129-\mathrm{I} & 0.0001 & 0.0001 \\ 134-\mathrm{Cs} & 99.5 & 99.5 \\ 137-\mathrm{Cs} & 99.5 & 99.5 \\ 137 \mathrm{~m}-\mathrm{Ba} & 99.5 & 99.5 \\ 14-\mathrm{C} & 0.0001 & 0.0001 \\ 151-\mathrm{Sm} & 99.5 & 99.5 \\ 152-\mathrm{Eu} & 99.5 & 99.5 \\ 154-\mathrm{Eu} & 99.5 & 99.5 \\ 155-\mathrm{Eu} & 99.5 & 99.5 \\ 232-\mathrm{Th} & 99.5 & 99.5 \\ 233-\mathrm{U} & 99.5 & 99.5 \\ 234-\mathrm{U} & 99.5 & 99.5 \\ 235-\mathrm{U} & 99.5 & 99.5 \\ 236-\mathrm{U} & 99.5 & 99.5 \\ 237-\mathrm{Np} & 99.5 & 99.5 \\ 238-\mathrm{Pu} & 99.5 & 99.5 \\ 238-\mathrm{U} & 99.5 & 99.5 \\ 239-\mathrm{Pu} & 99.5 & 99.5 \\ 240-\mathrm{Pu} & 99.5 & 99.5 \\ 241-\mathrm{Am} & 99.5 & 99.5 \\ 241-\mathrm{Pu} & 99.5 & 99.5 \\ 243-\mathrm{Cm} & 99.5 & 99.5 \\ 244-\mathrm{Cm} & 99.5 & 99.5\end{array}$


PVP-HEME-00001

Components

$3-\mathrm{H}$

60-Co

63-Ni

$90-\mathrm{Sr}$

90-Y

99-Tc

$\mathrm{Ag}+$

$\mathrm{Al}(\mathrm{OH}) 3$

$\mathrm{Al}(\mathrm{OH}) 4-$

$\mathrm{Al}+3$

$\mathrm{AlOOH}$

$\mathrm{As}+5$

$\mathrm{B}+3$

$\mathrm{Ba}+2$

$\mathrm{Bi}+3$

$\mathrm{C} 12 \mathrm{H} 4 \mathrm{Cl} 6$

$\mathrm{C} 2 \mathrm{H} 3 \mathrm{~N}$

C2O4-2

$\mathrm{Ca}+2$

$\mathrm{Cd}+2$

$\mathrm{Ce}+3$

$\mathrm{Cl}-$

$\mathrm{CO} 3-2$

Cr(TOTAL)

$\mathrm{Cu}+2$

F-

$\mathrm{Fe}+3$

$\mathrm{Hg}+2$

$\mathrm{K}+$

$\mathrm{La}+3$

$\mathrm{Li}+$

$\mathrm{Mg}+2$

$\mathrm{Mn}+4$

Mnp

$\mathrm{Na}+$

Nap+

$\mathrm{NH} 3$

$\mathrm{Ni}+2$

NO2-

NO3-

$\mathrm{OH}(\mathrm{BOUND})$

$\mathrm{OH}-$

$\mathrm{Pb}+2$

$\mathrm{Pd}+2$

PO4-3

PO4-3(HARD)

$\mathrm{Si}+4$
Liquid \%

to PVP-VSL-00001

0.0001

99.5

99.5

99.5

99.5

99.5

99.5

99.5

99.5

99.5

99.5

99.5

99.5

99.5

99.5

99.5

0

99.5

99.5

99.5

99.5

99.5

99.5

99.5

99.5

99.5

99.5

0.0001

99.5

99.5

99.5

99.5

99.5

99.5

99.5

99.5

0.0001

99.5

99.5

99.5

99.5

99.5

99.5

99.5

99.5

99.5

99.5
Solid \%

to PVP-VSL-00001

0.0001

99.5

99.5

99.5

99.5

99.5

99.5

99.5

99.5

99.5

99.5

99.5

99.5

99.5

99.5

99.5

0

99.5

99.5

99.5

99.5

99.5

99.5

99.5

99.5

99.5

99.5

0.0001

99.5

99.5

99.5

99.5

99.5

99.5

99.5

99.5

0.0001

99.5

99.5

99.5

99.5

99.5

99.5

99.5

99.5

99.5

99.5 
PVP-HEME-00001

Components

SO4-2

$\mathrm{Sr}+2$

$\mathrm{Ti}+4$

$\mathrm{Zn}+2$

$\mathrm{Zr}+4$
Liquid \%

to PVP-VSL-00001

99.5

99.5

99.5

99.5

99.5
Solid \%

to PVP-VSL-00001

99.5

99.5

99.5

99.5

99.5

\section{Table D-5 PVP-HEPA Splits}

PVP-HEPA-00001-2

\section{Components}

$125-\mathrm{Sb}$

126-Sn

134-Cs

137-Cs

$137 \mathrm{~m}-\mathrm{Ba}$

$151-\mathrm{Sm}$

152-Eu

154-Eu

155 -Eu

232-Th

233-U

234-U

235-U

236-U

237-Np

$238-\mathrm{Pu}$

238-U

239-Pu

240-Pu

241-Am

241-Pu

243-Cm

244-Cm

60-Co

63-Ni

90-Sr

90-Y

99-Tc

$\mathrm{Ag}+$

$\mathrm{Al}(\mathrm{OH}) 3$

$\mathrm{Al}+3$

$\mathrm{AlOOH}$

$\mathrm{As}+5$

$\mathrm{B}+3$

$\mathrm{Ba}+2$

$\mathrm{Bi}+3$

$\mathrm{C} 12 \mathrm{H} 4 \mathrm{Cl} 6$

C2H3N
Liquid \%

to Accumulate 99.99994001

99.99994001

99.99994001

100

100

99.99994001

99.99994001

99.99994001

99.99994001

99.99994001

99.99994001

99.99994001

99.99994001

99.99994001

99.99994001

99.99994001

99.99994001

99.99994001

99.99994001

99.99994001

99.99994001

99.99994001

99.99994001

99.99994001

99.99994001

100

100

99.99994001

99.99994001

100

99.99994001

100

99.99994001

99.99994001

99.99994001

99.99994001

98
Solid \%

to Accumulate

99.99994001

99.99994001

99.99994001

100

100

99.99994001

99.99994001

99.99994001

99.99994001

99.99994001

99.99994001

99.99994001

99.99994001

99.99994001

99.99994001

99.99994001

99.99994001

99.99994001

99.99994001

99.99994001

99.99994001

99.99994001

99.99994001

99.99994001

99.99994001

100

100

99.99994001

99.99994001

100

99.99994001

100

99.99994001

99.99994001

99.99994001

99.99994001

98 
PVP-HEPA-00001-2

\section{Components}

\section{C2O4-2}

$\mathrm{Ca}+2$

$\mathrm{Cd}+2$

$\mathrm{Ce}+3$

Cl-

$\mathrm{CO} 3-2$

Cr(TOTAL)

$\mathrm{Cu}+2$

F-

$\mathrm{Fe}+3$

$\mathrm{K}+$

$\mathrm{La}+3$

$\mathrm{Li}+$

$\mathrm{Mg}+2$

$\mathrm{Mn}+4$

Mnp

$\mathrm{Na}+$

Nap+

$\mathrm{Ni}+2$

$\mathrm{NO} 2-$

NO3-

$\mathrm{OH}-$

$\mathrm{Pb}+2$

$\mathrm{Pd}+2$

PO4-3

PO4-3(HARD)

$\mathrm{Si}+4$

SO4-2

$\mathrm{Sr}+2$

$\mathrm{Ti}+4$

$\mathrm{Zn}+2$

$\mathrm{Zr}+4$

Gas Components

$\mathrm{CO} 2$
Liquid \%

to Accumulate

99.99994001

99.99994001

99.99994001

99.99994001

99.99994001

99.99994001

99.99994001

99.99994001

99.99994001

99.99994001

99.99994001

99.99994001

99.99994001

99.99994001

99.99994001

100

100

100

99.99994001

99.99994001

99.99994001

99.99994001

99.99994001

99.99994001

99.99994001

100

99.99994001

99.99994001

99.99994001

99.99994001

99.99994001

99.99994001

Gas \% to

ACCUMULATE
Solid \%

to Accumulate

99.99994001

99.99994001

99.99994001

99.99994001

99.99994001

99.99994001

99.99994001

99.99994001

99.99994001

99.99994001

99.99994001

99.99994001

99.99994001

99.99994001

99.99994001

100

100

100

99.99994001

99.99994001

99.99994001

99.99994001

99.99994001

99.99994001

99.99994001

100

99.99994001

99.99994001

99.99994001

99.99994001

99.99994001

99.99994001

Table D-6 PVP-Thermal-Oxidizer Splits

PVP-OXID-00001

Components

C2O4-2

Gas Components

$\mathrm{CO} 2$
Liquid \% to Accumulate

99

Gas \%

to Accumulate
Solid \%

to Accumulate

99

\section{0}


Table D-7 PVP-Carbon-Absorber Splits

PVP-ABS-00001AB
Components
$\mathrm{C} 12 \mathrm{H} 4 \mathrm{Cl} 6$
$\mathrm{C} 2 \mathrm{H} 3 \mathrm{~N}$
$\mathrm{C} 2 \mathrm{O} 4-2$
$\mathrm{Hg}+2$

\section{Gas Components}

$\mathrm{C} 12 \mathrm{H} 4 \mathrm{Cl} 6$

$\mathrm{C} 2 \mathrm{H} 3 \mathrm{~N}$

$\mathrm{CO} 2$
Liquid \% to Accumulate

90

90

90

97.5

Gas \%

to Accumulate

90

90

0

Table D-8 PJV-VSL-00001 Splits

\begin{tabular}{|c|c|c|}
\hline $\begin{array}{l}\text { PJV-VSL-00001 } \\
\text { Components }\end{array}$ & $\begin{array}{r}\text { Liquid \% } \\
\text { to PJV-VSL-00002 }\end{array}$ & $\begin{array}{r}\text { Solid \% } \\
\text { to PJV-VSL-00002 }\end{array}$ \\
\hline $125-\mathrm{Sb}$ & 99.5 & 99.5 \\
\hline $126-\mathrm{Sn}$ & 99.5 & 99.5 \\
\hline 137-Cs & 99.5 & 99.5 \\
\hline $137 \mathrm{~m}-\mathrm{Ba}$ & 99.5 & 99.5 \\
\hline $151-\mathrm{Sm}$ & 99.5 & 99.5 \\
\hline 152-Eu & 99.5 & 99.5 \\
\hline 154-Eu & 99.5 & 99.5 \\
\hline $155-\mathrm{Eu}$ & 99.5 & 99.5 \\
\hline 232-Th & 99.5 & 99.5 \\
\hline $233-U$ & 99.5 & 99.5 \\
\hline $234-U$ & 99.5 & 99.5 \\
\hline $235-\mathrm{U}$ & 99.5 & 99.5 \\
\hline $236-\mathrm{U}$ & 99.5 & 99.5 \\
\hline 237-Np & 99.5 & 99.5 \\
\hline $238-\mathrm{Pu}$ & 99.5 & 99.5 \\
\hline $238-U$ & 99.5 & 99.5 \\
\hline 239-Pu & 99.5 & 99.5 \\
\hline $240-\mathrm{Pu}$ & 99.5 & 99.5 \\
\hline 241-Am & 99.5 & 99.5 \\
\hline 241-Pu & 99.5 & 99.5 \\
\hline 243-Cm & 99.5 & 99.5 \\
\hline 244-Cm & 99.5 & 99.5 \\
\hline 60-Co & 99.5 & 99.5 \\
\hline $63-\mathrm{Ni}$ & 99.5 & 99.5 \\
\hline $90-\mathrm{Sr}$ & 99.5 & 99.5 \\
\hline $90-\mathrm{Y}$ & 99.5 & 99.5 \\
\hline 99-Tc & 99.5 & 99.5 \\
\hline $\mathrm{Ag}+$ & 99.5 & 99.5 \\
\hline $\mathrm{Al}(\mathrm{OH}) 3$ & 99.5 & 99.5 \\
\hline $\mathrm{Al}+3$ & 99.5 & 99.5 \\
\hline $\mathrm{As}+5$ & 99.5 & 99.5 \\
\hline $\mathrm{B}+3$ & 99.5 & 99.5 \\
\hline $\mathrm{Ba}+2$ & 99.5 & 99.5 \\
\hline
\end{tabular}


PJV-VSL-00001

Components

$\mathrm{Bi}+3$

C2O4-2

$\mathrm{Ca}+2$

$\mathrm{Cd}+2$

$\mathrm{Ce}+3$

$\mathrm{Cl}-$

$\mathrm{CO} 3-2$

Cr(TOTAL)

$\mathrm{Cu}+2$

F-

$\mathrm{Fe}+3$

$\mathrm{K}+$

$\mathrm{La}+3$

$\mathrm{Li}+$

$\mathrm{Mg}+2$

$\mathrm{Mn}+4$

Mnp

$\mathrm{Na}+$

Nap +

$\mathrm{Ni}+2$

$\mathrm{NO} 2-$

NO3-

$\mathrm{OH}-$

$\mathrm{Pb}+2$

$\mathrm{Pd}+2$

PO4-3

$\mathrm{Si}+4$

SO4-2

$\mathrm{Sr}+2$

$\mathrm{Ti}+4$

$\mathrm{Zn}+2$

$\mathrm{Zr}+4$
Liquid \% to PJV-VSL-00002

99.5

99.5

99.5

99.5

99.5

99.5

99.5

99.5

99.5

99.5

99.5

99.5

99.5

99.5

99.5

99.5

99.5

99.5

99.5

99.5

99.5

99.5

99.5

99.5

99.5

99.5

99.5

99.5

99.5

99.5

99.5

99.5
Solid \%

to PJV-VSL-00002

99.5

99.5

99.5

99.5

99.5

99.5

99.5

99.5

99.5

99.5

99.5

99.5

99.5

99.5

99.5

99.5

99.5

99.5

99.5

99.5

99.5

99.5

99.5

99.5

99.5

99.5

99.5

99.5

99.5

99.5

99.5

99.5

\section{Table D-9 PJV-HEPA Splits}

PJV-HEPA
Components
$125-\mathrm{Sb}$
$126-\mathrm{Sn}$
$134-\mathrm{Cs}$
$137-\mathrm{Cs}$
$151-\mathrm{Sm}$
$152-\mathrm{Eu}$
$154-\mathrm{Eu}$
$155-\mathrm{Eu}$
$232-\mathrm{Th}$
$233-\mathrm{U}$
$234-\mathrm{U}$

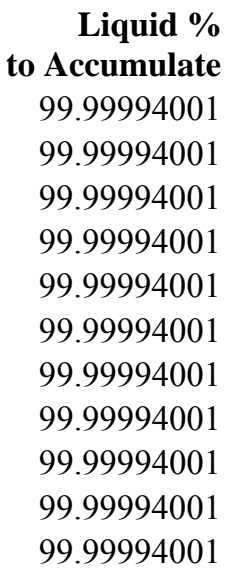

Solid \%

to Accumulate 99.99994001 99.99994001 99.99994001 99.99994001 99.99994001 99.99994001 99.99994001 99.99994001 99.99994001 99.99994001 99.99994001 
PJV-HEPA

\section{Components}

235-U

236-U

237-Np

$238-\mathrm{Pu}$

238-U

239-Pu

240-Pu

241-Am

241-Pu

243-Cm

244-Cm

60-Co

63-Ni

$90-\mathrm{Sr}$

99-Tc

$\mathrm{Ag}+$

$\mathrm{Al}(\mathrm{OH}) 3$

$\mathrm{Al}+3$

$\mathrm{As}+5$

$\mathrm{B}+3$

$\mathrm{Ba}+2$

$\mathrm{Bi}+3$

C2O4-2

$\mathrm{Ca}+2$

$\mathrm{Cd}+2$

$\mathrm{Ce}+3$

Cl-

CO3-2

Cr(TOTAL)

$\mathrm{Cu}+2$

F-

$\mathrm{Fe}+3$

K+

$\mathrm{La}+3$

$\mathrm{Li}+$

$\mathrm{Mg}+2$

$\mathrm{Mn}+4$

Mnp

$\mathrm{Na}+$

Nap+

$\mathrm{Ni}+2$

$\mathrm{NO} 2-$

NO3-

$\mathrm{OH}-$

$\mathrm{Pb}+2$

$\mathrm{Pd}+2$

PO4-3
Liquid \%

to Accumulate

99.99994001

99.99994001

99.99994001

99.99994001

99.99994001

99.99994001

99.99994001

99.99994001

99.99994001

99.99994001

99.99994001

99.99994001

99.99994001

99.99994001

99.99994001

99.99994001

100

99.99994001

99.99994001

99.99994001

99.99994001

99.99994001

99.99994001

99.99994001

99.99994001

99.99994001

99.99994001

99.99994001

99.99994001

99.99994001

99.99994001

99.99994001

99.99994001

99.99994001

99.99994001

99.99994001

99.99994001

100

100

100

99.99994001

99.99994001

99.99994001

99.99994001

99.99994001

99.99994001

99.99994001
Solid \%

to Accumulate

99.99994001

99.99994001

99.99994001

99.99994001

99.99994001

99.99994001

99.99994001

99.99994001

99.99994001

99.99994001

99.99994001

99.99994001

99.99994001

99.99994001

99.99994001

99.99994001

100

99.99994001

99.99994001

99.99994001

99.99994001

99.99994001

99.99994001

99.99994001

99.99994001

99.99994001

99.99994001

99.99994001

99.99994001

99.99994001

99.99994001

99.99994001

99.99994001

99.99994001

99.99994001

99.99994001

99.99994001

100

100

100

99.99994001

99.99994001

99.99994001

99.99994001

99.99994001

99.99994001

99.99994001 
PJV-HEPA

Components

$\mathrm{Si}+4$

SO4-2

$\mathrm{Sr}+2$

$\mathrm{Ti}+4$

$\mathrm{Zn}+2$

$\mathrm{Zr}+4$

Gas Components

$\mathrm{CO} 2$
Liquid \%

to Accumulate

99.99994001

99.99994001

99.99994001

99.99994001

99.99994001

99.99994001

Gas \%

to ACCUMULATE

Table D-10 TLP-SEP-00001 Splits

$\begin{array}{lr}\text { TLP-SEP-00001 } & \begin{array}{r}\text { Liquid/Solid Fractions } \\ \text { Components }\end{array} \\ 113 \mathrm{~m}-\mathrm{Cd} & 0.0000452 \\ 125-\mathrm{Sb} & 0.00025 \\ 126-\mathrm{Sn} & 0.00025 \\ 129-\mathrm{I} & 0.00231 \\ 134-\mathrm{Cs} & 3.57 \mathrm{E}-08 \\ 137-\mathrm{Cs} & 3.57 \mathrm{E}-08 \\ 137 \mathrm{~m}-\mathrm{Ba} & 3.57 \mathrm{E}-08 \\ 14-\mathrm{C} & 0.00099 \\ 151-\mathrm{Sm} & 2.67 \mathrm{E}-07 \\ 152-\mathrm{Eu} & 7.09 \mathrm{E}-07 \\ 154-\mathrm{Eu} & 7.09 \mathrm{E}-07 \\ 155-\mathrm{Eu} & 7.09 \mathrm{E}-07 \\ 229-\mathrm{Th} & 0.00099 \\ 232-\mathrm{Th} & 0.00099 \\ 232-\mathrm{U} & 0.00099 \\ 233-\mathrm{U} & 0.00099 \\ 234-\mathrm{U} & 0.00099 \\ 235-\mathrm{U} & 0.00099 \\ 236-\mathrm{U} & 0.00099 \\ 237-\mathrm{Np} & 0.00099 \\ 238-\mathrm{Pu} & 2.67 \mathrm{E}-07 \\ 238-\mathrm{U} & 0.00099 \\ 239-\mathrm{Pu} & 2.67 \mathrm{E}-07 \\ 240-\mathrm{Pu} & 2.67 \mathrm{E}-07 \\ 241-\mathrm{Am} & 7.09 \mathrm{E}-07 \\ 241-\mathrm{Pu} & 2.67 \mathrm{E}-07 \\ 242-\mathrm{Cm} & 0.00099 \\ 242-\mathrm{Pu} & 2.67 \mathrm{E}-07 \\ 243-\mathrm{Am} & 7.09 \mathrm{E}-07 \\ 243-\mathrm{Cm} & 0.00099 \\ 244-\mathrm{Cm} & 0.00099 \\ 3-\mathrm{H} & 0.5988 \\ 59-\mathrm{Ni} & 0.0000339 \\ 60-\mathrm{Co} & 0.00099 \\ & \\ & \end{array}$

\author{
Solid \% \\ to Accumulate \\ 99.99994001 \\ 99.99994001 \\ 99.99994001 \\ 99.99994001 \\ 99.99994001 \\ 99.99994001
}




\begin{tabular}{|c|c|}
\hline $\begin{array}{l}\text { TLP-SEP-00001 } \\
\text { Components }\end{array}$ & $\begin{array}{l}\text { Liquid/Solid Fractions } \\
\text { to TLP-DMST-00001 }\end{array}$ \\
\hline $63-\mathrm{Ni}$ & 0.0000339 \\
\hline $90-\mathrm{Sr}$ & $4.31 \mathrm{E}-06$ \\
\hline $90-\mathrm{Y}$ & $4.31 \mathrm{E}-06$ \\
\hline $93-\mathrm{Zr}$ & 0.00099 \\
\hline 99-Tc & 0.00099 \\
\hline $\mathrm{Ag}+$ & 0.00099 \\
\hline $\mathrm{Al}(\mathrm{OH}) 4-$ & 0.000658 \\
\hline $\mathrm{Al}+3$ & 0.000658 \\
\hline $\mathrm{As}+5$ & 0.00099 \\
\hline $\mathrm{B}+3$ & 0.00099 \\
\hline $\mathrm{Ba}+2$ & 0.00099 \\
\hline $\mathrm{Bi}+3$ & 0.00025 \\
\hline $\mathrm{C} 10 \mathrm{H} 12 \mathrm{~N} 2 \mathrm{O} 8-4$ & 0.001 \\
\hline $\mathrm{C} 10 \mathrm{H} 15 \mathrm{~N} 2 \mathrm{O} 7-3$ & 0.001 \\
\hline $\mathrm{C} 12 \mathrm{H} 27 \mathrm{O} 4 \mathrm{P}$ & 0.001 \\
\hline $\mathrm{C} 2 \mathrm{O} 4-2$ & 0.001 \\
\hline C6H5O7-3 & 0.09346 \\
\hline $\mathrm{Ca}+2$ & 0.001 \\
\hline $\mathrm{Cd}+2$ & 0.0000452 \\
\hline $\mathrm{Ce}+3$ & 0.00099 \\
\hline $\mathrm{Cl}-$ & 0.0019 \\
\hline $\mathrm{Co}+3$ & 0.00099 \\
\hline $\mathrm{CO} 3-2$ & 0.00099 \\
\hline $\mathrm{Cr}(\mathrm{OH}) 4-$ & 0.0000282 \\
\hline Cr(TOTAL) & 0.0000282 \\
\hline $\mathrm{Cs}^{+}$ & $3.57 \mathrm{E}-08$ \\
\hline $\mathrm{Cu}+2$ & 0.00099 \\
\hline F- & 0.0000411 \\
\hline $\mathrm{Fe}+3$ & 0.00099 \\
\hline $\mathrm{H}+$ & 0.1 \\
\hline $\mathrm{Hg}+2$ & 0.00099 \\
\hline $\mathrm{K}+$ & 0.000282 \\
\hline $\mathrm{La}+3$ & 0.00099 \\
\hline $\mathrm{Li}+$ & 0.000033 \\
\hline $\mathrm{Mg}+2$ & 0.00099 \\
\hline $\mathrm{Mn}+4$ & 0.00099 \\
\hline MnO4- & 0.00099 \\
\hline $\mathrm{Na}+$ & 0.000033 \\
\hline NH3 & 0.9 \\
\hline $\mathrm{Ni}+2$ & 0.0000339 \\
\hline NO2- & 0.000412 \\
\hline NO3- & 0.001733 \\
\hline OH(BOUND) & 0.00099 \\
\hline OH- & 0.00099 \\
\hline $\mathrm{Pb}+2$ & 0.00025 \\
\hline $\mathrm{Pd}+2$ & 0.0000339 \\
\hline PO4-3 & 0.0005 \\
\hline
\end{tabular}




TLP-SEP-00001
Components
$\mathrm{Pu}+4$
$\mathrm{Sb}+5$
$\mathrm{Si}+4$
$\mathrm{SO} 4-2$
$\mathrm{Sr}+2$
$\mathrm{Tc}+7$
$\mathrm{Th}+4$
$\mathrm{Ti}+4$
$\mathrm{U}(\mathrm{TOTAL})$
$\mathrm{Zn}+2$
$\mathrm{Zr}+4$
$\mathrm{C} 12 \mathrm{H} 4 \mathrm{Cl} 6$
$\mathrm{C} 2 \mathrm{H} 3 \mathrm{~N}$
$\mathrm{Mnp}$
$\mathrm{Nap}+$
Al(OH)3
PPG
DPPG
PDMS
DPDMS

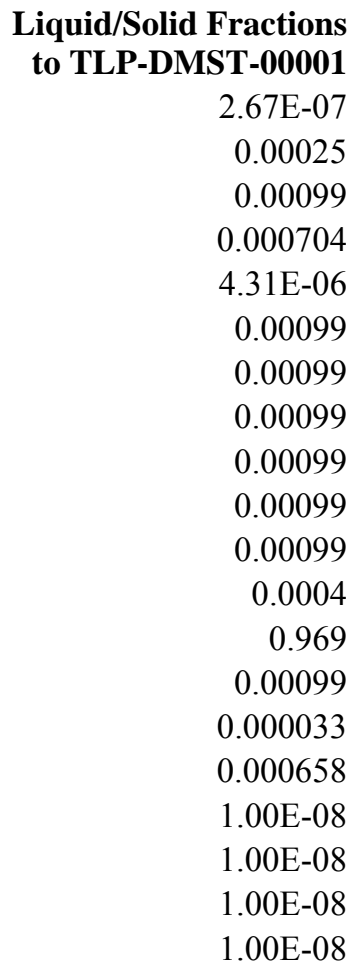

Table D-11 TLP-DMST-00001 Splits

$\begin{array}{lr}\begin{array}{l}\text { TLP-DMST-00001 } \\ \text { Components }\end{array} & \begin{array}{r}\text { Liquid/Solid Fractions } \\ \text { to PVP Vessel Vent }\end{array} \\ 113 \mathrm{~m}-\mathrm{Cd} & 0.00154 \\ 125-\mathrm{Sb} & 0.0000358 \\ 126-\mathrm{Sn} & 0.0000358 \\ 129-\mathrm{I} & 0.0011 \\ 134-\mathrm{Cs} & 0.0000427 \\ 137-\mathrm{Cs} & 0.0000427 \\ 137 \mathrm{~m}-\mathrm{Ba} & 0.0000427 \\ 14-\mathrm{C} & 0.00154 \\ 151-\mathrm{Sm} & 0.00032 \\ 152-\mathrm{Eu} & 0.00032 \\ 154-\mathrm{Eu} & 0.00032 \\ 155-\mathrm{Eu} & 0.00032 \\ 229-\mathrm{Th} & 0.00154 \\ 232-\mathrm{Th} & 0.00154 \\ 232-\mathrm{U} & 0.00154 \\ 233-\mathrm{U} & 0.00154 \\ 234-\mathrm{U} & 0.00154 \\ 235-\mathrm{U} & 0.00154 \\ 236-\mathrm{U} & 0.00154 \\ 237-\mathrm{Np} & 0.00154 \\ 238-\mathrm{Pu} & 0.00032 \\ 238-\mathrm{U} & 0.00154 \\ 239-\mathrm{Pu} & 0.00032\end{array}$




\begin{tabular}{|c|c|}
\hline $\begin{array}{l}\text { TLP-DMST-00001 } \\
\text { Components }\end{array}$ & $\begin{array}{r}\text { Liquid/Solid Fractions } \\
\text { to PVP Vessel Vent }\end{array}$ \\
\hline 240-Pu & 0.00032 \\
\hline 241-Am & 0.00032 \\
\hline 241-Pu & 0.00032 \\
\hline 242-Cm & 0.00154 \\
\hline 242-Pu & 0.00032 \\
\hline 243-Am & 0.00032 \\
\hline $243-\mathrm{Cm}$ & 0.00154 \\
\hline 244-Cm & 0.00154 \\
\hline $3-\mathrm{H}$ & 0.014 \\
\hline $59-\mathrm{Ni}$ & 0.00154 \\
\hline 60-Co & 0.00154 \\
\hline $63-\mathrm{Ni}$ & 0.00154 \\
\hline $90-\mathrm{Sr}$ & 0.0000855 \\
\hline $90-\mathrm{Y}$ & 0.0000855 \\
\hline $93-\mathrm{Zr}$ & 0.002 \\
\hline 99-Тc & 0.00154 \\
\hline $\mathrm{Ag}+$ & 0.00188 \\
\hline $\mathrm{Al}(\mathrm{OH}) 4-$ & 0.00154 \\
\hline $\mathrm{Al}+3$ & 0.00154 \\
\hline $\mathrm{As}+5$ & 0.00154 \\
\hline $\mathrm{B}+3$ & 0.00177 \\
\hline $\mathrm{Ba}+2$ & 0.002016 \\
\hline $\mathrm{Bi}+3$ & 0.0000358 \\
\hline $\mathrm{C} 10 \mathrm{H} 12 \mathrm{~N} 2 \mathrm{O} 8-4$ & 0.05814 \\
\hline $\mathrm{C} 10 \mathrm{H} 15 \mathrm{~N} 2 \mathrm{O} 7-3$ & 0.05814 \\
\hline $\mathrm{C} 12 \mathrm{H} 27 \mathrm{O} 4 \mathrm{P}$ & 0.05814 \\
\hline $\mathrm{C} 2 \mathrm{O} 4-2$ & 0.05814 \\
\hline C6H5O7-3 & $1.53 \mathrm{E}-06$ \\
\hline $\mathrm{Ca}+2$ & 0.05814 \\
\hline $\mathrm{Cd}+2$ & 0.00154 \\
\hline $\mathrm{Ce}+3$ & 0.00154 \\
\hline $\mathrm{Cl}-$ & 0.0001299 \\
\hline $\mathrm{Co}+3$ & 0.00154 \\
\hline $\mathrm{CO} 3-2$ & 0.00154 \\
\hline $\mathrm{Cr}(\mathrm{OH}) 4-$ & 0.000188 \\
\hline $\mathrm{Cr}(\mathrm{TOTAL})$ & 0.000188 \\
\hline $\mathrm{Cs}+$ & 0.0000427 \\
\hline $\mathrm{Cu}+2$ & 0.00188 \\
\hline F- & 0.00053 \\
\hline $\mathrm{Fe}+3$ & 0.002 \\
\hline $\mathrm{H}^{+}$ & 0.01 \\
\hline $\mathrm{Hg}+2$ & 0.00154 \\
\hline $\mathrm{K}+$ & 0.00157 \\
\hline $\mathrm{La}+3$ & 0.00154 \\
\hline $\mathrm{Li}+$ & 0.0016 \\
\hline $\mathrm{Mg}+2$ & 0.035 \\
\hline $\mathrm{Mn}+4$ & 0.001642 \\
\hline
\end{tabular}




$\begin{array}{lr}\begin{array}{l}\text { TLP-DMST-00001 } \\ \text { Components }\end{array} & \begin{array}{r}\text { Liquid/Solid Fractions } \\ \text { to PVP Vessel Vent }\end{array} \\ \mathrm{MnO} 4- & 0.001642 \\ \mathrm{Na}+ & 0.002 \\ \mathrm{NH} 3 & 0.0806 \\ \mathrm{Ni}+2 & 0.00154 \\ \mathrm{NO} 2- & 0.0000962 \\ \mathrm{NO}- & 0.0000962 \\ \mathrm{OH}(\mathrm{BOUND}) & 0.00154 \\ \mathrm{OH}- & 0.00154 \\ \mathrm{~Pb}+2 & 0.0000358 \\ \mathrm{Pd}+2 & 0.00154 \\ \mathrm{PO} 4-3 & 0.00154 \\ \mathrm{Pu}+4 & 0.00032 \\ \mathrm{Sb}+5 & 0.0000358 \\ \mathrm{Si}+4 & 0.00435 \\ \mathrm{SO} 4-2 & 0.0002375 \\ \mathrm{Sr}+2 & 0.0000855 \\ \mathrm{Tc}+7 & 0.00154 \\ \mathrm{Th}+4 & 0.00154 \\ \mathrm{Ti}+4 & 0.002 \\ \mathrm{U}(\mathrm{TOTAL}) & 0.00154 \\ \mathrm{Zn}+2 & 0.001621 \\ \mathrm{Zr}+4 & 0.002 \\ \mathrm{C} 12 \mathrm{H} 4 \mathrm{Cl} 6 & 0.0004 \\ \mathrm{C} 2 \mathrm{H} 3 \mathrm{~N} & 0.8314 \\ \mathrm{Mnp} & 0.002 \\ \mathrm{Nap}+ & 0.002 \\ \mathrm{Al}(\mathrm{OH}) 3 & 0.002\end{array}$

Table D-12 LAW Melter Splits

LMP-MLTR-00001/2

Components

106-Ru

$113 \mathrm{~m}-\mathrm{Cd}$

$125-\mathrm{Sb}$

126-Sn

129-I

134-Cs

137-Cs

$137 \mathrm{~m}-\mathrm{Ba}$

$14-\mathrm{C}$

151-Sm

152-Eu

154-Eu

$155-\mathrm{Eu}$

226-Ra

227-Ac

228-Ra

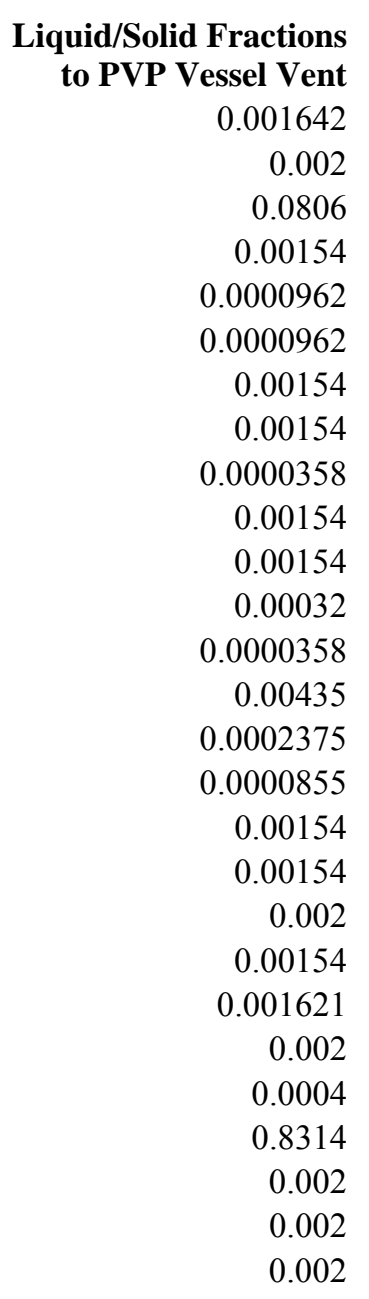

\section{Liquid \%}

to LOP-SCB-00001/2

1.852

0.855

24.39

0.943

0

24.39

24.39

24.39

100

0.943

0.943

0.943

0.943

24.39

0.943

24.39
Solid \% to LOP-SCB-00001/2

1.852

0.855

24.39

0.943

0

24.39

24.39

24.39

100

0.943

0.943

0.943

0.943

24.39

0.943

24.39 
LMP-MLTR-00001/2

Components

229-Th

231-Pa

232-Th

232-U

233-U

234-U

235-U

236-U

237-Np

238-Pu

238-U

239-Pu

240-Pu

241-Am

241-Pu

242-Cm

242-Pu

243-Am

243-Cm

244-Cm

3-H

$59-\mathrm{Ni}$

60-Co

$63-\mathrm{Ni}$

79-Se

90-Sr

$90-Y$

93-Zr

93m-Nb

99-Tc

$\mathrm{Ag}+$

$\mathrm{Al}(\mathrm{OH}) 3$

$\mathrm{Al}(\mathrm{OH}) 4-$

$\mathrm{Al}+3$

$\mathrm{As}+5$

$\mathrm{B}+3$

$\mathrm{Ba}+2$

$\mathrm{Be}+2$

$\mathrm{Bi}+3$

C10H12N2O8-4

C10H15N2O7-3

$\mathrm{C} 10 \mathrm{H} 8$

$\mathrm{C} 12 \mathrm{H} 27 \mathrm{O} 4 \mathrm{P}$

$\mathrm{C} 12 \mathrm{H} 4 \mathrm{Cl} 6$

$\mathrm{C} 12 \mathrm{H} 8$

$\mathrm{C} 13 \mathrm{H} 28$

$\mathrm{C} 2 \mathrm{Cl} 4$
Liquid \% to LOP-SCB-00001/2

0.943

0.943

0.943

0.943

0.943

0.943

0.943

0.943

0.943

0.943

0.943

0.943

0.943

1.852

0.943

0.943

0.943

1.852

0.943

0.943

100

0.941

0.943

0.941

24.39

0.943

0.943

0.021

0.943

62.5

1.852

0.192

0.192

0.192

24.39

1.0604

0.943

0.943

1.852

0

0

92.593

0

0

0

0

0
Solid \%

to LOP-SCB-00001/2

0.943

0.943

0.943

0.943

0.943

0.943

0.943

0.943

0.943

0.943

0.943

0.943

0.943

1.852

0.943

0.943

0.943

1.852

0.943

0.943

100

0.941

0.943

0.941

24.39

0.943

0.943

0.021

0.943

62.5

1.852

0.192

0.192

0.192

24.39

1.0604

0.943

0.943

1.852

0

92.593

0

0

0

0

0 
LMP-MLTR-00001/2

Components

$\mathrm{C} 2 \mathrm{H} 3 \mathrm{~N}$

$\mathrm{C} 2 \mathrm{H} 3 \mathrm{O} 2-$

$\mathrm{C} 2 \mathrm{H} 3 \mathrm{O} 3-$

$\mathrm{C} 2 \mathrm{H} 6 \mathrm{SiO}$

$\mathrm{C} 2 \mathrm{H} 8 \mathrm{~N} 2$

$\mathrm{C} 2 \mathrm{HCl} 3$

$\mathrm{C} 2 \mathrm{O} 4-2$

$\mathrm{C} 3 \mathrm{H} 6 \mathrm{O}$

$\mathrm{C} 4 \mathrm{H} 10 \mathrm{O}$

$\mathrm{C} 4 \mathrm{H} 7 \mathrm{NO} 4$

$\mathrm{C} 4 \mathrm{H} 8 \mathrm{O}$

$\mathrm{C} 5 \mathrm{H} 5 \mathrm{~N}$

$\mathrm{C} 6 \mathrm{H} 5 \mathrm{NO} 2$

C6H5O7-3

$\mathrm{C} 6 \mathrm{H} 6$

$\mathrm{C} 6 \mathrm{H} 6 \mathrm{O}$

$\mathrm{C} 7 \mathrm{H} 6 \mathrm{~N} 2 \mathrm{O} 4$

$\mathrm{C} 7 \mathrm{H} 8$

$\mathrm{C} 7 \mathrm{H} 8 \mathrm{O}$

$\mathrm{C} 8 \mathrm{H} 8 \mathrm{O}$

$\mathrm{Ca}+2$

$\mathrm{CCl} 4$

$\mathrm{Cd}+2$

$\mathrm{Ce}+3$

$\mathrm{CH} 2 \mathrm{Cl} 2$

$\mathrm{CHCl} 3$

$\mathrm{CHO} 2-$

Cl-

$\mathrm{CN}-$

$\mathrm{Co}+3$

$\mathrm{CO} 3-2$

$\mathrm{Cr}(\mathrm{OH}) 4-$

Cr(TOTAL)

$\mathrm{Cs}+$

$\mathrm{Cu}+2$

DPDMS

DPPG

F-

$\mathrm{Fe}+3$

$\mathrm{H}+$

$\mathrm{H} 2 \mathrm{CO} 3$

$\mathrm{H} 2 \mathrm{O}$

H2O(BOUND)

$\mathrm{H} 2 \mathrm{O} 2$

$\mathrm{HCO} 3-$

$\mathrm{Hg}+2$

$\mathrm{K}+$
Liquid \% to LOP-SCB-00001/2

0

0

$$
0
$$

0

0

0

0
0

20.04

0

0

0

\section{0}

0

0

0

\section{0}

0

0

0

0

0.125

0

0.855

0.943

0

0

0

0

1.852

0.943

0

7.143

7.143

14.286

9.091

0

0

0

0.185

0

0

0

0

0

0

0

5
Solid \%

to LOP-SCB-00001/2

\section{0}

0

0

0

0

0

0

20.04

0

0

0

0

0

0

0

0

0

0

0

0

0.125

0

0.855

0.943

0

0

0

0

1.852

0.943

0

7.143

7.143

14.286

9.091

0

0

0

0.185

0

0

0

0

0

0

0

5 
LMP-MLTR-00001/2

Components

$\mathrm{La}+3$

$\mathrm{Li}+$

LS10

$\mathrm{AlOOH}$

LS9

$\mathrm{Mg}+2$

$\mathrm{Mn}+4$

$\mathrm{MnO} 2$

MnO4-

Mnp

$\mathrm{Mo}+6$

$\mathrm{Na}+$

Nap+

$\mathrm{Nd}+3$

$\mathrm{NH} 3$

NH4+

$\mathrm{Ni}+2$

NO2-

$\mathrm{NO} 3-$

NO3-D

$\mathrm{O}-2$

OH(BOUND)

$\mathrm{OH}-$

$\mathrm{Pb}+2$

$\mathrm{Pd}+2$

PDMS

PO4-3

PO4-3(HARD)

PPG

$\mathrm{Pr}+3$

$\mathrm{Pu}+4$

$\mathrm{Rb}+$

$\mathrm{Rh}+3$

$\mathrm{Ru}+3$

$\mathrm{Sb}+5$

$\mathrm{Se}+6$

$\mathrm{Si}+4$

SO4-2

$\mathrm{Sr}+2$

$\mathrm{Ta}+5$

Tc +7

$\mathrm{Te}+6$

$\mathrm{Th}+4$

$\mathrm{Ti}+4$

$\mathrm{Tl}+3$

TOC

U(TOTAL)
Liquid \% to LOP-SCB-00001/2

0.943

0.952

0

\section{0}

0

0.016

0.943

0.943

0.943

0.943

1.852

1.282

1.282

0.943

0

0

0.943

0

0

0

0

0

0

1.852

0.943

0
1.19

1.19

1.19

0

0.943

0.943

1.852

0.943

0.943

24.39

24.39

0.116

0

0.943

0.943

62.5

24.39

0.943

0.294

24.39

0

0.943
Solid \%

to LOP-SCB-00001/2

0.943

0.952

0

0

0

0.016

0.943

0.943

0.943

0.943

1.852

1.282

1.282

0.943

0

0

0.943

0

0

0

0

0

0

1.852

0.943

0

1.19

1.19

0

0.943

0.943

1.852

0.943

0.943

24.39

24.39

0.116

0

0.943

0.943

62.5

24.39

0.943

0.294

24.39

0

0.943 


LMP-MLTR-00001/2
Components
$\mathrm{V}+5$
$\mathrm{~W}+6$
$\mathrm{Y}+3$
$\mathrm{Zn}+2$
$\mathrm{Zr}+4$

$\begin{array}{rr}\begin{array}{r}\text { Liquid \% } \\ \text { to LOP-SCB-00001/2 }\end{array} & \begin{array}{r}\text { Solid \% } \\ 1.852\end{array} \\ 0.943 & 1.852 \\ 0.943 & 0.943 \\ 0.286 & 0.943 \\ 0.022 & 0.286 \\ & 0.022\end{array}$

DFs are handled differently in the LAW melters. Refer to Section 4.8.15 for detailed discussions.

\section{Table D-13 LAW SBS Splits}

$\begin{array}{lrr}\begin{array}{l}\text { LOP-SCB-0001/2 } \\ \text { Components }\end{array} & \begin{array}{r}\text { Liquid \% } \\ \text { to LOP-VSL-00001/2 }\end{array} & \begin{array}{r}\text { Solid \% } \\ \text { to6-Ru }\end{array} \\ \text { 113m-Cd } & 72.22222222 & 72.22222222 \\ 125-\mathrm{Sb} & 81.13207547 & 81.13207547 \\ 126-\mathrm{Sn} & 99 & 99 \\ 129-\mathrm{I} & 98.82352941 & 98.82352941 \\ 134-\mathrm{Cs} & 0 & 0 \\ 137-\mathrm{Cs} & 79.59183673 & 79.59183673 \\ 137 \mathrm{~m}-\mathrm{Ba} & 79.59183673 & 79.59183673 \\ 14-\mathrm{C} & 79.59183673 & 79.59183673 \\ 151-\mathrm{Sm} & 0 & 0 \\ 152-\mathrm{Eu} & 98.82352941 & 98.82352941 \\ 154-\mathrm{Eu} & 98.82352941 & 98.82352941 \\ 155-\mathrm{Eu} & 98.82352941 & 98.82352941 \\ 226-\mathrm{Ra} & 98.82352941 & 98.82352941 \\ 227-\mathrm{Ac} & 98.82352941 & 98.82352941 \\ 228-\mathrm{Ra} & 98.82352941 & 98.82352941 \\ 229-\mathrm{Th} & 98.82352941 & 98.82352941 \\ 231-\mathrm{Pa} & 98.82352941 & 98.82352941 \\ 232-\mathrm{Th} & 98.82352941 & 98.82352941 \\ 232-\mathrm{U} & 98.82352941 & 98.82352941 \\ 233-\mathrm{U} & 98.82352941 & 98.82352941 \\ 234-\mathrm{U} & 98.82352941 & 98.82352941 \\ 235-\mathrm{U} & 98.82352941 & 98.82352941 \\ 236-\mathrm{U} & 98.82352941 & 98.82352941 \\ 237-\mathrm{Np} & 98.82352941 & 98.82352941 \\ 238-\mathrm{Pu} & 98.82352941 & 98.82352941 \\ 238-\mathrm{U} & 98.82352941 & 98.82352941 \\ 239-\mathrm{Pu} & 98.82352941 & 98.82352941 \\ 240-\mathrm{Pu} & 98.82352941 & 98.82352941 \\ 241-\mathrm{Am} & 98.82352941 & 98.82352941 \\ 241-\mathrm{Pu} & 98.82352941 & 98.82352941 \\ 242-\mathrm{Cm} & 98.82352941 & 98.82352941 \\ 242-\mathrm{Pu} & 98.82352941 & 98.82352941 \\ 243-\mathrm{Am} & 98.82352941 & 98.82352941 \\ 243-\mathrm{Cm} & 98.82352941 & 982352941 \\ 244-\mathrm{Cm} & 98.82352941 & \end{array}$




\section{LOP-SCB-00001/2}

Components

$3-\mathrm{H}$

$59-\mathrm{Ni}$

60-Co

$63-\mathrm{Ni}$

79-Se

$90-\mathrm{Sr}$

$90-Y$

$93-\mathrm{Zr}$

$93 \mathrm{~m}-\mathrm{Nb}$

99-Tc

$\mathrm{Ag}+$

$\mathrm{Al}(\mathrm{OH}) 3$

$\mathrm{Al}(\mathrm{OH}) 4-$

$\mathrm{Al}+3$

$\mathrm{AlOOH}$

$\mathrm{As}+5$

$\mathrm{B}+3$

$\mathrm{Ba}+2$

$\mathrm{Be}+2$

$\mathrm{Bi}+3$

C10H12N2O8-4

C10H15N2O7-3

$\mathrm{C} 10 \mathrm{H} 8$

$\mathrm{C} 12 \mathrm{H} 27 \mathrm{O} 4 \mathrm{P}$

$\mathrm{C} 12 \mathrm{H} 4 \mathrm{Cl} 6$

$\mathrm{C} 12 \mathrm{H} 8$

$\mathrm{C} 13 \mathrm{H} 28$

$\mathrm{C} 2 \mathrm{Cl} 4$

$\mathrm{C} 2 \mathrm{H} 3 \mathrm{~N}$

$\mathrm{C} 2 \mathrm{H} 3 \mathrm{O} 2-$

$\mathrm{C} 2 \mathrm{H} 3 \mathrm{O} 3-$

$\mathrm{C} 2 \mathrm{H} 6 \mathrm{SiO}$

C2H8N2

$\mathrm{C} 2 \mathrm{HCl} 3$

$\mathrm{C} 2 \mathrm{O} 4-2$

$\mathrm{C} 3 \mathrm{H} 6 \mathrm{O}$

$\mathrm{C} 4 \mathrm{H} 10 \mathrm{O}$

$\mathrm{C} 4 \mathrm{H} 7 \mathrm{NO} 4$

$\mathrm{C} 4 \mathrm{H} 8 \mathrm{O}$

$\mathrm{C} 5 \mathrm{H} 5 \mathrm{~N}$

$\mathrm{C} 6 \mathrm{H} 5 \mathrm{NO} 2$

C6H5O7-3

$\mathrm{C} 6 \mathrm{H} 6$

$\mathrm{C} 6 \mathrm{H} 6 \mathrm{O}$

C7H6N2O4

$\mathrm{C} 7 \mathrm{H} 8$

C7H8O
Liquid \%

to LOP-VSL-00001/2

0

75

89.36170213

75

88.0952381

99.851

99.851

96.552

98.82352941

79.59183673

98

99.04761905

99.04761905

99.04761905

0

95

56.522

99.71428571

98.82352941

98

0

0

0

0

94.50549451

\section{0}

0

0

94.50549451

0

0

0

0

0

87.95180723

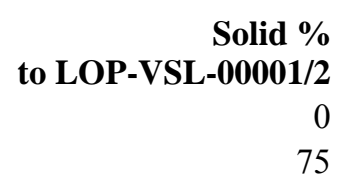

89.36170213

75

88.0952381

99.851

99.851

96.552

98.82352941

79.59183673

98

99.04761905

99.04761905

99.04761905

\section{0}

95

56.522

99.71428571

98.82352941

98

0

0

0

0

94.50549451

\section{0}

0

94.50549451

0

0

0

0

87.95180723

0

0

0

0

0

0

0

0

0

0

0

0 
LOP-SCB-00001/2

Components

$\mathrm{C} 8 \mathrm{H} 8 \mathrm{O}$

$\mathrm{Ca}+2$

$\mathrm{CCl} 4$

$\mathrm{Cd}+2$

$\mathrm{Ce}+3$

$\mathrm{CH} 2 \mathrm{Cl} 2$

$\mathrm{CHCl} 3$

$\mathrm{CHO} 2-$

Cl-

$\mathrm{CN}-$

$\mathrm{Co}+3$

$\mathrm{CO} 3-2$

$\mathrm{Cr}(\mathrm{OH}) 4-$

Cr(TOTAL)

$\mathrm{Cs}+$

$\mathrm{Cu}+2$

DPDMS

DPPG

F-

$\mathrm{Fe}+3$

$\mathrm{H}+$

$\mathrm{H} 2 \mathrm{CO} 3$

$\mathrm{H} 2 \mathrm{O}$

H2O(BOUND)

$\mathrm{H} 2 \mathrm{O} 2$

$\mathrm{HCO} 3-$

$\mathrm{Hg}+2$

$\mathrm{K}+$

$\mathrm{La}+3$

$\mathrm{Li}+$

LS10

LS9

$\mathrm{Mg}+2$

$\mathrm{Mn}+4$

$\mathrm{MnO} 2$

MnO4-

Mnp

$\mathrm{Mo}+6$

$\mathrm{Na}+$

Nap+

$\mathrm{Nd}+3$

$\mathrm{NH} 3$

$\mathrm{NH} 4+$

$\mathrm{Ni}+2$

NO2-

NO3-

NO3-D
Liquid \%

to LOP-VSL-00001/2

0

97.76785714

0

81.13207547

99.83870968

0

0

87.95180723

87.95180723

87.95180723

89.36170213

87.95180723

82.75862069

82.75862069

79.59183673

99.28571429

0

0

85.915

99.091

99.9

0

0

0

0

87.95180723

0

81.13207547

99.9

88.23529412

0

87.342

99.88235294

99.88235294

99.88235294

99.88235294

88.50574713

87.95180723

87.95180723

99.9

90

90

75

0.199600798

87.95180723
Solid \%

to LOP-VSL-00001/2

97.76785714

81.13207547

99.83870968

87.95180723

87.95180723

87.95180723

89.36170213

87.95180723

82.75862069

82.75862069

79.59183673

99.28571429

\section{0}

85.915

99.091

99.9

0

0

0

0

87.95180723

81.13207547

99.9

88.23529412

0

87.342

99.88235294

99.88235294

99.88235294

99.88235294

88.50574713

87.95180723

87.95180723

99.9

90

90

75

0.199600798

87.95180723 


LOP-SCB-00001/2
Components
$\mathrm{O}-2$
$\mathrm{OH}(\mathrm{BOUND})$
$\mathrm{OH}-$
$\mathrm{Pb}+2$
$\mathrm{Pd}+2$
$\mathrm{PDMS}$
$\mathrm{PO} 4-3$
$\mathrm{PO}-3$ (HARD)
$\mathrm{PPG}$
$\mathrm{Pr}+3$
$\mathrm{Pu}+4$
$\mathrm{Rb}+$
$\mathrm{Rh}+3$
$\mathrm{Ru}+3$
$\mathrm{Sb}+5$
$\mathrm{Se}+6$
$\mathrm{Si}+4$
$\mathrm{SO} 4-2$
$\mathrm{Sr}+2$
$\mathrm{Ta}+5$
$\mathrm{Tc}+7$
$\mathrm{Te}+6$
$\mathrm{Th}+4$
$\mathrm{Ti}+4$
$\mathrm{Tl}+3$
$\mathrm{TOC}$
$\mathrm{U}(\mathrm{TOTAL})$
$\mathrm{V}+5$
$\mathrm{~W}+6$
$\mathrm{Y}+3$
$\mathrm{Zn}+2$
$\mathrm{Zr}+4$

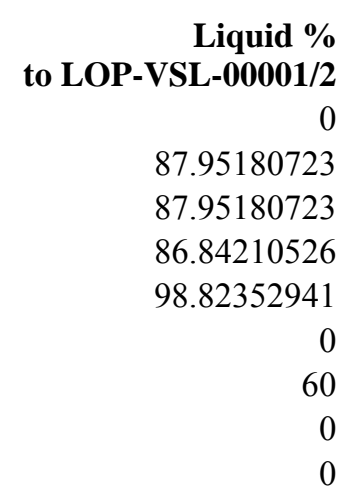

98.82352941

98.82352941

79.59183673

79.59183673

72.22222222

99

88.0952381

99.07407407

85.71428571

99.85074627

98.82352941

79.59183673

95

98.82352941

99.643

88.0952381

98.82352941

82.45614035

98.82352941

99.6875

96.62162162

96.552
Solid \%

to LOP-VSL-00001/2

87.95180723

87.95180723

86.84210526

98.82352941

0

60

0

0

98.82352941

98.82352941

79.59183673

79.59183673

72.22222222

99

88.0952381

99.07407407

85.71428571

99.85074627

98.82352941

79.59183673

95

98.82352941

99.643

88.0952381

98.82352941

82.45614035

98.82352941

99.6875

96.62162162

96.552

Table D-14 LAW WESP Splits

LOP-WESP-00001/2

Components

106-Ru

$113 \mathrm{~m}-\mathrm{Cd}$

$125-\mathrm{Sb}$

126-Sn

129-I

134-Cs

137-Cs

$137 \mathrm{~m}-\mathrm{Ba}$

14-C

151-Sm

152-Eu
Liquid \%

to RLD-VSL-00004

99.9

99.9

99.9

99.9

0

96

96

96

0

98

99.9
Solid \%

to RLD-VSL-00004

99.9

99.9

99.9

99.9

0

96

96

96

0

98

99.9 


\section{LOP-WESP-00001/2}

Components

154-Eu

$155-\mathrm{Eu}$

226-Ra

227-Ac

228-Ra

229-Th

231-Pa

232-Th

232-U

233-U

234-U

235-U

236-U

237-Np

238-Pu

238-U

239-Pu

240-Pu

241-Am

241-Pu

242-Cm

242-Pu

243-Am

243-Cm

244-Cm

$3-\mathrm{H}$

$59-\mathrm{Ni}$

60-Co

$63-\mathrm{Ni}$

79-Se

90-Sr

90-Y

93-Zr

$93 \mathrm{~m}-\mathrm{Nb}$

99-Tc

$\mathrm{Ag}+$

$\mathrm{Al}(\mathrm{OH}) 3$

$\mathrm{Al}(\mathrm{OH}) 4-$

$\mathrm{Al}+3$

$\mathrm{As}+5$

$\mathrm{B}+3$

$\mathrm{Ba}+2$

$\mathrm{Be}+2$

$\mathrm{Bi}+3$

C10H12N2O8-4

C10H15N2O7-3

$\mathrm{C} 10 \mathrm{H} 8$
Liquid \% to RLD-VSL-00004

99.9

99.9

99.9

99.9

99.9

99.9

98

99.9

99.9

99.9

99.9

99.9

99.9

99.9

99.9

99.9

99.9

99.9

99.9

99.9

99.9

99.9

99.9

99.9

99.9

0

99.9

99.9

99.9

99.9

99.9

99.9

33.333

98

99.9

99.9

99.9

99.9

79.167

99.9

56.522

99.9

99.9

99.9

0

0

0
Solid \%

to RLD-VSL-00004

99.9

99.9

99.9

99.9

99.9

99.9

98

99.9

99.9

99.9

99.9

99.9

99.9

99.9

99.9

99.9

99.9

99.9

99.9

99.9

99.9

99.9

99.9

99.9

99.9

0

99.9

99.9

99.9

99.9

99.9

99.9

33.333

98

99.9

99.9

99.9

99.9

79.167

99.9

56.522

99.9

99.9

99.9

0

0

0 
LOP-WESP-00001/2

Components

$\mathrm{C} 12 \mathrm{H} 27 \mathrm{O} 4 \mathrm{P}$

$\mathrm{C} 12 \mathrm{H} 4 \mathrm{Cl} 6$

$\mathrm{C} 12 \mathrm{H} 8$

$\mathrm{C} 13 \mathrm{H} 28$

$\mathrm{C} 2 \mathrm{Cl} 4$

$\mathrm{C} 2 \mathrm{H} 3 \mathrm{~N}$

$\mathrm{C} 2 \mathrm{H} 3 \mathrm{O} 2-$

$\mathrm{C} 2 \mathrm{H} 3 \mathrm{O} 3-$

$\mathrm{C} 2 \mathrm{H} 6 \mathrm{SiO}$

$\mathrm{C} 2 \mathrm{H} 8 \mathrm{~N} 2$

$\mathrm{C} 2 \mathrm{HCl} 3$

$\mathrm{C} 2 \mathrm{O} 4-2$

$\mathrm{C} 3 \mathrm{H} 6 \mathrm{O}$

$\mathrm{C} 4 \mathrm{H} 10 \mathrm{O}$

$\mathrm{C} 4 \mathrm{H} 7 \mathrm{NO} 4$

$\mathrm{C} 4 \mathrm{H} 8 \mathrm{O}$

$\mathrm{C} 5 \mathrm{H} 5 \mathrm{~N}$

$\mathrm{C} 6 \mathrm{H} 5 \mathrm{NO} 2$

$\mathrm{C} 6 \mathrm{H} 5 \mathrm{O} 7-3$

$\mathrm{C} 6 \mathrm{H} 6$

$\mathrm{C} 6 \mathrm{H} 6 \mathrm{O}$

C7H6N2O4

C7H8

$\mathrm{C} 7 \mathrm{H} 8 \mathrm{O}$

$\mathrm{C} 8 \mathrm{H} 8 \mathrm{O}$

$\mathrm{Ca}+2$

$\mathrm{CCl} 4$

$\mathrm{Cd}+2$

$\mathrm{Ce}+3$

$\mathrm{CH} 2 \mathrm{Cl} 2$

$\mathrm{CHCl} 3$

CHO2-

Cl-

$\mathrm{CN}-$

$\mathrm{Co}+3$

$\mathrm{CO} 3-2$

$\mathrm{Cr}(\mathrm{OH}) 4-$

Cr(TOTAL)

$\mathrm{Cs}+$

$\mathrm{Cu}+2$

DPDMS

DPPG

F-

$\mathrm{Fe}+3$

$\mathrm{H}+$

$\mathrm{H} 2 \mathrm{CO} 3$

$\mathrm{H} 2 \mathrm{O}$
Liquid \% to RLD-VSL-00004

99.796

0

0

0

99.796

0

0

0

0

0

99.796

0

0

0

0

0

0

0

0

0

0

0

0

0

85.714

96.296

99.9

0

0

99.796

58.333

96.296

99.9

96.296

88.764

88.764

96.154

99.9

0

0

76.19

88.636

0

0

99.9
Solid \%

to RLD-VSL-00004

0

99.796

0

0

0

99.796

0

0

0

0

0

99.796

0

0

0

0

0

0

0

0

0

0

0

0

0

85.714

0

96.296

99.9

0

0

99.796

58.333

96.296

99.9

96.296

88.764

88.764

96.154

99.9

0

0

76.19

88.636

0

0

99.9 
LOP-WESP-00001/2

Components

H2O(BOUND)

$\mathrm{H} 2 \mathrm{O} 2$

$\mathrm{HCO} 3-$

$\mathrm{Hg}+2$

$\mathrm{K}+$

$\mathrm{La}+3$

$\mathrm{Li}+$

LS10

$\mathrm{AlOOH}$

LS9

$\mathrm{Mg}+2$

$\mathrm{Mn}+4$

$\mathrm{MnO} 2$

$\mathrm{MnO} 4-$

Mnp

$\mathrm{Mo}+6$

$\mathrm{Na}+$

Nap+

$\mathrm{Nd}+3$

$\mathrm{NH} 3$

$\mathrm{NH} 4+$

$\mathrm{Ni}+2$

$\mathrm{NO} 2-$

NO3-

NO3-D

$\mathrm{O}-2$

OH(BOUND)

$\mathrm{OH}-$

$\mathrm{Pb}+2$

$\mathrm{Pd}+2$

PDMS

PO4-3

PO4-3(HARD)

PPG

$\mathrm{Pr}+3$

$\mathrm{Pu}+4$

$\mathrm{Rb}+$

$\mathrm{Rh}+3$

$\mathrm{Ru}+3$

$\mathrm{Sb}+5$

$\mathrm{Se}+6$

$\mathrm{Si}+4$

SO4-2

$\mathrm{Sr}+2$

$\mathrm{Ta}+5$

$\mathrm{Tc}+7$

$\mathrm{Te}+6$
Liquid \% to RLD-VSL-00004

0

0

96.296

0

96.667

99.9

94.737

0

0

0

56.522

99.9

99.9

99.9

99.9

99.9

96.296

96.296

99.9

23.077

99.9

99.9

3.846

96.296

0

0

99.9

99.9

93.333

99.9

0

83.051

0

0

99.9

99.9

99.9

99.9

99.9

99.9

99.9

88.889

91.667

99.9

99.9

99.9

99.9
Solid \%

to RLD-VSL-00004

0

96.296

0

96.667

99.9

94.737

0

0

56.522

99.9

99.9

99.9

99.9

99.9

96.296

96.296

99.9

23.077

99.9

99.9

3.846

96.296

0

0

99.9

99.9

93.333

99.9

0

83.051

0

0

99.9

99.9

99.9

99.9

99.9

99.9

99.9

88.889

91.667

99.9

99.9

99.9

99.9 


\section{LOP-WESP-00001/2}

Components

$\mathrm{Th}+4$

$\mathrm{Ti}+4$

$\mathrm{Tl}+3$

TOC

U(TOTAL)

$\mathrm{V}+5$

$\mathrm{W}+6$

$\mathrm{Y}+3$

$\mathrm{Zn}+2$

$\mathrm{Zr}+4$
Liquid \%

to RLD-VSL-00004

99.9

16.667

99.9

0

99.9

99.9

99.9

99.9

71.429

33.333
Solid \%

to RLD-VSL-00004

99.9

16.667

99.9

0

99.9

99.9

99.9

99.9

71.429

33.333

Table D-15 LAW HEPA Splits

LVP-HEPA-00001AB
Components
$106-\mathrm{Ru}$
$113 \mathrm{~m}-\mathrm{Cd}$
$125-\mathrm{Sb}$
$126-\mathrm{Sn}$
$129-\mathrm{I}$
$134-\mathrm{Cs}$
$137-\mathrm{Cs}$
$137 \mathrm{~m}-\mathrm{Ba}$
$14-\mathrm{C}$
$151-\mathrm{Sm}$
$152-\mathrm{Eu}$
$154-\mathrm{Eu}$
$155-\mathrm{Eu}$
$226-\mathrm{Ra}$
$227-\mathrm{Ac}$
$228-\mathrm{Ra}$
$229-\mathrm{Th}$
$231-\mathrm{Pa}$
$232-\mathrm{Th}$
$232-\mathrm{U}$
$233-\mathrm{U}$
$234-\mathrm{U}$
$235-\mathrm{U}$
$236-\mathrm{U}$
$237-\mathrm{Np}$
$238-\mathrm{Pu}$
$238-\mathrm{U}$
$239-\mathrm{Pu}$
$240-\mathrm{Pu}$
$241-\mathrm{Am}$
$241-\mathrm{Pu}$
$242-\mathrm{Cm}$
$242-\mathrm{Pu}$

\begin{tabular}{rr}
$\begin{array}{r}\text { Liquid \% } \\
\text { to Accumulate }\end{array}$ & $\begin{array}{r}\text { Solid \% } \\
\text { to Accumulate }\end{array}$ \\
99.99994 & 99.99994 \\
99.99994 & 99.99994 \\
99.99994 & 99.99994 \\
99.99994 & 99.99994 \\
0 & 0 \\
99.99994 & 99.99994 \\
99.99994 & 99.99994 \\
99.99994 & 99.99994 \\
0 & 0 \\
99.99994 & 99.99994 \\
99.99994 & 99.99994 \\
99.99994 & 99.99994 \\
99.99994 & 99.99994 \\
99.99994 & 99.99994 \\
99.99994 & 99.99994 \\
99.99994 & 99.99994 \\
99.99994 & 99.99994 \\
99.99994 & 99.99994 \\
99.99994 & 99.99994 \\
99.99994 & 99.99994 \\
99.99994 & 99.99994 \\
99.99994 & 99.99994 \\
99.99994 & 99.99994 \\
99.99994 & 99.99994 \\
99.99994 & 99.99994 \\
99.99994 & 99.99994 \\
99.99994 & 99.99994 \\
99.99994 & 99.99994 \\
99.99994 & 99.99994 \\
99.99994 & 99.99994 \\
99.99994 & 99.99994 \\
99.99994 & 99.99994 \\
99.99994 & 99.99994 \\
& \\
\hline
\end{tabular}


LVP-HEPA-00001AB

\section{Components}

243-Am

243-Cm

244-Cm

$3-\mathrm{H}$

$59-\mathrm{Ni}$

60-Co

$63-\mathrm{Ni}$

79-Se

$90-\mathrm{Sr}$

90-Y

93-Zr

$93 \mathrm{~m}-\mathrm{Nb}$

99-Tc

$\mathrm{Ag}+$

$\mathrm{Al}(\mathrm{OH}) 3$

$\mathrm{Al}(\mathrm{OH}) 4-$

$\mathrm{Al}+3$

$\mathrm{As}+5$

$\mathrm{B}+3$

$\mathrm{Ba}+2$

$\mathrm{Be}+2$

$\mathrm{Bi}+3$

C10H12N2O8-4

C10H15N2O7-3

$\mathrm{C} 10 \mathrm{H} 8$

$\mathrm{C} 12 \mathrm{H} 27 \mathrm{O} 4 \mathrm{P}$

$\mathrm{C} 12 \mathrm{H} 4 \mathrm{Cl} 6$

$\mathrm{C} 12 \mathrm{H} 8$

C13H28

$\mathrm{C} 2 \mathrm{Cl} 4$

$\mathrm{C} 2 \mathrm{H} 3 \mathrm{~N}$

$\mathrm{C} 2 \mathrm{H} 3 \mathrm{O} 2-$

$\mathrm{C} 2 \mathrm{H} 3 \mathrm{O} 3-$

$\mathrm{C} 2 \mathrm{H} 6 \mathrm{SiO}$

C2H8N2

$\mathrm{C} 2 \mathrm{HCl} 3$

C2O4-2

$\mathrm{C} 3 \mathrm{H} 6 \mathrm{O}$

C4H10O

$\mathrm{C} 4 \mathrm{H} 7 \mathrm{NO} 4$

$\mathrm{C} 4 \mathrm{H} 8 \mathrm{O}$

$\mathrm{C} 5 \mathrm{H} 5 \mathrm{~N}$

$\mathrm{C} 6 \mathrm{H} 5 \mathrm{NO} 2$

C6H5O7-3

C6H6

C6H6O

C7H6N2O4
Liquid \%

to Accumulate

99.99994

99.99994

99.99994

99.99994

99.99994

99.99994

99.99994

99.99994

99.99994

99.99994

99.99994

99.99994

99.99994

99.99994

99.99994

99.99994

99.99994

99.97

99.99994

99.99994

99.99994

99.99994

99.99994

99.99994

99.99994

99.99994

99.99994

99.99994

99.99994

99.99994

99.99994

99.99994

99.99994

99.99994

99.99994

99.99994

99.99994

99.99994

99.99994

99.99994

99.99994

99.99994

99.99994

99.99994

99.99994

99.99994

\section{Solid \%}

to Accumulate

99.99994

99.99994

99.99994

0

99.99994

99.99994

99.99994

99.99994

99.99994

99.99994

99.99994

99.99994

99.99994

99.99994

99.99994

99.99994

99.99994

99.99994

99.97

99.99994

99.99994

99.99994

99.99994

99.99994

99.99994

99.99994

99.99994

99.99994

99.99994

99.99994

99.99994

99.99994

99.99994

99.99994

99.99994

99.99994

99.99994

99.99994

99.99994

99.99994

99.99994

99.99994

99.99994

99.99994

99.99994

99.99994

99.99994 


\section{LVP-HEPA-00001AB}

Components

$\mathrm{C} 7 \mathrm{H} 8$

$\mathrm{C} 7 \mathrm{H} 8 \mathrm{O}$

$\mathrm{C} 8 \mathrm{H} 8 \mathrm{O}$

$\mathrm{Ca}+2$

$\mathrm{CCl} 4$

$\mathrm{Cd}+2$

$\mathrm{Ce}+3$

$\mathrm{CH} 2 \mathrm{Cl} 2$

$\mathrm{CHCl} 3$

$\mathrm{CHO} 2-$

Cl-

$\mathrm{CN}-$

$\mathrm{Co}+3$

$\mathrm{CO} 3-2$

$\mathrm{Cr}(\mathrm{OH}) 4-$

Cr(TOTAL)

$\mathrm{Cs}+$

$\mathrm{Cu}+2$

DPDMS

DPPG

F-

$\mathrm{Fe}+3$

$\mathrm{H}+$

$\mathrm{H} 2 \mathrm{CO} 3$

$\mathrm{H} 2 \mathrm{O}$

$\mathrm{H} 2 \mathrm{O}$ (BOUND)

$\mathrm{H} 2 \mathrm{O} 2$

$\mathrm{HCO} 3-$

$\mathrm{Hg}+2$

$\mathrm{K}+$

$\mathrm{La}+3$

$\mathrm{Li}+$

LS10

$\mathrm{AlOOH}$

LS9

$\mathrm{Mg}+2$

$\mathrm{Mn}+4$

$\mathrm{MnO} 2$

MnO4-

Mnp

$\mathrm{Mo}+6$

$\mathrm{Na}+$

Nap+

$\mathrm{Nd}+3$

$\mathrm{NH} 3$

NH4+

$\mathrm{Ni}+2$
Liquid \%

to Accumulate

99.99994

99.99994

99.99994

99.99994

99.99994

99.99994

99.99994

99.99994

99.99994

99.99994

99.99994

99.99994

99.99994

99.99994

99.99994

99.99994

99.99994

99.99994

99.99994

99.99994

99.99994

99.99994

99.99994

99.99994

99.99994

99.99994

99.99994

99.99994

99.99994

99.99994

99.99994

99.99994

99.99994

99.99994

99.99994

99.99994

99.99994

99.99994

99.99994

99.99994

99.99994

99.99994

99.99994

99.99994

99.99994

\section{Solid \%}

to Accumulate

99.99994

99.99994

99.99994

99.99994

99.99994

99.99994

99.99994

99.99994

99.99994

99.99994

99.99994

99.99994

99.99994

99.99994

99.99994

99.99994

99.99994

99.99994

99.99994

99.99994

99.99994

99.99994

99.99994

99.99994

99.99994

99.99994

99.99994

99.99994

99.99994

99.99994

99.99994

99.99994

99.99994

99.99994

99.99994

99.99994

99.99994

99.99994

99.99994

99.99994

99.99994

99.99994

99.99994

99.99994

99.99994 


\begin{tabular}{|c|c|c|}
\hline $\begin{array}{l}\text { LVP-HEPA-00001AB } \\
\text { Components }\end{array}$ & $\begin{array}{r}\text { Liquid \% } \\
\text { to Accumulate }\end{array}$ & $\begin{array}{r}\text { Solid \% } \\
\text { to Accumulate }\end{array}$ \\
\hline NO2- & 0 & 0 \\
\hline NO3- & 99.99994 & 99.99994 \\
\hline NO3-D & 99.99994 & 99.99994 \\
\hline $\mathrm{O}-2$ & 99.99994 & 99.99994 \\
\hline OH(BOUND) & 99.99994 & 99.99994 \\
\hline $\mathrm{OH}-$ & 99.99994 & 99.99994 \\
\hline $\mathrm{Pb}+2$ & 99.99994 & 99.99994 \\
\hline $\mathrm{Pd}+2$ & 99.99994 & 99.99994 \\
\hline PDMS & 99.99994 & 99.99994 \\
\hline PO4-3 & 99.99994 & 99.99994 \\
\hline PO4-3(HARD) & 99.99994 & 99.99994 \\
\hline PPG & 99.99994 & 99.99994 \\
\hline $\mathrm{Pr}+3$ & 99.99994 & 99.99994 \\
\hline $\mathrm{Pu}+4$ & 99.99994 & 99.99994 \\
\hline $\mathrm{Rb}+$ & 99.99994 & 99.99994 \\
\hline $\mathrm{Rh}+3$ & 99.99994 & 99.99994 \\
\hline $\mathrm{Ru}+3$ & 99.99994 & 99.99994 \\
\hline $\mathrm{Sb}+5$ & 99.99994 & 99.99994 \\
\hline $\mathrm{Se}+6$ & 99.99994 & 99.99994 \\
\hline $\mathrm{Si}+4$ & 99.99994 & 99.99994 \\
\hline SO4-2 & 99.99994 & 99.99994 \\
\hline $\mathrm{Sr}+2$ & 99.99994 & 99.99994 \\
\hline $\mathrm{Ta}+5$ & 99.99994 & 99.99994 \\
\hline $\mathrm{Tc}+7$ & 99.99994 & 99.99994 \\
\hline $\mathrm{Te}+6$ & 99.99994 & 99.99994 \\
\hline $\mathrm{Th}+4$ & 99.99994 & 99.99994 \\
\hline $\mathrm{Ti}+4$ & 99.99994 & 99.99994 \\
\hline $\mathrm{Tl}+3$ & 99.99994 & 99.99994 \\
\hline TOC & 99.99994 & 99.99994 \\
\hline U(TOTAL) & 99.99994 & 99.99994 \\
\hline$V+5$ & 99.99994 & 99.99994 \\
\hline $\mathrm{W}+6$ & 99.99994 & 99.99994 \\
\hline $\mathrm{Y}+3$ & 99.99994 & 99.99994 \\
\hline $\mathrm{Zn}+2$ & 99.99994 & 99.99994 \\
\hline $\mathrm{Zr}+4$ & 99.99994 & 99.99994 \\
\hline & Gas \% & \\
\hline Gas Components & to ACCUMULATE & \\
\hline 129-I & 0 & \\
\hline $\mathrm{C} 12 \mathrm{H} 4 \mathrm{Cl} 6$ & 99.99994 & \\
\hline $\mathrm{C} 2 \mathrm{H} 3 \mathrm{~N}$ & 99.99994 & \\
\hline $\mathrm{CO} 2$ & 0 & \\
\hline $\mathrm{H} 2 \mathrm{O}$ & 0 & \\
\hline $\mathrm{HCl}$ & 69.512 & \\
\hline $\mathrm{HF}$ & 26.471 & \\
\hline $\mathrm{Hg}$ & 99.99994 & \\
\hline $\mathrm{N} 2$ & 0 & \\
\hline NH3 & 99.99994 & \\
\hline NO & 0 & \\
\hline
\end{tabular}


LVP-HEPA-00001AB

Components

$\mathrm{NO} 2$

$\mathrm{O} 2$

$\mathrm{P} 2 \mathrm{O} 5$

$\mathrm{SO} 2$

$\mathrm{SO} 3$
Liquid \% to Accumulate

0

0

99.99994

90

99.99994

Table D-16 LAW Carbon Absorber Splits

LVP-ADBR-00001AB

Components

106-Ru

$113 \mathrm{~m}-\mathrm{Cd}$

$125-\mathrm{Sb}$

126-Sn

129-I

134-Cs

137-Cs

$137 \mathrm{~m}-\mathrm{Ba}$

14-C

151-Sm

152-Eu

154-Eu

155-Eu

226-Ra

227-Ac

228-Ra

229-Th

231-Pa

232-Th

232-U

233-U

234-U

235-U

236-U

237-Np

238-Pu

238-U

239-Pu

240-Pu

241-Am

241-Pu

242-Cm

242-Pu

243-Am

243-Cm

244-Cm

3-H

$59-\mathrm{Ni}$
Liquid \%

to Accumulate

0

0

0

0

0

0

\section{0}

0

0

0

0

0

0

0

0

0

0

0

0

0

0

0

0

0

0

0

0

0

0

0

0

0

0

0

0

0

0

0
Solid \%

to Accumulate
Solid \%

to Accumulate

0

0

0

0

0

0

0

0

0

0

0

0

0

0

0

0

0

0

0

0

0

0

0

0

0

0

0

0

0

0

0

0

0

0

0

0

0

0 
LVP-ADBR-00001AB

Components

60-Co

$63-\mathrm{Ni}$

79-Se

$90-\mathrm{Sr}$

$90-\mathrm{Y}$

$93-\mathrm{Zr}$

$93 \mathrm{~m}-\mathrm{Nb}$

99-Tc

$\mathrm{Ag}+$

$\mathrm{Al}(\mathrm{OH}) 3$

$\mathrm{Al}(\mathrm{OH}) 4-$

$\mathrm{Al}+3$

As +5

$\mathrm{B}+3$

$\mathrm{Ba}+2$

$\mathrm{Be}+2$

$\mathrm{Bi}+3$

C10H12N2O8-4

C10H15N2O7-3

$\mathrm{C} 10 \mathrm{H} 8$

$\mathrm{C} 12 \mathrm{H} 27 \mathrm{O} 4 \mathrm{P}$

$\mathrm{C} 12 \mathrm{H} 4 \mathrm{Cl} 6$

$\mathrm{C} 12 \mathrm{H} 8$

$\mathrm{C} 13 \mathrm{H} 28$

$\mathrm{C} 2 \mathrm{Cl} 4$

$\mathrm{C} 2 \mathrm{H} 3 \mathrm{~N}$

$\mathrm{C} 2 \mathrm{H} 3 \mathrm{O} 2-$

$\mathrm{C} 2 \mathrm{H} 3 \mathrm{O} 3-$

$\mathrm{C} 2 \mathrm{H} 6 \mathrm{SiO}$

C2H8N2

$\mathrm{C} 2 \mathrm{HCl} 3$

C2O4-2

$\mathrm{C} 3 \mathrm{H} 6 \mathrm{O}$

$\mathrm{C} 4 \mathrm{H} 10 \mathrm{O}$

$\mathrm{C} 4 \mathrm{H} 7 \mathrm{NO} 4$

$\mathrm{C} 4 \mathrm{H} 8 \mathrm{O}$

$\mathrm{C} 5 \mathrm{H} 5 \mathrm{~N}$

$\mathrm{C} 6 \mathrm{H} 5 \mathrm{NO} 2$

C6H5O7-3

C6H6

$\mathrm{C} 6 \mathrm{H} 6 \mathrm{O}$

C7H6N2O4

$\mathrm{C} 7 \mathrm{H} 8$

$\mathrm{C} 7 \mathrm{H} 8 \mathrm{O}$

$\mathrm{C} 8 \mathrm{H} 8 \mathrm{O}$

$\mathrm{Ca}+2$

$\mathrm{CCl} 4$
Liquid \% to Accumulate

0

0

0

0

0

0

0

0

0

0

0

0

0

0

0

0

0

0

0

0

0

0

0

0

0

0

0

0

0

0

0

0

0

0

0

0

0

0

0

0

0

0

0

0
Solid \%

to Accumulate

0

0

0

0

0

0

0

0

0

0

0

0

0

0

0

0

0

0

0

0

0

0

0

0

0

0

0

0

0

0

0

0

0

0

0

0

0

0

0

0

0

0

0

0

0

0

0 
LVP-ADBR-00001AB

Components

$\mathrm{Cd}+2$

$\mathrm{Ce}+3$

$\mathrm{CH} 2 \mathrm{Cl} 2$

$\mathrm{CHCl} 3$

$\mathrm{CHO} 2-$

$\mathrm{Cl}-$

$\mathrm{CN}-$

$\mathrm{Co}+3$

$\mathrm{CO} 3-2$

$\mathrm{Cr}(\mathrm{OH}) 4-$

Cr(TOTAL)

Cs+

$\mathrm{Cu}+2$

DPDMS

DPPG

F-

$\mathrm{Fe}+3$

$\mathrm{H}+$

$\mathrm{H} 2 \mathrm{CO} 3$

$\mathrm{H} 2 \mathrm{O}$

H2O(BOUND)

$\mathrm{H} 2 \mathrm{O} 2$

$\mathrm{HCO} 3-$

$\mathrm{Hg}+2$

$\mathrm{K}+$

$\mathrm{La}+3$

$\mathrm{Li}+$

LS10

$\mathrm{AlOOH}$

LS9

$\mathrm{Mg}+2$

$\mathrm{Mn}+4$

$\mathrm{MnO} 2$

MnO4-

Mnp

$\mathrm{Mo}+6$

$\mathrm{Na}+$

Nap+

$\mathrm{Nd}+3$

NH3

NH4+

$\mathrm{Ni}+2$

$\mathrm{NO} 2-$

NO3-

NO3-D

$\mathrm{O}-2$

OH(BOUND)
Liquid \% to Accumulate

0

0

0

0

0

0

0

0

0

0

0

0

0

0

0

0

0

0

0

0

0

0

0

0

0

0

0

0

0

0

0

0

0

0

0

0

0

0

0

0

0

0

0

0
Solid \%

to Accumulate

0

0

0

0

0

0

0

0

0

0

0

0

0

0

0

0

0

0

0

0

0

0

0

0

0

0

0

0

0

0

0

0

0

0

0

0

0

0

0

0

0

0

0

0

0

0 
LVP-ADBR-00001AB

Components

$\mathrm{OH}-$

$\mathrm{Pb}+2$

$\mathrm{Pd}+2$

PDMS

PO4-3

PO4-3(HARD)

PPG

$\operatorname{Pr}+3$

$\mathrm{Pu}+4$

$\mathrm{Rb}+$

$\mathrm{Rh}+3$

$\mathrm{Ru}+3$

$\mathrm{Sb}+5$

$\mathrm{Se}+6$

$\mathrm{Si}+4$

SO4-2

$\mathrm{Sr}+2$

$\mathrm{Ta}+5$

$\mathrm{Tc}+7$

$\mathrm{Te}+6$

$\mathrm{Th}+4$

$\mathrm{Ti}+4$

$\mathrm{Tl}+3$

TOC

U(TOTAL)

$\mathrm{V}+5$

$\mathrm{W}+6$

$\mathrm{Y}+3$

$\mathrm{Zn}+2$

$\mathrm{Zr}+4$

Gas Components

129-I

$\mathrm{C} 12 \mathrm{H} 4 \mathrm{Cl} 6$

$\mathrm{C} 2 \mathrm{H} 3 \mathrm{~N}$

$\mathrm{CO} 2$

$\mathrm{H} 2 \mathrm{O}$

$\mathrm{HCl}$

$\mathrm{HF}$

$\mathrm{Hg}$

N2

$\mathrm{NH} 3$

$\mathrm{NO}$

$\mathrm{NO} 2$

$\mathrm{O} 2$

$\mathrm{P} 2 \mathrm{O} 5$

$\mathrm{SO} 2$

SO3
Liquid \% to Accumulate

0

0

0

0

0

0

0

0

0

0

0

0

0

0

0

0

0

0

0

0

0

0

0

0

0

0

0

0

Gas \%

to ACCUMULATE

99

0

0

0

0

96.997

96.997

99.583

0

0

0

0

0

0

0

0 
Table D-17 LAW Catalytic-Oxidizer-Reducer Splits

$\begin{array}{lr}\begin{array}{l}\text { LVP-SCR-00001-2 } \\ \text { Components } \\ \text { C2O4-2 }\end{array} & \begin{array}{r}\text { Liquid \% } \\ \text { to Accumulate }\end{array} \\ \text { Gas Components } & \begin{array}{r}99.999 \\ \text { Gas \% }\end{array} \\ \text { NO } & \text { to Accumulate } \\ \text { NO2 } & 0 \\ \end{array}$

Table D-18 LAW Caustic Scrubber Splits

\begin{tabular}{|c|c|c|}
\hline $\begin{array}{l}\text { LVP-SCB-00001 } \\
\text { Components }\end{array}$ & $\begin{array}{r}\text { Liquid \% } \\
\text { to LVP-TK-00001 }\end{array}$ & $\begin{array}{r}\text { Solid \% } \\
\text { to LVP-TK-00001 }\end{array}$ \\
\hline $106-\mathrm{Ru}$ & 0 & 0 \\
\hline $113 \mathrm{~m}-\mathrm{Cd}$ & 0 & 0 \\
\hline $125-\mathrm{Sb}$ & 0 & 0 \\
\hline $126-\mathrm{Sn}$ & 0 & 0 \\
\hline $129-\mathrm{I}$ & 0 & 0 \\
\hline $134-\mathrm{Cs}$ & 0 & 0 \\
\hline $137-\mathrm{Cs}$ & 0 & 0 \\
\hline $137 \mathrm{~m}-\mathrm{Ba}$ & 0 & 0 \\
\hline $14-\mathrm{C}$ & 0 & 0 \\
\hline $151-\mathrm{Sm}$ & 0 & 0 \\
\hline $152-\mathrm{Eu}$ & 0 & 0 \\
\hline $154-\mathrm{Eu}$ & 0 & 0 \\
\hline $155-\mathrm{Eu}$ & 0 & 0 \\
\hline 226-Ra & 0 & 0 \\
\hline 227-Ac & 0 & 0 \\
\hline 228-Ra & 0 & 0 \\
\hline 229-Th & 0 & 0 \\
\hline 231-Pa & 0 & 0 \\
\hline 232-Th & 0 & 0 \\
\hline $232-U$ & 0 & 0 \\
\hline $233-U$ & 0 & 0 \\
\hline 234-U & 0 & 0 \\
\hline $235-\mathrm{U}$ & 0 & 0 \\
\hline $236-U$ & 0 & 0 \\
\hline 237-Np & 0 & 0 \\
\hline 238-Pu & 0 & 0 \\
\hline $238-U$ & 0 & 0 \\
\hline 239-Pu & 0 & 0 \\
\hline $240-\mathrm{Pu}$ & 0 & 0 \\
\hline 241-Am & 0 & 0 \\
\hline 241-Pu & 0 & 0 \\
\hline $242-\mathrm{Cm}$ & 0 & 0 \\
\hline $242-\mathrm{Pu}$ & 0 & 0 \\
\hline 243-Am & 0 & 0 \\
\hline $243-\mathrm{Cm}$ & 0 & 0 \\
\hline 244-Cm & 0 & 0 \\
\hline
\end{tabular}


LVP-SCB-00001

Components

3-H

$59-\mathrm{Ni}$

60-Co

$63-\mathrm{Ni}$

79-Se

90-Sr

90-Y

93-Zr

$93 \mathrm{~m}-\mathrm{Nb}$

99-Tc

$\mathrm{Ag}+$

$\mathrm{Al}(\mathrm{OH}) 3$

$\mathrm{Al}(\mathrm{OH}) 4-$

$\mathrm{Al}+3$

$\mathrm{As}+5$

$\mathrm{B}+3$

$\mathrm{Ba}+2$

$\mathrm{Be}+2$

$\mathrm{Bi}+3$

C10H12N2O8-4

C10H15N2O7-3

$\mathrm{C} 10 \mathrm{H} 8$

$\mathrm{C} 12 \mathrm{H} 27 \mathrm{O} 4 \mathrm{P}$

$\mathrm{C} 12 \mathrm{H} 4 \mathrm{Cl} 6$

$\mathrm{C} 12 \mathrm{H} 8$

$\mathrm{C} 13 \mathrm{H} 28$

$\mathrm{C} 2 \mathrm{Cl} 4$

$\mathrm{C} 2 \mathrm{H} 3 \mathrm{~N}$

$\mathrm{C} 2 \mathrm{H} 3 \mathrm{O} 2-$

$\mathrm{C} 2 \mathrm{H} 3 \mathrm{O} 3-$

$\mathrm{C} 2 \mathrm{H} 6 \mathrm{SiO}$

C2H8N2

$\mathrm{C} 2 \mathrm{HCl} 3$

C2O4-2

$\mathrm{C} 3 \mathrm{H} 6 \mathrm{O}$

$\mathrm{C} 4 \mathrm{H} 10 \mathrm{O}$

$\mathrm{C} 4 \mathrm{H} 7 \mathrm{NO} 4$

$\mathrm{C} 4 \mathrm{H} 8 \mathrm{O}$

C5H5N

$\mathrm{C} 6 \mathrm{H} 5 \mathrm{NO} 2$

C6H5O7-3

$\mathrm{C} 6 \mathrm{H} 6$

C6H6O

C7H6N2O4

$\mathrm{C} 7 \mathrm{H} 8$

$\mathrm{C} 7 \mathrm{H} 8 \mathrm{O}$

$\mathrm{C} 8 \mathrm{H} 8 \mathrm{O}$
Liquid \% to LVP-TK-00001

0

0

0

0

0

0

0

0

0

0

0

0

0

0

0

0

0

0

0

0

0

0

0

0

0

0

0

0

0

0

0

0

0

0

0

0

0

0

0

0

0

0

0

0

0

0

0
Solid \%

to LVP-TK-00001

\section{0}

0

0

0

0

0

0

0

0

0

0

0

0

0

0

0

0

0

0

0

0

0

0

0

0

0

0

0

0

0

0

0

0

0

0

0

0

0

0

0

0

0

0

0

0

0

0 
LVP-SCB-00001

Components

$\mathrm{Ca}+2$

$\mathrm{CCl} 4$

$\mathrm{Cd}+2$

$\mathrm{Ce}+3$

$\mathrm{CH} 2 \mathrm{Cl} 2$

$\mathrm{CHCl} 3$

$\mathrm{CHO} 2-$

$\mathrm{Cl}-$

$\mathrm{CN}-$

$\mathrm{Co}+3$

$\mathrm{CO} 3-2$

$\mathrm{Cr}(\mathrm{OH}) 4-$

Cr(TOTAL)

$\mathrm{Cs}+$

$\mathrm{Cu}+2$

DPDMS

DPPG

F-

$\mathrm{Fe}+3$

$\mathrm{H}+$

$\mathrm{H} 2 \mathrm{CO} 3$

$\mathrm{H} 2 \mathrm{O}$

$\mathrm{H} 2 \mathrm{O} 2$

$\mathrm{HCO} 3-$

$\mathrm{Hg}+2$

$\mathrm{K}+$

$\mathrm{La}+3$

$\mathrm{Li}+$

$\mathrm{H} 2 \mathrm{O}(\mathrm{BOUND})$

LS10

NO3-D

$\mathrm{AlOOH}$

LS9

$\mathrm{Mg}+2$

$\mathrm{Mn}+4$

$\mathrm{MnO} 2$

$\mathrm{MnO} 4-$

$\mathrm{Mnp}$

$\mathrm{Mo}+6$

$\mathrm{Na}+$

$\mathrm{Nap}+$

$\mathrm{Nd}+3$

$\mathrm{NH} 3$

$\mathrm{NH} 4+$

$\mathrm{Ni}+2$

$\mathrm{NO} 2-$

NO3-
Liquid \% to LVP-TK-00001

0

0

0

0

0

0

0

0

0

0

0

0

0

0

0

0

99.99

0

0

0

0

0

0

0

0

0

0

0

0

0

0

0

0

0

0

0

0

0

0

0

0

0
Solid \%

to LVP-TK-00001

0

0

0

0

0

0

0

0

0

0

0

0

0

0

0

0

0

0

0

0

0

99.99

0

0

0

0

0

0

0

0

0

0

0

0

0

0

0

0

0

0

0

0

0

0

0

0

0 


LVP-SCB-00001
Components
O-2
OH(BOUND)
OH-
$\mathrm{Pb}+2$
$\mathrm{Pd}+2$
$\mathrm{PDMS}$
$\mathrm{PO} 4-3$
$\mathrm{PO}-3$ (HARD)
$\mathrm{PPG}$
$\mathrm{Pr}+3$
$\mathrm{Pu}+4$
$\mathrm{Rb}+$
$\mathrm{Rh}+3$
$\mathrm{Ru}+3$
$\mathrm{Sb}+5$
$\mathrm{Se}+6$
$\mathrm{Si}+4$
$\mathrm{SO} 4-2$
$\mathrm{Sr}+2$
$\mathrm{Ta}+5$
$\mathrm{Tc}+7$
$\mathrm{Te}+6$
$\mathrm{Th}+4$
$\mathrm{Ti}+4$
$\mathrm{Tl}+3$
$\mathrm{TOC}$
$\mathrm{U}(\mathrm{TOTAL})$
$\mathrm{V}+5$
$\mathrm{~W}+6$
$\mathrm{Y}+3$
$\mathrm{Zn}+2$
$\mathrm{Zr}+4$

Liquid \% to LVP-TK-00001

0

0

0

0

0

0

0

0

0

0

0

0

0

0

9.091

0

0

0

0

0

0

0

0

0

0

0

0

0

0

0

0

0

Table D-19 HLW Melter Splits

HMP-MLTR-00001/2

Components

106-Ru

$113 \mathrm{~m}-\mathrm{Cd}$

$125-\mathrm{Sb}$

$126-\mathrm{Sn}$

129-I

134-Cs

137-Cs

$137 \mathrm{~m}-\mathrm{Ba}$

14-C

151-Sm

152-Eu
Liquid \% to HOP-SCB-00001/2

24.39

7.692

0.37

1.282

0

2.128

2.128

0.943

100

1.282

1.282
Solid \%

to LVP-TK-00001

0

0

0

0

0

0

0

0

0

0

0

0

0

9.091

0

0

0

0

0

0

0

0

0

0

0

0

0

0

0

0

0 


HMP-MLTR-00001/2
Components
$154-\mathrm{Eu}$
$155-\mathrm{Eu}$
$226-\mathrm{Ra}$
$227-\mathrm{Ac}$
$228-\mathrm{Ra}$
$229-\mathrm{Th}$
$231-\mathrm{Pa}$
$232-\mathrm{Th}$
$232-\mathrm{U}$
$233-\mathrm{U}$
$234-\mathrm{U}$
$235-\mathrm{U}$
$236-\mathrm{U}$
$237-\mathrm{Np}$
$238-\mathrm{Pu}$
$238-\mathrm{U}$
$239-\mathrm{Pu}$
$240-\mathrm{Pu}$
$241-\mathrm{Am}$
$241-\mathrm{Pu}$
$242-\mathrm{Cm}$
$242-\mathrm{Pu}$
$243-\mathrm{Am}$
$243-\mathrm{Cm}$
$244-\mathrm{Cm}$
$3-\mathrm{H}$
$59-\mathrm{Ni}$
$60-\mathrm{Co}$
$63-\mathrm{Ni}$
$79-\mathrm{Se}$
$90-\mathrm{Sr}$
$90-\mathrm{Y}$
$93-\mathrm{Zr}$
$93 \mathrm{~m}-\mathrm{Nb}$
99-Tc
$\mathrm{Ag}+$
$\mathrm{C} 10 \mathrm{H} 10 \mathrm{H} 12 \mathrm{~N} 2 \mathrm{OH}) 3$
$\mathrm{C} 10 \mathrm{H} 8$
$\mathrm{Al}(\mathrm{OH}) 4-$
$\mathrm{Al}+3$
$\mathrm{As}+5$
$\mathrm{~B}+3$

HMP-MLTR-00001/2

$155-\mathrm{Eu}$

226-Ra

229-Th

$231-\mathrm{Pa}$

233-U

236-U

237-Np

$238-\mathrm{Pu}$

238-U

239-Pu

241-Am

241-Pu

$242-\mathrm{Pu}$

243-Am

$244-\mathrm{Cm}$

$3-\mathrm{H}$

60-Co

$63-\mathrm{N}$

90-Y

$93 \mathrm{~m}-\mathrm{Nb}$

99-Tc

$\mathrm{Al}(\mathrm{OH}) 3$

$\mathrm{Al}(\mathrm{OH}) 4$

$\mathrm{Al}+3$

As +5

$\mathrm{B}+3$

$\mathrm{Ba}+2$

C10H12N2O8-4

$\mathrm{C} 10 \mathrm{H} 8$
Liquid \% to HOP-SCB-00001/2

1.282

1.282

25.641

1.282

25.641

1.282

1.282

1.282

1.282

1.282

1.282

1.282

1.282

1.282

1.282

1.282

1.282

1.282

1.961

1.282

1.282

1.282

1.961

1.282

1.282

100

0.847

1.282

0.847

50

1.111

0.137

0.599

1.282

62.5

0.8

0.769

0.769

0.769

1.852

2.0284

0.943

1.282

0.37

0

0

95.147
Solid \%

to HOP-SCB-00001/2

1.282

1.282

25.641

1.282

25.641

1.282

1.282

1.282

1.282

1.282

1.282

1.282

1.282

1.282

1.282

1.282

1.282

1.282

1.961

1.282

1.282

1.282

1.961

1.282

1.282

100

0.847

1.282

0.847

50

1.111

0.137

0.599

1.282

62.5

0.8

0.769

0.769

0.769

1.852

2.0284

0.943

1.282

0.37

0

0

95.147 
HMP-MLTR-00001/2

Components

$\mathrm{C} 12 \mathrm{H} 27 \mathrm{O} 4 \mathrm{P}$

$\mathrm{C} 12 \mathrm{H} 4 \mathrm{Cl} 6$

$\mathrm{C} 12 \mathrm{H} 8$

$\mathrm{C} 13 \mathrm{H} 28$

$\mathrm{C} 2 \mathrm{Cl} 4$

$\mathrm{C} 2 \mathrm{H} 3 \mathrm{~N}$

$\mathrm{C} 2 \mathrm{H} 3 \mathrm{O} 2-$

$\mathrm{C} 2 \mathrm{H} 3 \mathrm{O} 3-$

$\mathrm{C} 2 \mathrm{H} 6 \mathrm{SiO}$

$\mathrm{C} 2 \mathrm{H} 8 \mathrm{~N} 2$

$\mathrm{C} 2 \mathrm{HCl} 3$

$\mathrm{C} 2 \mathrm{O} 4-2$

$\mathrm{C} 3 \mathrm{H} 6 \mathrm{O}$

$\mathrm{C} 4 \mathrm{H} 10 \mathrm{O}$

$\mathrm{C} 4 \mathrm{H} 7 \mathrm{NO} 4$

$\mathrm{C} 4 \mathrm{H} 8 \mathrm{O}$

$\mathrm{C} 5 \mathrm{H} 5 \mathrm{~N}$

$\mathrm{C} 6 \mathrm{H} 5 \mathrm{NO} 2$

$\mathrm{C} 6 \mathrm{H} 5 \mathrm{O} 7-3$

$\mathrm{C} 6 \mathrm{H} 6$

$\mathrm{C} 6 \mathrm{H} 6 \mathrm{O}$

C7H6N2O4

C7H8

$\mathrm{C} 7 \mathrm{H} 8 \mathrm{O}$

$\mathrm{C} 8 \mathrm{H} 8 \mathrm{O}$

$\mathrm{Ca}+2$

$\mathrm{CCl} 4$

$\mathrm{Cd}+2$

$\mathrm{Ce}+3$

$\mathrm{CH} 2 \mathrm{Cl} 2$

$\mathrm{CHCl} 3$

CHO2-

Cl-

$\mathrm{CN}-$

$\mathrm{Co}+3$

$\mathrm{CO} 3-2$

$\mathrm{Cr}(\mathrm{OH}) 4-$

Cr(TOTAL)

$\mathrm{Cs}+$

$\mathrm{Cu}+2$

DPDMS

DPPG

F-

$\mathrm{Fe}+3$

$\mathrm{H}+$

$\mathrm{H} 2 \mathrm{CO} 3$

$\mathrm{H} 2 \mathrm{O}$
Liquid \%

to HOP-SCB-00001/2

0

0

0

0

0

0

0

0

0

0

62.893

0

0

0

0

0

0

0

0

0

0

0

0

1.163

0

0.781

1.282

0

0

0

0

1.961

1.282

0

1.282

1.282

2.128

0.8

0

0

0

0.99

0

0

0
Solid \%

to HOP-SCB-00001/2

\section{0}

0

0

0

0

0

0

0

0

0

0

0

62.893

0

0

0

0

0

0

0

0

0

0

0

0

1.163

0

0.781

1.282

0

0

0

0

1.961

1.282

0

1.282

1.282

2.128

0.8

0

0

0

0.99

0

0

0 
HMP-MLTR-00001/2

Components

$\mathrm{H} 2 \mathrm{O}(\mathrm{BOUND})$

$\mathrm{H} 2 \mathrm{O} 2$

$\mathrm{HCO} 3-$

$\mathrm{Hg}+2$

$\mathrm{K}+$

$\mathrm{La}+3$

$\mathrm{Li}+$

LS10

$\mathrm{AlOOH}$

LS9

$\mathrm{Mg}+2$

$\mathrm{Mn}+4$

$\mathrm{MnO} 2$

$\mathrm{MnO} 4-$

Mnp

$\mathrm{Mo}+6$

$\mathrm{Na}+$

Nap+

$\mathrm{Nd}+3$

NH3

NH4+

$\mathrm{Ni}+2$

$\mathrm{NO} 2-$

NO3-

NO3-D

$\mathrm{O}-2$

OH(BOUND)

$\mathrm{OH}-$

$\mathrm{Pb}+2$

$\mathrm{Pd}+2$

PDMS

PO4-3

PO4-3(HARD)

PPG

$\mathrm{Pr}+3$

$\mathrm{Pu}+4$

$\mathrm{Rb}+$

$\mathrm{Rh}+3$

$\mathrm{Ru}+3$

$\mathrm{Sb}+5$

$\mathrm{Se}+6$

$\mathrm{Si}+4$

SO4-2

$\mathrm{Sr}+2$

$\mathrm{Ta}+5$

$\mathrm{Tc}+7$

$\mathrm{Te}+6$
Liquid \% to HOP-SCB-0001/2

0

0

0

0

3.125

1.282

0.49

0

0

0

1.786

0.483

0.483

0.483

0.483

1.667

0.99

0.99

1.282

0

0

0.847

0

0

0

0

0

0

1.587

1.282

0

1.053

1.053

0

1.282

1.282

1.961

1.282

24.39

0.37

50

0.455

0

1.111

1.282

62.5

7.143
Solid \%

to HOP-SCB-00001/2

0

0

0

3.125

1.282

0.49

0

0

0

1.786

0.483

0.483

0.483

0.483

1.667

0.99

0.99

1.282

0

0

0.847

0

0

0

0

0

0

1.587

1.282

0

1.053

1.053

0

1.282

1.282

1.961

1.282

24.39

0.37

50

0.455

0

1.111

1.282

62.5

7.143 
HMP-MLTR-00001/2

Components

$\mathrm{Th}+4$

$\mathrm{Ti}+4$

$\mathrm{Tl}+3$

TOC

U(TOTAL)

$\mathrm{V}+5$

$\mathrm{W}+6$

$\mathrm{Y}+3$

$\mathrm{Zn}+2$

$\mathrm{Zr}+4$
Liquid \% to HOP-SCB-0001/2

1.282

0.313

25.641

0

1.282

1.961

1.282

0.137

0.935

0.599
Solid \%

to HOP-SCB-00001/2

1.282

0.313

25.641

0

1.282

1.961

1.282

0.137

0.935

0.599

Table D-20 HLW SBS Splits

HOP-SCB-00001/2
Components
$106-\mathrm{Ru}$
$113 \mathrm{~m}-\mathrm{Cd}$
$125-\mathrm{Sb}$
$126-\mathrm{Sn}$
$129-\mathrm{I}$
$134-\mathrm{Cs}$
$137-\mathrm{Cs}$
$137 \mathrm{~m}-\mathrm{Ba}$
$14-\mathrm{C}$
$151-\mathrm{Sm}$
$152-\mathrm{Eu}$
$154-\mathrm{Eu}$
$155-\mathrm{Eu}$
$226-\mathrm{Ra}$
$227-\mathrm{Ac}$
$228-\mathrm{Ra}$
$229-\mathrm{Th}$
$231-\mathrm{Pa}$
$232-\mathrm{Th}$
$232-\mathrm{U}$
$233-\mathrm{U}$
$234-\mathrm{U}$
$235-\mathrm{U}$
$236-\mathrm{U}$
$237-\mathrm{Np}$
$238-\mathrm{Pu}$
$238-\mathrm{U}$
$239-\mathrm{Pu}$
$240-\mathrm{Pu}$
$241-\mathrm{Am}$
$241-\mathrm{Pu}$
$242-\mathrm{Cm}$
$242-\mathrm{Pu}$

Liquid \% to HOP-VSL-00903/904

65.517

90.991

94.764

98

0

69.697

69.697

69.697

0.1

98

98

98

98

98

98

98

98

98

98

98

98

98

98

98

98

98

98

98

98

98

98

98

98
Solid \%

to HOP-VSL-00903/904

65.517

90.991

94.764

98

0

69.697

69.697

69.697

0.1

98

98

98

98

98

98

98

98

98

98

98

98

98

98

98

98

98

98

98

98

98

98

98

98 
HOP-SCB-00001/2

Components

243-Am

243-Cm

244-Cm

$3-\mathrm{H}$

$59-\mathrm{Ni}$

60-Co

$63-\mathrm{Ni}$

79-Se

90-Sr

90-Y

93-Zr

$93 \mathrm{~m}-\mathrm{Nb}$

99-Tc

$\mathrm{Ag}+$

$\mathrm{Al}(\mathrm{OH}) 3$

$\mathrm{Al}(\mathrm{OH}) 4-$

$\mathrm{Al}+3$

$\mathrm{As}+5$

$\mathrm{B}+3$

$\mathrm{Ba}+2$

$\mathrm{Be}+2$

$\mathrm{Bi}+3$

C10H12N2O8-4

C10H15N2O7-3

$\mathrm{C} 10 \mathrm{H} 8$

$\mathrm{C} 12 \mathrm{H} 27 \mathrm{O} 4 \mathrm{P}$

$\mathrm{C} 12 \mathrm{H} 4 \mathrm{Cl} 6$

$\mathrm{C} 12 \mathrm{H} 8$

$\mathrm{C} 13 \mathrm{H} 28$

$\mathrm{C} 2 \mathrm{Cl} 4$

$\mathrm{C} 2 \mathrm{H} 3 \mathrm{~N}$

$\mathrm{C} 2 \mathrm{H} 3 \mathrm{O} 2-$

$\mathrm{C} 2 \mathrm{H} 3 \mathrm{O} 3-$

$\mathrm{C} 2 \mathrm{H} 6 \mathrm{SiO}$

$\mathrm{C} 2 \mathrm{H} 8 \mathrm{~N} 2$

$\mathrm{C} 2 \mathrm{HCl} 3$

C2O4-2

$\mathrm{C} 3 \mathrm{H} 6 \mathrm{O}$

$\mathrm{C} 4 \mathrm{H} 10 \mathrm{O}$

$\mathrm{C} 4 \mathrm{H} 7 \mathrm{NO} 4$

$\mathrm{C} 4 \mathrm{H} 8 \mathrm{O}$

C5H5N

$\mathrm{C} 6 \mathrm{H} 5 \mathrm{NO} 2$

$\mathrm{C} 6 \mathrm{H} 5 \mathrm{O} 7-3$

C6H6

C6H6O

C7H6N2O4
Liquid \%

to HOP-VSL-00903/904

98

98

98

0

98.957

88.506

98.957

79.167

97.11

97.11

99.311

98

98

98

99.552

99.552

99.552

81.132

98.953

90.654

98

98

0

0

0

0

94.505

0

0

0

94.505

\section{0}

0

0

0

0

94.505

0

0

0

0

0

0

0

0

0

0
Solid \%

to HOP-VSL-00903/904

98

98

98

0

98.957

88.506

98.957

79.167

97.11

97.11

99.311

98

98

98

99.552

99.552

99.552

81.132

98.953

90.654

98

98

0

0

0

0

94.505

0

0

94.505

0

0

0

0

0

94.505

0

0

0

0

0

0

0

0

0

0 
HOP-SCB-00001/2

Components

$\mathrm{C} 7 \mathrm{H} 8$

$\mathrm{C} 7 \mathrm{H} 8 \mathrm{O}$

$\mathrm{C} 8 \mathrm{H} 8 \mathrm{O}$

$\mathrm{Ca}+2$

$\mathrm{CCl} 4$

$\mathrm{Cd}+2$

$\mathrm{Ce}+3$

$\mathrm{CH} 2 \mathrm{Cl} 2$

$\mathrm{CHCl} 3$

CHO2-

$\mathrm{Cl}-$

$\mathrm{CN}-$

$\mathrm{Co}+3$

$\mathrm{CO} 3-2$

$\mathrm{Cr}(\mathrm{OH}) 4-$

Cr(TOTAL)

$\mathrm{Cs}+$

$\mathrm{Cu}+2$

DPDMS

DPPG

F-

$\mathrm{Fe}+3$

$\mathrm{H}+$

$\mathrm{H} 2 \mathrm{CO} 3$

$\mathrm{H} 2 \mathrm{O}$

H2O(BOUND)

$\mathrm{H} 2 \mathrm{O} 2$

$\mathrm{HCO} 3-$

$\mathrm{Hg}+2$

$\mathrm{K}+$

$\mathrm{La}+3$

$\mathrm{Li}+$

LS10

$\mathrm{AlOOH}$

LS9

$\mathrm{Mg}+2$

$\mathrm{Mn}+4$

$\mathrm{MnO} 2$

MnO4-

Mnp

$\mathrm{Mo}+6$

$\mathrm{Na}+$

Nap+

$\mathrm{Nd}+3$

NH3

NH4+

$\mathrm{Ni}+2$
Liquid \%

to HOP-VSL-00903/904

0

0

97.199

90.991

99.808

0

0

0

75.61

93.631

88.506

93.631

81.818

81.818

69.697

92.248

0

0

98.246

99.66

99.95

93.631

0

0

0

93.631

0

88.889

99.9

94.975

0

0

0

99.057

98.054

98.054

98.054

98.054

90

93.631

93.631

99.882

90

90

98.957
Solid \%

to HOP-VSL-00903/904

0

0

0

97.199

0

90.991

99.808

0

0

0

75.61

93.631

88.506

93.631

81.818

81.818

69.697

92.248

0

0

98.246

99.66

99.95

93.631

0

0

0

93.631

0

88.889

99.9

94.975

0

0

0

99.057

98.054

98.054

98.054

98.054

90

93.631

93.631

99.882

90

90

98.957 


HOP-SCB-0001/2
Components
NO2-
NO3-
NO3-D
O-2
OH(BOUND)
OH-
$\mathrm{Pb}+2$
$\mathrm{Pd}+2$
$\mathrm{PDMS}$
$\mathrm{PO} 4-3$
$\mathrm{PO} 4-3(\mathrm{HARD})$
$\mathrm{PPG}$
$\mathrm{Pr}+3$
$\mathrm{Pu}+4$
$\mathrm{Rb}+$
$\mathrm{Rh}+3$
$\mathrm{Ru}+3$
$\mathrm{Sb}+5$
$\mathrm{Se}+6$
$\mathrm{Si}+4$
$\mathrm{SO} 4-2$
$\mathrm{Sr}+2$
$\mathrm{Ta}+5$
$\mathrm{Tc}+7$
$\mathrm{Te}+6$
$\mathrm{Th}+4$
$\mathrm{Ti}+4$
$\mathrm{Tl}+3$
$\mathrm{TOC}$
$\mathrm{U}(\mathrm{TOTAL})$
$\mathrm{V}+5$
$\mathrm{~W}+6$
$\mathrm{Y}+3$
$\mathrm{Zn}+2$
$\mathrm{Zr}+4$

Liquid \% to HOP-VSL-00903/904

93.631

93.631

0

0

93.631

93.631

89.362

98

0

88.235

\section{0}

0

98

98

98

98

65.517

94.764

79.167

99.615

74.359

97.11

98

98

72.222

98

96.951

98

0

98

81.132

98

99.643

98.851

99.311
Solid \%

to HOP-VSL-00903/904

93.631

93.631

\section{0}

0

93.631

93.631

89.362

98

0

88.235

0

0

98

98

98

98

65.517

94.764

79.167

99.615

74.359

97.11

98

98

72.222

98

96.951

98

0

98

81.132

98

99.643

98.851

99.311

Table D-21 HLW WESP Splits

HOP-WESP-00001/2
Components
$106-\mathrm{Ru}$
$113 \mathrm{~m}-\mathrm{Cd}$
$125-\mathrm{Sb}$
$126-\mathrm{Sn}$
$129-\mathrm{I}$
$134-\mathrm{Cs}$
$137-\mathrm{Cs}$
$137 \mathrm{~m}-\mathrm{Ba}$

$\begin{array}{rr}\begin{array}{r}\text { Liquid \% } \\ \text { to HOP-SCB-00001/2 }\end{array} & \begin{array}{r}\text { Solid \% } \\ \text { 95.4954955 }\end{array} \\ 79.16666667 & 95.4954955 \\ 82.45614035 & 79.16666667 \\ 99.9 & 82.45614035 \\ 0 & 99.9 \\ 94.56521739 & 0 \\ 94.56521739 & 94.56521739 \\ 94.56521739 & 94.56521739 \\ & 94.56521739\end{array}$




HOP-WESP-00001/2
Components
$14-\mathrm{C}$
$151-\mathrm{Sm}$
$152-\mathrm{Eu}$
$154-\mathrm{Eu}$
$155-\mathrm{Eu}$
$226-\mathrm{Ra}$
$227-\mathrm{Ac}$
$228-\mathrm{Ra}$
$229-\mathrm{Th}$
$231-\mathrm{Pa}$
$232-\mathrm{Th}$
$232-\mathrm{U}$
$233-\mathrm{U}$
$234-\mathrm{U}$
$235-\mathrm{U}$
$236-\mathrm{U}$
$237-\mathrm{Np}$
$238-\mathrm{Pu}$
$238-\mathrm{U}$
$239-\mathrm{Pu}$
$240-\mathrm{Pu}$
$241-\mathrm{Am}$
$241-\mathrm{Pu}$
$242-\mathrm{Cm}$
$242-\mathrm{Pu}$
$243-\mathrm{Am}$
$243-\mathrm{Cm}$
$244-\mathrm{Cm}$
$3-\mathrm{H}$
$59-\mathrm{Ni}$
$60-\mathrm{Co}$
$63-\mathrm{Ni}$
$79-\mathrm{Se}$
$90-\mathrm{Sr}$
$90-\mathrm{Y}$
$93-\mathrm{Zr}$
$93 \mathrm{~m}-\mathrm{Nb}$
$99-\mathrm{Tc}$
$\mathrm{Ag}+$
$\mathrm{Al}(\mathrm{OH}) 3$
$\mathrm{Al}(\mathrm{OH}) 4-$
$\mathrm{Al}+3$
$\mathrm{As}+5$
$\mathrm{~B}+3$
$\mathrm{Ba}+2$
$\mathrm{Be}+2$
$\mathrm{Bi}+3$

\begin{tabular}{|c|c|}
\hline $\begin{array}{r}\text { Liquid \% } \\
\text { to HOP-SCB-00001/2 }\end{array}$ & $\begin{array}{r}\text { Solid \% } \\
\text { to HOP-SCB-00001/2 }\end{array}$ \\
\hline 0 & 0 \\
\hline 99.9 & 99.9 \\
\hline 99.9 & 99.9 \\
\hline 99.9 & 99.9 \\
\hline 99.9 & 99.9 \\
\hline 99.9 & 99.9 \\
\hline 99.9 & 99.9 \\
\hline 99.9 & 99.9 \\
\hline 99.9 & 99.9 \\
\hline 99.9 & 99.9 \\
\hline 99.9 & 99.9 \\
\hline 99.9 & 99.9 \\
\hline 99.9 & 99.9 \\
\hline 99.9 & 99.9 \\
\hline 99.9 & 99.9 \\
\hline 99.9 & 99.9 \\
\hline 99.9 & 99.9 \\
\hline 99.9 & 99.9 \\
\hline 99.9 & 99.9 \\
\hline 99.9 & 99.9 \\
\hline 99.9 & 99.9 \\
\hline 99.9 & 99.9 \\
\hline 99.9 & 99.9 \\
\hline 99.9 & 99.9 \\
\hline 99.9 & 99.9 \\
\hline 99.9 & 99.9 \\
\hline 99.9 & 99.9 \\
\hline 99.9 & 99.9 \\
\hline 0 & 0 \\
\hline 28.57142857 & 28.57142857 \\
\hline 99.9 & 99.9 \\
\hline 28.57142857 & 28.57142857 \\
\hline 97.2299169 & 97.2299169 \\
\hline 44.444 & 44.444 \\
\hline 44.444 & 44.444 \\
\hline 44.44444444 & 44.44444444 \\
\hline 99.9 & 99.9 \\
\hline 99.9 & 99.9 \\
\hline 99.9 & 99.9 \\
\hline 65.51724138 & 65.51724138 \\
\hline 65.51724138 & 65.51724138 \\
\hline 65.51724138 & 65.51724138 \\
\hline 96.06299213 & 96.06299213 \\
\hline 92 & 92 \\
\hline 99.9 & 99.9 \\
\hline 99.9 & 99.9 \\
\hline 99.9 & 99.9 \\
\hline
\end{tabular}


HOP-WESP-00001/2

Components

C10H12N2O8-4

$\mathrm{C} 10 \mathrm{H} 15 \mathrm{~N} 2 \mathrm{O} 7-3$

$\mathrm{C} 10 \mathrm{H} 8$

$\mathrm{C} 12 \mathrm{H} 27 \mathrm{O} 4 \mathrm{P}$

$\mathrm{C} 12 \mathrm{H} 4 \mathrm{Cl} 6$

$\mathrm{C} 12 \mathrm{H} 8$

$\mathrm{C} 13 \mathrm{H} 28$

$\mathrm{C} 2 \mathrm{Cl} 4$

$\mathrm{C} 2 \mathrm{H} 3 \mathrm{~N}$

$\mathrm{C} 2 \mathrm{H} 3 \mathrm{O} 2-$

$\mathrm{C} 2 \mathrm{H} 3 \mathrm{O} 3-$

$\mathrm{C} 2 \mathrm{H} 6 \mathrm{SiO}$

$\mathrm{C} 2 \mathrm{H} 8 \mathrm{~N} 2$

$\mathrm{C} 2 \mathrm{HCl} 3$

$\mathrm{C} 2 \mathrm{O} 4-2$

$\mathrm{C} 3 \mathrm{H} 6 \mathrm{O}$

$\mathrm{C} 4 \mathrm{H} 10 \mathrm{O}$

$\mathrm{C} 4 \mathrm{H} 7 \mathrm{NO} 4$

$\mathrm{C} 4 \mathrm{H} 8 \mathrm{O}$

C5H5N

$\mathrm{C} 6 \mathrm{H} 5 \mathrm{NO} 2$

C6H5O7-3

$\mathrm{C} 6 \mathrm{H} 6$

$\mathrm{C} 6 \mathrm{H} 6 \mathrm{O}$

$\mathrm{C} 7 \mathrm{H} 6 \mathrm{~N} 2 \mathrm{O} 4$

C7H8

C7H8O

$\mathrm{C} 8 \mathrm{H} 8 \mathrm{O}$

$\mathrm{Ca}+2$

$\mathrm{CCl} 4$

$\mathrm{Cd}+2$

$\mathrm{Ce}+3$

$\mathrm{CH} 2 \mathrm{Cl} 2$

$\mathrm{CHCl} 3$

CHO2-

Cl-

$\mathrm{CN}-$

$\mathrm{Co}+3$

$\mathrm{CO} 3-2$

$\mathrm{Cr}(\mathrm{OH}) 4-$

Cr(TOTAL)

$\mathrm{Cs}+$

$\mathrm{Cu}+2$

F-

$\mathrm{Fe}+3$

$\mathrm{H}+$

$\mathrm{H} 2 \mathrm{CO} 3$
Liquid \% to HOP-SCB-00001/2

0

99.58333333

0

0

0

99.58333333

0

0

\section{0}

0

0

0

99.58333333

0

0

0

0

0

0

0

0

0

0

0

0

0

73.68421053

79.16666667

99.9

0

0

0

89.47368421

97.2826087

88.50574713

97.2826087

88.0952381

88.0952381

94.56521739

33.33333333

50

88.37209302

99.9

99.58333333
Solid \%

to HOP-SCB-00001/2

\section{0}

0

0

0

99.58333333

0

0

0

99.58333333

0

0

0

0

0

99.58333333

0

0

0

0

0

0

0

0

0

0

0

0

0

73.68421053

79.16666667

99.9

0

0

0

89.47368421

97.2826087

88.50574713

97.2826087

88.0952381

88.0952381

94.56521739

33.33333333

50

88.37209302

99.9

99.58333333 
HOP-WESP-00001/2

Components

$\mathrm{H} 2 \mathrm{O}$

$\mathrm{H} 2 \mathrm{O} 2$

$\mathrm{HCO} 3-$

$\mathrm{Hg}+2$

$\mathrm{K}+$

$\mathrm{La}+3$

$\mathrm{Li}+$

H2O(BOUND)

LS10

PPG

DPPG

PDMS

DPDMS

NO3-D

$\mathrm{AlOOH}$

LS9

$\mathrm{Mg}+2$

$\mathrm{Mn}+4$

$\mathrm{MnO} 2$

MnO4-

Mnp

$\mathrm{Mo}+6$

$\mathrm{Na}+$

Nap+

$\mathrm{Nd}+3$

$\mathrm{NH} 3$

NH4+

$\mathrm{Ni}+2$

$\mathrm{NO} 2-$

NO3-

$\mathrm{O}-2$

OH(BOUND)

$\mathrm{OH}-$

$\mathrm{Pb}+2$

$\mathrm{Pd}+2$

PO4-3

PO4-3(HARD)

$\mathrm{Pr}+3$

$\mathrm{Pu}+4$

$\mathrm{Rb}+$

$\mathrm{Rh}+3$

$\mathrm{Ru}+3$

$\mathrm{Sb}+5$

$\mathrm{Se}+6$

$\mathrm{Si}+4$

SO4-2

$\mathrm{Sr}+2$
Liquid \% to HOP-SCB-00001/2

0

0

97.2826087

82.45614035

99.9

95

0

0

0

0

0

0

0

0

0

28.57142857

37.5

37.5

37.5

37.5

99.9

97.2826087

97.2826087

99.9

9.90990991

9.90990991

28.57142857

97.2826087

97.2826087

0

99.9

99.9

81.81818182

99.9

61.53846154

0

99.9

99.9

99.9

99.9

95.4954955

82.45614035

97.2299169

89.47368421

98.32214765

44.44444444
Solid \%

to HOP-SCB-00001/2

\section{0}

0

97.2826087

82.45614035

99.9

95

0

0

0

0

0

0

0

0

0

28.57142857

37.5

37.5

37.5

37.5

99.9

97.2826087

97.2826087

99.9

9.90990991

9.90990991

28.57142857

97.2826087

97.2826087

99.9

99.9

81.81818182

99.9

61.53846154

0

99.9

99.9

99.9

99.9

95.4954955

82.45614035

97.2299169

89.47368421

98.32214765

44.44444444 


\section{HOP-WESP-00001/2}

Components

$\mathrm{Ta}+5$

$\mathrm{Tc}+7$

$\mathrm{Te}+6$

$\mathrm{Th}+4$

$\mathrm{Ti}+4$

$\mathrm{Tl}+3$

TOC

U(TOTAL)

$\mathrm{V}+5$

$\mathrm{W}+6$

$\mathrm{Y}+3$

$\mathrm{Zn}+2$

$\mathrm{Zr}+4$
Liquid \% to HOP-SCB-00001/2

99.9

99.9

99.28469242

99.9

33.33333333

99.9

0

99.9

99.9

99.9

99.9

86.666666667

44.44444444
Solid \%

to HOP-SCB-00001/2

99.9

99.9

99.28469242

99.9

33.33333333

99.9

0

99.9

99.9

99.9

99.9

86.666666667

44.44444444

Table D-22 HLW HEME Splits

HOP-HEME-00001/2
Components
$106-\mathrm{Ru}$
$113 \mathrm{~m}-\mathrm{Cd}$
$125-\mathrm{Sb}$
$126-\mathrm{Sn}$
$129-\mathrm{I}$
$134-\mathrm{Cs}$
$137-\mathrm{Cs}$
$137 \mathrm{~m}-\mathrm{Ba}$
$14-\mathrm{C}$
$151-\mathrm{Sm}$
$152-\mathrm{Eu}$
$154-\mathrm{Eu}$
$155-\mathrm{Eu}$
$226-\mathrm{Ra}$
$227-\mathrm{Ac}$
$228-\mathrm{Ra}$
$229-\mathrm{Th}$
$231-\mathrm{Pa}$
$232-\mathrm{Th}$
$232-\mathrm{U}$
$233-\mathrm{U}$
$234-\mathrm{U}$
$235-\mathrm{U}$
$236-\mathrm{U}$
$237-\mathrm{Np}$
$238-\mathrm{Pu}$
$238-\mathrm{U}$
$239-\mathrm{Pu}$
$240-\mathrm{Pu}$
$241-\mathrm{Am}$

Liquid \% to HOP-SCB-00001/2 99.6969697 99.6875 99.62962963 99.67741935

0 99.6875 99.688

99.688

99.67741935

99.67741935

99.67741935

99.67741935

99.67741935

99.67741935

99.67741935

99.67741935

99.67741935

99.67741935

99.67741935

99.67741935

99.67741935

99.67741935

99.67741935

99.67741935

99.67741935

99.67741935

99.67741935

99.67741935

99.67741935
Solid \%

to HOP-SCB-00001/2

99.6969697

99.6875

99.62962963

99.67741935

99.6875

99.688

99.688

0

99.67741935

99.67741935

99.67741935

99.67741935

99.67741935

99.67741935

99.67741935

99.67741935

99.67741935

99.67741935

99.67741935

99.67741935

99.67741935

99.67741935

99.67741935

99.67741935

99.67741935

99.67741935

99.67741935

99.67741935

99.67741935 


HOP-HEME-00001/2
Components
241-Pu
242-Cm
242-Pu
243-Am
243-Cm
244-Cm
3-H
59-Ni
$60-\mathrm{Co}$
$63-\mathrm{Ni}$
79-Se
90-Sr
90-Y
93-Zr
93m-Nb
99-Tc
$\mathrm{Ag}+$
$\mathrm{Al}(\mathrm{OH}) 3$
$\mathrm{Al}(\mathrm{OH}) 4-$
$\mathrm{Al}+3$
$\mathrm{As}+5$
$\mathrm{~B}+3$
$\mathrm{Ba}+2$
$\mathrm{Be}+2$
$\mathrm{Bi}+3$
$\mathrm{C} 10 \mathrm{H} 12 \mathrm{~N} 2 \mathrm{O} 8-4$
$\mathrm{C} 10 \mathrm{H} 15 \mathrm{~N} 2 \mathrm{O} 7-3$
$\mathrm{C} 10 \mathrm{H} 8$
$\mathrm{C} 6 \mathrm{H} 5 \mathrm{H} 5 \mathrm{NO} 2$
$\mathrm{C} 12 \mathrm{H} 27 \mathrm{O} 4 \mathrm{P}$
$\mathrm{C} 12 \mathrm{H} 4 \mathrm{Cl} 6$
$\mathrm{C} 12 \mathrm{H} 8$
$\mathrm{C} 13 \mathrm{H} 28$
$\mathrm{C} 2 \mathrm{Cl} 4$
$\mathrm{C} 2 \mathrm{H} 3 \mathrm{~N}$
$\mathrm{C} 2 \mathrm{H} 3 \mathrm{O} 2-$
$\mathrm{C} 2 \mathrm{H} 3 \mathrm{O} 3-$
$\mathrm{C} 2 \mathrm{H} 6 \mathrm{SiO}$
$\mathrm{C} 2 \mathrm{H} 8 \mathrm{~N} 2$
$\mathrm{C} 2 \mathrm{HCl} 3$
$\mathrm{C} 2 \mathrm{O} 4-2$

\begin{tabular}{|c|c|}
\hline $\begin{array}{r}\text { Liquid \% } \\
\text { to HOP-SCB-00001/2 }\end{array}$ & $\begin{array}{r}\text { Solid \% } \\
\text { to HOP-SCB-00001/2 }\end{array}$ \\
\hline 99.67741935 & 99.67741935 \\
\hline 99.67741935 & 99.67741935 \\
\hline 99.67741935 & 99.67741935 \\
\hline 99.67741935 & 99.67741935 \\
\hline 99.67741935 & 99.67741935 \\
\hline 99.67741935 & 99.67741935 \\
\hline 0 & 0 \\
\hline 99.666666667 & 99.66666667 \\
\hline 99.6969697 & 99.6969697 \\
\hline 99.666666667 & 99.66666667 \\
\hline 99.6875 & 99.6875 \\
\hline 99.667 & 99.667 \\
\hline 99.667 & 99.667 \\
\hline 99.65517241 & 99.65517241 \\
\hline 99.67741935 & 99.67741935 \\
\hline 99.67741935 & 99.67741935 \\
\hline 99.67741935 & 99.67741935 \\
\hline 99.67741935 & 99.67741935 \\
\hline 99.67741935 & 99.67741935 \\
\hline 99.67741935 & 99.67741935 \\
\hline 99.6969697 & 99.6969697 \\
\hline 99.677 & 99.677 \\
\hline 99.67741935 & 99.67741935 \\
\hline 99.67741935 & 99.67741935 \\
\hline 99.67741935 & 99.67741935 \\
\hline 0 & 0 \\
\hline 0 & 0 \\
\hline 0 & 0 \\
\hline 0 & 0 \\
\hline 99.79591837 & 99.79591837 \\
\hline 0 & 0 \\
\hline 0 & 0 \\
\hline 0 & 0 \\
\hline 99.79591837 & 99.79591837 \\
\hline 0 & 0 \\
\hline 0 & 0 \\
\hline 0 & 0 \\
\hline 0 & 0 \\
\hline 0 & 0 \\
\hline 99.79591837 & 99.79591837 \\
\hline 0 & 0 \\
\hline 0 & 0 \\
\hline 0 & 0 \\
\hline 0 & 0 \\
\hline 0 & 0 \\
\hline 0 & 0 \\
\hline 0 & 0 \\
\hline
\end{tabular}


HOP-HEME-00001/2

Components

C6H6

$\mathrm{C} 6 \mathrm{H} 6 \mathrm{O}$

C7H6N2O4

$\mathrm{C} 7 \mathrm{H} 8$

$\mathrm{C} 7 \mathrm{H} 8 \mathrm{O}$

$\mathrm{C} 8 \mathrm{H} 8 \mathrm{O}$

$\mathrm{Ca}+2$

$\mathrm{CCl} 4$

$\mathrm{Cd}+2$

$\mathrm{Ce}+3$

$\mathrm{CH} 2 \mathrm{Cl} 2$

$\mathrm{CHCl} 3$

$\mathrm{CHO} 2-$

Cl-

$\mathrm{CN}-$

$\mathrm{Co}+3$

$\mathrm{CO} 3-2$

$\mathrm{Cr}(\mathrm{OH}) 4-$

Cr(TOTAL)

$\mathrm{Cs}+$

$\mathrm{Cu}+2$

DPDMS

DPPG

F-

$\mathrm{Fe}+3$

$\mathrm{H}+$

$\mathrm{H} 2 \mathrm{CO} 3$

$\mathrm{H} 2 \mathrm{O}$

H2O(BOUND)

$\mathrm{H} 2 \mathrm{O} 2$

$\mathrm{HCO} 3-$

$\mathrm{Hg}+2$

$\mathrm{K}+$

$\mathrm{La}+3$

$\mathrm{Li}+$

LS10

$\mathrm{AlOOH}$

LS9

$\mathrm{Mg}+2$

$\mathrm{Mn}+4$

$\mathrm{MnO} 2$

$\mathrm{MnO} 4-$

Mnp

$\mathrm{Mo}+6$

$\mathrm{Na}+$

$\mathrm{Nap}+$

$\mathrm{Nd}+3$
Liquid \% to HOP-SCB-00001/2

0

0

99.66666667

99.6874

99.666666667

\section{0}

\section{0}

0

99.6875

99.6875

99.6969697

99.6875

99.70588235

99.70588235

99.6875

99.67741935

0

0

99.6969697

99.67741935

99.9

99.6875

0

\section{0}

0

99.6875

99.6875

99.666666667

99.6875

0

0

0

99.666666667

99.66666667

99.66666667

99.66666667

99.66666667

99.6969697

99.6875

99.6875

99.666666667
Solid \%

to HOP-SCB-00001/2

0

0

0

0

0

0

99.66666667

99.6875

99.66666667

0

0

0

99.6875

99.6875

99.6969697

99.6875

99.70588235

99.70588235

99.6875

99.67741935

0

0

99.6969697

99.67741935

99.9

99.6875

0

0

0

99.6875

99.6875

99.666666667

99.6875

0

0

0

99.666666667

99.66666667

99.666666667

99.66666667

99.66666667

99.6969697

99.6875

99.6875

99.66666667 
HOP-HEME-00001/2 Components

$\mathrm{NH} 3$

NH4+

$\mathrm{Ni}+2$

$\mathrm{NO} 2-$

NO3-

NO3-D

$\mathrm{O}-2$

$\mathrm{OH}(\mathrm{BOUND})$

$\mathrm{OH}-$

$\mathrm{Pb}+2$

$\mathrm{Pd}+2$

PDMS

PO4-3

PO4-3(HARD)

PPG

$\mathrm{Pr}+3$

$\mathrm{Pu}+4$

$\mathrm{Rb}+$

$\mathrm{Rh}+3$

$\mathrm{Ru}+3$

$\mathrm{Sb}+5$

$\mathrm{Se}+6$

$\mathrm{Si}+4$

SO4-2

$\mathrm{Sr}+2$

$\mathrm{Ta}+5$

$\mathrm{Tc}+7$

$\mathrm{Te}+6$

$\mathrm{Th}+4$

$\mathrm{Ti}+4$

$\mathrm{Tl}+3$

TOC

U(TOTAL)

$\mathrm{V}+5$

$\mathrm{W}+6$

$\mathrm{Y}+3$

$\mathrm{Zn}+2$

$\mathrm{Zr}+4$
Liquid \%

to HOP-SCB-00001/2

9.90990991

9.90990991

99.66666667

99.6875

99.6875

\section{0}

99.6875

99.6875

99.6875

99.67741935

99.6875

0

0

99.67741935

99.67741935

99.67741935

99.67741935

99.6969697

99.62962963

99.6875

99.67741935

99.6875

99.66666667

99.67741935

99.67741935

99.6875

99.67741935

99.65517241

99.67741935

99.67741935

99.70588235

99.67741935

99.67741935

99.67741935

99.65517241

\section{Solid \%}

to HOP-SCB-00001/2

9.90990991

9.90990991

99.66666667

99.6875

99.6875

0

0

99.6875

99.6875

99.6875

99.67741935

99.6875

0

99.67741935

99.67741935

99.67741935

99.67741935

99.6969697

99.62962963

99.6875

99.67741935

99.6875

99.66666667

99.67741935

99.67741935

99.6875

99.67741935

99.65517241

99.67741935

99.67741935

99.70588235

99.67741935

99.67741935

99.67741935

99.65517241 
Table D-23 HLW HEPA Splits

\section{HOP-HEPA-00001-2AB}

HOP-HEPA-00007-8AB

Components

106-Ru

$113 \mathrm{~m}-\mathrm{Cd}$

$125-\mathrm{Sb}$

126-Sn

$129-\mathrm{I}$

134-Cs

137-Cs

$137 \mathrm{~m}-\mathrm{Ba}$

14-C

$151-\mathrm{Sm}$

152-Eu

$154-\mathrm{Eu}$

$155-\mathrm{Eu}$

226-Ra

227-Ac

228-Ra

229-Th

231-Pa

232-Th

232-U

233-U

234-U

235-U

236-U

237-Np

238-Pu

238-U

239-Pu

240-Pu

241-Am

241-Pu

242-Cm

242-Pu

243-Am

243-Cm

244-Cm

3-H

$59-\mathrm{Ni}$

60-Co

$63-\mathrm{Ni}$

79-Se

90-Sr

90-Y

93-Zr

$93 \mathrm{~m}-\mathrm{Nb}$

99-Tc

$\begin{array}{rr}\begin{array}{r}\text { Liquid \% } \\ \text { to Accumulate }\end{array} & \begin{array}{r}\text { Solid \% } \\ \text { to Accumulate }\end{array} \\ 99.99994 & 99.99994 \\ 99.99994 & 99.99994 \\ 99.99994 & 99.99994 \\ 99.99994 & 99.99994 \\ 0 & 0 \\ 99.99994 & 99.99994 \\ 99.99994 & 99.99994 \\ 99.99994 & 99.99994 \\ 0 & 0 \\ 99.99994 & 99.99994 \\ 99.99994 & 99.99994 \\ 99.99994 & 99.99994 \\ 99.99994 & 99.99994 \\ 99.99994 & 99.99994 \\ 99.99994 & 99.99994 \\ 99.99994 & 99.99994 \\ 99.99994 & 99.99994 \\ 99.99994 & 99.99994 \\ 99.99994 & 99.99994 \\ 99.99994 & 99.99994 \\ 99.99994 & 99.99994 \\ 99.99994 & 99.99994 \\ 99.99994 & 99.99994 \\ 99.99994 & 99.99994 \\ 99.99994 & 99.99994 \\ 99.99994 & 99.99994 \\ 99.99994 & 99.99994 \\ 99.99994 & 99.99994 \\ 99.99994 & 99.99994 \\ 99.99994 & 99.99994 \\ 99.99994 & 99.99994 \\ 99.99994 & 99.99994 \\ 99.99994 & 99.99994 \\ 99.99994 & 99.99994 \\ 99.99994 & 99.99994 \\ 99.99994 & 99.99994 \\ 0 & 99.99994 \\ 99.99994 & 99.99994 \\ 99.99994 & 99.99994 \\ 99.99994 & 99.99994 \\ 99.99994 & 99.99994 \\ 99.99994 & 99.99994 \\ 99.99994 & 99.99994 \\ 99.99994 & 999994 \\ 99.99994 & \\ 99.99994 & \end{array}$




HOP-HEPA-00001-2AB
HOP-HEPA-0007-8AB
Components
$\mathrm{Ag}+$
$\mathrm{Al}(\mathrm{OH}) 3$
$\mathrm{Al}(\mathrm{OH}) 4-$
$\mathrm{Al}+3$
$\mathrm{As}+5$
$\mathrm{~B}+3$
$\mathrm{Ba}+2$
$\mathrm{Be}+2$
$\mathrm{Bi}+3$
$\mathrm{C} 10 \mathrm{H} 12 \mathrm{~N} 2 \mathrm{O} 8-4$
$\mathrm{C} 10 \mathrm{H} 15 \mathrm{~N} 2 \mathrm{O}-3$
$\mathrm{C} 10 \mathrm{H} 8$
$\mathrm{C} 12 \mathrm{H} 27 \mathrm{O} 4 \mathrm{P}$
$\mathrm{C} 12 \mathrm{H} 4 \mathrm{Cl} 6$
$\mathrm{C} 12 \mathrm{H} 8$
$\mathrm{C} 13 \mathrm{H} 28$
$\mathrm{C} 2 \mathrm{Cl} 4$
$\mathrm{C} 2 \mathrm{H} 3 \mathrm{~N}$
$\mathrm{C} 2 \mathrm{H} 3 \mathrm{O} 2-$
$\mathrm{C} 2 \mathrm{H} 3 \mathrm{O} 3-$
$\mathrm{C} 2 \mathrm{H} 6 \mathrm{SiO}$
$\mathrm{C} 2 \mathrm{H} 8 \mathrm{~N} 2$
$\mathrm{C} 2 \mathrm{HCl} 3$
$\mathrm{C} 2 \mathrm{O} 4-2$
$\mathrm{C} 3 \mathrm{H} 6 \mathrm{O}$
$\mathrm{C} 4 \mathrm{H} 10 \mathrm{O}$
$\mathrm{C} 4 \mathrm{H} 7 \mathrm{NO} 4$
$\mathrm{C} 4 \mathrm{H} 8 \mathrm{O}$
$\mathrm{C} 5 \mathrm{H} 5 \mathrm{~N}$
$\mathrm{C} 6 \mathrm{H} 5 \mathrm{NO} 2$
$\mathrm{C} 6 \mathrm{H} 5 \mathrm{O}-3$
$\mathrm{C} 6 \mathrm{H} 6$
$\mathrm{C} 6 \mathrm{H} 6 \mathrm{O}$
$\mathrm{C} 7 \mathrm{H} 6 \mathrm{~N} 2 \mathrm{O} 4$
$\mathrm{C} 7 \mathrm{H} 8$
$\mathrm{C} 7 \mathrm{H} 8 \mathrm{O}$
$\mathrm{C} 8 \mathrm{H} 8 \mathrm{O}$
$\mathrm{Ca}+2$
$\mathrm{CC} 14$
$\mathrm{Cd}+2$
$\mathrm{Ce}+3$
$\mathrm{CH} 2 \mathrm{Cl} 2$
$\mathrm{CHCl} 3$
$\mathrm{CHO} 2-$
$\mathrm{Co}+3$

\begin{tabular}{rr}
$\begin{array}{r}\text { Liquid \% } \\
\text { to Accumulate }\end{array}$ & $\begin{array}{r}\text { Solid \% } \\
\text { to Accumulate }\end{array}$ \\
99.99994 & 99.99994 \\
99.99994 & 99.99994 \\
99.99994 & 99.99994 \\
99.99994 & 99.99994 \\
99.99994 & 99.99994 \\
99.97 & 99.97 \\
99.99994 & 99.99994 \\
99.99994 & 99.99994 \\
99.99994 & 99.99994 \\
99.99994 & 99.99994 \\
99.99994 & 99.99994 \\
99.99994 & 99.99994 \\
99.99994 & 99.99994 \\
99.99994 & 99.99994 \\
99.99994 & 99.99994 \\
99.99994 & 99.99994 \\
99.99994 & 99.99994 \\
99.99994 & 99.99994 \\
99.99994 & 99.99994 \\
99.99994 & 99.99994 \\
99.99994 & 99.99994 \\
99.99994 & 99.99994 \\
99.99994 & 99.99994 \\
99.99994 & 99.99994 \\
99.99994 & 99.99994 \\
99.99994 & 99.99994 \\
99.99994 & 99.99994 \\
99.99994 & 99.99994 \\
99.99994 & 99.99994 \\
99.99994 & 99.99994 \\
99.99994 & 99.99994 \\
99.99994 & 99.99994 \\
99.99994 & 99.99994 \\
99.99994 & 99.99994 \\
99.99994 & 99.99994 \\
99.99994 & 99.99994 \\
99.99994 & 99.99994 \\
99.99994 & 99.99994 \\
99.99994 & 99.99994 \\
99.99994 & 99.99994 \\
99.99994 & 99.99994 \\
99.99994 & 99.99994 \\
99.99994 & 99.99994 \\
99.99994 & 99.99994 \\
99.99994 & 99.99994 \\
99.99994 & 99.99994 \\
99.99994 & \\
\hline &
\end{tabular}




HOP-HEPA-00001-2AB
HOP-HEPA-00007-8AB
Components
$\mathrm{CO} 3-2$
$\mathrm{Cr}(\mathrm{OH}) 4-$
$\mathrm{Cr}(\mathrm{TOTAL})$
$\mathrm{Cs}+$
$\mathrm{Cu}+2$
$\mathrm{DPDMS}$
$\mathrm{DPPG}$
$\mathrm{F}-$
$\mathrm{Fe}+3$
$\mathrm{H}+$
$\mathrm{H} 2 \mathrm{CO} 3$
$\mathrm{H} 2 \mathrm{O}$
$\mathrm{H} 2 \mathrm{O}(\mathrm{BOUND})$
$\mathrm{H} 2 \mathrm{O} 2$
$\mathrm{HCO} 3-$
$\mathrm{Hg}+2$
$\mathrm{~K}+$
$\mathrm{La}+3$
$\mathrm{Li}+$
$\mathrm{LS} 10$
$\mathrm{AlOOH}$
$\mathrm{LS} 9$
$\mathrm{Mg}+2$
$\mathrm{Mn}+4$
$\mathrm{MnO} 2$
$\mathrm{MnO} 4-$
$\mathrm{Mnp}$
$\mathrm{Mo}+6$
$\mathrm{Na}+$
$\mathrm{Nap}+$
$\mathrm{Nd}+3$
$\mathrm{NH} 3$
$\mathrm{NH} 4+$
$\mathrm{Ni}+2$
$\mathrm{NO} 2-$
$\mathrm{Pr}+3$
$\mathrm{NO} 3-$
$\mathrm{NO} 3-\mathrm{D}-$
$\mathrm{O}-2$
$\mathrm{OH}(\mathrm{BOUND}$
$\mathrm{OH}-$
$\mathrm{Pb}+2$
$\mathrm{Pd}+2$

\begin{tabular}{|c|c|}
\hline $\begin{array}{r}\text { Liquid \% } \\
\text { to Accumulate }\end{array}$ & $\begin{array}{r}\text { Solid \% } \\
\text { to Accumulate }\end{array}$ \\
\hline 99.99994 & 99.99994 \\
\hline 99.99994 & 99.99994 \\
\hline 99.99994 & 99.99994 \\
\hline 99.99994 & 99.99994 \\
\hline 99.99994 & 99.99994 \\
\hline 99.99994 & 99.99994 \\
\hline 99.99994 & 99.99994 \\
\hline 99.99994 & 99.99994 \\
\hline 99.99994 & 99.99994 \\
\hline 99.99994 & 99.99994 \\
\hline 99.99994 & 99.99994 \\
\hline 0 & 0 \\
\hline 99.99994 & 99.99994 \\
\hline 99.99994 & 99.99994 \\
\hline 99.99994 & 99.99994 \\
\hline 0 & 0 \\
\hline 99.99994 & 99.99994 \\
\hline 99.99994 & 99.99994 \\
\hline 99.99994 & 99.99994 \\
\hline 99.99994 & 99.99994 \\
\hline 99.99994 & 99.99994 \\
\hline 99.99994 & 99.99994 \\
\hline 99.99994 & 99.99994 \\
\hline 99.99994 & 99.99994 \\
\hline 99.99994 & 99.99994 \\
\hline 99.99994 & 99.99994 \\
\hline 99.99994 & 99.99994 \\
\hline 99.99994 & 99.99994 \\
\hline 99.99994 & 99.99994 \\
\hline 99.99994 & 99.99994 \\
\hline 99.99994 & 99.99994 \\
\hline 99.99994 & 99.99994 \\
\hline 99.99994 & 99.99994 \\
\hline 99.99994 & 99.99994 \\
\hline 0 & 0 \\
\hline 99.99994 & 99.99994 \\
\hline 99.99994 & 99.99994 \\
\hline 99.99994 & 99.99994 \\
\hline 99.99994 & 99.99994 \\
\hline 99.99994 & 99.99994 \\
\hline 99.99994 & 99.99994 \\
\hline 99.99994 & 99.99994 \\
\hline 99.99994 & 99.99994 \\
\hline 99.99994 & 99.99994 \\
\hline 99.99994 & 99.99994 \\
\hline 99.99994 & 99.99994 \\
\hline 99.99994 & 99.99994 \\
\hline
\end{tabular}


HOP-HEPA-00001-2AB HOP-HEPA-00007-8AB

Components

$\mathrm{Pu}+4$

$\mathrm{Rb}+$

$\mathrm{Rh}+3$

$\mathrm{Ru}+3$

$\mathrm{Sb}+5$

$\mathrm{Se}+6$

$\mathrm{Si}+4$

SO4-2

$\mathrm{Sr}+2$

$\mathrm{Ta}+5$

$\mathrm{Tc}+7$

$\mathrm{Te}+6$

$\mathrm{Th}+4$

$\mathrm{Ti}+4$

$\mathrm{Tl}+3$

TOC

U(TOTAL)

$\mathrm{V}+5$

$\mathrm{W}+6$

$\mathrm{Y}+3$

$\mathrm{Zn}+2$

$\mathrm{Zr}+4$

Gas Components 129-I

$\mathrm{C} 12 \mathrm{H} 4 \mathrm{Cl} 6$

$\mathrm{C} 2 \mathrm{H} 3 \mathrm{~N}$

$\mathrm{CO} 2$

$\mathrm{H} 2 \mathrm{O}$

$\mathrm{HCl}$

$\mathrm{HF}$

$\mathrm{Hg}$

N2

$\mathrm{NH} 3$

$\mathrm{NO}$

$\mathrm{NO} 2$

$\mathrm{O} 2$

$\mathrm{P} 2 \mathrm{O} 5$

$\mathrm{SO} 2$

SO3
Liquid \%

to Accumulate

99.99994

99.99994

99.99994

99.99994

99.99994

99.99994

99.99994

99.99994

99.99994

99.99994

99.99994

99.99994

99.99994

99.99994

99.99994

99.99994

99.99994

99.99994

99.99994

99.99994

99.99994

99.99994

Gas \%

to ACCUMULATE

99.99994

99.99994

0

0

0

0

99.99994

99.99994

\section{0}

0

0

99.99994

0

99.99994
Solid \%

to Accumulate

99.99994

99.99994

99.99994

99.99994

99.99994

99.99994

99.99994

99.99994

99.99994

99.99994

99.99994

99.99994

99.99994

99.99994

99.99994

99.99994

99.99994

99.99994

99.99994

99.99994

99.99994

99.99994

\section{0}

( . 


\section{Table D-24 HLW Carbon Absorber Splits}

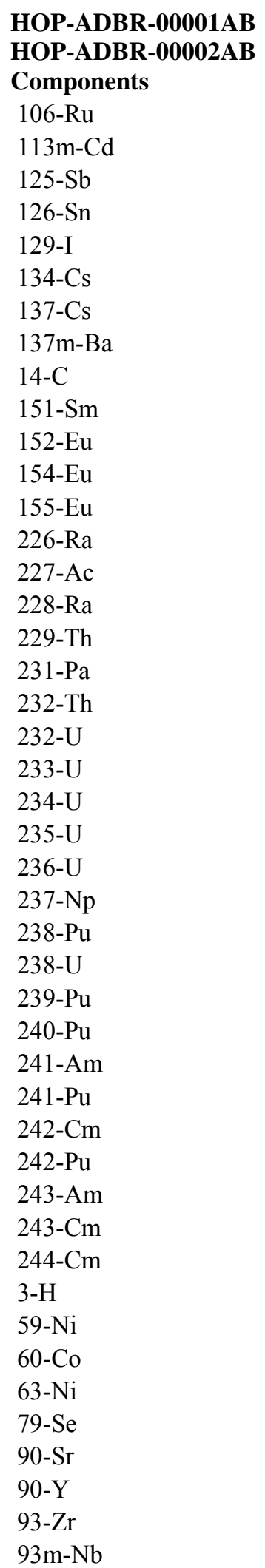

Liquid \%

to Accumulate

0

0

0

0

0

0

0

0

0

0

0

0

0

0

0

0

0

0

0

0

0

0

0

0

0

0

0

0

0

0

0

0

0

0

0

0

0

0

0

0

0

0

0

0

0
Solid \%

to Accumulate

0

0

0

0

0

0

0

0

0

0

0

0

0

0

0

0

0

0

0

0

0

0

0

0

0

0

0

0

0

0

0

0

0

0

0

0

0

0

0

0

0

0

0

0

0 
HOP-ADBR-00001AB HOP-ADBR-00002AB

Components

99-Tc

$\mathrm{Ag}+$

$\mathrm{Al}(\mathrm{OH}) 3$

$\mathrm{Al}(\mathrm{OH}) 4-$

$\mathrm{Al}+3$

$\mathrm{As}+5$

$\mathrm{B}+3$

$\mathrm{Ba}+2$

$\mathrm{Be}+2$

$\mathrm{Bi}+3$

C10H12N2O8-4

C10H15N2O7-3

$\mathrm{C} 10 \mathrm{H} 8$

$\mathrm{C} 12 \mathrm{H} 27 \mathrm{O} 4 \mathrm{P}$

$\mathrm{C} 12 \mathrm{H} 4 \mathrm{Cl} 6$

$\mathrm{C} 12 \mathrm{H} 8$

C13H 28

$\mathrm{C} 2 \mathrm{Cl} 4$

$\mathrm{C} 2 \mathrm{H} 3 \mathrm{~N}$

$\mathrm{C} 2 \mathrm{H} 3 \mathrm{O} 2-$

$\mathrm{C} 2 \mathrm{H} 3 \mathrm{O} 3-$

$\mathrm{C} 2 \mathrm{H} 6 \mathrm{SiO}$

$\mathrm{C} 2 \mathrm{H} 8 \mathrm{~N} 2$

$\mathrm{C} 2 \mathrm{HCl} 3$

C2O4-2

$\mathrm{C} 3 \mathrm{H} 6 \mathrm{O}$

$\mathrm{C} 4 \mathrm{H} 10 \mathrm{O}$

C4H7NO4

$\mathrm{C} 4 \mathrm{H} 8 \mathrm{O}$

C5H5N

$\mathrm{C} 6 \mathrm{H} 5 \mathrm{NO} 2$

C6H5O7-3

C6H6

C6H6O

C7H6N2O4

$\mathrm{C} 7 \mathrm{H} 8$

$\mathrm{C} 7 \mathrm{H} 8 \mathrm{O}$

$\mathrm{C} 8 \mathrm{H} 8 \mathrm{O}$

$\mathrm{Ca}+2$

$\mathrm{CCl} 4$

$\mathrm{Cd}+2$

$\mathrm{Ce}+3$

$\mathrm{CH} 2 \mathrm{Cl} 2$

$\mathrm{CHCl} 3$

CHO2-

$\mathrm{Cl}-$

$\mathrm{CN}-$
Liquid \%

to Accumulate

0

0

0

0

0

0

0

0

0

0

0

0

0

0

90

0

0

0

0

0

0

0

0

0

0

0

0

0

0

0

0

0

0

0

0

0

0

0

0

0

0

0

0

0

0

0

0
Solid \%

to Accumulate

0

0

0

0

0

0

0

0

0

0

0

0

0

0

90

0

0

0

0

0

0

0

0

0

0

0

0

0

0

0

0

0

0

0

0

0

0

0

0

0

0

0

0

0

0

0

0 
HOP-ADBR-00001AB HOP-ADBR-00002AB

Components

$\mathrm{Co}+3$

$\mathrm{CO} 3-2$

$\mathrm{Cr}(\mathrm{OH}) 4-$

Cr(TOTAL)

$\mathrm{Cs}+$

$\mathrm{Cu}+2$

DPDMS

DPPG

F-

$\mathrm{Fe}+3$

$\mathrm{H}+$

$\mathrm{H} 2 \mathrm{CO} 3$

$\mathrm{H} 2 \mathrm{O}$

$\mathrm{H} 2 \mathrm{O}(\mathrm{BOUND})$

$\mathrm{H} 2 \mathrm{O} 2$

$\mathrm{HCO} 3-$

$\mathrm{Hg}+2$

$\mathrm{K}+$

$\mathrm{La}+3$

$\mathrm{Li}+$

LS10

$\mathrm{AlOOH}$

LS9

$\mathrm{Mg}+2$

$\mathrm{Mn}+4$

$\mathrm{MnO} 2$

$\mathrm{MnO} 4-$

Mnp

$\mathrm{Mo}+6$

$\mathrm{Na}+$

$\mathrm{Nap}+$

$\mathrm{Nd}+3$

NH3

NH4+

$\mathrm{Ni}+2$

$\mathrm{NO} 2-$

NO3-

NO3-D

$\mathrm{O}-2$

$\mathrm{OH}(\mathrm{BOUND})$

$\mathrm{OH}-$

$\mathrm{Pb}+2$

$\mathrm{Pd}+2$

PDMS

PO4-3

PO4-3(HARD)

PPG
Liquid \%

to Accumulate

0

\section{0}

0

0

0

0

0

0

0

0

0

0

0

0

0

0

0

0

0

0

0

0

0

0

0

0

0

0

0

0

0

0

0

0

0

0

0

0

0

0

0

0

0

0

0

0

0
Solid \%

to Accumulate

0

0

0

0

0

0

0

0

0

0

0

0

0

0

0

0

0

0

0

0

0

0

0

0

0

0

0

0

0

0

0

0

0

0

0

0

0

0

0

0

0

0

0

0

0

0

0 
HOP-ADBR-00001AB HOP-ADBR-00002AB

Components

$\operatorname{Pr}+3$

$\mathrm{Pu}+4$

$\mathrm{Rb}+$

$\mathrm{Rh}+3$

$\mathrm{Ru}+3$

$\mathrm{Sb}+5$

$\mathrm{Se}+6$

$\mathrm{Si}+4$

SO4-2

$\mathrm{Sr}+2$

$\mathrm{Ta}+5$

$\mathrm{Tc}+7$

$\mathrm{Te}+6$

$\mathrm{Th}+4$

$\mathrm{Ti}+4$

$\mathrm{Tl}+3$

TOC

$\mathrm{U}(\mathrm{TOTAL})$

$\mathrm{V}+5$

$\mathrm{W}+6$

$\mathrm{Y}+3$

$\mathrm{Zn}+2$

$\mathrm{Zr}+4$

Gas Components

129-I

$\mathrm{C} 12 \mathrm{H} 4 \mathrm{Cl} 6$

$\mathrm{C} 2 \mathrm{H} 3 \mathrm{~N}$

$\mathrm{CO} 2$

$\mathrm{H} 2 \mathrm{O}$

$\mathrm{HCl}$

$\mathrm{HF}$

$\mathrm{Hg}$

N2

$\mathrm{NH} 3$

NO

$\mathrm{NO} 2$

$\mathrm{O} 2$

$\mathrm{P} 2 \mathrm{O} 5$

$\mathrm{SO} 2$

SO3
Liquid \%

to Accumulate

0

0

0

0

0

0

0

0

0

0

0

0

0

0

0

0

0

0

0

0

0

0

0

Gas \%

to ACCUMULATE

0

90

0

0

0

0

0

99.9

0

0

0

0

0

0

0

0 
Table D-25 HLW Silver Mordenite Splits

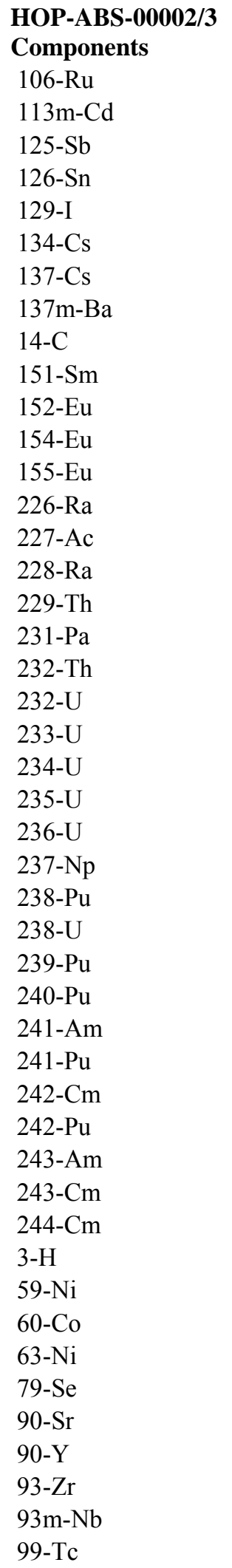

Liquid \% to Accumulate 0

0

0

0

0

0

0

0

0

0

0

0

0

0

0

0

0

0

0

0

0

0

0

$$
0
$$

0

0

0

0

0

0

0

0

0

0

0

0

0

0

0

0

0

0

0

0

0

0

0
Solid \%

to Accumulate

0

0

0

0

0

0

0

0

0

0

0

0

0

0

0

0

0

0

0

0

0

0

0

0

0

0

0

0

0

0

0

0

0

0

0

0

0

0

0

0

0

0

0

0

0

0 
HOP-ABS-00002/3

Components

$\mathrm{Ag}+$

$\mathrm{Al}(\mathrm{OH}) 3$

$\mathrm{Al}(\mathrm{OH}) 4-$

$\mathrm{Al}+3$

As +5

$\mathrm{B}+3$

$\mathrm{Ba}+2$

$\mathrm{Be}+2$

$\mathrm{Bi}+3$

C10H12N2O8-4

$\mathrm{C} 10 \mathrm{H} 15 \mathrm{~N} 2 \mathrm{O} 7-3$

$\mathrm{C} 10 \mathrm{H} 8$

$\mathrm{C} 12 \mathrm{H} 27 \mathrm{O} 4 \mathrm{P}$

$\mathrm{C} 12 \mathrm{H} 4 \mathrm{Cl} 6$

$\mathrm{C} 12 \mathrm{H} 8$

C13H 28

$\mathrm{C} 2 \mathrm{Cl} 4$

$\mathrm{C} 2 \mathrm{H} 3 \mathrm{~N}$

$\mathrm{C} 2 \mathrm{H} 3 \mathrm{O} 2-$

$\mathrm{C} 2 \mathrm{H} 3 \mathrm{O} 3-$

$\mathrm{C} 2 \mathrm{H} 6 \mathrm{SiO}$

$\mathrm{C} 2 \mathrm{H} 8 \mathrm{~N} 2$

$\mathrm{C} 2 \mathrm{HCl} 3$

$\mathrm{C} 2 \mathrm{O} 4-2$

$\mathrm{C} 3 \mathrm{H} 6 \mathrm{O}$

C4H10O

$\mathrm{C} 4 \mathrm{H} 7 \mathrm{NO} 4$

$\mathrm{C} 4 \mathrm{H} 8 \mathrm{O}$

C5H5N

$\mathrm{C} 6 \mathrm{H} 5 \mathrm{NO} 2$

C6H5O7-3

$\mathrm{C} 6 \mathrm{H} 6$

$\mathrm{C} 6 \mathrm{H} 6 \mathrm{O}$

C7H6N2O4

$\mathrm{C} 7 \mathrm{H} 8$

$\mathrm{C} 7 \mathrm{H} 8 \mathrm{O}$

C8H8O

$\mathrm{Ca}+2$

$\mathrm{CCl} 4$

$\mathrm{Cd}+2$

$\mathrm{Ce}+3$

$\mathrm{CH} 2 \mathrm{Cl} 2$

$\mathrm{CHCl} 3$

CHO2-

Cl-

$\mathrm{CN}-$

$\mathrm{Co}+3$
Liquid \% to Accumulate

0

0

0

0

0

0

0

0

0

0

0

0

0

0

0

0

0

0

0

0

0

0

0

0

0

0

0

0

0

0

0

0

0

0

0

0

0

0

0

0

0

0

0

0
Solid \%

to Accumulate

0

0

0

0

0

0

0

0

0

0

0

0

0

0

0

0

0

0

0

0

0

0

0

0

0

0

0

0

0

0

0

0

0

0

0

0

0

0

0

0

0

0

0

0

0

0

0 
HOP-ABS-00002/3

Components

$\mathrm{CO} 3-2$

$\mathrm{Cr}(\mathrm{OH}) 4-$

Cr(TOTAL)

$\mathrm{Cs}+$

$\mathrm{Cu}+2$

F-

$\mathrm{Fe}+3$

$\mathrm{H}+$

$\mathrm{H} 2 \mathrm{CO} 3$

$\mathrm{H} 2 \mathrm{O}$

$\mathrm{H} 2 \mathrm{O} 2$

$\mathrm{HCO} 3-$

$\mathrm{Hg}+2$

$\mathrm{K}+$

$\mathrm{La}+3$

$\mathrm{Li}+$

H2O(BOUND)

LS10

PO4-3(HARD)

PPG

DPPG

PDMS

DPDMS

NO3-D

$\mathrm{AlOOH}$

LS9

$\mathrm{Mg}+2$

$\mathrm{Mn}+4$

$\mathrm{MnO} 2$

MnO4-

Mnp

$\mathrm{Mo}+6$

$\mathrm{Na}+$

Nap+

$\mathrm{Nd}+3$

$\mathrm{NH} 3$

NH4+

$\mathrm{Ni}+2$

NO2-

NO3-

$\mathrm{O}-2$

OH(BOUND)

$\mathrm{OH}-$

$\mathrm{Pb}+2$

$\mathrm{Pd}+2$

PO4-3

$\mathrm{Pr}+3$
Liquid \% to Accumulate

0

0

0

0

0

0

0

0

0

0

0

0

0

0

0

0

0

0

0

0

0

0

0

0

0

0

0

0

0

0

0

0

0

0

0

0

0

0

0

0

0

0

0

0
Solid \%

to Accumulate

0

0

0

0

0

0

0

0

0

0

0

0

0

0

0

0

0

0

0

0

0

0

0

0

0

0

0

0

0

0

0

0

0

0

0

0

0

0

0

0

0

0

0

0

0

0

0 
HOP-ABS-00002/3

Components

$\mathrm{Pu}+4$

$\mathrm{Rb}+$

$\mathrm{Rh}+3$

$\mathrm{Ru}+3$

$\mathrm{Sb}+5$

$\mathrm{Se}+6$

$\mathrm{Si}+4$

$\mathrm{SO} 4-2$

$\mathrm{Sr}+2$

$\mathrm{Ta}+5$

Tc+7

$\mathrm{Te}+6$

$\mathrm{Th}+4$

$\mathrm{Ti}+4$

$\mathrm{Tl}+3$

TOC

U(TOTAL)

$\mathrm{V}+5$

$\mathrm{W}+6$

$\mathrm{Y}+3$

$\mathrm{Zn}+2$

$\mathrm{Zr}+4$

Gas Components

129-I

$\mathrm{C} 12 \mathrm{H} 4 \mathrm{Cl} 6$

$\mathrm{C} 2 \mathrm{H} 3 \mathrm{~N}$

$\mathrm{CO} 2$

$\mathrm{Hg}$

$\mathrm{H} 2 \mathrm{O}$

$\mathrm{HCl}$

$\mathrm{HF}$

$\mathrm{N} 2$

$\mathrm{NH} 3$

$\mathrm{NO}$

$\mathrm{NO} 2$

$\mathrm{O} 2$

$\mathrm{P} 2 \mathrm{O} 5$

$\mathrm{SO} 2$

$\mathrm{SO} 3$
Liquid \% to Accumulate

0

0

0

0

0

0

0

0

0

0

0

0

0

0

0

0

0

0

0

Gas \%

to ACCUMULATE

99.9

0

0

0

0

99.9

99.9

0

0

0

0

0

0

0

0

Table D-26 HLW NOx Scrubber Splits

HOP-SCR-00001/2

Components

C2O4-2

Gas Components
Liquid \%

to Accumulate

Gas \%

to Accumulate
Solid \%

to Accumulate

0
Solid \%
to Accumulate

0

0

0

0

0

0

0

0

0

0

0

0

0

0

0

0

0

0

0

0

0 


$\begin{array}{ll}\mathrm{CO} 2 & 0 \\ \mathrm{H} 2 \mathrm{O} & 0 \\ \mathrm{HCl} & 0 \\ \mathrm{HF} & 0 \\ \mathrm{~N} 2 & 0 \\ \mathrm{NH} 3 & 0 \\ \mathrm{NO} & 0 \\ \mathrm{NO} 2 & 0 \\ \mathrm{O} 2 & 0 \\ \mathrm{P} 2 \mathrm{O} 5 & 0 \\ \mathrm{SO} 2 & 0\end{array}$

\section{Table D-27 HLW PJV HEPA Splits}

PJV-HEPA-00004-5

Components

106-Ru

Gas Components

$\mathrm{CO} 2$
Liquid \%

to Accumulate

Gas \%

to Accumulate

0
Solid \%

to Accumulate 
24590-WTP-MDD-PR-01-002, Rev 12

Dynamic (G2) Model Design Document

\section{Appendix E}

\section{Vessel Parameter Table}




\section{Appendix E \\ Vessel Parameter Table}

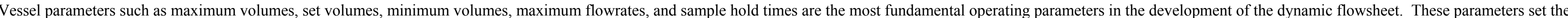
physical limits on how vessels are filled, emptied, sampled, and pumped. Maximum operating temperatures are assigned to some equipment of interests where hydrogen generation rates are calculated.

\section{Table E-1 Vessel Parameters}

\begin{tabular}{|c|c|c|c|c|c|c|c|c|c|}
\hline \begin{tabular}{|l|} 
Equipment Tag No. \\
\end{tabular} & Equipment name & $\begin{array}{r}\text { Overflow } \\
\text { Volume } \\
\text { (gal) }\end{array}$ & $\begin{array}{r}\text { Maximum } \\
\text { Volume } \\
\text { (gal) }\end{array}$ & $\begin{array}{r}\text { Set Volume } \\
\text { (gal) }\end{array}$ & $\begin{array}{r}\text { Minimum } \\
\text { Volume } \\
\text { (gal) }\end{array}$ & $\begin{array}{r}\text { Maximum } \\
\text { Flowrate } \\
\text { (gpm) }\end{array}$ & $\begin{array}{r}\text { Sample } \\
\text { Hold Time } \\
\text { (hours) }\end{array}$ & $\begin{array}{r}\text { Maximum } \\
\text { Temperature } \\
\left({ }^{\circ} \mathrm{F}\right)\end{array}$ & Descriptions \\
\hline CNP-EVAP-00001 & $\begin{array}{l}\text { Cesium Evaporator Separator } \\
\text { Vessel }\end{array}$ & $\mathrm{n} / \mathrm{a}$ & 2,130 & 2,128 & 130 & 20 & $\mathrm{n} / \mathrm{a}$ & 140 & Add 2,128 gallons of 5M nitric acid initially and after each discharge \\
\hline \begin{tabular}{|l|} 
CNP-VSL-00003 \\
\end{tabular} & $\begin{array}{l}\text { Eluate Contingency Storage } \\
\text { Vessel }\end{array}$ & 18,750 & 16,127 & 13,127 & 3,627 & 62 & 0.00 & 190 & Per design values \\
\hline \begin{tabular}{|l|} 
CNP-VSL-00004 \\
\end{tabular} & Recovered Nitric Acid Vessel & 10,064 & 7,342 & 5,342 & 1,342 & 20 & 0.00 & $\mathrm{n} / \mathrm{a}$ & Flow rate $=10 \mathrm{gpm}$ for IX elution, $20 \mathrm{gpm}$ for resin outlet flush \\
\hline \begin{tabular}{|l|} 
CRP-VSL-00001 \\
\end{tabular} & Cesium Resin Addition Vessel & 2,500 & 2,146 & $\mathrm{n} / \mathrm{a}$ & 0 & 60 & 0.00 & $\mathrm{n} / \mathrm{a}$ & Set volume is not used \\
\hline \begin{tabular}{|l|} 
CXP-VSL-00001 \\
\end{tabular} & Cesium IX Feed Vessel & 92,843 & 84,431 & $\mathrm{n} / \mathrm{a}$ & 5,931 & 30 & 0.00 & $\mathrm{n} / \mathrm{a}$ & Eliminated from design \\
\hline \begin{tabular}{|l|l|} 
CXP-VSL-00004 \\
\end{tabular} & $\begin{array}{l}\text { Cesium IX Caustic Rinse } \\
\text { Collection Vessel }\end{array}$ & $\mathrm{n} / \mathrm{a}$ & $\mathrm{n} / \mathrm{a}$ & $\mathrm{n} / \mathrm{a}$ & $\mathrm{n} / \mathrm{a}$ & $\mathrm{n} / \mathrm{a}$ & 0.00 & $\mathrm{n} / \mathrm{a}$ & Combined with UFP-VSL-00062A/B/C into a Supertank (see UFP-VSL-00062A for details) \\
\hline CXP-VSL-00005 & Reagent & 1,003 & 454 & 404 & 304 & 30 & $\mathrm{n} / \mathrm{a}$ & $\mathrm{n} / \mathrm{a}$ & Supply diluted caustic for IXC displacement operation \\
\hline CXP-VSL-00026A & Treated LAW Collection Vessel & 33,335 & 29,768 & 28,768 & 2,268 & 34 & 7.5 & $\mathrm{n} / \mathrm{a}$ & Flow rate $=34 \mathrm{gpm}$ for single input, $30 \mathrm{gpm}$ for dual pumping \\
\hline CXP-VSL-00026B & Treated LAW Collection Vessel & 33,335 & 29,768 & 28,768 & 2,268 & 34 & 7.5 & $\mathrm{n} / \mathrm{a}$ & Flow rate $=34 \mathrm{gpm}$ for single input, $30 \mathrm{gpm}$ for dual pumping \\
\hline CXP-VSL-00026C & Treated LAW Collection Vessel & 33,335 & 29,768 & 28,768 & 2,268 & 34 & 7.5 & $\mathrm{n} / \mathrm{a}$ & Flow rate $=34 \mathrm{gpm}$ for single input, $30 \mathrm{gpm}$ for dual pumping \\
\hline \begin{tabular}{|l|l|l} 
FEP-DMST-00001A \\
\end{tabular} & Waste Feed Evaporator Demister & $\mathrm{n} / \mathrm{a}$ & 1,300 & 850 & 50 & 80 & 0.00 & $\mathrm{n} / \mathrm{a}$ & Per design values \\
\hline FEP-DMST-00001B & Waste Feed Evaporator Demister & $\mathrm{n} / \mathrm{a}$ & 1,300 & 850 & 50 & 80 & 0.00 & $\mathrm{n} / \mathrm{a}$ & Reserved capability \\
\hline FEP-SEP-00001A & Waste Feed Evaporator & $\mathrm{n} / \mathrm{a}$ & 4,819 & 2,820 & 0 & $28 / 45$ & $\mathrm{n} / \mathrm{a}$ & $\mathrm{n} / \mathrm{a}$ & $\begin{array}{l}\text { Boiloff rate }=\text { maximum boiloff }(30) \text { - reflux }(2) \\
\text { Concentrate flow rate }=45 \text { gpm }\end{array}$ \\
\hline \begin{tabular}{|l|} 
FEP-SEP-00001B \\
\end{tabular} & Waste Feed Evaporator & $\mathrm{n} / \mathrm{a}$ & 4,819 & 2,820 & 0 & $28 / 45$ & $\mathrm{n} / \mathrm{a}$ & $\mathrm{n} / \mathrm{a}$ & Reserved capability \\
\hline FEP-VSL-00005A & $\begin{array}{l}\text { Waste Feed Evaporator } \\
\text { Condensate Vessel }\end{array}$ & 4,144 & 1,280 & 1,080 & 310 & 60 & 0.00 & $\mathrm{n} / \mathrm{a}$ & Per design values \\
\hline \begin{tabular}{|l|} 
FEP-VSL-00005B \\
\end{tabular} & $\begin{array}{l}\text { Waste Feed Evaporator } \\
\text { Condensate Vessel }\end{array}$ & 4,144 & 1,280 & 1,080 & 310 & 60 & 0.00 & $\mathrm{n} / \mathrm{a}$ & Reserved capability \\
\hline FEP-VSL-00017A & $\begin{array}{l}\text { Waste Feed Evaporator Feed } \\
\text { Vessel }\end{array}$ & 69,292 & 55,692 & 50,692 & 5,692 & 50 & 0.00 & 122 & $\begin{array}{l}\text { Actual flow rate varies to satisfy boiloff rate, evaporator concentrate } \mathrm{SpG} \text { and wt\% solids } \\
\text { requirements }\end{array}$ \\
\hline \begin{tabular}{|l|} 
FEP-VSL-00017B \\
\end{tabular} & $\begin{array}{l}\text { Waste Feed Evaporator Feed } \\
\text { Vessel }\end{array}$ & 69,292 & 55,692 & 50,692 & 5,692 & 50 & 0.00 & 122 & $\begin{array}{l}\text { Actual flow rate varies to satisfy boiloff rate, evaporator concentrate } \mathrm{SpG} \text { and wt\% solids } \\
\text { requirements }\end{array}$ \\
\hline FRP-VSL-00002A & Waste Feed Receipt Vessel & 403,592 & 379,391 & 309,391 & 4,391 & 125 & 14.20 & 120 & Per design values \\
\hline \begin{tabular}{|l|} 
FRP-VSL-00002B \\
\end{tabular} & Waste Feed Receipt Vessel & 403,592 & 379,391 & 369,391 & 4,391 & 125 & 14.20 & 120 & Per design values \\
\hline \begin{tabular}{|l|} 
FRP-VSL-00002C \\
\end{tabular} & Waste Feed Receipt Vessel & 403,592 & 379,391 & 369,391 & 4,391 & 125 & 14.20 & 120 & Per design values \\
\hline \begin{tabular}{|l|} 
FRP-VSL-00002D \\
\end{tabular} & Waste Feed Receipt Vessel & 403,592 & 379,391 & 369,391 & 4,391 & 125 & 14.20 & 120 & Per design values \\
\hline GFR-TK-00022 & $\begin{array}{l}\text { LAW Glass Former Feed } \\
\text { Hopper }\end{array}$ & $\mathrm{n} / \mathrm{a}$ & 3,516 & & 3,516 & 49 & 0.0 & $\mathrm{n} / \mathrm{a}$ & Per design values \\
\hline \begin{tabular}{|l} 
GFR-TK-00023 \\
\end{tabular} & $\begin{array}{l}\text { LAW Glass Former Feed } \\
\text { Hopper }\end{array}$ & $\mathrm{n} / \mathrm{a}$ & 3,516 & & 3,516 & 49 & 0.0 & $\mathrm{n} / \mathrm{a}$ & Per design values \\
\hline
\end{tabular}




\begin{tabular}{|c|c|c|c|c|c|c|c|c|c|}
\hline Equipment Tag No. & Equipment name & $\begin{array}{r}\text { Overflow } \\
\text { Volume } \\
\text { (gal) }\end{array}$ & $\begin{array}{r}\text { Maximum } \\
\text { Volume } \\
\text { (gal) }\end{array}$ & $\begin{array}{r}\text { Set Volume } \\
\text { (gal) }\end{array}$ & $\begin{array}{r}\text { Minimum } \\
\text { Volume } \\
\text { (gal) }\end{array}$ & $\begin{array}{r}\text { Maximum } \\
\text { Flowrate } \\
(\text { gpm) }\end{array}$ & $\begin{array}{r}\text { Sample } \\
\text { Hold Time } \\
\text { (hours) }\end{array}$ & $\begin{array}{r}\text { Maximum } \\
\text { Temperature } \\
\left({ }^{\circ} \mathrm{F}\right) \\
\end{array}$ & Descriptions \\
\hline GFR-TK-00025 & $\begin{array}{l}\text { HLW Glass Former Feed } \\
\text { Hopper }\end{array}$ & $\mathrm{n} / \mathrm{a}$ & 2,394 & & 2,394 & 33.3 & 0.0 & $\mathrm{n} / \mathrm{a}$ & Per design values \\
\hline GFR-TK-00031 & $\begin{array}{l}\text { HLW Glass Former Feed } \\
\text { Hopper }\end{array}$ & $\mathrm{n} / \mathrm{a}$ & 2,394 & & 2,394 & 33.3 & 0.0 & $\mathrm{n} / \mathrm{a}$ & Per design values \\
\hline HDH-VSL-00001 & $\begin{array}{l}\text { Canister Rinse Bogie Decon } \\
\text { Vessel }\end{array}$ & 360 & 360 & 360 & 0 & 80 & 0.00 & $\mathrm{n} / \mathrm{a}$ & Per design values \\
\hline HDH-VSL-00002 & $\begin{array}{l}\text { Canister Decontamination Vessel } \\
\end{array}$ & 210 & 215 & 215 & 0 & 48 & 0.00 & $\mathrm{n} / \mathrm{a}$ & Decon vessel discharge logic is modified to work with the updated volume. \\
\hline HDH-VSL-00003 & Waste Neutralization Vessel & 4,142 & 3,647 & 3,647 & 675 & 58 & 0.00 & $\mathrm{n} / \mathrm{a}$ & Per design values \\
\hline HDH-VSL-00004 & Canister Decontamination Vessel & 213 & 215 & 215 & 0 & 48 & 0.00 & $\mathrm{n} / \mathrm{a}$ & Decon vessel discharge logic is modified to work with the updated volume. \\
\hline HFP-VSL-00001 & $\begin{array}{l}\text { HLW Melter Feed Preparation } \\
\text { Vessel }\end{array}$ & 7,650 & 5,661 & Variable & 1,356 & 44 & $12.79,9.59$ & 140 & Set volume is dynamically calculated \\
\hline HFP-VSL-00002 & HLW Melter Feed Vessel & 7,646 & 7,208 & 1,563 & 1,543 & Variable & 0.00 & 140 & $\begin{array}{l}\text { Lower set volume triggers pumping from MFPV while continue emptying, flow rate is } \\
\text { dynamically calculated to meet IHLW glass production rate }\end{array}$ \\
\hline HFP-VSL-00005 & $\begin{array}{l}\text { HLW Melter Feed Preparation } \\
\text { Vessel }\end{array}$ & 7,650 & 5,661 & Variable & 1,356 & 44 & $12.79,9.59$ & 140 & Set volume is dynamically calculated \\
\hline HFP-VSL-00006 & HLW Melter Feed Vessel & 7,646 & 7,208 & 1,563 & 1,543 & Variable & 0.00 & 140 & $\begin{array}{l}\text { Lower set volume triggers pumping from MFPV while continue emptying, flow rate is } \\
\text { dynamically calculated to meet IHLW glass production rate }\end{array}$ \\
\hline HLP-VSL-00022 & HLW Feed Receipt Vessel & 231,161 & 174,486 & 170,000 & 29,486 & 155 & 16.4 & 150 & Per design values \\
\hline HLP-VSL-00027A & HLW Lag Storage Vessel & 111,376 & 92,600 & 61,800 & 10,600 & 90 & 0.00 & 138 & Per design values \\
\hline HLP-VSL-00027B & HLW Lag Storage Vessel & 111,376 & 92,600 & 61,800 & 10,600 & 90 & 0.00 & 138 & Secondary choice for receiving CNP evaporator concentrate \\
\hline HLP-VSL-00028 & HLW Feed Blend Vessel & 122,233 & 103,000 & 100,000 & 27,000 & 56 & 12.45 & 141 & Primary choice for receiving CNP evaporator concentrate \\
\hline IHLW1 & IHLW Canister & $\mathrm{n} / \mathrm{a}$ & 300 & $\mathrm{n} / \mathrm{a}$ & 0 & $\mathrm{n} / \mathrm{a}$ & $\mathrm{n} / \mathrm{a}$ & $\mathrm{n} / \mathrm{a}$ & Used to track HLW canister count and composition \\
\hline IHLW2 & IHLW Canister & $\mathrm{n} / \mathrm{a}$ & 300 & $\mathrm{n} / \mathrm{a}$ & 0 & $\mathrm{n} / \mathrm{a}$ & $\mathrm{n} / \mathrm{a}$ & $\mathrm{n} / \mathrm{a}$ & Used to track HLW canister count and composition \\
\hline HMP-MLTR-00001 & HLW Melter 1 & 1,217 & 1,092 & 1,067 & 1,067 & 250 & $\mathrm{n} / \mathrm{a}$ & $\mathrm{n} / \mathrm{a}$ & Flow rate is set high enough to ensure instant glass pouring \\
\hline HMP-MLTR-00002 & HLW Melter 2 & 1,217 & 1,092 & 1,067 & 1,067 & 250 & $\mathrm{n} / \mathrm{a}$ & $\mathrm{n} / \mathrm{a}$ & Flow rate is set high enough to ensure instant glass pouring \\
\hline $\begin{array}{l}\text { HOP-SCB-00001 } \\
\text { (combined) }\end{array}$ & HLW Submerged Bed Scrubber & 11,227 & 9,137 & 8,137 & 1,758 & 80 & $\mathrm{n} / \mathrm{a}$ & 140 & $\begin{array}{l}\text { Column is modeled to also assume the function of HOP-VSL-00903. Maximum accumulation } \\
\text { time }=24 \text { hours }\end{array}$ \\
\hline $\begin{array}{l}\text { HOP-SCB-00002 } \\
\text { (combined) }\end{array}$ & HLW Submerged Bed Scrubber & 11,227 & 9,137 & 8,137 & 1,758 & 80 & $\mathrm{n} / \mathrm{a}$ & 140 & $\begin{array}{l}\text { Column is modeled to also assume the function of HOP-VSL-00904. Maximum accumulation } \\
\text { time }=24 \text { hours }\end{array}$ \\
\hline HOP-VSL-00903 & $\begin{array}{l}\text { HLW SBS Condensate Receiver } \\
\text { Vessel }\end{array}$ & 7,898 & 5,808 & $\mathrm{n} / \mathrm{a}$ & 1,758 & 80 & $\mathrm{n} / \mathrm{a}$ & $\mathrm{n} / \mathrm{a}$ & Combined with HOP-SCB-00001 \\
\hline HOP-VSL-00904 & $\begin{array}{l}\text { HLW SBS Condensate Receiver } \\
\text { Vessel }\end{array}$ & 7,898 & 5,808 & $\mathrm{n} / \mathrm{a}$ & 1,758 & 80 & $\mathrm{n} / \mathrm{a}$ & $\mathrm{n} / \mathrm{a}$ & Combined with HOP-SCB-00002 \\
\hline ILAW1 & ILAW Container & $\mathrm{n} / \mathrm{a}$ & 564 & $\mathrm{n} / \mathrm{a}$ & 0 & $\mathrm{n} / \mathrm{a}$ & $\mathrm{n} / \mathrm{a}$ & $\mathrm{n} / \mathrm{a}$ & Used to track LAW container count and composition \\
\hline ILAW2 & ILAW Container & $\mathrm{n} / \mathrm{a}$ & 564 & $\mathrm{n} / \mathrm{a}$ & 0 & $\mathrm{n} / \mathrm{a}$ & $\mathrm{n} / \mathrm{a}$ & $\mathrm{n} / \mathrm{a}$ & Used to track LAW container count and composition \\
\hline $\begin{array}{ll}\text { LCP-VSL-00001 } \\
\end{array}$ & $\begin{array}{l}\text { LAW Concentrate Receipt } \\
\text { Vessel }\end{array}$ & 14,877 & 12,855 & 12,855 & 3,740 & 88 & 14.5 & $\mathrm{n} / \mathrm{a}$ & $\begin{array}{l}\text { Set volume is lower set point and is dynamically calculated to ensure that partial batch transfer to } \\
\text { MFPV will not occur }\end{array}$ \\
\hline $\begin{array}{ll}\text { LCP-VSL-00002 } \\
\end{array}$ & $\begin{array}{l}\text { LAW Concentrate Receipt } \\
\text { Vessel }\end{array}$ & 14,877 & 12,855 & 12,855 & 3,740 & 88 & 14.5 & $\mathrm{n} / \mathrm{a}$ & $\begin{array}{l}\text { Set volume is lower set point and is dynamically calculated to ensure that partial batch transfer to } \\
\text { MFPV will not occur }\end{array}$ \\
\hline
\end{tabular}




\begin{tabular}{|c|c|c|c|c|c|c|c|c|c|}
\hline Equipment Tag No. & Equipment name & $\begin{array}{r}\text { Overflow } \\
\text { Volume } \\
\text { (gal) }\end{array}$ & $\begin{array}{r}\text { Maximum } \\
\text { Volume } \\
\text { (gal) }\end{array}$ & $\begin{array}{r}\text { Set Volume } \\
\text { (gal) }\end{array}$ & $\begin{array}{r}\text { Minimum } \\
\text { Volume } \\
\text { (gal) }\end{array}$ & $\begin{array}{r}\text { Maximum } \\
\text { Flowrate } \\
(\mathrm{gpm})\end{array}$ & $\begin{array}{r}\text { Sample } \\
\text { Hold Time } \\
\text { (hours) }\end{array}$ & \begin{tabular}{|r} 
Maximum \\
Temperature \\
$\left({ }^{\circ} \mathrm{F}\right)$
\end{tabular} & Descriptions \\
\hline LFP-VSL-00001 & $\begin{array}{l}\text { LAW Melter Feed Preparation } \\
\text { Vessel }\end{array}$ & 7,489 & 5,132 & Variable & 1,732 & 50 & 0.75 & 140 & Set point is dynamically calculated \\
\hline LFP-VSL-00002 & LAW Melter Feed Vessel & 5,786 & 4,377 & 1,000 & 977 & Variable & 0.00 & 140 & $\begin{array}{l}\text { Set volume is lower set point, flow rate is dynamically calculated to meet ILAW glass production } \\
\text { rate }\end{array}$ \\
\hline LFP-VSL-00003 & $\begin{array}{l}\text { LAW Melter Feed Preparation } \\
\text { Vessel }\end{array}$ & 7,489 & 5,132 & Variable & 1,732 & 50 & 0.75 & 140 & Set point is dynamically calculated \\
\hline LFP-VSL-00004 & LAW Melter Feed Vessel & 5,786 & 4,377 & 1,000 & 977 & Variable & 0.00 & 140 & $\begin{array}{l}\text { Set volume is lower set point, flow rate is dynamically calculated to meet ILAW glass production } \\
\text { rate }\end{array}$ \\
\hline LMP-MLTR-00001 & LAW Melter 1 & 2,318 & 2,016 & 1,875 & 1,875 & 250 & $\mathrm{n} / \mathrm{a}$ & $\mathrm{n} / \mathrm{a}$ & Flow rate is set high enough to ensure instant glass pouring \\
\hline LMP-MLTR-00002 & LAW Melter 2 & 2,318 & 2,016 & 1,875 & 1,875 & 250 & $\mathrm{n} / \mathrm{a}$ & $\mathrm{n} / \mathrm{a}$ & Flow rate is set high enough to ensure instant glass pouring \\
\hline $\begin{array}{l}\text { LOP-SCB-00001 } \\
\text { (combined volumes) }\end{array}$ & LAW Submerged Bed Scrubber & 10,135 & 9,980 & 8,980 & 451 & 80 & $\mathrm{n} / \mathrm{a}$ & $\mathrm{n} / \mathrm{a}$ & $\begin{array}{l}\text { Column is modeled to also assume the function of LOP-VSL-00001. Maximum accumulation } \\
\text { time }=48 \text { hours }\end{array}$ \\
\hline $\begin{array}{l}\text { LOP-SCB-00002 } \\
\text { (combined volumes) }\end{array}$ & LAW Submerged Bed Scrubber & 10,135 & 9,980 & 8,980 & 451 & 80 & $\mathrm{n} / \mathrm{a}$ & $\mathrm{n} / \mathrm{a}$ & $\begin{array}{l}\text { Column is modeled to also assume the function of LOP-VSL-00002. Maximum accumulation } \\
\text { time }=48 \text { hours }\end{array}$ \\
\hline LOP-VSL-00001 & LAW SBS Condensate Vessel & 6,806 & 6,651 & $\mathrm{n} / \mathrm{a}$ & 451 & 80 & $\mathrm{n} / \mathrm{a}$ & $\mathrm{n} / \mathrm{a}$ & Combined with LOP-SCB-00001 \\
\hline LOP-VSL-00002 & LAW SBS Condensate Vessel & 6,806 & 6,651 & $\mathrm{n} / \mathrm{a}$ & 451 & 80 & $\mathrm{n} / \mathrm{a}$ & $\mathrm{n} / \mathrm{a}$ & Combined with LOP-SCB-00002 \\
\hline LVP-TK-00001 & Caustic Collection Vessel & 7,325 & 6,032 & 6,032 & 2,032 & 200 & 0.00 & $\mathrm{n} / \mathrm{a}$ & Per design values \\
\hline PVP-SCB-00002 & Vessel Vent Caustic Scrubber & 3,564 & 1,960 & 1,960 & 0 & 120 & $\mathrm{n} / \mathrm{a}$ & $\mathrm{n} / \mathrm{a}$ & SBS operates at constant level at 3,300 gallons \\
\hline PVP-VSL-00001 & $\begin{array}{l}\text { Vessel Vent HEME Drain } \\
\text { Collection Vessel }\end{array}$ & 1,650 & 1,547 & 1,047 & 747 & 50 & 0.00 & $\mathrm{n} / \mathrm{a}$ & Per design values \\
\hline PWD-VSL-00015 & Acidic/Alkaline Effluent Vessel & 105,864 & 87,148 & 82,148 & 12,148 & 88 & 0.00 & $\mathrm{n} / \mathrm{a}$ & 10,000 gallon headspace is reserved for $\mathrm{pH}$ adjustment \\
\hline PWD-VSL-00016 & Acidic/Alkaline Effluent Vessel & 105,864 & 87,148 & 82,148 & 12,148 & 88 & 0.00 & $\mathrm{n} / \mathrm{a}$ & 10,000 gallon headspace is reserved for $\mathrm{pH}$ adjustment \\
\hline PWD-VSL-00033 & Ultimate Overflow Vessel & 31,476 & 20,386 & 10,386 & 5,386 & 88 & 0.00 & 141 & Dual set points are implemented to allow contingency storage \\
\hline PWD-VSL-00043 & \begin{tabular}{|l|} 
HLW Effluent Transfer Vessel \\
\end{tabular} & 26,504 & 20,301 & 10,301 & 5,301 & 88 & 0.00 & 141 & Dual set points are implemented to allow contingency storage \\
\hline PWD-VSL-00044 & Plant Wash Vessel & 88,637 & 73,644 & 68,644 & 13,644 & 88 & 0.00 & $\mathrm{n} / \mathrm{a}$ & 10,000 gallon headspace is reserved for $\mathrm{pH}$ adjustment \\
\hline RDP-VSL-00002A & Spent Resin Slurry Vessel & 12,688 & 7,525 & 7,508 & 8 & 140 & 0.00 & 123 & None \\
\hline RDP-VSL-00002B & Spent Resin Slurry Vessel & 12,688 & 7,525 & 7,085 & 8 & 140 & 0.25 & 123 & None \\
\hline RDP-VSL-00002C & Spent Resin Slurry Vessel & 12,688 & 7,525 & $\mathrm{n} / \mathrm{a}$ & 8 & 140 & 0.00 & 123 & None \\
\hline RLD-TK-00006A & Process Condensate Vessel & 313,500 & 292,500 & 200,000 & 27,500 & 1,000 & 0.00 & $\mathrm{n} / \mathrm{a}$ & Per design values \\
\hline RLD-TK-00006B & Process Condensate Vessel & 313,500 & 292,500 & 200,000 & 27,500 & 170 & 0.00 & $\mathrm{n} / \mathrm{a}$ & Per design values \\
\hline RLD-VSL-00003 & Plant Wash Vessel & 22,650 & 19,164 & $\mathrm{n} / \mathrm{a}$ & 3,164 & 130 & 0.00 & $\mathrm{n} / \mathrm{a}$ & Contingency vessel, not used in model \\
\hline RLD-VSL-00004 & $\begin{array}{l}\text { C3/C5 Drains/Sump Collection } \\
\text { Vessel }\end{array}$ & 6,291 & 5,887 & 5,387 & 1,387 & 75 & 0.00 & $\mathrm{n} / \mathrm{a}$ & Per design values \\
\hline RLD-VSL-00005 & $\begin{array}{l}\text { SBS Condensate Collection } \\
\text { Vessel }\end{array}$ & 23,400 & 20,300 & 17,300 & 4,300 & 130 & 0.00 & 166 & Per design values \\
\hline RLD-VSL-00007 & Acidic Waste Storage Vessel & 14,612 & 11,662 & $8,662.0$ & 1,794 & 48 & 7.8 & $\mathrm{n} / \mathrm{a}$ & Per design values \\
\hline RLD-VSL-00008 & Plant Wash and Drains Vessel & 10,624 & 8,281 & $\mathrm{n} / \mathrm{a}$ & 1,416 & 48 & 7.8 & $\mathrm{n} / \mathrm{a}$ & Contingency vessel, not used in model \\
\hline RLD-VSL-00017A & Alkaline Effluent Vessel & 23,742 & 21,870 & 21,870 & 1,870 & 130 & 10.30 & $\mathrm{n} / \mathrm{a}$ & Per design values \\
\hline RLD-VSL-00017B & \begin{tabular}{|l|} 
Alkaline Effluent Vessel \\
\end{tabular} & 23,742 & 21,870 & 21,870 & 1,870 & 130 & 10.30 & $\mathrm{n} / \mathrm{a}$ & Per design values \\
\hline TCP-VSL-00001 & \begin{tabular}{|l} 
Treated LAW Concentrate \\
Storage Vessel
\end{tabular} & 128,845 & 102,621 & 75,000 & 9,621 & 88 & 0.00 & $\mathrm{n} / \mathrm{a}$ & Per design values \\
\hline
\end{tabular}




\begin{tabular}{|c|c|c|c|c|c|c|c|c|c|}
\hline Equipment Tag No. & Equipment name & $\begin{array}{r}\text { Overflow } \\
\text { Volume } \\
\text { (gal) }\end{array}$ & $\begin{array}{r}\text { Maximum } \\
\text { Volume } \\
\text { (gal) }\end{array}$ & \begin{tabular}{r|r} 
Set Volume \\
(gal)
\end{tabular} & $\begin{array}{r}\text { Minimum } \\
\text { Volume } \\
\text { (gal) }\end{array}$ & $\begin{array}{r}\text { Maximum } \\
\text { Flowrate } \\
\text { (gpm) }\end{array}$ & $\begin{array}{r}\text { Sample } \\
\text { Hold Time } \\
\text { (hours) }\end{array}$ & $\begin{array}{r}\text { Maximum } \\
\text { Temperature } \\
\left({ }^{\circ} \mathrm{F}\right)\end{array}$ & Descriptions \\
\hline TLP-DMST-00001 & $\begin{array}{l}\text { Treated LAW Evaporator } \\
\text { Demister }\end{array}$ & $\mathrm{n} / \mathrm{a}$ & 1,300 & 350 & 50 & 66 & 0.00 & $\mathrm{n} / \mathrm{a}$ & Per design values \\
\hline TLP-SEP-00001 & Treated LAW Evaporator & $\mathrm{n} / \mathrm{a}$ & 3,820 & 2,820 & 0 & $28 / 34$ & $\mathrm{n} / \mathrm{a}$ & $\mathrm{n} / \mathrm{a}$ & $\begin{array}{l}\text { Boiloff rate }=\text { maximum boiloff }(30) \text { - reflux (2) } \\
\text { Concentrate flow rate }=34 \mathrm{gpm}\end{array}$ \\
\hline TLP-VSL-00002 & $\begin{array}{l}\text { Treated LAW Evaporator } \\
\text { Condensate Vessel }\end{array}$ & 1,705 & 495 & 385 & 131 & 34 & 0.00 & $\mathrm{n} / \mathrm{a}$ & Per design values \\
\hline TLP-VSL-00009A & $\begin{array}{l}\text { LAW SBS Condensate Receipt } \\
\text { Vessel }\end{array}$ & 114,064 & 87,450 & 77,450 & 7,450 & 10 & 0.00 & $\mathrm{n} / \mathrm{a}$ & Discharge at fixed flow rate $(\mathrm{max}=40 \mathrm{gpm})$ \\
\hline TLP-VSL-00009B & \begin{tabular}{|l|} 
LAW SBS Condensate Receipt \\
Vessel
\end{tabular} & 114,064 & 87,450 & 77,450 & 7,450 & 10 & 0.00 & $\mathrm{n} / \mathrm{a}$ & Discharge at fixed flow rate $(\max =40 \mathrm{gpm})$ \\
\hline UFP-VSL-00001A & $\begin{array}{l}\text { Ultrafiltration Feed Preparation } \\
\text { Vessel }\end{array}$ & 64,373 & 52,704 & 28,300 & 7,704 & 140 & 0.00 & 194 & $\begin{array}{l}\text { UFP2 leaching case: set volume }=18,300 \mathrm{gal} \\
\text { UFP1 leaching case: set volume }=28,300 \mathrm{gal}\end{array}$ \\
\hline UFP-VSL-00001B & $\begin{array}{l}\text { Ultrafiltration Feed Preparation } \\
\text { Vessel }\end{array}$ & 64,373 & 52,704 & 28,300 & 7,704 & 140 & 0.00 & 194 & $\begin{array}{l}\text { UFP2 leaching case: set volume }=18,300 \mathrm{gal} \\
\text { UFP1 leaching case: set volume }=28,300 \mathrm{gal}\end{array}$ \\
\hline UFP-VSL-00002A & Ultrafiltration Feed Vessel & 33,767 & 29,900 & $\begin{array}{l}16,000 \\
19,000\end{array}$ & 500 & Variable/55/94 & 12.00 & 194 & $\begin{array}{l}\text { UFP2 leaching case: set volume }=16,000 \text { gal } \\
\text { UFP1 leaching case: set volume }=19,000 \text { gal } \\
\text { Variable flow rate for solid concentration and wash }(\max =110 \mathrm{gpm}) \\
55 \text { gpm for Non-Newtonian fluids } \\
94 \text { gpm for Newtonian fluids }\end{array}$ \\
\hline UFP-VSL-00002B & Ultrafiltration Feed Vessel & 33,767 & 29,900 & $\begin{array}{r}26,000 / 16,000 \\
19,000\end{array}$ & 500 & Variable/55/94 & 12.00 & 194 & $\begin{array}{l}\text { UP2 leaching case: set volume }=26,000 \text { gal for initial solids concentration (see Section 4.8.3) } \\
\text { UPP2 leaching case: set volume }=16,600 \text { gal for after feed split (see Section 4.8.3) } \\
\text { UFP1 leaching case: set volume }=19,000 \text { gal } \\
\text { Variable flow rate for solid concentration and wash ( } \max =110 \mathrm{gpm}) \\
55 \text { gpm for Non-Newtonian fluids } \\
94 \text { gpm for Newtonian fluids }\end{array}$ \\
\hline UFP-VSL-00062A & $\begin{array}{l}\text { Ultrafiltration Permeate } \\
\text { Collection Vessel }\end{array}$ & 99,081 & 78,775 & 11,800 & 11,755 & $70 / 30$ & 0.00 & 133 & $\begin{array}{l}\text { Represents the combined UFP-VSL-00062A/B/C and CXP-VSL-00004. Net discharge flowrate } \\
=30 \mathrm{gpm} \text {. }\end{array}$ \\
\hline UFP-VSL-00062B & $\begin{array}{l}\text { Ultrafiltration Permeate } \\
\text { Collection Vessel } \\
\end{array}$ & $\mathrm{n} / \mathrm{a}$ & n/a & $\mathrm{n} / \mathrm{a}$ & $\mathrm{n} / \mathrm{a}$ & n/a & 0.00 & $\mathrm{n} / \mathrm{a}$ & Combined with UFP-VSL-00062A/B/C into a Supertank (see UFP-VSL-00062A for details) \\
\hline UFP-VSL-00062C & \begin{tabular}{|l|} 
Ultrafiltration Permeate \\
Collection Vessel
\end{tabular} & $\mathrm{n} / \mathrm{a}$ & n/a & $\mathrm{n} / \mathrm{a}$ & $\mathrm{n} / \mathrm{a}$ & $\mathrm{n} / \mathrm{a}$ & 0.00 & $\mathrm{n} / \mathrm{a}$ & Combined with UFP-VSL-00062A/B/C into a Supertank (see UFP-VSL-00062A for details) \\
\hline
\end{tabular}


24590-WTP-MDD-PR-01-002, Rev 12

Dynamic (G2) Model Design Document

\section{Appendix F}

\section{Workspace Screenshots}




\section{Appendix F \\ Workspace Screenshots}

The WTP process systems are organized graphically in workspaces within which unit operations, chemical reagent sources, and their connections are shown. Also shown on the workspaces are the names of the unit operations and the directions of material flow for the process streams. 
Figure F-1 Waste Treatment Plant Overview

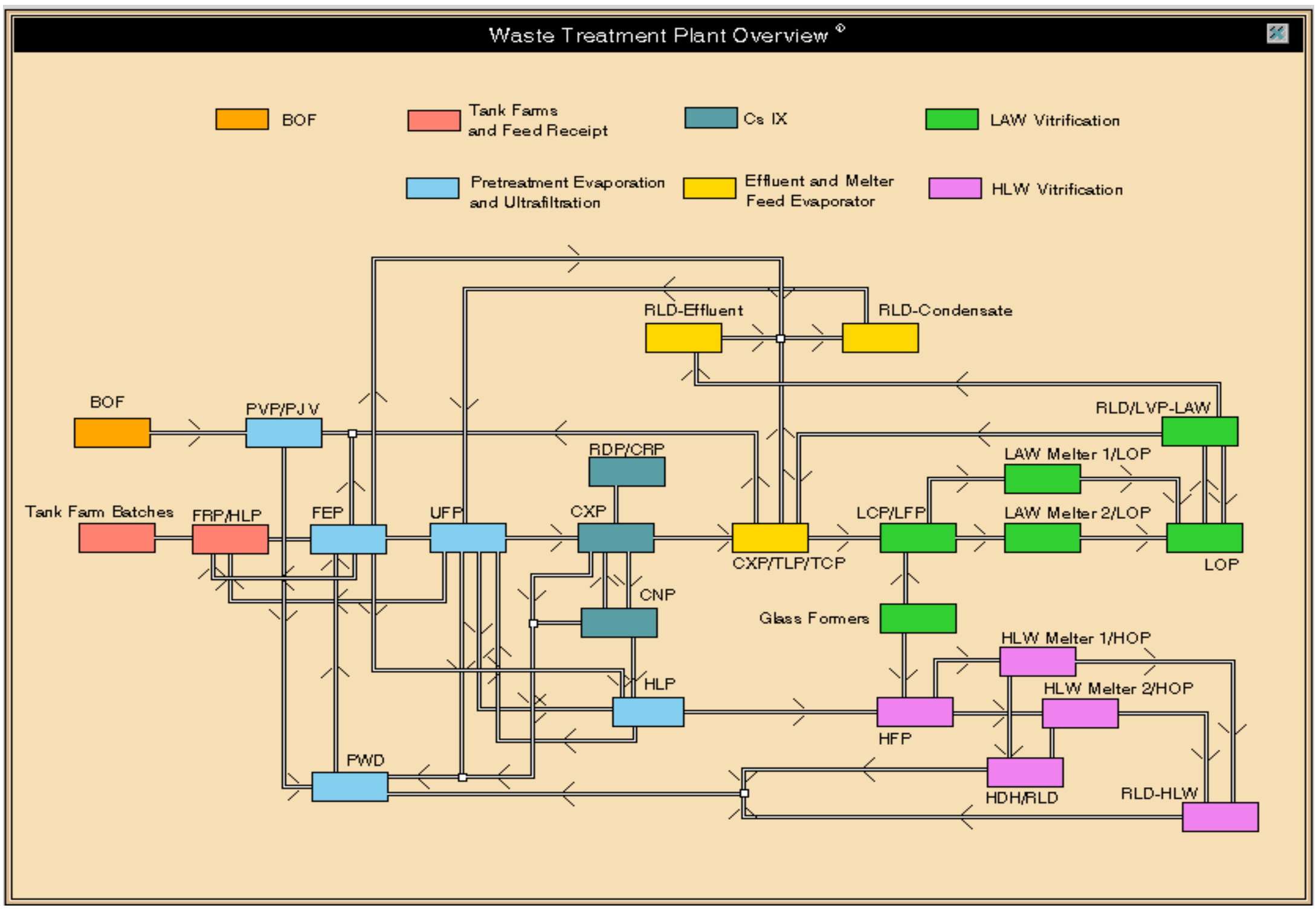


Figure F-2 Tank Farm Wastes and Water Sources

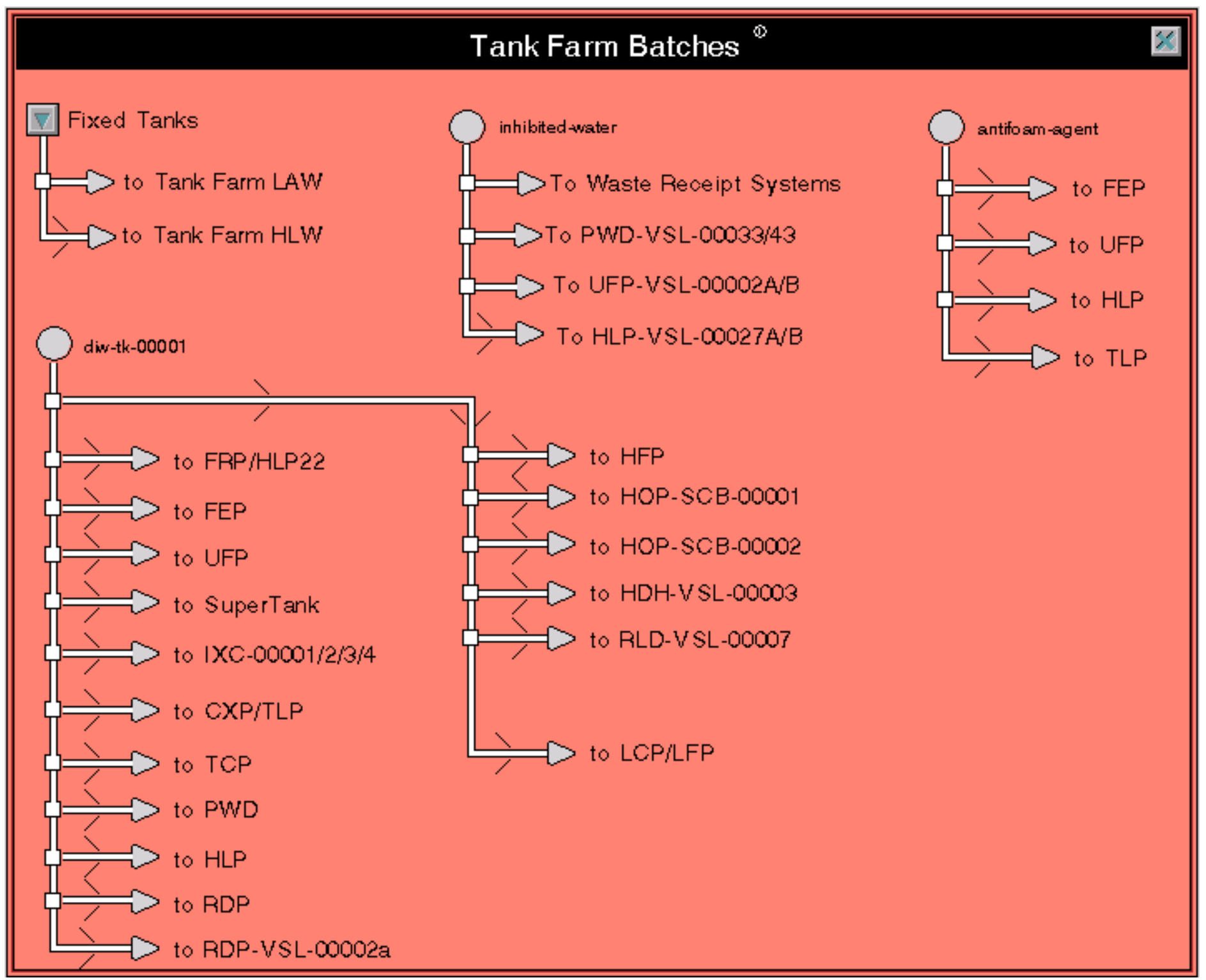




\section{Figure F-3 Waste Feed Receipt Process}

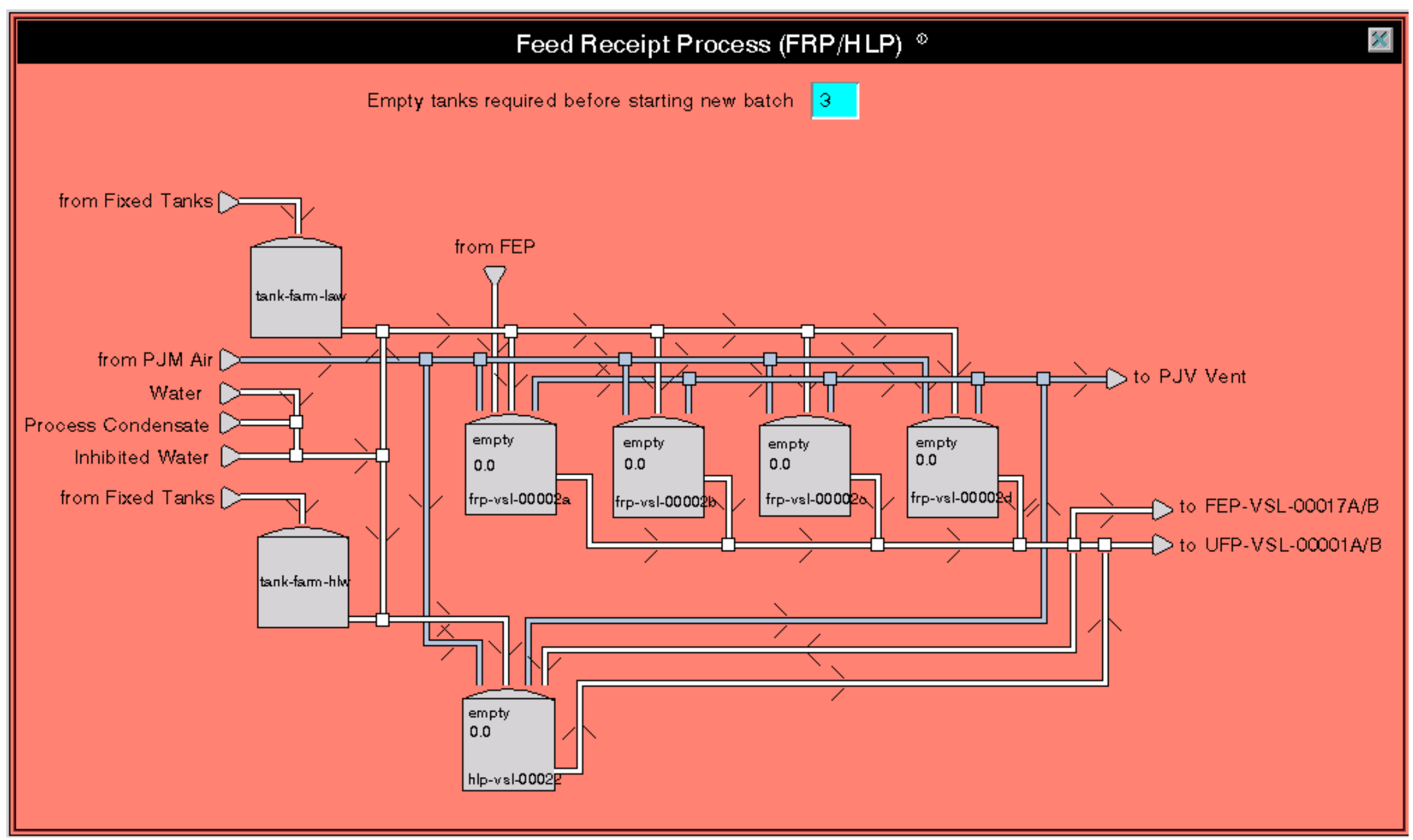




\section{Figure F-4 Waste Feed Evaporation Process}

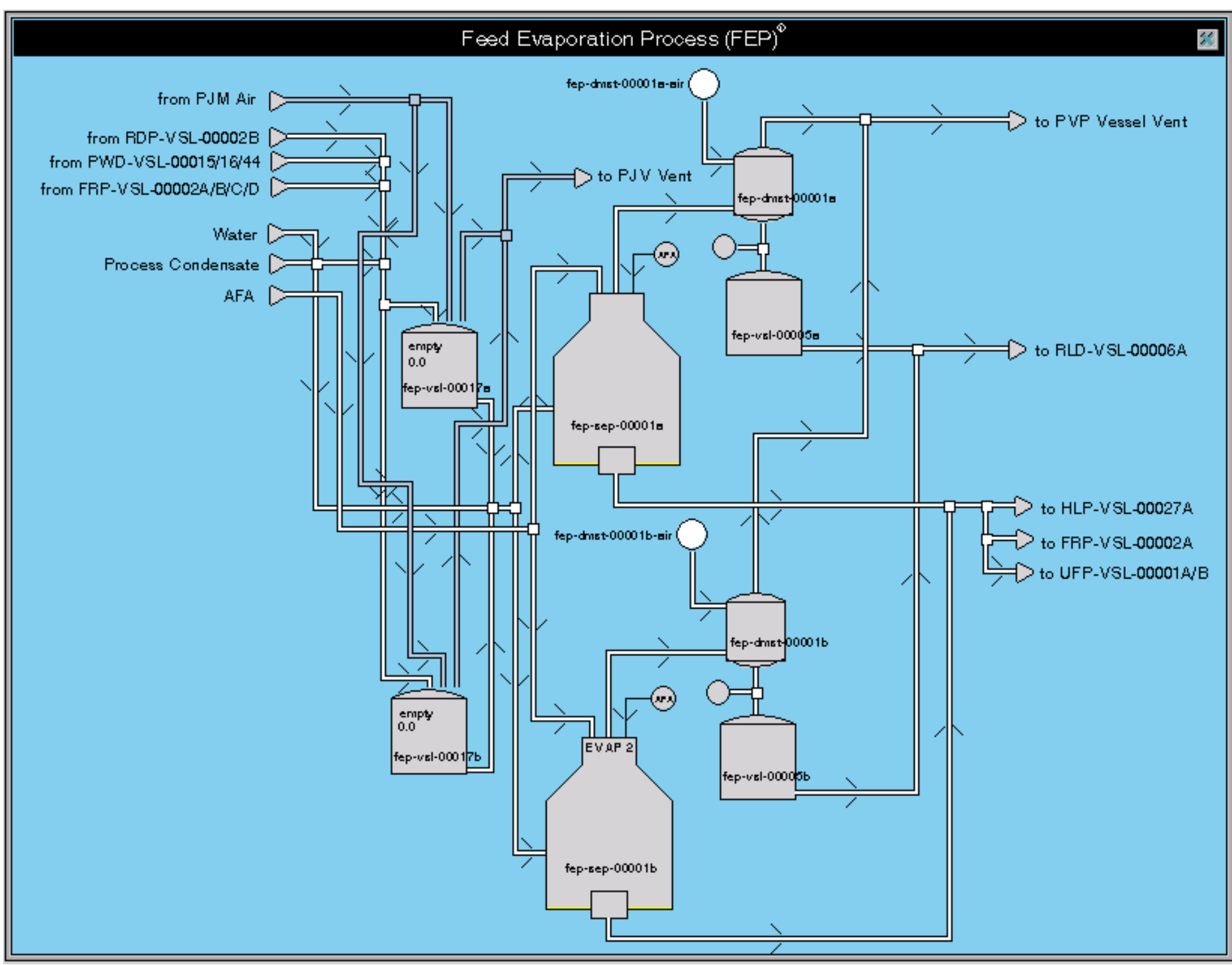




\section{Figure F-5 Ultrafiltration Process}

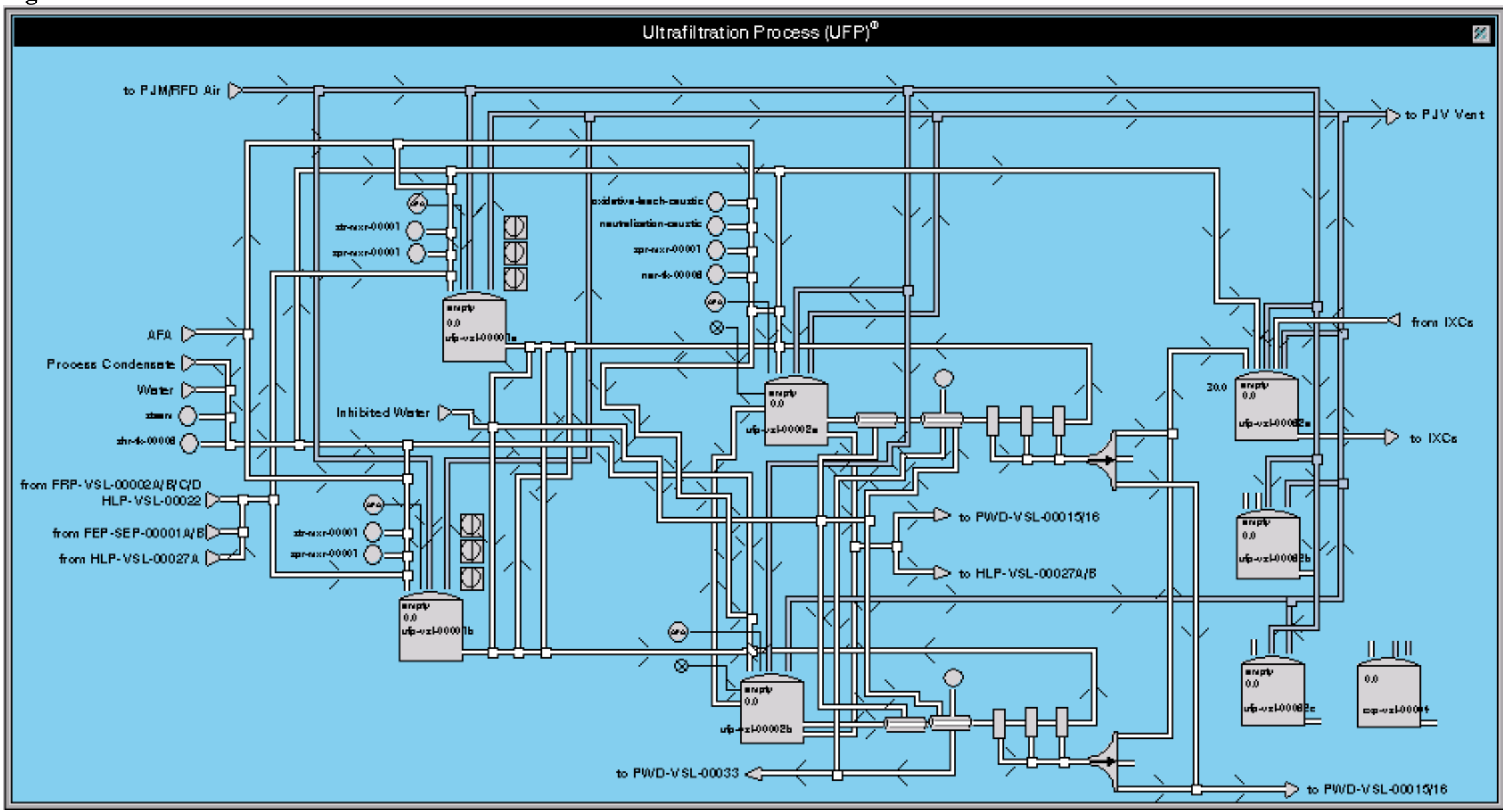




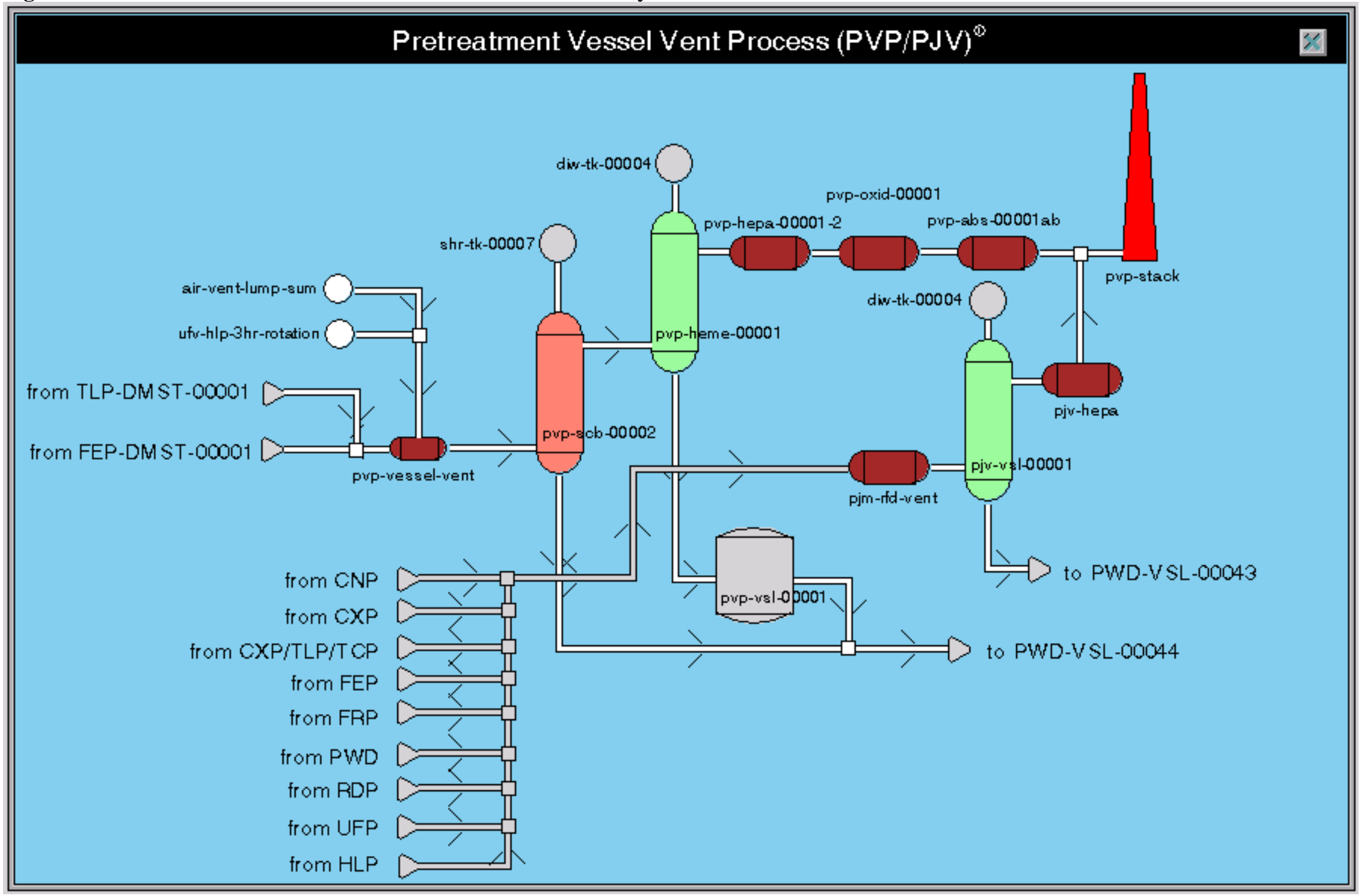




\section{Figure F-7 Plant Wash and Disposal System}

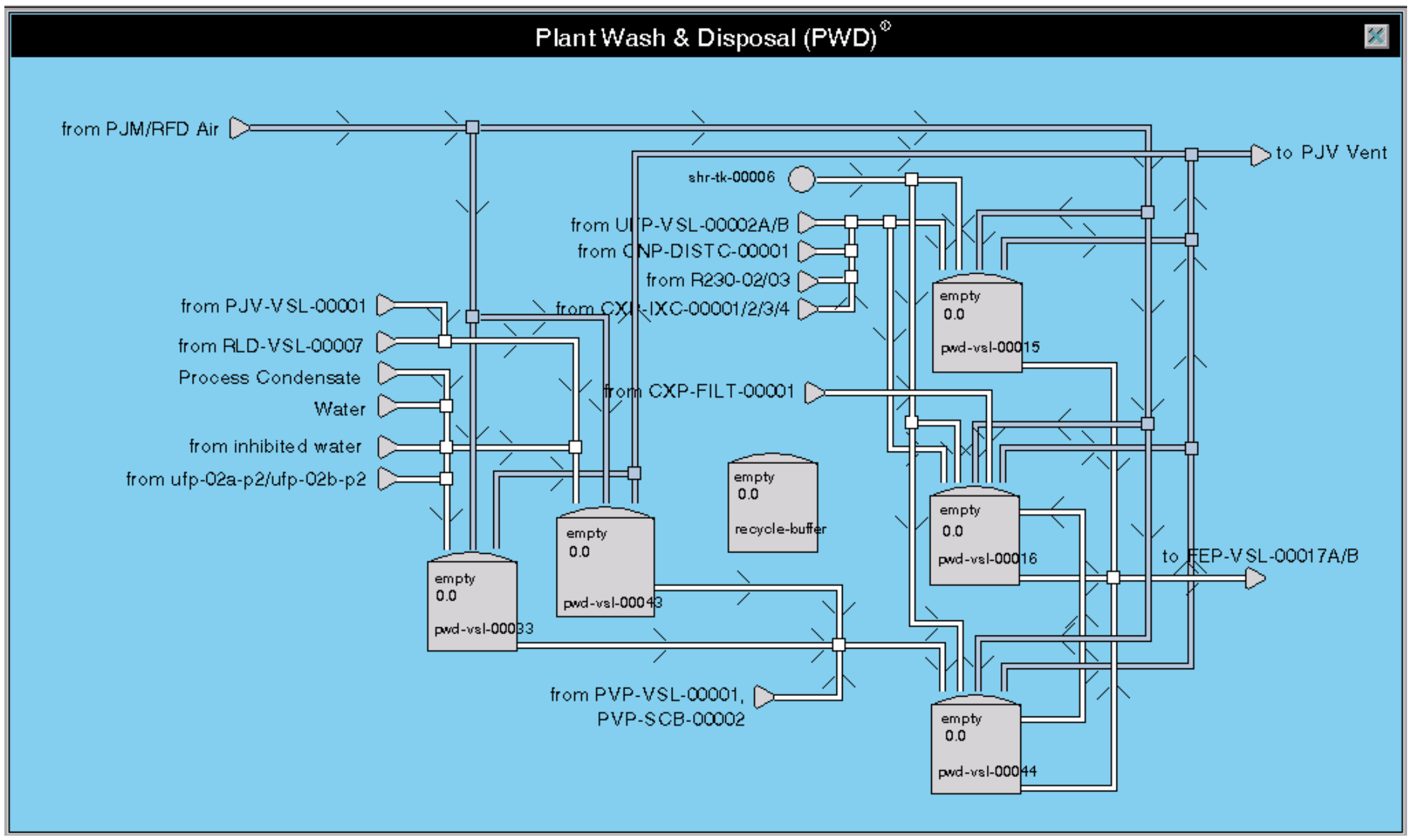




\section{Figure F-8 Cesium Removal Using Ion Exchange Process}

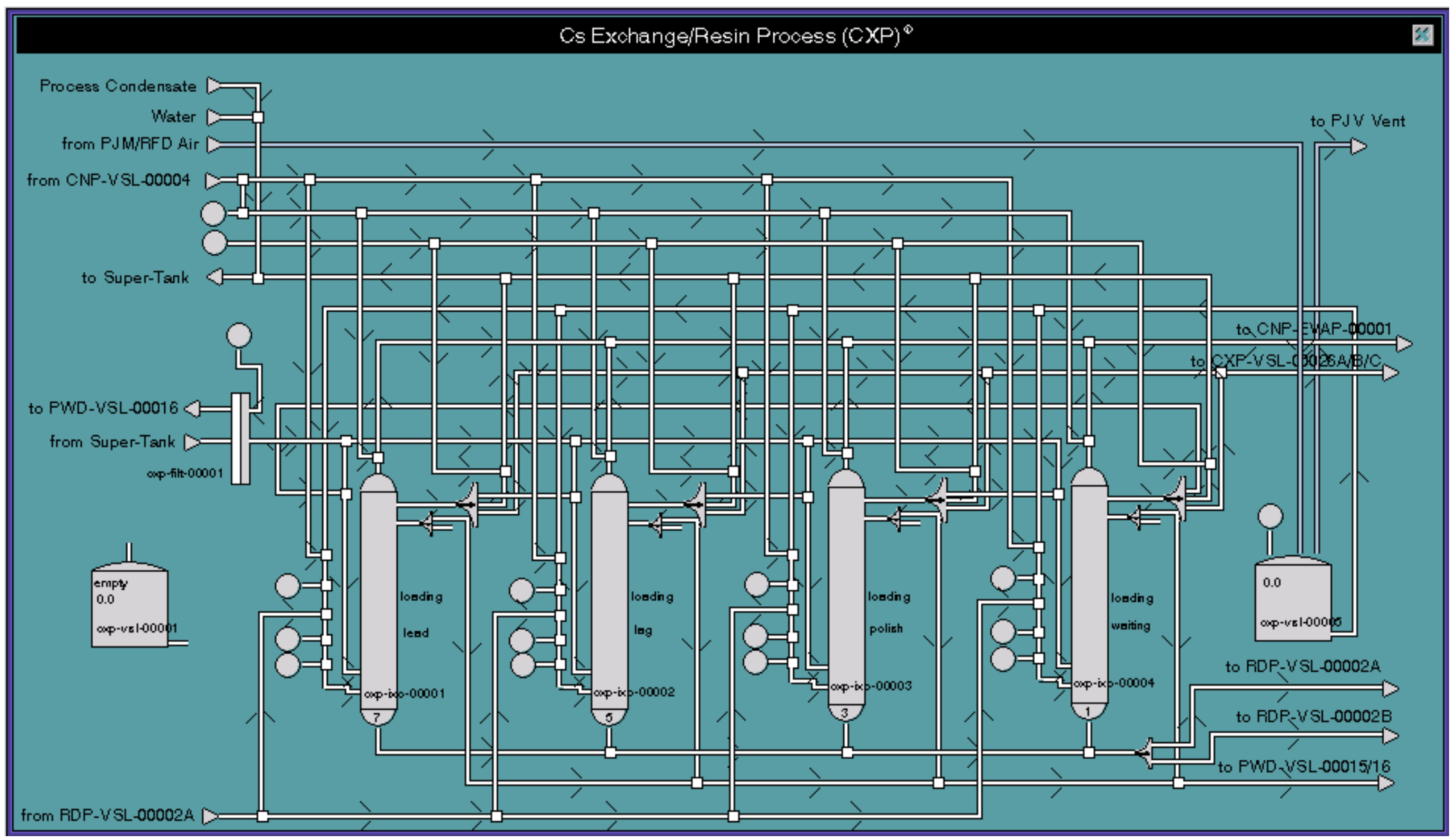


Figure F-9 Cesium Fresh Resin Addition and Spent Resin Collection and Dewatering Process

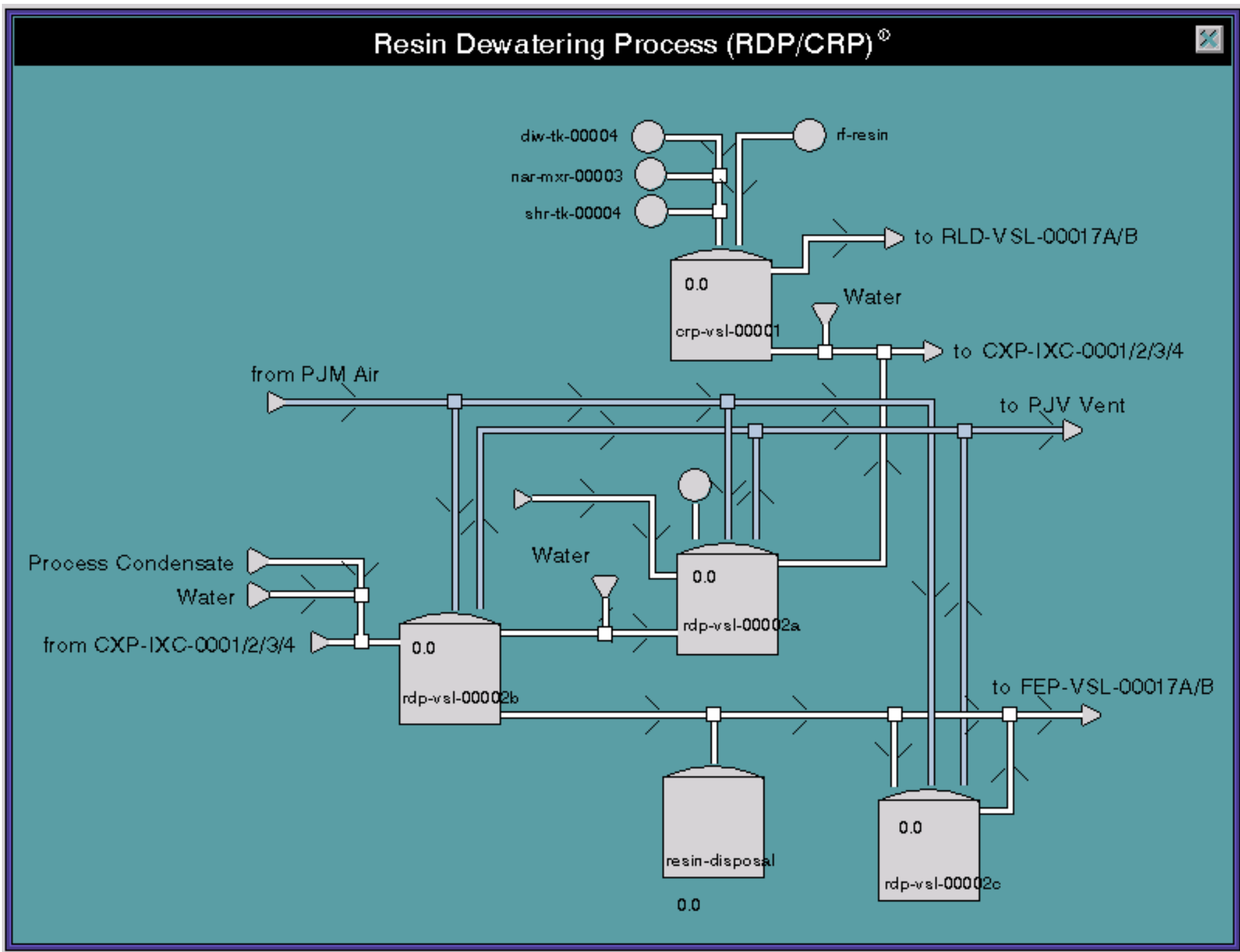


Figure F-10 Cesium Nitric Acid Recovery Process

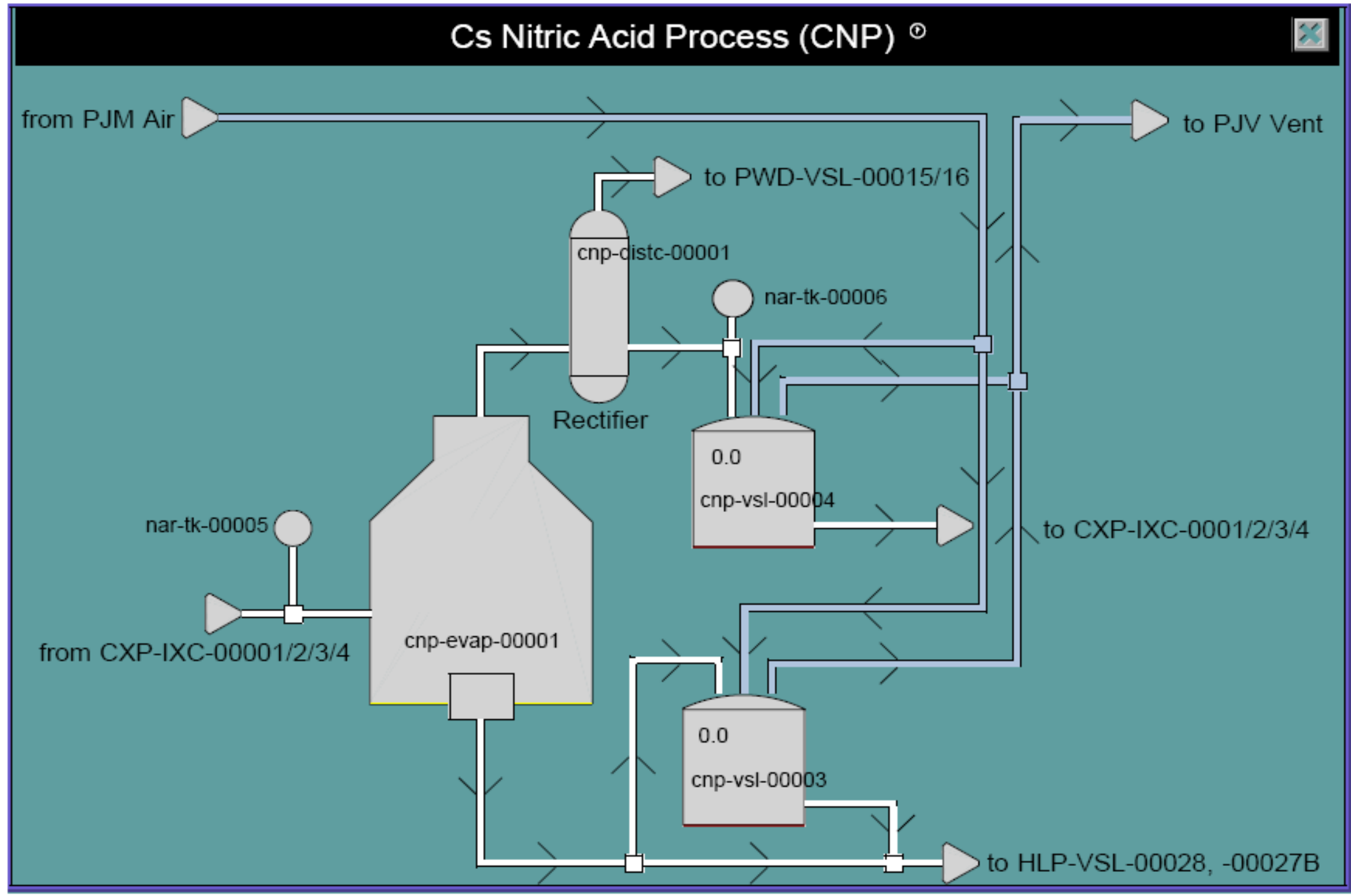




\section{Figure F-11 Treated LAW Evaporation and Concentrate Storage Process}

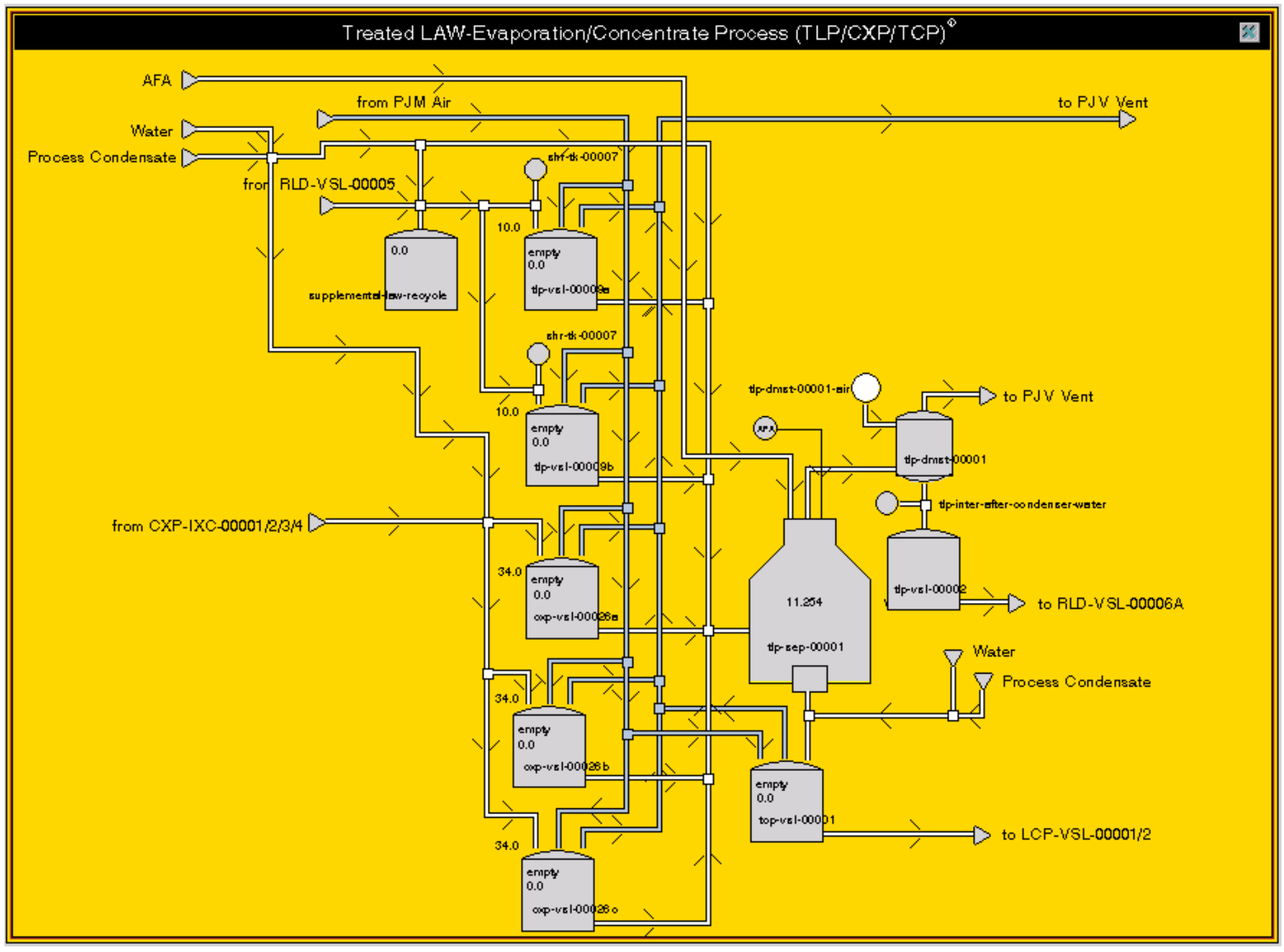


Figure F-12 LAW Melter Feed Process

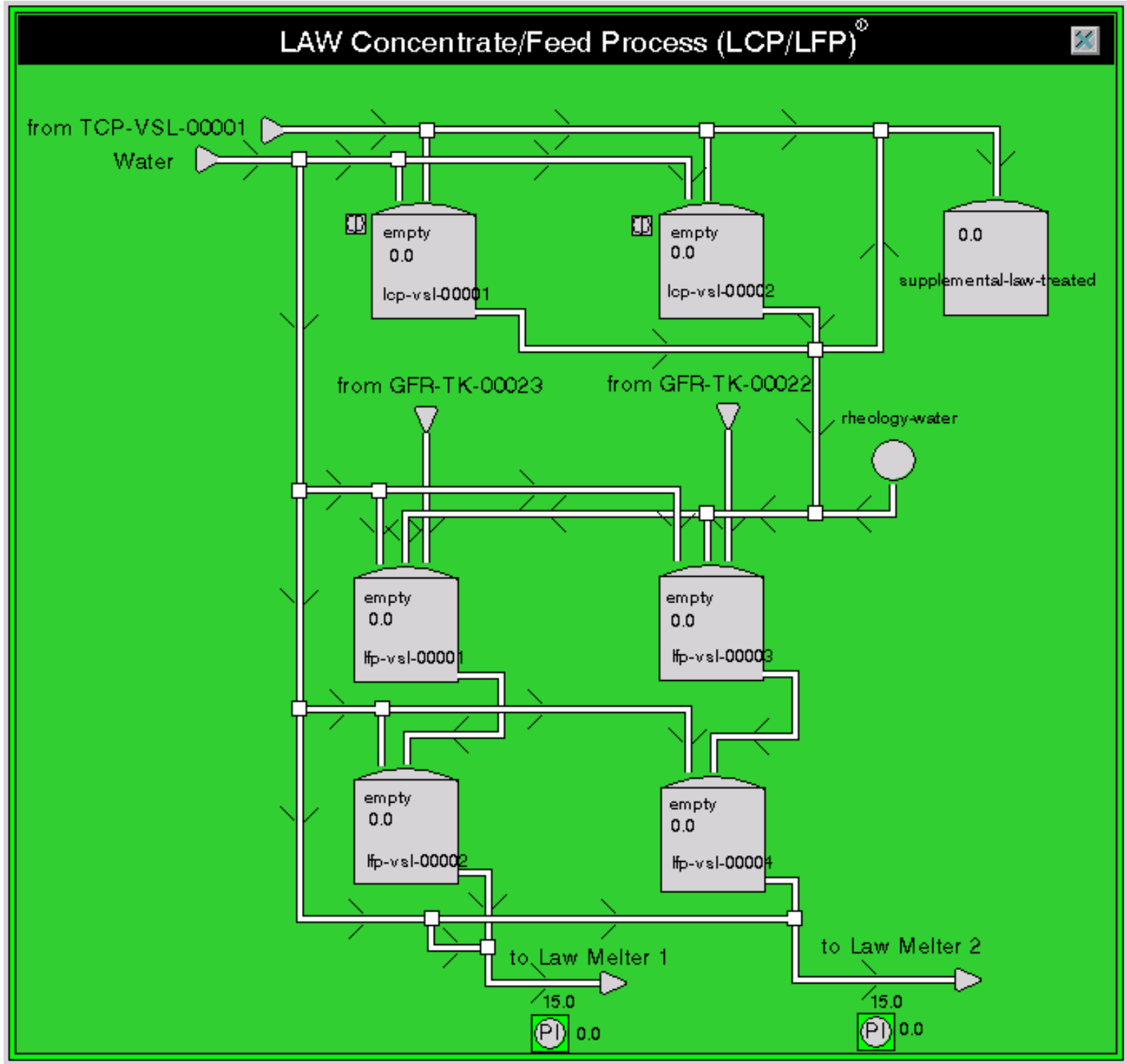


Figure F-13 LAW Melter 1 and Associated Primary Offgas Process

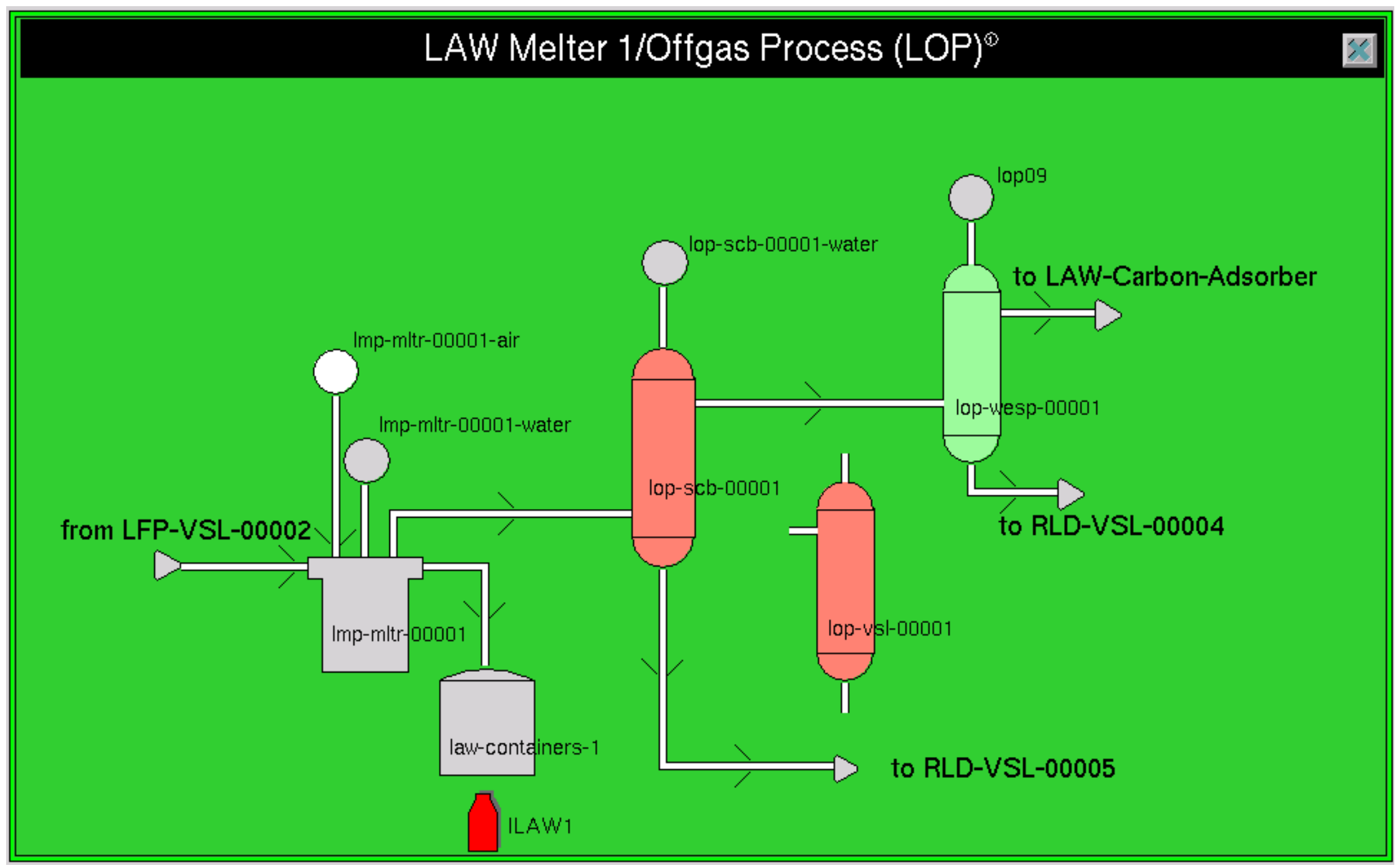


Figure F-14 LAW Melter 2 and Associated Primary Offgas Process

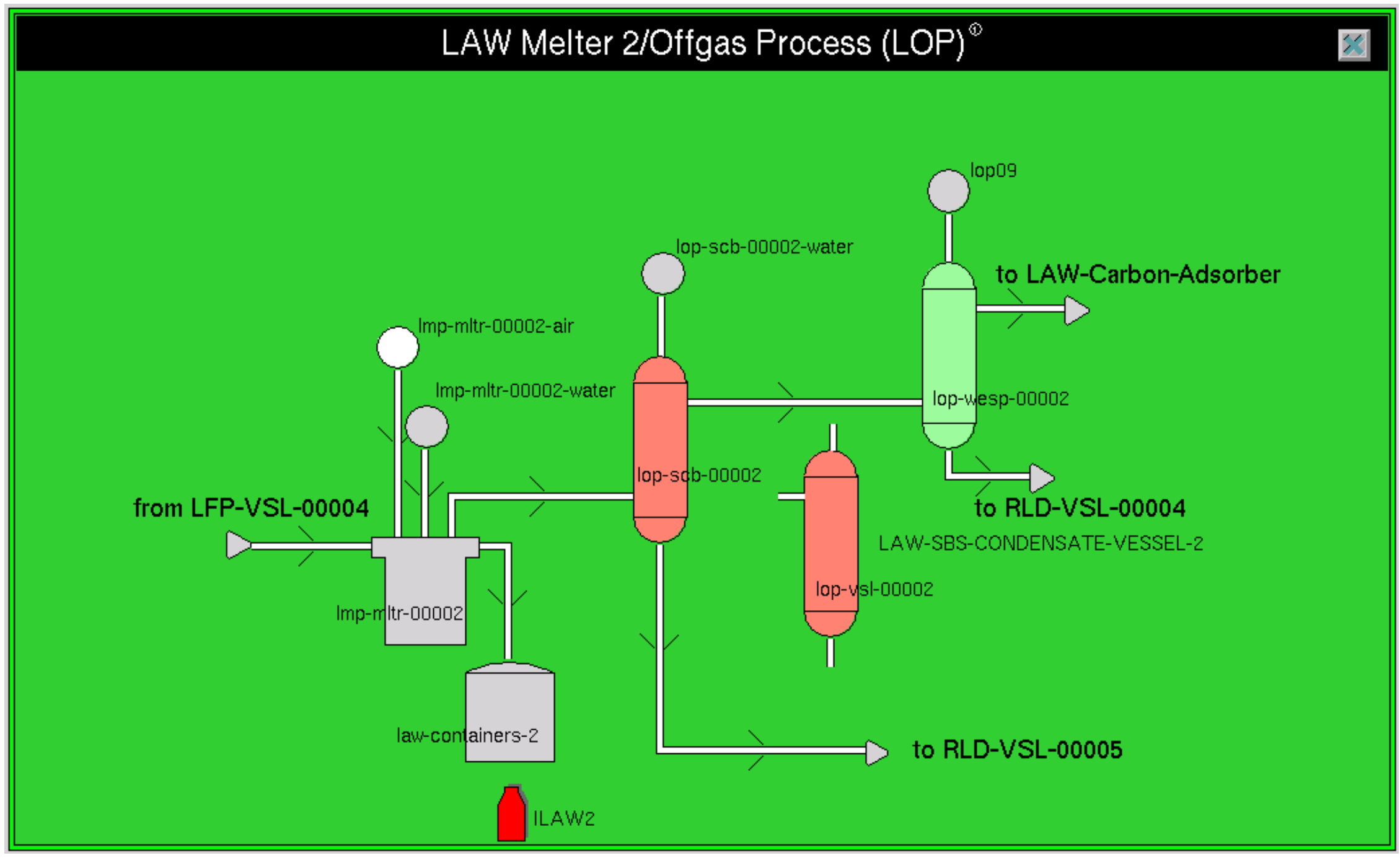


Figure F-15 LAW Secondary Offgas/Vessel Vent Process

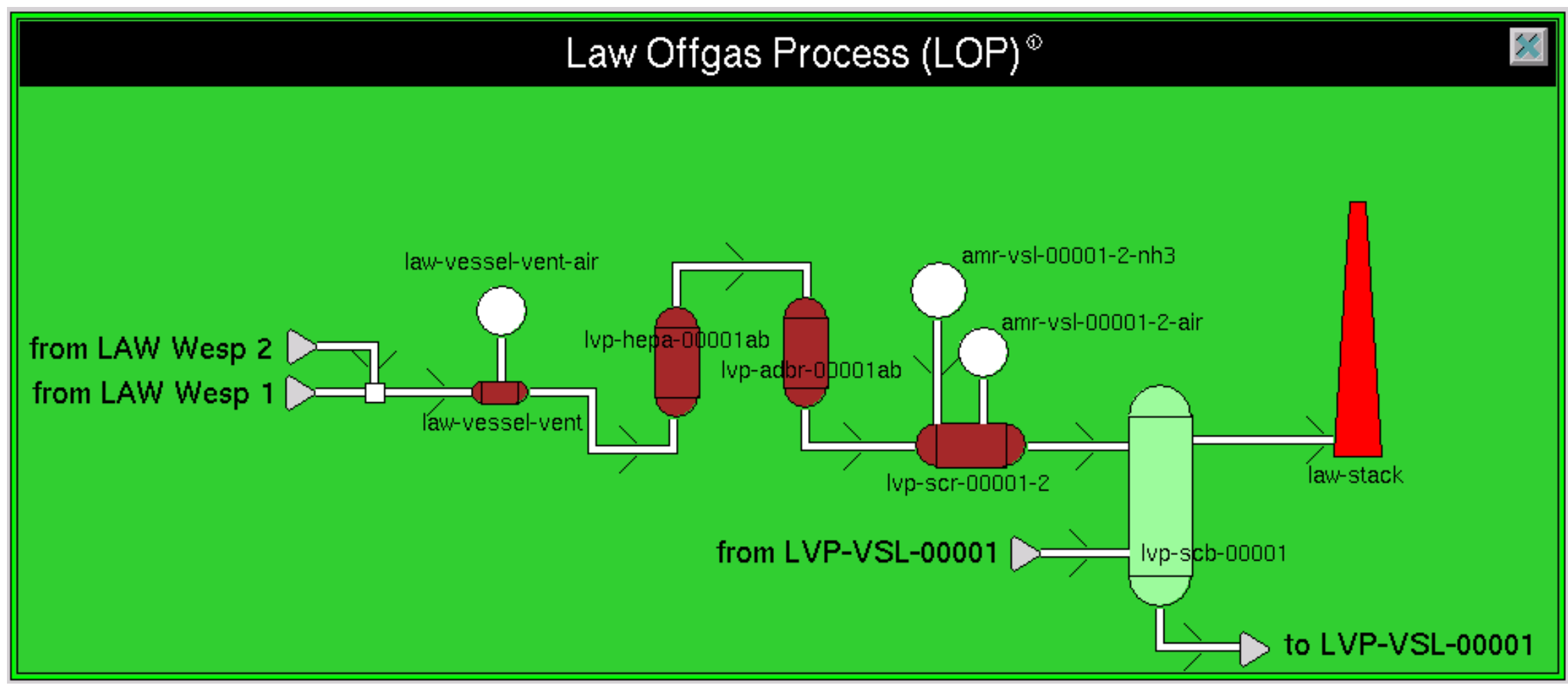


Figure F-16 LAW Radioactive Liquid Waste Disposal

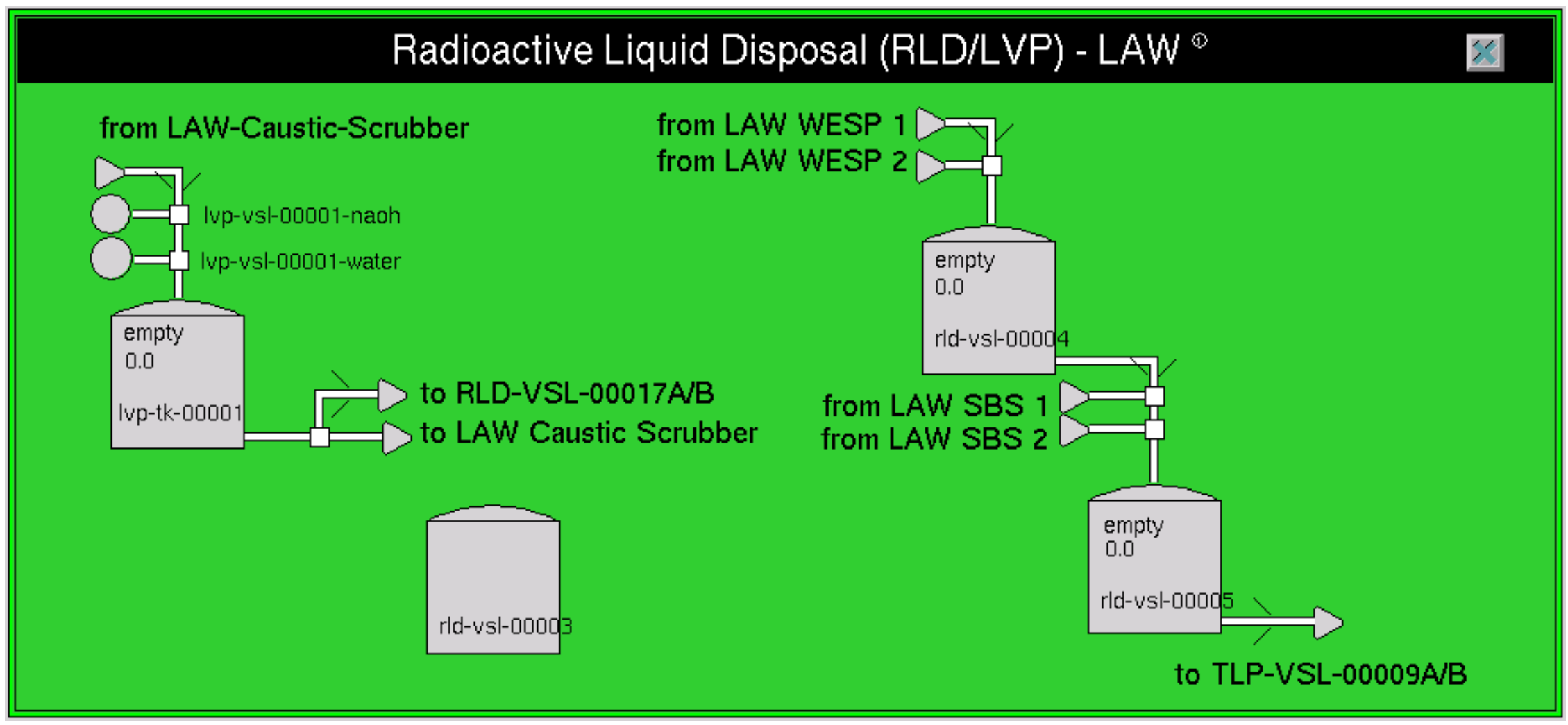




\section{Figure F-17 HLW Lag Storage and Feed Blending Process}

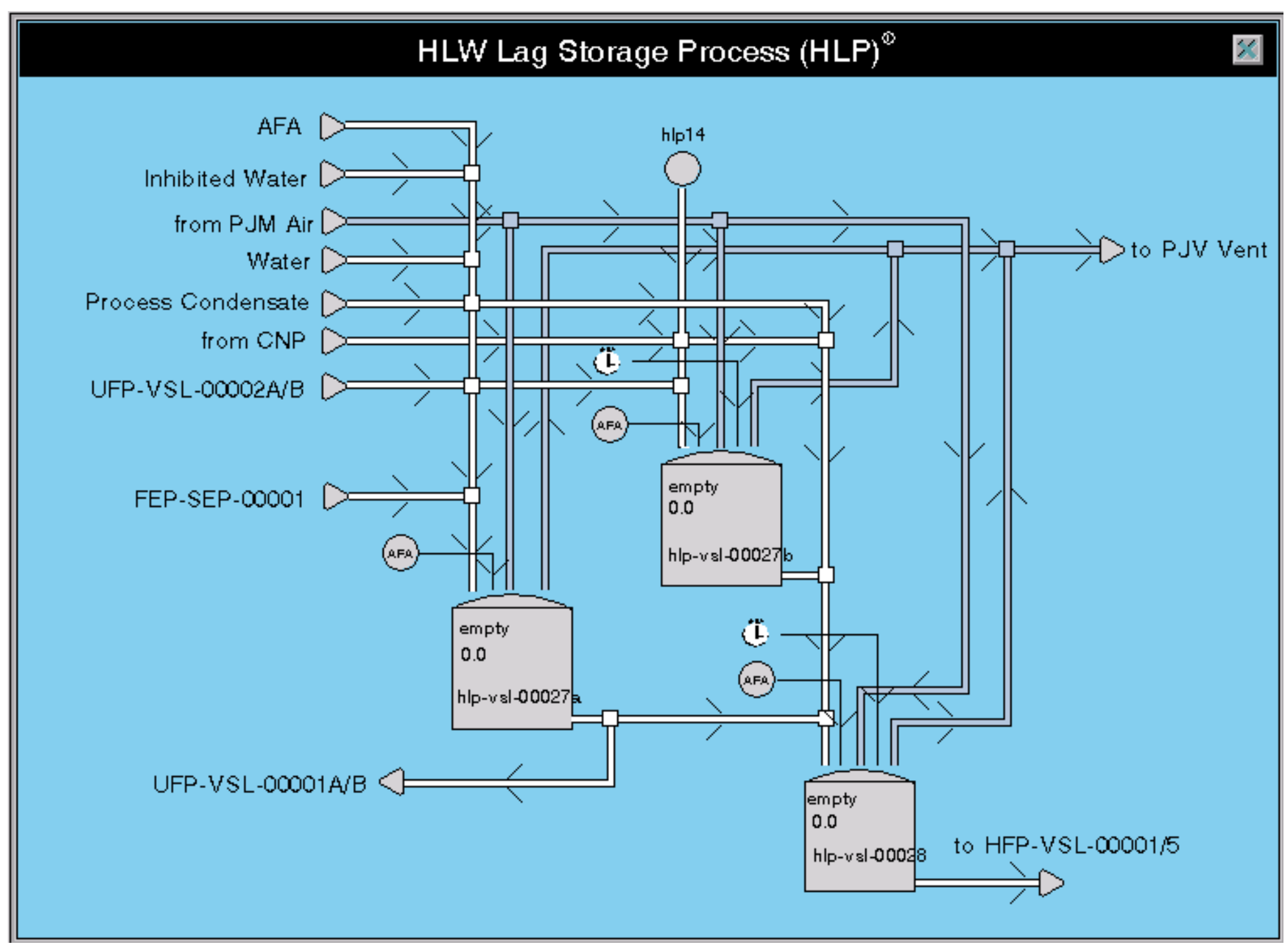




\section{Figure F-18 HLW Melter Feed Process}

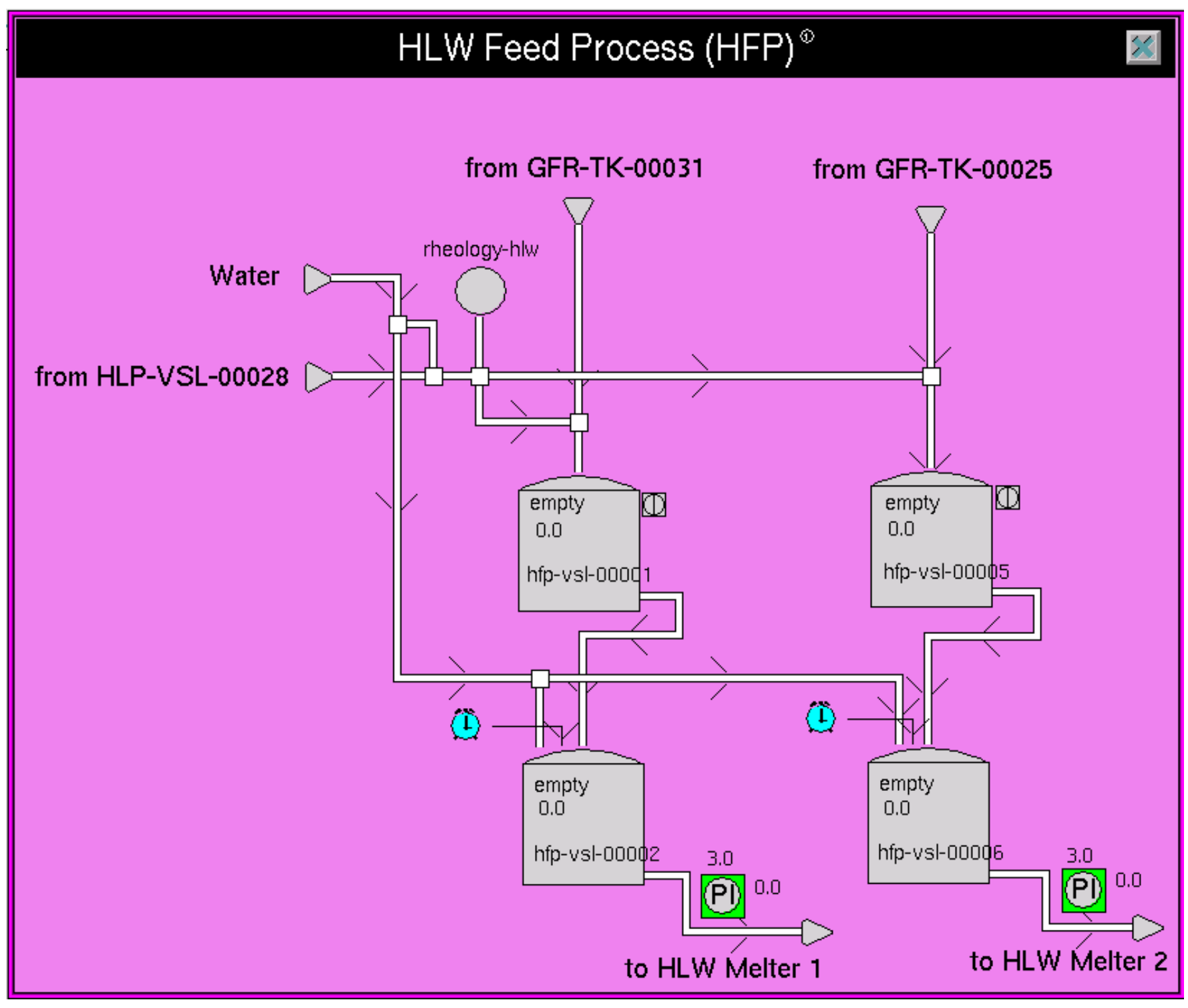


Figure F-19 HLW Melter 1 and Associated Offgas Process

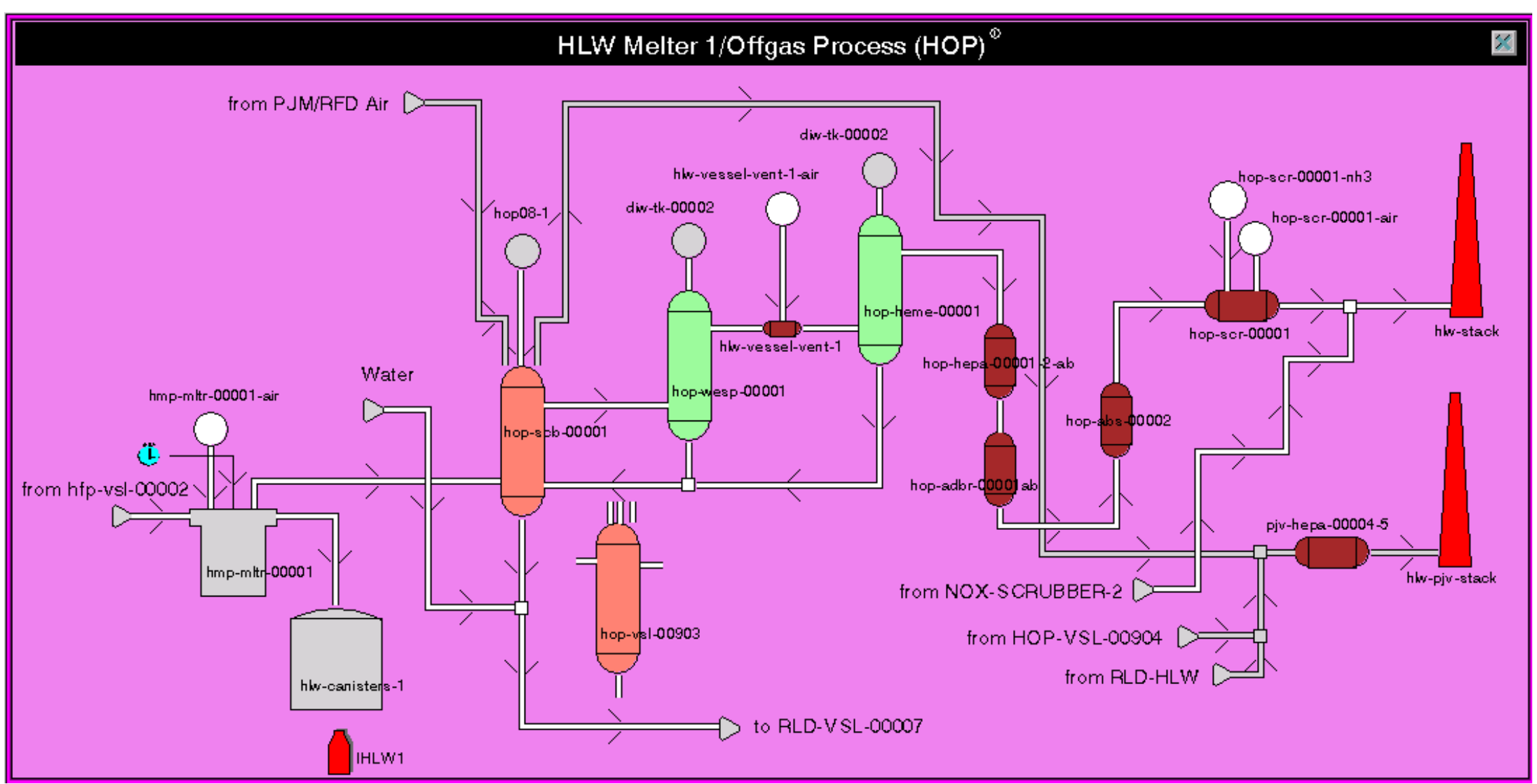


Figure F-20 HLW Melter 2 and Associated Offgas Process

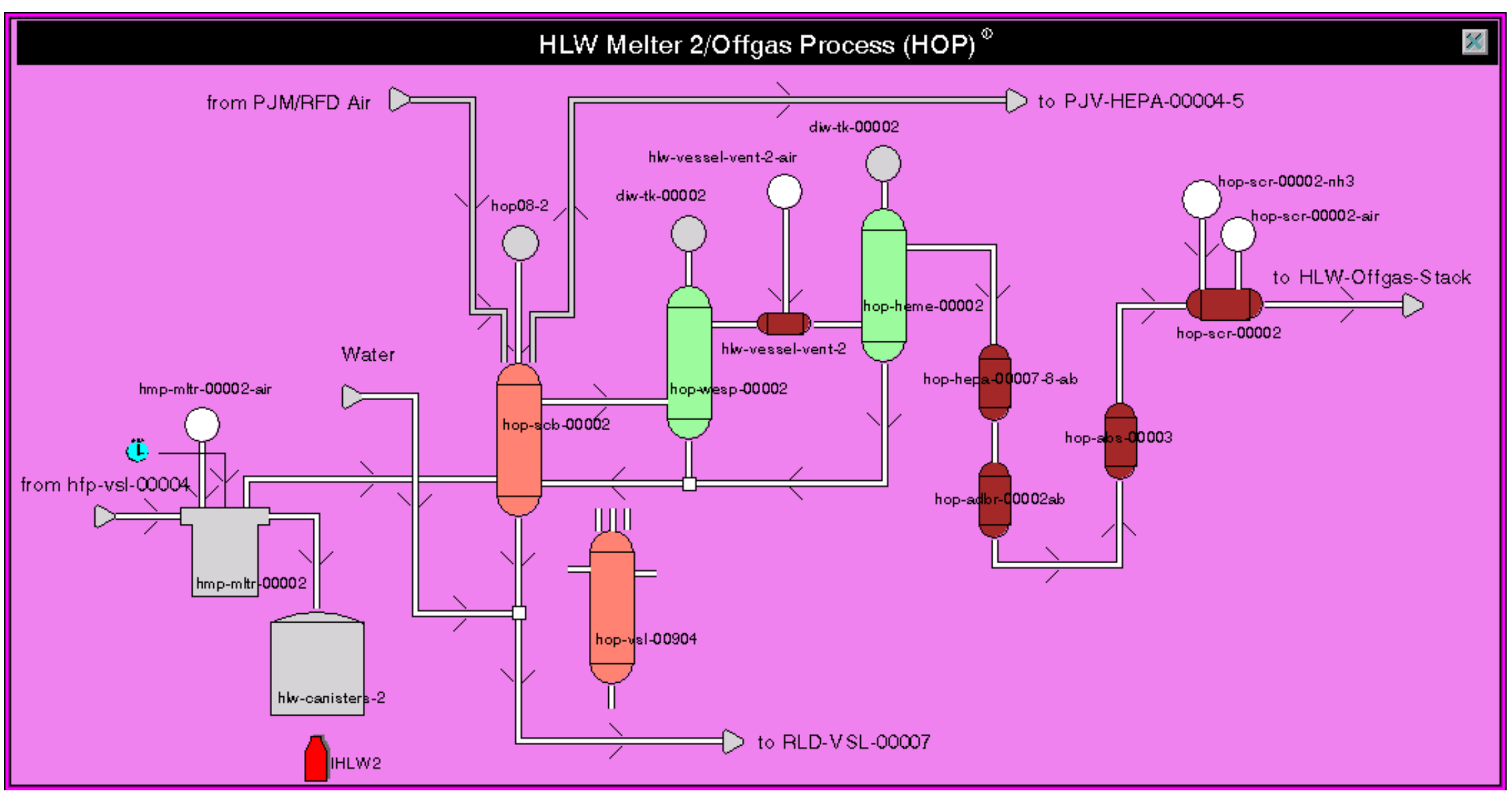


Figure F-21 HLW Canister Decontamination Handling

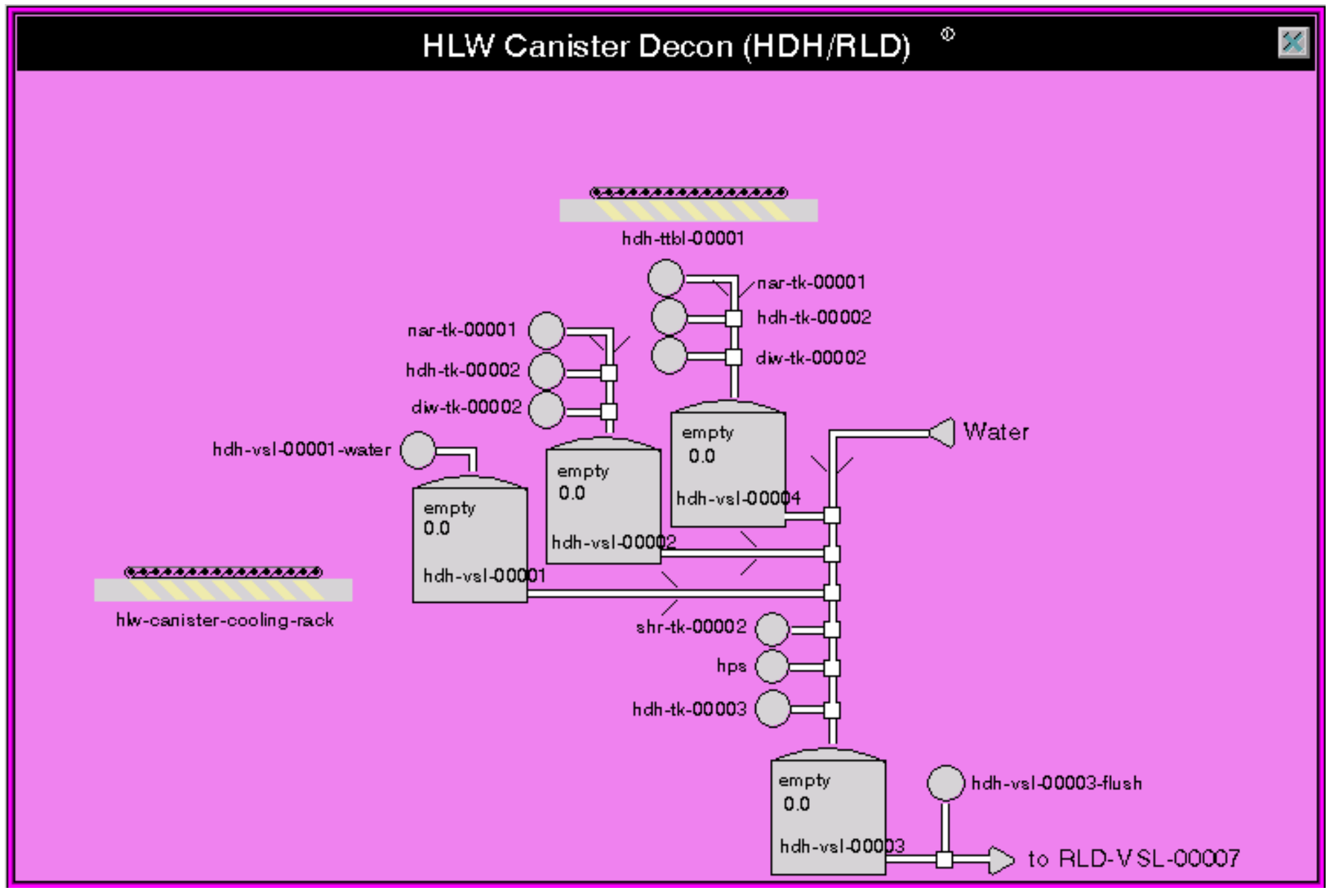




\section{Figure F-22 HLW Radioactive Liquid Disposal}

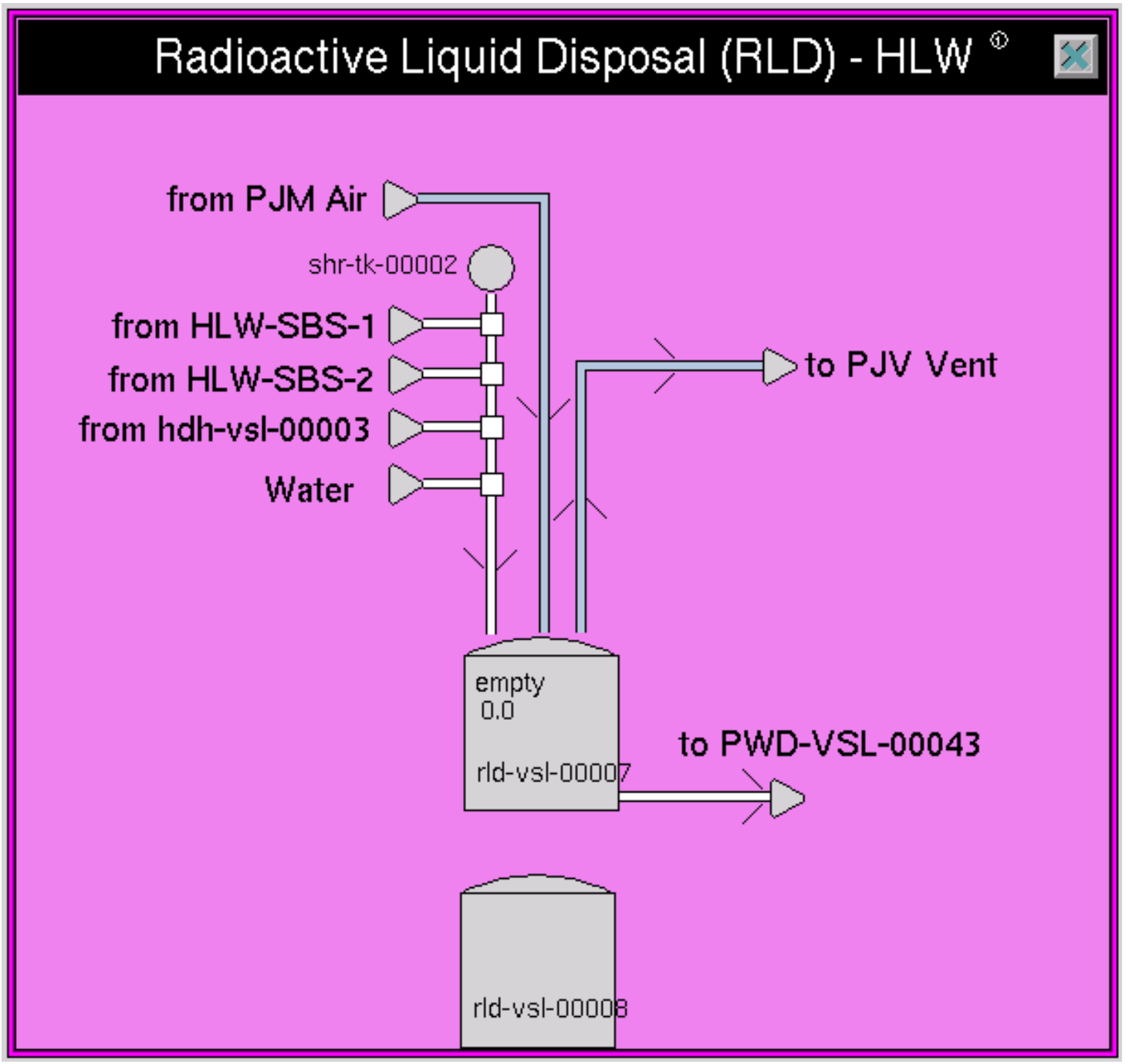




\section{Figure F-23 Glass Formers Reagent System}

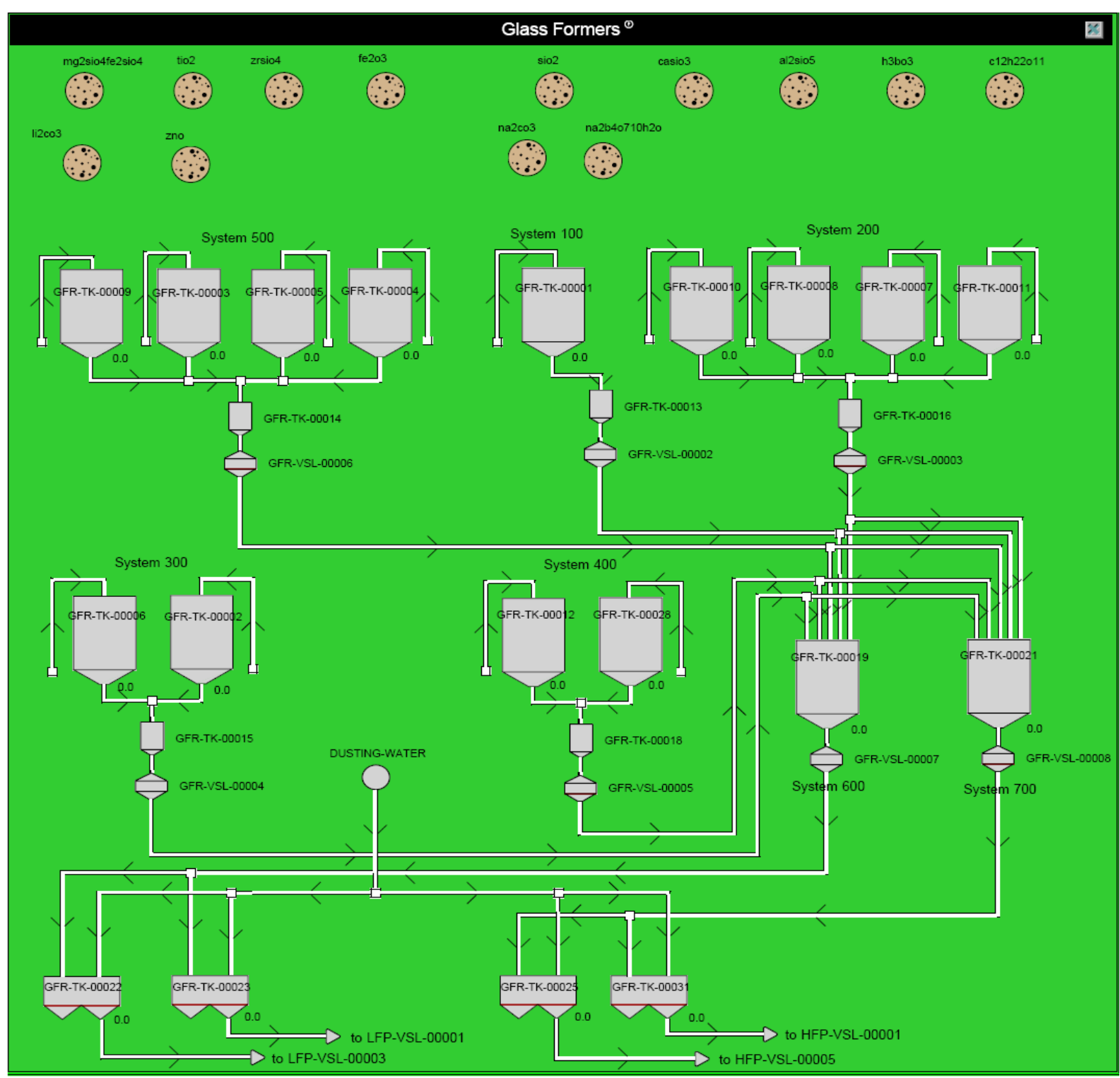


Figure F-24 Radioactive Liquid Waste Disposal - Effluent

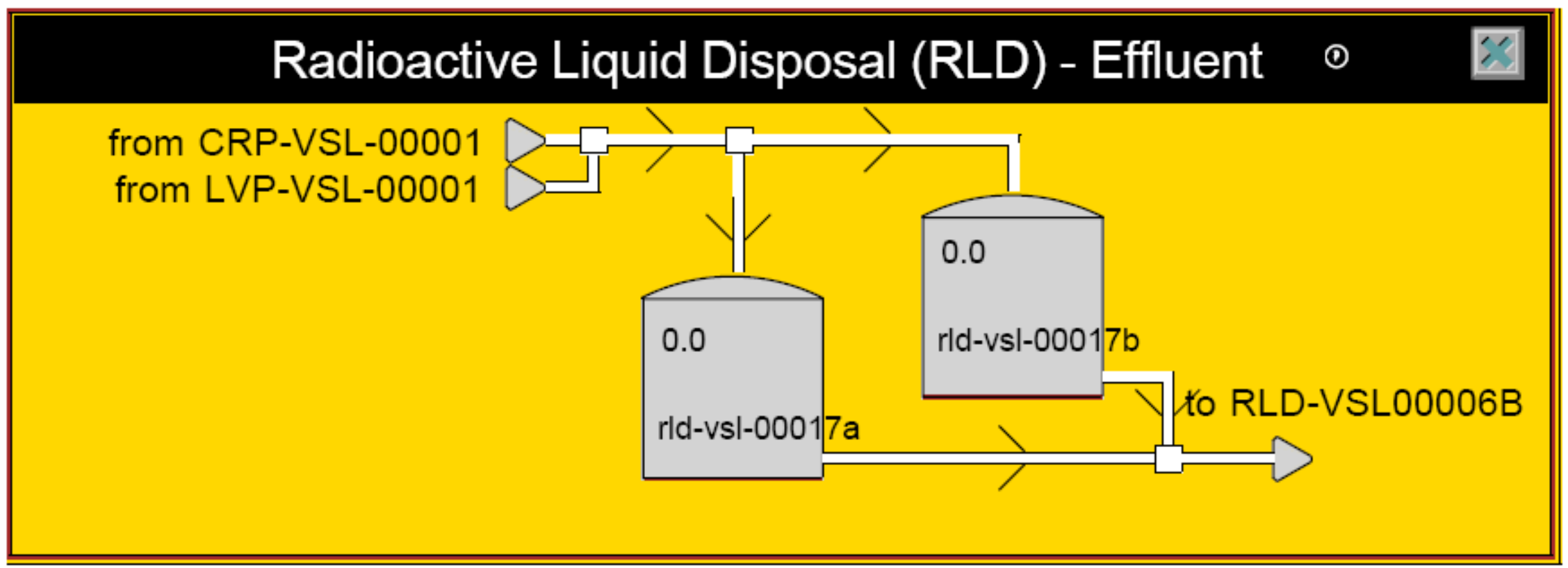


Figure F-25 Radioactive Liquid Waste Disposal - Pretreatment

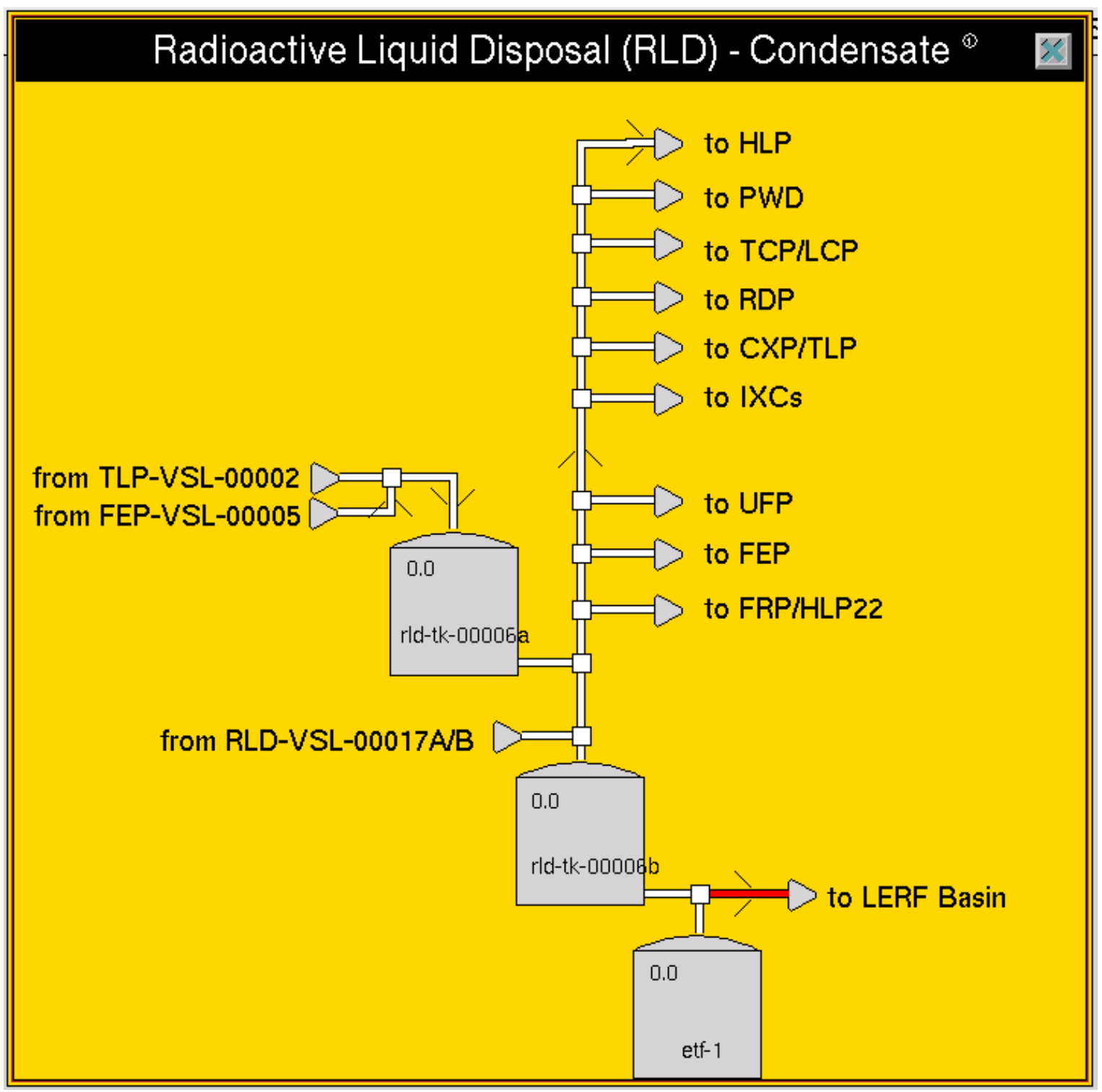


Figure F-26 PJM/RFD Air Sources

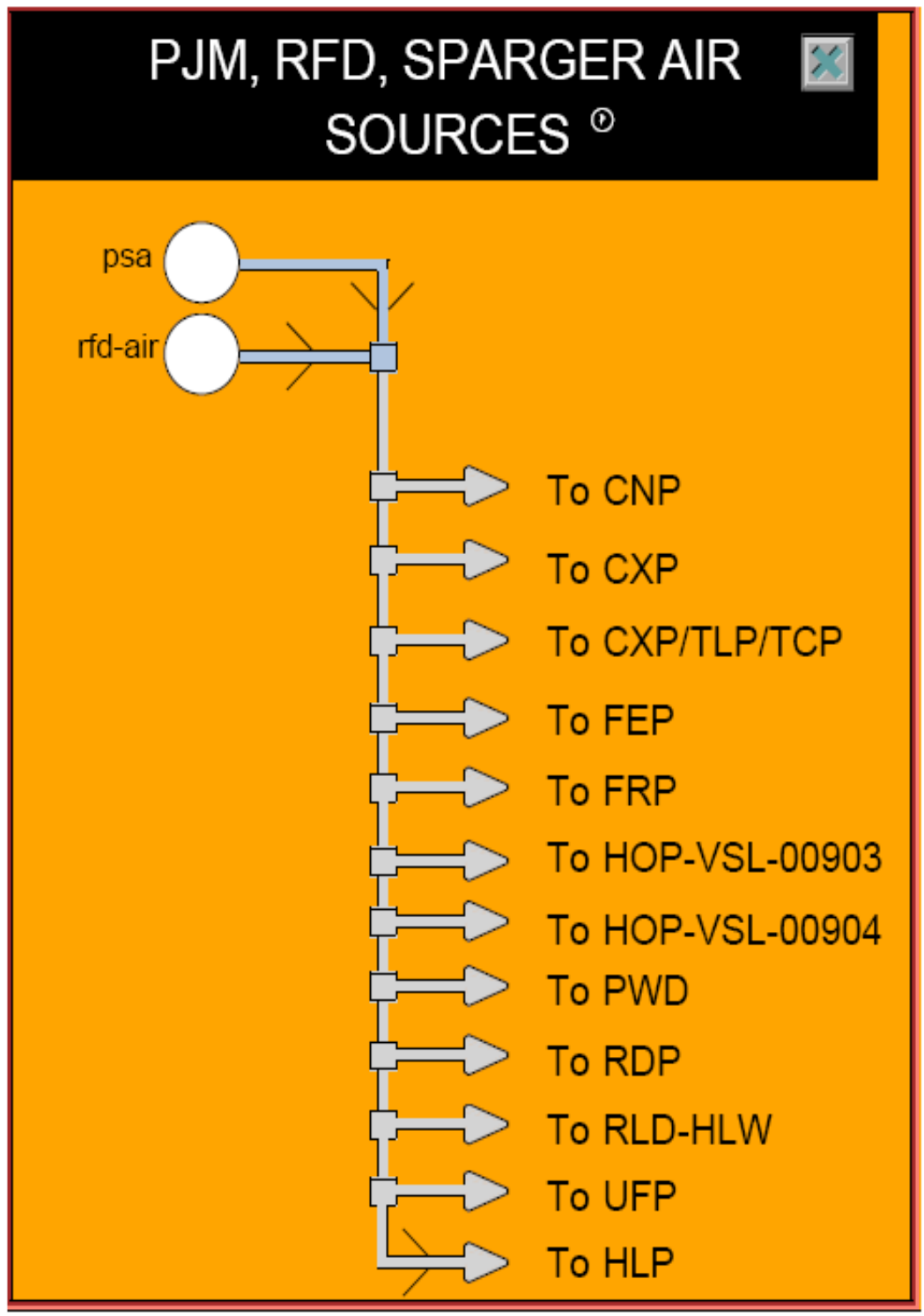




\section{Appendix G}

\section{Dynamic Air Utilization Modeling}




\section{Appendix G \\ Dynamic Air Utilization Modeling}

Dynamic air utilization is a legacy modeling capability from Dynamic (G2) Model Version 4.0 that simulates the sparging, PJM and RFD operations, purge flows, bubbler flows, and sump inbleed flows. This modeling capability needs to be verified and validated before it can be used as the WTP process flowsheet has evolved significantly since June 8, 2005. Flowrates for the vessels are shown in Table G-1 where operational logic is also summarized. It is assumed that sparger air is humidified so that it does not evaporate the waste. It is also assumed that none of the air additions will lead to evaporation. Sparger air, purge air, bubbler air, and sump inbleed air will go to the PVP system. PJM suction and discharge air along with the RFD air will go to the PJV system.

\begin{tabular}{|c|c|c|c|}
\hline \multicolumn{4}{|c|}{ PJM/RFD Air Flowrates } \\
\hline Vessel Name & $\begin{array}{l}\text { PJM Air Flowrate } \\
\text { (acfm) }\end{array}$ & $\begin{array}{l}\text { RFD Air Flowrate } \\
\text { (acfm) }\end{array}$ & Operational Logic \\
\hline RDP-VSL-00002A & 570.0 & & Run when the vessel is discharging \\
\hline RDP-VSL-00002B & 570.0 & & Run when the vessel is discharging \\
\hline RDP-VSL-00002C & 570.0 & & Run when the vessel is discharging \\
\hline FEP-VSL-00017A & 1007.0 & & Run after vessel is ready to discharge and until vessel reaches minimum volume \\
\hline FEP-VSL-00017B & 1007.0 & & Run after vessel is ready to discharge and until vessel reaches minimum volume \\
\hline FRP-VSL-00002A & 1660.0 & & Run from hold time initiation until vessel reaches minimum volume \\
\hline FRP-VSL-00002B & 1660.0 & & Run from hold time initiation until vessel reaches minimum volume \\
\hline FRP-VSL-00002C & 1660.0 & & Run from hold time initiation until vessel reaches minimum volume \\
\hline FRP-VSL-00002D & 1660.0 & & Run from hold time initiation until vessel reaches minimum volume \\
\hline HLP-VSL-00022 & 1536.0 & & Run if volume is above minimum volume \\
\hline HLP-VSL-00027A & 1463.0 & & $\begin{array}{l}\text { Run at } 100 \% \text { capacity if volume is above minimum volume, run at } 33 \% \text { capacity if } \\
\text { volume is below minimum volume but greater than zero. }\end{array}$ \\
\hline HLP-VSL-00027B & 1463.0 & & $\begin{array}{l}\text { Run at } 100 \% \text { capacity if volume is above minimum volume, run at } 33 \% \text { capacity if } \\
\text { volume is below minimum volume but greater than zero. }\end{array}$ \\
\hline HLP-VSL-00028 & 1463.0 & & $\begin{array}{l}\text { Run at } 100 \% \text { capacity if volume is above minimum volume, run at } 33 \% \text { capacity if } \\
\text { volume is below minimum volume but greater than zero. }\end{array}$ \\
\hline UFP-VSL-00001A & 884.0 & & Run after vessel is ready to discharge and until vessel reaches minimum volume \\
\hline UFP-VSL-00001B & 884.0 & & Run after vessel is ready to discharge and until vessel reaches minimum volume \\
\hline UFP-VSL-00002A & 1294.0 & & $\begin{array}{l}\text { Run at } 100 \% \text { capacity if volume is above minimum volume, run at } 33 \% \text { capacity if } \\
\text { volume is below minimum volume but greater than zero. }\end{array}$ \\
\hline
\end{tabular}


24590-WTP-MDD-PR-01-002, Rev 12

\begin{tabular}{|c|c|c|c|}
\hline Vessel Name & $\begin{array}{l}\text { PJM Air Flowrate } \\
\text { (acfm) }\end{array}$ & $\begin{array}{l}\text { RFD Air Flowrate } \\
\text { (acfm) }\end{array}$ & Operational Logic \\
\hline UFP-VSL-00002B & 1294.0 & & $\begin{array}{l}\text { Run at } 100 \% \text { capacity if volume is above minimum volume, run at } 33 \% \text { capacity if } \\
\text { volume is below minimum volume but greater than zero. }\end{array}$ \\
\hline UFP-VSL-00062A & 626.0 & 258.0 & $\begin{array}{l}\text { PJM runs after vessel is ready to discharge until vessel reaches minimum volume, } \\
\text { RFD runs when vessel is discharging }\end{array}$ \\
\hline UFP-VSL-00062B & 626.0 & 258.0 & $\begin{array}{l}\text { PJM runs after vessel is ready to discharge until vessel reaches minimum volume, } \\
\text { RFD runs when vessel is discharging }\end{array}$ \\
\hline UFP-VSL-00062C & 626.0 & 254.0 & $\begin{array}{l}\text { PJM runs after vessel is ready to discharge until vessel reaches minimum volume, } \\
\text { RFD runs when vessel is discharging }\end{array}$ \\
\hline CXP-VSL-00004 & 133.0 & 81.0 & Both PJM and RFD run when solution is pumped to the IX column for displacement \\
\hline CNP-VSL-00004 & 512.0 & & Run when the vessel is discharging \\
\hline CNP-VSL-00003 & 450.0 & & Run if volume is above minimum volume \\
\hline PWD-VSL-00044 & 859.0 & 175.0 & $\begin{array}{l}\text { PJM runs from hold time initiation until vessel reaches minimum volume, RFD runs } \\
\text { when vessel is discharging }\end{array}$ \\
\hline PWD-VSL-00033 & 1140.0 & 363.0 & $\begin{array}{l}\text { PJM runs from hold time initiation until vessel reaches minimum volume, RFD runs } \\
\text { when vessel is discharging }\end{array}$ \\
\hline PWD-VSL-00043 & 1140.0 & 400.0 & $\begin{array}{l}\text { PJM runs from hold time initiation until vessel reaches minimum volume, RFD runs } \\
\text { when vessel is discharging }\end{array}$ \\
\hline PWD-VSL-00015 & 983.0 & 217.0 & $\begin{array}{l}\text { PJM runs from hold time initiation until vessel reaches minimum volume, RFD runs } \\
\text { when vessel is discharging }\end{array}$ \\
\hline PWD-VSL-00016 & 983.0 & 186.0 & $\begin{array}{l}\text { PJM runs from hold time initiation until vessel reaches minimum volume, RFD runs } \\
\text { when vessel is discharging }\end{array}$ \\
\hline TCP-VSL-00001 & 983.0 & & Run after vessel is ready to discharge and until vessel reaches minimum volume \\
\hline CXP-VSL-00026A & 613.0 & & Run from hold time initiation until vessel reaches minimum volume \\
\hline CXP-VSL-00026B & 613.0 & & Run from hold time initiation until vessel reaches minimum volume \\
\hline CXP-VSL-00026C & 613.0 & & Run from hold time initiation until vessel reaches minimum volume \\
\hline TLP-VSL-00009A & 727.0 & & Run after vessel is ready to discharge and until vessel reaches minimum volume \\
\hline TLP-VSL-00009B & 727.0 & & Run after vessel is ready to discharge and until vessel reaches minimum volume \\
\hline
\end{tabular}




\section{Appendix $\mathbf{H}$}

\section{Transfer, Sampling, and Time Triggered Line Flushes}




\section{Appendix $\mathbf{H}$ \\ Transfer, Sampling, and Time Triggered Line Flushes}

Line flushes occur in the Pretreatment, LAW, and HLW facilities. The sources of line flush include process condensate and process water. For the Pretreatment line flush operations, process condensate from RLD-TK-00006A is the preferred source if sufficient volume is available at the time when it is needed. Process water is the secondary source and will only be used if the primary source is unavailable or insufficient. For the LAW and HLW line flush operations, process water is the only source. Logic triggering the flushes are discussed in Sections 4.7 and 4.8 as appropriate. Table $\mathrm{H}-1$ presents the sequential transfer route number (STRN), origin of flush, destination of flush, and associated flush volume. In most cases, an origin of flush is associated with multiple destinations of flushes as the results of dead legs and piping configurations. The G2 model assumes that flushing takes place instantly.

Table H-1 HPAV and Plugging Line Flush Volumes

\begin{tabular}{|l|l|l|l|}
\hline STRN & Origin of Flush & Destination of Flush & $\begin{array}{l}\text { Flush Volume } \\
\text { (Gal) }\end{array}$ \\
\hline CXP-43 & CXP-VSL-00026A & CXP-VSL-00026A & 73.89 \\
\hline CXP-43 & same & TLP-SEP-00001 & 15.37 \\
\hline CXP-43 & same & TCP-VSL-00001 & 56.31 \\
\hline CXP-44 & CXP-VSL-00026B & CXP-VSL-00026B & 84.66 \\
\hline CXP-44 & same & TLP-SEP-00001 & 15.37 \\
\hline CXP-44 & same & TCP-VSL-00001 & 56.31 \\
\hline CXP-45 & CXP-VSL-00026C & CXP-VSL-00026C & 65.18 \\
\hline CXP-45 & same & TLP-SEP-00001 & 15.37 \\
\hline CXP-45 & same & TCP-VSL-00001 & 56.31 \\
\hline FEP-02 & FEP-VSL-00017A & FEP-VSL-00017A & 64.25 \\
\hline FEP-04 & FEP-VSL-00017A & FEP-SEP-00001A & 39.99 \\
\hline FEP-04 & same & FEP-VSL-00017A & 33.77 \\
\hline FEP-04 & same & FRP-VSL-00002A & 55.79 \\
\hline FEP-04 & same & FEP-SEP-00001B & 18.23 \\
\hline FEP-07 & FEP-VSL-00017A & FEP-SEP-00001B & 40.64 \\
\hline FEP-07 & same & FEP-VSL-00017A & 30.63 \\
\hline FEP-07 & same & FEP-SEP-00001A & 11.44 \\
\hline FEP-07 & same & FRP-VSL-00002A & 54.97 \\
\hline FEP-10 & FEP-VSL-00017B & FEP-VSL-00017B & 65.36 \\
\hline FEP-12 & FEP-VSL-00017B & FEP-SEP-00001A & 39.06 \\
\hline FEP-12 & same & FEP-VSL-00017B & 35.40 \\
\hline FEP-12 & same & FEP-SEP-00001B & 15.51 \\
\hline FEP-12 & same & FRP-VSL-00002A & 53.40 \\
\hline FEP-15 & FEP-VSL-00017B & FEP-SEP-00001B & 47.68 \\
\hline FEP-15 & same & FEP-VSL-00017B & 30.63 \\
\hline FEP-15 & same & FEP-SEP-00001A & 11.44 \\
\hline FEP-15 & same & FRP-VSL-00002A & 54.97 \\
\hline FEP-17 & FEP-SEP-00001A & UFP-VSL-00001A & 34.93 \\
\hline FEP-17 & same & UFP-VSL-00001B & 14.96 \\
\hline FEP-17 & same & FRP-VSL-00002A & 53.10 \\
\hline & &
\end{tabular}




\begin{tabular}{|c|c|c|c|}
\hline STRN & Origin of Flush & Destination of Flush & $\begin{array}{l}\text { Flush Volume } \\
\text { (Gal) }\end{array}$ \\
\hline FEP-18 & FEP-SEP-00001A & UFP-VSL-00001B & 38.85 \\
\hline FEP-18 & same & UFP-VSL-00001B & 11.83 \\
\hline FEP-18 & same & FRP-VSL-00002A & 53.10 \\
\hline FEP-20 & FEP-SEP-00001B & UFP-VSL-00001A & 37.99 \\
\hline FEP-20 & same & UFP-VSL-00001B & 10.84 \\
\hline FEP-20 & same & FRP-VSL-00002A & 50.57 \\
\hline FEP-21 & FEP-SEP-00001B & UFP-VSL-00001B & 42.04 \\
\hline FEP-21 & same & UFP-VSL-00001A & 7.59 \\
\hline FEP-21 & same & FRP-VSL-00002A & 50.57 \\
\hline FRP-01 & FRP-VSL-00002A & FEP-VSL-00017A & 228.79 \\
\hline FRP-01 & same & FEP-VSL-00017B & 139.54 \\
\hline FRP-01 & same & UFP-VSL-00001A & 156.12 \\
\hline FRP-01 & same & UFP-VSL-00001B & 158.19 \\
\hline FRP-01 & same & FRP-VSL-00002B & 73.45 \\
\hline FRP-02 & FRP-VSL-00002A & FEP-VSL-00017B & 255.27 \\
\hline FRP-02 & same & FEP-VSL-00017A & 118.36 \\
\hline FRP-02 & same & UFP-VSL-00001A & 156.12 \\
\hline FRP-02 & same & UFP-VSL-00001B & 158.19 \\
\hline FRP-02 & same & FRP-VSL-00002B & 73.45 \\
\hline FRP-03 & FRP-VSL-00002A & UFP-VSL-00001A & 276.00 \\
\hline FRP-03 & same & FEP-VSL-00017A & 118.36 \\
\hline FRP-03 & same & FEP-VSL-00017B & 139.54 \\
\hline FRP-03 & same & UFP-VSL-00001B & 158.19 \\
\hline FRP-03 & same & FRP-VSL-00002B & 73.45 \\
\hline FRP-04 & FRP-VSL-00002A & UFP-VSL-00001B & 278.59 \\
\hline FRP-04 & same & FEP-VSL-00017A & 118.36 \\
\hline FRP-04 & same & FEP-VSL-00017B & 139.54 \\
\hline FRP-04 & same & UFP-VSL-00001A & 156.12 \\
\hline FRP-04 & same & FRP-VSL-00002B & 73.45 \\
\hline FRP-05 & FRP-VSL-00002B & FEP-VSL-00017A & 261.26 \\
\hline FRP-05 & same & FEP-VSL-00017B & 139.77 \\
\hline FRP-05 & same & UFP-VSL-00001A & 156.35 \\
\hline FRP-05 & same & UFP-VSL-00001B & 158.42 \\
\hline FRP-05 & same & FRP-VSL-00002B & 73.45 \\
\hline FRP-06 & FRP-VSL-00002B & FEP-VSL-00017B & 259.53 \\
\hline FRP-06 & same & FEP-VSL-00017A & 141.15 \\
\hline FRP-06 & same & UFP-VSL-00001A & 156.35 \\
\hline FRP-06 & same & UFP-VSL-00001B & 158.42 \\
\hline FRP-06 & same & FRP-VSL-00002B & 73.45 \\
\hline FRP-07 & FRP-VSL-00002B & UFP-VSL-00001A & 280.25 \\
\hline FRP-07 & same & FEP-VSL-00017A & 141.15 \\
\hline FRP-07 & same & FEP-VSL-00017B & 139.77 \\
\hline FRP-07 & same & UFP-VSL-00001B & 158.42 \\
\hline FRP-07 & same & FRP-VSL-00002B & 73.45 \\
\hline FRP-08 & FRP-VSL-00002B & UFP-VSL-00001B & 282.84 \\
\hline
\end{tabular}




\begin{tabular}{|c|c|c|c|}
\hline STRN & Origin of Flush & Destination of Flush & $\begin{array}{l}\text { Flush Volume } \\
\text { (Gal) }\end{array}$ \\
\hline FRP-08 & same & FEP-VSL-00017A & 141.15 \\
\hline FRP-08 & same & FEP-VSL-00017B & 139.77 \\
\hline FRP-08 & same & UFP-VSL-00001A & 156.35 \\
\hline FRP-08 & same & FRP-VSL-00002C & 73.45 \\
\hline FRP-09 & FRP-VSL-00002C & FEP-VSL-00017A & 245.10 \\
\hline FRP-09 & same & FEP-VSL-00017B & 139.54 \\
\hline FRP-09 & same & UFP-VSL-00001A & 156.12 \\
\hline FRP-09 & same & UFP-VSL-00001B & 158.19 \\
\hline FRP-09 & same & FRP-VSL-00002C & 73.45 \\
\hline FRP-10 & FRP-VSL-00002C & FEP-VSL-00017B & 243.37 \\
\hline FRP-10 & same & FEP-VSL-00017A & 140.92 \\
\hline FRP-10 & same & UFP-VSL-00001A & 156.12 \\
\hline FRP-10 & same & UFP-VSL-00001B & 158.19 \\
\hline FRP-10 & same & FRP-VSL-00002C & 73.45 \\
\hline FRP-11 & FRP-VSL-00002C & UFP-VSL-00001A & 264.09 \\
\hline FRP-11 & same & FEP-VSL-00017A & 140.92 \\
\hline FRP-11 & same & FEP-VSL-00017B & 139.54 \\
\hline FRP-11 & same & UFP-VSL-00001B & 158.19 \\
\hline FRP-11 & same & FRP-VSL-00002C & 73.45 \\
\hline FRP-12 & FRP-VSL-00002C & UFP-VSL-00001B & 266.68 \\
\hline FRP-12 & same & FEP-VSL-00017A & 140.92 \\
\hline FRP-12 & same & FEP-VSL-00017B & 139.54 \\
\hline FRP-12 & same & UFP-VSL-00001A & 156.12 \\
\hline FRP-12 & same & FRP-VSL-00002C & 73.45 \\
\hline FRP-13 & FRP-VSL-00002D & FEP-VSL-00017A & 296.68 \\
\hline FRP-13 & same & FEP-VSL-00017B & 139.54 \\
\hline FRP-13 & same & UFP-VSL-00001A & 156.12 \\
\hline FRP-13 & same & UFP-VSL-00001B & 158.19 \\
\hline FRP-13 & same & FRP-VSL-00002C & 73.45 \\
\hline FRP-14 & FRP-VSL-00002D & FEP-VSL-00017B & 294.95 \\
\hline FRP-14 & same & FEP-VSL-00017A & 140.92 \\
\hline FRP-14 & same & UFP-VSL-00001A & 156.12 \\
\hline FRP-14 & same & UFP-VSL-00001B & 158.19 \\
\hline FRP-14 & same & FRP-VSL-00002C & 73.45 \\
\hline FRP-15 & FRP-VSL-00002D & UFP-VSL-00001A & 315.68 \\
\hline FRP-15 & same & FEP-VSL-00017A & 140.92 \\
\hline FRP-15 & same & FEP-VSL-00017B & 139.54 \\
\hline FRP-15 & same & UFP-VSL-00001B & 158.19 \\
\hline FRP-15 & same & FRP-VSL-00002C & 73.45 \\
\hline FRP-16 & FRP-VSL-00002D & UFP-VSL-00001B & 318.27 \\
\hline FRP-16 & same & FEP-VSL-00017A & 140.92 \\
\hline FRP-16 & same & FEP-VSL-00017B & 139.54 \\
\hline FRP-16 & same & UFP-VSL-00001A & 156.12 \\
\hline FRP-16 & same & FRP-VSL-00002C & 73.45 \\
\hline FRP-17 & FRP-VSL-00002A & FRP-VSL-00002B & 172.66 \\
\hline
\end{tabular}




\begin{tabular}{|c|c|c|c|}
\hline STRN & Origin of Flush & Destination of Flush & $\begin{array}{l}\text { Flush Volume } \\
\text { (Gal) }\end{array}$ \\
\hline FRP-19 & FRP-VSL-00002B & FRP-VSL-00002B & 155.80 \\
\hline FRP-21 & FRP-VSL-00002C & FRP-VSL-00002C & 158.28 \\
\hline FRP-23 & FRP-VSL-00002D & FRP-VSL-00002C & 208.87 \\
\hline FRP-41 & Tank-Farm-HLW & HLP-VSL-00022 & 254.33 \\
\hline FRP-41 & same & FRP-VSL-00002D & 138.18 \\
\hline FRP-41 & same & FRP-VSL-00002C & 40.77 \\
\hline FRP-41 & same & FRP-VSL-00002B & 52.71 \\
\hline FRP-41 & same & FRP-VSL-00002A & 56.41 \\
\hline FRP-44 & Tank-Farm-LAW & FRP-VSL-00002A & 283.70 \\
\hline FRP-44 & same & HLP-VSL-00022 & 96.56 \\
\hline FRP-44 & same & FRP-VSL-00002D & 53.53 \\
\hline FRP-44 & same & FRP-VSL-00002C & 31.71 \\
\hline FRP-44 & same & FRP-VSL-00002B & 43.65 \\
\hline FRP-45 & Tank-Farm-LAW & FRP-VSL-00002B & 279.07 \\
\hline FRP-45 & same & HLP-VSL-00022 & 96.56 \\
\hline FRP-45 & same & FRP-VSL-00002D & 53.53 \\
\hline FRP-45 & same & FRP-VSL-00002C & 31.71 \\
\hline FRP-45 & same & FRP-VSL-00002A & 47.36 \\
\hline FRP-46 & Tank-Farm-LAW & FRP-VSL-00002C & 264.14 \\
\hline FRP-46 & same & HLP-VSL-00022 & 96.56 \\
\hline FRP-46 & same & FRP-VSL-00002D & 53.53 \\
\hline FRP-46 & same & FRP-VSL-00002B & 43.65 \\
\hline FRP-46 & same & FRP-VSL-00002A & 47.36 \\
\hline FRP-47 & Tank-Farm-LAW & FRP-VSL-00002D & 291.42 \\
\hline FRP-47 & same & HLP-VSL-00022 & 96.56 \\
\hline FRP-47 & same & FRP-VSL-00002C & 31.71 \\
\hline FRP-47 & same & FRP-VSL-00002B & 43.65 \\
\hline FRP-47 & same & FRP-VSL-00002A & 47.36 \\
\hline HLP-02 & HLP-VSL-00022 & HLP-VSL-00022 & 262.79 \\
\hline HLP-03 & HLP-VSL-00022 & UFP-VSL-00001A & 211.27 \\
\hline HLP-03 & same & HLP-VSL-00022 & 67.70 \\
\hline HLP-03 & same & HLP-VSL-00022 & 72.53 \\
\hline HLP-03 & same & UFP-VSL-00001B & 133.32 \\
\hline HLP-03 & same & FEP-VSL-00017A & 159.11 \\
\hline HLP-03 & same & FEP-VSL-00017B & 127.11 \\
\hline HLP-03 & same & FRP-VSL-00002A & 315.04 \\
\hline HLP-04 & HLP-VSL-00022 & UFP-VSL-00001B & 210.40 \\
\hline HLP-04 & same & HLP-VSL-00022 & 67.70 \\
\hline HLP-04 & same & HLP-VSL-00022 & 72.53 \\
\hline HLP-04 & same & UFP-VSL-00001A & 134.01 \\
\hline HLP-04 & same & FEP-VSL-00017A & 159.11 \\
\hline HLP-04 & same & FEP-VSL-00017B & 127.11 \\
\hline HLP-04 & same & FRP-VSL-00002A & 315.04 \\
\hline HLP-05 & HLP-VSL-00022 & FEP-VSL-00017A & 242.64 \\
\hline HLP-05 & same & HLP-VSL-00022 & 67.70 \\
\hline
\end{tabular}




\begin{tabular}{|c|c|c|c|}
\hline STRN & Origin of Flush & Destination of Flush & $\begin{array}{l}\text { Flush Volume } \\
\text { (Gal) }\end{array}$ \\
\hline HLP-05 & same & HLP-VSL-00022 & 72.53 \\
\hline HLP-05 & same & UFP-VSL-00001A & 134.01 \\
\hline HLP-05 & same & UFP-VSL-00001B & 133.32 \\
\hline HLP-05 & same & FEP-VSL-00017B & 127.11 \\
\hline HLP-05 & same & FRP-VSL-00002A & 315.04 \\
\hline HLP-06 & HLP-VSL-00022 & FEP-VSL-00017B & 202.63 \\
\hline HLP-06 & same & HLP-VSL-00022 & 67.70 \\
\hline HLP-06 & same & HLP-VSL-00022 & 72.53 \\
\hline HLP-06 & same & UFP-VSL-00001A & 134.01 \\
\hline HLP-06 & same & UFP-VSL-00001B & 133.32 \\
\hline HLP-06 & same & FEP-VSL-00017A & 159.11 \\
\hline HLP-06 & same & FRP-VSL-00002A & 315.04 \\
\hline HLP-11 & HLP-VSL-00028 & HLP-VSL-00028 & 326.91 \\
\hline HLP-13 & HLP-VSL-00028 & HFP-VSL-00005 & 1.00 \\
\hline HLP-13 & same & HLP-VSL-00028 & 51.64 \\
\hline HLP-13 & same & HLP-VSL-00028 & 88.31 \\
\hline HLP-13 & same & PWD-VSL-00043 & 923.91 \\
\hline HLP-15 & HLP-VSL-00028 & HFP-VSL-00001 & 1.00 \\
\hline HLP-15 & same & HLP-VSL-00028 & 51.64 \\
\hline HLP-15 & same & HLP-VSL-00028 & 88.31 \\
\hline HLP-15 & same & PWD-VSL-00043 & 905.64 \\
\hline HLP-20 & HLP-VSL-00027A & HLP-VSL-00027A & 300.61 \\
\hline HLP-22 & HLP-VSL-00027A & HLP-VSL-00028 & 144.43 \\
\hline HLP-22 & same & HLP-VSL-00027A & 93.55 \\
\hline HLP-22 & same & HLP-VSL-00027A & 48.64 \\
\hline HLP-22 & same & HLP-VSL-00027B & 23.70 \\
\hline HLP-22 & same & PWD-VSL-00033 & 30.71 \\
\hline HLP-24 & HLP-VSL-00027B & HLP-VSL-00027B & 245.31 \\
\hline HLP-26 & HLP-VSL-00027B & HLP-VSL-00028 & 119.72 \\
\hline HLP-26 & same & HLP-VSL-00027B & 91.05 \\
\hline HLP-26 & same & HLP-VSL-00027B & 40.66 \\
\hline HLP-26 & same & HLP-VSL-00027A & 45.65 \\
\hline HLP-26 & same & PWD-VSL-00033 & 30.71 \\
\hline RDP-21 & RDP-VSL-00002B & RDP-VSL-00002B & 431.74 \\
\hline TCP-03 & TCP-VSL-00001 & LCP-VSL-00001 & 642.60 \\
\hline TCP-03 & same & PWD-VSL-00033 & 495.00 \\
\hline TCP-03 & same & TLP-VSL-00009A & 50.43 \\
\hline TCP-03 & same & TLP-VSL-00009B & 58.22 \\
\hline TCP-03 & same & TCP-VSL-00001 & 122.42 \\
\hline TCP-04 & TCP-VSL-00001 & LCP-VSL-00002 & 664.10 \\
\hline TCP-04 & same & PWD-VSL-00033 & 495.00 \\
\hline TCP-04 & same & TLP-VSL-00009A & 43.60 \\
\hline TCP-04 & same & TLP-VSL-00009B & 51.39 \\
\hline TCP-04 & same & TCP-VSL-00001 & 111.83 \\
\hline TLP-07 & TLP-VSL-00009A & TLP-VSL-00009A & 74.76 \\
\hline
\end{tabular}




\begin{tabular}{|c|c|c|c|}
\hline STRN & Origin of Flush & Destination of Flush & $\begin{array}{l}\text { Flush Volume } \\
\text { (Gal) }\end{array}$ \\
\hline TLP-08 & TLP-VSL-00009B & TLP-VSL-00009A & 77.87 \\
\hline TLP-10 & TLP-SEP-00001 & TCP-VSL-00001 & 86.36 \\
\hline UFP-02 & UFP-VSL-00001A & UFP-VSL-00001A & 139.21 \\
\hline UFP-05 & UFP-VSL-00001A & UFP-VSL-00002B & 96.76 \\
\hline UFP-05 & same & UFP-VSL-00001A & 26.41 \\
\hline UFP-05 & same & UFP-VSL-00001A & 65.78 \\
\hline UFP-05 & same & FEP-VSL-00017B & 50.28 \\
\hline UFP-05 & same & FEP-VSL-00017A & 63.02 \\
\hline UFP-05 & same & UFP-VSL-00002A & 54.96 \\
\hline UFP-06 & UFP-VSL-00001A & UFP-VSL-00002A & 80.69 \\
\hline UFP-06 & same & UFP-VSL-00001A & 26.41 \\
\hline UFP-06 & same & UFP-VSL-00001A & 65.78 \\
\hline UFP-06 & same & FEP-VSL-00017B & 50.28 \\
\hline UFP-06 & same & FEP-VSL-00017A & 63.02 \\
\hline UFP-06 & same & UFP-VSL-00002B & 67.81 \\
\hline UFP-08 & UFP-VSL-00001B & UFP-VSL-00001B & 220.72 \\
\hline UFP-11 & UFP-VSL-00001B & UFP-VSL-00002B & 152.79 \\
\hline UFP-11 & same & UFP-VSL-00001B & 36.07 \\
\hline UFP-11 & same & UFP-VSL-00001B & 104.96 \\
\hline UFP-11 & same & FEP-VSL-00017B & 87.85 \\
\hline UFP-11 & same & FEP-VSL-00017A & 100.59 \\
\hline UFP-11 & same & UFP-VSL-00002A & 90.23 \\
\hline UFP-12 & UFP-VSL-00001B & UFP-VSL-00002A & 134.99 \\
\hline UFP-12 & same & UFP-VSL-00001B & 36.07 \\
\hline UFP-12 & same & UFP-VSL-00001B & 104.96 \\
\hline UFP-12 & same & FEP-VSL-00017B & 87.85 \\
\hline UFP-12 & same & FEP-VSL-00017A & 100.59 \\
\hline UFP-12 & same & UFP-VSL-00002B & 104.46 \\
\hline UFP-13 ${ }^{\text {a) }}$ & UFP-VSL-00002A & PWD-VSL-00043 & 0.00 \\
\hline UFP-13 & same & UFP-VSL-00002A & 0.00 \\
\hline UFP-13 & same & HLP-VSL-00027A & 0.00 \\
\hline UFP-15 ${ }^{\text {a) }}$ & UFP-VSL-00002A & HLP-VSL-00027A & 126.90 \\
\hline UFP-15 & same & UFP-VSL-00002A & 152.25 \\
\hline UFP-15 & same & UFP-VSL-00002A & $1,520.30$ \\
\hline UFP-15 & same & HLP-VSL-00027B & 202.60 \\
\hline UFP-15 & same & HLP-VSL-00028 & 186.92 \\
\hline UFP-16 ${ }^{\text {a) }}$ & UFP-VSL-00002A & HLP-VSL-00027B & 103.30 \\
\hline UFP-16 & same & UFP-VSL-00002A & 152.25 \\
\hline UFP-16 & same & UFP-VSL-00002A & $1,520.30$ \\
\hline UFP-16 & same & HLP-VSL-00027A & 220.64 \\
\hline UFP-16 & same & HLP-VSL-00028 & 202.60 \\
\hline UFP-18 ${ }^{\text {a) }}$ & UFP-VSL-00002B & PWD-VSL-00043 & 0.00 \\
\hline UFP-18 & same & UFP-VSL-00002B & 0.00 \\
\hline UFP-18 & same & HLP-VSL-00027A & 0.00 \\
\hline UFP-20 a) & UFP-VSL-00002B & HLP-VSL-00027A & 126.90 \\
\hline
\end{tabular}




\begin{tabular}{|c|c|c|c|}
\hline STRN & Origin of Flush & Destination of Flush & $\begin{array}{l}\text { Flush Volume } \\
\text { (Gal) }\end{array}$ \\
\hline UFP-20 & same & UFP-VSL-00002B & 114.22 \\
\hline UFP-20 & same & UFP-VSL-00002B & $1,552.25$ \\
\hline UFP-20 & same & HLP-VSL-00027B & 192.02 \\
\hline UFP-20 & same & HLP-VSL-00028 & 174.85 \\
\hline UFP-21 ${ }^{\text {a) }}$ & UFP-VSL-00002B & HLP-VSL-00027B & 103.30 \\
\hline UFP-21 & same & UFP-VSL-00002B & 114.22 \\
\hline UFP-21 & same & UFP-VSL-00002B & $1,552.25$ \\
\hline UFP-21 & same & HLP-VSL-00027A & 211.56 \\
\hline UFP-21 & same & HLP-VSL-00028 & 174.85 \\
\hline HLW-04 & HFP-VSL-00001 & HFP-VSL-00002 & 7.72 \\
\hline HLW-06 & HFP-VSL-00001 & HFP-VSL-00001 & 29.70 \\
\hline HLW-17 & HFP-VSL-00005 & HFP-VSL-00006 & 6.62 \\
\hline HLW-19 & HFP-VSL-00005 & HFP-VSL-00005 & 13.43 \\
\hline HLW-29 & HOP-SCB-00001 & RLD-VSL-00007 & 36.35 \\
\hline HLW-35 & HOP-VSL-00903 & RLD-VSL-00007 & 64.76 \\
\hline HLW-37 & HOP-HEME-00001 & HOP-VSL-00903 & 26.77 \\
\hline HLW-39 & HOP-SCB-00002 & RLD-VSL-00007 & 35.94 \\
\hline HLW-44 & HOP-VSL-00904 & RLD-VSL-00007 & 79.85 \\
\hline HLW-47 & HOP-HEME-00002 & HOP-VSL-00904 & 12.55 \\
\hline HLW-51 & RLD-VSL-00007 & PWD-VSL-00043 & 119.09 \\
\hline HLW-55 & RLD-VSL-00007 & RLD-VSL-00007 & 29.46 \\
\hline HLW-81 & HDH-VSL-00003 & RLD-VSL-00007 & 94.55 \\
\hline HLW-85 & HDH-VSL-00001 & HDH-VSL-00003 & 34.94 \\
\hline LCP-1 & LCP-VSL-00001 & LCP-VSL-00001 & 28.00 \\
\hline LCP-2 & LCP-VSL-00002 & LCP-VSL-00002 & 43.00 \\
\hline LFP-1 & LCP-VSL-00001 & LFP-VSL-00001 & 15.00 \\
\hline LFP-1 & same & LCP-VSL-00001 & 10.00 \\
\hline LFP-2 & LCP-VSL-00001 & LFP-VSL-00003 & 26.00 \\
\hline LFP-2 & same & LCP-VSL-00001 & 10.00 \\
\hline LFP-3 & LCP-VSL-00002 & LFP-VSL-00001 & 11.00 \\
\hline LFP-3 & same & LCP-VSL-00002 & 10.00 \\
\hline LFP-4 & LCP-VSL-00002 & LFP-VSL-00003 & 16.00 \\
\hline LFP-4 & same & LCP-VSL-00002 & 10.00 \\
\hline LFP-5 & LFP-VSL-00001 & LFP-VSL-00001 & 79.00 \\
\hline LFP-6 & LFP-VSL-00003 & LFP-VSL-00003 & 111.00 \\
\hline LFP-7 & LFP-VSL-00001 & LFP-VSL-00002 & 18.00 \\
\hline LFP-7 & same & LFP-VSL-00001 & 27.00 \\
\hline LFP-8 & LFP-VSL-00003 & LFP-VSL-00004 & 18.00 \\
\hline LFP-8 & same & LFP-VSL-00003 & 28.00 \\
\hline LFP-9 & LFP-VSL-00002 & LMP-MLTR-00001 & 6.00 \\
\hline LFP-9 & same & LFP-VSL-00002 & 12.00 \\
\hline LFP-10 & LFP-VSL-00004 & LMP-MLTR-00002 & 6.00 \\
\hline LFP-10 & same & LFP-VSL-00004 & 12.00 \\
\hline LFP-11 & LFP-VSL-00002 & LMP-MLTR-00001 & 6.00 \\
\hline LFP-12 & LFP-VSL-00004 & LMP-MLTR-00002 & 6.00 \\
\hline
\end{tabular}




\begin{tabular}{|l|l|l|l|}
\hline STRN & Origin of Flush & Destination of Flush & $\begin{array}{l}\text { Flush Volume } \\
\text { (Gal) }\end{array}$ \\
\hline HLP27A $^{\text {b) }}$ & FEP-SEP-00001A & HLP-VSL-00027A & 130.00 \\
\hline FRP2A ${ }^{\text {b) }}$ & FEP-SEP-00001A & FRP-VSL-00002A & 130.00 \\
\hline UFP1 $^{\text {b) }}$ & HLP-VSL-00027A & UFP-VSL-00001A & 200.00 \\
\hline UFP1 $^{\text {b) }}$ & HLP-VSL-00027A & UFP-VSL-00001B & 200.00 \\
\hline
\end{tabular}

Notes:

a) The transfer line flushes have been replaced by the power flush, positive displacement pump forward feeding, and circulation loop draining operations discussed in Section 4.8.3.

b) Reserved line flush operations for upfront leaching flowsheet. 


\section{Appendix I}

\section{Feed Vector Charge Balance}




\section{Appendix I \\ Feed Vector Charge Balance}

Tank Farm supplied feed vectors must be charge balanced before they can be applied to the Dynamic (G2) Model. The feed vector component arrays must also be expanded to account for the new components used for charge balancing and also for other components specific to the WTP operations. Table I-1 provides a side-by-side comparison of the liquid/solid components given by the Tank Farm and utilized in the G2 Model.

The charge balance operations include: 1) converting all liquid phase $\mathrm{Al}+3$ to aluminate, 2) converting leachable solid phase $\mathrm{Al}+3$ to gibbsite, and 3) converting unleachable solid phase PO4-3 to PO43 (HARD). Liquid phase aluminum exists in the form of aluminate and therefore all the $\mathrm{Al}+3$ (1) in the feed vector are converted to aluminate according to the following reaction:

$\mathrm{Al}+3(\mathrm{l})+4 \mathrm{OH}($ Bound $)(\mathrm{l}) \rightarrow \mathrm{Al}(\mathrm{OH}) 4-(\mathrm{l})$

If liquid phase $\mathrm{OH}$ (Bound) is limiting, liquid phase $\mathrm{CO} 3-2, \mathrm{NO} 2-$, and NO3- will be used to substitute the deficit part of the $\mathrm{OH}$ (Bound). The anions for substitution will be consumed in the order specified above. Unlike the other anions used for charge balancing, CO3-2 carries two negative charges, which means that $2 \mathrm{~kg}$-moles of CO3-2 will convert $1 \mathrm{~kg}$-mole of $\mathrm{Al}+3$.

The leachable part of the $\mathrm{Al}+3$ (s) in the feed vector is converted to gibbsite according to the following reaction:

$\mathrm{Al}+3(\mathrm{~s})+3 \mathrm{OH}(\mathrm{Bound})(\mathrm{s}) \rightarrow \mathrm{Al}(\mathrm{OH}) 3(\mathrm{~s})$

If solid phase $\mathrm{OH}$ (Bound) is limiting, the deficit amount will be added forcefully. It shall be noted that the charge balance operations may cause the overall mass to alter, which has typically been insignificant.

All HLW batch clusters that are greater than $10 \mathrm{wt} \%$ solids by mass are diluted with preceding or succeeding LAW batches to $10 \mathrm{wt} \%$. The new leach factors for each modified batch are the weighted average by total solids mass between the original HLW batch and the portion of the LAW batch(es) added. Each LAW batch is delivered with whatever volume is left after diluting HLW batches.

Table I-1 Comparison of Feed Vector Components

\begin{tabular}{|l|l|}
\hline Tank Farm Components & Dynamic (G2) Model Components \\
\hline $106-\mathrm{Ru}$ & $106-\mathrm{Ru}$ \\
\hline $113 \mathrm{~m}-\mathrm{Cd}$ & $113 \mathrm{~m}-\mathrm{Cd}$ \\
\hline $125-\mathrm{Sb}$ & $125-\mathrm{Sb}$ \\
\hline $126-\mathrm{Sn}$ & $126-\mathrm{Sn}$ \\
\hline $129-\mathrm{I}$ & $129-\mathrm{I}$ \\
\hline $134-\mathrm{Cs}$ & $134-\mathrm{Cs}$ \\
\hline $137-\mathrm{Cs}$ & $137-\mathrm{Cs}$ \\
\hline $137 \mathrm{~m}-\mathrm{Ba}$ & $137 \mathrm{~m}-\mathrm{Ba}$ \\
\hline $14-\mathrm{C}$ & $14-\mathrm{C}$ \\
\hline $151-\mathrm{Sm}$ & $151-\mathrm{Sm}$ \\
\hline $152-\mathrm{Eu}$ & $152-\mathrm{Eu}$ \\
\hline $154-\mathrm{Eu}$ & $154-\mathrm{Eu}$ \\
\hline
\end{tabular}




\begin{tabular}{|c|c|}
\hline Tank Farm Components & Dynamic (G2) Model Components \\
\hline 155-Eu & 155-Eu \\
\hline $226-\mathrm{Ra}$ & 226-Ra \\
\hline $227-\mathrm{Ac}$ & $227-A c$ \\
\hline 228-Ra & 228-Ra \\
\hline 229-Th & 229-Th \\
\hline $231-\mathrm{Pa}$ & 231-Pa \\
\hline 232-Th & 232-Th \\
\hline $232-\mathrm{U}$ & $232-\mathrm{U}$ \\
\hline $233-\mathrm{U}$ & $233-U$ \\
\hline $234-\mathrm{U}$ & 234-U \\
\hline $235-\mathrm{U}$ & $235-\mathrm{U}$ \\
\hline $236-\mathrm{U}$ & $236-U$ \\
\hline $237-\mathrm{Np}$ & $237-\mathrm{Np}$ \\
\hline $238-\mathrm{Pu}$ & 238-Pu \\
\hline $238-\mathrm{U}$ & $238-U$ \\
\hline $239-\mathrm{Pu}$ & 239-Pu \\
\hline $240-\mathrm{Pu}$ & $240-\mathrm{Pu}$ \\
\hline 241-Am & 241-Am \\
\hline $241-\mathrm{Pu}$ & $241-\mathrm{Pu}$ \\
\hline $242-\mathrm{Cm}$ & $242-\mathrm{Cm}$ \\
\hline $242-\mathrm{Pu}$ & 242-Pu \\
\hline 243-Am & 243-Am \\
\hline $243-\mathrm{Cm}$ & $243-\mathrm{Cm}$ \\
\hline $244-\mathrm{Cm}$ & 244-Cm \\
\hline $3-\mathrm{H}$ & $3-\mathrm{H}$ \\
\hline $59-\mathrm{Ni}$ & $59-\mathrm{Ni}$ \\
\hline 60-Co & $60-\mathrm{Co}$ \\
\hline $63-\mathrm{Ni}$ & $63-\mathrm{Ni}$ \\
\hline 79-Se & 79-Se \\
\hline $90-\mathrm{Sr}$ & 90-Sr \\
\hline $90-\mathrm{Y}$ & $90-\mathrm{Y}$ \\
\hline $93-\mathrm{Zr}$ & $93-\mathrm{Zr}$ \\
\hline $93 \mathrm{~m}-\mathrm{Nb}$ & $93 \mathrm{~m}-\mathrm{Nb}$ \\
\hline 99-Tc & 99-Tc \\
\hline$\overline{\mathrm{Ag}+}$ & $\mathrm{Ag}+$ \\
\hline $\mathrm{Al}(\mathrm{OH}) 4-$ & $\mathrm{Al}(\mathrm{OH}) 4-$ \\
\hline $\mathrm{Al}+3$ & $\mathrm{Al}+3$ \\
\hline $\mathrm{As}+5$ & $\mathrm{As}+5$ \\
\hline $\mathrm{B}+3$ & $\mathrm{~B}+3$ \\
\hline $\mathrm{Ba}+2$ & $\mathrm{Ba}+2$ \\
\hline $\mathrm{Be}+2$ & $\mathrm{Be}+2$ \\
\hline $\mathrm{Bi}+3$ & $\mathrm{Bi}+3$ \\
\hline C10H12N2O8-4 & C10H12N2O8-4 \\
\hline $\mathrm{C} 10 \mathrm{H} 15 \mathrm{~N} 2 \mathrm{O} 7-3$ & $\mathrm{C} 10 \mathrm{H} 15 \mathrm{~N} 2 \mathrm{O} 7-3$ \\
\hline $\mathrm{C} 12 \mathrm{H} 27 \mathrm{O} 4 \mathrm{P}$ & $\mathrm{C} 12 \mathrm{H} 27 \mathrm{O} 4 \mathrm{P}$ \\
\hline \begin{tabular}{|l}
$\mathrm{C} 13 \mathrm{H} 28$ \\
\end{tabular} & $\mathrm{C} 13 \mathrm{H} 28$ \\
\hline $\mathrm{C} 2 \mathrm{Cl} 4$ & $\mathrm{C} 2 \mathrm{Cl} 4$ \\
\hline C2H3O2- & C2H3O2- \\
\hline
\end{tabular}




\begin{tabular}{|c|c|}
\hline Tank Farm Components & Dynamic (G2) Model Components \\
\hline C2H3O3- & $\mathrm{C} 2 \mathrm{H} 3 \mathrm{O} 3-$ \\
\hline $\mathrm{C} 2 \mathrm{HCl} 3$ & $\mathrm{C} 2 \mathrm{HCl} 3$ \\
\hline $\mathrm{C} 2 \mathrm{O} 4-2$ & $\mathrm{C} 2 \mathrm{O} 4-2$ \\
\hline $\mathrm{C} 3 \mathrm{H} 6 \mathrm{O}$ & $\mathrm{C} 3 \mathrm{H} 6 \mathrm{O}$ \\
\hline $\mathrm{C} 4 \mathrm{H} 10 \mathrm{O}$ & $\mathrm{C} 4 \mathrm{H} 10 \mathrm{O}$ \\
\hline $\mathrm{C} 4 \mathrm{H} 8 \mathrm{O}$ & $\mathrm{C} 4 \mathrm{H} 8 \mathrm{O}$ \\
\hline C5H5N & C5H5N \\
\hline C6H5NO2 & C6H5NO2 \\
\hline C6H5O7-3 & C6H5O7-3 \\
\hline C6H6 & C6H6 \\
\hline C7H6N2O4 & $\mathrm{C} 7 \mathrm{H} 6 \mathrm{~N} 2 \mathrm{O} 4$ \\
\hline $\mathrm{C} 7 \mathrm{H} 8 \mathrm{O}$ & $\mathrm{C} 7 \mathrm{H} 8 \mathrm{O}$ \\
\hline $\mathrm{Ca}+2$ & $\mathrm{Ca}+2$ \\
\hline $\mathrm{Cd}+2$ & $\mathrm{Cd}+2$ \\
\hline $\mathrm{Ce}+3$ & $\mathrm{Ce}+3$ \\
\hline $\mathrm{CH} 2 \mathrm{Cl} 2$ & $\mathrm{CH} 2 \mathrm{Cl} 2$ \\
\hline $\mathrm{CHCl} 3$ & $\mathrm{CHCl} 3$ \\
\hline CHO2- & $\mathrm{CHO} 2-$ \\
\hline $\mathrm{Cl}-$ & $\mathrm{Cl}-$ \\
\hline $\mathrm{CN}-$ & $\mathrm{CN}-$ \\
\hline $\mathrm{Co}+3$ & $\mathrm{Co}+3$ \\
\hline $\mathrm{CO} 3-2$ & $\mathrm{CO} 3-2$ \\
\hline $\mathrm{Cr}(\mathrm{OH}) 4-$ & $\mathrm{Cr}(\mathrm{OH}) 4-$ \\
\hline Cr(TOTAL) & Cr(TOTAL) \\
\hline$\overline{\mathrm{Cs}+}$ & $\mathrm{Cs}+$ \\
\hline $\mathrm{Cu}+2$ & $\mathrm{Cu}+2$ \\
\hline F- & F- \\
\hline $\mathrm{Fe}+3$ & $\mathrm{Fe}+3$ \\
\hline $\mathrm{H}+$ & $\mathrm{H}+$ \\
\hline $\mathrm{H} 2 \mathrm{O}$ & $\mathrm{H} 2 \mathrm{O}$ \\
\hline $\mathrm{H} 2 \mathrm{O} 2$ & $\mathrm{H} 2 \mathrm{O} 2$ \\
\hline $\mathrm{Hg}+2$ & $\mathrm{Hg}+2$ \\
\hline $\mathrm{K}+$ & $\mathrm{K}+$ \\
\hline $\mathrm{La}+3$ & $\mathrm{La}+3$ \\
\hline \begin{tabular}{|l}
$\mathrm{Li}+$ \\
\end{tabular} & $\mathrm{Li}+$ \\
\hline $\mathrm{Mg}+2$ & $\mathrm{Mg}+2$ \\
\hline $\mathrm{Mn}+4$ & $\mathrm{Mn}+4$ \\
\hline $\begin{array}{l}\mathrm{MnO} 2 \\
\end{array}$ & $\mathrm{MnO} 2$ \\
\hline MnO4- & MnO4- \\
\hline $\mathrm{Mo}+6$ & $\mathrm{Mo}+6$ \\
\hline $\mathrm{Na}+$ & $\mathrm{Na}+$ \\
\hline $\mathrm{Nd}+3$ & $\mathrm{Nd}+3$ \\
\hline \begin{tabular}{|l}
$\mathrm{NH} 3$ \\
\end{tabular} & NH3 \\
\hline NH4+ & $\mathrm{Ni}+2$ \\
\hline $\mathrm{Ni}+2$ & NO2- \\
\hline NO2- & NO3- \\
\hline NO3- & $\mathrm{OH}(\mathrm{BOUND})$ \\
\hline$\overline{\mathrm{O}(\mathrm{BOUND})}$ & $\mathrm{OH}-$ \\
\hline
\end{tabular}




\begin{tabular}{|c|c|}
\hline Tank Farm Components & Dynamic (G2) Model Components \\
\hline $\mathrm{OH}(\mathrm{BOUND})$ & $\mathrm{Pb}+2$ \\
\hline $\mathrm{OH}-$ & $\mathrm{Pd}+2$ \\
\hline $\mathrm{Pb}+2$ & PO4-3 \\
\hline $\mathrm{Pd}+2$ & $\operatorname{Pr}+3$ \\
\hline PO4-3 & $\mathrm{Pu}+4$ \\
\hline $\mathrm{Pr}+3$ & $\mathrm{Rb}+$ \\
\hline $\mathrm{Pu}+4$ & $\mathrm{Rh}+3$ \\
\hline $\mathrm{Rb}+$ & $\mathrm{Ru}+3$ \\
\hline $\mathrm{Rh}+3$ & $\mathrm{Sb}+5$ \\
\hline $\mathrm{Ru}+3$ & $\mathrm{Se}+6$ \\
\hline $\mathrm{Sb}+5$ & $\mathrm{Si}+4$ \\
\hline $\mathrm{Se}+6$ & SO4-2 \\
\hline $\mathrm{Si}+4$ & $\mathrm{Sr}+2$ \\
\hline \begin{tabular}{|l|} 
SO4-2 \\
\end{tabular} & $\mathrm{Ta}+5$ \\
\hline $\mathrm{Sr}+2$ & $\mathrm{Tc}+7$ \\
\hline $\mathrm{Ta}+5$ & $\mathrm{Te}+6$ \\
\hline $\mathrm{Tc}+7$ & $\mathrm{Th}+4$ \\
\hline $\mathrm{Te}+6$ & $\mathrm{Ti}+4$ \\
\hline $\mathrm{Th}+4$ & $\mathrm{Tl}+3$ \\
\hline $\mathrm{Ti}+4$ & TOC \\
\hline $\mathrm{Tl}+3$ & U(TOTAL) \\
\hline TOC & $\mathrm{V}+5$ \\
\hline U(TOTAL) & $\mathrm{W}+6$ \\
\hline $\mathrm{V}+5$ & $\mathrm{Y}+3$ \\
\hline $\mathrm{W}+6$ & $\mathrm{Zn}+2$ \\
\hline $\mathrm{Y}+3$ & $\mathrm{Zr}+4$ \\
\hline $\mathrm{Zn}+2$ & $\mathrm{C} 10 \mathrm{H} 8$ \\
\hline \multirow[t]{21}{*}{$\mathrm{Zr}+4$} & $\mathrm{C} 12 \mathrm{H} 4 \mathrm{Cl} 6$ \\
\hline & $\mathrm{C} 12 \mathrm{H} 8$ \\
\hline & $\mathrm{C} 2 \mathrm{H} 3 \mathrm{~N}$ \\
\hline & $\mathrm{C} 2 \mathrm{H} 6 \mathrm{SiO}$ \\
\hline & $\mathrm{C} 2 \mathrm{H} 8 \mathrm{~N} 2$ \\
\hline & $\mathrm{H} 2 \mathrm{O}(\mathrm{BOUND})$ \\
\hline & $\mathrm{C} 4 \mathrm{H} 7 \mathrm{NO} 4$ \\
\hline & C6H6O \\
\hline & C7H8 \\
\hline & $\mathrm{C} 8 \mathrm{H} 8 \mathrm{O}$ \\
\hline & $\mathrm{CCl} 4$ \\
\hline & $\mathrm{H} 2 \mathrm{CO} 3$ \\
\hline & HCO3- \\
\hline & Mnp \\
\hline & Nap + \\
\hline & NH4+ \\
\hline & $\mathrm{O}-2$ \\
\hline & $\mathrm{Al}(\mathrm{OH}) 3$ \\
\hline & PO4-3(HARD) \\
\hline & PPG \\
\hline & DPPG \\
\hline
\end{tabular}




\begin{tabular}{|l|l|}
\hline Tank Farm Components & Dynamic (G2) Model Components \\
\hline & PDMS \\
\hline & DPDMS \\
\hline & NO3-D \\
\hline & AlOOH \\
\hline & LS9 \\
\hline & LS10 \\
\hline
\end{tabular}




\section{Appendix $\mathbf{J}$}

\section{Mass Balance Flowsheet Diagrams and Volume Throughput}




\section{Appendix J Mass Balance Flowsheet Diagrams and Volume Throughput}

Simplified mass balance flowsheet diagrams are developed to support flowsheet developments and model validations. These diagrams present the cumulative masses for process streams on the systems level. The diagrams are presented in four Excel spreadsheets, namely: PT-1, PT-2, LAW, and HLW as shown in Figures J-1 through J-4. Types of mass balance and flowsheet diagrams include: Total Mass, Total Volume, Radioactivity, Aluminum, Chromium, Iron, Sodium, Oxalate, Cesium 137, Strontium 90, Iodine 129, Technetium 99, Mercury, Water, AFA, and Phosphate. The mass balance is obtained using the G2 totalizer data for process streams into and out of the systems. Table J-1 shows the totalizer files and associated systems, stream names, stream numbers, and waste types. The Visual C\# routine "G2MBC" is used to perform the calculations. Source codes of the G2MBC are presented in Table J-2. Similarities and differences between the totalizer files in Tables J-1 and N-1 shall be distinguished. Table J-1 provides the totalizer files for process streams on the systems level such that internal streams are excluded. Table N-1 lists the totalizer files for every process streams in the flowsheet including those internal.

In addition to the mass balance flowsheet diagrams above, volume throughputs for some key unit operations are also of particular interests in performance analysis. These volume throughputs are extracted from G2 totalizer files for the process streams specified in the input using the Visual C\# routine "TVExtractor". Table J-3 shows the results of an example application where process condensate utilization by various unit operations are summarized. Source codes of the TVExtractor are presented in Table J-4. 


\section{Figure J-1 Mass Balance Flowsheet Diagram (PT-1)}

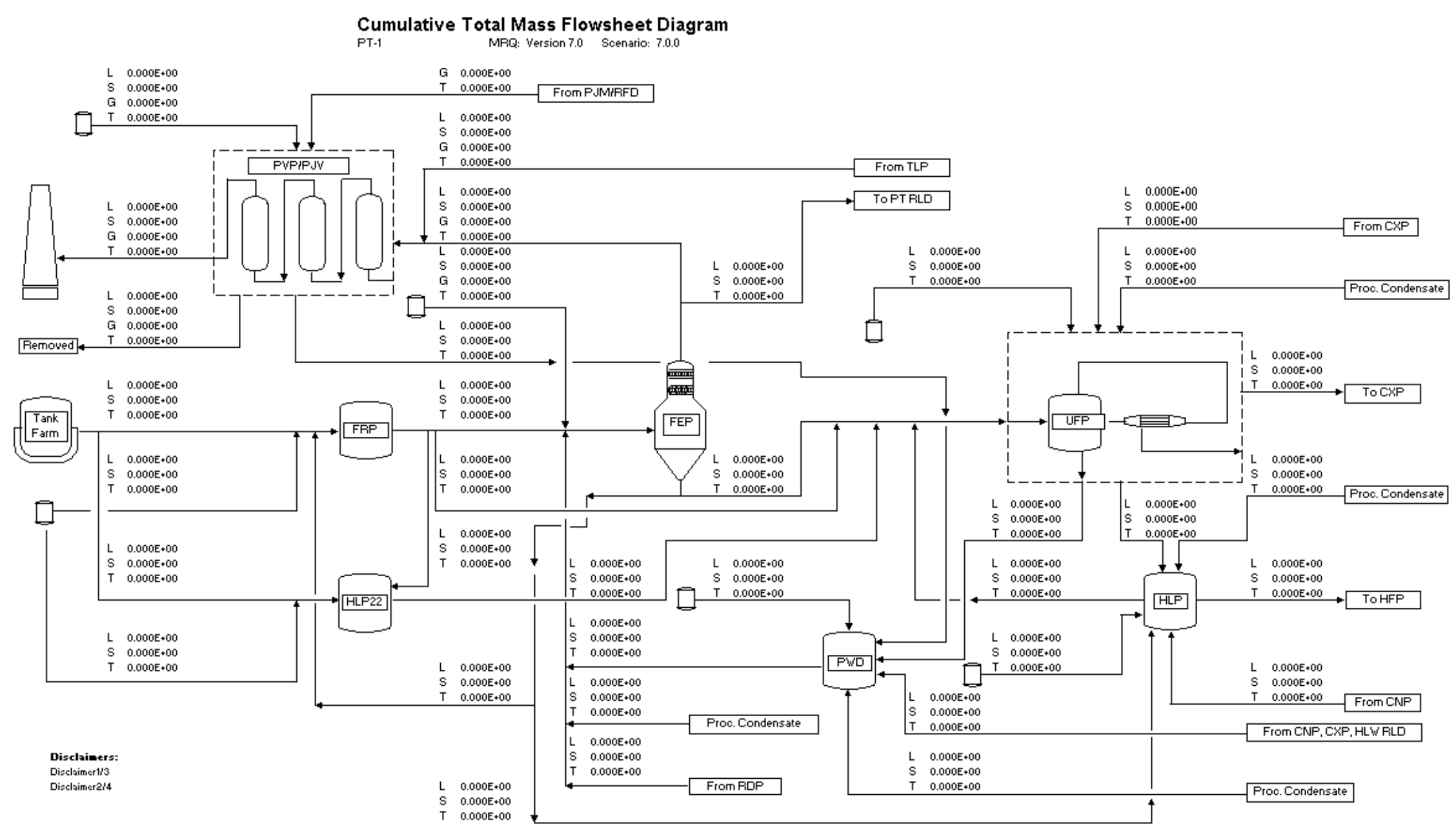




\section{Figure J-2 Mass Balance Flowsheet Diagram (PT-2)}

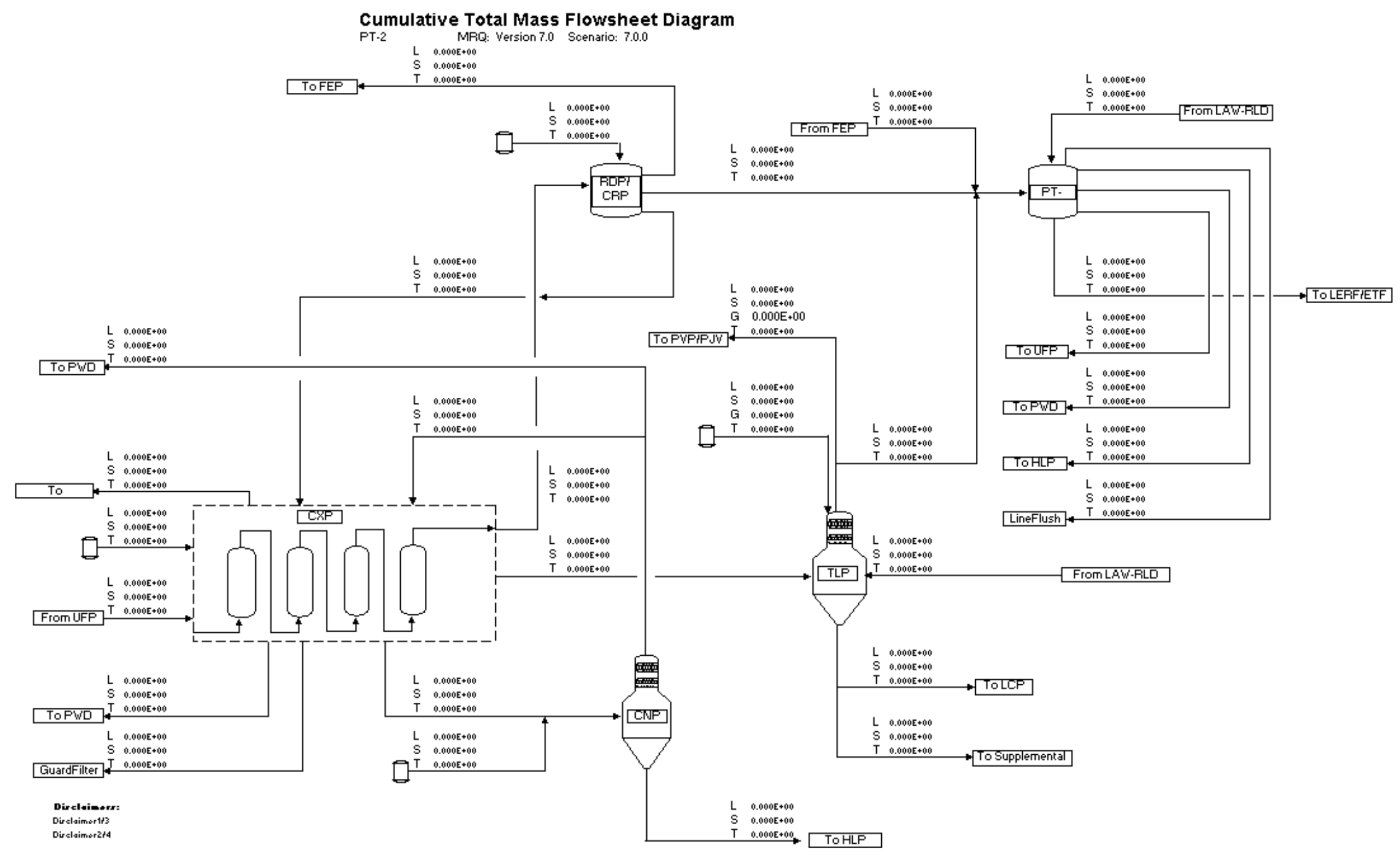




\section{Figure J-3 Mass Balance Flowsheet Diagram (LAW)}

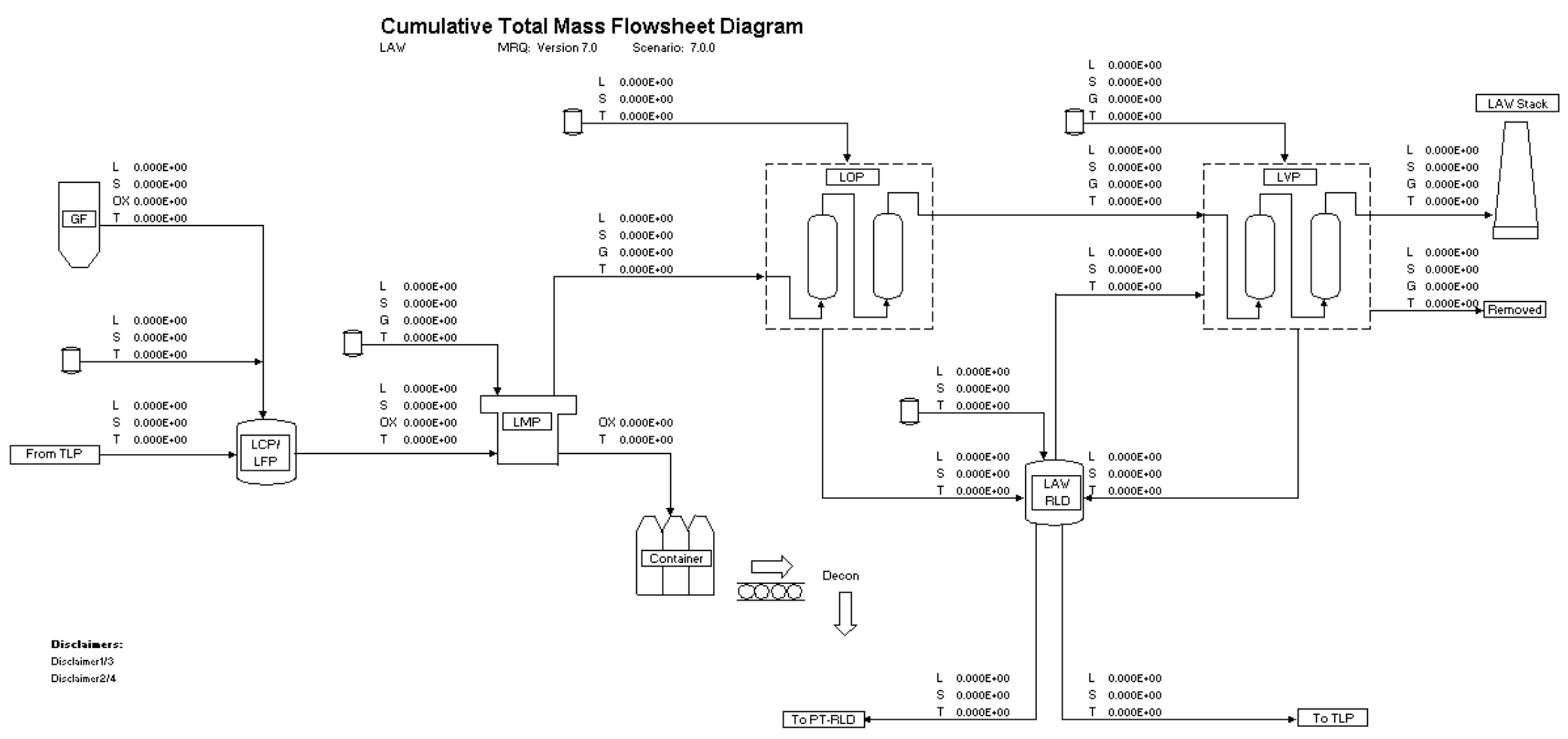


Figure J-4 Mass Balance Flowsheet Diagram (HLW)

Cumulative Total Mass Flowsheet Diagram

HLW MRQ: Wersion 7.0 Scenario: 7.0 .0

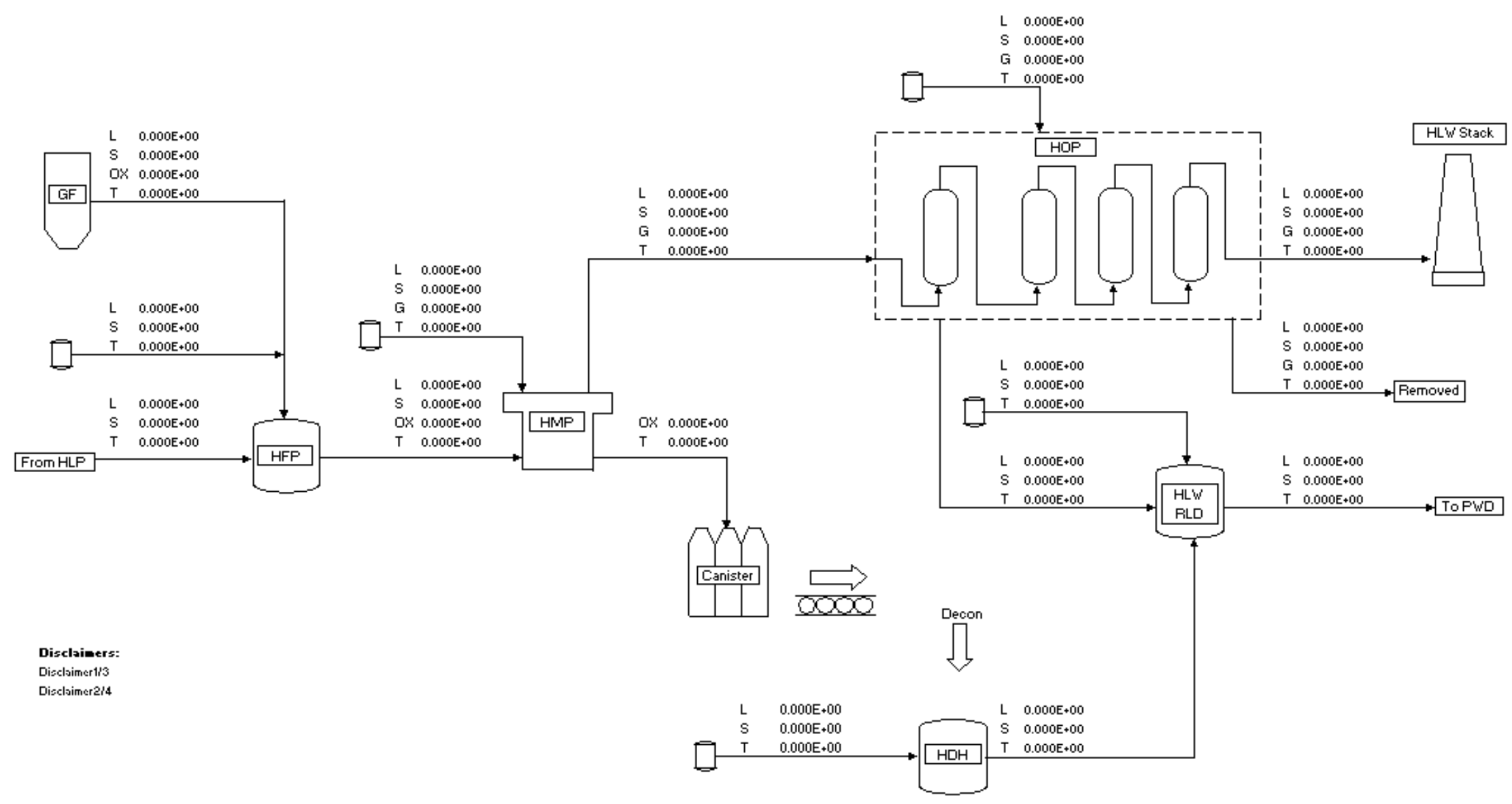


Table J-1 Flowsheet Diagram Stream Makeup File

\begin{tabular}{|c|c|c|c|c|}
\hline $\begin{array}{l}\text { System } \\
\text { Name }\end{array}$ & $\begin{array}{l}\text { Stream } \\
\text { Name }\end{array}$ & $\begin{array}{l}\text { Stream } \\
\text { ID }\end{array}$ & $\begin{array}{l}\text { Totalizer } \\
\text { File Name }\end{array}$ & $\begin{array}{l}\text { Waste } \\
\text { Type }\end{array}$ \\
\hline FRP & TankFarm_FRP & 1 & TANK-FARM-LAW_FRP-VSL-00002A.csv & Waste \\
\hline FRP & TankFarm_FRP & 1 & TANK-FARM-LAW_FRP-VSL-00002B.csV & Waste \\
\hline FRP & TankFarm_FRP & 1 & TANK-FARM-LAW_FRP-VSL-00002C.csV & Waste \\
\hline FRP & TankFarm_FRP & 1 & TANK-FARM-LAW_FRP-VSL-00002D.csv & Waste \\
\hline FRP & ChemAdd_FRP & 2 & DIW-TK-00001_FRP-VSL-00002A.csV & ChemAdd \\
\hline FRP & ChemAdd_FRP & 2 & DIW-TK-00001_FRP-VSL-00002B.csV & ChemAdd \\
\hline FRP & ChemAdd_FRP & 2 & DIW-TK-00001_FRP-VSL-00002C.csV & ChemAdd \\
\hline FRP & ChemAdd_FRP & 2 & DIW-TK-00001_FRP-VSL-00002D.csV & ChemAdd \\
\hline FRP & ChemAdd_FRP & 2 & INHIBITED-WATER_FRP-VSL-00002A.csv & ChemAdd \\
\hline FRP & ChemAdd_FRP & 2 & INHIBITED-WATER_FRP-VSL-00002B.csv & ChemAdd \\
\hline FRP & ChemAdd_FRP & 2 & INHIBITED-WATER_FRP-VSL-00002C.csV & ChemAdd \\
\hline FRP & ChemAdd_FRP & 2 & INHIBITED-WATER_FRP-VSL-00002D.csv & ChemAdd \\
\hline$\overline{\text { FRP }}$ & ChemAdd_FRP & 2 & PSA_FRP-VSL-00002A.csV & ChemAdd \\
\hline FRP & ChemAdd_FRP & 2 & PSA_FRP-VSL-00002B.csV & ChemAdd \\
\hline FRP & ChemAdd_FRP & 2 & PSA_FRP-VSL-00002C.csV & ChemAdd \\
\hline FRP & ChemAdd_FRP & 2 & PSA_FRP-VSL-00002D.csV & ChemAdd \\
\hline FRP & ChemAdd_FRP & 2 & RFD-AIR_FRP-VSL-00002A.csv & ChemAdd \\
\hline$\overline{\text { FRP }}$ & ChemAdd_FRP & 2 & RFD-AIR_FRP-VSL-00002B.csV & ChemAdd \\
\hline FRP & ChemAdd_FRP & 2 & RFD-AIR_FRP-VSL-00002C.csV & ChemAdd \\
\hline FRP & ChemAdd_FRP & 2 & RFD-AIR_FRP-VSL-00002D.csV & ChemAdd \\
\hline FRP & ChemAdd_FRP & 2 & RLD-TK-00006A_FRP-VSL-00002A.csV & ChemAdd \\
\hline FRP & ChemAdd_FRP & 2 & RLD-TK-00006A_FRP-VSL-00002B.csv & ChemAdd \\
\hline$\overline{\text { FRP }}$ & ChemAdd_FRP & 2 & RLD-TK-00006A_FRP-VSL-00002C.csV & ChemAdd \\
\hline FRP & ChemAdd_FRP & 2 & RLD-TK-00006A_FRP-VSL-00002D.csv & ChemAdd \\
\hline HLP22 & TankFarm_HLP22 & 3 & TANK-FARM-HLW_HLP-VSL-00022.csv & Waste \\
\hline HLP22 & ChemAdd_HLP22 & 4 & DIW-TK-00001_HLP-VSL-00022.csv & ChemAdd \\
\hline HLP22 & ChemAdd_HLP22 & 4 & INHIBITED-WATER_HLP-VSL-00022.csv & ChemAdd \\
\hline HLP22 & ChemAdd_HLP22 & 4 & PSA_HLP-VSL-00022.csv & ChemAdd \\
\hline HLP22 & ChemAdd_HLP22 & 4 & RFD-AIR_HLP-VSL-00022.csv & ChemAdd \\
\hline HLP22 & ChemAdd_HLP22 & 4 & RLD-TK-00006A_HLP-VSL-00022.csV & ChemAdd \\
\hline FEP & FRP_FEP & 5 & FRP-VSL-00002A_FEP-VSL-00017A.csv & Waste \\
\hline FEP & FRP_FEP & 5 & FRP-VSL-00002A_FEP-VSL-00017B.csv & Waste \\
\hline FEP & FRP_FEP & 5 & FRP-VSL-00002B_FEP-VSL-00017A.csv & Waste \\
\hline FEP & FRP_FEP & 5 & FRP-VSL-00002B_FEP-VSL-00017B.csv & Waste \\
\hline FEP & FRP_FEP & 5 & FRP-VSL-00002C_FEP-VSL-00017A.csv & Waste \\
\hline FEP & FRP_FEP & 5 & FRP-VSL-00002C_FEP-VSL-00017B.csv & Waste \\
\hline FEP & FRP_FEP & 5 & FRP-VSL-00002D_FEP-VSL-00017A.csv & Waste \\
\hline FEP & FRP_FEP & 5 & FRP-VSL-00002D_FEP-VSL-00017B.csv & Waste \\
\hline HLP22 & FRP_HLP22 & 6 & FRP-VSL-00002A_HLP-VSL-00022.csV & Waste \\
\hline HLP22 & FRP_HLP22 & 6 & FRP-VSL-00002B_HLP-VSL-00022.csv & Waste \\
\hline HLP22 & FRP_HLP22 & 6 & FRP-VSL-00002C_HLP-VSL-00022.csv & Waste \\
\hline HLP22 & FRP_HLP22 & 6 & FRP-VSL-00002D_HLP-VSL-00022.csv & Waste \\
\hline FEP & PWD_FEP & 7 & PWD-VSL-00015_FEP-VSL-00017A.csv & Recycle \\
\hline FEP & PWD_FEP & 7 & PWD-VSL-00015_FEP-VSL-00017B.csV & Recycle \\
\hline FEP & PWD_FEP & 7 & PWD-VSL-00016_FEP-VSL-00017A.csv & Recycle \\
\hline
\end{tabular}




\begin{tabular}{|c|c|c|c|c|}
\hline $\begin{array}{l}\text { System } \\
\text { Name }\end{array}$ & $\begin{array}{l}\text { Stream } \\
\text { Name }\end{array}$ & $\begin{array}{l}\text { Stream } \\
\text { ID }\end{array}$ & $\begin{array}{l}\text { Totalizer } \\
\text { File Name }\end{array}$ & $\begin{array}{l}\text { Waste } \\
\text { Type }\end{array}$ \\
\hline FEP & PWD_FEP & 7 & PWD-VSL-00016_FEP-VSL-00017B.csV & Recycle \\
\hline FEP & PWD_FEP & 7 & PWD-VSL-00044_FEP-VSL-00017A.csV & Recycle \\
\hline FEP & PWD_FEP & 7 & PWD-VSL-00044_FEP-VSL-00017B.csv & Recycle \\
\hline FEP & ProCond_FEP & 8 & RLD-TK-00006A_FEP-SEP-00001A.csv & ProCond \\
\hline FEP & ProCond_FEP & 8 & RLD-TK-00006A_FEP-SEP-00001B.csv & ProCond \\
\hline FEP & ProCond_FEP & 8 & RLD-TK-00006A_FEP-VSL-00017A.csv & ProCond \\
\hline FEP & ProCond_FEP & 8 & RLD-TK-00006A_FEP-VSL-00017B.csV & ProCond \\
\hline FEP & RDP_FEP & 9 & RDP-VSL-00002B_FEP-VSL-00017A.csv & Waste \\
\hline FEP & RDP_FEP & 9 & RDP-VSL-00002B_FEP-VSL-00017B.csv & Waste \\
\hline FEP & RDP_FEP & 9 & RDP-VSL-00002C_FEP-VSL-00017A.csv & Waste \\
\hline FEP & RDP_FEP & 9 & RDP-VSL-00002C_FEP-VSL-00017B.csv & Waste \\
\hline FEP & ChemAdd_FEP & 10 & ANTIFOAM-AGENT_FEP-SEP-00001A.csV & ChemAdd \\
\hline FEP & ChemAdd_FEP & 10 & ANTIFOAM-AGENT_FEP-SEP-00001B.csv & ChemAdd \\
\hline FEP & ChemAdd_FEP & 10 & DIW-TK-00001_FEP-SEP-00001A.csv & ChemAdd \\
\hline FEP & ChemAdd_FEP & 10 & DIW-TK-00001_FEP-SEP-00001B.csV & ChemAdd \\
\hline FEP & ChemAdd_FEP & 10 & DIW-TK-00001_FEP-VSL-00017A.csv & ChemAdd \\
\hline FEP & ChemAdd_FEP & 10 & DIW-TK-00001_FEP-VSL-00017B.csV & ChemAdd \\
\hline FEP & ChemAdd_FEP & 10 & $\begin{array}{l}\text { FEPA-INTER-AFTER-CONDENSER- } \\
\text { WATER FEP-VSL-00005A.csv }\end{array}$ & ChemAdd \\
\hline FEP & ChemAdd_FEP & 10 & $\begin{array}{l}\text { FEPB-INTER-AFTER-CONDENSER- } \\
\text { WATER FEP-VSL-00005B.csv }\end{array}$ & ChemAdd \\
\hline FEP & ChemAdd_FEP & 10 & $\begin{array}{l}\text { FEP-DMST-00001A-AIR_FEP-DMST- } \\
\text { 00001A.csv }\end{array}$ & ChemAdd \\
\hline FEP & ChemAdd_FEP & 10 & $\begin{array}{l}\text { FEP-DMST-00001B-AIR_FEP-DMST- } \\
\text { 00001B.csv }\end{array}$ & ChemAdd \\
\hline FEP & ChemAdd_FEP & 10 & PSA_FEP-VSL-00017A.csv & ChemAdd \\
\hline FEP & ChemAdd_FEP & 10 & PSA_FEP-VSL-00017B.csv & ChemAdd \\
\hline FEP & ChemAdd FEP & 10 & RFD-AIR FEP-VSL-00017A.csv & ChemAdd \\
\hline FEP & ChemAdd_FEP & 10 & RFD-AIR_FEP-VSL-00017B.csV & ChemAdd \\
\hline UFP & FEP_UFP & 11 & FEP-SEP-00001A_UFP-VSL-00001A.csV & Waste \\
\hline UFP & FEP_UFP & 11 & FEP-SEP-00001A_UFP-VSL-00001B.csv & Waste \\
\hline UFP & FEP_UFP & 11 & FEP-SEP-00001B_UFP-VSL-00001A.csv & Waste \\
\hline UFP & FEP_UFP & 11 & FEP-SEP-00001B_UFP-VSL-00001B.csv & Waste \\
\hline UFP & FRP_UFP & 12 & FRP-VSL-00002A_UFP-VSL-00001A.csv & Waste \\
\hline UFP & FRP_UFP & 12 & FRP-VSL-00002A_UFP-VSL-00001B.csv & Waste \\
\hline UFP & FRP_UFP & 12 & FRP-VSL-00002B_UFP-VSL-00001A.csv & Waste \\
\hline UFP & FRP_UFP & 12 & FRP-VSL-00002B_UFP-VSL-00001B.csv & Waste \\
\hline UFP & FRP_UFP & 12 & FRP-VSL-00002C_UFP-VSL-00001A.csv & Waste \\
\hline UFP & FRP_UFP & 12 & FRP-VSL-00002C_UFP-VSL-00001B.csV & Waste \\
\hline UFP & FRP_UFP & 12 & FRP-VSL-00002D_UFP-VSL-00001A.csV & Waste \\
\hline UFP & FRP_UFP & 12 & FRP-VSL-00002D_UFP-VSL-00001B.csv & Waste \\
\hline UFP & HLP22_UFP & 13 & HLP-VSL-00022_UFP-VSL-00001A.csv & Waste \\
\hline UFP & HLP22_UFP & 13 & HLP-VSL-00022_UFP-VSL-00001B.csV & Waste \\
\hline UFP & ProCond_UFP & 14 & RLD-TK-00006A_UFP-VSL-00001A.csv & ProCond \\
\hline UFP & ProCond_UFP & 14 & RLD-TK-00006A_UFP-VSL-00001B.csv & ProCond \\
\hline UFP & ProCond_UFP & 14 & RLD-TK-00006A_UFP-VSL-00002A.csv & ProCond \\
\hline UFP & ProCond_UFP & 14 & RLD-TK-00006A_UFP-VSL-00002B.csV & ProCond \\
\hline
\end{tabular}




\begin{tabular}{|c|c|c|c|c|}
\hline $\begin{array}{l}\text { System } \\
\text { Name }\end{array}$ & $\begin{array}{l}\text { Stream } \\
\text { Name }\end{array}$ & $\begin{array}{l}\text { Stream } \\
\text { ID }\end{array}$ & $\begin{array}{l}\text { Totalizer } \\
\text { File Name }\end{array}$ & $\begin{array}{l}\text { Waste } \\
\text { Type }\end{array}$ \\
\hline UFP & ProCond_UFP & 14 & RLD-TK-00006A_UFP-VSL-00062A.csV & ProCond \\
\hline UFP & ChemAdd_UFP & 15 & ANTIFOAM-AGENT_UFP-VSL-00001A.csV & ChemAdd \\
\hline UFP & ChemAdd_UFP & 15 & ANTIFOAM-AGENT_UFP-VSL-00001B.csv & ChemAdd \\
\hline UFP & ChemAdd_UFP & 15 & ANTIFOAM-AGENT_UFP-VSL-00002A.csv & ChemAdd \\
\hline UFP & ChemAdd_UFP & 15 & ANTIFOAM-AGENT_UFP-VSL-00002B.csv & ChemAdd \\
\hline UFP & ChemAdd_UFP & 15 & DIW-TK-00001_UFP-VSL-00001A.csv & ChemAdd \\
\hline UFP & ChemAdd_UFP & 15 & DIW-TK-00001_UFP-VSL-00001B.csV & ChemAdd \\
\hline UFP & ChemAdd_UFP & 15 & DIW-TK-00001_UFP-VSL-00002A.csv & ChemAdd \\
\hline UFP & ChemAdd_UFP & 15 & DIW-TK-00001_UFP-VSL-00002B.csv & ChemAdd \\
\hline UFP & ChemAdd_UFP & 15 & DIW-TK-00001_UFP-VSL-00062A.csv & ChemAdd \\
\hline UFP & ChemAdd_UFP & 15 & INHIBITED-WATER_UFP-02A-P1.csv & ChemAdd \\
\hline UFP & ChemAdd_UFP & 15 & INHIBITED-WATER_UFP-02A-P2.csv & ChemAdd \\
\hline UFP & ChemAdd_UFP & 15 & INHIBITED-WATER_UFP-02B-P1.csv & ChemAdd \\
\hline UFP & ChemAdd_UFP & 15 & INHIBITED-WATER_UFP-02B-P2.csv & ChemAdd \\
\hline UFP & ChemAdd_UFP & 15 & INHIBITED-WATER_UFP-VSL-00002A.csV & ChemAdd \\
\hline UFP & ChemAdd_UFP & 15 & INHIBITED-WATER_UFP-VSL-00002B.csV & ChemAdd \\
\hline UFP & ChemAdd_UFP & 15 & NAR-TK-00006_UFP-VSL-00002A.csV & ChemAdd \\
\hline UFP & ChemAdd_UFP & 15 & NAR-TK-00006_UFP-VSL-00002B.csv & ChemAdd \\
\hline UFP & ChemAdd_UFP & 15 & $\begin{array}{l}\text { NEUTRALIZATION-CAUSTIC_UFP-VSL- } \\
\text { 00002A.csv }\end{array}$ & ChemAdd \\
\hline UFP & ChemAdd_UFP & 15 & $\begin{array}{l}\text { NEUTRALIZATION-CAUSTIC_UFP-VSL- } \\
\text { 00002B.csv }\end{array}$ & ChemAdd \\
\hline UFP & ChemAdd_UFP & 15 & $\begin{array}{l}\text { OXIDATIVE-LEACH-CAUSTIC_UFP-VSL- } \\
\text { 00002A.csv }\end{array}$ & ChemAdd \\
\hline UFP & ChemAdd_UFP & 15 & $\begin{array}{l}\text { OXIDATIVE-LEACH-CAUSTIC_UFP-VSL- } \\
\text { 00002B.csv }\end{array}$ & ChemAdd \\
\hline UFP & ChemAdd_UFP & 15 & PSA_UFP-VSL-00001A.csV & ChemAdd \\
\hline UFP & ChemAdd_UFP & 15 & PSA_UFP-VSL-00001B.csV & ChemAdd \\
\hline UFP & ChemAdd_UFP & 15 & PSA_UFP-VSL-00002A.csv & ChemAdd \\
\hline UFP & ChemAdd_UFP & 15 & PSA_UFP-VSL-00002B.csV & ChemAdd \\
\hline UFP & ChemAdd_UFP & 15 & PSA_UFP-VSL-00062A.csv & ChemAdd \\
\hline UFP & ChemAdd_UFP & 15 & RFD-AIR_UFP-VSL-00001A.csV & ChemAdd \\
\hline UFP & ChemAdd_UFP & 15 & RFD-AIR_UFP-VSL-00001B.csV & ChemAdd \\
\hline UFP & ChemAdd_UFP & 15 & RFD-AIR_UFP-VSL-00002A.csv & ChemAdd \\
\hline UFP & ChemAdd_UFP & 15 & RFD-AIR_UFP-VSL-00002B.csV & ChemAdd \\
\hline UFP & ChemAdd_UFP & 15 & RFD-AIR_UFP-VSL-00062A.csV & ChemAdd \\
\hline UFP & ChemAdd_UFP & 15 & SHR-TK-00006_UFP-VSL-00001A.csv & ChemAdd \\
\hline UFP & ChemAdd_UFP & 15 & SHR-TK-00006_UFP-VSL-00001B.csv & ChemAdd \\
\hline UFP & ChemAdd_UFP & 15 & SHR-TK-00006_UFP-VSL-00002A.csv & ChemAdd \\
\hline UFP & ChemAdd_UFP & 15 & SHR-TK-00006_UFP-VSL-00002B.csV & ChemAdd \\
\hline UFP & ChemAdd_UFP & 15 & SHR-TK-00006_UFP-VSL-00062A.csv & ChemAdd \\
\hline UFP & ChemAdd_UFP & 15 & SPR-MXR-00001_UFP-VSL-00001A.csv & ChemAdd \\
\hline UFP & ChemAdd_UFP & 15 & SPR-MXR-00001_UFP-VSL-00001B.csV & ChemAdd \\
\hline UFP & ChemAdd_UFP & 15 & SPR-MXR-00001_UFP-VSL-00002A.csv & ChemAdd \\
\hline UFP & ChemAdd_UFP & 15 & SPR-MXR-00001_UFP-VSL-00002B.csv & ChemAdd \\
\hline UFP & ChemAdd_UFP & 15 & STEAM_UFP-VSL-00001A.csv & ChemAdd \\
\hline UFP & ChemAdd_UFP & 15 & STEAM_UFP-VSL-00001B.csv & ChemAdd \\
\hline
\end{tabular}


24590-WTP-MDD-PR-01-002, Rev 12 Dynamic (G2) Model Design Document

\begin{tabular}{|c|c|c|c|c|}
\hline $\begin{array}{l}\text { System } \\
\text { Name }\end{array}$ & $\begin{array}{l}\text { Stream } \\
\text { Name }\end{array}$ & $\begin{array}{c}\text { Stream } \\
\text { ID }\end{array}$ & $\begin{array}{l}\text { Totalizer } \\
\text { File Name }\end{array}$ & $\begin{array}{l}\text { Waste } \\
\text { Type }\end{array}$ \\
\hline UFP & ChemAdd UFP & 15 & STEAM UFP-VSL-00002A.csV & ChemAdd \\
\hline UFP & ChemAdd_UFP & 15 & STEAM_UFP-VSL-00002B.csv & ChemAdd \\
\hline UFP & ChemAdd_UFP & 15 & STEAM_UFP-VSL-00062A.csv & ChemAdd \\
\hline UFP & ChemAdd_UFP & 15 & STR-MXR-00001_UFP-VSL-00001A.csV & ChemAdd \\
\hline UFP & ChemAdd_UFP & 15 & STR-MXR-00001_UFP-VSL-00001B.csv & ChemAdd \\
\hline UFP & ChemAdd_UFP & 15 & UFP-02-P2-NAOH_UFP-02A-P2.csv & ChemAdd \\
\hline UFP & ChemAdd_UFP & 15 & UFP-02-P2-NAOH_UFP-02B-P2.csv & ChemAdd \\
\hline PWD & UFP_PWD & 16 & UFP-02A-P2_PWD-VSL-00015.csV & Waste \\
\hline PWD & UFP_PWD & 16 & UFP-02A-P2_PWD-VSL-00016.csv & Waste \\
\hline PWD & UFP_PWD & 16 & UFP-02B-P2_PWD-VSL-00015.csv & Waste \\
\hline PWD & UFP_PWD & 16 & UFP-02B-P2_PWD-VSL-00016.csv & Waste \\
\hline PWD & UFP_PWD & 16 & UFP-02A-P2_PWD-VSL-00033.csV & Waste \\
\hline PWD & UFP_PWD & 16 & UFP-02B-P2_PWD-VSL-00033.csv & Waste \\
\hline PWD & UFP_PWD & 16 & UFP-VSL-00002A_PWD-VSL-00015.csV & Waste \\
\hline PWD & UFP_PWD & 16 & UFP-VSL-00002A_PWD-VSL-00016.csV & Waste \\
\hline PWD & UFP_PWD & 16 & UFP-VSL-00002B_PWD-VSL-00015.csv & Waste \\
\hline PWD & UFP_PWD & 16 & UFP-VSL-00002B_PWD-VSL-00016.csV & Waste \\
\hline PWD & PVP_PWD & 17 & PJV-VSL-00001_PWD-VSL-00043.csv & Waste \\
\hline PWD & PVP_PWD & 17 & PVP-SCB-00002_PWD-VSL-00044.csv & Waste \\
\hline PWD & PVP_PWD & 17 & PVP-VSL-00001_PWD-VSL-00044.csV & Waste \\
\hline PWD & Recycle_PWD & 18 & CNP-EVAP-00001_PWD-VSL-00015.csv & Waste \\
\hline PWD & Recycle_PWD & 18 & CNP-EVAP-00001_PWD-VSL-00016.csV & Waste \\
\hline PWD & Recycle_PWD & 18 & CXP-IXC-00001_PWD-VSL-00015.csv & Waste \\
\hline PWD & Recycle_PWD & 18 & CXP-IXC-00001_PWD-VSL-00016.csv & Waste \\
\hline PWD & Recycle_PWD & 18 & CXP-IXC-00002_PWD-VSL-00015.csV & Waste \\
\hline PWD & Recycle_PWD & 18 & CXP-IXC-00002_PWD-VSL-00016.csv & Waste \\
\hline PWD & Recycle_PWD & 18 & CXP-IXC-00003_PWD-VSL-00015.csv & Waste \\
\hline PWD & Recycle_PWD & 18 & CXP-IXC-00003_PWD-VSL-00016.csv & Waste \\
\hline PWD & Recycle_PWD & 18 & CXP-IXC-00004_PWD-VSL-00015.csv & Waste \\
\hline PWD & Recycle_PWD & 18 & CXP-IXC-00004_PWD-VSL-00016.csV & Waste \\
\hline PWD & Recycle_PWD & 18 & RLD-VSL-00007_PWD-VSL-00043.csv & Waste \\
\hline PWD & Recycle_PWD & 18 & CXP-FILT-00001_PWD-VSL-00016.csV & Waste \\
\hline PWD & ProCond_PWD & 19 & RLD-TK-00006A_PWD-VSL-00033.csv & ProCond \\
\hline PWD & ProCond_PWD & 19 & RLD-TK-00006A_PWD-VSL-00043.csv & ProCond \\
\hline PWD & ChemAdd_PWD & 20 & DIW-TK-00001_PWD-VSL-00033.csv & ChemAdd \\
\hline PWD & ChemAdd_PWD & 20 & DIW-TK-00001_PWD-VSL-00043.csV & ChemAdd \\
\hline PWD & ChemAdd_PWD & 20 & INHIBITED-WATER_PWD-VSL-00033.csv & ChemAdd \\
\hline PWD & ChemAdd_PWD & 20 & INHIBITED-WATER_PWD-VSL-00043.csv & ChemAdd \\
\hline PWD & ChemAdd_PWD & 20 & PSA_PWD-VSL-00015.csv & ChemAdd \\
\hline PWD & ChemAdd_PWD & 20 & PSA_PWD-VSL-00016.csv & ChemAdd \\
\hline PWD & ChemAdd_PWD & 20 & PSA_PWD-VSL-00033.csv & ChemAdd \\
\hline PWD & ChemAdd_PWD & 20 & PSA_PWD-VSL-00043.csv & ChemAdd \\
\hline PWD & ChemAdd_PWD & 20 & PSA_PWD-VSL-00044.csv & ChemAdd \\
\hline PWD & ChemAdd_PWD & 20 & RFD-AIR_PWD-VSL-00015.csv & ChemAdd \\
\hline PWD & ChemAdd_PWD & 20 & RFD-AIR_PWD-VSL-00016.csv & ChemAdd \\
\hline PWD & ChemAdd_PWD & 20 & RFD-AIR_PWD-VSL-00033.csv & ChemAdd \\
\hline
\end{tabular}




\begin{tabular}{|c|c|c|c|c|}
\hline $\begin{array}{l}\text { System } \\
\text { Name }\end{array}$ & $\begin{array}{l}\text { Stream } \\
\text { Name }\end{array}$ & $\begin{array}{l}\text { Stream } \\
\text { ID }\end{array}$ & $\begin{array}{l}\text { Totalizer } \\
\text { File Name }\end{array}$ & $\begin{array}{l}\text { Waste } \\
\text { Type }\end{array}$ \\
\hline PWD & ChemAdd PWD & 20 & RFD-AIR PWD-VSL-00043.csv & ChemAdd \\
\hline PWD & ChemAdd_PWD & 20 & RFD-AIR_PWD-VSL-00044.csV & ChemAdd \\
\hline PWD & ChemAdd_PWD & 20 & SHR-TK-00006_PWD-VSL-00015.csv & ChemAdd \\
\hline PWD & ChemAdd_PWD & 20 & SHR-TK-00006_PWD-VSL-00016.csV & ChemAdd \\
\hline PWD & ChemAdd_PWD & 20 & SHR-TK-00006_PWD-VSL-00044.csv & ChemAdd \\
\hline HLP & UFP_HLP & 21 & UFP-VSL-00002A_HLP-VSL-00027A.csV & Waste \\
\hline HLP & UFP_HLP & 21 & UFP-VSL-00002A_HLP-VSL-00027B.csV & Waste \\
\hline HLP & UFP_HLP & 21 & UFP-VSL-00002B_HLP-VSL-00027A.csv & Waste \\
\hline HLP & UFP_HLP & 21 & UFP-VSL-00002B_HLP-VSL-00027B.csv & Waste \\
\hline HLP & ProCond_HLP & 22 & RLD-TK-00006A_HLP-VSL-00027A.csv & ProCond \\
\hline HLP & ProCond_HLP & 22 & RLD-TK-00006A_HLP-VSL-00027B.csv & ProCond \\
\hline HLP & ProCond_HLP & 22 & RLD-TK-00006A_HLP-VSL-00028.csV & ProCond \\
\hline HLP & CNP_HLP & 23 & CNP-EVAP-00001_HLP-VSL-00027B.csv & Waste \\
\hline HLP & CNP_HLP & 23 & CNP-EVAP-00001_HLP-VSL-00028.csv & Waste \\
\hline HLP & CNP_HLP & 23 & CNP-VSL-00003_HLP-VSL-00027B.csv & Waste \\
\hline HLP & CNP_HLP & 23 & CNP-VSL-00003_HLP-VSL-00028.csV & Waste \\
\hline HLP & ChemAdd_HLP & 24 & ANTIFOAM-AGENT_HLP-VSL-00027A.csV & ChemAdd \\
\hline HLP & ChemAdd_HLP & 24 & ANTIFOAM-AGENT_HLP-VSL-00027B.csv & ChemAdd \\
\hline HLP & ChemAdd_HLP & 24 & ANTIFOAM-AGENT_HLP-VSL-00028.csv & ChemAdd \\
\hline HLP & ChemAdd_HLP & 24 & DIW-TK-00001_HLP-VSL-00027A.csV & ChemAdd \\
\hline HLP & ChemAdd_HLP & 24 & DIW-TK-00001_HLP-VSL-00027B.csv & ChemAdd \\
\hline HLP & ChemAdd_HLP & 24 & DIW-TK-00001_HLP-VSL-00028.csV & ChemAdd \\
\hline HLP & ChemAdd_HLP & 24 & HLP14_HLP-VSL-00027B.csv & ChemAdd \\
\hline HLP & ChemAdd_HLP & 24 & HLP14_HLP-VSL-00028.csv & ChemAdd \\
\hline HLP & ChemAdd_HLP & 24 & INHIBITED-WATER_HLP-VSL-00027A.csv & ChemAdd \\
\hline HLP & ChemAdd_HLP & 24 & INHIBITED-WATER_HLP-VSL-00027B.csv & ChemAdd \\
\hline HLP & ChemAdd_HLP & 24 & INHIBITED-WATER_HLP-VSL-00028.csv & ChemAdd \\
\hline HLP & ChemAdd_HLP & 24 & PSA_HLP-VSL-00027A.csV & ChemAdd \\
\hline HLP & ChemAdd_HLP & 24 & PSA_HLP-VSL-00027B.csV & ChemAdd \\
\hline HLP & ChemAdd_HLP & 24 & PSA_HLP-VSL-00028.csV & ChemAdd \\
\hline HLP & ChemAdd_HLP & 24 & RFD-AIR_HLP-VSL-00027A.csv & ChemAdd \\
\hline HLP & ChemAdd_HLP & 24 & RFD-AIR_HLP-VSL-00027B.csV & ChemAdd \\
\hline HLP & ChemAdd_HLP & 24 & RFD-AIR_HLP-VSL-00028.csv & ChemAdd \\
\hline PVP-PJV & FEP_PVP/PJV & 25 & FEP-DMST-00001A_PVP-VESSEL-VENT.csv & Waste \\
\hline PVP-PJV & FEP_PVP/PJV & 25 & FEP-DMST-00001B_PVP-VESSEL-VENT.csV & Waste \\
\hline PVP-PJV & TLP_PVP/PJV & 26 & TLP-DMST-00001_PVP-VESSEL-VENT.csv & Waste \\
\hline PVP-PJV & PJM/RFD_PVP/PJV & 27 & CNP-VSL-00004_PJM-RFD-VENT.csv & ChemAdd \\
\hline PVP-PJV & PJM/RFD_PVP/PJV & 27 & CNP-VSL-00003_PJM-RFD-VENT.csv & ChemAdd \\
\hline PVP-PJV & PJM/RFD_PVP/PJV & 27 & CXP-VSL-00005_PJM-RFD-VENT.csv & ChemAdd \\
\hline PVP-PJV & PJM/RFD_PVP/PJV & 27 & CXP-VSL-00026A_PJM-RFD-VENT.csv & ChemAdd \\
\hline PVP-PJV & PJM/RFD_PVP/PJV & 27 & CXP-VSL-00026B_PJM-RFD-VENT.csV & ChemAdd \\
\hline PVP-PJV & PJM/RFD_PVP/PJV & 27 & CXP-VSL-00026C_PJM-RFD-VENT.csv & ChemAdd \\
\hline PVP-PJV & PJM/RFD_PVP/PJV & 27 & FEP-VSL-00017A_PJM-RFD-VENT.csv & ChemAdd \\
\hline PVP-PJV & PJM/RFD_PVP/PJV & 27 & FEP-VSL-00017B_PJM-RFD-VENT.csv & ChemAdd \\
\hline PVP-PJV & PJM/RFD_PVP/PJV & 27 & FRP-VSL-00002A_PJM-RFD-VENT.csv & ChemAdd \\
\hline PVP-PJV & PJM/RFD_PVP/PJV & 27 & FRP-VSL-00002B_PJM-RFD-VENT.csv & ChemAdd \\
\hline
\end{tabular}




\begin{tabular}{|c|c|c|c|c|}
\hline $\begin{array}{l}\text { System } \\
\text { Name }\end{array}$ & $\begin{array}{l}\text { Stream } \\
\text { Name }\end{array}$ & $\begin{array}{c}\text { Stream } \\
\text { ID }\end{array}$ & $\begin{array}{l}\text { Totalizer } \\
\text { File Name }\end{array}$ & $\begin{array}{l}\text { Waste } \\
\text { Type }\end{array}$ \\
\hline PVP-PJV & PJM/RFD PVP/PJV & 27 & FRP-VSL-00002C PJM-RFD-VENT.csv & ChemAdd \\
\hline PVP-PJV & PJM/RFD_PVP/PJV & 27 & FRP-VSL-00002D_PJM-RFD-VENT.csV & ChemAdd \\
\hline PVP-PJV & PJM/RFD_PVP/PJV & 27 & HLP-VSL-00022_PJM-RFD-VENT.csv & ChemAdd \\
\hline PVP-PJV & PJM/RFD_PVP/PJV & 27 & HLP-VSL-00027A_PJM-RFD-VENT.csV & ChemAdd \\
\hline PVP-PJV & PJM/RFD_PVP/PJV & 27 & HLP-VSL-00027B_PJM-RFD-VENT.csv & ChemAdd \\
\hline PVP-PJV & PJM/RFD_PVP/PJV & 27 & HLP-VSL-00028_PJM-RFD-VENT.csV & ChemAdd \\
\hline PVP-PJV & PJM/RFD_PVP/PJV & 27 & PWD-VSL-00015_PJM-RFD-VENT.csV & ChemAdd \\
\hline PVP-PJV & PJM/RFD_PVP/PJV & 27 & PWD-VSL-00016_PJM-RFD-VENT.csv & ChemAdd \\
\hline PVP-PJV & PJM/RFD_PVP/PJV & 27 & PWD-VSL-00033_PJM-RFD-VENT.csv & ChemAdd \\
\hline PVP-PJV & PJM/RFD_PVP/PJV & 27 & PWD-VSL-00043_PJM-RFD-VENT.csV & ChemAdd \\
\hline PVP-PJV & PJM/RFD_PVP/PJV & 27 & PWD-VSL-00044_PJM-RFD-VENT.csv & ChemAdd \\
\hline PVP-PJV & PJM/RFD_PVP/PJV & 27 & RDP-VSL-00002A_PJM-RFD-VENT.csV & ChemAdd \\
\hline PVP-PJV & PJM/RFD_PVP/PJV & 27 & RDP-VSL-00002B_PJM-RFD-VENT.csv & ChemAdd \\
\hline PVP-PJV & PJM/RFD_PVP/PJV & 27 & RDP-VSL-00002C_PJM-RFD-VENT.csv & ChemAdd \\
\hline PVP-PJV & PJM/RFD_PVP/PJV & 27 & TCP-VSL-00001_PJM-RFD-VENT.csv & ChemAdd \\
\hline PVP-PJV & PJM/RFD_PVP/PJV & 27 & TLP-VSL-00009A_PJM-RFD-VENT.csv & ChemAdd \\
\hline PVP-PJV & PJM/RFD_PVP/PJV & 27 & TLP-VSL-00009B_PJM-RFD-VENT.csV & ChemAdd \\
\hline PVP-PJV & PJM/RFD_PVP/PJV & 27 & UFP-VSL-00001A_PJM-RFD-VENT.csv & ChemAdd \\
\hline PVP-PJV & PJM/RFD_PVP/PJV & 27 & UFP-VSL-00001B_PJM-RFD-VENT.csV & ChemAdd \\
\hline PVP-PJV & PJM/RFD_PVP/PJV & 27 & UFP-VSL-00002A_PJM-RFD-VENT.csV & ChemAdd \\
\hline PVP-PJV & PJM/RFD_PVP/PJV & 27 & UFP-VSL-00002B_PJM-RFD-VENT.csv & ChemAdd \\
\hline PVP-PJV & PJM/RFD_PVP/PJV & 27 & UFP-VSL-00062A_PJM-RFD-VENT.csv & ChemAdd \\
\hline PVP-PJV & ChemAdd_PVP/PJV & 28 & $\begin{array}{l}\text { AIR-VENT-LUMP-SUM_PVP-VESSEL- } \\
\text { VENT.csV }\end{array}$ & ChemAdd \\
\hline PVP-PJV & ChemAdd_PVP/PJV & 28 & DIW-TK-00004_PJV-VSL-00001.csv & ChemAdd \\
\hline PVP-PJV & ChemAdd_PVP/PJV & 28 & DIW-TK-00004_PVP-HEME-00001.csv & ChemAdd \\
\hline PVP-PJV & ChemAdd_PVP/PJV & 28 & SHR-TK-00007_PVP-SCB-00002.csv & ChemAdd \\
\hline PVP-PJV & ChemAdd_PVP/PJV & 28 & $\begin{array}{l}\text { UFV-HLP-3HR-ROTATION_PVP-VESSEL- } \\
\text { VENT.csv }\end{array}$ & ChemAdd \\
\hline PVP-PJV & PT-Stack & 29 & PJV-HEPA_PVP-STACK.csV & OffGas \\
\hline PVP-PJV & PT-Stack & 29 & PVP-ABS-00001AB_PVP-STACK.csv & OffGas \\
\hline CXP & UFP_CXP & 30 & UFP-VSL-00062A_CXP-FILT-00001.csv & Waste \\
\hline CXP & RDP/CRP_CXP & 31 & CRP-VSL-00001_CXP-IXC-00001.csv & ChemAdd \\
\hline CXP & RDP/CRP_CXP & 31 & CRP-VSL-00001_CXP-IXC-00002.csv & ChemAdd \\
\hline CXP & RDP/CRP_CXP & 31 & CRP-VSL-00001_CXP-IXC-00003.csv & ChemAdd \\
\hline CXP & RDP/CRP_CXP & 31 & CRP-VSL-00001_CXP-IXC-00004.csv & ChemAdd \\
\hline CXP & RDP/CRP_CXP & 31 & RDP-VSL-00002A_CXP-IXC-00001.csv & ChemAdd \\
\hline CXP & RDP/CRP_CXP & 31 & RDP-VSL-00002A_CXP-IXC-00002.csv & ChemAdd \\
\hline CXP & RDP/CRP_CXP & 31 & RDP-VSL-00002A_CXP-IXC-00003.csv & ChemAdd \\
\hline CXP & RDP/CRP_CXP & 31 & RDP-VSL-00002A_CXP-IXC-00004.csv & ChemAdd \\
\hline CXP & CNP_CXP & 32 & CNP-VSL-00004_CXP-IXC-00001.csV & ChemAdd \\
\hline CXP & CNP_CXP & 32 & CNP-VSL-00004_CXP-IXC-00002.csv & ChemAdd \\
\hline CXP & CNP_CXP & 32 & CNP-VSL-00004_CXP-IXC-00003.csv & ChemAdd \\
\hline CXP & CNP_CXP & 32 & CNP-VSL-00004_CXP-IXC-00004.csv & ChemAdd \\
\hline CXP & ChemAdd_CXP & 33 & DIW-IXC-RINSE-1_CXP-IXC-00001.csv & ChemAdd \\
\hline CXP & ChemAdd_CXP & 33 & DIW-IXC-RINSE-1_CXP-IXC-00002.csv & ChemAdd \\
\hline
\end{tabular}




\begin{tabular}{|c|c|c|c|c|}
\hline $\begin{array}{l}\text { System } \\
\text { Name }\end{array}$ & $\begin{array}{l}\text { Stream } \\
\text { Name }\end{array}$ & $\begin{array}{l}\text { Stream } \\
\text { ID }\end{array}$ & $\begin{array}{l}\text { Totalizer } \\
\text { File Name }\end{array}$ & $\begin{array}{l}\text { Waste } \\
\text { Type }\end{array}$ \\
\hline CXP & ChemAdd_CXP & 33 & DIW-IXC-RINSE-1_CXP-IXC-00003.csv & ChemAdd \\
\hline CXP & ChemAdd CXP & 33 & DIW-IXC-RINSE-1 CXP-IXC-00004.csv & ChemAdd \\
\hline CXP & ChemAdd_CXP & 33 & DIW-IXC-RINSE-2_CXP-IXC-00001.csv & ChemAdd \\
\hline CXP & ChemAdd_CXP & 33 & DIW-IXC-RINSE-2_CXP-IXC-00002.csv & ChemAdd \\
\hline CXP & ChemAdd_CXP & 33 & DIW-IXC-RINSE-2_CXP-IXC-00003.csv & ChemAdd \\
\hline CXP & ChemAdd_CXP & 33 & DIW-IXC-RINSE-2_CXP-IXC-00004.csv & ChemAdd \\
\hline CXP & ChemAdd_CXP & 33 & SHR-TK-00005_CXP-IXC-00001.csv & ChemAdd \\
\hline CXP & ChemAdd_CXP & 33 & SHR-TK-00005_CXP-IXC-00002.csv & ChemAdd \\
\hline CXP & ChemAdd_CXP & 33 & SHR-TK-00005_CXP-IXC-00003.csv & ChemAdd \\
\hline CXP & ChemAdd_CXP & 33 & SHR-TK-00005_CXP-IXC-00004.csv & ChemAdd \\
\hline CXP & ChemAdd_CXP & 33 & DIW-TK-00001_CXP-IXC-00001.csv & ChemAdd \\
\hline CXP & ChemAdd_CXP & 33 & DIW-TK-00001_CXP-IXC-00002.csv & ChemAdd \\
\hline CXP & ChemAdd_CXP & 33 & DIW-TK-00001_CXP-IXC-00003.csv & ChemAdd \\
\hline CXP & ChemAdd_CXP & 33 & DIW-TK-00001_CXP-IXC-00004.csv & ChemAdd \\
\hline CXP & ChemAdd_CXP & 33 & SHR-TK-00005_CXP-FILT-00001.csv & ChemAdd \\
\hline CXP & ChemAdd_CXP & 33 & PSA_CXP-VSL-00005.csv & ChemAdd \\
\hline CXP & ChemAdd_CXP & 33 & RFD-AIR_CXP-VSL-00005.csv & ChemAdd \\
\hline CXP & ChemAdd_CXP & 33 & SHR-TK-00005_CXP-VSL-00005.csv & ChemAdd \\
\hline RDP-CRP & CXP_RDP/CRP & 34 & CXP-IXC-00001_RDP-VSL-00002A.csv & Waste \\
\hline RDP-CRP & CXP_RDP/CRP & 34 & CXP-IXC-00001_RDP-VSL-00002B.csv & Waste \\
\hline RDP-CRP & CXP_RDP/CRP & 34 & CXP-IXC-00002_RDP-VSL-00002A.csv & Waste \\
\hline RDP-CRP & CXP_RDP/CRP & 34 & CXP-IXC-00002_RDP-VSL-00002B.csv & Waste \\
\hline RDP-CRP & CXP_RDP/CRP & 34 & CXP-IXC-00003_RDP-VSL-00002A.csv & Waste \\
\hline RDP-CRP & CXP_RDP/CRP & 34 & CXP-IXC-00003_RDP-VSL-00002B.csv & Waste \\
\hline RDP-CRP & CXP_RDP/CRP & 34 & CXP-IXC-00004_RDP-VSL-00002A.csv & Waste \\
\hline RDP-CRP & CXP_RDP/CRP & 34 & CXP-IXC-00004_RDP-VSL-00002B.csv & Waste \\
\hline RDP-CRP & ChemAdd_RDP/CRP & 35 & DIW-TK-00001_RDP-VSL-00002A.csv & ChemAdd \\
\hline RDP-CRP & ChemAdd_RDP/CRP & 35 & DIW-TK-00001_RDP-VSL-00002B.csV & ChemAdd \\
\hline RDP-CRP & ChemAdd_RDP/CRP & 35 & DIW-TK-00004_CRP-VSL-00001.csv & ChemAdd \\
\hline RDP-CRP & ChemAdd_RDP/CRP & 35 & NAR-MXR-00003_CRP-VSL-00001.csv & ChemAdd \\
\hline RDP-CRP & ChemAdd_RDP/CRP & 35 & PSA_RDP-VSL-00002A.csV & ChemAdd \\
\hline RDP-CRP & ChemAdd_RDP/CRP & 35 & PSA_RDP-VSL-00002B.csV & ChemAdd \\
\hline RDP-CRP & ChemAdd_RDP/CRP & 35 & PSA_RDP-VSL-00002C.csV & ChemAdd \\
\hline RDP-CRP & ChemAdd_RDP/CRP & 35 & $\begin{array}{l}\text { RDP-VSL-00002A-CAUSTIC_RDP-VSL- } \\
\text { 00002A.csv }\end{array}$ & ChemAdd \\
\hline RDP-CRP & ChemAdd_RDP/CRP & 35 & RFD-AIR_RDP-VSL-00002A.csV & ChemAdd \\
\hline RDP-CRP & ChemAdd_RDP/CRP & 35 & RFD-AIR_RDP-VSL-00002B.csV & ChemAdd \\
\hline RDP-CRP & ChemAdd_RDP/CRP & 35 & RFD-AIR_RDP-VSL-00002C.csv & ChemAdd \\
\hline RDP-CRP & ChemAdd_RDP/CRP & 35 & RF-RESIN_CRP-VSL-00001.csV & ChemAdd \\
\hline RDP-CRP & ChemAdd_RDP/CRP & 35 & RLD-TK-00006A_RDP-VSL-00002B.csv & ChemAdd \\
\hline RDP-CRP & ChemAdd_RDP/CRP & 35 & SHR-TK-00004_CRP-VSL-00001.csV & ChemAdd \\
\hline $\mathrm{CNP}$ & CXP_CNP & 36 & CXP-IXC-00001_CNP-EVAP-00001.csv & Waste \\
\hline $\mathrm{CNP}$ & CXP_CNP & 36 & CXP-IXC-00002_CNP-EVAP-00001.csv & Waste \\
\hline CNP & CXP_CNP & 36 & CXP-IXC-00003_CNP-EVAP-00001.csv & Waste \\
\hline $\mathrm{CNP}$ & CXP_CNP & 36 & CXP-IXC-00004_CNP-EVAP-00001.csv & Waste \\
\hline $\mathrm{CNP}$ & CXP_CNP & 36 & CNP-VSL-00004_CNP-EVAP-00001.csv & Waste \\
\hline
\end{tabular}




\begin{tabular}{|c|c|c|c|c|}
\hline $\begin{array}{l}\text { System } \\
\text { Name }\end{array}$ & $\begin{array}{l}\text { Stream } \\
\text { Name }\end{array}$ & $\begin{array}{l}\text { Stream } \\
\text { ID }\end{array}$ & $\begin{array}{l}\text { Totalizer } \\
\text { File Name }\end{array}$ & $\begin{array}{l}\text { Waste } \\
\text { Type }\end{array}$ \\
\hline CNP & ChemAdd_CNP & 37 & NAR-TK-00005_CNP-EVAP-00001.csv & ChemAdd \\
\hline $\mathrm{CNP}$ & ChemAdd CNP & 37 & NAR-TK-00006 CNP-VSL-00004.csV & ChemAdd \\
\hline CNP & ChemAdd_CNP & 37 & SHR-TK-00001_CNP-EVAP-00001.csv & ChemAdd \\
\hline CNP & ChemAdd_CNP & 37 & PSA_CNP-VSL-00004.csv & ChemAdd \\
\hline CNP & ChemAdd_CNP & 37 & PSA_CNP-VSL-00003.csv & ChemAdd \\
\hline CNP & ChemAdd_CNP & 37 & RFD-AIR_CNP-VSL-00004.csv & ChemAdd \\
\hline CNP & ChemAdd_CNP & 37 & RFD-AIR_CNP-VSL-00003.csv & ChemAdd \\
\hline TLP & CXP_TLP & 38 & CXP-IXC-00001_CXP-VSL-00026A.csv & Waste \\
\hline TLP & CXP_TLP & 38 & CXP-IXC-00001_CXP-VSL-00026B.csv & Waste \\
\hline TLP & CXP_TLP & 38 & CXP-IXC-00001_CXP-VSL-00026C.csv & Waste \\
\hline TLP & CXP_TLP & 38 & CXP-IXC-00002_CXP-VSL-00026A.csv & Waste \\
\hline TLP & CXP_TLP & 38 & CXP-IXC-00002_CXP-VSL-00026B.csv & Waste \\
\hline TLP & CXP_TLP & 38 & CXP-IXC-00002_CXP-VSL-00026C.csv & Waste \\
\hline TLP & CXP_TLP & 38 & CXP-IXC-00003_CXP-VSL-00026A.csv & Waste \\
\hline TLP & CXP_TLP & 38 & CXP-IXC-00003_CXP-VSL-00026B.csv & Waste \\
\hline TLP & CXP_TLP & 38 & CXP-IXC-00003_CXP-VSL-00026C.csv & Waste \\
\hline TLP & CXP_TLP & 38 & CXP-IXC-00004_CXP-VSL-00026A.csv & Waste \\
\hline TLP & CXP_TLP & 38 & CXP-IXC-00004_CXP-VSL-00026B.csv & Waste \\
\hline TLP & CXP_TLP & 38 & CXP-IXC-00004_CXP-VSL-00026C.csv & Waste \\
\hline TLP & LAWRLD_TLP & 39 & $\begin{array}{l}\text { RLD-VSL-00005_SUPPLEMENTAL-LAW- } \\
\text { RECYCLE.csv }\end{array}$ & Waste \\
\hline TLP & LAWRLD_TLP & 39 & RLD-VSL-00005_TLP-VSL-00009A.csV & Waste \\
\hline TLP & LAWRLD_TLP & 39 & RLD-VSL-00005_TLP-VSL-00009B.csV & Waste \\
\hline TLP & ChemAdd_TLP & 40 & ANTIFOAM-AGENT_TLP-SEP-00001.csv & ChemAdd \\
\hline TLP & ChemAdd_TLP & 40 & DIW-TK-00001_CXP-VSL-00026A.csv & ChemAdd \\
\hline TLP & ChemAdd_TLP & 40 & DIW-TK-00001_CXP-VSL-00026B.csv & ChemAdd \\
\hline TLP & ChemAdd_TLP & 40 & DIW-TK-00001_CXP-VSL-00026C.csV & ChemAdd \\
\hline TLP & ChemAdd_TLP & 40 & $\begin{array}{l}\text { DIW-TK-00001_SUPPLEMENTAL-LAW- } \\
\text { RECYCLE.csv }\end{array}$ & ChemAdd \\
\hline TLP & ChemAdd_TLP & 40 & DIW-TK-00001_TCP-VSL-00001.csV & ChemAdd \\
\hline TLP & ChemAdd_TLP & 40 & DIW-TK-00001_TLP-SEP-00001.csv & ChemAdd \\
\hline TLP & ChemAdd_TLP & 40 & DIW-TK-00001_TLP-VSL-00009A.csV & ChemAdd \\
\hline TLP & ChemAdd_TLP & 40 & DIW-TK-00001_TLP-VSL-00009B.csV & ChemAdd \\
\hline TLP & ChemAdd_TLP & 40 & PSA_CXP-VSL-00026A.csv & ChemAdd \\
\hline TLP & ChemAdd_TLP & 40 & PSA_CXP-VSL-00026B.csV & ChemAdd \\
\hline TLP & ChemAdd_TLP & 40 & PSA_CXP-VSL-00026C.csV & ChemAdd \\
\hline TLP & ChemAdd_TLP & 40 & PSA_TCP-VSL-00001.csv & ChemAdd \\
\hline TLP & ChemAdd_TLP & 40 & PSA_TLP-VSL-00009A.csV & ChemAdd \\
\hline TLP & ChemAdd_TLP & 40 & PSA_TLP-VSL-00009B.csV & ChemAdd \\
\hline TLP & ChemAdd_TLP & 40 & RFD-AIR_CXP-VSL-00026A.csV & ChemAdd \\
\hline TLP & ChemAdd_TLP & 40 & RFD-AIR_CXP-VSL-00026B.csv & ChemAdd \\
\hline TLP & ChemAdd_TLP & 40 & RFD-AIR_CXP-VSL-00026C.csv & ChemAdd \\
\hline TLP & ChemAdd_TLP & 40 & RFD-AIR_TCP-VSL-00001.csv & ChemAdd \\
\hline TLP & ChemAdd_TLP & 40 & RFD-AIR_TLP-VSL-00009A.csV & ChemAdd \\
\hline TLP & ChemAdd_TLP & 40 & RFD-AIR_TLP-VSL-00009B.csv & ChemAdd \\
\hline TLP & ChemAdd_TLP & 40 & RLD-TK-00006A_CXP-VSL-00026A.csV & ChemAdd \\
\hline
\end{tabular}




\begin{tabular}{|c|c|c|c|c|}
\hline $\begin{array}{l}\text { System } \\
\text { Name }\end{array}$ & $\begin{array}{l}\text { Stream } \\
\text { Name }\end{array}$ & $\begin{array}{c}\text { Stream } \\
\text { ID }\end{array}$ & $\begin{array}{l}\text { Totalizer } \\
\text { File Name }\end{array}$ & $\begin{array}{l}\text { Waste } \\
\text { Type }\end{array}$ \\
\hline TLP & ChemAdd_TLP & 40 & RLD-TK-00006A_CXP-VSL-00026B.csV & ChemAdd \\
\hline TLP & ChemAdd_TLP & 40 & RLD-TK-00006A_CXP-VSL-00026C.csV & ChemAdd \\
\hline TLP & ChemAdd_TLP & 40 & $\begin{array}{l}\text { RLD-TK-00006A_SUPPLEMENTAL-LAW- } \\
\text { RECYCLE.csv }\end{array}$ & ChemAdd \\
\hline TLP & ChemAdd_TLP & 40 & RLD-TK-00006A_TCP-VSL-00001.csv & ChemAdd \\
\hline TLP & ChemAdd_TLP & 40 & RLD-TK-00006A_TLP-SEP-00001.csV & ChemAdd \\
\hline TLP & ChemAdd_TLP & 40 & RLD-TK-00006A_TLP-VSL-00009A.csv & ChemAdd \\
\hline TLP & ChemAdd_TLP & 40 & RLD-TK-00006A_TLP-VSL-00009B.csv & ChemAdd \\
\hline TLP & ChemAdd_TLP & 40 & SHR-TK-00007_TLP-VSL-00009A.csv & ChemAdd \\
\hline TLP & ChemAdd_TLP & 40 & SHR-TK-00007_TLP-VSL-00009B.csv & ChemAdd \\
\hline TLP & ChemAdd_TLP & 40 & $\begin{array}{l}\text { TLP-DMST-00001-AIR_TLP-DMST- } \\
\text { 00001.csv }\end{array}$ & ChemAdd \\
\hline TLP & ChemAdd_TLP & 40 & $\begin{array}{l}\text { TLP-INTER-AFTER-CONDENSER- } \\
\text { WATER_TLP-VSL-00002.csv }\end{array}$ & ChemAdd \\
\hline PT-RLD & FEP_PTRLD & 41 & FEP-VSL-00005A_RLD-TK-00006A.csv & Waste \\
\hline PT-RLD & FEP_PTRLD & 41 & FEP-VSL-00005B_RLD-TK-00006A.csv & Waste \\
\hline PT-RLD & CRP_PTRLD & 42 & CRP-VSL-00001_RLD-VSL-00017A.csv & Waste \\
\hline PT-RLD & CRP_PTRLD & 42 & CRP-VSL-00001_RLD-VSL-00017B.csV & Waste \\
\hline PT-RLD & TLP_PTRLD & 43 & TLP-VSL-00002_RLD-TK-00006A.csv & Waste \\
\hline PT-RLD & LAWRLD_PTRLD & 44 & LVP-TK-00001_RLD-VSL-00017A.csv & Waste \\
\hline PT-RLD & LAWRLD_PTRLD & 44 & LVP-TK-00001_RLD-VSL-00017B.csv & Waste \\
\hline PT-RLD & PTRLD_ETF & 45 & RLD-TK-00006B_ETF-1.csv & Waste \\
\hline LCP-LFP & TLP_LCP & 46 & TCP-VSL-00001_LCP-VSL-00001.csv & Waste \\
\hline LCP-LFP & TLP_LCP & 46 & TCP-VSL-00001_LCP-VSL-00002.csV & Waste \\
\hline LCP-LFP & TLP_SupLAW & 47 & $\begin{array}{l}\text { TCP-VSL-00001_SUPPLEMENTAL-LAW- } \\
\text { TREATED.csv }\end{array}$ & Waste \\
\hline LCP-LFP & TLP_SupLAW & 47 & $\begin{array}{l}\text { LCP-VSL-00001_SUPPLEMENTAL-LAW- } \\
\text { TREATED.csv }\end{array}$ & Waste \\
\hline LCP-LFP & TLP_SupLAW & 47 & $\begin{array}{l}\text { LCP-VSL-00002_SUPPLEMENTAL-LAW- } \\
\text { TREATED.csv }\end{array}$ & Waste \\
\hline LCP-LFP & Water_LCP/LFP & 48 & DIW-TK-00001_LCP-VSL-00001.csv & ChemAdd \\
\hline LCP-LFP & Water_LCP/LFP & 48 & DIW-TK-00001_LCP-VSL-00002.csv & ChemAdd \\
\hline LCP-LFP & Water_LCP/LFP & 48 & DIW-TK-00001_LFP-VSL-00001.csV & ChemAdd \\
\hline LCP-LFP & Water_LCP/LFP & 48 & DIW-TK-00001_LFP-VSL-00002.csv & ChemAdd \\
\hline LCP-LFP & Water_LCP/LFP & 48 & DIW-TK-00001_LFP-VSL-00003.csv & ChemAdd \\
\hline LCP-LFP & Water_LCP/LFP & 48 & DIW-TK-00001_LFP-VSL-00004.csv & ChemAdd \\
\hline LCP-LFP & Water_LCP/LFP & 48 & RHEOLOGY-WATER_LFP-VSL-00001.csv & ChemAdd \\
\hline LCP-LFP & Water_LCP/LFP & 48 & RHEOLOGY-WATER_LFP-VSL-00003.csv & ChemAdd \\
\hline LCP-LFP & GFormer_LFP & 49 & LFP-VSL-00001-MIXER_LFP-VSL-00001.csV & ChemAdd \\
\hline LCP-LFP & GFormer_LFP & 49 & LFP-VSL-00003-MIXER_LFP-VSL-00003.csV & ChemAdd \\
\hline LMP & LFP_LMP & 50 & LFP-VSL-00002_LMP-MLTR-00001.csv & Waste \\
\hline LMP & LFP_LMP & 50 & LFP-VSL-00004_LMP-MLTR-00002.csv & Waste \\
\hline LMP & ChemAdd_LMP & 51 & DIW-TK-00001_LMP-MLTR-00002.csv & ChemAdd \\
\hline LMP & ChemAdd_LMP & 51 & DIW-TK-00001_LMP-MLTR-00001.csv & ChemAdd \\
\hline LMP & ChemAdd_LMP & 51 & $\begin{array}{l}\text { LMP-MLTR-00001-AIR_LMP-MLTR- } \\
\text { 00001.csv }\end{array}$ & ChemAdd \\
\hline LMP & ChemAdd_LMP & 51 & $\begin{array}{l}\text { LMP-MLTR-00001-WATER_LMP-MLTR- } \\
\text { 00001.csv }\end{array}$ & ChemAdd \\
\hline
\end{tabular}




\begin{tabular}{|c|c|c|c|c|}
\hline $\begin{array}{l}\text { System } \\
\text { Name }\end{array}$ & $\begin{array}{l}\text { Stream } \\
\text { Name }\end{array}$ & $\begin{array}{c}\text { Stream } \\
\text { ID }\end{array}$ & $\begin{array}{l}\text { Totalizer } \\
\text { File Name }\end{array}$ & $\begin{array}{l}\text { Waste } \\
\text { Type }\end{array}$ \\
\hline LMP & ChemAdd_LMP & 51 & $\begin{array}{l}\text { LMP-MLTR-00002-AIR_LMP-MLTR- } \\
\text { 00002.csv }\end{array}$ & ChemAdd \\
\hline LMP & ChemAdd_LMP & 51 & $\begin{array}{l}\text { LMP-MLTR-00002-WATER_LMP-MLTR- } \\
\text { 00002.csv }\end{array}$ & ChemAdd \\
\hline LMP & LMP_ILAW & 52 & $\begin{array}{l}\text { LMP-MLTR-00001_LAW-CONTAINERS- } \\
\text { 1.csv }\end{array}$ & ILAW \\
\hline LMP & LMP_ILAW & 52 & $\begin{array}{l}\text { LMP-MLTR-00002_LAW-CONTAINERS- } \\
\text { 2.csv }\end{array}$ & ILAW \\
\hline LOP & LMP_LOP & 53 & LMP-MLTR-00001_LOP-SCB-00001.csv & OffGas \\
\hline LOP & LMP_LOP & 53 & LMP-MLTR-00002_LOP-SCB-00002.csv & OffGas \\
\hline LOP & ChemAdd_LOP & 54 & LOP09_LOP-WESP-00001.csv & ChemAdd \\
\hline LOP & ChemAdd_LOP & 54 & LOP09_LOP-WESP-00002.csv & ChemAdd \\
\hline LOP & ChemAdd_LOP & 54 & $\begin{array}{l}\text { LOP-SCB-00001-WATER_LOP-SCB- } \\
\text { 00001.csv }\end{array}$ & ChemAdd \\
\hline LOP & ChemAdd_LOP & 54 & $\begin{array}{l}\text { LOP-SCB-00002-WATER_LOP-SCB- } \\
00002 . c s v\end{array}$ & ChemAdd \\
\hline LVP & LOP_LVP & 55 & LOP-WESP-00001_LAW-VESSEL-VENT.csv & OffGas \\
\hline LVP & LOP_LVP & 55 & LOP-WESP-00002_LAW-VESSEL-VENT.csv & OffGas \\
\hline LVP & ChemAdd_LVP & 56 & $\begin{array}{l}\text { AMR-VSL-00001-2-AIR_LVP-SCR-00001- } \\
\text { 2.csv }\end{array}$ & ChemAdd \\
\hline LVP & ChemAdd_LVP & 56 & $\begin{array}{l}\text { AMR-VSL-00001-2-NH3_LVP-SCR-00001- } \\
\text { 2.csv }\end{array}$ & ChemAdd \\
\hline LVP & ChemAdd_LVP & 56 & $\begin{array}{l}\text { LAW-VESSEL-VENT-AIR_LAW-VESSEL- } \\
\text { VENT.csV }\end{array}$ & ChemAdd \\
\hline LVP & LAWRLD_LVP & 57 & LVP-TK-00001_LVP-SCB-00001.csv & Waste \\
\hline LVP & LAW-Stack & 58 & LVP-SCB-00001_LAW-STACK.csv & OffGas \\
\hline LAW-RLD & LVP_LAWRLD & 59 & LVP-SCB-00001_LVP-TK-00001.csV & Waste \\
\hline LAW-RLD & LOP_LAWRLD & 60 & LOP-SCB-00001_RLD-VSL-00005.csv & Waste \\
\hline LAW-RLD & LOP_LAWRLD & 60 & LOP-SCB-00002_RLD-VSL-00005.csV & Waste \\
\hline LAW-RLD & LOP_LAWRLD & 60 & LOP-WESP-00001_RLD-VSL-00004.csv & Waste \\
\hline LAW-RLD & LOP_LAWRLD & 60 & LOP-WESP-00002_RLD-VSL-00004.csV & Waste \\
\hline LAW-RLD & ChemAdd_LAWRLD & 61 & LVP-VSL-00001-NAOH_LVP-TK-00001.csV & ChemAdd \\
\hline LAW-RLD & ChemAdd_LAWRLD & 61 & LVP-VSL-00001-WATER_LVP-TK-00001.csv & ChemAdd \\
\hline HFP & HLP_HFP & 62 & HLP-VSL-00028_HFP-VSL-00001.csv & Waste \\
\hline HFP & HLP_HFP & 62 & HLP-VSL-00028_HFP-VSL-00005.csv & Waste \\
\hline HFP & ChemAdd_HFP & 63 & DIW-TK-00001_HFP-VSL-00001.csv & ChemAdd \\
\hline HFP & ChemAdd_HFP & 63 & DIW-TK-00001_HFP-VSL-00002.csv & ChemAdd \\
\hline HFP & ChemAdd_HFP & 63 & DIW-TK-00001_HFP-VSL-00005.csv & ChemAdd \\
\hline HFP & ChemAdd_HFP & 63 & DIW-TK-00001_HFP-VSL-00006.csv & ChemAdd \\
\hline HFP & ChemAdd_HFP & 63 & RHEOLOGY-HLW_HFP-VSL-00001.csv & ChemAdd \\
\hline HFP & ChemAdd_HFP & 63 & RHEOLOGY-HLW_HFP-VSL-00005.csv & ChemAdd \\
\hline HFP & GFormer_HFP & 64 & $\begin{array}{l}\text { HFP-VSL-00001-MIXER_HFP-VSL- } \\
\text { 00001.csV }\end{array}$ & ChemAdd \\
\hline HFP & GFormer_HFP & 64 & $\begin{array}{l}\text { HFP-VSL-00005-MIXER_HFP-VSL- } \\
\text { 00005.csv }\end{array}$ & ChemAdd \\
\hline HMP & HFP_HMP & 65 & HFP-VSL-00002_HMP-MLTR-00001.csv & Waste \\
\hline HMP & HFP_HMP & 65 & HFP-VSL-00006_HMP-MLTR-00002.csv & Waste \\
\hline HMP & ChemAdd_HMP & 66 & HMP-MLTR-00001-AIR_HMP-MLTR- & ChemAdd \\
\hline
\end{tabular}




\begin{tabular}{|c|c|c|c|c|}
\hline $\begin{array}{l}\text { System } \\
\text { Name }\end{array}$ & $\begin{array}{l}\text { Stream } \\
\text { Name }\end{array}$ & $\begin{array}{l}\text { Stream } \\
\text { ID }\end{array}$ & $\begin{array}{l}\text { Totalizer } \\
\text { File Name }\end{array}$ & $\begin{array}{l}\text { Waste } \\
\text { Type }\end{array}$ \\
\hline & & & 00001.csv & \\
\hline HMP & ChemAdd_HMP & 66 & $\begin{array}{l}\text { HMP-MLTR-00002-AIR_HMP-MLTR- } \\
\text { 00002.csv }\end{array}$ & ChemAdd \\
\hline HMP & HMP_IHLW & 67 & HMP-MLTR-00001_HLW-CANISTERS-1.csv & IHLW \\
\hline HMP & HMP_IHLW & 67 & HMP-MLTR-00002_HLW-CANISTERS-2.csv & IHLW \\
\hline HOP & HMP_HOP & 68 & HMP-MLTR-00001_HOP-SCB-00001.csv & Waste \\
\hline HOP & HMP_HOP & 68 & HMP-MLTR-00002_HOP-SCB-00002.csv & Waste \\
\hline HOP & ChemAdd_HOP & 69 & DIW-TK-00002_HOP-HEME-00001.csv & ChemAdd \\
\hline HOP & ChemAdd_HOP & 69 & DIW-TK-00002_HOP-HEME-00002.csv & ChemAdd \\
\hline HOP & ChemAdd_HOP & 69 & DIW-TK-00002_HOP-WESP-00001.csv & ChemAdd \\
\hline HOP & ChemAdd_HOP & 69 & DIW-TK-00002_HOP-WESP-00002.csv & ChemAdd \\
\hline HOP & ChemAdd_HOP & 69 & $\begin{array}{l}\text { HLW-VESSEL-VENT-1-AIR_HLW-VESSEL- } \\
\text { VENT-1.csv }\end{array}$ & ChemAdd \\
\hline HOP & ChemAdd_HOP & 69 & $\begin{array}{l}\text { HLW-VESSEL-VENT-2-AIR_HLW-VESSEL- } \\
\text { VENT-2.csv }\end{array}$ & ChemAdd \\
\hline HOP & ChemAdd_HOP & 69 & HOP08-1_HOP-SCB-00001.csv & ChemAdd \\
\hline HOP & ChemAdd_HOP & 69 & HOP08-2_HOP-SCB-00002.csv & ChemAdd \\
\hline HOP & ChemAdd_HOP & 69 & HOP-SCR-00001-AIR_HOP-SCR-00001.csv & ChemAdd \\
\hline HOP & ChemAdd_HOP & 69 & HOP-SCR-00001-NH3_HOP-SCR-00001.csv & ChemAdd \\
\hline HOP & ChemAdd_HOP & 69 & HOP-SCR-00002-AIR_HOP-SCR-00002.csv & ChemAdd \\
\hline HOP & ChemAdd_HOP & 69 & HOP-SCR-00002-NH3_HOP-SCR-00002.csv & ChemAdd \\
\hline HOP & ChemAdd_HOP & 69 & PSA_HOP-SCB-00001.csv & ChemAdd \\
\hline HOP & ChemAdd_HOP & 69 & PSA_HOP-SCB-00002.csv & ChemAdd \\
\hline HOP & ChemAdd_HOP & 69 & RFD-AIR_HOP-SCB-00001.csv & ChemAdd \\
\hline HOP & ChemAdd_HOP & 69 & RFD-AIR_HOP-SCB-00002.csv & ChemAdd \\
\hline HOP & ChemAdd_HOP & 69 & RLD-VSL-00007_PJV-HEPA-00004-5.csV & ChemAdd \\
\hline HOP & HLW-Stacks & 70 & HOP-SCR-00001_HLW-STACK.csv & OffGas \\
\hline HOP & HLW-Stacks & 70 & HOP-SCR-00002_HLW-STACK.csv & OffGas \\
\hline HOP & HLW-Stacks & 70 & PJV-HEPA-00004-5_HLW-PJV-STACK.csV & OffGas \\
\hline RLD-HLW & HOP_HLWRLD & 71 & HOP-SCB-00001_RLD-VSL-00007.csv & Waste \\
\hline RLD-HLW & HOP_HLWRLD & 71 & HOP-SCB-00002_RLD-VSL-00007.csv & Waste \\
\hline RLD-HLW & HDH_HLWRLD & 72 & HDH-VSL-00003_RLD-VSL-00007.csv & Waste \\
\hline RLD-HLW & ChemAdd_HLWRLD & 73 & DIW-TK-00001_RLD-VSL-00007.csv & ChemAdd \\
\hline RLD-HLW & ChemAdd_HLWRLD & 73 & $\begin{array}{l}\text { HDH-VSL-00003-FLUSH_RLD-VSL- } \\
\text { 00007.csv }\end{array}$ & ChemAdd \\
\hline RLD-HLW & ChemAdd_HLWRLD & 73 & PSA_RLD-VSL-00007.csv & ChemAdd \\
\hline RLD-HLW & ChemAdd_HLWRLD & 73 & RFD-AIR_RLD-VSL-00007.csv & ChemAdd \\
\hline RLD-HLW & ChemAdd_HLWRLD & 73 & SHR-TK-00002_RLD-VSL-00007.csv & ChemAdd \\
\hline $\mathrm{HDH}$ & ChemAdd_HDH & 74 & DIW-TK-00001_HDH-VSL-00003.csv & ChemAdd \\
\hline $\mathrm{HDH}$ & ChemAdd_HDH & 74 & DIW-TK-00002_HDH-VSL-00002.csv & ChemAdd \\
\hline $\mathrm{HDH}$ & ChemAdd_HDH & 74 & DIW-TK-00002_HDH-VSL-00004.csv & ChemAdd \\
\hline $\mathrm{HDH}$ & ChemAdd_HDH & 74 & HDH-TK-00002_HDH-VSL-00002.csv & ChemAdd \\
\hline $\mathrm{HDH}$ & ChemAdd_HDH & 74 & HDH-TK-00002_HDH-VSL-00004.csv & ChemAdd \\
\hline $\mathrm{HDH}$ & ChemAdd_HDH & 74 & HDH-TK-00003_HDH-VSL-00003.csv & ChemAdd \\
\hline $\mathrm{HDH}$ & ChemAdd_HDH & 74 & $\begin{array}{l}\text { HDH-VSL-00001-WATER_HDH-VSL- } \\
\text { 00001.csv }\end{array}$ & ChemAdd \\
\hline $\mathrm{HDH}$ & ChemAdd_HDH & 74 & HPS_HDH-VSL-00003.csV & ChemAdd \\
\hline
\end{tabular}




\begin{tabular}{|c|c|c|c|c|}
\hline $\begin{array}{l}\text { System } \\
\text { Name }\end{array}$ & $\begin{array}{l}\text { Stream } \\
\text { Name }\end{array}$ & $\begin{array}{l}\text { Stream } \\
\text { ID }\end{array}$ & $\begin{array}{l}\text { Totalizer } \\
\text { File Name }\end{array}$ & $\begin{array}{l}\text { Waste } \\
\text { Type }\end{array}$ \\
\hline $\mathrm{HDH}$ & ChemAdd HDH & 74 & NAR-TK-00001 HDH-VSL-00002.csv & ChemAdd \\
\hline $\mathrm{HDH}$ & ChemAdd_HDH & 74 & NAR-TK-00001_HDH-VSL-00004.csV & ChemAdd \\
\hline $\mathrm{HDH}$ & ChemAdd_HDH & 74 & SHR-TK-00002_HDH-VSL-00003.csv & ChemAdd \\
\hline PWD & HLWRLD_Recycle & 75 & RLD-VSL-00007_PWD-VSL-00043.csV & Waste \\
\hline PWD & CNP_Recycle & 76 & CNP-EVAP-00001_PWD-VSL-00015.csv & Waste \\
\hline PWD & CNP_Recycle & 76 & CNP-EVAP-00001_PWD-VSL-00016.csv & Waste \\
\hline PWD & CXP_Recycle & 77 & CXP-IXC-00001_PWD-VSL-00015.csV & Waste \\
\hline PWD & CXP_Recycle & 77 & CXP-IXC-00001_PWD-VSL-00016.csv & Waste \\
\hline PWD & CXP_Recycle & 77 & CXP-IXC-00002_PWD-VSL-00015.csV & Waste \\
\hline PWD & CXP_Recycle & 77 & CXP-IXC-00002_PWD-VSL-00016.csv & Waste \\
\hline PWD & CXP_Recycle & 77 & CXP-IXC-00003_PWD-VSL-00015.csV & Waste \\
\hline PWD & CXP_Recycle & 77 & CXP-IXC-00003_PWD-VSL-00016.csV & Waste \\
\hline PWD & CXP_Recycle & 77 & CXP-IXC-00004_PWD-VSL-00015.csv & Waste \\
\hline PWD & CXP_Recycle & 77 & CXP-IXC-00004_PWD-VSL-00016.csv & Waste \\
\hline PWD & CXP_Recycle & 77 & CXP-FILT-00001_PWD-VSL-00016.csv & Waste \\
\hline HLP27A & FEP_HLP27A & 78 & FEP-SEP-00001A_HLP-VSL-00027A.csv & Waste \\
\hline HLP27A & FEP_HLP27A & 78 & FEP-SEP-00001B_HLP-VSL-00027A.csV & Waste \\
\hline FRP2A & FEP_FRP2A & 79 & FEP-SEP-00001A_FRP-VSL-00002A.csv & Waste \\
\hline FRP2A & FEP_FRP2A & 79 & FEP-SEP-00001B_FRP-VSL-00002A.csv & Waste \\
\hline UFP & HLP27A_UFP1 & 80 & HLP-VSL-00027A_UFP-VSL-00001A.csv & Waste \\
\hline UFP & HLP27A_UFP1 & 80 & HLP-VSL-00027A_UFP-VSL-00001B.csv & Waste \\
\hline PT-RLD & LineFlush & 81 & RLD-TK-00006A_CXP-VSL-00026A.csV & ProCond \\
\hline PT-RLD & LineFlush & 81 & RLD-TK-00006A_CXP-VSL-00026B.csv & ProCond \\
\hline PT-RLD & LineFlush & 81 & RLD-TK-00006A_CXP-VSL-00026C.csv & ProCond \\
\hline PT-RLD & LineFlush & 81 & RLD-TK-00006A FEP-SEP-00001A.csv & ProCond \\
\hline PT-RLD & LineFlush & 81 & RLD-TK-00006A_FEP-SEP-00001B.csv & ProCond \\
\hline PT-RLD & LineFlush & 81 & RLD-TK-00006A_FEP-VSL-00017A.csv & ProCond \\
\hline PT-RLD & LineFlush & 81 & RLD-TK-00006A_FEP-VSL-00017B.csv & ProCond \\
\hline PT-RLD & LineFlush & 81 & RLD-TK-00006A_FRP-VSL-00002A.csV & ProCond \\
\hline PT-RLD & LineFlush & 81 & RLD-TK-00006A_FRP-VSL-00002B.csV & ProCond \\
\hline PT-RLD & LineFlush & 81 & RLD-TK-00006A_FRP-VSL-00002C.csv & ProCond \\
\hline PT-RLD & LineFlush & 81 & RLD-TK-00006A_FRP-VSL-00002D.csV & ProCond \\
\hline PT-RLD & LineFlush & 81 & RLD-TK-00006A_HLP-VSL-00022.csv & ProCond \\
\hline PT-RLD & LineFlush & 81 & RLD-TK-00006A_RDP-VSL-00002B.csv & ProCond \\
\hline PT-RLD & LineFlush & 81 & $\begin{array}{l}\text { RLD-TK-00006A_SUPPLEMENTAL-LAW- } \\
\text { RECYCLE.csv }\end{array}$ & ProCond \\
\hline PT-RLD & LineFlush & 81 & RLD-TK-00006A_TCP-VSL-00001.csv & ProCond \\
\hline PT-RLD & LineFlush & 81 & RLD-TK-00006A_TLP-SEP-00001.csV & ProCond \\
\hline PT-RLD & LineFlush & 81 & RLD-TK-00006A_TLP-VSL-00009A.csV & ProCond \\
\hline PT-RLD & LineFlush & 81 & RLD-TK-00006A_TLP-VSL-00009B.csv & ProCond \\
\hline CXP & GuardFilter_Backflush & 82 & CXP-FILT-00001_PWD-VSL-00016.csV & Waste \\
\hline LVP & LVP_Removed & 83 & $\begin{array}{l}\text { LVP-ADBR-00001AB_LVP-ADBR- } \\
\text { 00001AB.csv }\end{array}$ & OffGas \\
\hline LVP & LVP_Removed & 83 & $\begin{array}{l}\text { LVP-HEPA-00001AB_LVP-HEPA- } \\
\text { 00001AB.csv }\end{array}$ & OffGas \\
\hline LVP & LVP_Removed & 83 & LVP-SCR-00001-2_LVP-SCR-00001-2.csV & OffGas \\
\hline
\end{tabular}


24590-WTP-MDD-PR-01-002, Rev 12 Dynamic (G2) Model Design Document

\begin{tabular}{|l|l|c|l|c|}
\hline $\begin{array}{l}\text { System } \\
\text { Name }\end{array}$ & $\begin{array}{l}\text { Stream } \\
\text { Name }\end{array}$ & $\begin{array}{c}\text { Stream } \\
\text { ID }\end{array}$ & $\begin{array}{l}\text { Totalizer } \\
\text { File Name }\end{array}$ & $\begin{array}{c}\text { Waste } \\
\text { Type }\end{array}$ \\
\hline HOP & HOP_Removed & 84 & HOP-ABS-00002_HOP-ABS-00002.csv & OffGas \\
\hline HOP & HOP_Removed & 84 & HOP-ABS-00003_HOP-ABS-00003.csv & OffGas \\
\hline HOP & HOP_Removed & 84 & $\begin{array}{l}\text { HOP-ADBR-00001AB_HOP-ADBR- } \\
\text { 00001AB.csv }\end{array}$ & OffGas \\
\hline HOP & HOP_Removed & 84 & $\begin{array}{l}\text { HOP-ADBR-00002AB_HOP-ADBR- } \\
\text { 00002AB.csv }\end{array}$ & OffGas \\
\hline HOP & HOP_Removed & 84 & $\begin{array}{l}\text { HOP-HEPA-00001-2-AB_HOP-HEPA-00001- } \\
\text { 2-AB.csv }\end{array}$ & OffGas \\
\hline HOP & HOP_Removed & 84 & $\begin{array}{l}\text { HOP-HEPA-00007-8-AB_HOP-HEPA-00007- } \\
\text { 8-AB.csv }\end{array}$ & OffGas \\
\hline HOP & HOP_Removed & 84 & HOP-SCR-00001_HOP-SCR-00001.csv & OffGas \\
\hline HOP & HOP_Removed & 84 & HOP-SCR-00002_HOP-SCR-00002.csv & OffGas \\
\hline HOP & HOP_Removed & 84 & PJV-HEPA-00004-5_PJV-HEPA-00004-5.csv & OffGas \\
\hline PVP & PVP_Removed & 85 & PJV-HEPA_PJV-HEPA.csV & OffGas \\
\hline PVP & PVP_Removed & 85 & PVP-ABS-00001AB_PVP-ABS-00001AB.csv & OffGas \\
\hline PVP & PVP_Removed & 85 & PVP-HEPA-00001-2_PVP-HEPA-00001-2.csv & OffGas \\
\hline PVP & PVP_Removed & 85 & PVP-OXID-00001_PVP-OXID-00001.csv & OffGas \\
\hline UFP & IXC_Displacement & 86 & CXP-IXC-00001_UFP-VSL-00062A.csv & Waste \\
\hline UFP & IXC_Displacement & 86 & CXP-IXC-00002_UFP-VSL-00062A.csv & Waste \\
\hline UFP & IXC_Displacement & 86 & CXP-IXC-00003_UFP-VSL-00062A.csv & Waste \\
\hline UFP & IXC_Displacement & 86 & CXP-IXC-00004_UFP-VSL-00062A.csv & Waste \\
\hline UFP & IXC_Displacement & 86 & IX-DI-WATER_UFP-VSL-00062A.csv & Waste \\
\hline
\end{tabular}




\section{Table J-2 Visual C\# Routine for Mass Balance Flowsheet Diagrams}

using System;

using System.I0;

using system. Collections;

using System.Collections.Generic;

using System.ComponentModel;

using System.Data;

using System.Drawing;

using system. Text;

using System. Windows. Forms;

using Microsoft.office.Interop.Excel;

using System. Threading;

namespace G2MBC_GUI

public partial class G2MassBalanceCalculator : Form

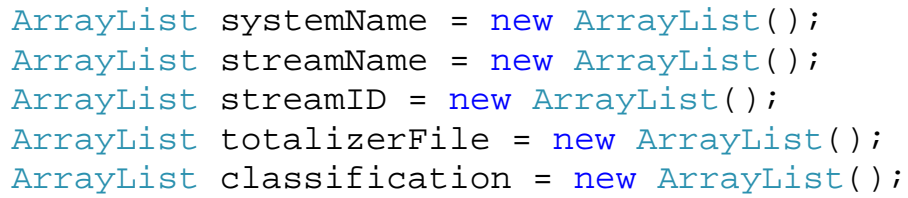

int streamCount;

double[] liqMassTotal, solMassTotal, gasMassTotal, oxMassTotal, massTotal;

double[] liqvolTotal, solvolTotal, volTotal;

double $[$,$] liqMassComp, solMassComp, oxMassComp, gasMassComp;$

string[] lsCompName, oxCompName, gasCompName;

string outputHeader;

string sysName = "SystemName \t";

string strmName = "StreamName $\backslash t "$;

string strmID = "StreamID $\backslash t "$;

string classi = "Classification \t";

string oldName $=" "$;

string liqMassLine = "Liq. Mass (kgs) \t";

string solMassLine $=$ "Sol. Mass (kgs) \t";

string gasMassLine $=$ "Gas Mass (kgs) \t";

string oxMassLine $=" 0 x$. Mass $(\mathrm{kgs}) \backslash \mathrm{t} "$; 


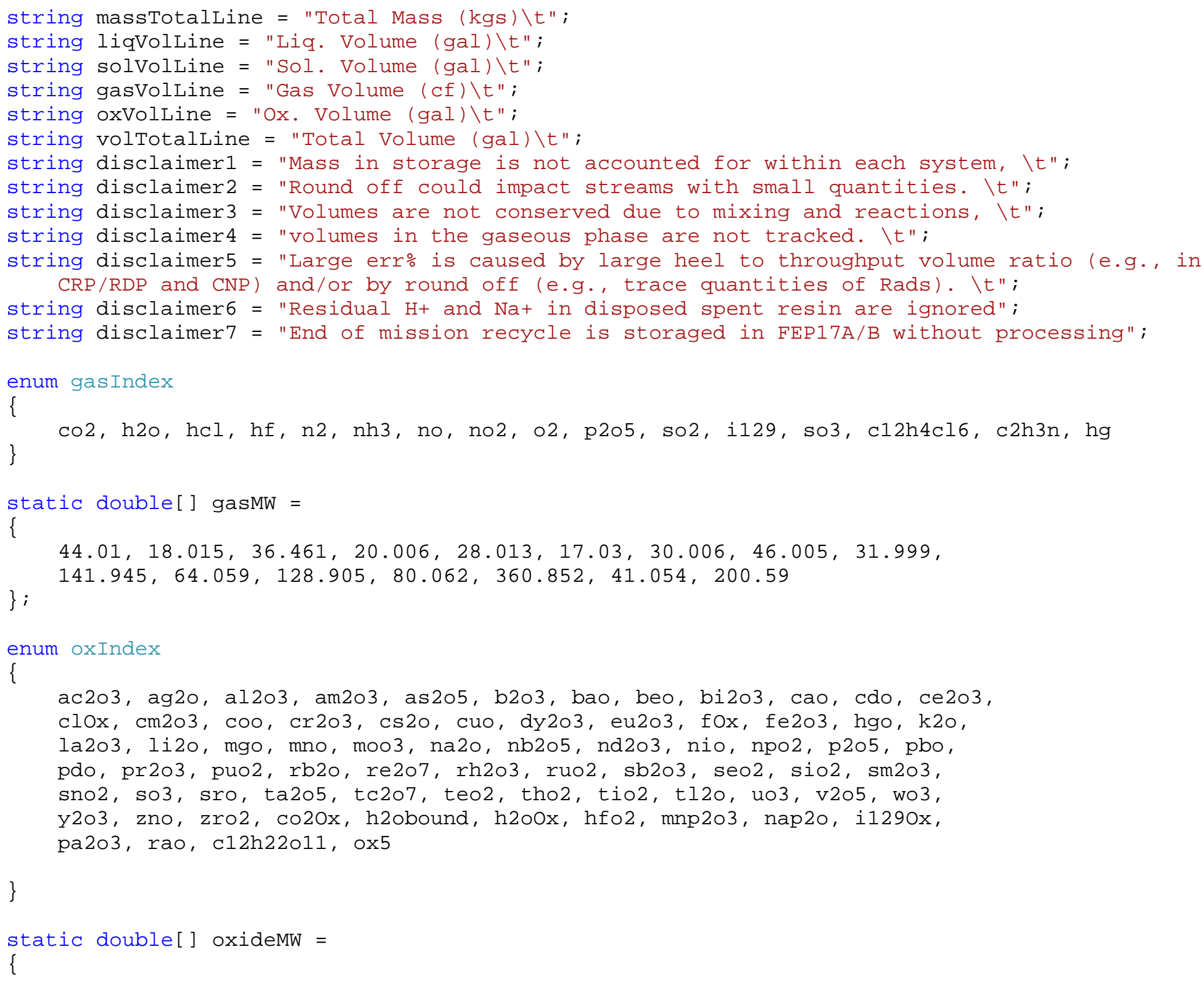




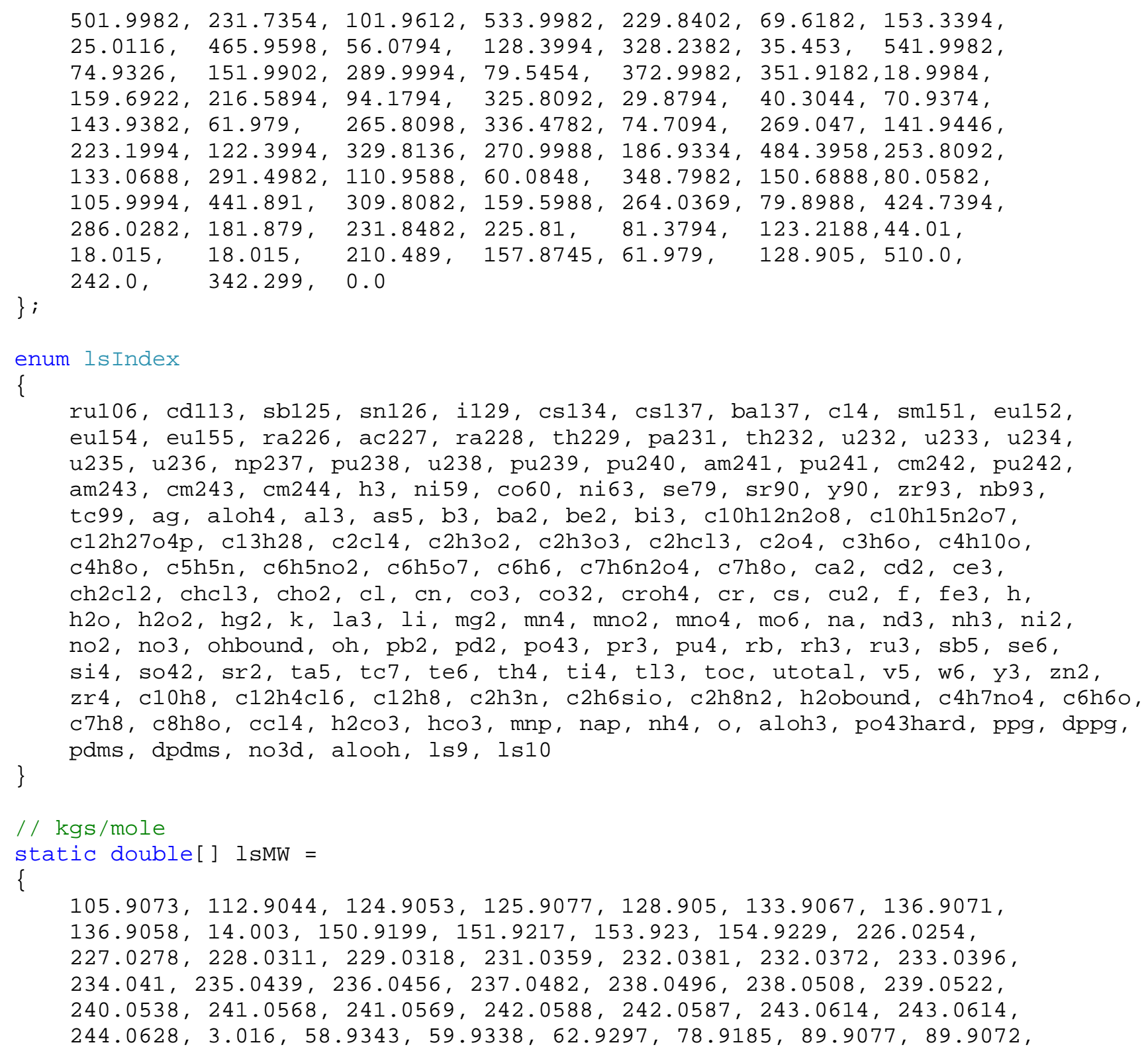




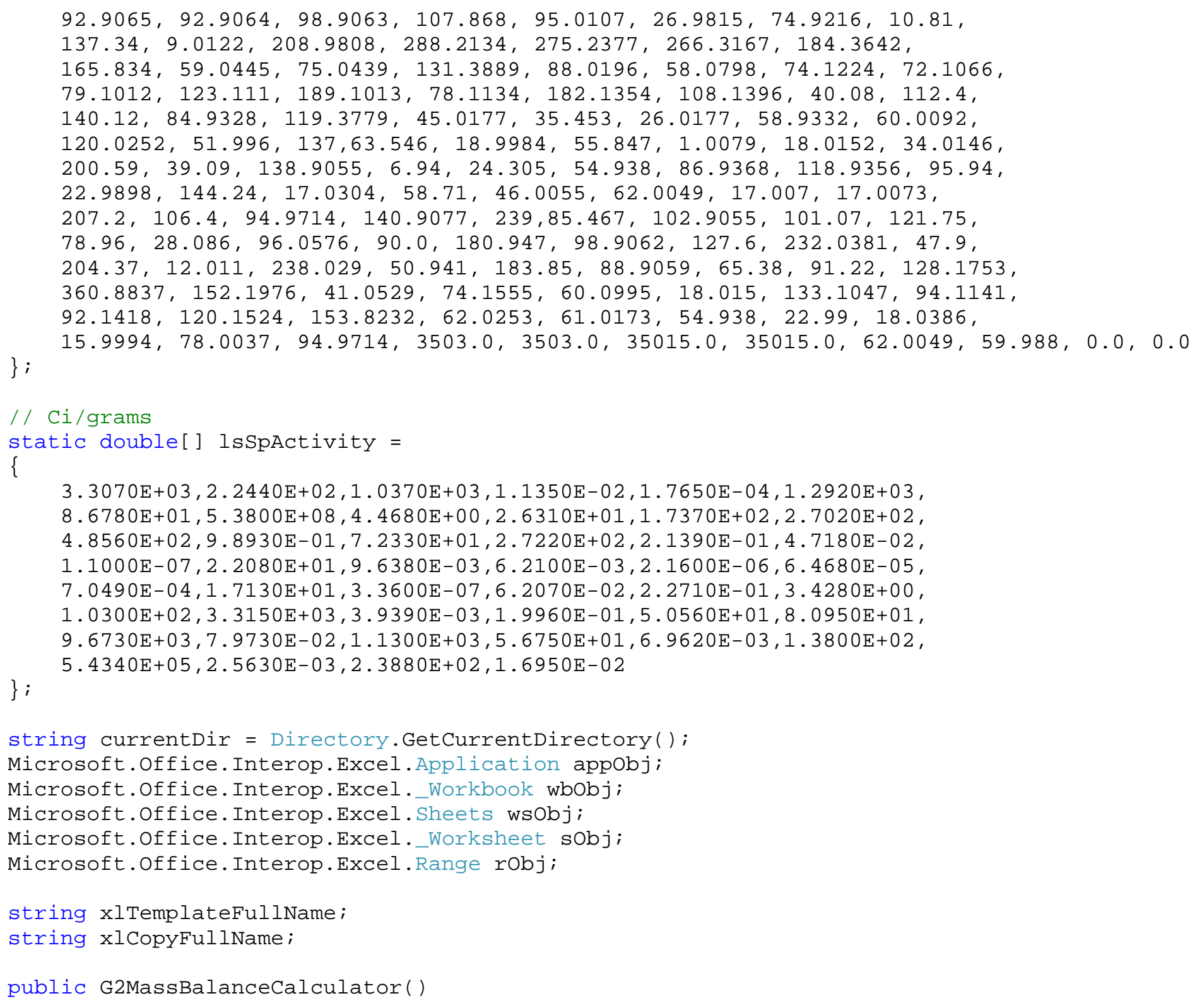

$3.3070 \mathrm{E}+03,2.2440 \mathrm{E}+02,1.0370 \mathrm{E}+03,1.1350 \mathrm{E}-02,1.7650 \mathrm{E}-04,1.2920 \mathrm{E}+03$,

8.6780E+01, $5.3800 \mathrm{E}+08,4.4680 \mathrm{E}+00,2.6310 \mathrm{E}+01,1.7370 \mathrm{E}+02,2.7020 \mathrm{E}+02$, $4.8560 \mathrm{E}+02,9.8930 \mathrm{E}-01,7.2330 \mathrm{E}+01,2.7220 \mathrm{E}+02,2.1390 \mathrm{E}-01,4.7180 \mathrm{E}-02$, $1.1000 \mathrm{E}-07,2.2080 \mathrm{E}+01,9.6380 \mathrm{E}-03,6.2100 \mathrm{E}-03,2.1600 \mathrm{E}-06,6.4680 \mathrm{E}-05$, 7. $0490 \mathrm{E}-04,1.7130 \mathrm{E}+01,3.3600 \mathrm{E}-07,6.2070 \mathrm{E}-02,2.2710 \mathrm{E}-01,3.4280 \mathrm{E}+\odot \odot$, $1.0300 \mathrm{E}+02,3.3150 \mathrm{E}+03,3.9390 \mathrm{E}-03,1.9960 \mathrm{E}-01,5.0560 \mathrm{E}+01,8.0950 \mathrm{E}+01$, $9.6730 \mathrm{E}+03,7.9730 \mathrm{E}-02,1.1300 \mathrm{E}+03,5.6750 \mathrm{E}+01,6.9620 \mathrm{E}-03,1.3800 \mathrm{E}+02$, \}

$5.4340 \mathrm{E}+05,2.5630 \mathrm{E}-03,2.3880 \mathrm{E}+02,1.6950 \mathrm{E}-\odot 2$

string currentDir = Directory.GetCurrentDirectory( );

Microsoft.Office.Interop.Excel.Application appobj;

Microsoft. Office. Interop.Excel. Workbook wbobj;

Microsoft. Office. Interop.Excel. Sheets wsObj;

Microsoft. Office. Interop.Excel. Worksheet sobj;

Microsoft.Office.Interop.Excel. Range robj;

string xlTemplateFullName;

string xlCopyFullName;

public G2MassBalanceCalculator() 


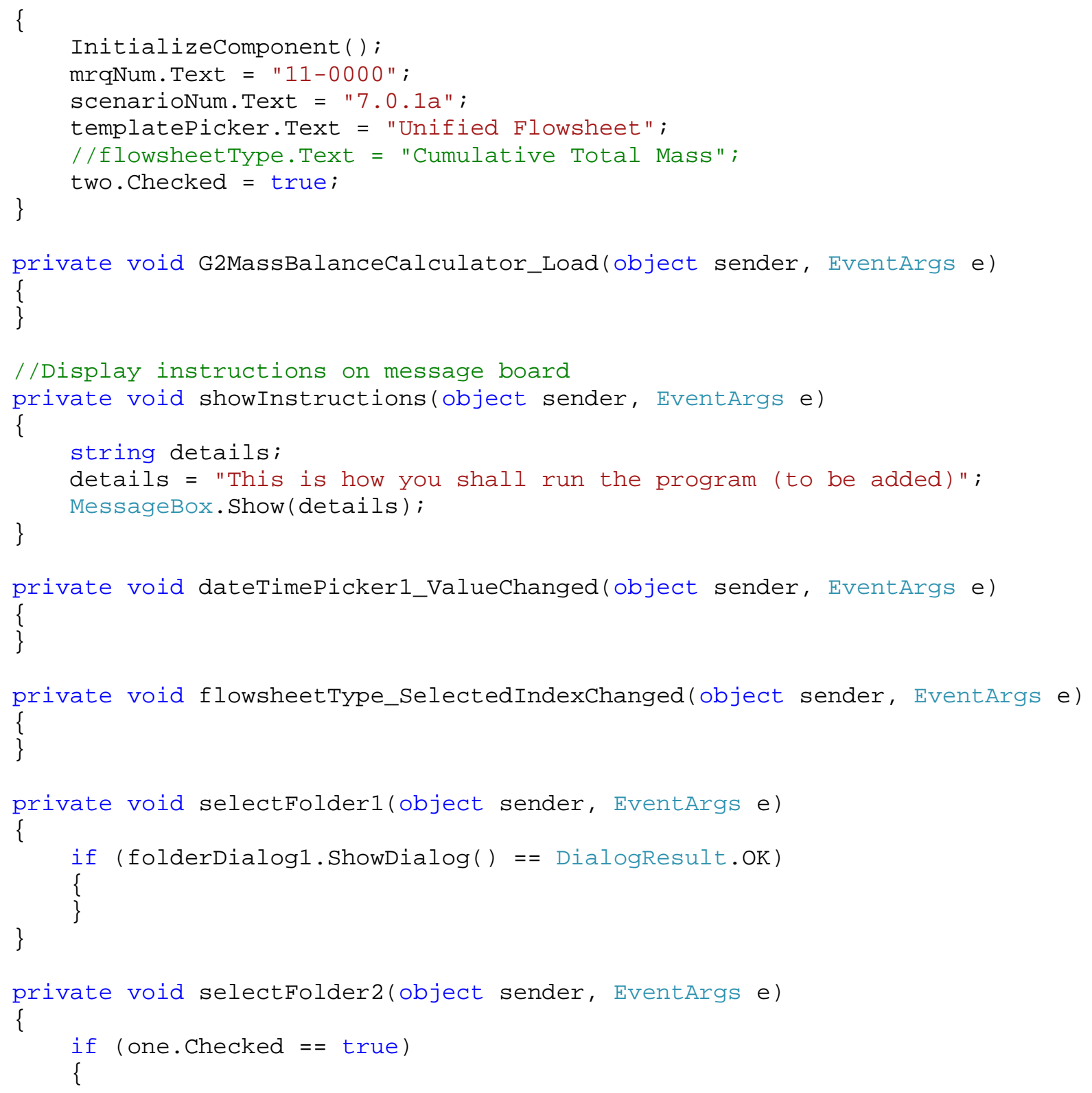




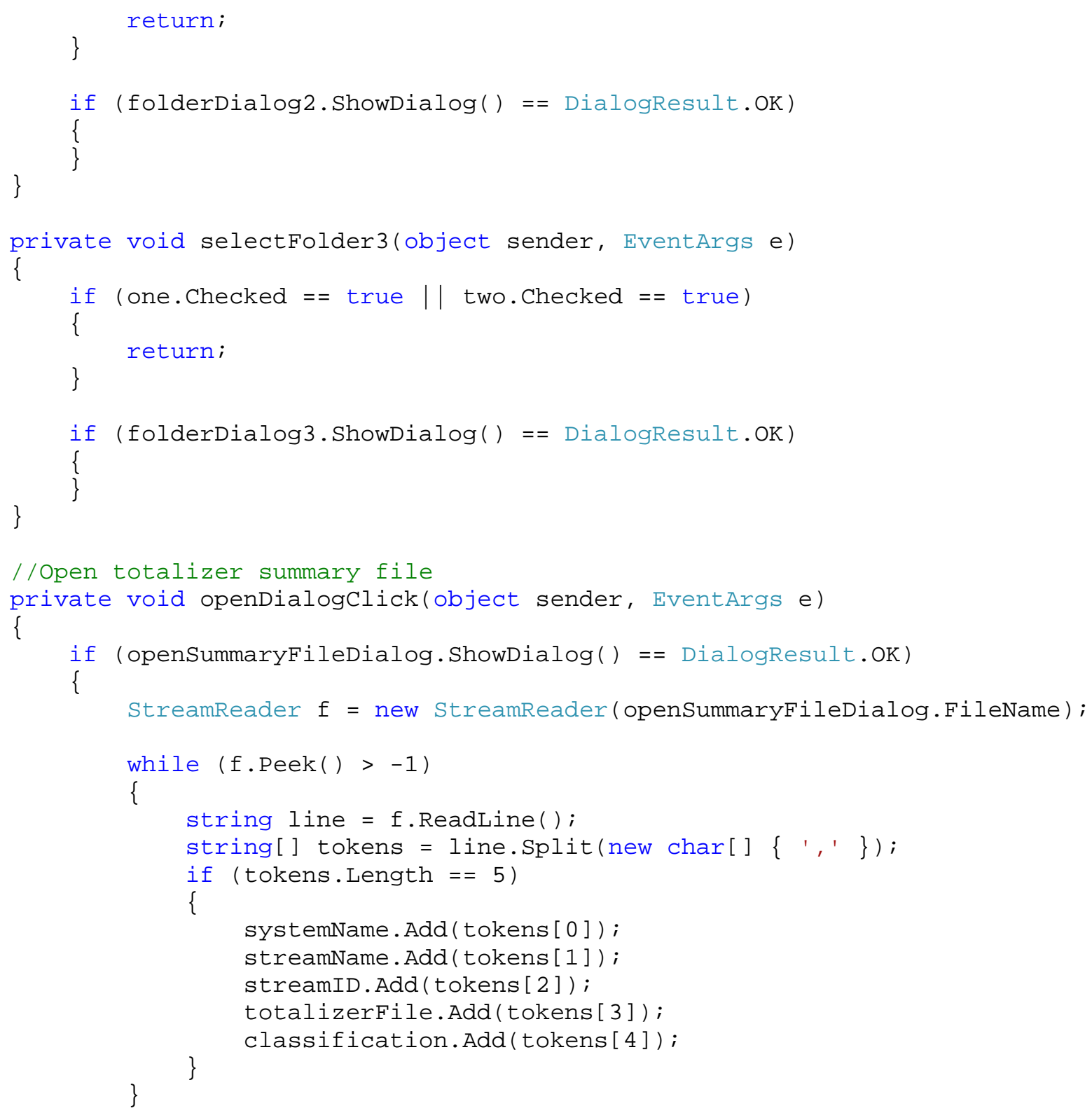




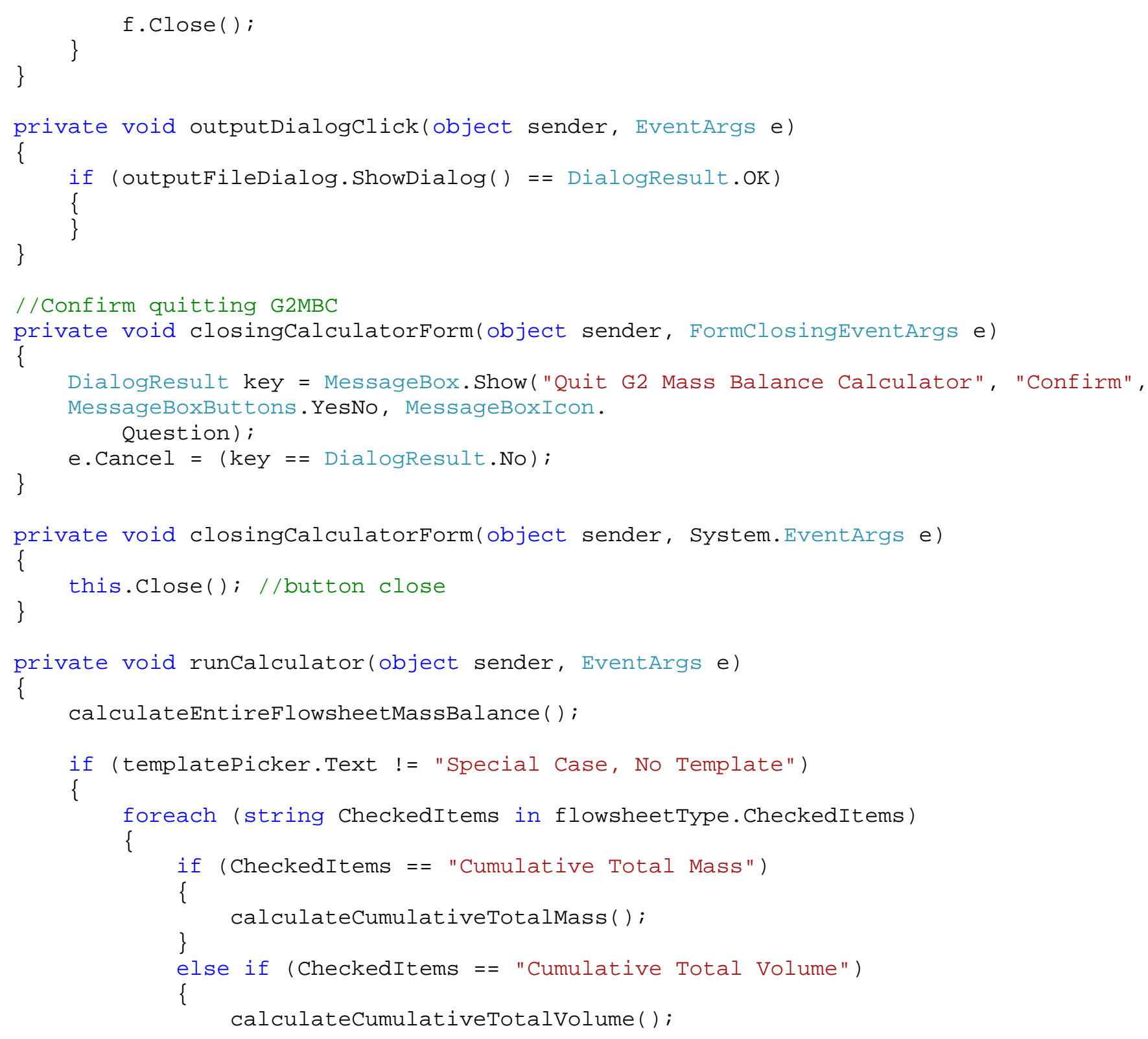




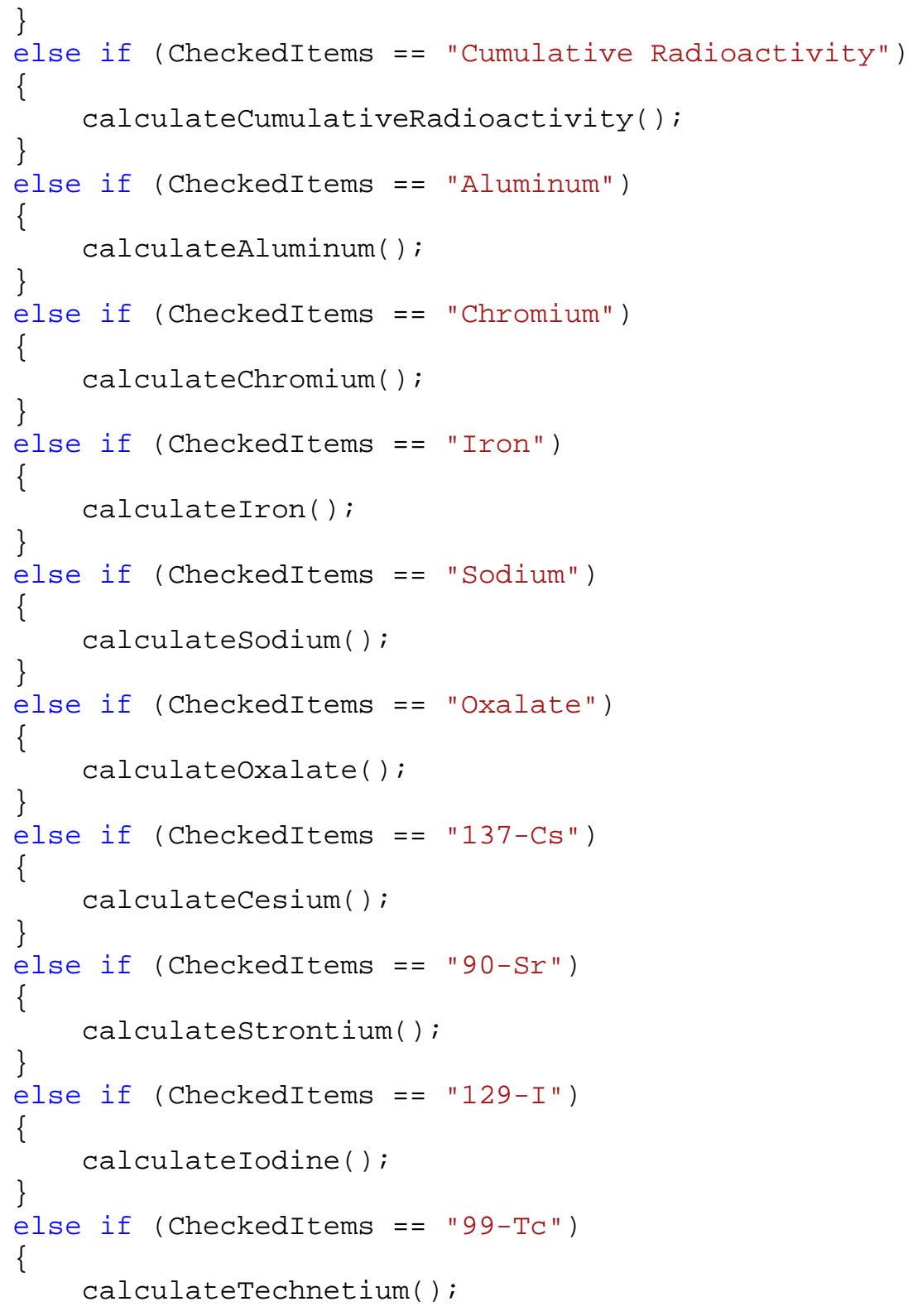




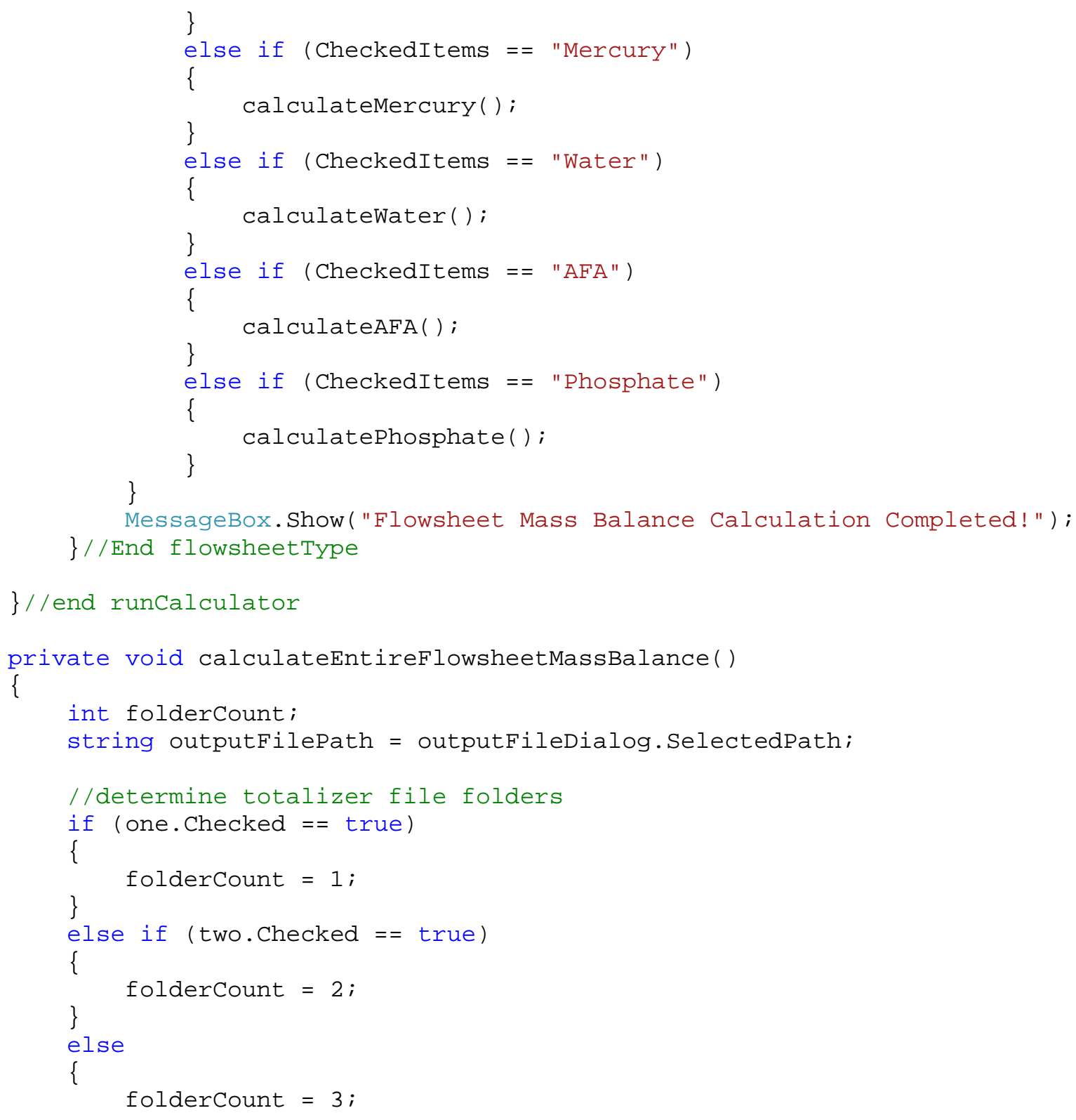


//initialize calculation arrays

streamCount $=$ int. Parse $((\operatorname{string})(\operatorname{streamID}[\operatorname{streamID} \cdot$ Count -1$]))$;

liqMassTotal = new double [streamCount];

solMassTotal $=$ new double [streamCount];

gasMassTotal = new double [streamCount];

oxMassTotal = new double [streamCount];

massTotal = new double [streamcount];

liqVolTotal = new double[streamCount];

solvolTotal = new double [streamCount];

volTotal = new double [streamCount];

liqMassComp = new double[lsMW. Length, streamCount];

solMassComp = new double[lsMW. Length, streamCount];

oxMassComp $=$ new double [oxideMW. Length, streamCount];

gasMasscomp $=$ new double[gasMW. Length, streamCount];

lsCompName = new string[1sMW. Length];

oxCompName $=$ new string[oxideMw. Length];

gasCompName $=$ new string[gasMW. Length];

//repeat calculation for totalizer file folders

for (int folder $=1$; folder $!=$ folderCount +1 ; folder++)

\{

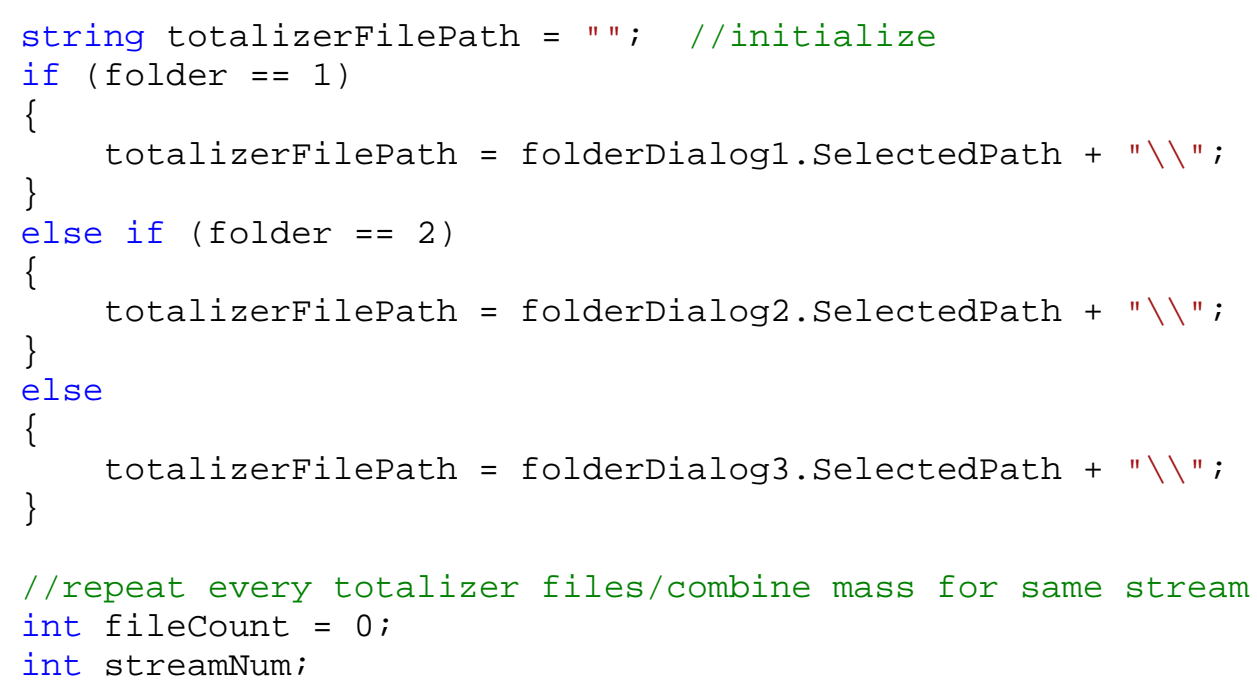




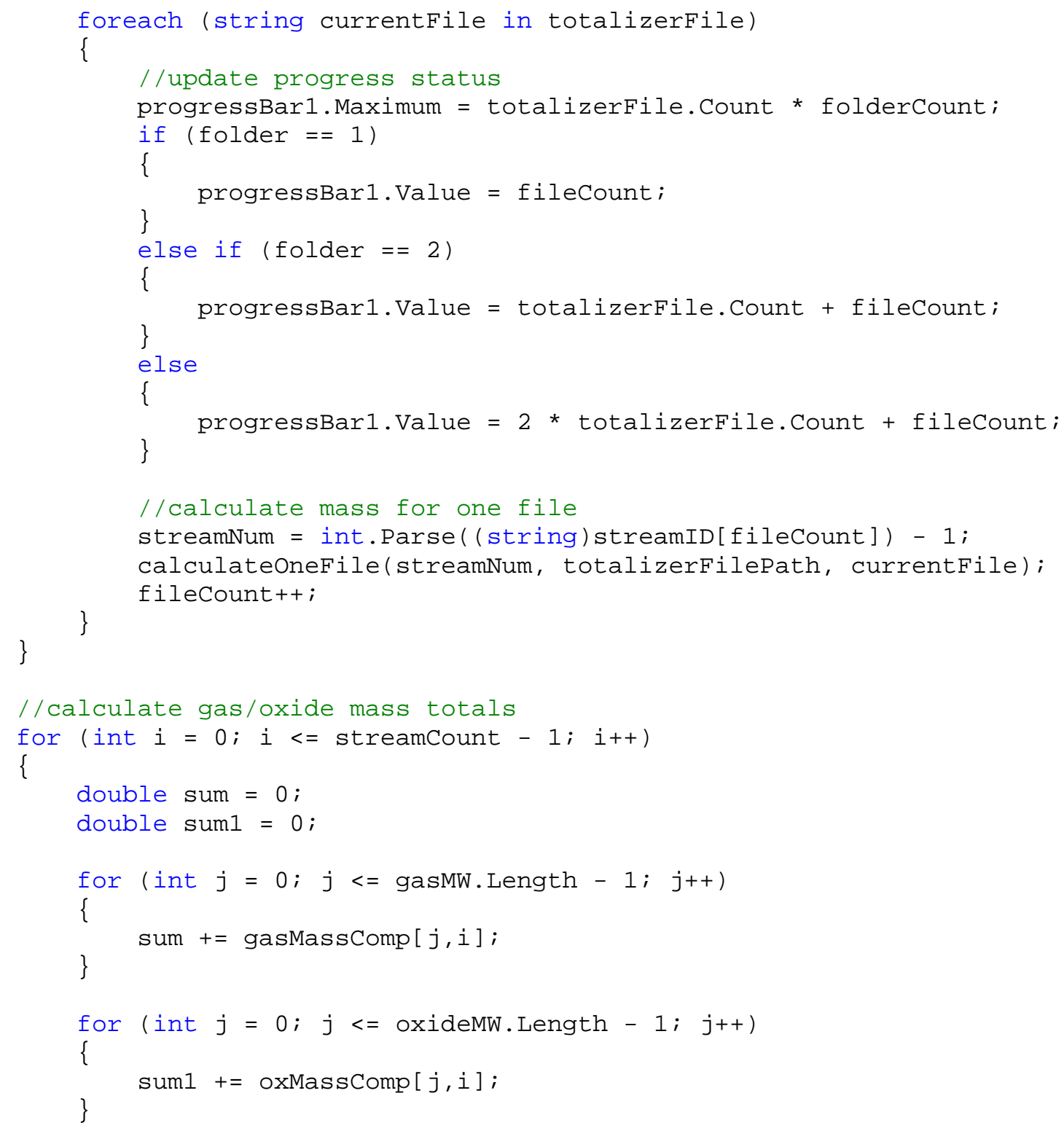




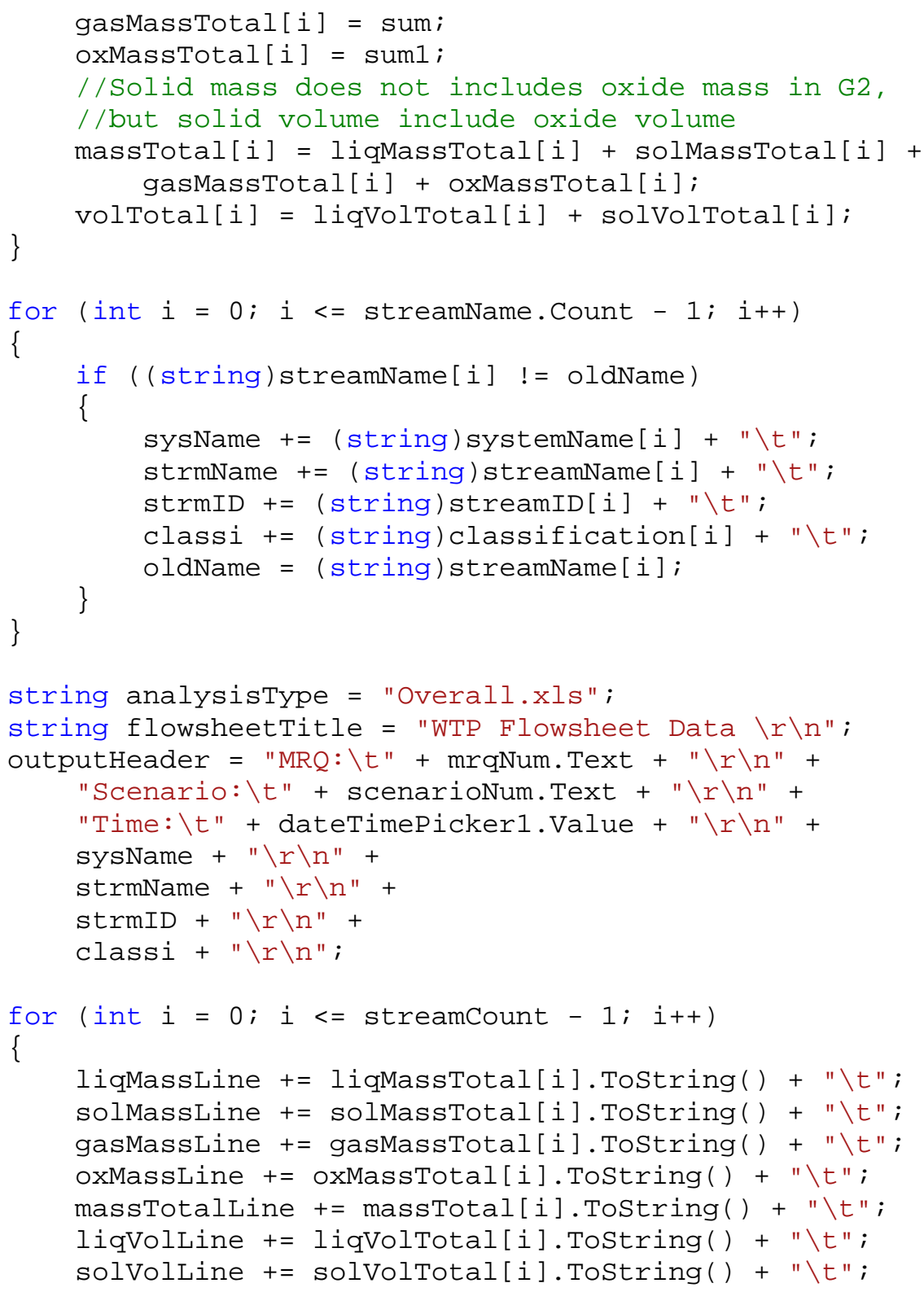




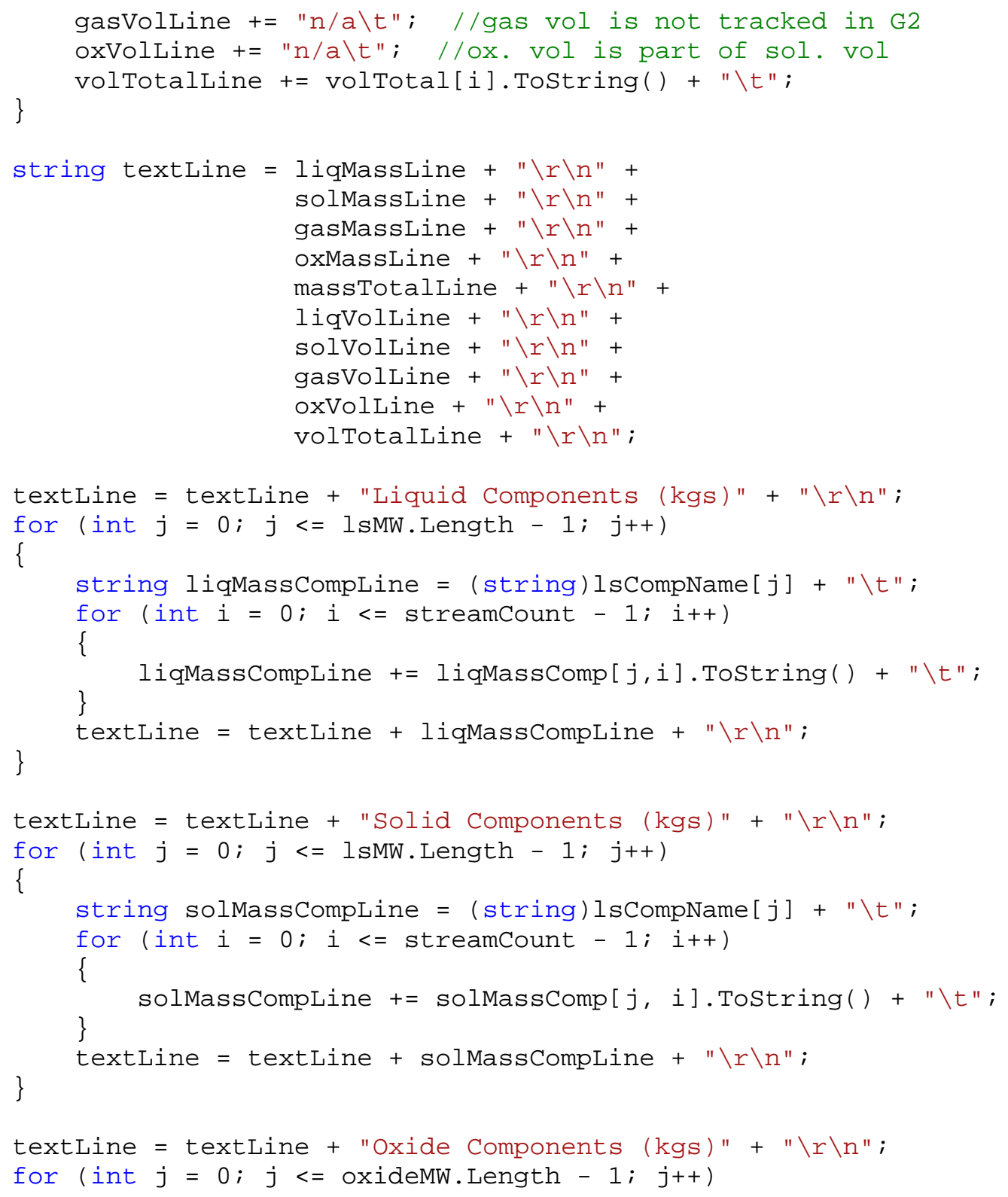




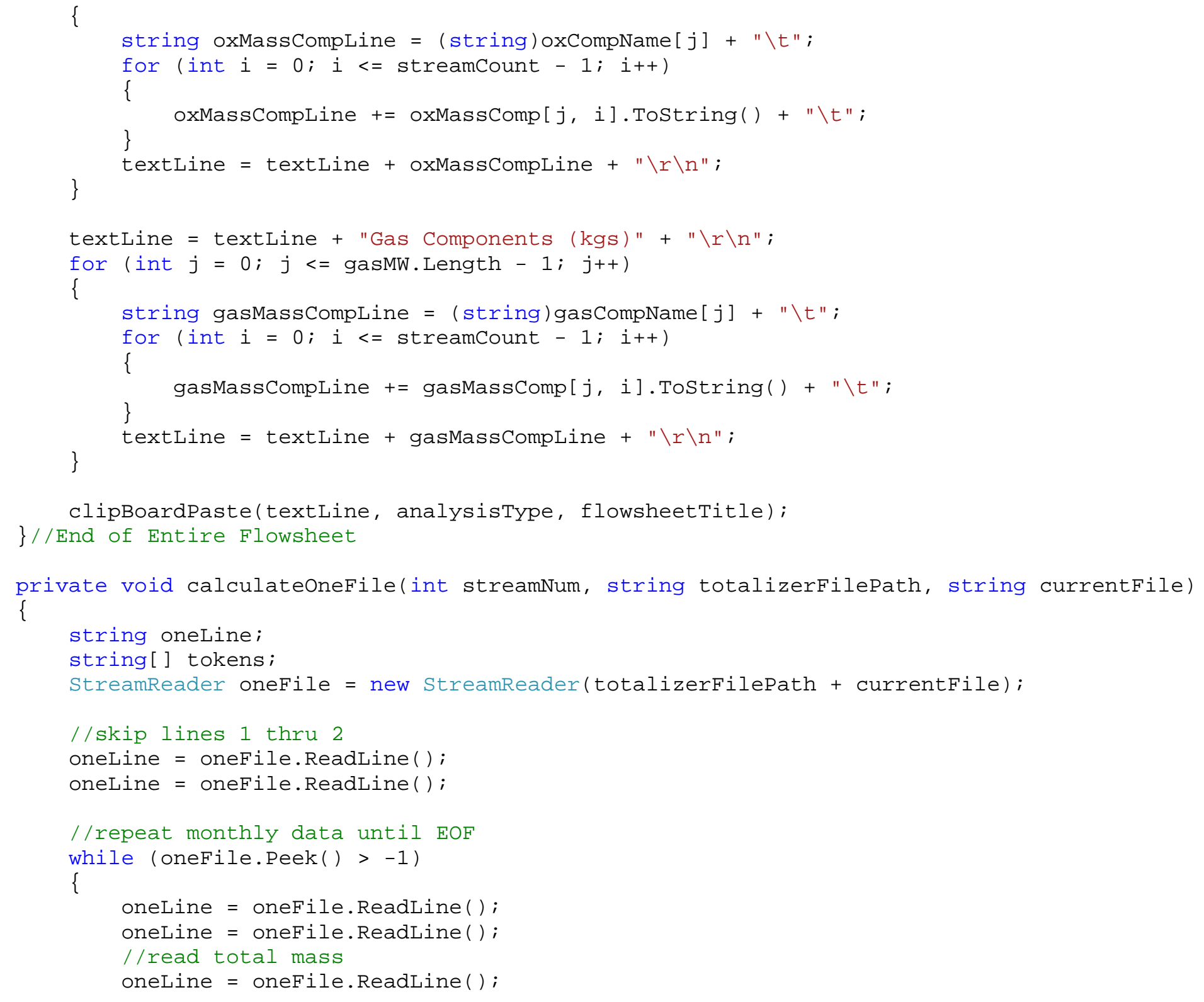




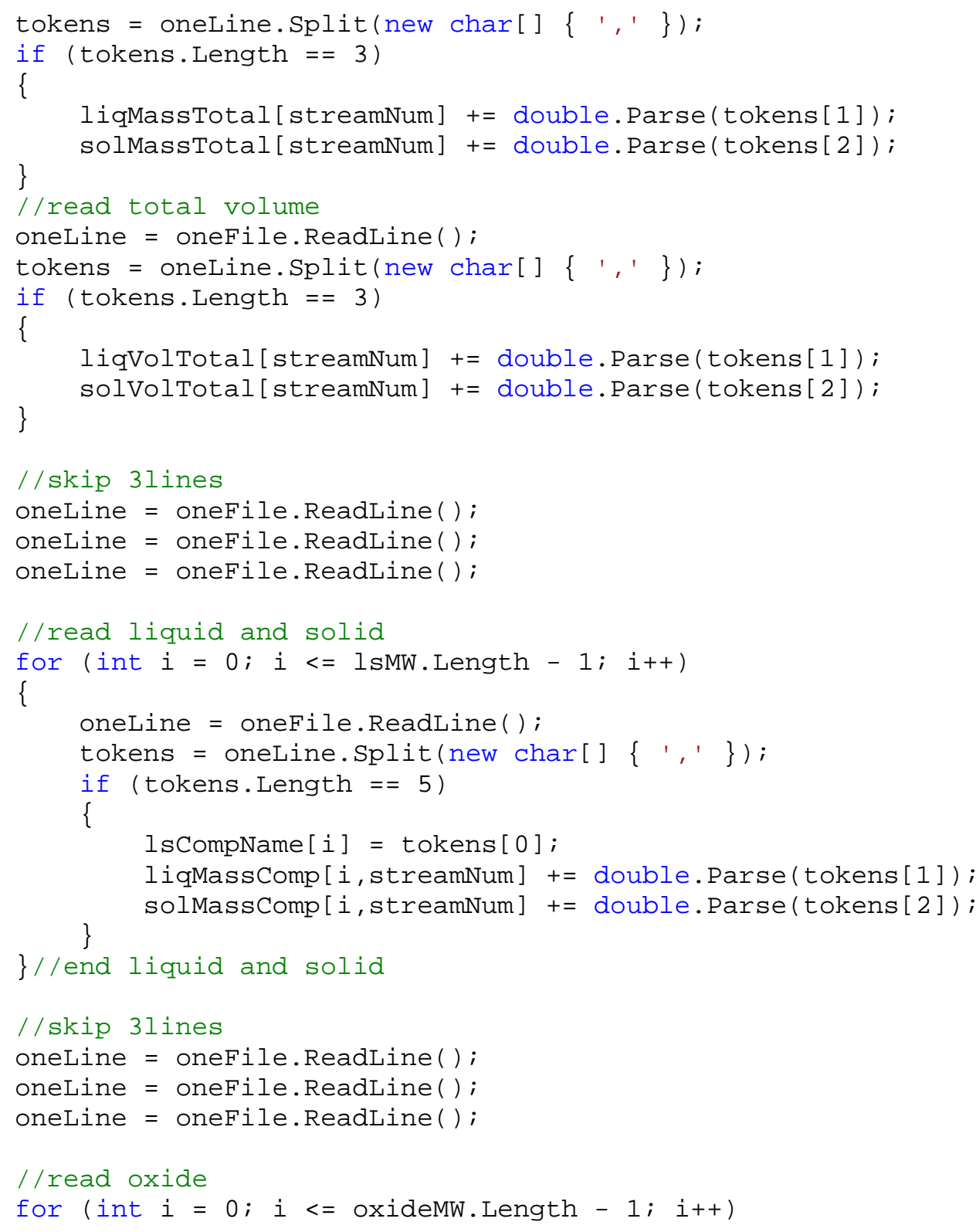




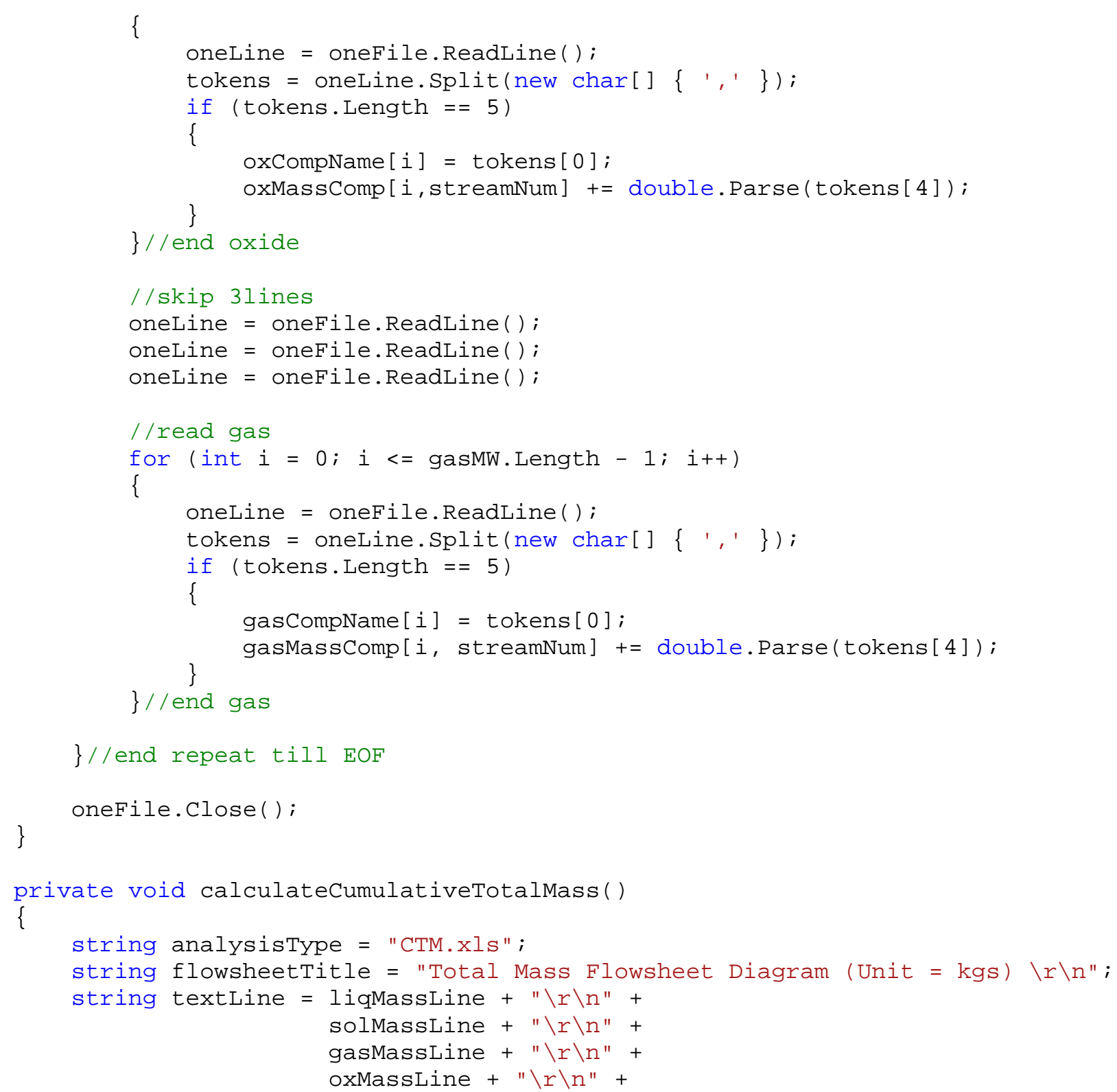




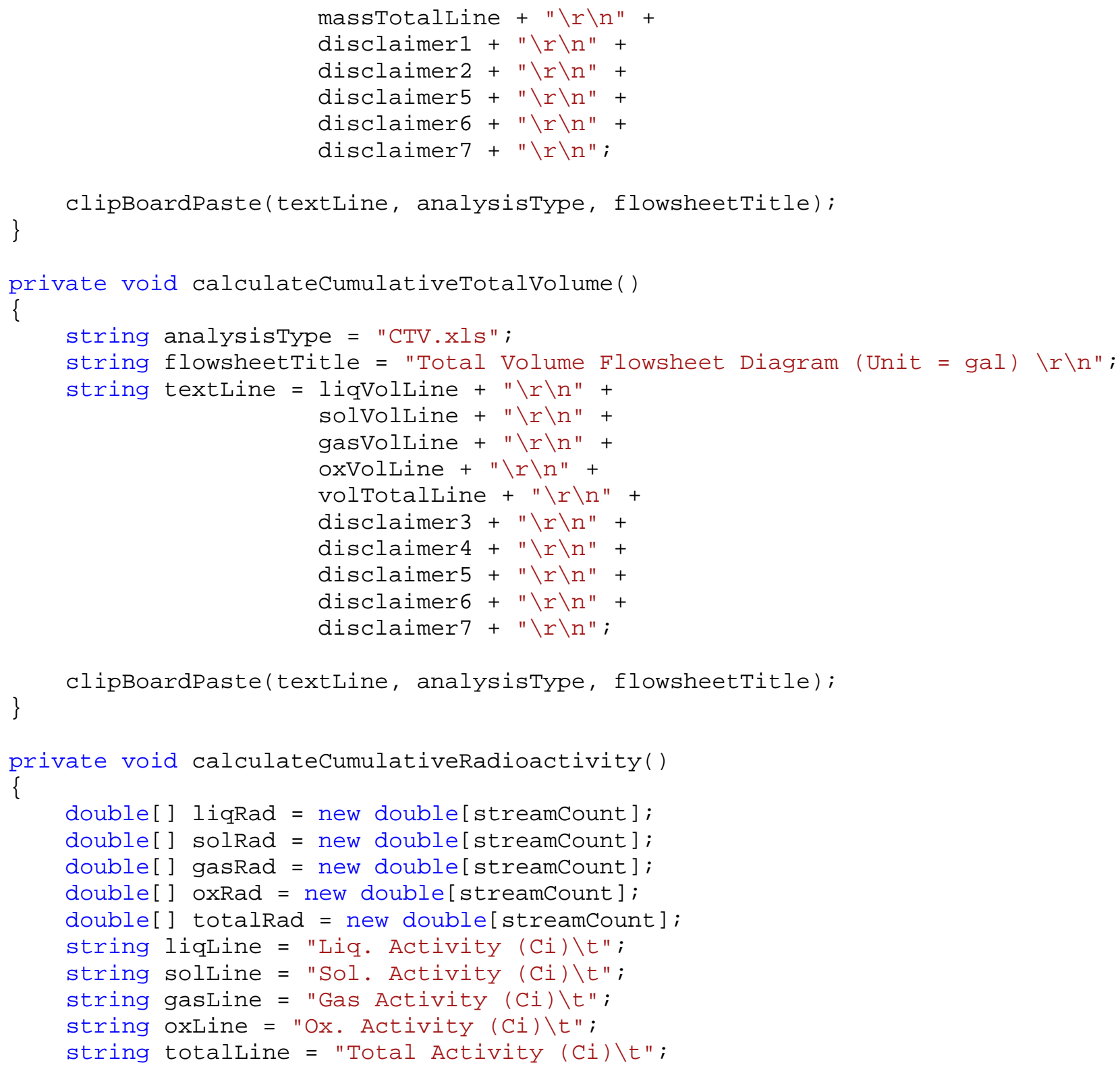

private void calculatecumulativeRadioactivity()

\{

double[] liqRad = new double[streamcount];

double [] solRad = new double [streamCount] ;

double [] gasRad = new double [streamcount];

double [] oxRad = new double[streamcount];

double [] totalRad $=$ new double [streamcount]

string liqLine = "Liq. Activity (Ci)\t";

string solLine = "Sol. Activity (Ci)\t";

string gasLine = "Gas Activity (Ci)\t";

string oxLine $=$ "Ox. Activity (Ci) $\backslash \mathrm{t} "$;

string totalLine = "Total Activity (Ci)\t"; 


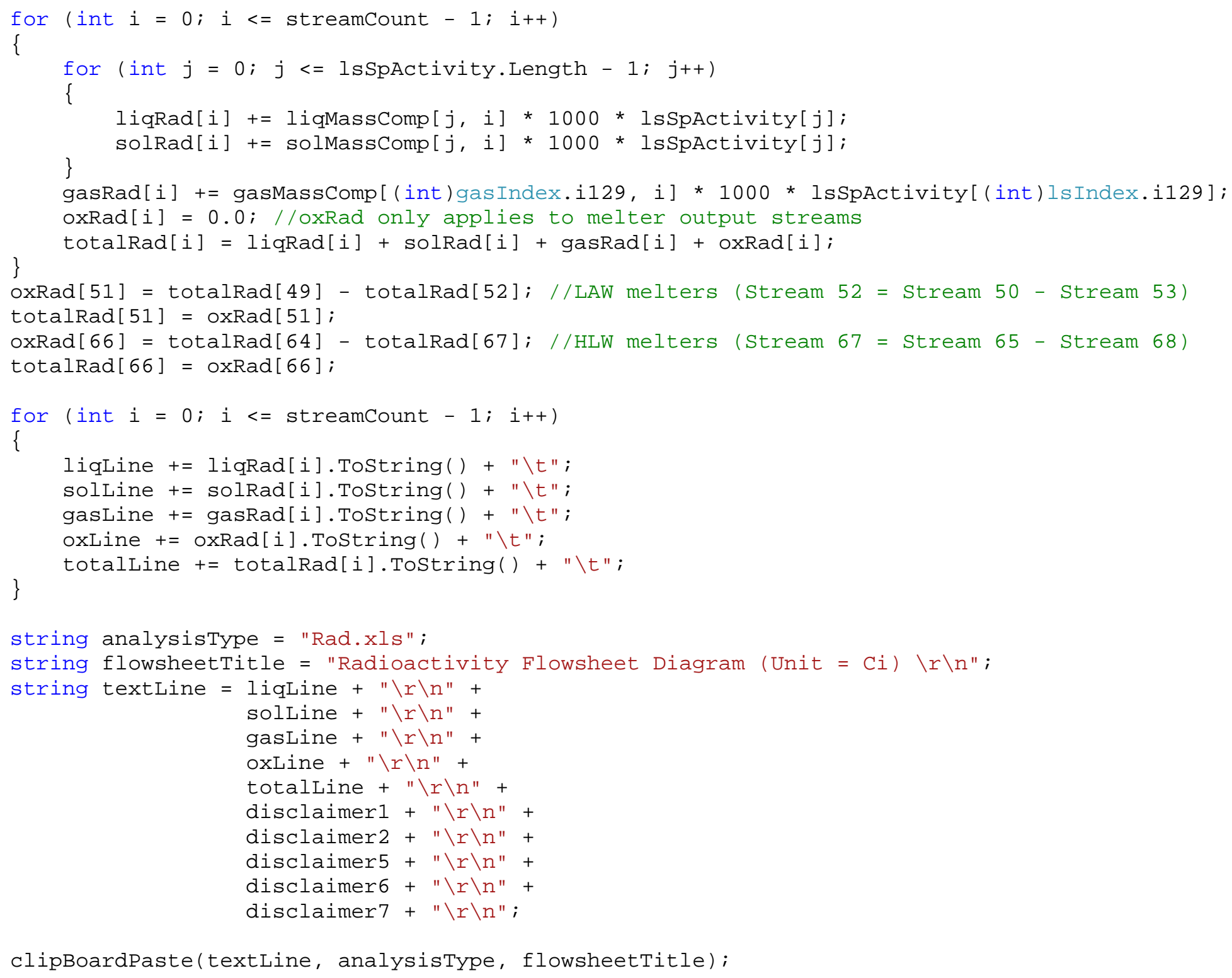

clipBoardPaste(textLine, analysisType, flowsheetTitle); 
private void calculateAluminum()

\{

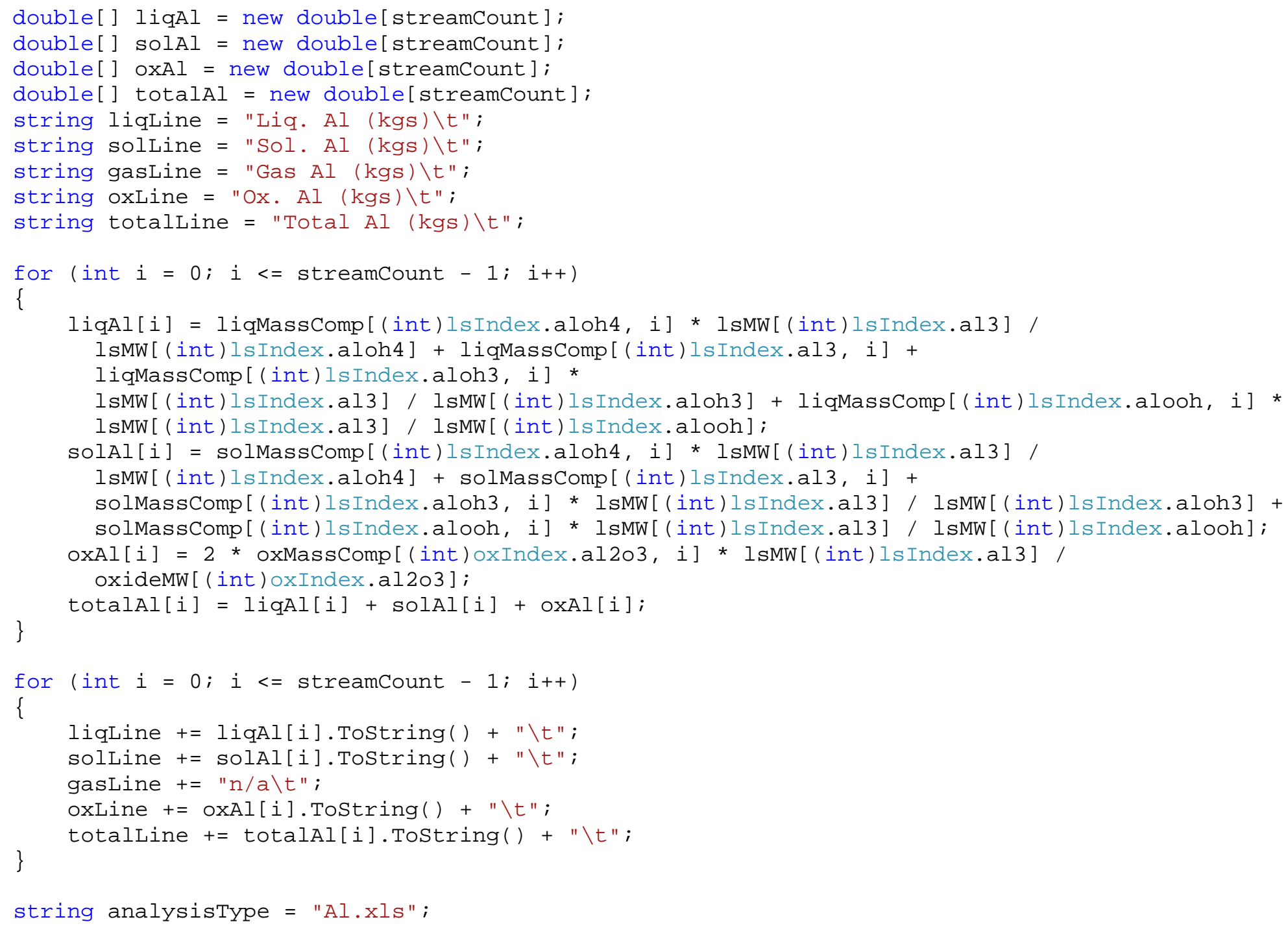


string flowsheetTitle = "Aluminum Mass Flowsheet Diagram (Unit = kgs) \r\n";

string textLine = liqLine + "\r\n" +

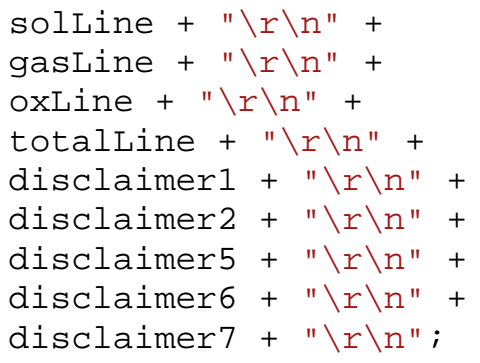

clipBoardPaste(textLine, analysisType, flowsheetTitle);

private void calculatechromium()

\{

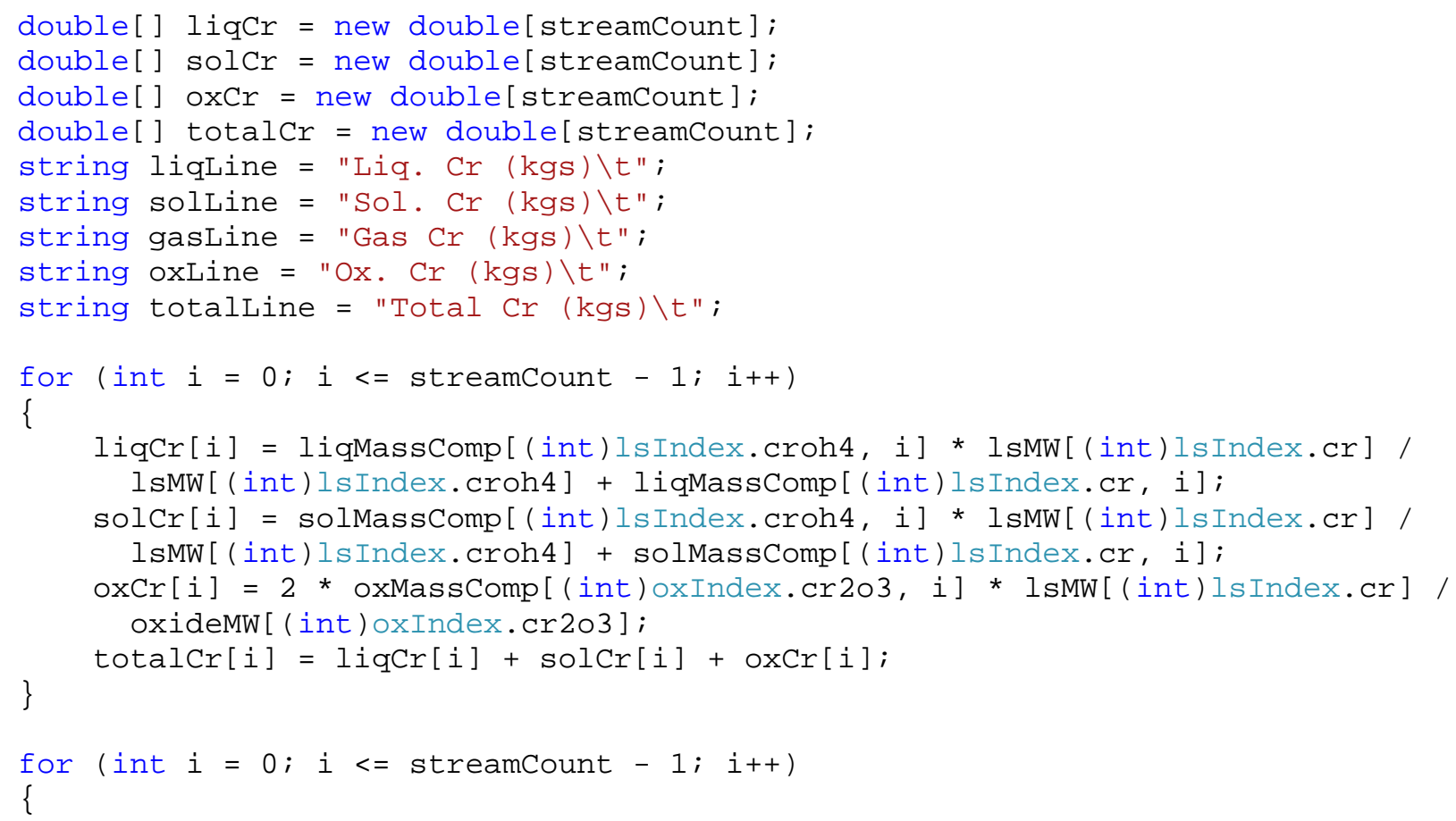




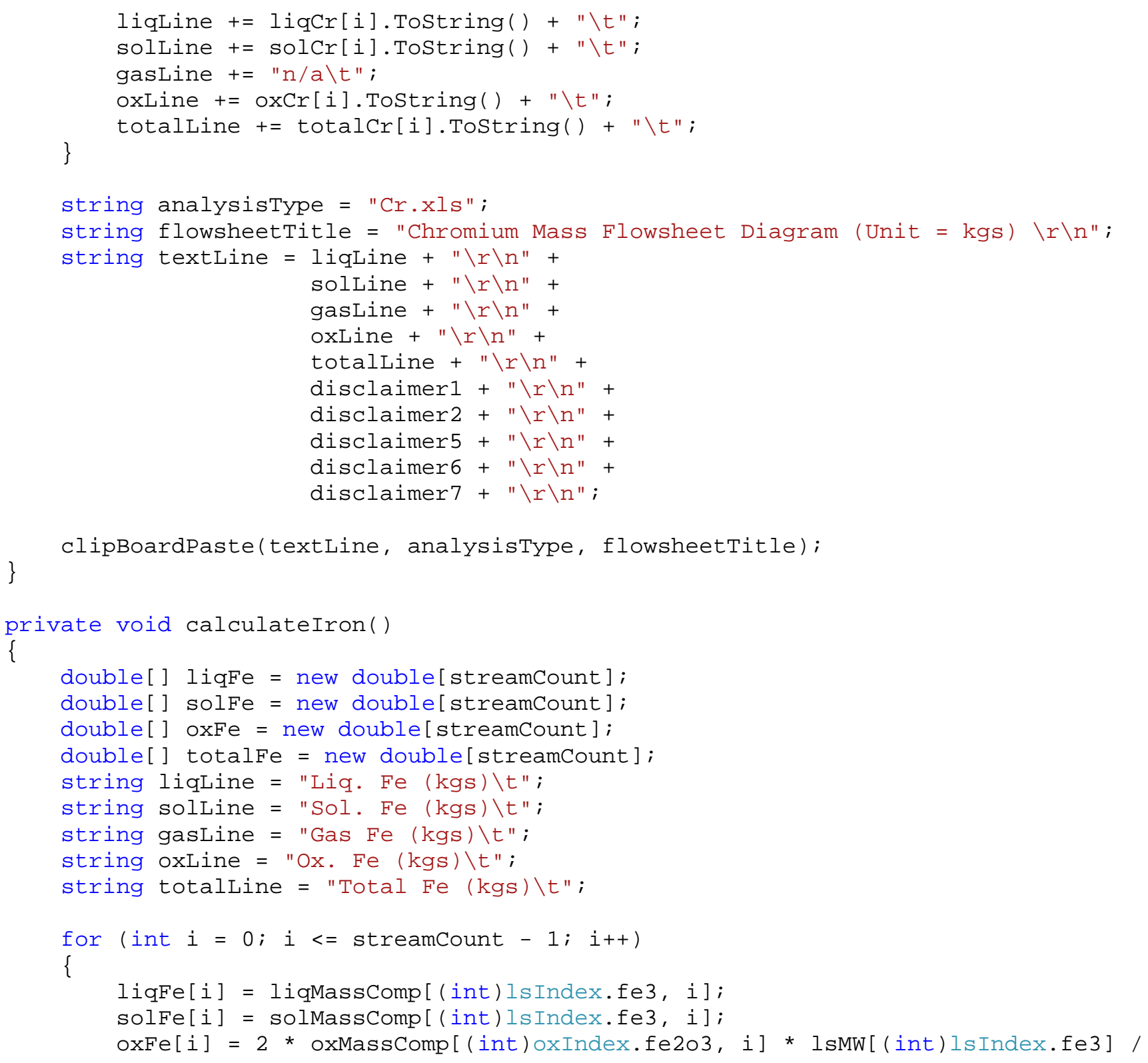




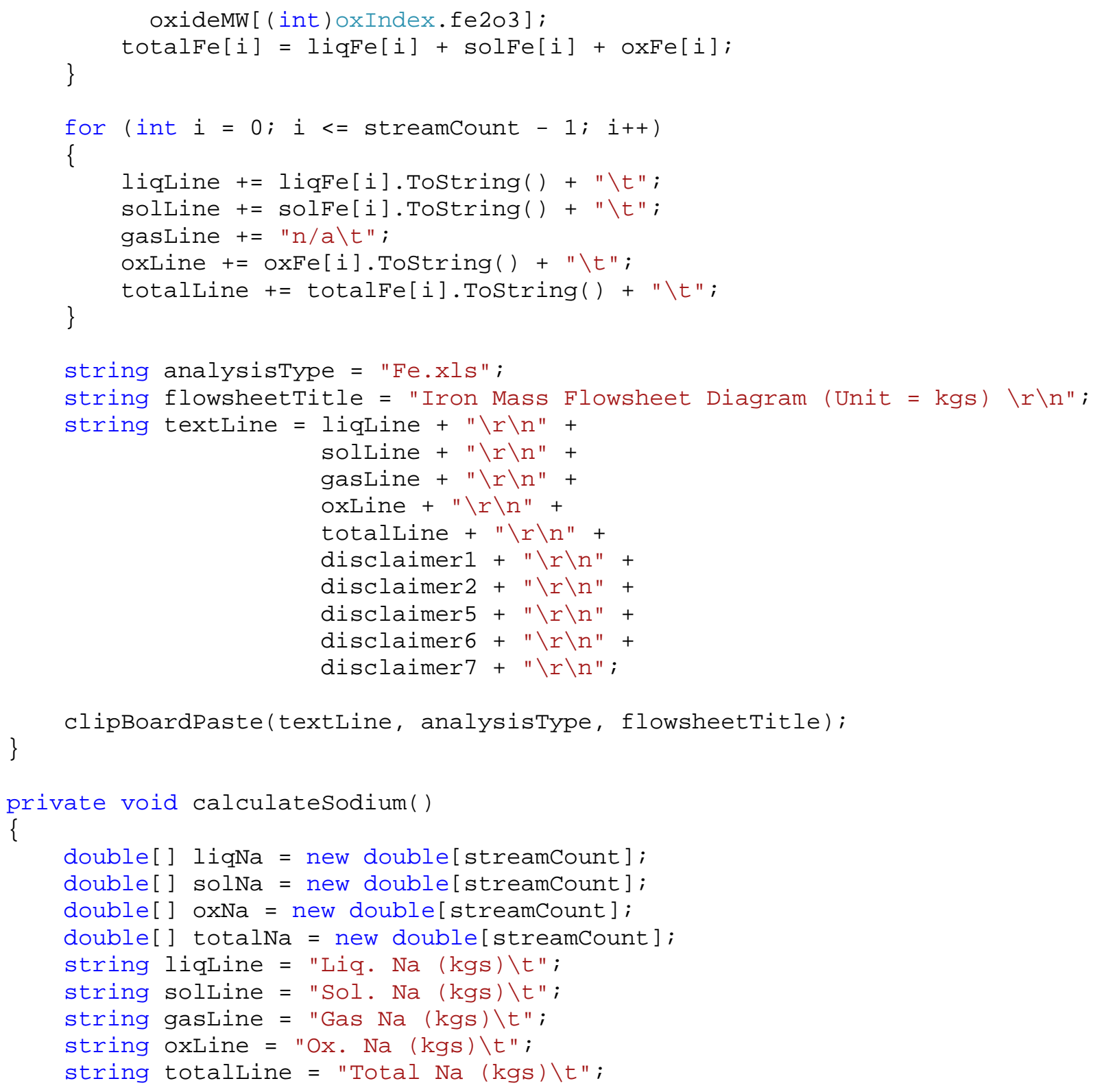

clipBoardPaste(textLine, analysisType, flowsheetTitle);

private void calculateSodium()

\{

double[] liqNa = new double[streamCount];

double[] solNa = new double[streamCount];

double[] oxNa = new double[streamCount];

double[] totalNa = new double[streamCount];

string liqLine = "Liq. $\mathrm{Na}$ (kgs) \t";

string solLine = "Sol. $\mathrm{Na}$ (kgs) \t";

string gasLine = "Gas $\mathrm{Na}$ ( kgs) \t";

string oxLine $=" 0 x . \mathrm{Na}(\mathrm{kgs}) \backslash \mathrm{t} "$;

string totalLine $=$ "Total $\mathrm{Na}$ (kgs) \t"; 


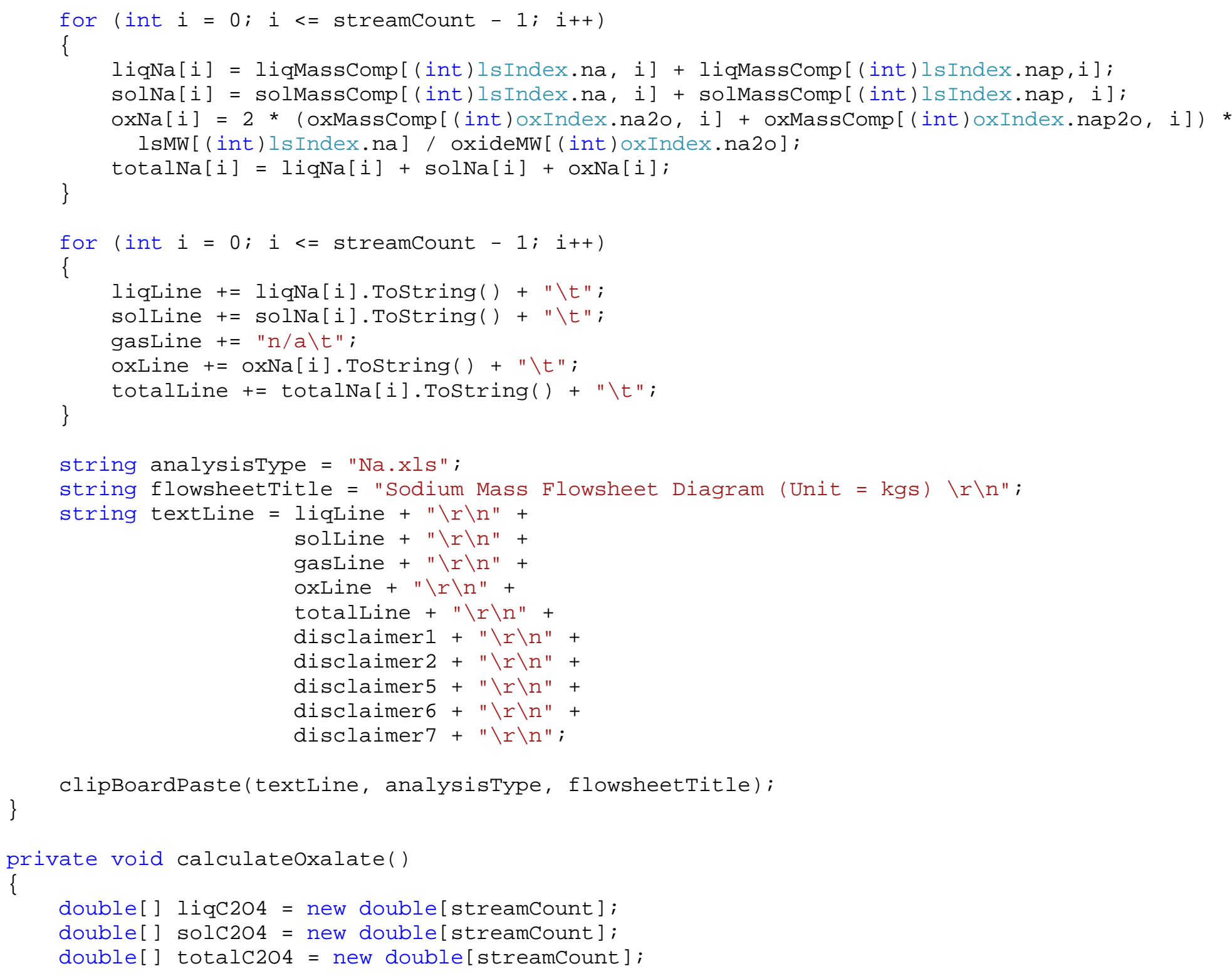




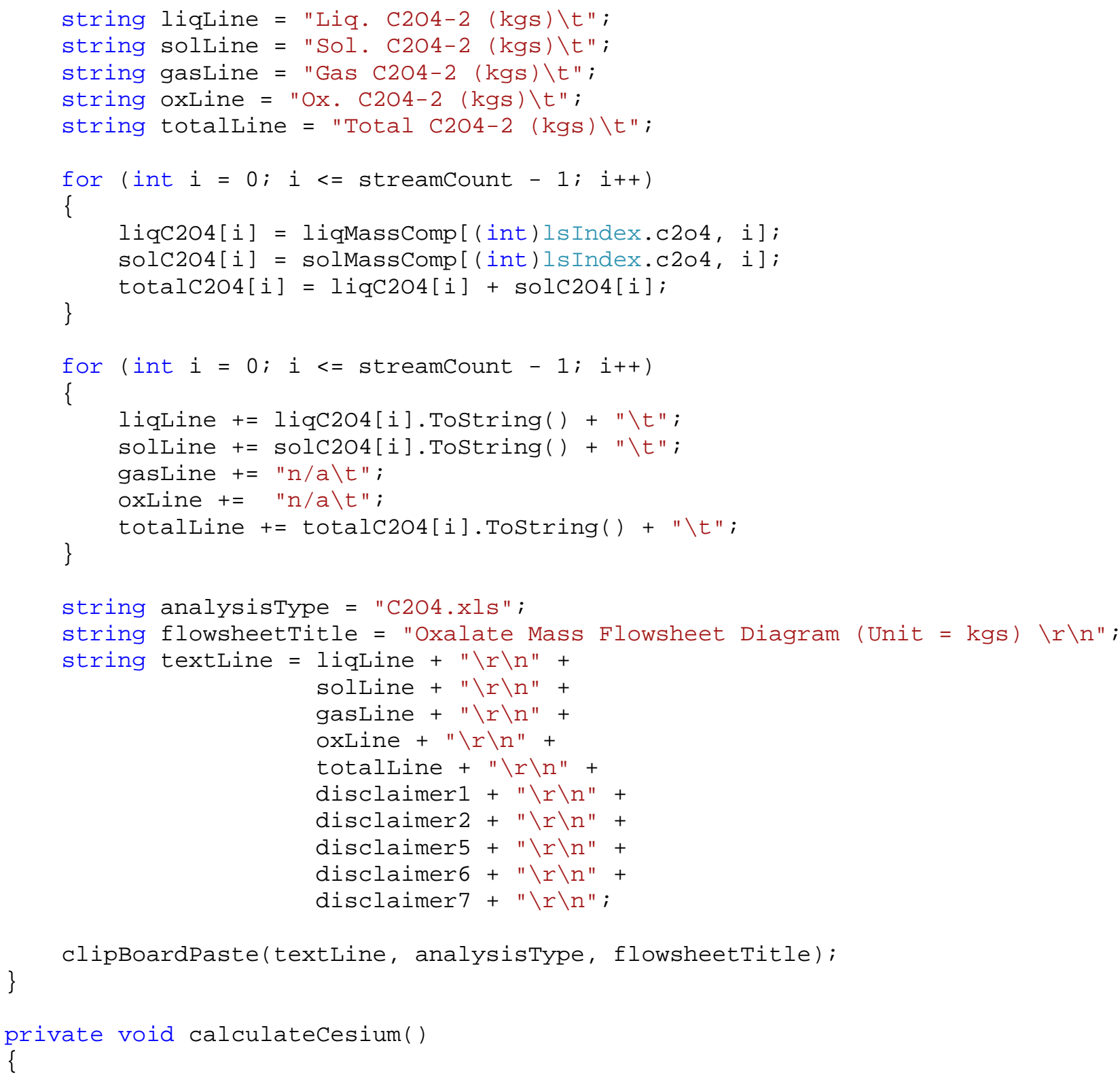




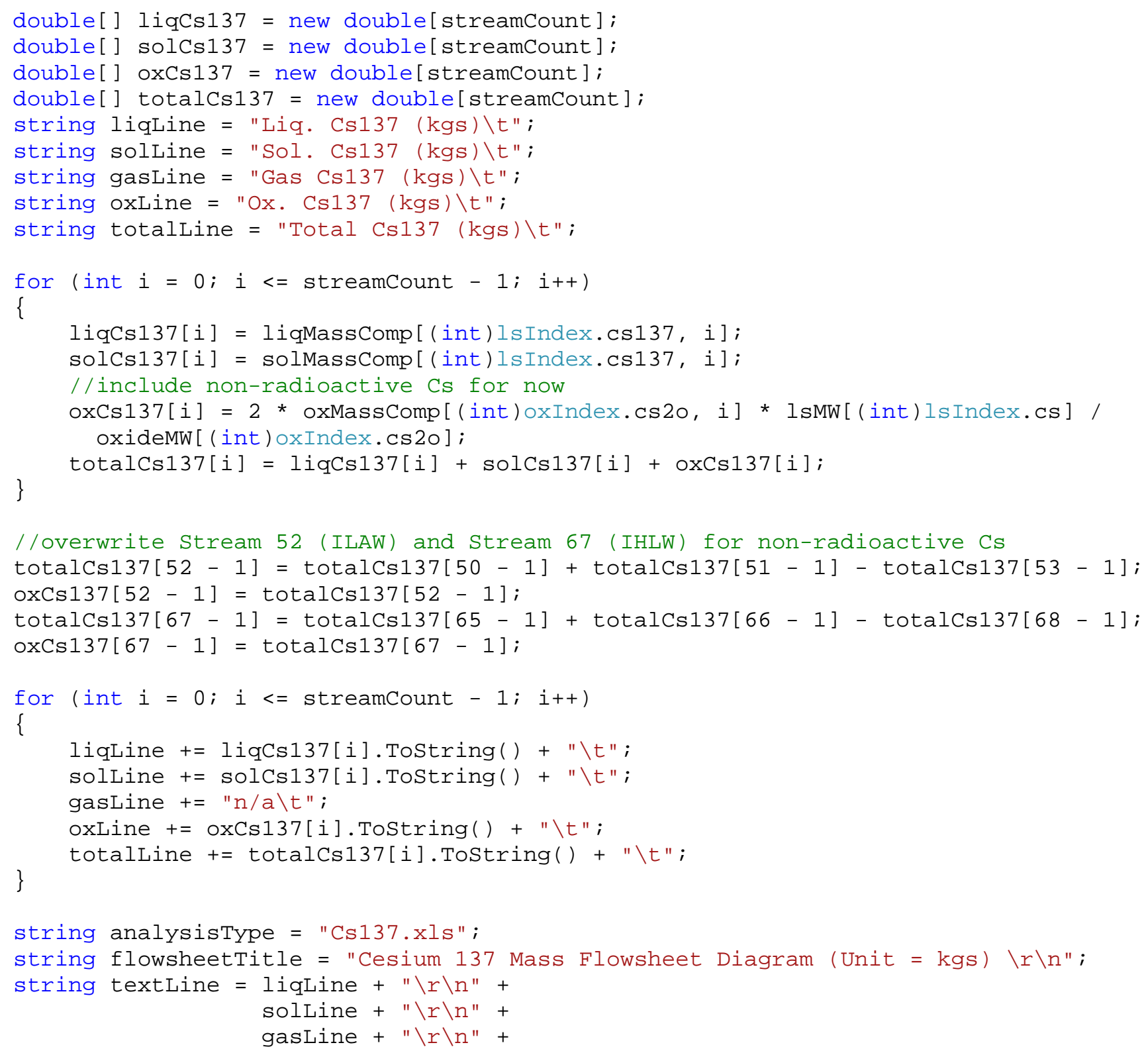




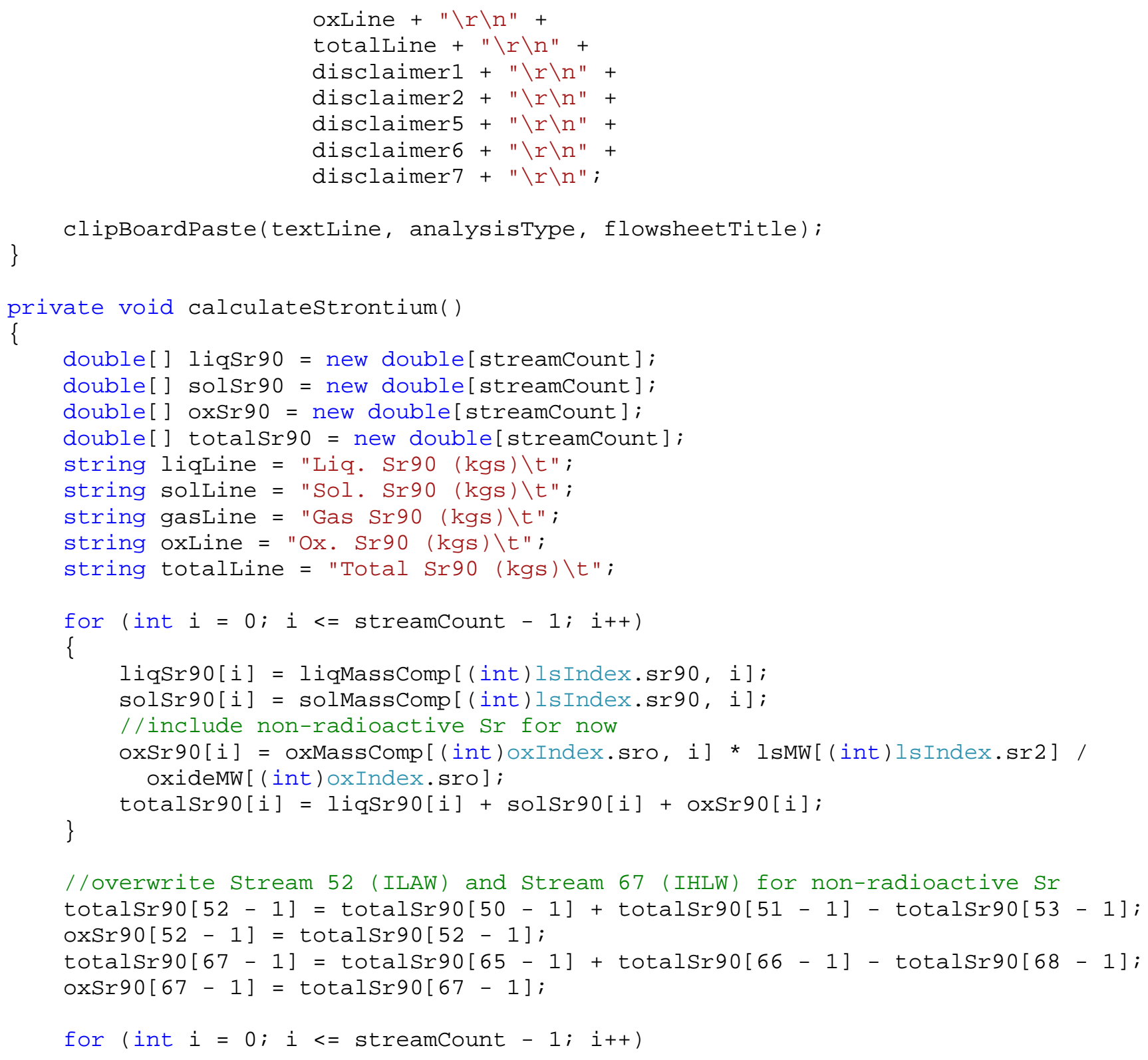




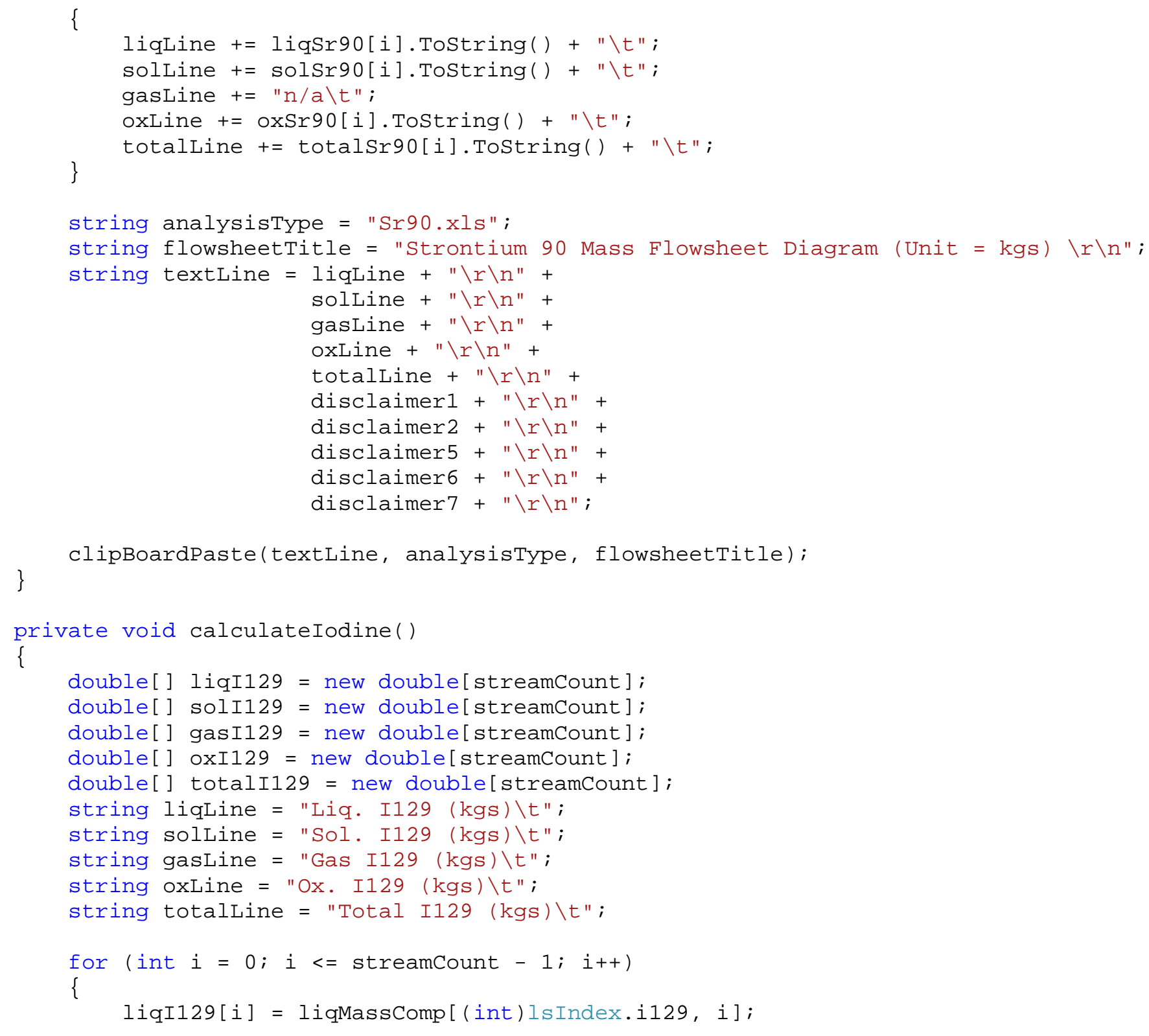

clipBoardPaste(textLine, analysisType, flowsheetTitle);

private void calculatelodine()

\{ 


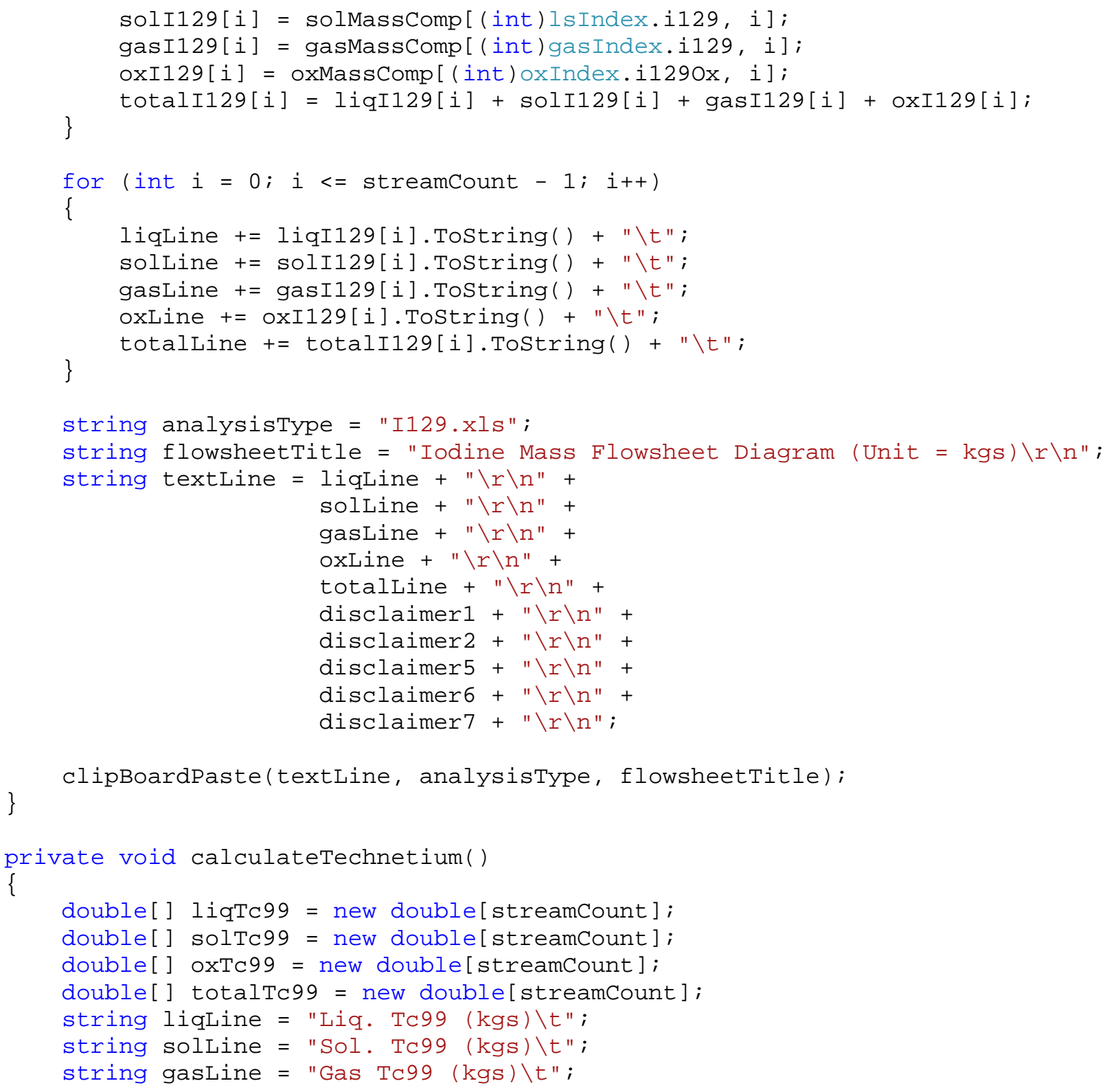




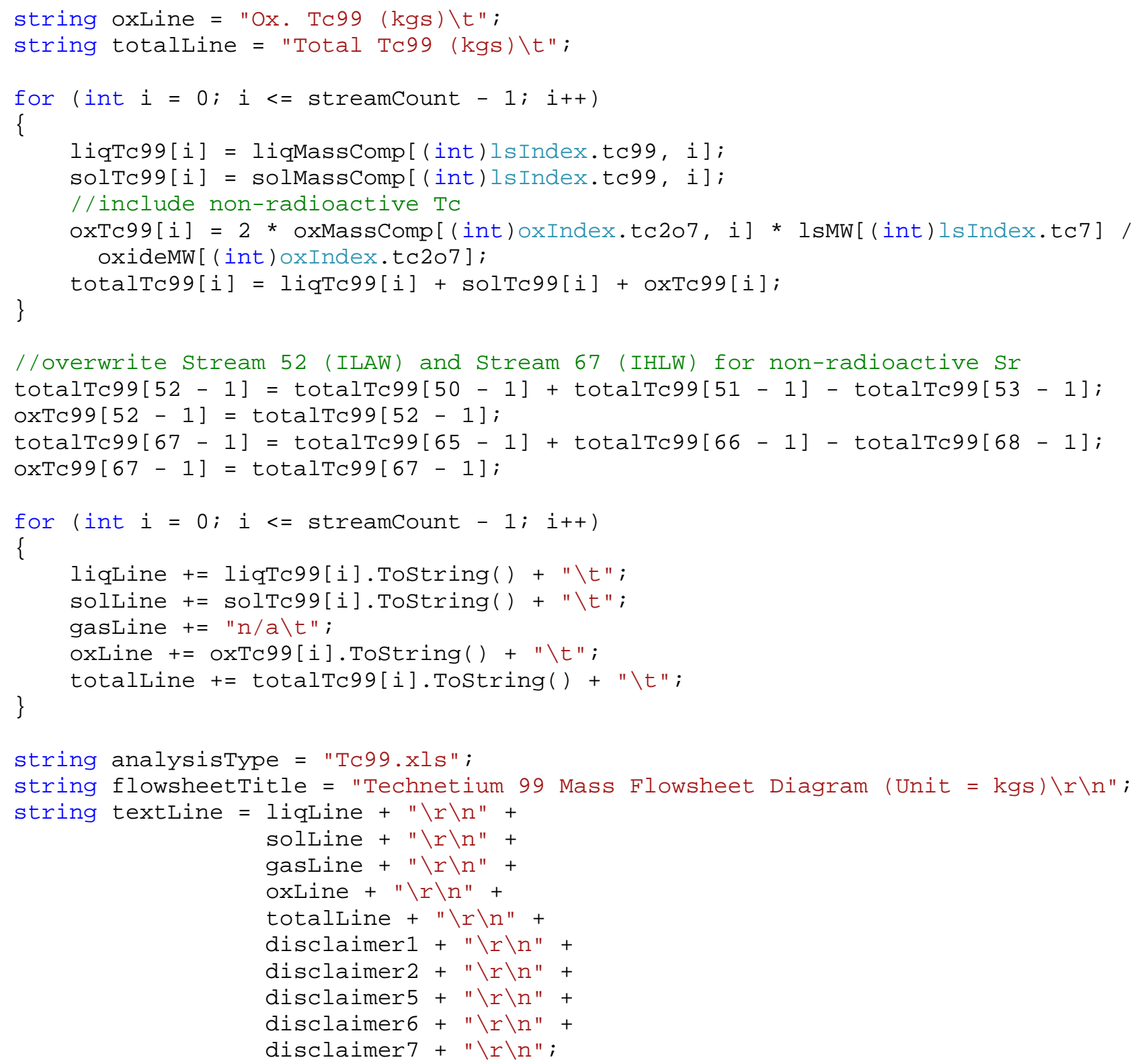



\}

clipBoardPaste(textLine, analysistype, flowsheetTitle);

private void calculateMercury()

\{

double [] liqHg = new double[streamCount]

double [] solHg = new double[streamCount];

double[] gasHg = new double[streamCount];

double[] oxHg = new double[streamCount];

double[] totalHg = new double[streamcount];

string liqLine = "Liq. Hg (kgs) \t";

string solLine = "Sol. Hg (kgs) \t";

string gasLine $=$ "Gas $\mathrm{Hg}(\mathrm{kgs}) \backslash \mathrm{t} "$

string oxLine $=" 0 \mathrm{x} \cdot \mathrm{Hg}(\mathrm{kgs}) \backslash \mathrm{t} "$;

string totalLine $=$ "Total $\mathrm{Hg}$ (kgs) \t";

for (int $i=0 ; i<=$ streamCount $-1 ; i++$ )

\{

liqHg[i] = liqMassComp[(int)lsIndex.hg2, i] ;

solHg $[i]=$ solMassComp [(int)lsIndex.hg2, $i] ;$

gasHg[i] = gasMassComp [(int)gasIndex.hg, i]

$\operatorname{oxHg}[i]=\operatorname{oxMassComp}[($ int $)$ oxIndex.hgo, i] * lsMw[(int)lsIndex.hg2 $] /$ oxideMW [ (int) oxIndex.hgo];

\}

totalHg[i] = liqHg[i] + solHg[i] + gasHg[i] +oxHg[i];

for (int $i=0 ; i<=$ streamCount $-1 ; i++$ )

\{

liqLine $+=$ liqHg[i].ToString ()$+" \backslash t "$;

solLine $+=$ solHg[i].ToString ()$+" \backslash t "$;

gasLine $+=$ gasHg[i].ToString ()$+$ "\t";

oxLine $+=$ oxHg[i]. ToString ()$+" \backslash t "$;

\}

totalLine $+=$ totalHg[i].Tostring ()$+" \backslash t "$;

string analysisType $=" H g \cdot x l s "$;

string flowsheetTitle $=$ "Mercury Mass Flowsheet Diagram (Unit $=$ kgs) $\backslash r \backslash n "$;

string textLine = liqLine + "\r $\backslash n "+$

solLine + "\r\n" + 


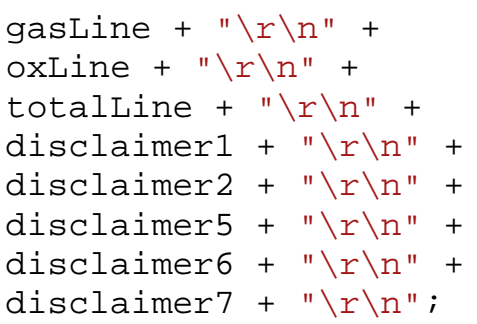




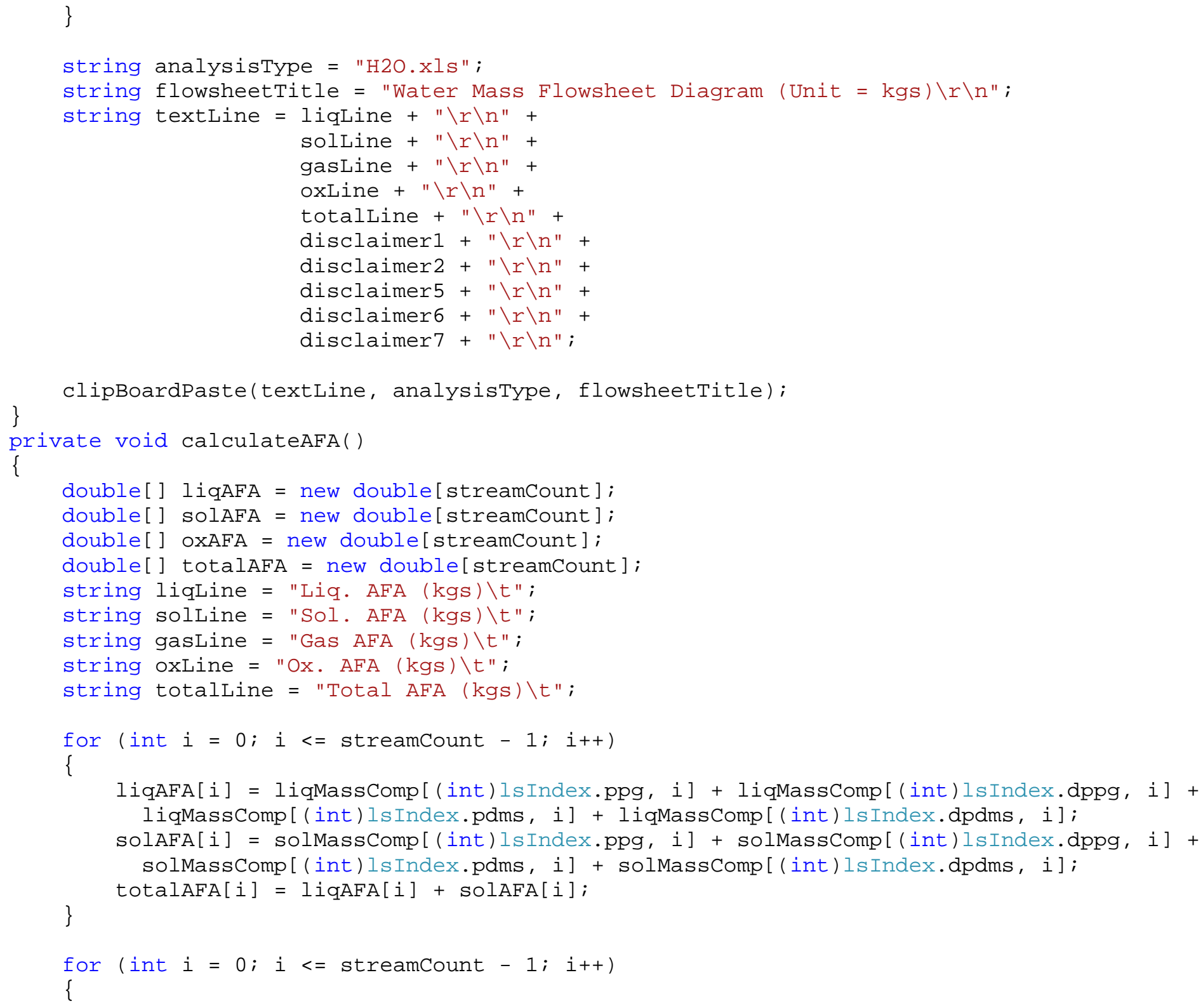




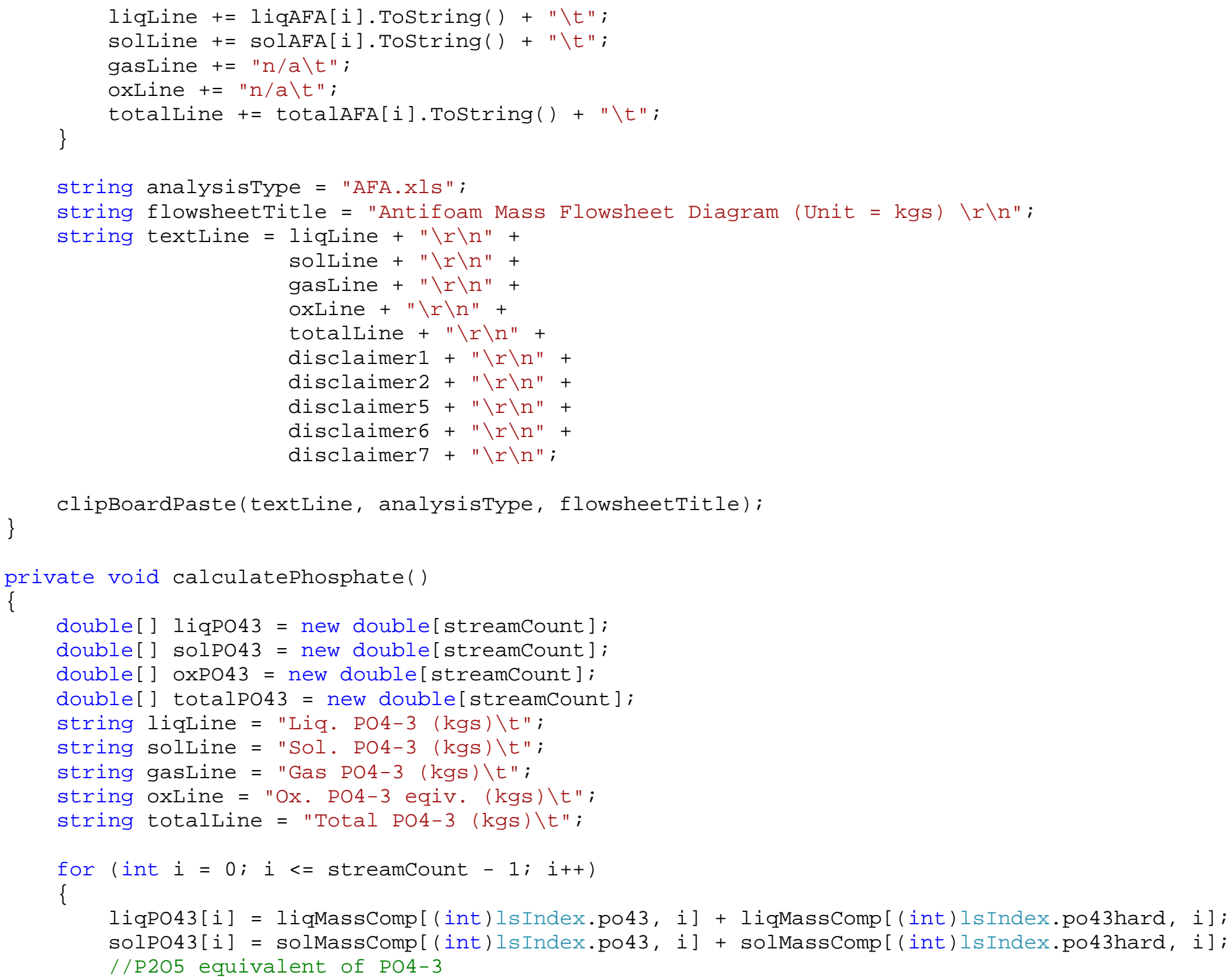




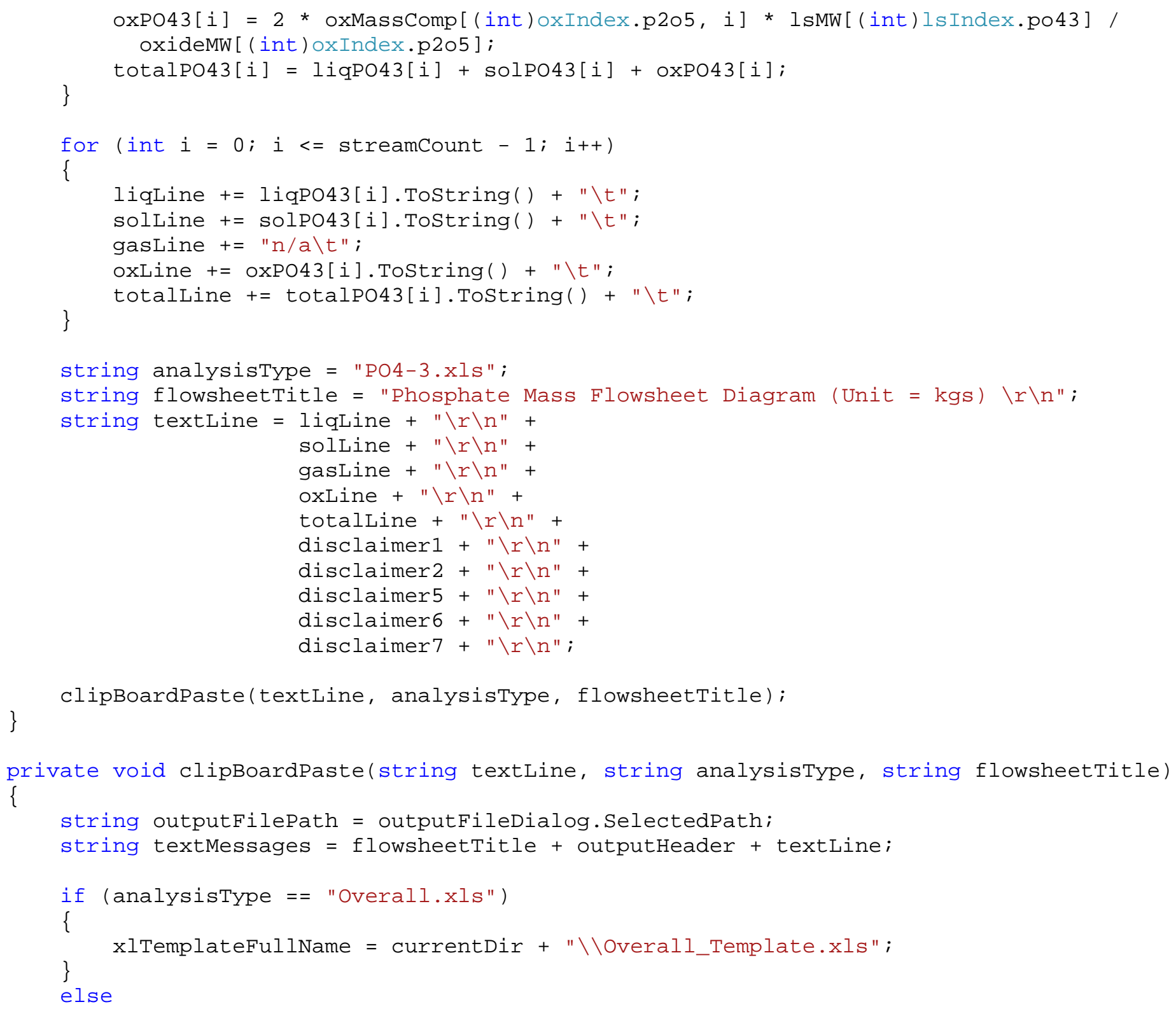




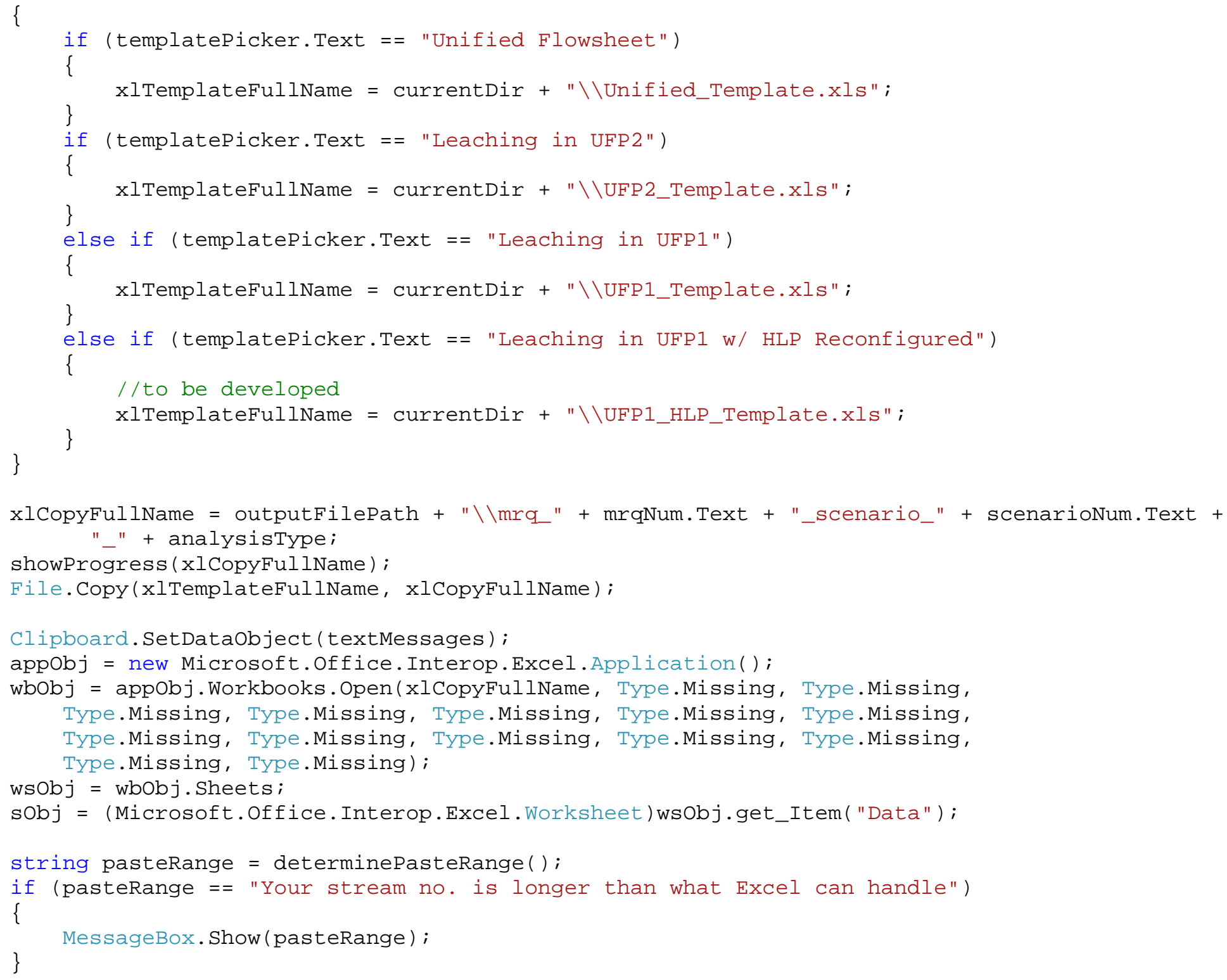




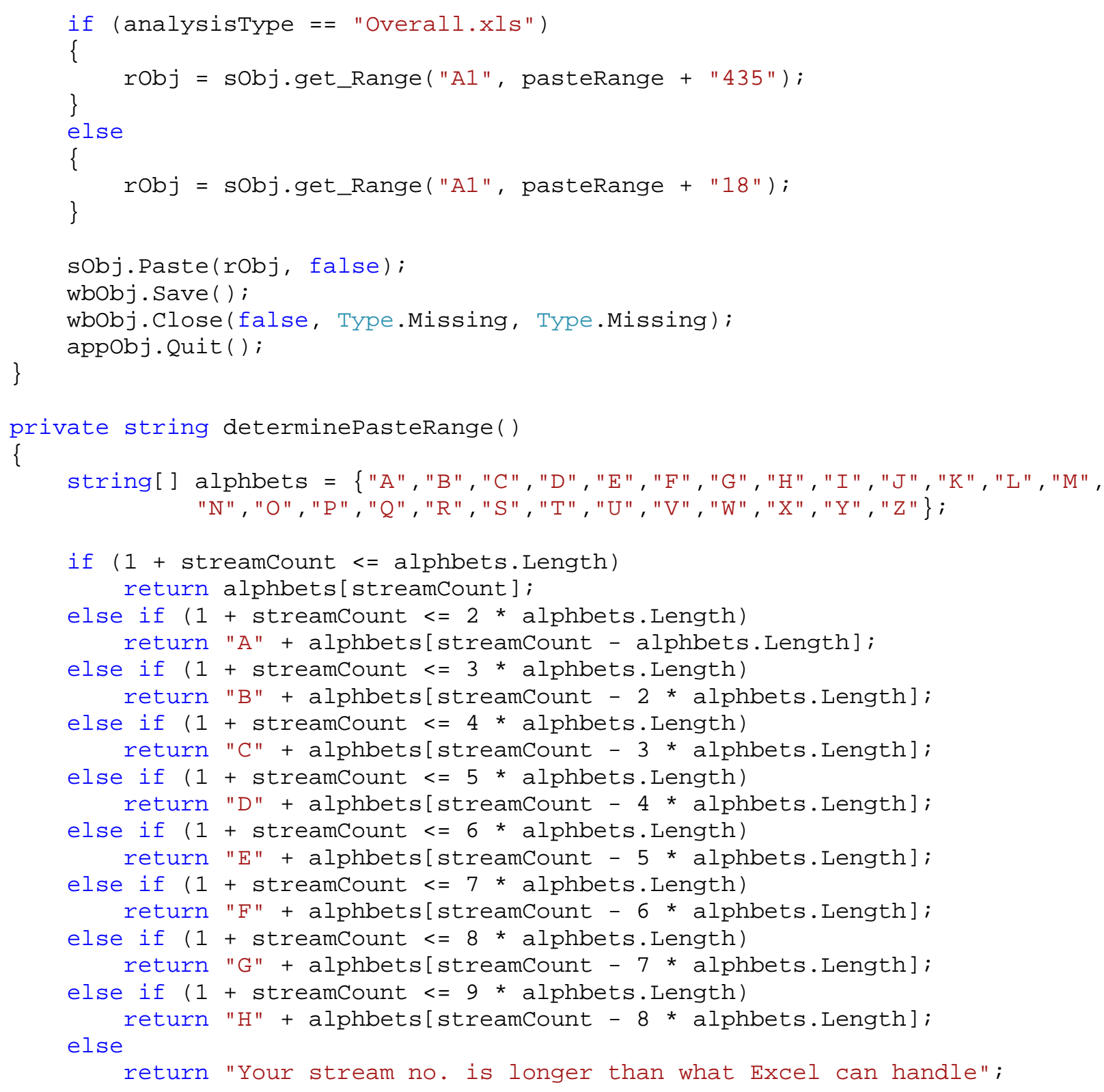


24590-WTP-MDD-PR-01-002, Rev 12

Table J-3 Volume Throughput Data for RLD-VSL-0006A by TVExtractor

\begin{tabular}{|c|c|c|c|c|c|c|c|c|c|c|}
\hline Upstream & Downstream & $\begin{array}{r}\text { Output } \\
\text { Time } \\
\end{array}$ & $\begin{array}{r}\text { 30-May- } \\
18 \\
\end{array}$ & $\begin{array}{r}\text { 29-Jun- } \\
18 \\
\end{array}$ & $\begin{array}{r}\text { 29-Jul- } \\
18 \\
\end{array}$ & $\begin{array}{r}28 \text {-Aug- } \\
18 \\
\end{array}$ & $\begin{array}{r}27-S e p- \\
18 \\
\end{array}$ & $\begin{array}{r}27-O c t- \\
18 \\
\end{array}$ & $\begin{array}{r}26-\text { Nov- } \\
18 \\
\end{array}$ & $\begin{array}{r}26-\mathrm{Dec}- \\
18 \\
\end{array}$ \\
\hline RLD6A & FRP2A & (gal) & 490 & 977 & 871 & 853 & 700 & 758 & 868 & 741 \\
\hline RLD6A & FRP2B & (gal) & 2,309 & 1,721 & 546 & 521 & 2,332 & 3,631 & 1,207 & 1,667 \\
\hline RLD6A & FRP2C & (gal) & 963 & 1,771 & 3,166 & 4,049 & 2,566 & 1,404 & 2,946 & 3,996 \\
\hline RLD6A & FRP2D & (gal) & 276 & 276 & 138 & 437 & 415 & 276 & 276 & 437 \\
\hline RLD6A & HLP22 & (gal) & 0 & 0 & 0 & 290 & 97 & 0 & 68 & 386 \\
\hline RLD6A & FEP17A & (gal) & 5,933 & 7,038 & 7,423 & 7,613 & 8,214 & 9,081 & 8,128 & 8,825 \\
\hline RLD6A & FEP17B & (gal) & 418 & 756 & 645 & 489 & 655 & 604 & 655 & 453 \\
\hline RLD6A & FEP-EVAP & (gal) & 277 & 553 & 632 & 395 & 434 & 395 & 474 & 356 \\
\hline RLD6A & UFP1A & (gal) & 4,179 & 4,252 & 4,833 & 4,886 & 5,658 & 6,808 & 5,097 & 5,658 \\
\hline RLD6A & UFP1B & (gal) & 4,274 & 4,129 & 4,274 & 5,181 & 5,115 & 6,233 & 4,970 & 6,167 \\
\hline RLD6A & UFP2A & (gal) & 140,468 & 206,172 & 203,466 & 161,067 & 203,087 & 172,480 & 220,407 & 209,067 \\
\hline RLD6A & UFP2B & (gal) & 174,820 & 105,139 & 194 & 97 & 97 & 194 & 80,134 & 81,433 \\
\hline RLD6A & SUPERTANK & (gal) & 0 & 0 & 0 & 0 & 0 & 32,700 & 73,800 & 77,750 \\
\hline RLD6A & RDP2B & (gal) & 0 & 0 & 0 & 0 & 0 & 0 & 0 & 0 \\
\hline RLD6A & TLP9A & (gal) & 1,153 & 1,998 & 1,923 & 1,826 & 1,947 & 1,281 & 1,315 & 1,187 \\
\hline RLD6A & TLP9B & (gal) & 1,257 & 1,973 & 1,973 & 1,863 & 1,915 & 1,315 & 1,264 & 1,206 \\
\hline RLD6A & CXP26A & (gal) & 591 & 591 & 739 & 739 & 739 & 887 & 813 & 961 \\
\hline RLD6A & CXP26B & (gal) & 677 & 762 & 847 & 847 & 931 & 1,016 & 931 & 931 \\
\hline RLD6A & CXP26C & (gal) & 521 & 587 & 587 & 717 & 652 & 847 & 782 & 782 \\
\hline RLD6A & TLP-EVAP & (gal) & 369 & 415 & 446 & 476 & 492 & 569 & 553 & 553 \\
\hline RLD6A & TCP1 & (gal) & 4,299 & 5,904 & 6,281 & 6,017 & 6,185 & 5,326 & 4,959 & 4,979 \\
\hline RLD6A & HLP27A & (gal) & 94 & 0 & 94 & 94 & 94 & 0 & 94 & 0 \\
\hline RLD6A & HLP27B & (gal) & 336 & 336 & 427 & 0 & 245 & 182 & 0 & 336 \\
\hline RLD6A & HLP28 & (gal) & 2,084 & 4,458 & 4,442 & 2,244 & 2,104 & 3,178 & 4,063 & 2,639 \\
\hline RLD6A & PWD33 & (gal) & 31 & 31 & 61 & 0 & 0 & 61 & 0 & 31 \\
\hline RLD6A & PWD43 & (gal) & 476 & 2,501 & 2,382 & 1,072 & 1,667 & 1,667 & 1,905 & 1,429 \\
\hline
\end{tabular}




\section{Table J-4 Visual C\# Routine for Totalizer Value Extractor}

using System;

using System.I0;

using System.Collections;

using System.Collections.Generic;

using System.ComponentModel;

using system. Data;

using System.Drawing;

using system. Text;

using System. Windows. Forms;

using Microsoft.office.Interop.Excel;

using System. Threading;

namespace G2MBC_GUI

public partial class TVExtractor : Form

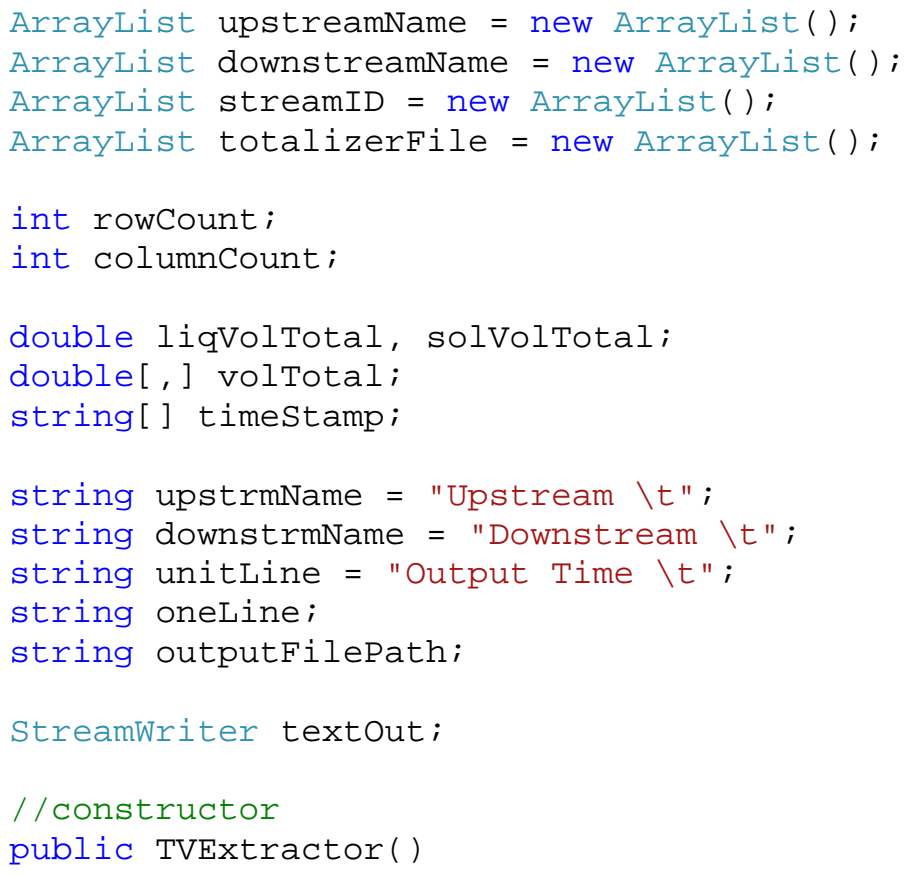




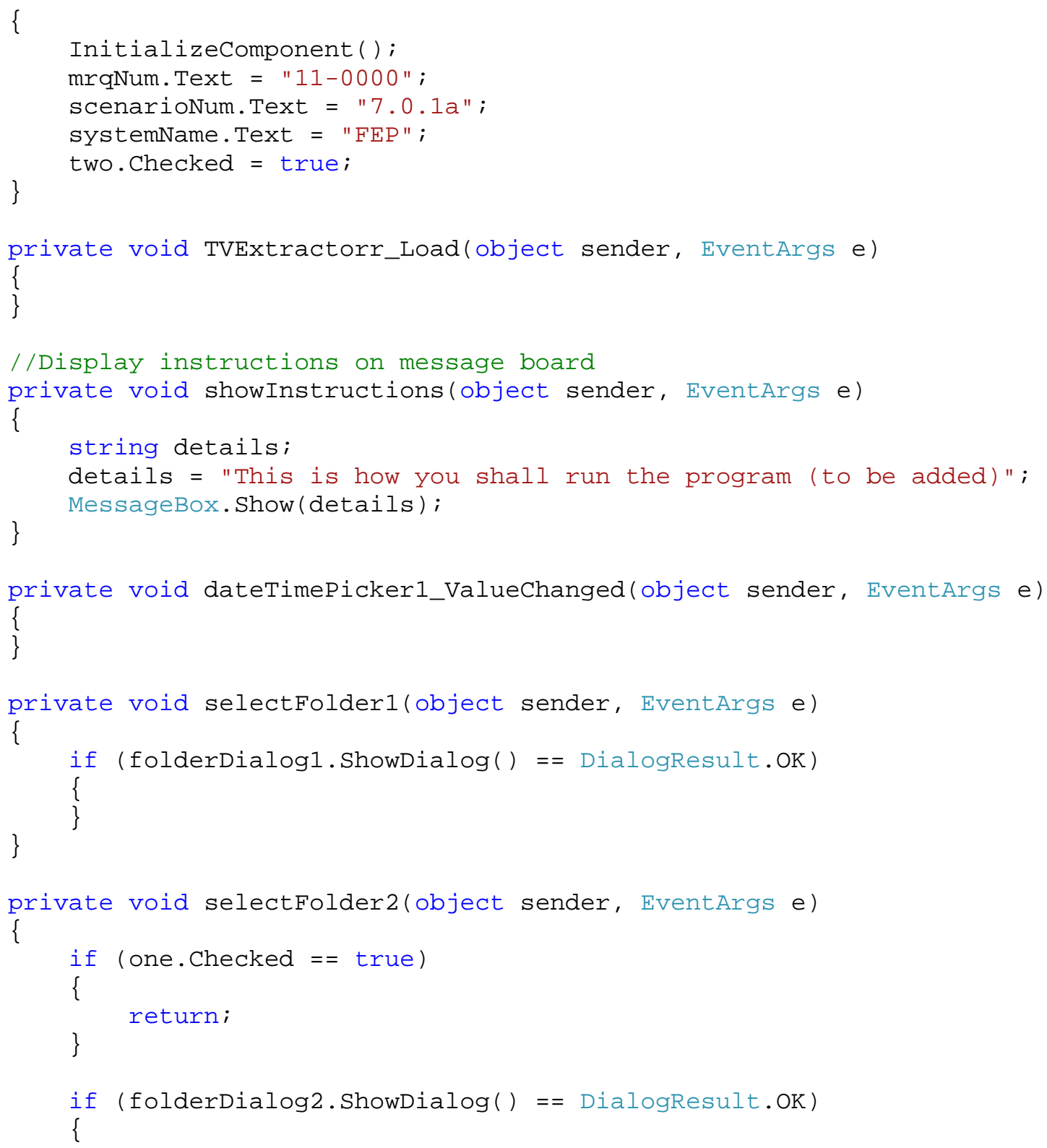




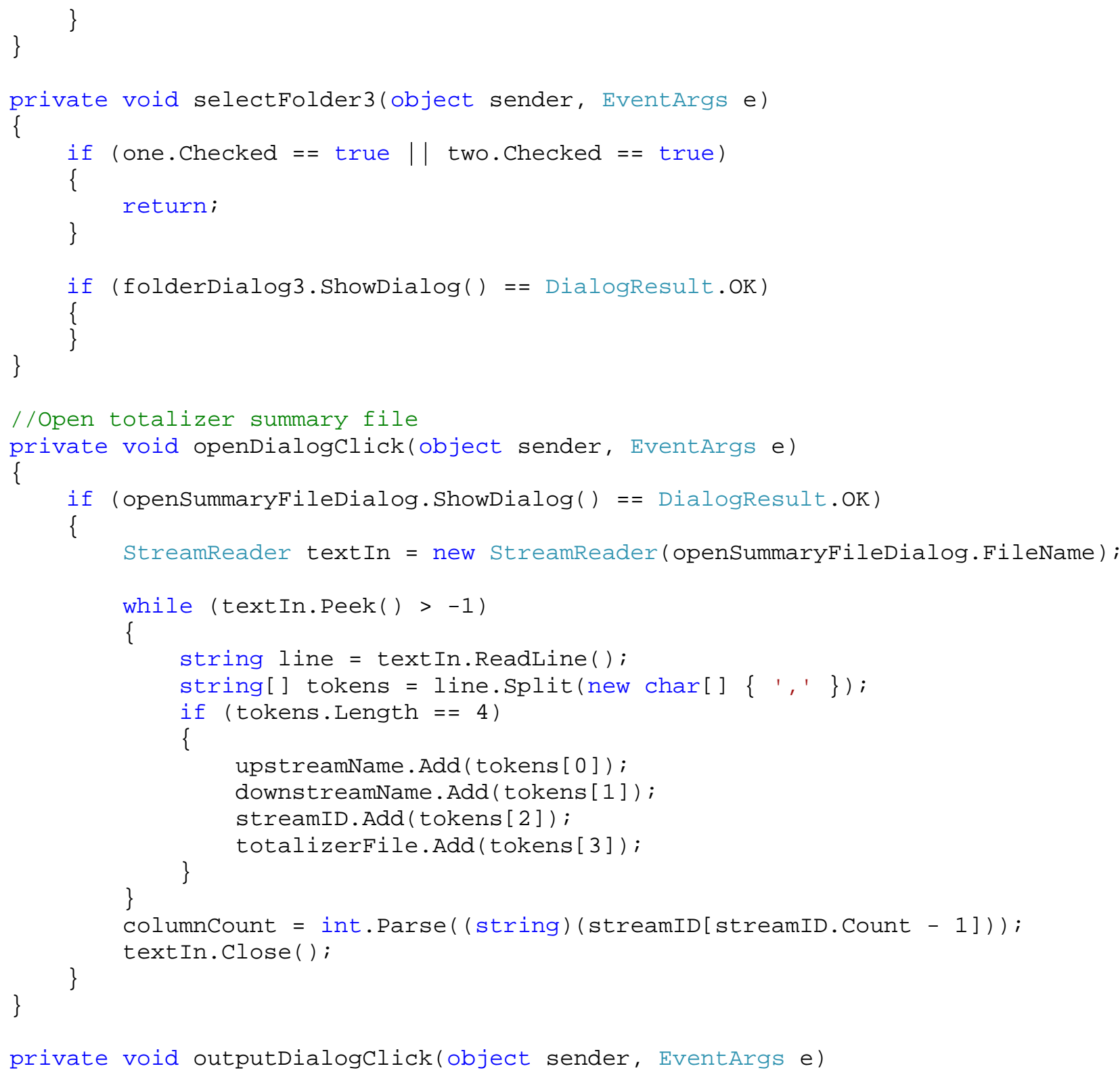




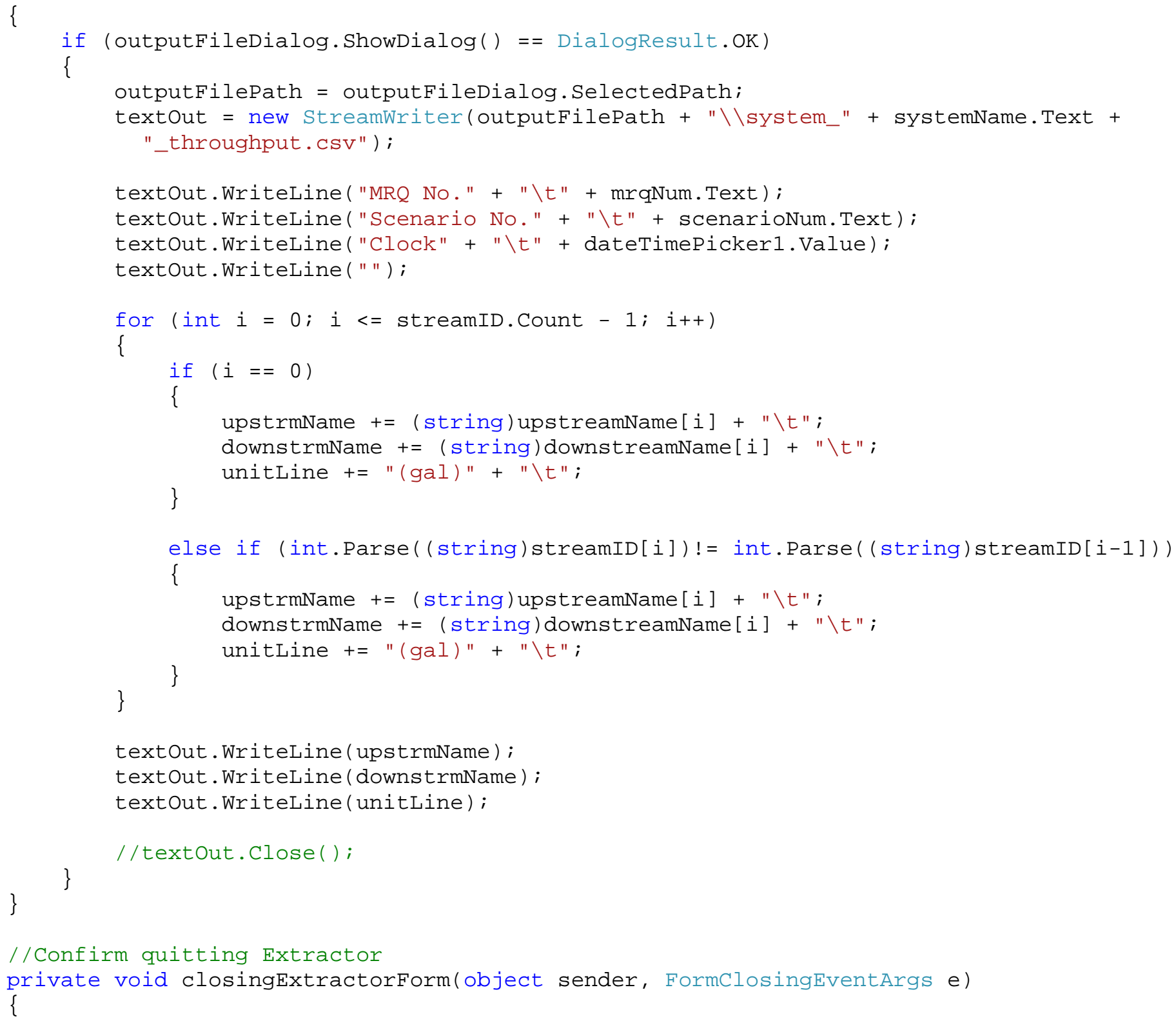


DialogResult key = MessageBox.Show("Quit TVExtractor", "Confirm", MessageBoxButtons.YesNo, MessageBoxIcon.

Question);

\}

e. Cancel $=($ key $==$ DialogResult. No $)$;

private void extractEntirestreamMakeup(object sender, EventArgs e)

\{

int foldercount;

string outputFilePath = outputFileDialog.SelectedPath;

volTotal $=$ new double $[999$, columnCount $] ;$

timestamp = new string[999];

//determine totalizer file folders

if (one. Checked $==$ true)

\{

\}

folderCount $=1$;

else if (two. Checked $==$ true)

\{ \}

foldercount $=2$;

else

\{

folderCount $=3$;

//repeat calculation for totalizer file folders

for (int folder $=1$; folder $!=$ folderCount +1 ; folder ++ )

\{

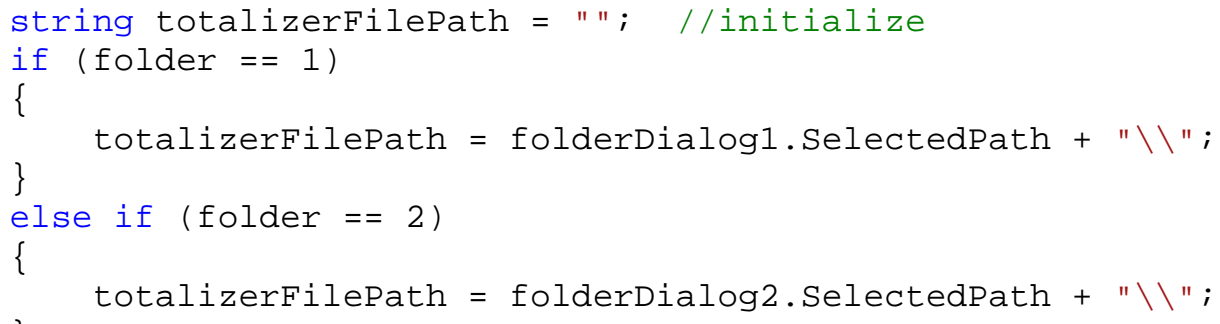




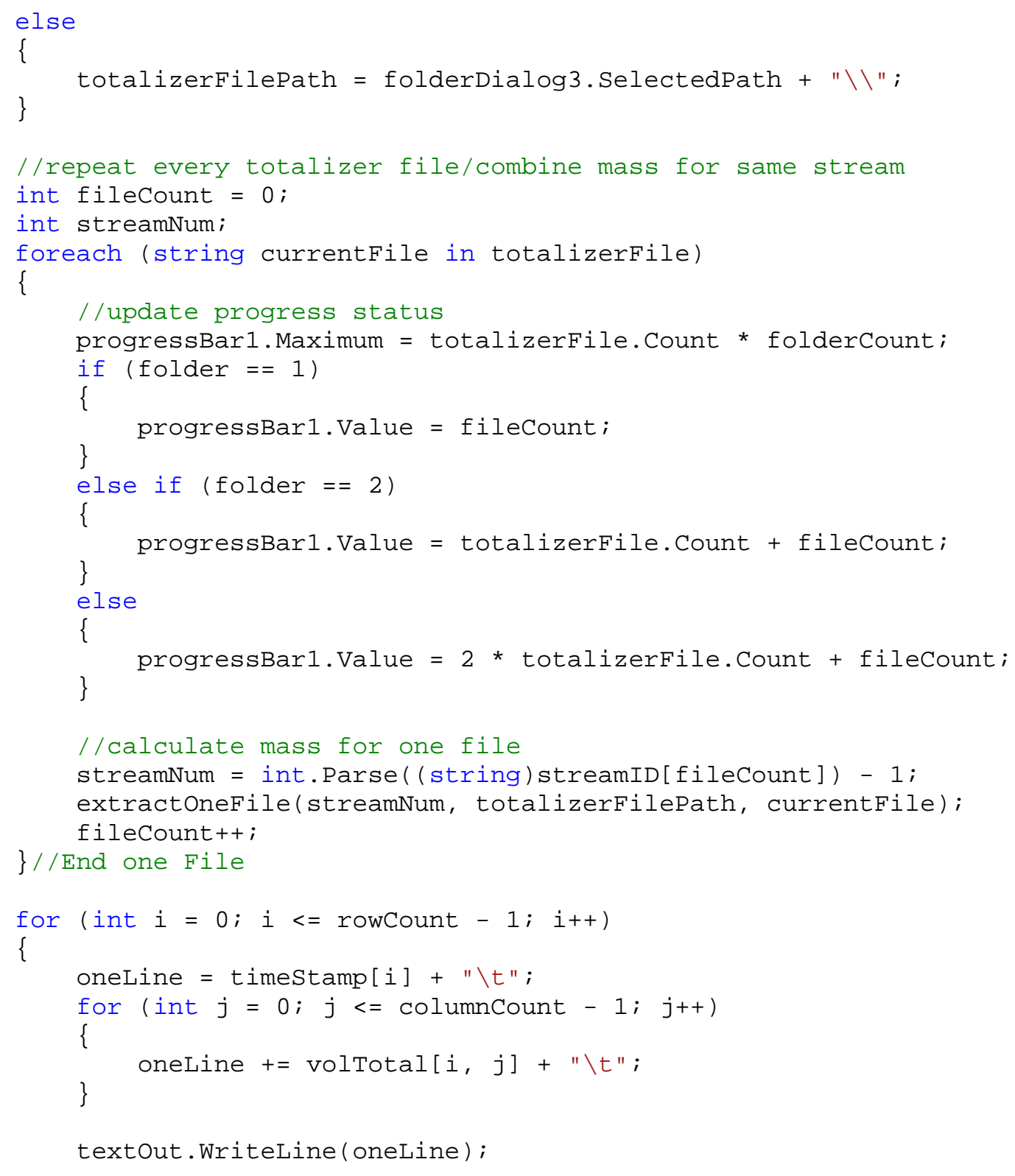




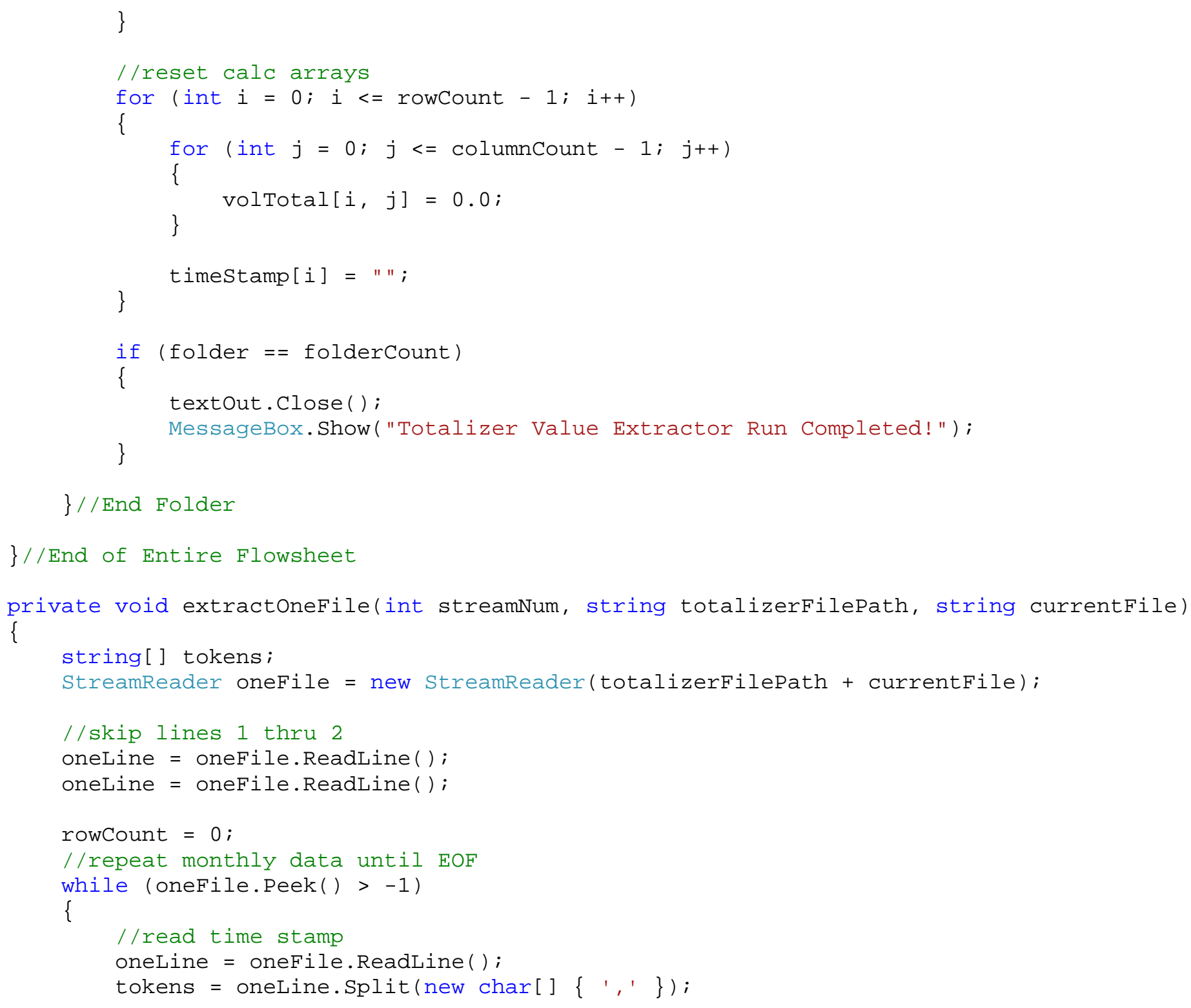




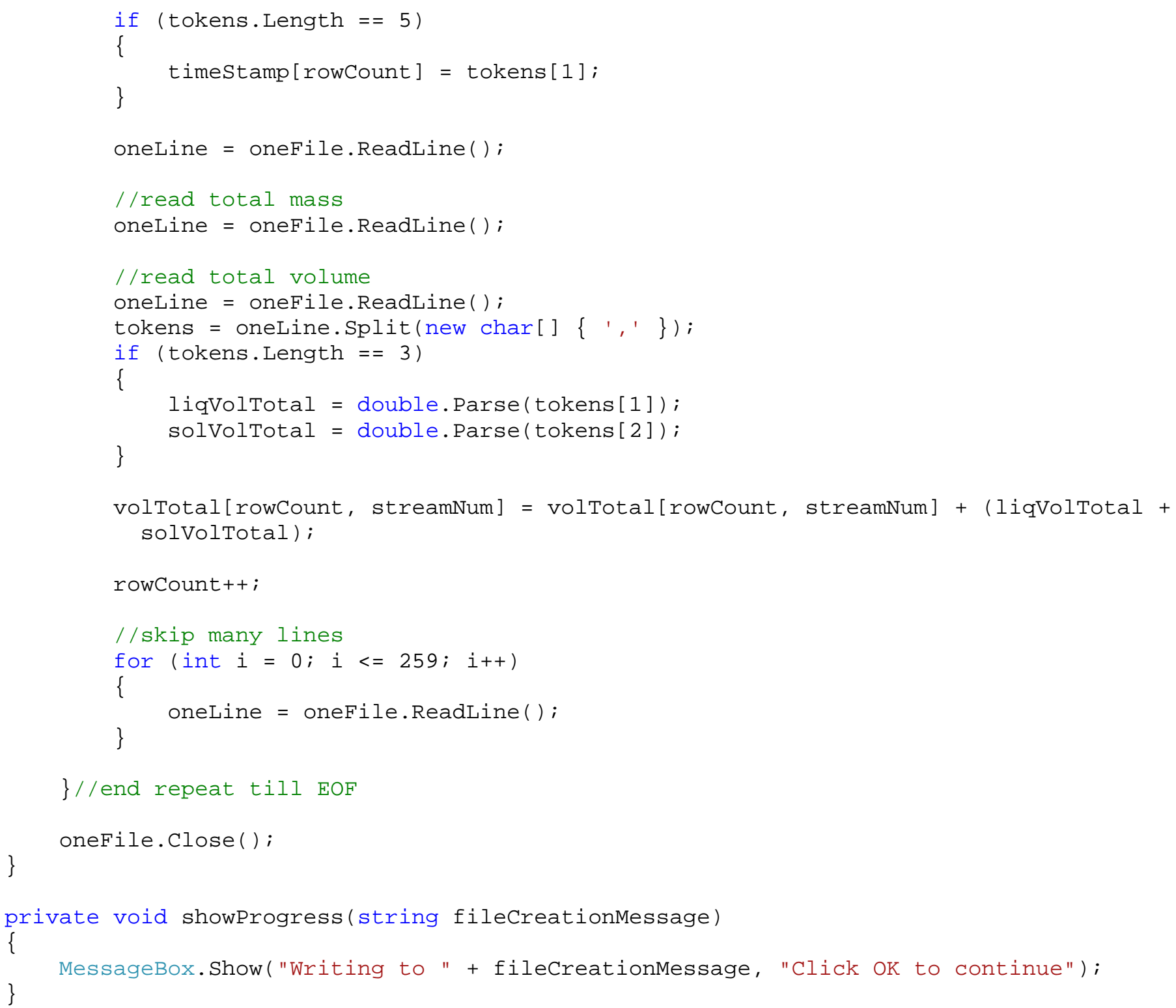


private void closingTVEForm(object sender, System.EventArgs e)

\{ \}

this.Close(); //button close

\}//end Form 
24590-WTP-MDD-PR-01-002, Rev 12

Dynamic (G2) Model Design Document

\section{Appendix K}

\section{Envelope C Reactions}




\section{Appendix K \\ Envelope C Reactions}

Although not in the baseline flowsheet, the Dynamic (G2) Model reserves the capability for performing

Envelope $\mathrm{C}$ reactions in the UFP1 vessels where strontium and transuranic elements (TRU) are precipitated with the additions of $19 \mathrm{M}$ NapOH, 1M NapMnpO4, and 1M Sr(NO3)2. The reactions are tank specific and are shown below:

\section{Reactions Common to AN-102 and AN-107}

\begin{tabular}{|c|c|}
\hline Reaction & $\begin{array}{l}\text { Conversion } \\
\text { (Fraction) }\end{array}$ \\
\hline $\mathrm{Sr}+2(\mathrm{l})+\mathrm{CO} 3-2(\mathrm{l})-->\mathrm{Sr}+2(\mathrm{~s})+\mathrm{CO} 3-2(\mathrm{~s})$ & 0.98 \\
\hline $90-\mathrm{Sr}(\mathrm{l})+\mathrm{CO} 3-2(\mathrm{l})-->90-\mathrm{Sr}(\mathrm{s})+\mathrm{CO} 3-2(\mathrm{~s})$ & 0.98 \\
\hline $\begin{array}{l}14.5 \mathrm{Nap}+(\mathrm{l})+13 \mathrm{Mnp}(\mathrm{l})+52 \mathrm{O}-2(\mathrm{l})+10.5 \mathrm{Na}+(\mathrm{l})+6.5 \mathrm{CHO} 2-(\mathrm{l})+2 \mathrm{C} 2 \mathrm{O} 4-2(\mathrm{l})+4.5 \mathrm{OH}-(\mathrm{l})+241- \\
\mathrm{Am}(\mathrm{l})-->14.5 \mathrm{Nap}+(\mathrm{l})+13 \mathrm{Mnp}(\mathrm{s})+27 \mathrm{O}-2(\mathrm{~s})+6.5 \mathrm{O} 2(\mathrm{~g})+4 \mathrm{Na}+(\mathrm{s})+6.5 \mathrm{Na}+(\mathrm{l})+10.5 \mathrm{CO}-2(\mathrm{l})+ \\
5.5 \mathrm{H} 2 \mathrm{O}(\mathrm{l})+241-\mathrm{Am}(\mathrm{s})\end{array}$ & 0.86 \\
\hline $\begin{array}{l}14.5 \mathrm{Nap}+(\mathrm{l})+13 \mathrm{Mnp}(\mathrm{l})+52 \mathrm{O}-2(\mathrm{l})+10.5 \mathrm{Na}+(\mathrm{l})+6.5 \mathrm{CHO} 2-(\mathrm{l})+2 \mathrm{C} 2 \mathrm{O} 4-2(\mathrm{l})+4.5 \mathrm{OH}-(\mathrm{l})+243- \\
\mathrm{Am}(\mathrm{l})-->14.5 \mathrm{Nap}+(\mathrm{l})+13 \mathrm{Mnp}(\mathrm{s})+27 \mathrm{O}-2(\mathrm{~s})+6.5 \mathrm{O} 2(\mathrm{~g})+4 \mathrm{Na}+(\mathrm{s})+6.5 \mathrm{Na}+(\mathrm{l})+10.5 \mathrm{CO}-2(\mathrm{l})+ \\
5.5 \mathrm{H} 2 \mathrm{O}(\mathrm{l})+243-\mathrm{Am}(\mathrm{s})\end{array}$ & 0.86 \\
\hline $\begin{array}{l}14.5 \mathrm{Nap}+(\mathrm{l})+13 \mathrm{Mnp}(\mathrm{l})+52 \mathrm{O}-2(\mathrm{l})+10.5 \mathrm{Na}+(\mathrm{l})+6.5 \mathrm{CHO} 2-(\mathrm{l})+2 \mathrm{C} 2 \mathrm{O} 4-2(\mathrm{l})+4.5 \mathrm{OH}-(\mathrm{l})+242- \\
\mathrm{Cm}(\mathrm{l})-->14.5 \mathrm{Nap}+(\mathrm{l})+13 \mathrm{Mnp}(\mathrm{s})+27 \mathrm{O}-2(\mathrm{~s})+6.5 \mathrm{O} 2(\mathrm{~g})+4 \mathrm{Na}+(\mathrm{s})+6.5 \mathrm{Na}+(\mathrm{l})+10.5 \mathrm{CO} 3-2(\mathrm{l})+ \\
5.5 \mathrm{H} 2 \mathrm{O}(\mathrm{l})+242-\mathrm{Cm}(\mathrm{s})\end{array}$ & 0.83 \\
\hline $\begin{array}{l}14.5 \mathrm{Nap}+(\mathrm{l})+13 \mathrm{Mnp}(\mathrm{l})+52 \mathrm{O}-2(\mathrm{l})+10.5 \mathrm{Na}+(\mathrm{l})+6.5 \mathrm{CHO} 2-(\mathrm{l})+2 \mathrm{C} 2 \mathrm{O} 4-2(\mathrm{l})+4.5 \mathrm{OH}-(\mathrm{l})+243- \\
\mathrm{Cm}(\mathrm{l})-->14.5 \mathrm{Nap}+(\mathrm{l})+13 \mathrm{Mnp}(\mathrm{s})+27 \mathrm{O}-2(\mathrm{~s})+6.5 \mathrm{O} 2(\mathrm{~g})+4 \mathrm{Na}+(\mathrm{s})+6.5 \mathrm{Na}+(\mathrm{l})+10.5 \mathrm{CO} 3-2(\mathrm{l})+ \\
5.5 \mathrm{H} 2 \mathrm{O}(\mathrm{l})+243-\mathrm{Cm}(\mathrm{s})\end{array}$ & 0.83 \\
\hline $\begin{array}{l}\text { 14.5Nap+ (l) + 13Mnp (l) + 52O-2 (l) + 10.5Na+ (l) +6.5CHO2-(l) + 2C2O4-2 (l) + 4.5OH- (l) + 244- } \\
\mathrm{Cm}(\mathrm{l})-->14.5 \mathrm{Nap}+(\mathrm{l})+13 \mathrm{Mnp}(\mathrm{s})+27 \mathrm{O}-2(\mathrm{~s})+6.5 \mathrm{O} 2(\mathrm{~g})+4 \mathrm{Na}+(\mathrm{s})+6.5 \mathrm{Na}+(\mathrm{l})+10.5 \mathrm{CO} 3-2(\mathrm{l})+ \\
5.5 \mathrm{H} 2 \mathrm{O}(\mathrm{l})+244-\mathrm{Cm}(\mathrm{s})\end{array}$ & 0.83 \\
\hline $\begin{array}{l}14.5 \mathrm{Nap}+(\mathrm{l})+13 \mathrm{Mnp}(\mathrm{l})+52 \mathrm{O}-2(\mathrm{l})+10.5 \mathrm{Na}+(\mathrm{l})+6.5 \mathrm{CHO} 2-(\mathrm{l})+2 \mathrm{C} 2 \mathrm{O} 4-2(\mathrm{l})+4.5 \mathrm{OH}-(\mathrm{l})+152- \\
\mathrm{Eu}(\mathrm{l})--->14.5 \mathrm{Nap}+(\mathrm{l})+13 \mathrm{Mnp}(\mathrm{s})+27 \mathrm{O}-2(\mathrm{~s})+6.5 \mathrm{O} 2(\mathrm{~g})+4 \mathrm{Na}+(\mathrm{s})+6.5 \mathrm{Na}+(\mathrm{l})+10.5 \mathrm{CO} 3-2(\mathrm{l})+ \\
5.5 \mathrm{H} 2 \mathrm{O}(\mathrm{l})+152-\mathrm{Eu}(\mathrm{s})\end{array}$ & 0.91 \\
\hline $\begin{array}{l}\text { 14.5Nap+ (l) + 13Mnp (l) + 52O-2 (l) + 10.5Na+ (l) + 6.5CHO2- (l) + 2C2O4-2 (l) + 4.5OH- (l) + 154- } \\
\mathrm{Eu}(\mathrm{l})--->14.5 \mathrm{Nap}+(\mathrm{l})+13 \mathrm{Mnp}(\mathrm{s})+27 \mathrm{O}-2(\mathrm{~s})+6.5 \mathrm{O} 2(\mathrm{~g})+4 \mathrm{Na}+(\mathrm{s})+6.5 \mathrm{Na}+(\mathrm{l})+10.5 \mathrm{CO} 3-2(\mathrm{l})+ \\
5.5 \mathrm{H} 2 \mathrm{O}(\mathrm{l})+154-\mathrm{Eu}(\mathrm{s})\end{array}$ & 0.91 \\
\hline $\begin{array}{l}\text { 14.5Nap+ (l) + 13 Mnp (l) + 52O-2 (l) + 10.5Na+ (l) + 6.5 CHO2- (l) + 2C2O4-2 (l) + 4.5OH- (l) + 155- } \\
\text { Eu (l) --- 14.5Nap+ (l) + 13Mnp (s) + 27O-2 (s) + 6.5O2 (g) + 4Na }+(\mathrm{s})+6.5 \mathrm{Na}+(\mathrm{l})+10.5 \mathrm{CO}-2(\mathrm{l})+ \\
5.5 \mathrm{H} 2 \mathrm{O}(\mathrm{l})+155-\mathrm{Eu}(\mathrm{s})\end{array}$ & 0.91 \\
\hline 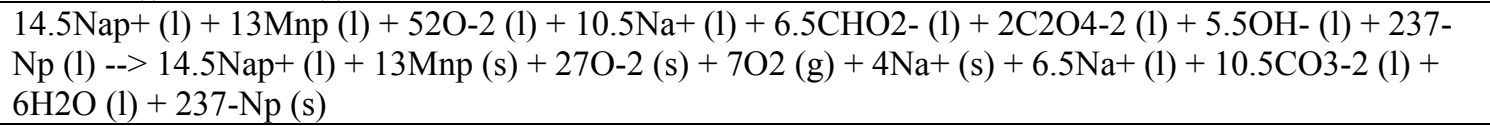 & 0.78 \\
\hline $\begin{array}{l}\text { 14.5Nap+(l) + 13Mnp (l) + 52O-2 (l) }+10.5 \mathrm{Na}+(\mathrm{l})+6.5 \mathrm{CHO} 2-(\mathrm{l})+2 \mathrm{C} 2 \mathrm{O} 4-2(\mathrm{l})+5.5 \mathrm{OH}-(\mathrm{l})+\mathrm{Pu}+4 \\
(\mathrm{l})---14.5 \mathrm{Nap}+(\mathrm{l})+13 \mathrm{Mnp}(\mathrm{s})+27 \mathrm{O}-2(\mathrm{~s})+7 \mathrm{O} 2(\mathrm{~g})+4 \mathrm{Na}+(\mathrm{s})+6.5 \mathrm{Na}+(\mathrm{l})+10.5 \mathrm{CO} 3-2(\mathrm{l})+ \\
6 \mathrm{H} 2 \mathrm{O}(\mathrm{l})+\mathrm{Pu}+4(\mathrm{~s})\end{array}$ & 0.78 \\
\hline $\begin{array}{l}\text { 14.5Nap+ (l) + 13Mnp (l) + 52O-2 (l) + 10.5Na+ (l) + 6.5CHO2- (l) + 2C2O4-2 (l) + 4.5OH- (l) + 238- } \\
\mathrm{Pu}(\mathrm{l})-->14.5 \mathrm{Nap}+(\mathrm{l})+13 \mathrm{Mnp}(\mathrm{s})+27 \mathrm{O}-2(\mathrm{~s})+6.5 \mathrm{O} 2(\mathrm{~g})+4 \mathrm{Na}+(\mathrm{s})+6.5 \mathrm{Na}+(\mathrm{l})+10.5 \mathrm{CO} 3-2(\mathrm{l})+ \\
5.5 \mathrm{H} 2 \mathrm{O}(\mathrm{l})+238-\mathrm{Pu}(\mathrm{s})\end{array}$ & 0.78 \\
\hline $\begin{array}{l}\text { 14.5Nap+ (l) + 13Mnp (l) + 52O-2 (l) + 10.5Na }+(\mathrm{l})+6.5 \mathrm{CHO} 2-(\mathrm{l})+2 \mathrm{C} 2 \mathrm{O} 4-2(\mathrm{l})+4.5 \mathrm{OH}-(\mathrm{l})+239- \\
\mathrm{Pu}(\mathrm{l})-->14.5 \mathrm{Nap}+(\mathrm{l})+13 \mathrm{Mnp}(\mathrm{s})+27 \mathrm{O}-2(\mathrm{~s})+6.5 \mathrm{O} 2(\mathrm{~g})+4 \mathrm{Na}+(\mathrm{s})+6.5 \mathrm{Na}+(\mathrm{l})+10.5 \mathrm{CO} 3-2(\mathrm{l})+ \\
5.5 \mathrm{H} 2 \mathrm{O}(\mathrm{l})+239-\mathrm{Pu}(\mathrm{s})\end{array}$ & 0.78 \\
\hline $\begin{array}{l}\text { 14.5Nap+ (l) + 13Mnp (l) }+52 \mathrm{O}-2(\mathrm{l})+10.5 \mathrm{Na}+(\mathrm{l})+6.5 \mathrm{CHO} 2-(\mathrm{l})+2 \mathrm{C} 2 \mathrm{O} 4-2(\mathrm{l}) \\
\mathrm{Pu}(\mathrm{l})-->14.5 \mathrm{Nap}+(\mathrm{l})+13 \mathrm{Mnp}(\mathrm{s})+27 \mathrm{O}-2(\mathrm{~s})+6.5 \mathrm{O} 2(\mathrm{~g})+4 \mathrm{Na}+(\mathrm{s})+6.5 \mathrm{Na}+\end{array}$ & 0.78 \\
\hline
\end{tabular}




\begin{tabular}{|c|c|}
\hline Reaction & $\begin{array}{c}\text { Conversion } \\
\text { (Fraction) }\end{array}$ \\
\hline \multicolumn{2}{|l|}{$5.5 \mathrm{H} 2 \mathrm{O}(\mathrm{l})+240-\mathrm{Pu}(\mathrm{s})$} \\
\hline $\begin{array}{l}14.5 \mathrm{Nap}+(\mathrm{l})+13 \mathrm{Mnp}(\mathrm{l})+52 \mathrm{O}-2(\mathrm{l})+10.5 \mathrm{Na}+(\mathrm{l})+6.5 \mathrm{CHO} 2-(\mathrm{l})+2 \mathrm{C} 2 \mathrm{O} 4-2(\mathrm{l})+4.5 \mathrm{OH}-(\mathrm{l})+241- \\
\mathrm{Pu}(\mathrm{l})-->14.5 \mathrm{Nap}+(\mathrm{l})+13 \mathrm{Mnp}(\mathrm{s})+27 \mathrm{O}-2(\mathrm{~s})+6.5 \mathrm{O} 2(\mathrm{~g})+4 \mathrm{Na}+(\mathrm{s})+6.5 \mathrm{Na}+(\mathrm{l})+10.5 \mathrm{CO} 3-2(\mathrm{l})+ \\
5.5 \mathrm{H} 2 \mathrm{O}(\mathrm{l})+241-\mathrm{Pu}(\mathrm{s})\end{array}$ & 0.78 \\
\hline $\begin{array}{l}14.5 \mathrm{Nap}+(\mathrm{l})+13 \mathrm{Mnp}(\mathrm{l})+52 \mathrm{O}-2(\mathrm{l})+10.5 \mathrm{Na}+(\mathrm{l})+6.5 \mathrm{CHO} 2-(\mathrm{l})+2 \mathrm{C} 2 \mathrm{O} 4-2(\mathrm{l})+4.5 \mathrm{OH}-(\mathrm{l})+242- \\
\mathrm{Pu}(\mathrm{l})-->14.5 \mathrm{Nap}+(\mathrm{l})+13 \mathrm{Mnp}(\mathrm{s})+27 \mathrm{O}-2(\mathrm{~s})+6.5 \mathrm{O} 2(\mathrm{~g})+4 \mathrm{Na}+(\mathrm{s})+6.5 \mathrm{Na}+(\mathrm{l})+10.5 \mathrm{CO} 3-2(\mathrm{l})+ \\
5.5 \mathrm{H} 2 \mathrm{O}(\mathrm{l})+242-\mathrm{Pu}(\mathrm{s})\end{array}$ & 0.78 \\
\hline 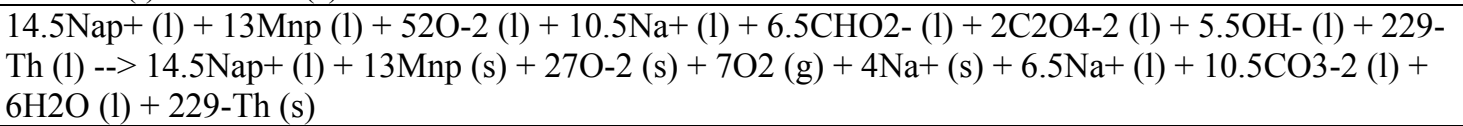 & 0.78 \\
\hline $\begin{array}{l}14.5 \mathrm{Nap}+(\mathrm{l})+13 \mathrm{Mnp}(\mathrm{l})+52 \mathrm{O}-2(\mathrm{l})+10.5 \mathrm{Na}+(\mathrm{l})+6.5 \mathrm{CHO} 2-(\mathrm{l})+2 \mathrm{C} 2 \mathrm{O} 4-2(\mathrm{l})+5.5 \mathrm{OH}-(\mathrm{l})+231- \\
\mathrm{Pa}(\mathrm{l})-->14.5 \mathrm{Nap}+(\mathrm{l})+13 \mathrm{Mnp}(\mathrm{s})+27 \mathrm{O}-2(\mathrm{~s})+7 \mathrm{O} 2(\mathrm{~g})+4 \mathrm{Na}+(\mathrm{s})+6.5 \mathrm{Na}+(\mathrm{l})+10.5 \mathrm{CO}-2(\mathrm{l})+ \\
6 \mathrm{H} 2 \mathrm{O}(\mathrm{l})+231-\mathrm{Pa}(\mathrm{s})\end{array}$ & 0.78 \\
\hline $\begin{array}{l}14.5 \mathrm{Nap}+(\mathrm{l})+13 \mathrm{Mnp}(\mathrm{l})+52 \mathrm{O}-2(\mathrm{l})+10.5 \mathrm{Na}+(\mathrm{l})+6.5 \mathrm{CHO} 2-(\mathrm{l})+2 \mathrm{C} 2 \mathrm{O} 4-2(\mathrm{l})+5.5 \mathrm{OH}-(\mathrm{l})+232- \\
\mathrm{Th}(\mathrm{l})-->14.5 \mathrm{Nap}+(\mathrm{l})+13 \mathrm{Mnp}(\mathrm{s})+27 \mathrm{O}-2(\mathrm{~s})+7 \mathrm{O} 2(\mathrm{~g})+4 \mathrm{Na}+(\mathrm{s})+6.5 \mathrm{Na}+(\mathrm{l})+10.5 \mathrm{CO}-2(\mathrm{l})+ \\
6 \mathrm{H} 2 \mathrm{O}(\mathrm{l})+232-\mathrm{Th}(\mathrm{s})\end{array}$ & 0.78 \\
\hline $\begin{array}{l}14.5 \mathrm{Nap}+(\mathrm{l})+13 \mathrm{Mnp}(\mathrm{l})+52 \mathrm{O}-2(\mathrm{l})+10.5 \mathrm{Na}+(\mathrm{l})+6.5 \mathrm{CHO} 2-(\mathrm{l})+2 \mathrm{C} 2 \mathrm{O} 4-2(\mathrm{l})+5.5 \mathrm{OH}-(\mathrm{l})+232- \\
\mathrm{U}(\mathrm{l})-->14.5 \mathrm{Nap}+(\mathrm{l})+13 \mathrm{Mnp}(\mathrm{s})+27 \mathrm{O}-2(\mathrm{~s})+7 \mathrm{O} 2(\mathrm{~g})+4 \mathrm{Na}+(\mathrm{s})+6.5 \mathrm{Na}+(\mathrm{l})+10.5 \mathrm{CO} 3-2(\mathrm{l})+ \\
6 \mathrm{H} 2 \mathrm{O}(\mathrm{l})+232-\mathrm{U}(\mathrm{s})\end{array}$ & 0.78 \\
\hline $\begin{array}{l}14.5 \mathrm{Nap}+(\mathrm{l})+13 \mathrm{Mnp}(\mathrm{l})+52 \mathrm{O}-2(\mathrm{l})+10.5 \mathrm{Na}+(\mathrm{l})+6.5 \mathrm{CHO} 2-(\mathrm{l})+2 \mathrm{C} 2 \mathrm{O} 4-2(\mathrm{l})+5.5 \mathrm{OH}-(\mathrm{l})+233- \\
\mathrm{U}(\mathrm{l})-->14.5 \mathrm{Nap}+(\mathrm{l})+13 \mathrm{Mnp}(\mathrm{s})+27 \mathrm{O}-2(\mathrm{~s})+7 \mathrm{O} 2(\mathrm{~g})+4 \mathrm{Na}+(\mathrm{s})+6.5 \mathrm{Na}+(\mathrm{l})+10.5 \mathrm{CO} 3-2(\mathrm{l})+ \\
6 \mathrm{H} 2 \mathrm{O}(\mathrm{l})+233-\mathrm{U}(\mathrm{s})\end{array}$ & 0.78 \\
\hline $\begin{array}{l}14.5 \mathrm{Nap}+(\mathrm{l})+13 \mathrm{Mnp}(\mathrm{l})+52 \mathrm{O}-2(\mathrm{l})+10.5 \mathrm{Na}+(\mathrm{l})+6.5 \mathrm{CHO} 2-(\mathrm{l})+2 \mathrm{C} 2 \mathrm{O} 4-2(\mathrm{l})+5.5 \mathrm{OH}-(\mathrm{l})+234- \\
\mathrm{U}(\mathrm{l})-->14.5 \mathrm{Nap}+(\mathrm{l})+13 \mathrm{Mnp}(\mathrm{s})+27 \mathrm{O}-2(\mathrm{~s})+7 \mathrm{O} 2(\mathrm{~g})+4 \mathrm{Na}+(\mathrm{s})+6.5 \mathrm{Na}+(\mathrm{l})+10.5 \mathrm{CO} 3-2(\mathrm{l})+ \\
6 \mathrm{H} 2 \mathrm{O}(\mathrm{l})+234-\mathrm{U}(\mathrm{s})\end{array}$ & 0.78 \\
\hline $\begin{array}{l}14.5 \mathrm{Nap}+(\mathrm{l})+13 \mathrm{Mnp}(\mathrm{l})+52 \mathrm{O}-2(\mathrm{l})+10.5 \mathrm{Na}+(\mathrm{l})+6.5 \mathrm{CHO} 2-(\mathrm{l})+2 \mathrm{C} 2 \mathrm{O} 4-2(\mathrm{l})+5.5 \mathrm{OH}-(\mathrm{l})+235- \\
\mathrm{U}(\mathrm{l})-->14.5 \mathrm{Nap}+(\mathrm{l})+13 \mathrm{Mnp}(\mathrm{s})+27 \mathrm{O}-2(\mathrm{~s})+7 \mathrm{O} 2(\mathrm{~g})+4 \mathrm{Na}+(\mathrm{s})+6.5 \mathrm{Na}+(\mathrm{l})+10.5 \mathrm{CO} 3-2(\mathrm{l})+ \\
6 \mathrm{H} 2 \mathrm{O}(\mathrm{l})+235-\mathrm{U}(\mathrm{s})\end{array}$ & 0.78 \\
\hline $\begin{array}{l}14.5 \mathrm{Nap}+(\mathrm{l})+13 \mathrm{Mnp}(\mathrm{l})+52 \mathrm{O}-2(\mathrm{l})+10.5 \mathrm{Na}+(\mathrm{l})+6.5 \mathrm{CHO} 2-(\mathrm{l})+2 \mathrm{C} 2 \mathrm{O} 4-2(\mathrm{l})+5.5 \mathrm{OH}-(\mathrm{l})+236- \\
\mathrm{U}(\mathrm{l})-->14.5 \mathrm{Nap}+(\mathrm{l})+13 \mathrm{Mnp}(\mathrm{s})+27 \mathrm{O}-2(\mathrm{~s})+7 \mathrm{O} 2(\mathrm{~g})+4 \mathrm{Na}+(\mathrm{s})+6.5 \mathrm{Na}+(\mathrm{l})+10.5 \mathrm{CO} 3-2(\mathrm{l})+ \\
6 \mathrm{H} 2 \mathrm{O}(\mathrm{l})+236-\mathrm{U}(\mathrm{s})\end{array}$ & 0.78 \\
\hline $\begin{array}{l}14.5 \mathrm{Nap}+(\mathrm{l})+13 \mathrm{Mnp}(\mathrm{l})+52 \mathrm{O}-2(\mathrm{l})+10.5 \mathrm{Na}+(\mathrm{l})+6.5 \mathrm{CHO} 2-(\mathrm{l})+2 \mathrm{C} 2 \mathrm{O} 4-2(\mathrm{l})+5.5 \mathrm{OH}-(\mathrm{l})+238- \\
\mathrm{U}(\mathrm{l})-->14.5 \mathrm{Nap}+(\mathrm{l})+13 \mathrm{Mnp}(\mathrm{s})+27 \mathrm{O}-2(\mathrm{~s})+7 \mathrm{O} 2(\mathrm{~g})+4 \mathrm{Na}+(\mathrm{s})+6.5 \mathrm{Na}+(\mathrm{l})+10.5 \mathrm{CO} 3-2(\mathrm{l})+ \\
6 \mathrm{H} 2 \mathrm{O}(\mathrm{l})+238-\mathrm{U}(\mathrm{s})\end{array}$ & 0.78 \\
\hline $\begin{array}{l}14.5 \mathrm{Nap}+(\mathrm{l})+13 \mathrm{Mnp}(\mathrm{l})+52 \mathrm{O}-2(\mathrm{l})+10.5 \mathrm{Na}+(\mathrm{l})+6.5 \mathrm{CHO} 2-(\mathrm{l})+2 \mathrm{C} 2 \mathrm{O} 4-2(\mathrm{l})+5.5 \mathrm{OH}-(\mathrm{l})+238- \\
\mathrm{U}(\mathrm{l})-->14.5 \mathrm{Nap}+(\mathrm{l})+13 \mathrm{Mnp}(\mathrm{s})+27 \mathrm{O}-2(\mathrm{~s})+7 \mathrm{O} 2(\mathrm{~g})+4 \mathrm{Na}+(\mathrm{s})+6.5 \mathrm{Na}+(\mathrm{l})+10.5 \mathrm{CO} 3-2(\mathrm{l})+ \\
6 \mathrm{H} 2 \mathrm{O}(\mathrm{l})+238-\mathrm{U}(\mathrm{s})\end{array}$ & 0.78 \\
\hline $\begin{array}{l}15 \mathrm{Nap}+(\mathrm{l})+14 \mathrm{Mnp}(\mathrm{l})+56 \mathrm{O}-2(\mathrm{l})+11 \mathrm{Na}+(\mathrm{l})+7 \mathrm{CHO} 2-(\mathrm{l})+2 \mathrm{C} 2 \mathrm{O} 4-2(\mathrm{l})+\mathrm{OH}-(\mathrm{l})-->4 \mathrm{Nap}+(\mathrm{s})+ \\
14 \mathrm{Mnp}(\mathrm{s})+27 \mathrm{O}-2(\mathrm{~s})+7.5 \mathrm{O} 2(\mathrm{~g})+11 \mathrm{Nap}+(\mathrm{l})+11 \mathrm{Na}+(\mathrm{l})+11 \mathrm{CO}-2(\mathrm{l})+4 \mathrm{H} 2 \mathrm{O}(\mathrm{l})\end{array}$ & 0.99 \\
\hline
\end{tabular}

$\underline{\text { Reactions Specific to AN-102 }}$ 


\begin{tabular}{|l|c|}
\hline Reaction & $\begin{array}{c}\text { Conversion } \\
\text { (Fraction) }\end{array}$ \\
\hline $\mathrm{Al}+3(\mathrm{~s})+3 \mathrm{OH}-(\mathrm{s}) \rightarrow \mathrm{Al}+3(\mathrm{l})+3 \mathrm{OH}-(\mathrm{l})$ & 0.72 \\
\hline $\mathrm{Al}+3(\mathrm{~s})+3 \mathrm{OH}(\mathrm{Bound})(\mathrm{s}) \rightarrow \mathrm{Al}+3(\mathrm{l})+3 \mathrm{OH}-(\mathrm{l})$ & 0.72 \\
\hline $\mathrm{Ca}+2(\mathrm{l})+\mathrm{CO} 3-2(\mathrm{l}) \rightarrow \mathrm{Ca}+2(\mathrm{~s})+\mathrm{CO} 3-2(\mathrm{~s})$ & 0.47 \\
\hline $\mathrm{Cr}(\mathrm{OH} 4)-(\mathrm{l})+3 \mathrm{OH}-(\mathrm{l}) \rightarrow \mathrm{Cr}(\mathrm{OH}) 4-(\mathrm{s})+3 \mathrm{OH}-(\mathrm{s})$ & 0.33 \\
\hline $\mathrm{Cr}(\mathrm{Total})(\mathrm{l})+3 \mathrm{OH}-(\mathrm{l}) \rightarrow \mathrm{Cr}(\mathrm{Total})(\mathrm{s})+3 \mathrm{OH}-(\mathrm{s})$ & 0.33 \\
\hline $\mathrm{Fe}+3(\mathrm{l})+3 \mathrm{OH}-(\mathrm{l}) \rightarrow \mathrm{Fe}+3(\mathrm{~s})+3 \mathrm{OH}-(\mathrm{s})$ & 0.83 \\
\hline $\mathrm{Pb}+2(\mathrm{l})+\mathrm{CO}-2(1) \rightarrow \mathrm{Pb}+2(\mathrm{~s})+\mathrm{CO} 3-2(\mathrm{~s})$ & 0.17 \\
\hline
\end{tabular}

$\underline{\text { Reactions Specific to AN-107 }}$

\begin{tabular}{|l|c|}
\hline Reaction & $\begin{array}{c}\text { Conversion } \\
\text { (Fraction) }\end{array}$ \\
\hline $\mathrm{Al}+3(\mathrm{l})+3 \mathrm{OH}-(\mathrm{l}) \rightarrow \mathrm{Al}+3(\mathrm{~s})+3 \mathrm{OH}-(\mathrm{s})$ & 0.20 \\
\hline $\mathrm{Ca}+2(\mathrm{l})+\mathrm{CO} 3-2(\mathrm{l}) \rightarrow \mathrm{Ca}+2(\mathrm{~s})+\mathrm{CO} 3-2(\mathrm{~s})$ & 0.43 \\
\hline $\mathrm{Cr}(\mathrm{OH} 4)-(\mathrm{l})+3 \mathrm{OH}-(\mathrm{l}) \rightarrow \mathrm{Cr}(\mathrm{OH}) 4-(\mathrm{s})+3 \mathrm{OH}-(\mathrm{s})$ & 0.50 \\
\hline $\mathrm{Cr}(\mathrm{Total})(\mathrm{l})+3 \mathrm{OH}-(\mathrm{l}) \rightarrow \mathrm{Cr}(\mathrm{Total})(\mathrm{s})+3 \mathrm{OH}-(\mathrm{s})$ & 0.50 \\
\hline $\mathrm{Fe}+3(\mathrm{l})+3 \mathrm{OH}-(\mathrm{l}) \rightarrow \mathrm{Fe}+3(\mathrm{~s})+3 \mathrm{OH}-(\mathrm{s})$ & 0.99 \\
\hline $\mathrm{Pb}+2(\mathrm{l})+\mathrm{CO} 3-2(\mathrm{l}) \rightarrow \mathrm{Pb}+2(\mathrm{~s})+\mathrm{CO} 3-2(\mathrm{~s})$ & 0.60 \\
\hline
\end{tabular}




\section{Appendix L}

\section{UFP2 Leaching with Boehmite}




\section{Appendix L UFP2 Leaching with Boehmite}

The Dynamic (G2) Model Version 7.0 is designed with the option to perform boehmite leaching in the UFP2 vessels. The boehmite leaching option can be selected by setting the leaching-scenario variable to UFP2 and the boehmite-leaching variable to true on the Model Run Graphic User Interface. The boehmite leaching flowsheet differs from the baseline backend leaching flowsheet in how caustic leach reactions are performed. Operating logic and parameters applied to the boehmite leaching flowsheet are shown in Figures L-1 through L-12. Development of the boehmite leaching logic are discussed in the BARD.

\section{Parame ter Preparation}

Utilize IHLW' Shell V2.0 Bounding Conditions:

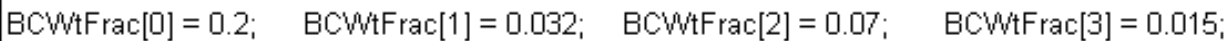

BCWtFrac:[4] = 0.005; BCWtFrac $[5]=0.012 ; \quad \mathrm{BCW}$ tFrac $[6]=0.02 ; \quad \mathrm{BCW}$ tFrac $[7]=0.174 ;$

BCWtFrac $[8]=0.06 ; \quad \mathrm{BCW} \mathrm{tFrac}[9]=0.06 ; \quad \mathrm{BCW} \mathrm{tFrac}[10]=0.07 ; \quad \mathrm{BCW} / \mathrm{tFac}[11]=0.214 ;$

BCWtFrac [12] = 0.03; BCWtFrac[13] = 0.0025; BCWtFrac [14] = 0.025; BCW/Frac [15] = 0.025;

$\mathrm{BCW}$ tFrac $[16]=0.05 ; \mathrm{BCW} \mathrm{tFrac}[17]=0.53 ; \quad \mathrm{BCW} \mathrm{tFrac}[18]=0.005 ; \quad \mathrm{BCW} \mathrm{tFrac}[19]=0.045 ;$

$\mathrm{BCW}$ tFrac $[20]=0.06 ; \mathrm{BCW}$ tF rac $[21]=0.063 ; \quad \mathrm{BCW} \mathrm{tFrac}[22]=0.135 ; \quad \mathrm{BCW}$ tF rac $[23]=0.045 ;$

\begin{tabular}{|c|c|c|}
\hline \multicolumn{3}{|l|}{ W She } \\
\hline 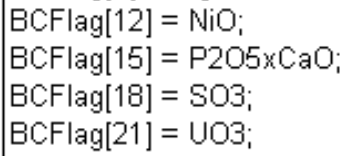 & $\begin{array}{l}\text { BCFlag[1] = Bi2O3; } \\
\text { BCFlag[4] = Cl; } \\
\text { BCFlag[7] = Fe203; } \\
\text { BCFlag[10] = MnO; } \\
\text { BCFlag[13] = NobleMetal; } \\
\text { BCFlag[16] = PbO; } \\
\text { BCFlag[19] = GO }\end{array}$ & 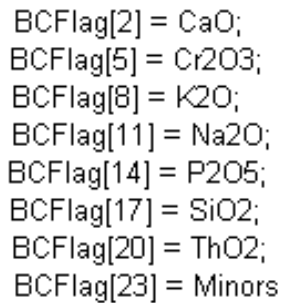 \\
\hline
\end{tabular}

Figure L-1 Boehmite Leaching Decision Logic (Part 1) 


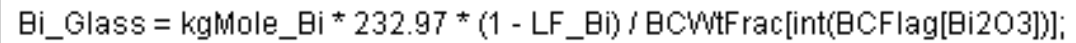

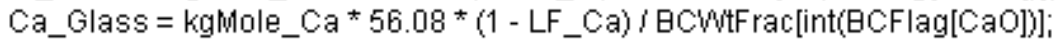

Co_Glass = (2kglwole_Cd) * 128.41 i BChitFrac[int(BCFlag[CdO])];

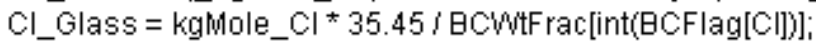

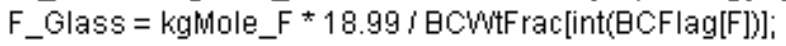

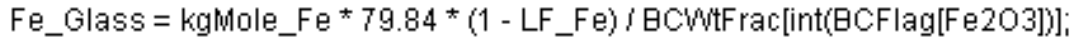

K_Glass = kglwole_K* 47.10 i BCWtF rac:[int(BCFlag[K2O])];

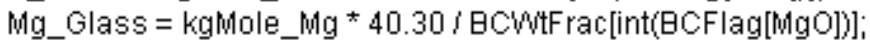

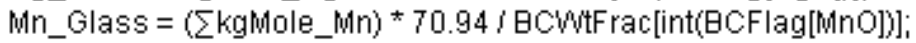

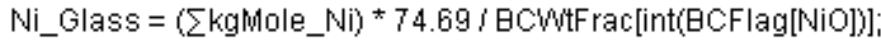

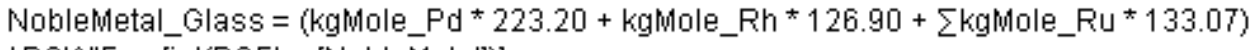

i BChitFrac [intigCFlag[Noblemetal]];

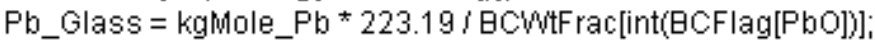

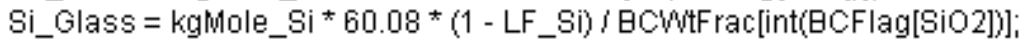

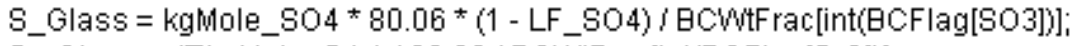

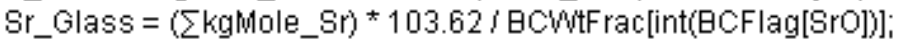

Th_Glass $=$ (¿kgMole_Th) $* 265.03$ / BChtFrac [int(BCFlag[Tho2]]];

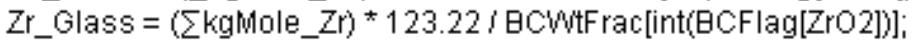

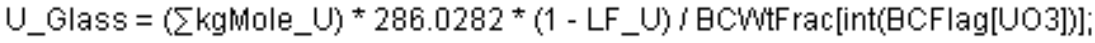

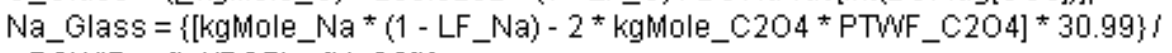

BChtF rac[int(ECFlag[iva20]];

P_Glass = (kghole_PO4HARD $+\mathrm{Ppf}^{*}$ kghole_PO4)*70.97 i

BChitFrac[int(ECFlag[P205])];

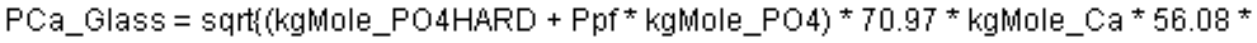

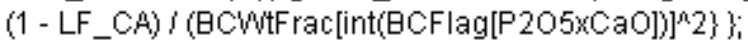

EG_Majors = Max (Bi_Glass, Ca_Glass, Cd_Glass, Cl_Glass, F_Glass, Fe_Glass,

K_Glass,

Mg_Glass, Wn_Glass, Ni_Glass, Noblemetal_Glass, Pb_Glass, Si_Glass, S_Glass,

Sr_Glass, Th_Glass, Zr_Glass, U_Glass, Na_Glass, P_Glass, PCa_Glass);

PTWF_C204 = 1.0;

$P p f=0.03$;

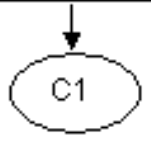

Figure L-2 Boehmite Leaching Decision Logic (Part 2) 


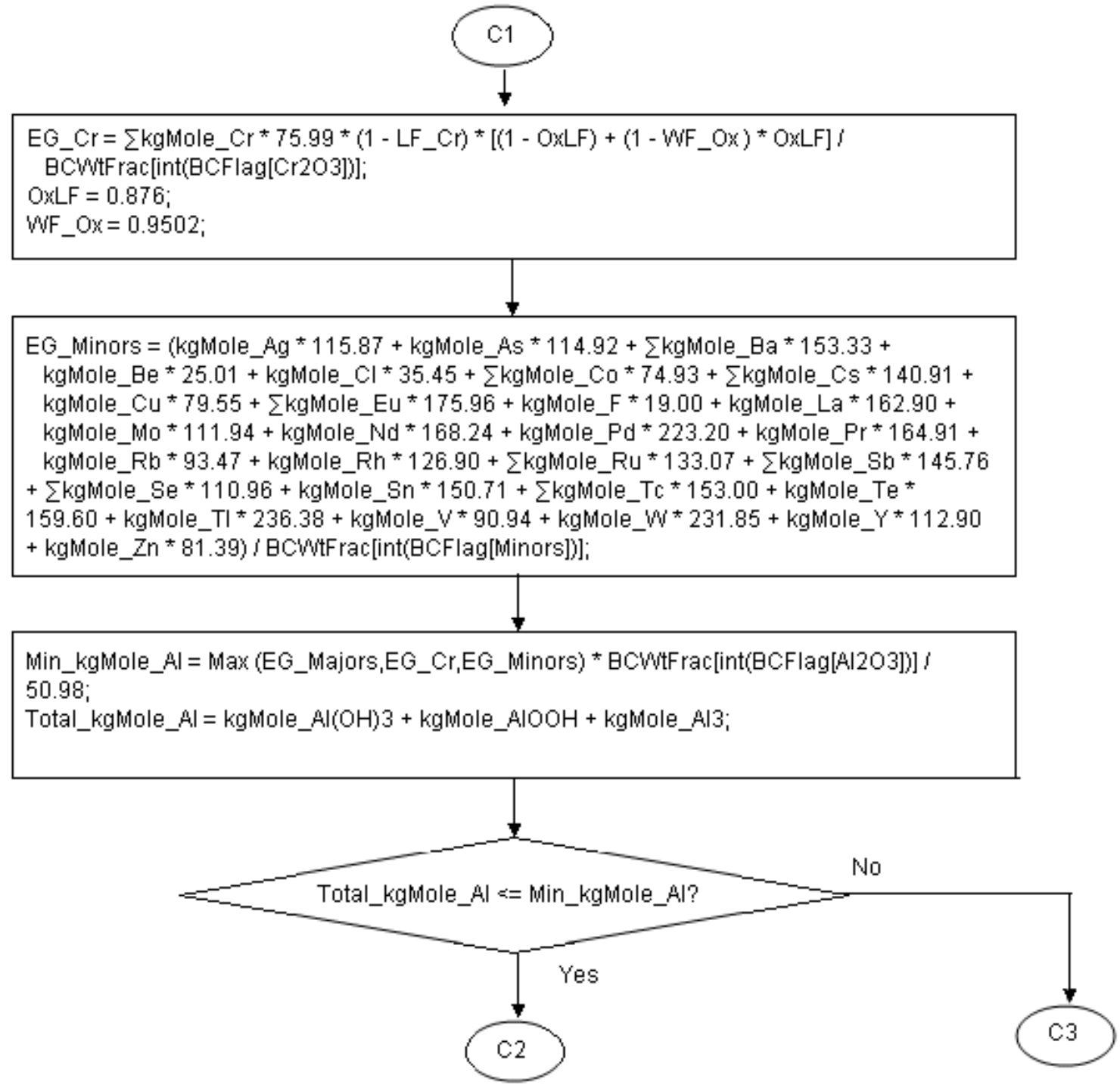

Figure L-3 Boehmite Leaching Decision Logic (Part 3) 


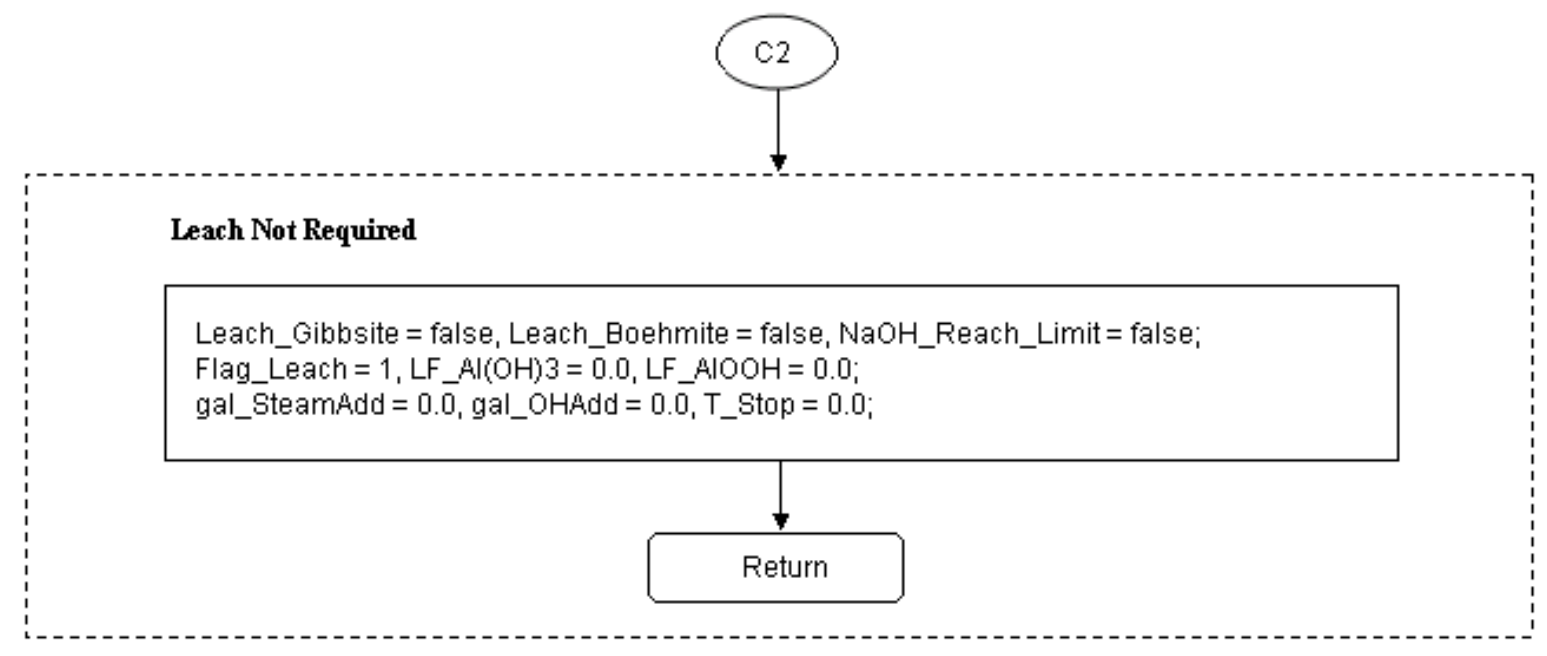

Figure L-4 Boehmite Leaching Decision Logic (Part 4) 


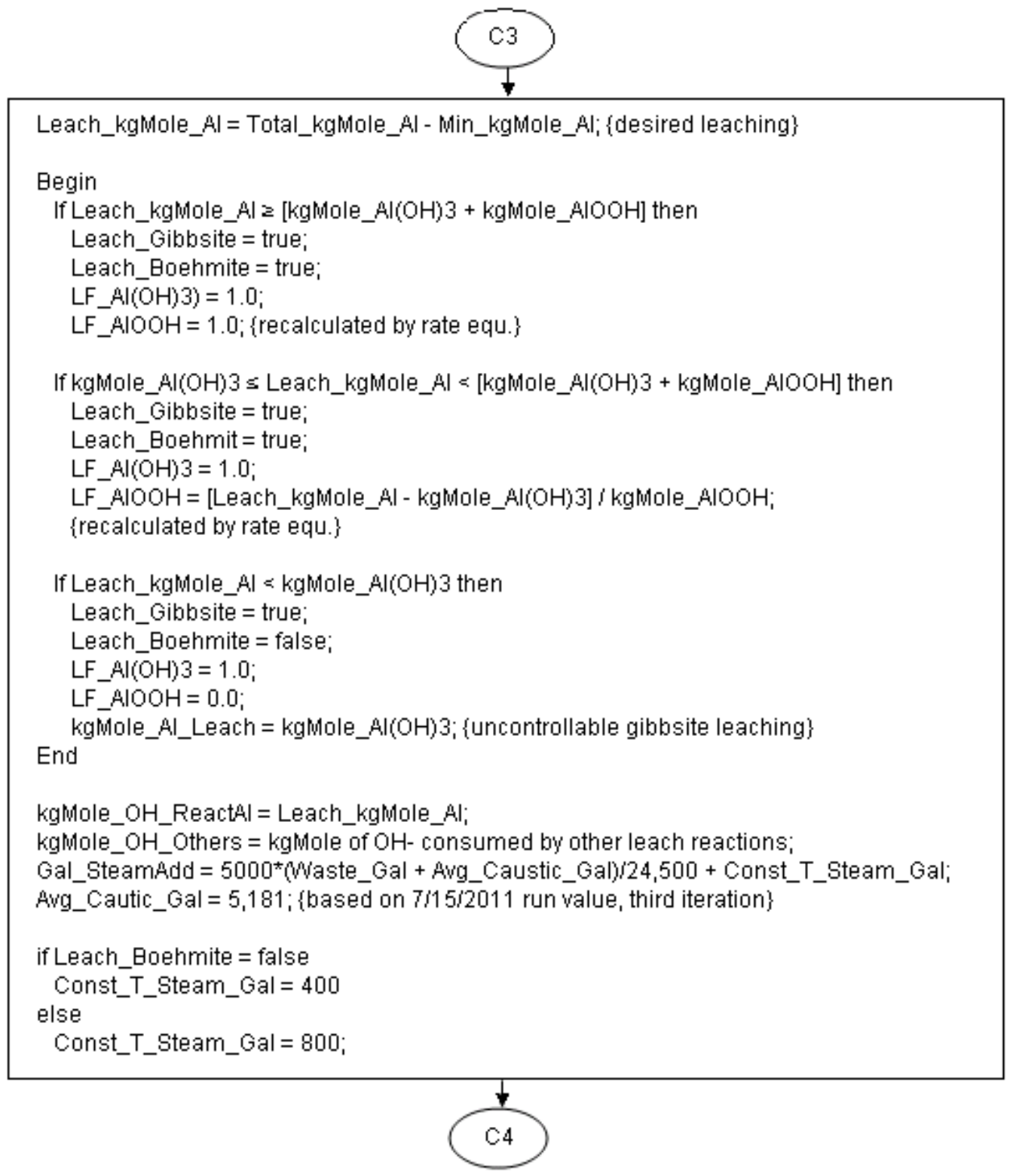

Figure L-5 Boehmite Leaching Decision Logic (Part 5) 


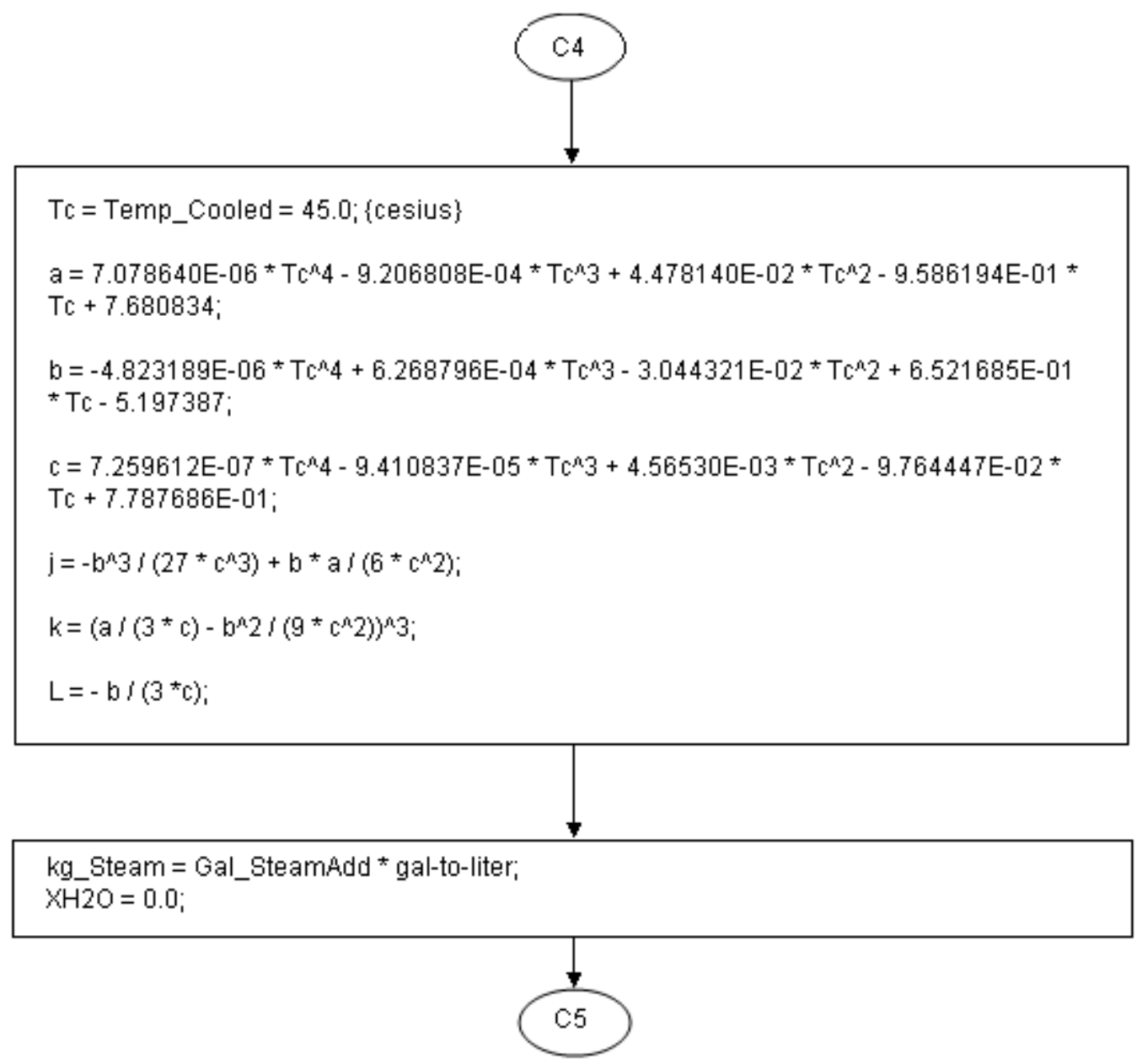

Figure L-6 Boehmite Leaching Decision Logic (Part 6) 


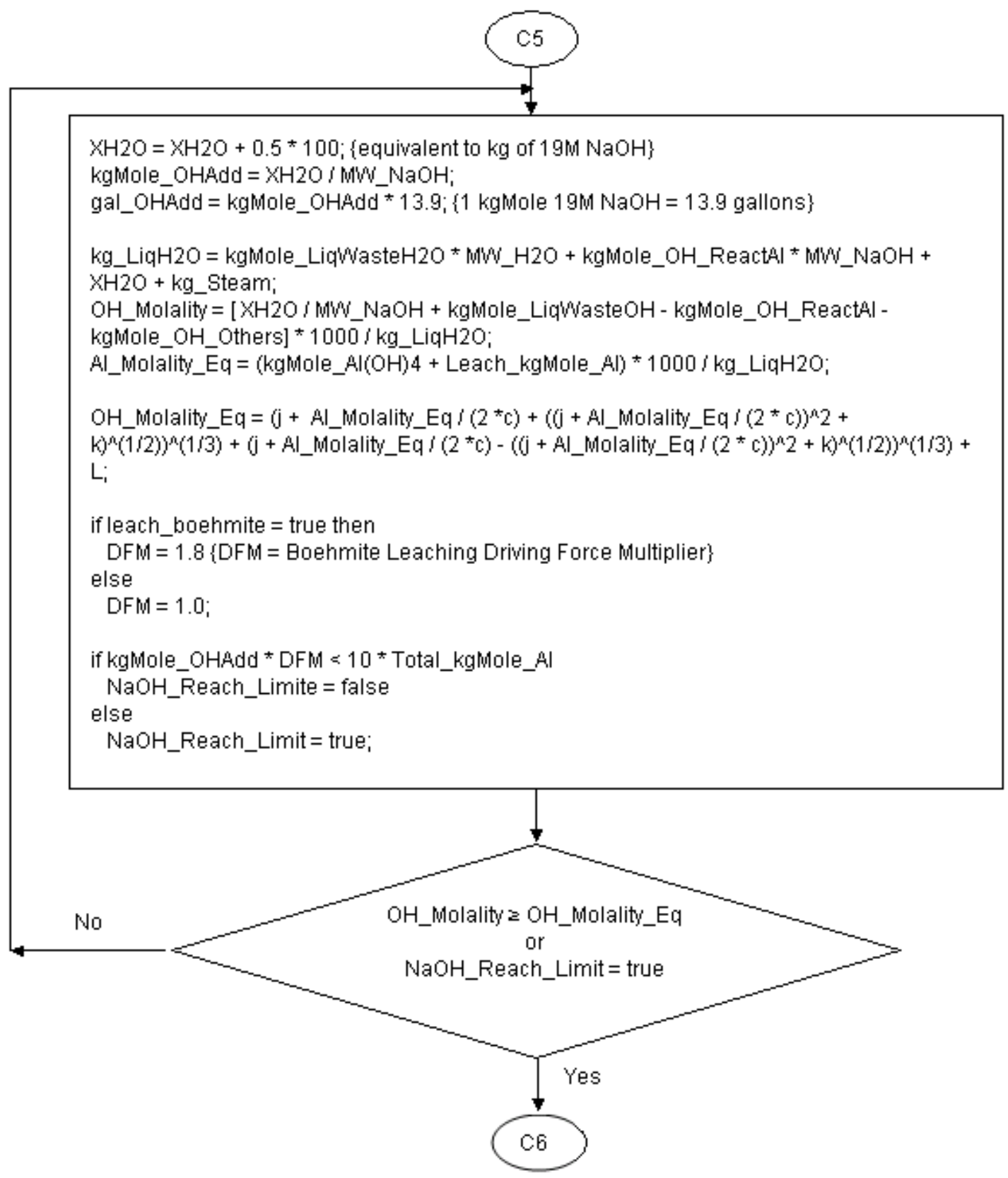

Figure L-7 Boehmite Leaching Decision Logic (Part 7) 


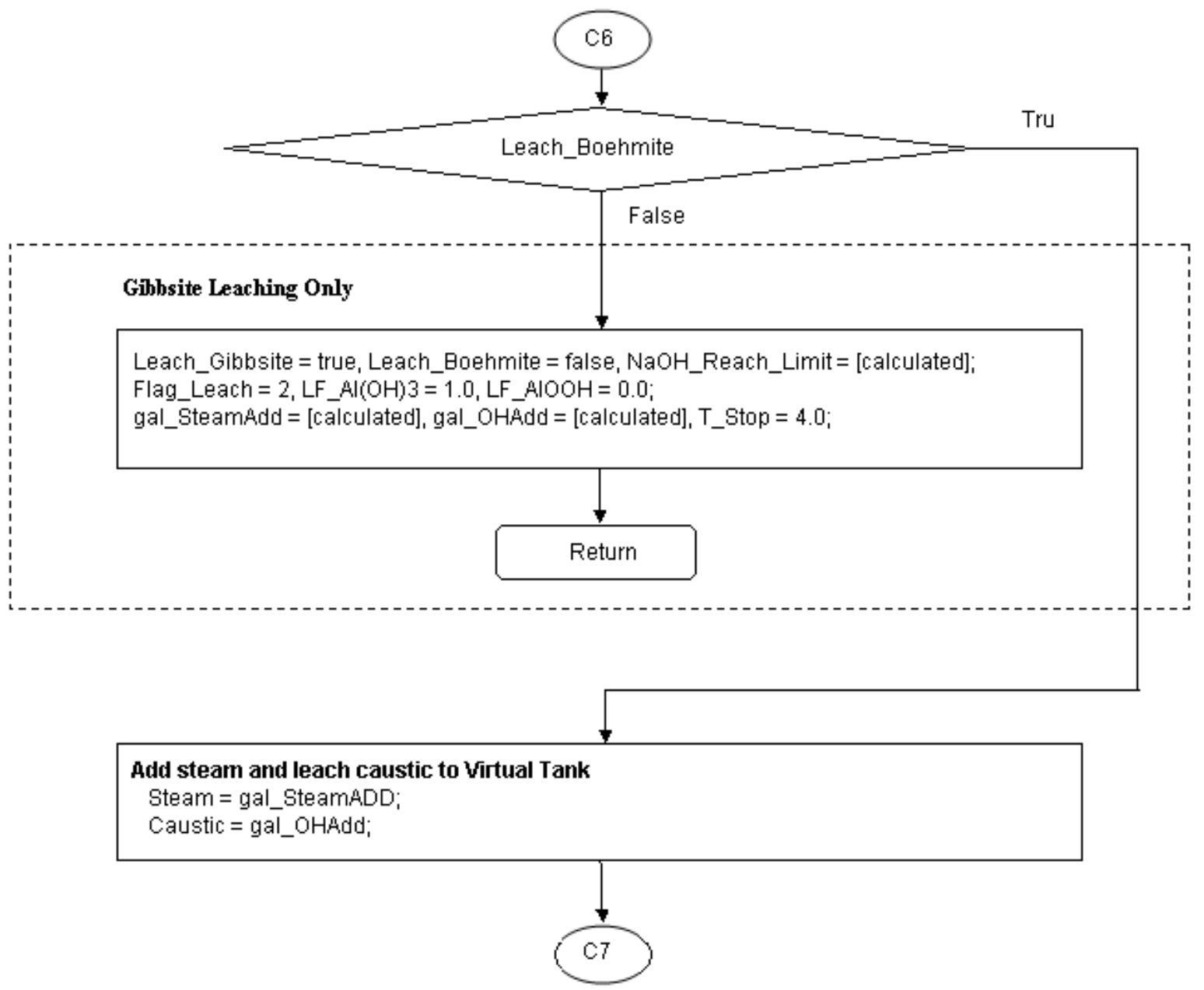

Figure L-8 Boehmite Leaching Decision Logic (Part 8) 


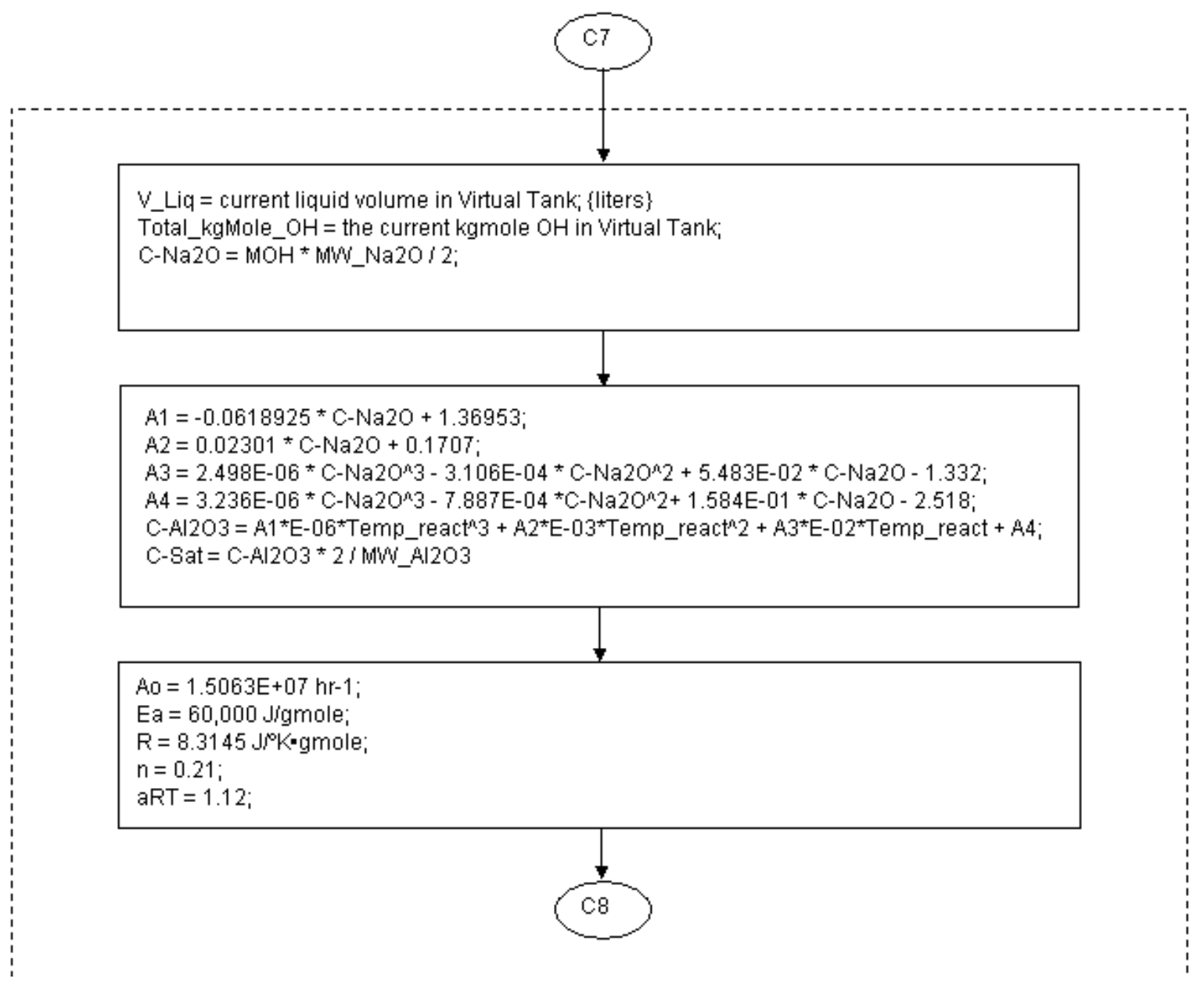

Figure L-9 Boehmite Leaching Decision Logic (Part 9) 


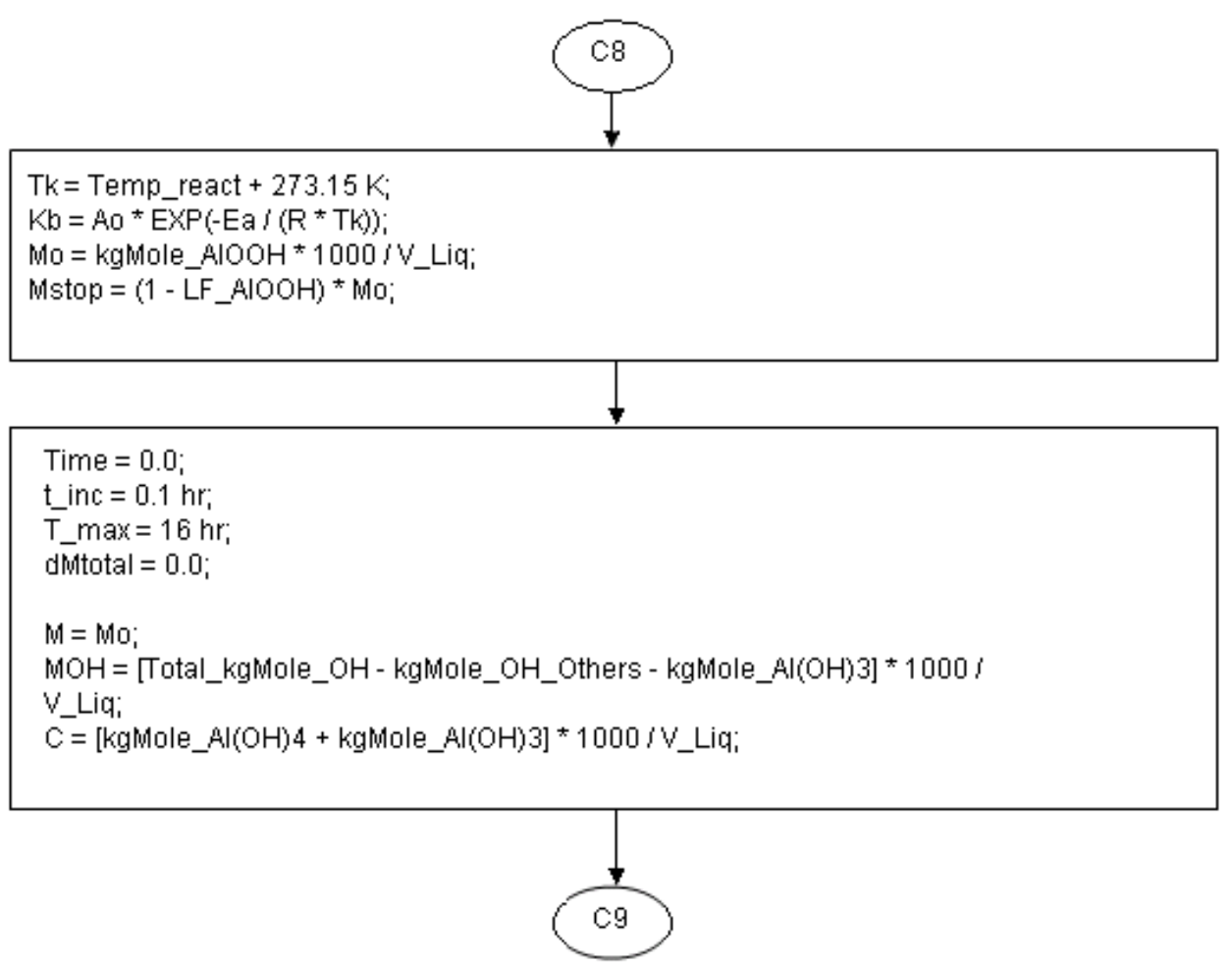

Figure L-10 Boehmite Leaching Decision Logic (Part 10) 


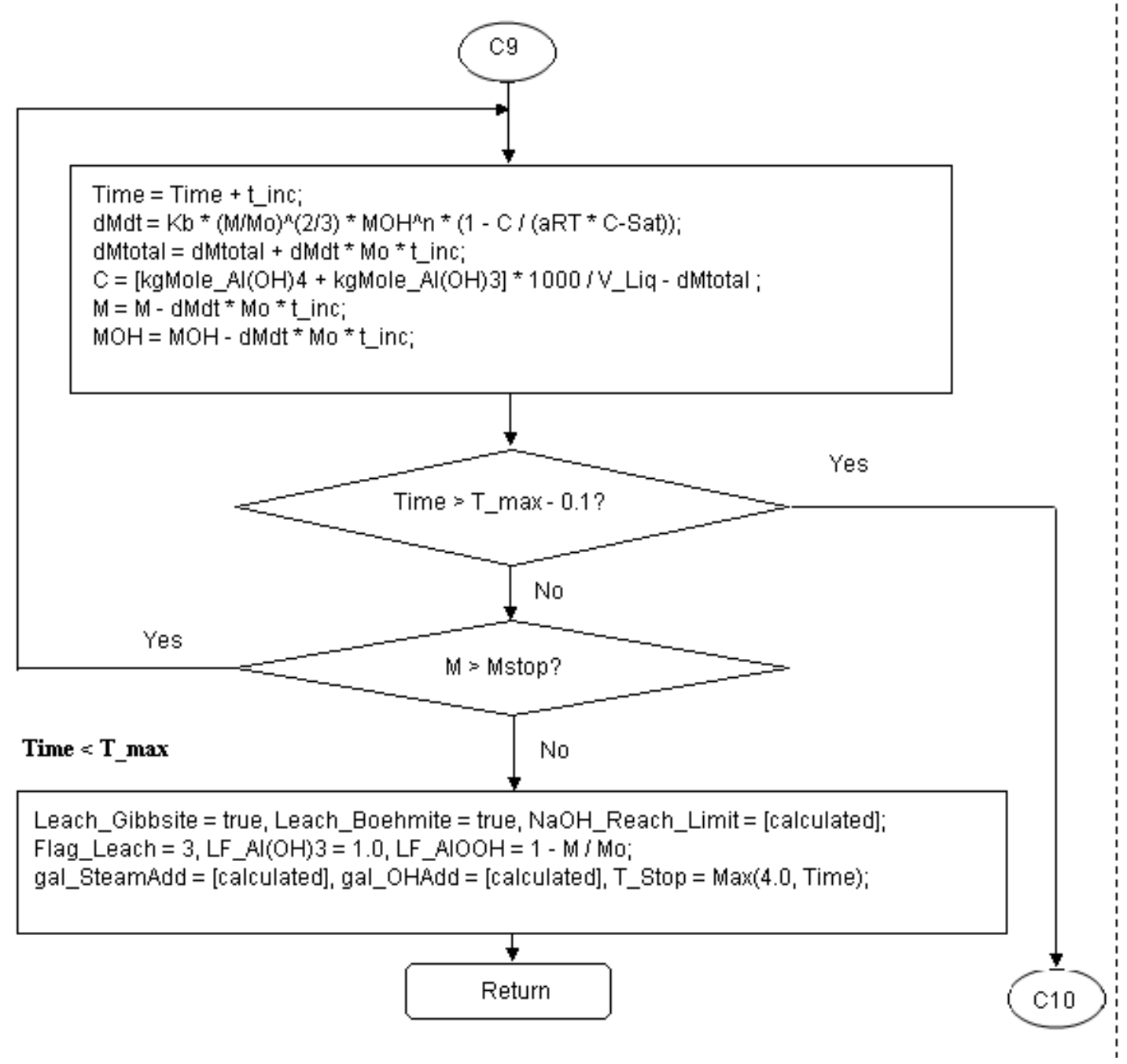

Figure L-11 Boehmite Leaching Decision Logic (Part 11) 


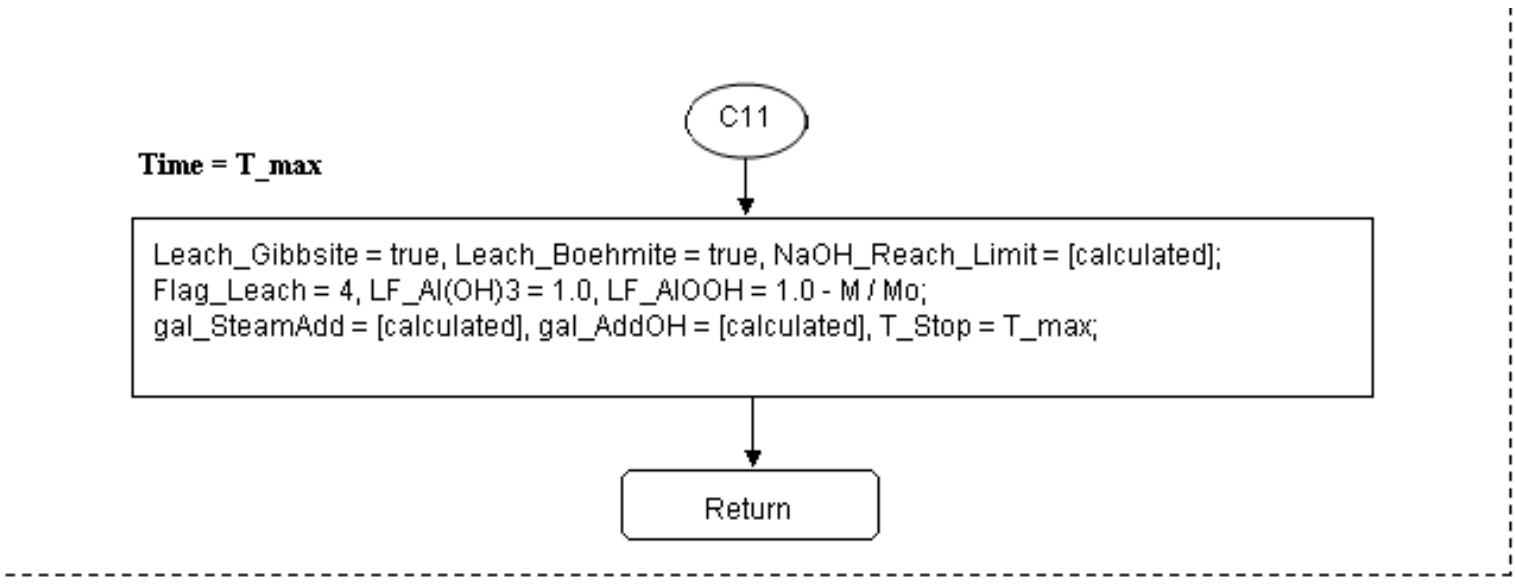

Figure L-12 Boehmite Leaching Decision Logic (Part 12) 


\section{Appendix M}

\section{UFP1 Leaching with Gibbsite}




\section{Appendix M UFP1 Leaching with Gibbsite}

The Dynamic (G2) Model Version 7.0 is designed with the option to perform caustic leach in the UFP1 vessels (upfront leaching) which can be selected by setting the leaching-scenario variable to UFP1 on the Model Run Graphic User Interface. The upfront leaching flowsheet differs from the baseline backend leaching flowsheet primary in where caustic leach reactions are performed, but also in feed blending, recycle handling, and solid processes. Operating logic and parameters applied to the upfront leaching flowsheet are described as follows:

\section{$\underline{\text { Recycle Handling }}$}

When the frontend leaching operations are chosen, HLP-VSL-00027A is configured to only receive, store, and transfer FEP evaporator concentrates. A contingent volume is set between the lower set point (31,196 gallons) and the upper set point ( 85,100 gallons). The vessel can be discharged when its volume is above the lower set point. The vessel can receive and discharge when its volume is within the contingency zone. HLP-VSL-00027A can only be discharged when its volume is above the upper set point. The recycle concentrate in this vessel is used for the feed blending operations described below in this Appendix.

\section{Feed Blending}

Feed blending logic are implemented in the UFP1 vessels to: a) improve the uniformity of ultrafiltration feeds, b) better balance the overall utilization of LAW and HLW feeds, and c) alleviate the pressure of water lock on the recycle loop. Normal feed blending is performed by filling a UFP1 vessel with a predetermined volume of HLW feed then topping it off with recycled concentrate or LAW feed. Other feed blendings include those described below:

1) When a UFP1 vessel becomes "empty", it will receive feeds from upstream sources in the following order: a) HLW feed from HLP-VSL-00022, b) recycle concentrate from HLP-VSL-00027A, or c) LAW feed from FRP-VSL-00002A/B/C/D. It will use the next priority feed if the higher priority feed is not available at the time of request.

2) When HLW feed is delivered to the UFP1 vessel, the mass of gibbsite will be determined. The transfer will stop if $25 \mathrm{~kg}$-moles of $\mathrm{Al}(\mathrm{OH}) 3$ has been accumulated in the vessel which will then be topped off to its set volume with recycle concentrate or LAW feed. The set volume of the UFP1 vessel is 28,300 gallons.

3) The UFP1 vessel will be filled with HLW feed to its set volume if the $25 \mathrm{~kg}$-moles of $\mathrm{Al}(\mathrm{OH}) 3 \mathrm{goal}$ is not reached. When this happens, topping off the vessel with recycle concentrate or LAW feed is not required.

4) If the UFP1 vessel reaches the $25 \mathrm{~kg}$-mole $\mathrm{Al}(\mathrm{OH}) 3$ goal before it fills to the set volume and no recycle concentrate and LAW feed are available at the time, the UFP1 vessel will be set to perform the caustic leach operations.

5) To achieve a better balance for the use of LAW and HLW feeds, a LAW batch will be sent through the UFP system without blending in the beginning of each UFP2 cycle. The LAW feed will be transferred to the available UFP1 vessel to its set volume at 28,300 gallons. 
6) To alleviate the pressure of water lock on the recycle loop, an upper set volume is placed in HLPVSL-00027A at 84,584 gallons, when reached will trigger a discharge, e.g., the next available UFP1 vessel will receive a full batch of recycle concentrate.

Line flush is received in the UFP1 vessel after each batch transfer from HLP-VSL-00022, HLP-VSL00027A, or FRP-VSL-00002A/B/C/D. Caustic leach decisions will be made based on the waste compositions in the vessel.

\section{Caustic Leach}

Generally caustic leach can reduce the HLW glass produced as most of the WTP solids are aluminum limiting. For waste batches that are limited by other Specification 1 species, caustic leach is unnecessary as it increases processing time and add sodium to the plant. A caustic leach decision logic has been developed by the WTP Process Engineering and Technology Department that determines if a waste batch is aluminum limiting and consequently if the caustic leach can reduce HLW glass by $10 \%$ or more. Wash factors as well as the subsequent oxidative leach have also been considered. Figures 5 through 8 in Section 4.8.3 provide a logic diagram that determines if caustic leach is required for the waste batch. The following actions could take place depending on the caustic leach decisions:

1) The feed will be heated, caustic leached, and cooled, or

2) The feed will be adjusted for sodium concentration by dilution and hydroxide concentration by caustic addition.

Caustic leach operations include heating, reaction, and cooling. A pre-determined volume of $19 \mathrm{M} \mathrm{NaOH}$ $\left(\mathrm{Na}=\right.$ waste sodium) will also be added during heating. Vessel contents will be heated to $85{ }^{\circ} \mathrm{C}$ with a steam condensate volume and a heating time determined by Equations L-1 and L-2, respectively.

$\mathrm{V}_{\mathrm{S}}=9,000 *\left(\mathrm{~V}_{\mathrm{W}}+\mathrm{V}_{\mathrm{ACV}}\right) / 39,000$

$\mathrm{T}_{\mathrm{H}}=9 *\left(\mathrm{~V}_{\mathrm{W}}+\mathrm{V}_{\mathrm{ACV}}\right) / 39,000$

Where:

$\mathrm{V}_{\mathrm{S}} \quad=$ Steam condensate volume (gallons)

$\mathrm{V}_{\mathrm{W}} \quad=$ Waste volume before heating (gallons)

$\mathrm{V}_{\mathrm{ACV}} \quad=$ Average caustic volume (gallons)

$\mathrm{T}_{\mathrm{H}} \quad=$ Heating time (hours)

The average caustic volume (2,133 gallons) is obtained by averaging the values for the entire mission from a trial run. The caustic volume for leaching is determined based on Equation 13 with an additional $10 \%$ margin to account for dilution later during wash. The heated solutions are allowed to react for 8 hours during which an additional 400 gallons of steam condensate is added to maintain temperature. Leaching reactions are the same as those discussed in the backend leaching flowsheet in Section 4.8.3. Cooling takes 2 hours. Steam and chill water supply can support parallel heating and parallel cooling operations in the two UFP1 vessels.

For waste batches not requiring caustic leach, water will be added if necessary to lower sodium concentration to $6 \mathrm{M}$. Caustic (19M) will also be added if necessary to keep hydroxide concentration to 
above $0.5 \mathrm{M}$. Vessel contents will be heated to $45^{\circ} \mathrm{C}$ with a steam condensate volume and a heating time determined by Equations 28 and 29, respectively. Solubility is applied at $45^{\circ} \mathrm{C}$ after pre-heating.

\section{$\underline{\text { Solid Processes }}$}

Set volumes of the UFP2 vessels are placed at 19,000 gallons, e.g., solid concentratin is achieved in an 1000 -gallon increment between 18,000 - 19,000 gallons. Solid concentration stops when the $20 \mathrm{wt} \%$ target value is reached. Solid wash, oxidative leach, and oxidative wash processes are the same as those backend leaching flowsheet, but treated solids can only be discharged to HLP-VSL-00027B. 
24590-WTP-MDD-PR-01-002, Rev 12

Dynamic (G2) Model Design Document

\section{Appendix N}

\section{Totalizer Files}




\section{Appendix N Totalizer Files}

The totalizer data provides the cumulative mass in kilograms for every process stream in a 30-day time interval. The process streams are identified by the following convention: "upstream_downstream". The data includes the mass in the liquid phase, solid phase, gas phase, and in the oxide form as appropriate. Table N-1 provides a summary of totalizer files available for Dynamic (G2) Model Version 7.0.

Table N-1 G2 Version 6.0 Totalizer Files

\begin{tabular}{|c|c|c|}
\hline Upstream Equipment & Downstream Equipment & Totalizer File \\
\hline RDP-VSL-00002A & PJM-RFD-VENT & RDP-VSL-00002A PJM-RFD-VENT \\
\hline RDP-VSL-00002A & CXP-IXC-00004 & RDP-VSL-00002A_CXP-IXC-00004 \\
\hline RDP-VSL-00002A & CXP-IXC-00003 & RDP-VSL-00002A_CXP-IXC-00003 \\
\hline RDP-VSL-00002A & CXP-IXC-00002 & RDP-VSL-00002A_CXP-IXC-00002 \\
\hline RDP-VSL-00002A & CXP-IXC-00001 & RDP-VSL-00002A_CXP-IXC-00001 \\
\hline $\begin{array}{l}\text { RDP-VSL-00002A- } \\
\text { CAUSTIC }\end{array}$ & RDP-VSL-00002A & RDP-VSL-00002A-CAUSTIC_RDP-VSL-00002A \\
\hline NAR-MXR-00003 & CRP-VSL-00001 & NAR-MXR-00003_CRP-VSL-00001 \\
\hline SHR-TK-00004 & CRP-VSL-00001 & SHR-TK-00004_CRP-VSL-00001 \\
\hline DIW-TK-00004 & CRP-VSL-00001 & DIW-TK-00004 CRP-VSL-00001 \\
\hline CRP-VSL-00001 & CXP-IXC-00004 & CRP-VSL-00001_CXP-IXC-00004 \\
\hline CRP-VSL-00001 & CXP-IXC-00003 & CRP-VSL-00001_CXP-IXC-00003 \\
\hline CRP-VSL-00001 & CXP-IXC-00002 & CRP-VSL-00001_CXP-IXC-00002 \\
\hline CRP-VSL-00001 & CXP-IXC-00001 & CRP-VSL-00001_CXP-IXC-00001 \\
\hline CRP-VSL-00001 & RLD-VSL-00017A & CRP-VSL-00001_RLD-VSL-00017A \\
\hline CRP-VSL-00001 & RLD-VSL-00017B & CRP-VSL-00001_RLD-VSL-00017B \\
\hline RDP-VSL-00002B & PJM-RFD-VENT & RDP-VSL-00002B_PJM-RFD-VENT \\
\hline RDP-VSL-00002B & FEP-VSL-00017B & RDP-VSL-00002B_FEP-VSL-00017B \\
\hline RDP-VSL-00002B & FEP-VSL-00017A & RDP-VSL-00002B_FEP-VSL-00017A \\
\hline RDP-VSL-00002B & RDP-VSL-00002C & RDP-VSL-00002B_RDP-VSL-00002C \\
\hline RDP-VSL-00002B & RESIN-DISPOSAL & RDP-VSL-00002B_RESIN-DISPOSAL \\
\hline RDP-VSL-00002B & RDP-VSL-00002A & RDP-VSL-00002B_RDP-VSL-00002A \\
\hline RDP-VSL-00002C & PJM-RFD-VENT & RDP-VSL-00002C_PJM-RFD-VENT \\
\hline RDP-VSL-00002C & FEP-VSL-00017B & RDP-VSL-00002C_FEP-VSL-00017B \\
\hline RDP-VSL-00002C & FEP-VSL-00017A & RDP-VSL-00002C_FEP-VSL-00017A \\
\hline RF-RESIN & CRP-VSL-00001 & RF-RESIN_CRP-VSL-00001 \\
\hline $\begin{array}{l}\text { TLP-INTER-AFTER- } \\
\text { CONDENSER-WATER }\end{array}$ & TLP-VSL-00002 & $\begin{array}{l}\text { TLP-INTER-AFTER-CONDENSER-WATER_TLP- } \\
\text { VSL-00002 }\end{array}$ \\
\hline TLP-SEP-00001 & TLP-DMST-00001 & TLP-SEP-00001_TLP-DMST-00001 \\
\hline TLP-SEP-00001 & TCP-VSL-00001 & TLP-SEP-00001_TCP-VSL-00001 \\
\hline CXP-VSL-00026A & PJM-RFD-VENT & CXP-VSL-00026A_PJM-RFD-VENT \\
\hline CXP-VSL-00026A & TLP-SEP-00001 & CXP-VSL-00026A_TLP-SEP-00001 \\
\hline CXP-VSL-00026C & PJM-RFD-VENT & CXP-VSL-00026C_PJM-RFD-VENT \\
\hline CXP-VSL-00026C & TLP-SEP-00001 & CXP-VSL-00026C_TLP-SEP-00001 \\
\hline CXP-VSL-00026B & PJM-RFD-VENT & CXP-VSL-00026B_PJM-RFD-VENT \\
\hline CXP-VSL-00026B & TLP-SEP-00001 & CXP-VSL-00026B_TLP-SEP-00001 \\
\hline TCP-VSL-00001 & PJM-RFD-VENT & TCP-VSL-00001 PJM-RFD-VENT \\
\hline
\end{tabular}




\begin{tabular}{|c|c|c|}
\hline Upstream Equipment & Downstream Equipment & Totalizer File \\
\hline TCP-VSL-00001 & LCP-VSL-00001 & TCP-VSL-00001_LCP-VSL-00001 \\
\hline TCP-VSL-00001 & $\begin{array}{l}\text { SUPPLEMENTAL-LAW- } \\
\text { TREATED }\end{array}$ & $\begin{array}{l}\text { TCP-VSL-00001_SUPPLEMENTAL-LAW- } \\
\text { TREATED }\end{array}$ \\
\hline TCP-VSL-00001 & LCP-VSL-00002 & TCP-VSL-00001_LCP-VSL-00002 \\
\hline TLP-VSL-00002 & RLD-TK-00006A & TLP-VSL-00002_RLD-TK-00006A \\
\hline TLP-DMST-00001 & TLP-VSL-00002 & TLP-DMST-00001 TLP-VSL-00002 \\
\hline TLP-DMST-00001 & PVP-VESSEL-VENT & TLP-DMST-00001_PVP-VESSEL-VENT \\
\hline TLP-DMST-00001-AIR & TLP-DMST-00001 & TLP-DMST-00001-AIR_TLP-DMST-00001 \\
\hline TLP-VSL-00009A & PJM-RFD-VENT & TLP-VSL-00009A_PJM-RFD-VENT \\
\hline TLP-VSL-00009A & TLP-SEP-00001 & TLP-VSL-00009A_TLP-SEP-00001 \\
\hline SHR-TK-00007 & TLP-VSL-00009A & SHR-TK-00007_TLP-VSL-00009A \\
\hline TLP-VSL-00009B & PJM-RFD-VENT & TLP-VSL-00009B_PJM-RFD-VENT \\
\hline TLP-VSL-00009B & TLP-SEP-00001 & TLP-VSL-00009B_TLP-SEP-00001 \\
\hline SHR-TK-00007 & TLP-VSL-00009B & SHR-TK-00007_TLP-VSL-00009B \\
\hline RLD-TK-00006A & HLP-VSL-00027B & RLD-TK-00006A_HLP-VSL-00027B \\
\hline RLD-TK-00006A & HLP-VSL-00027A & RLD-TK-00006A_HLP-VSL-00027A \\
\hline RLD-TK-00006A & HLP-VSL-00028 & RLD-TK-00006A_HLP-VSL-00028 \\
\hline RLD-TK-00006A & PWD-VSL-00033 & RLD-TK-00006A_PWD-VSL-00033 \\
\hline RLD-TK-00006A & PWD-VSL-00043 & RLD-TK-00006A_PWD-VSL-00043 \\
\hline RLD-TK-00006A & TCP-VSL-00001 & RLD-TK-00006A_TCP-VSL-00001 \\
\hline RLD-TK-00006A & RDP-VSL-00002B & RLD-TK-00006A_RDP-VSL-00002B \\
\hline RLD-TK-00006A & TLP-SEP-00001 & RLD-TK-00006A_TLP-SEP-00001 \\
\hline RLD-TK-00006A & TLP-VSL-00009A & RLD-TK-00006A_TLP-VSL-00009A \\
\hline RLD-TK-00006A & TLP-VSL-00009B & RLD-TK-00006A_TLP-VSL-00009B \\
\hline RLD-TK-00006A & $\begin{array}{l}\text { SUPPLEMENTAL-LAW- } \\
\text { RECYCLE }\end{array}$ & $\begin{array}{l}\text { RLD-TK-00006A_SUPPLEMENTAL-LAW- } \\
\text { RECYCLE }\end{array}$ \\
\hline RLD-TK-00006A & CXP-VSL-00026A & RLD-TK-00006A_CXP-VSL-00026A \\
\hline RLD-TK-00006A & CXP-VSL-00026C & RLD-TK-00006A_CXP-VSL-00026C \\
\hline RLD-TK-00006A & CXP-VSL-00026B & RLD-TK-00006A_CXP-VSL-00026B \\
\hline RLD-TK-00006A & UFP-VSL-00062A & RLD-TK-00006A_UFP-VSL-00062A \\
\hline RLD-TK-00006A & UFP-VSL-00002A & RLD-TK-00006A_UFP-VSL-00002A \\
\hline RLD-TK-00006A & UFP-VSL-00001A & RLD-TK-00006A_UFP-VSL-00001A \\
\hline RLD-TK-00006A & UFP-VSL-00002B & RLD-TK-00006A_UFP-VSL-00002B \\
\hline RLD-TK-00006A & UFP-VSL-00001B & RLD-TK-00006A_UFP-VSL-00001B \\
\hline RLD-TK-00006A & FEP-VSL-00017B & RLD-TK-00006A_FEP-VSL-00017B \\
\hline RLD-TK-00006A & FEP-VSL-00017A & RLD-TK-00006A_FEP-VSL-00017A \\
\hline RLD-TK-00006A & FEP-SEP-00001A & RLD-TK-00006A_FEP-SEP-00001A \\
\hline RLD-TK-00006A & FEP-SEP-00001B & RLD-TK-00006A_FEP-SEP-00001B \\
\hline RLD-TK-00006A & FRP-VSL-00002D & RLD-TK-00006A_FRP-VSL-00002D \\
\hline RLD-TK-00006A & FRP-VSL-00002C & RLD-TK-00006A_FRP-VSL-00002C \\
\hline RLD-TK-00006A & FRP-VSL-00002B & RLD-TK-00006A_FRP-VSL-00002B \\
\hline RLD-TK-00006A & FRP-VSL-00002A & RLD-TK-00006A_FRP-VSL-00002A \\
\hline RLD-TK-00006A & HLP-VSL-00022 & RLD-TK-00006A_HLP-VSL-00022 \\
\hline RLD-TK-00006A & RLD-TK-00006B & RLD-TK-00006A_RLD-TK-00006B \\
\hline RLD-TK-00006B & ETF-1 & RLD-TK-00006B_ETF-1 \\
\hline RLD-VSL-00017A & RLD-TK-00006B & RLD-VSL-00017A_RLD-TK-00006B \\
\hline RLD-VSL-00017B & RLD-TK-00006B & RLD-VSL-00017B_RLD-TK-00006B \\
\hline
\end{tabular}




\begin{tabular}{|c|c|c|}
\hline Upstream Equipment & Downstream Equipment & Totalizer File \\
\hline UFP-VSL-00002B & UFP-VSL-00002A & UFP-VSL-00002B_UFP-VSL-00002A \\
\hline UFP-VSL-00002B & PWD-VSL-00015 & UFP-VSL-00002B_PWD-VSL-00015 \\
\hline UFP-VSL-00002B & PWD-VSL-00016 & UFP-VSL-00002B_PWD-VSL-00016 \\
\hline UFP-VSL-00002B & HLP-VSL-00027B & UFP-VSL-00002B_HLP-VSL-00027B \\
\hline UFP-VSL-00002B & HLP-VSL-00027A & UFP-VSL-00002B_HLP-VSL-00027A \\
\hline UFP-VSL-00002B & PJM-RFD-VENT & UFP-VSL-00002B_PJM-RFD-VENT \\
\hline UFP-VSL-00002B & UFP-02B-P1 & UFP-VSL-00002B_UFP-02B-P1 \\
\hline UFP-VSL-00002A & UFP-02A-P1 & UFP-VSL-00002A_UFP-02A-P1 \\
\hline UFP-VSL-00002A & PWD-VSL-00015 & UFP-VSL-00002A_PWD-VSL-00015 \\
\hline UFP-VSL-00002A & PWD-VSL-00016 & UFP-VSL-00002A_PWD-VSL-00016 \\
\hline UFP-VSL-00002A & HLP-VSL-00027B & UFP-VSL-00002A_HLP-VSL-00027B \\
\hline UFP-VSL-00002A & HLP-VSL-00027A & UFP-VSL-00002A_HLP-VSL-00027A \\
\hline UFP-VSL-00002A & PJM-RFD-VENT & UFP-VSL-00002A_PJM-RFD-VENT \\
\hline NAR-TK-00006 & UFP-VSL-00002A & NAR-TK-00006_UFP-VSL-00002A \\
\hline NAR-TK-00006 & UFP-VSL-00002B & NAR-TK-00006_UFP-VSL-00002B \\
\hline SPR-MXR-00001 & UFP-VSL-00001A & SPR-MXR-00001_UFP-VSL-00001A \\
\hline STR-MXR-00001 & UFP-VSL-00001A & STR-MXR-00001_UFP-VSL-00001A \\
\hline SPR-MXR-00001 & UFP-VSL-00001B & SPR-MXR-00001_UFP-VSL-00001B \\
\hline STR-MXR-00001 & UFP-VSL-00001B & STR-MXR-00001_UFP-VSL-00001B \\
\hline UFP-VSL-00062A & CXP-FILT-00001 & UFP-VSL-00062A_CXP-FILT-00001 \\
\hline UFP-VSL-00062A & PJM-RFD-VENT & UFP-VSL-00062A_PJM-RFD-VENT \\
\hline UFP-VSL-00062B & PJM-RFD-VENT & UFP-VSL-00062B_PJM-RFD-VENT \\
\hline UFP-VSL-00001A & PJM-RFD-VENT & UFP-VSL-00001A_PJM-RFD-VENT \\
\hline UFP-VSL-00001A & UFP-VSL-00002A & UFP-VSL-00001A_UFP-VSL-00002A \\
\hline UFP-VSL-00001A & UFP-VSL-00002B & UFP-VSL-00001A_UFP-VSL-00002B \\
\hline UFP-VSL-00001B & PJM-RFD-VENT & UFP-VSL-00001B_PJM-RFD-VENT \\
\hline UFP-VSL-00001B & UFP-VSL-00002B & UFP-VSL-00001B_UFP-VSL-00002B \\
\hline UFP-VSL-00001B & UFP-VSL-00002A & UFP-VSL-00001B_UFP-VSL-00002A \\
\hline STEAM & UFP-VSL-00062A & STEAM_UFP-VSL-00062A \\
\hline STEAM & UFP-VSL-00002A & STEAM_UFP-VSL-00002A \\
\hline STEAM & UFP-VSL-00001A & STEAM_UFP-VSL-00001A \\
\hline STEAM & UFP-VSL-00002B & STEAM_UFP-VSL-00002B \\
\hline STEAM & UFP-VSL-00001B & STEAM_UFP-VSL-00001B \\
\hline UFP-VSL-00062C & PJM-RFD-VENT & UFP-VSL-00062C_PJM-RFD-VENT \\
\hline UFP-02A-P1 & UFP-02A-P2 & UFP-02A-P1_UFP-02A-P2 \\
\hline UFP-02A-P2 & PWD-VSL-00033 & UFP-02A-P2_PWD-VSL-00033 \\
\hline UFP-02A-P2 & UFP-VSL-00002A & UFP-02A-P2_UFP-VSL-00002A \\
\hline UFP-02A-P2 & PWD-VSL-00015 & UFP-02A-P2_PWD-VSL-00015 \\
\hline UFP-02A-P2 & PWD-VSL-00016 & UFP-02A-P2_PWD-VSL-00016 \\
\hline UFP-02A-P2 & UFP-VSL-00062A & UFP-02A-P2_UFP-VSL-00062A \\
\hline UFP-02-P2-NAOH & UFP-02A-P2 & UFP-02-P2-NAOH_UFP-02A-P2 \\
\hline UFP-02B-P1 & UFP-02B-P2 & UFP-02B-P1_UFP-02B-P2 \\
\hline UFP-02B-P2 & PWD-VSL-00033 & UFP-02B-P2_PWD-VSL-00033 \\
\hline UFP-02B-P2 & UFP-VSL-00002B & UFP-02B-P2_UFP-VSL-00002B \\
\hline UFP-02B-P2 & PWD-VSL-00015 & UFP-02B-P2_PWD-VSL-00015 \\
\hline UFP-02B-P2 & PWD-VSL-00016 & UFP-02B-P2_PWD-VSL-00016 \\
\hline UFP-02B-P2 & UFP-VSL-00062A & UFP-02B-P2_UFP-VSL-00062A \\
\hline
\end{tabular}




\begin{tabular}{|c|c|c|}
\hline Upstream Equipment & Downstream Equipment & Totalizer File \\
\hline UFP-02-P2-NAOH & UFP-02B-P2 & UFP-02-P2-NAOH_UFP-02B-P2 \\
\hline SPR-MXR-00001 & UFP-VSL-00002A & SPR-MXR-00001_UFP-VSL-00002A \\
\hline SPR-MXR-00001 & UFP-VSL-00002B & SPR-MXR-00001_UFP-VSL-00002B \\
\hline SHR-TK-00006 & UFP-VSL-00062A & SHR-TK-00006_UFP-VSL-00062A \\
\hline SHR-TK-00006 & UFP-VSL-00002A & SHR-TK-00006_UFP-VSL-00002A \\
\hline SHR-TK-00006 & UFP-VSL-00001A & SHR-TK-00006_UFP-VSL-00001A \\
\hline SHR-TK-00006 & UFP-VSL-00002B & SHR-TK-00006_UFP-VSL-00002B \\
\hline SHR-TK-00006 & UFP-VSL-00001B & SHR-TK-00006_UFP-VSL-00001B \\
\hline $\begin{array}{l}\text { NEUTRALIZATION- } \\
\text { CAUSTIC }\end{array}$ & UFP-VSL-00002A & NEUTRALIZATION-CAUSTIC_UFP-VSL-00002A \\
\hline $\begin{array}{l}\text { NEUTRALIZATION- } \\
\text { CAUSTIC }\end{array}$ & UFP-VSL-00002B & NEUTRALIZATION-CAUSTIC_UFP-VSL-00002B \\
\hline $\begin{array}{l}\text { OXIDATIVE-LEACH- } \\
\text { CAUSTIC }\end{array}$ & UFP-VSL-00002A & $\begin{array}{l}\text { OXIDATIVE-LEACH-CAUSTIC_UFP-VSL- } \\
00002 \mathrm{~A}\end{array}$ \\
\hline $\begin{array}{l}\text { OXIDATIVE-LEACH- } \\
\text { CAUSTIC }\end{array}$ & UFP-VSL-00002B & $\begin{array}{l}\text { OXIDATIVE-LEACH-CAUSTIC_UFP-VSL- } \\
\text { 00002B }\end{array}$ \\
\hline DIW-TK-00001 & RDP-VSL-00002B & DIW-TK-00001_RDP-VSL-00002B \\
\hline DIW-TK-00001 & RDP-VSL-00002A & DIW-TK-00001_RDP-VSL-00002A \\
\hline DIW-TK-00001 & HLP-VSL-00027B & DIW-TK-00001_HLP-VSL-00027B \\
\hline DIW-TK-00001 & HLP-VSL-00027A & DIW-TK-00001_HLP-VSL-00027A \\
\hline DIW-TK-00001 & HLP-VSL-00028 & DIW-TK-00001_HLP-VSL-00028 \\
\hline DIW-TK-00001 & PWD-VSL-00033 & DIW-TK-00001_PWD-VSL-00033 \\
\hline DIW-TK-00001 & PWD-VSL-00043 & DIW-TK-00001_PWD-VSL-00043 \\
\hline DIW-TK-00001 & TCP-VSL-00001 & DIW-TK-00001_TCP-VSL-00001 \\
\hline DIW-TK-00001 & TLP-SEP-00001 & DIW-TK-00001_TLP-SEP-00001 \\
\hline DIW-TK-00001 & TLP-VSL-00009A & DIW-TK-00001_TLP-VSL-00009A \\
\hline DIW-TK-00001 & TLP-VSL-00009B & DIW-TK-00001_TLP-VSL-00009B \\
\hline DIW-TK-00001 & $\begin{array}{l}\text { SUPPLEMENTAL-LAW- } \\
\text { RECYCLE }\end{array}$ & $\begin{array}{l}\text { DIW-TK-00001_SUPPLEMENTAL-LAW- } \\
\text { RECYCLE }\end{array}$ \\
\hline DIW-TK-00001 & CXP-VSL-00026A & DIW-TK-00001_CXP-VSL-00026A \\
\hline DIW-TK-00001 & CXP-VSL-00026C & DIW-TK-00001_CXP-VSL-00026C \\
\hline DIW-TK-00001 & CXP-VSL-00026B & DIW-TK-00001_CXP-VSL-00026B \\
\hline DIW-TK-00001 & CXP-IXC-00004 & DIW-TK-00001_CXP-IXC-00004 \\
\hline DIW-TK-00001 & CXP-IXC-00003 & DIW-TK-00001_CXP-IXC-00003 \\
\hline DIW-TK-00001 & CXP-IXC-00002 & DIW-TK-00001_CXP-IXC-00002 \\
\hline DIW-TK-00001 & CXP-IXC-00001 & DIW-TK-00001_CXP-IXC-00001 \\
\hline DIW-TK-00001 & UFP-VSL-00062A & DIW-TK-00001_UFP-VSL-00062A \\
\hline DIW-TK-00001 & UFP-VSL-00002A & DIW-TK-00001_UFP-VSL-00002A \\
\hline DIW-TK-00001 & UFP-VSL-00001A & DIW-TK-00001_UFP-VSL-00001A \\
\hline DIW-TK-00001 & UFP-VSL-00002B & DIW-TK-00001_UFP-VSL-00002B \\
\hline DIW-TK-00001 & UFP-VSL-00001B & DIW-TK-00001_UFP-VSL-00001B \\
\hline DIW-TK-00001 & FEP-VSL-00017B & DIW-TK-00001_FEP-VSL-00017B \\
\hline DIW-TK-00001 & FEP-VSL-00017A & DIW-TK-00001_FEP-VSL-00017A \\
\hline DIW-TK-00001 & FEP-SEP-00001A & DIW-TK-00001_FEP-SEP-00001A \\
\hline DIW-TK-00001 & FEP-SEP-00001B & DIW-TK-00001_FEP-SEP-00001B \\
\hline DIW-TK-00001 & FRP-VSL-00002D & DIW-TK-00001_FRP-VSL-00002D \\
\hline DIW-TK-00001 & FRP-VSL-00002C & DIW-TK-00001_FRP-VSL-00002C \\
\hline DIW-TK-00001 & FRP-VSL-00002B & DIW-TK-00001_FRP-VSL-00002B \\
\hline
\end{tabular}




\begin{tabular}{|c|c|c|}
\hline Upstream Equipment & Downstream Equipment & Totalizer File \\
\hline DIW-TK-00001 & FRP-VSL-00002A & DIW-TK-00001_FRP-VSL-00002A \\
\hline DIW-TK-00001 & HLP-VSL-00022 & DIW-TK-00001_HLP-VSL-00022 \\
\hline DIW-TK-00001 & HDH-VSL-00003 & DIW-TK-00001_HDH-VSL-00003 \\
\hline DIW-TK-00001 & LCP-VSL-00002 & DIW-TK-00001_LCP-VSL-00002 \\
\hline DIW-TK-00001 & LCP-VSL-00001 & DIW-TK-00001_LCP-VSL-00001 \\
\hline DIW-TK-00001 & LFP-VSL-00003 & DIW-TK-00001_LFP-VSL-00003 \\
\hline DIW-TK-00001 & LFP-VSL-00001 & DIW-TK-00001_LFP-VSL-00001 \\
\hline DIW-TK-00001 & LFP-VSL-00004 & DIW-TK-00001_LFP-VSL-00004 \\
\hline DIW-TK-00001 & LFP-VSL-00002 & DIW-TK-00001_LFP-VSL-00002 \\
\hline DIW-TK-00001 & LMP-MLTR-00002 & DIW-TK-00001_LMP-MLTR-00002 \\
\hline DIW-TK-00001 & LMP-MLTR-00001 & DIW-TK-00001_LMP-MLTR-00001 \\
\hline DIW-TK-00001 & RLD-VSL-00007 & DIW-TK-00001_RLD-VSL-00007 \\
\hline DIW-TK-00001 & HFP-VSL-00005 & DIW-TK-00001_HFP-VSL-00005 \\
\hline DIW-TK-00001 & HFP-VSL-00001 & DIW-TK-00001_HFP-VSL-00001 \\
\hline DIW-TK-00001 & HFP-VSL-00006 & DIW-TK-00001_HFP-VSL-00006 \\
\hline DIW-TK-00001 & HFP-VSL-00002 & DIW-TK-00001_HFP-VSL-00002 \\
\hline INHIBITED-WATER & PWD-VSL-00033 & INHIBITED-WATER_PWD-VSL-00033 \\
\hline INHIBITED-WATER & PWD-VSL-00043 & INHIBITED-WATER_PWD-VSL-00043 \\
\hline INHIBITED-WATER & UFP-02A-P2 & INHIBITED-WATER_UFP-02A-P2 \\
\hline INHIBITED-WATER & UFP-02B-P2 & INHIBITED-WATER_UFP-02B-P2 \\
\hline INHIBITED-WATER & UFP-02A-P1 & INHIBITED-WATER_UFP-02A-P1 \\
\hline INHIBITED-WATER & UFP-02B-P1 & INHIBITED-WATER_UFP-02B-P1 \\
\hline INHIBITED-WATER & UFP-VSL-00002B & INHIBITED-WATER_UFP-VSL-00002B \\
\hline INHIBITED-WATER & UFP-VSL-00002A & INHIBITED-WATER_UFP-VSL-00002A \\
\hline INHIBITED-WATER & HLP-VSL-00027B & INHIBITED-WATER_HLP-VSL-00027B \\
\hline INHIBITED-WATER & HLP-VSL-00027A & INHIBITED-WATER_HLP-VSL-00027A \\
\hline INHIBITED-WATER & HLP-VSL-00028 & INHIBITED-WATER_HLP-VSL-00028 \\
\hline INHIBITED-WATER & FRP-VSL-00002D & INHIBITED-WATER_FRP-VSL-00002D \\
\hline INHIBITED-WATER & FRP-VSL-00002C & INHIBITED-WATER_FRP-VSL-00002C \\
\hline INHIBITED-WATER & FRP-VSL-00002B & INHIBITED-WATER_FRP-VSL-00002B \\
\hline INHIBITED-WATER & FRP-VSL-00002A & INHIBITED-WATER_FRP-VSL-00002A \\
\hline INHIBITED-WATER & HLP-VSL-00022 & INHIBITED-WATER_HLP-VSL-00022 \\
\hline ANTIFOAM-AGENT & TLP-SEP-00001 & ANTIFOAM-AGENT_TLP-SEP-00001 \\
\hline ANTIFOAM-AGENT & HLP-VSL-00027B & ANTIFOAM-AGENT_HLP-VSL-00027B \\
\hline ANTIFOAM-AGENT & HLP-VSL-00027A & ANTIFOAM-AGENT_HLP-VSL-00027A \\
\hline ANTIFOAM-AGENT & HLP-VSL-00028 & ANTIFOAM-AGENT_HLP-VSL-00028 \\
\hline ANTIFOAM-AGENT & UFP-VSL-00002A & ANTIFOAM-AGENT_UFP-VSL-00002A \\
\hline ANTIFOAM-AGENT & UFP-VSL-00002B & ANTIFOAM-AGENT_UFP-VSL-00002B \\
\hline ANTIFOAM-AGENT & UFP-VSL-00001A & ANTIFOAM-AGENT_UFP-VSL-00001A \\
\hline ANTIFOAM-AGENT & UFP-VSL-00001B & ANTIFOAM-AGENT_UFP-VSL-00001B \\
\hline ANTIFOAM-AGENT & FEP-SEP-00001B & ANTIFOAM-AGENT_FEP-SEP-00001B \\
\hline ANTIFOAM-AGENT & FEP-SEP-00001A & ANTIFOAM-AGENT_FEP-SEP-00001A \\
\hline LFP-VSL-00003-MIXER & LFP-VSL-00003 & LFP-VSL-00003-MIXER_LFP-VSL-00003 \\
\hline RUTILE & $\begin{array}{l}\text { SYSTEM-500-WEIGH- } \\
\text { HOPPER }\end{array}$ & RUTILE_SYSTEM-500-W̄WEIGH-HOPPER \\
\hline OLIVINE & $\begin{array}{l}\text { SYSTEM-500-WEIGH- } \\
\text { HOPPER }\end{array}$ & OLIVINE_SYSTEM-500-WEIGH-HOPPER \\
\hline
\end{tabular}




\begin{tabular}{|c|c|c|}
\hline Upstream Equipment & Downstream Equipment & Totalizer File \\
\hline ZIRCON-SAND & $\begin{array}{l}\text { SYSTEM-500-WEIGH- } \\
\text { HOPPER }\end{array}$ & ZIRCON-SAND_SYSTEM-500-WEIGH-HOPPER \\
\hline IRON-OXIDE & $\begin{array}{l}\text { SYSTEM-500-WEIGH- } \\
\text { HOPPER }\end{array}$ & IRON-OXIDE_SYSTEM-500-WEIGH-HOPPER \\
\hline SILICA & $\begin{array}{l}\text { SYSTEM-100-WEIGH- } \\
\text { HOPPER }\end{array}$ & SILICA_SYSTEM-100-WEIGH-HOPPER \\
\hline WOLLASTONITE & $\begin{array}{l}\text { SYSTEM-200-WEIGH- } \\
\text { HOPPER }\end{array}$ & $\begin{array}{l}\text { WOLLASTONITE_SYSTEM-200-WEIGH- } \\
\text { HOPPER }\end{array}$ \\
\hline KYANITE & $\begin{array}{l}\text { SYSTEM-200-WEIGH- } \\
\text { HOPPER }\end{array}$ & KYANITE_SYSTEM-200-WEIGH-HOPPER \\
\hline BORIC-ACID & $\begin{array}{l}\text { SYSTEM-200-WEIGH- } \\
\text { HOPPER }\end{array}$ & BORIC-ACID_SYSTEM-200-WEIGH-HOPPER \\
\hline SUGAR & $\begin{array}{l}\text { SYSTEM-200-WEIGH- } \\
\text { HOPPER }\end{array}$ & SUGAR_SYSTEM-200-WEIGH-HOPPER \\
\hline $\begin{array}{l}\text { SYSTEM-100-WEIGH- } \\
\text { HOPPER }\end{array}$ & $\begin{array}{l}\text { SYSTEM-100- } \\
\text { TRANSPORTER }\end{array}$ & $\begin{array}{l}\text { SYSTEM-100-WEIGH-HOPPER_SYSTEM-100- } \\
\text { TRANSPORTER }\end{array}$ \\
\hline $\begin{array}{l}\text { SYSTEM-500-WEIGH- } \\
\text { HOPPER }\end{array}$ & $\begin{array}{l}\text { SYSTEM-500- } \\
\text { TRANSPORTER }\end{array}$ & $\begin{array}{l}\text { SYSTEM-500-WEIGH-HOPPER_SYSTEM-500- } \\
\text { TRANSPORTER }\end{array}$ \\
\hline $\begin{array}{l}\text { SYSTEM-200-WEIGH- } \\
\text { HOPPER }\end{array}$ & $\begin{array}{l}\text { SYSTEM-200- } \\
\text { TRANSPORTER }\end{array}$ & $\begin{array}{l}\text { SYSTEM-200-WEIGH-HOPPER_SYSTEM-200- } \\
\text { TRANSPORTER }\end{array}$ \\
\hline $\begin{array}{l}\text { SYSTEM-500- } \\
\text { TRANSPORTER }\end{array}$ & LAW-BLENDING-SILO & $\begin{array}{l}\text { SYSTEM-500-TRANSPORTER_LAW- } \\
\text { BLENDING-SILO }\end{array}$ \\
\hline $\begin{array}{l}\text { SYSTEM-500- } \\
\text { TRANSPORTER }\end{array}$ & HLW-BLENDING-SILO & $\begin{array}{l}\text { SYSTEM-500-TRANSPORTER_HLW- } \\
\text { BLENDING-SILO }\end{array}$ \\
\hline $\begin{array}{l}\text { SYSTEM-100- } \\
\text { TRANSPORTER }\end{array}$ & LAW-BLENDING-SILO & $\begin{array}{l}\text { SYSTEM-100-TRANSPORTER_LAW- } \\
\text { BLENDING-SILO }\end{array}$ \\
\hline $\begin{array}{l}\text { SYSTEM-100- } \\
\text { TRANSPORTER }\end{array}$ & HLW-BLENDING-SILO & $\begin{array}{l}\text { SYSTEM-100-TRANSPORTER_HLW- } \\
\text { BLENDING-SILO }\end{array}$ \\
\hline $\begin{array}{l}\text { SYSTEM-200- } \\
\text { TRANSPORTER }\end{array}$ & LAW-BLENDING-SILO & $\begin{array}{l}\text { SYSTEM-200-TRANSPORTER_LAW- } \\
\text { BLENDING-SILO }\end{array}$ \\
\hline $\begin{array}{l}\text { SYSTEM-200- } \\
\text { TRANSPORTER }\end{array}$ & HLW-BLENDING-SILO & $\begin{array}{l}\text { SYSTEM-200-TRANSPORTER_HLW- } \\
\text { BLENDING-SILO }\end{array}$ \\
\hline HLW-BLENDING-SILO & HLW-TRANSPORTER & HLW-BLENDING-SILO_HLW-TRANSPORTER \\
\hline LAW-BLENDING-SILO & LAW-TRANSPORTER & LAW-BLENDING-SILO_LAW-TRANSPORTER \\
\hline HLW-TRANSPORTER & HFP-VSL-00005-MIXER & HLW-TRANSPORTER_HFP-VSL-00005-MIXER \\
\hline HLW-TRANSPORTER & HFP-VSL-00001-MIXER & HLW-TRANSPORTER_HFP-VSL-00001-MIXER \\
\hline LITHIUM-CARBONATE & $\begin{array}{l}\text { SYSTEM-300-WEIGH- } \\
\text { HOPPER }\end{array}$ & $\begin{array}{l}\text { LITHIUM-CARBONATE_SYSTEM-300-WEIGH- } \\
\text { HOPPER }\end{array}$ \\
\hline ZINC-OXIDE & $\begin{array}{l}\text { SYSTEM-300-WEIGH- } \\
\text { HOPPER }\end{array}$ & ZINC-OXIDE_SYSTEM-300-WEIGH-HOPPER \\
\hline SODA-ASH & $\begin{array}{l}\text { SYSTEM-400-WEIGH- } \\
\text { HOPPER }\end{array}$ & SODA-ASH_SYSTEM-400-WEIGH-HOPPER \\
\hline BORAX & $\begin{array}{l}\text { SYSTEM-400-WEIGH- } \\
\text { HOPPER }\end{array}$ & BORAX_SYSTEM-400-WEIGH-HOPPER \\
\hline $\begin{array}{l}\text { SYSTEM-300-WEIGH- } \\
\text { HOPPER }\end{array}$ & $\begin{array}{l}\text { SYSTEM-300- } \\
\text { TRANSPORTER }\end{array}$ & $\begin{array}{l}\text { SYSTEM-300-WEIGH-HOPPER_SYSTEM-300- } \\
\text { TRANSPORTER }\end{array}$ \\
\hline $\begin{array}{l}\text { SYSTEM-400-WEIGH- } \\
\text { HOPPER }\end{array}$ & $\begin{array}{l}\text { SYSTEM-400- } \\
\text { TRANSPORTER }\end{array}$ & $\begin{array}{l}\text { SYSTEM-400-WEIGH-HOPPER_SYSTEM-400- } \\
\text { TRANSPORTER }\end{array}$ \\
\hline LAW-TRANSPORTER & LFP-VSL-00003-MIXER & LAW-TRANSPORTER_LFP-VSL-00003-MIXER \\
\hline LAW-TRANSPORTER & LFP-VSL-00001-MIXER & LAW-TRANSPORTER_LFP-VSL-00001-MIXER \\
\hline $\begin{array}{l}\text { SYSTEM-300- } \\
\text { TRANSPORTER }\end{array}$ & LAW-BLENDING-SILO & $\begin{array}{l}\text { SYSTEM-300-TRANSPORTER_LAW- } \\
\text { BLENDING-SILO }\end{array}$ \\
\hline
\end{tabular}




\begin{tabular}{|c|c|c|}
\hline Upstream Equipment & Downstream Equipment & Totalizer File \\
\hline $\begin{array}{l}\text { SYSTEM-300- } \\
\text { TRANSPORTER }\end{array}$ & HLW-BLENDING-SILO & $\begin{array}{l}\text { SYSTEM-300-TRANSPORTER_HLW- } \\
\text { BLENDING-SILO }\end{array}$ \\
\hline $\begin{array}{l}\text { SYSTEM-400- } \\
\text { TRANSPORTER }\end{array}$ & LAW-BLENDING-SILO & $\begin{array}{l}\text { SYSTEM-400-TRANSPORTER_LAW- } \\
\text { BLENDING-SILO }\end{array}$ \\
\hline $\begin{array}{l}\text { SYSTEM-400- } \\
\text { TRANSPORTER }\end{array}$ & HLW-BLENDING-SILO & $\begin{array}{l}\text { SYSTEM-400-TRANSPORTER_HLW- } \\
\text { BLENDING-SILO }\end{array}$ \\
\hline DUSTING-WATER & HFP-VSL-00001-MIXER & DUSTING-WATER_HFP-VSL-00001-MIXER \\
\hline DUSTING-WATER & HFP-VSL-00005-MIXER & DUSTING-WATER_HFP-VSL-00005-MIXER \\
\hline DUSTING-WATER & LFP-VSL-00003-MIXER & DUSTING-WATER_LFP-VSL-00003-MIXER \\
\hline DUSTING-WATER & LFP-VSL-00001-MIXER & DUSTING-WATER_LFP-VSL-00001-MIXER \\
\hline HFP-VSL-00001-MIXER & HFP-VSL-00001 & HFP-VSL-00001-MIXER_HFP-VSL-00001 \\
\hline HFP-VSL-00005-MIXER & HFP-VSL-00005 & HFP-VSL-00005-MIXER_HFP-VSL-00005 \\
\hline LFP-VSL-00001-MIXER & LFP-VSL-00001 & LFP-VSL-00001-MIXER_LFP-VSL-00001 \\
\hline LCP-VSL-00001 & $\begin{array}{l}\text { SUPPLEMENTAL-LAW- } \\
\text { TREATED }\end{array}$ & $\begin{array}{l}\text { LCP-VSL-00001_SUPPLEMENTAL-LAW- } \\
\text { TREATED }\end{array}$ \\
\hline LCP-VSL-00001 & LFP-VSL-00001 & LCP-VSL-00001_LFP-VSL-00001 \\
\hline LCP-VSL-00001 & LFP-VSL-00003 & LCP-VSL-00001_LFP-VSL-00003 \\
\hline LCP-VSL-00002 & $\begin{array}{l}\text { SUPPLEMENTAL-LAW- } \\
\text { TREATED }\end{array}$ & $\begin{array}{l}\text { LCP-VSL-00002_SUPPLEMENTAL-LAW- } \\
\text { TREATED }\end{array}$ \\
\hline LCP-VSL-00002 & LFP-VSL-00001 & LCP-VSL-00002_LFP-VSL-00001 \\
\hline LCP-VSL-00002 & LFP-VSL-00003 & LCP-VSL-00002_LFP-VSL-00003 \\
\hline LFP-VSL-00003 & LFP-VSL-00004 & LFP-VSL-00003_LFP-VSL-00004 \\
\hline LFP-VSL-00002 & LMP-MLTR-00001 & LFP-VSL-00002_LMP-MLTR-00001 \\
\hline LFP-VSL-00004 & LMP-MLTR-00002 & LFP-VSL-00004_LMP-MLTR-00002 \\
\hline LFP-VSL-00001 & LFP-VSL-00002 & LFP-VSL-00001_LFP-VSL-00002 \\
\hline RHEOLOGY-WATER & LFP-VSL-00001 & RHEOLOGY-WATER_LFP-VSL-00001 \\
\hline RHEOLOGY-WATER & LFP-VSL-00003 & RHEOLOGY-WATER_LFP-VSL-00003 \\
\hline HLP-VSL-00027B & PJM-RFD-VENT & HLP-VSL-00027B_PJM-RFD-VENT \\
\hline HLP-VSL-00027B & HLP-VSL-00028 & HLP-VSL-00027B_HLP-VSL-00028 \\
\hline HLP-VSL-00027A & PJM-RFD-VENT & HLP-VSL-00027A_PJM-RFD-VENT \\
\hline HLP-VSL-00027A & HLP-VSL-00028 & HLP-VSL-00027A_HLP-VSL-00028 \\
\hline HLP-VSL-00027A & UFP-VSL-00001A & HLP-VSL-00027A_UFP-VSL-00001A \\
\hline HLP-VSL-00027A & UFP-VSL-00001B & HLP-VSL-00027A_UFP-VSL-00001B \\
\hline HLP-VSL-00028 & PJM-RFD-VENT & HLP-VSL-00028_PJM-RFD-VENT \\
\hline HLP-VSL-00028 & HFP-VSL-00005 & HLP-VSL-00028_HFP-VSL-00005 \\
\hline HLP-VSL-00028 & HFP-VSL-00001 & HLP-VSL-00028_HFP-VSL-00001 \\
\hline HLP14 & HLP-VSL-00028 & HLP14_HLP-VSL-00028 \\
\hline HLP14 & HLP-VSL-00027B & HLP14_HLP-VSL-00027B \\
\hline FRP-VSL-00002A & PJM-RFD-VENT & FRP-VSL-00002A_PJM-RFD-VENT \\
\hline FRP-VSL-00002A & HLP-VSL-00022 & FRP-VSL-00002A_HLP-VSL-00022 \\
\hline FRP-VSL-00002A & UFP-VSL-00001A & FRP-VSL-00002A_UFP-VSL-00001A \\
\hline FRP-VSL-00002A & UFP-VSL-00001B & FRP-VSL-00002A_UFP-VSL-00001B \\
\hline FRP-VSL-00002A & FEP-VSL-00017B & FRP-VSL-00002A_FEP-VSL-00017B \\
\hline FRP-VSL-00002A & FEP-VSL-00017A & FRP-VSL-00002A_FEP-VSL-00017A \\
\hline FRP-VSL-00002B & PJM-RFD-VENT & FRP-VSL-00002B_PJM-RFD-VENT \\
\hline FRP-VSL-00002B & HLP-VSL-00022 & FRP-VSL-00002B_HLP-VSL-00022 \\
\hline FRP-VSL-00002B & UFP-VSL-00001A & FRP-VSL-00002B_UFP-VSL-00001A \\
\hline FRP-VSL-00002B & UFP-VSL-00001B & FRP-VSL-00002B_UFP-VSL-00001B \\
\hline
\end{tabular}




\begin{tabular}{|c|c|c|}
\hline Upstream Equipment & Downstream Equipment & Totalizer File \\
\hline FRP-VSL-00002B & FEP-VSL-00017B & FRP-VSL-00002B_FEP-VSL-00017B \\
\hline FRP-VSL-00002B & FEP-VSL-00017A & FRP-VSL-00002B_FEP-VSL-00017A \\
\hline FRP-VSL-00002D & PJM-RFD-VENT & FRP-VSL-00002D_PJM-RFD-VENT \\
\hline FRP-VSL-00002D & HLP-VSL-00022 & FRP-VSL-00002D_HLP-VSL-00022 \\
\hline FRP-VSL-00002D & UFP-VSL-00001A & FRP-VSL-00002D_UFP-VSL-00001A \\
\hline FRP-VSL-00002D & UFP-VSL-00001B & FRP-VSL-00002D_UFP-VSL-00001B \\
\hline FRP-VSL-00002D & FEP-VSL-00017B & FRP-VSL-00002D_FEP-VSL-00017B \\
\hline FRP-VSL-00002D & FEP-VSL-00017A & FRP-VSL-00002D_FEP-VSL-00017A \\
\hline FRP-VSL-00002C & PJM-RFD-VENT & FRP-VSL-00002C_PJM-RFD-VENT \\
\hline FRP-VSL-00002C & HLP-VSL-00022 & FRP-VSL-00002C_HLP-VSL-00022 \\
\hline FRP-VSL-00002C & UFP-VSL-00001A & FRP-VSL-00002C_UFP-VSL-00001A \\
\hline FRP-VSL-00002C & UFP-VSL-00001B & FRP-VSL-00002C_UFP-VSL-00001B \\
\hline FRP-VSL-00002C & FEP-VSL-00017B & FRP-VSL-00002C_FEP-VSL-00017B \\
\hline FRP-VSL-00002C & FEP-VSL-00017A & FRP-VSL-00002C_FEP-VSL-00017A \\
\hline TANK-FARM-LAW & FRP-VSL-00002D & TANK-FARM-LAW_FRP-VSL-00002D \\
\hline TANK-FARM-LAW & FRP-VSL-00002C & TANK-FARM-LAW_FRP-VSL-00002C \\
\hline TANK-FARM-LAW & FRP-VSL-00002B & TANK-FARM-LAW_FRP-VSL-00002B \\
\hline TANK-FARM-LAW & FRP-VSL-00002A & TANK-FARM-LAW_FRP-VSL-00002A \\
\hline HLP-VSL-00022 & PJM-RFD-VENT & HLP-VSL-00022_PJM-RFD-VENT \\
\hline HLP-VSL-00022 & UFP-VSL-00001A & HLP-VSL-00022_UFP-VSL-00001A \\
\hline HLP-VSL-00022 & UFP-VSL-00001B & HLP-VSL-00022_UFP-VSL-00001B \\
\hline TANK-FARM-HLW & HLP-VSL-00022 & TANK-FARM-HLW_HLP-VSL-00022 \\
\hline CNP-VSL-00004 & PJM-RFD-VENT & CNP-VSL-00004_PJM-RFD-VENT \\
\hline CNP-VSL-00004 & CXP-IXC-00004 & CNP-VSL-00004_CXP-IXC-00004 \\
\hline CNP-VSL-00004 & CXP-IXC-00003 & CNP-VSL-00004_CXP-IXC-00003 \\
\hline CNP-VSL-00004 & CXP-IXC-00002 & CNP-VSL-00004_CXP-IXC-00002 \\
\hline CNP-VSL-00004 & CXP-IXC-00001 & CNP-VSL-00004_CXP-IXC-00001 \\
\hline CNP-VSL-00004 & CNP-EVAP-00001 & CNP-VSL-00004_CNP-EVAP-00001 \\
\hline CNP-EVAP-00001 & CNP-VSL-00004 & CNP-EVAP-00001_CNP-VSL-00004 \\
\hline CNP-EVAP-00001 & PWD-VSL-00015 & CNP-EVAP-00001_PWD-VSL-00015 \\
\hline CNP-EVAP-00001 & PWD-VSL-00016 & CNP-EVAP-00001_PWD-VSL-00016 \\
\hline CNP-EVAP-00001 & HLP-VSL-00028 & CNP-EVAP-00001_HLP-VSL-00028 \\
\hline CNP-EVAP-00001 & HLP-VSL-00027B & CNP-EVAP-00001_HLP-VSL-00027B \\
\hline CNP-EVAP-00001 & CNP-VSL-00003 & CNP-EVAP-00001_CNP-VSL-00003 \\
\hline NAR-TK-00006 & CNP-VSL-00004 & NAR-TK-00006_CNP-VSL-00004 \\
\hline CNP-VSL-00003 & PJM-RFD-VENT & CNP-VSL-00003_PJM-RFD-VENT \\
\hline CNP-VSL-00003 & HLP-VSL-00028 & CNP-VSL-00003_HLP-VSL-00028 \\
\hline CNP-VSL-00003 & HLP-VSL-00027B & CNP-VSL-00003_HLP-VSL-00027B \\
\hline NAR-TK-00005 & CNP-EVAP-00001 & NAR-TK-00005_CNP-EVAP-00001 \\
\hline PWD-VSL-00015 & PJM-RFD-VENT & PWD-VSL-00015_PJM-RFD-VENT \\
\hline PWD-VSL-00015 & FEP-VSL-00017B & PWD-VSL-00015_FEP-VSL-00017B \\
\hline PWD-VSL-00015 & FEP-VSL-00017A & PWD-VSL-00015_FEP-VSL-00017A \\
\hline PWD-VSL-00016 & PJM-RFD-VENT & PWD-VSL-00016_PJM-RFD-VENT \\
\hline PWD-VSL-00016 & FEP-VSL-00017B & PWD-VSL-00016_FEP-VSL-00017B \\
\hline PWD-VSL-00016 & FEP-VSL-00017A & PWD-VSL-00016_FEP-VSL-00017A \\
\hline PWD-VSL-00044 & PWD-VSL-00016 & PWD-VSL-00044_PWD-VSL-00016 \\
\hline PWD-VSL-00044 & PJM-RFD-VENT & PWD-VSL-00044_PJM-RFD-VENT \\
\hline
\end{tabular}




\begin{tabular}{|c|c|c|}
\hline Upstream Equipment & Downstream Equipment & Totalizer File \\
\hline PWD-VSL-00044 & FEP-VSL-00017B & PWD-VSL-00044_FEP-VSL-00017B \\
\hline PWD-VSL-00044 & FEP-VSL-00017A & PWD-VSL-00044_FEP-VSL-00017A \\
\hline PWD-VSL-00043 & PJM-RFD-VENT & PWD-VSL-00043_PJM-RFD-VENT \\
\hline PWD-VSL-00043 & PWD-VSL-00044 & PWD-VSL-00043_PWD-VSL-00044 \\
\hline PWD-VSL-00033 & PJM-RFD-VENT & PWD-VSL-00033 PJM-RFD-VENT \\
\hline PWD-VSL-00033 & PWD-VSL-00044 & PWD-VSL-00033_PWD-VSL-00044 \\
\hline SHR-TK-00006 & PWD-VSL-00015 & SHR-TK-00006 PWD-VSL-00015 \\
\hline SHR-TK-00006 & PWD-VSL-00016 & SHR-TK-00006_PWD-VSL-00016 \\
\hline SHR-TK-00006 & PWD-VSL-00044 & SHR-TK-00006_PWD-VSL-00044 \\
\hline RLD-VSL-00007 & PJV-HEPA-00004-5 & RLD-VSL-00007_PJV-HEPA-00004-5 \\
\hline RLD-VSL-00007 & PWD-VSL-00043 & RLD-VSL-00007_PWD-VSL-00043 \\
\hline SHR-TK-00002 & RLD-VSL-00007 & SHR-TK-00002_RLD-VSL-00007 \\
\hline DIW-IXC-RINSE-1 & CXP-IXC-00002 & DIW-IXC-RINSE-1_CXP-IXC-00002 \\
\hline DIW-IXC-RINSE-2 & CXP-IXC-00002 & DIW-IXC-RINSE-2_CXP-IXC-00002 \\
\hline SHR-TK-00005 & CXP-IXC-00002 & SHR-TK-00005_CXP-IXC-00002 \\
\hline CXP-IXC-00002 & CNP-EVAP-00001 & CXP-IXC-00002_CNP-EVAP-00001 \\
\hline CXP-IXC-00002 & RDP-VSL-00002A & CXP-IXC-00002_RDP-VSL-00002A \\
\hline CXP-IXC-00002 & RDP-VSL-00002B & CXP-IXC-00002 RDP-VSL-00002B \\
\hline CXP-IXC-00002 & UFP-VSL-00062A & CXP-IXC-00002_UFP-VSL-00062A \\
\hline CXP-IXC-00002 & CXP-VSL-00026A & CXP-IXC-00002_CXP-VSL-00026A \\
\hline CXP-IXC-00002 & CXP-VSL-00026C & CXP-IXC-00002_CXP-VSL-00026C \\
\hline CXP-IXC-00002 & CXP-VSL-00026B & CXP-IXC-00002_CXP-VSL-00026B \\
\hline CXP-IXC-00002 & CXP-IXC-00003 & CXP-IXC-00002_CXP-IXC-00003 \\
\hline CXP-IXC-00002 & PWD-VSL-00015 & CXP-IXC-00002_PWD-VSL-00015 \\
\hline CXP-IXC-00002 & PWD-VSL-00016 & CXP-IXC-00002_PWD-VSL-00016 \\
\hline CXP-IXC-00002 & CXP-IXC-00002 & CXP-IXC-00002_CXP-IXC-00002 \\
\hline DIW-IXC-RINSE-1 & CXP-IXC-00001 & DIW-IXC-RINSE-1_CXP-IXC-00001 \\
\hline DIW-IXC-RINSE-2 & CXP-IXC-00001 & DIW-IXC-RINSE-2_CXP-IXC-00001 \\
\hline SHR-TK-00005 & CXP-IXC-00001 & SHR-TK-00005_CXP-IXC-00001 \\
\hline CXP-IXC-00001 & CNP-EVAP-00001 & CXP-IXC-00001_CNP-EVAP-00001 \\
\hline CXP-IXC-00001 & RDP-VSL-00002A & CXP-IXC-00001_RDP-VSL-00002A \\
\hline CXP-IXC-00001 & RDP-VSL-00002B & CXP-IXC-00001_RDP-VSL-00002B \\
\hline CXP-IXC-00001 & UFP-VSL-00062A & CXP-IXC-00001_UFP-VSL-00062A \\
\hline CXP-IXC-00001 & CXP-VSL-00026A & CXP-IXC-00001_CXP-VSL-00026A \\
\hline CXP-IXC-00001 & CXP-VSL-00026C & CXP-IXC-00001_CXP-VSL-00026C \\
\hline CXP-IXC-00001 & CXP-VSL-00026B & CXP-IXC-00001_CXP-VSL-00026B \\
\hline CXP-IXC-00001 & CXP-IXC-00002 & CXP-IXC-00001_CXP-IXC-00002 \\
\hline CXP-IXC-00001 & PWD-VSL-00015 & CXP-IXC-00001_PWD-VSL-00015 \\
\hline CXP-IXC-00001 & PWD-VSL-00016 & CXP-IXC-00001_PWD-VSL-00016 \\
\hline CXP-IXC-00001 & CXP-IXC-00001 & CXP-IXC-00001_CXP-IXC-00001 \\
\hline DIW-IXC-RINSE-1 & CXP-IXC-00004 & DIW-IXC-RINSE-1_CXP-IXC-00004 \\
\hline DIW-IXC-RINSE-2 & CXP-IXC-00004 & DIW-IXC-RINSE-2_CXP-IXC-00004 \\
\hline SHR-TK-00005 & CXP-IXC-00004 & SHR-TK-00005_CXP-IXC-00004 \\
\hline CXP-IXC-00004 & CNP-EVAP-00001 & CXP-IXC-00004_CNP-EVAP-00001 \\
\hline CXP-IXC-00004 & RDP-VSL-00002A & CXP-IXC-00004_RDP-VSL-00002A \\
\hline CXP-IXC-00004 & RDP-VSL-00002B & CXP-IXC-00004_RDP-VSL-00002B \\
\hline CXP-IXC-00004 & UFP-VSL-00062A & CXP-IXC-00004_UFP-VSL-00062A \\
\hline
\end{tabular}




\begin{tabular}{|c|c|c|}
\hline Upstream Equipment & Downstream Equipment & Totalizer File \\
\hline CXP-IXC-00004 & CXP-VSL-00026A & CXP-IXC-00004_CXP-VSL-00026A \\
\hline CXP-IXC-00004 & CXP-VSL-00026C & CXP-IXC-00004_CXP-VSL-00026C \\
\hline CXP-IXC-00004 & CXP-VSL-00026B & CXP-IXC-00004_CXP-VSL-00026B \\
\hline CXP-IXC-00004 & CXP-IXC-00001 & CXP-IXC-00004_CXP-IXC-00001 \\
\hline CXP-IXC-00004 & PWD-VSL-00015 & CXP-IXC-00004 PWD-VSL-00015 \\
\hline CXP-IXC-00004 & PWD-VSL-00016 & CXP-IXC-00004_PWD-VSL-00016 \\
\hline CXP-IXC-00004 & CXP-IXC-00004 & CXP-IXC-00004 CXP-IXC-00004 \\
\hline DIW-IXC-RINSE-1 & CXP-IXC-00003 & DIW-IXC-RINSE-1_CXP-IXC-00003 \\
\hline DIW-IXC-RINSE-2 & CXP-IXC-00003 & DIW-IXC-RINSE-2_CXP-IXC-00003 \\
\hline SHR-TK-00005 & CXP-IXC-00003 & SHR-TK-00005_CXP-IXC-00003 \\
\hline CXP-IXC-00003 & CNP-EVAP-00001 & CXP-IXC-00003_CNP-EVAP-00001 \\
\hline CXP-IXC-00003 & RDP-VSL-00002A & CXP-IXC-00003_RDP-VSL-00002A \\
\hline CXP-IXC-00003 & RDP-VSL-00002B & CXP-IXC-00003_RDP-VSL-00002B \\
\hline CXP-IXC-00003 & UFP-VSL-00062A & CXP-IXC-00003_UFP-VSL-00062A \\
\hline CXP-IXC-00003 & CXP-VSL-00026A & CXP-IXC-00003_CXP-VSL-00026A \\
\hline CXP-IXC-00003 & CXP-VSL-00026C & CXP-IXC-00003_CXP-VSL-00026C \\
\hline CXP-IXC-00003 & CXP-VSL-00026B & CXP-IXC-00003_CXP-VSL-00026B \\
\hline CXP-IXC-00003 & CXP-IXC-00004 & CXP-IXC-00003 CXP-IXC-00004 \\
\hline CXP-IXC-00003 & PWD-VSL-00015 & CXP-IXC-00003_PWD-VSL-00015 \\
\hline CXP-IXC-00003 & PWD-VSL-00016 & CXP-IXC-00003_PWD-VSL-00016 \\
\hline CXP-IXC-00003 & CXP-IXC-00003 & CXP-IXC-00003_CXP-IXC-00003 \\
\hline CXP-VSL-00005 & PJM-RFD-VENT & CXP-VSL-00005_PJM-RFD-VENT \\
\hline CXP-VSL-00005 & CXP-IXC-00001 & CXP-VSL-00005_CXP-IXC-00001 \\
\hline CXP-VSL-00005 & CXP-IXC-00002 & CXP-VSL-00005_CXP-IXC-00002 \\
\hline CXP-VSL-00005 & CXP-IXC-00003 & CXP-VSL-00005_CXP-IXC-00003 \\
\hline CXP-VSL-00005 & CXP-IXC-00004 & CXP-VSL-00005_CXP-IXC-00004 \\
\hline SHR-TK-00005 & CXP-VSL-00005 & SHR-TK-00005_CXP-VSL-00005 \\
\hline IX-DI-WATER & UFP-VSL-00062A & IX-DI-WATER_UFP-VSL-00062A \\
\hline SHR-TK-00001 & CNP-EVAP-00001 & SHR-TK-00001_CNP-EVAP-00001 \\
\hline CXP-FILT-00001 & PWD-VSL-00016 & CXP-FILT-00001_PWD-VSL-00016 \\
\hline CXP-FILT-00001 & CXP-IXC-00004 & CXP-FILT-00001_CXP-IXC-00004 \\
\hline CXP-FILT-00001 & CXP-IXC-00003 & CXP-FILT-00001_CXP-IXC-00003 \\
\hline CXP-FILT-00001 & CXP-IXC-00002 & CXP-FILT-00001_CXP-IXC-00002 \\
\hline CXP-FILT-00001 & CXP-IXC-00001 & CXP-FILT-00001_CXP-IXC-00001 \\
\hline SHR-TK-00005 & CXP-FILT-00001 & SHR-TK-00005_CXP-FILT-00001 \\
\hline PVP-HEME-00001 & PVP-VSL-00001 & PVP-HEME-00001_PVP-VSL-00001 \\
\hline PVP-HEME-00001 & PVP-HEPA-00001-2 & PVP-HEME-00001_PVP-HEPA-00001-2 \\
\hline PVP-VESSEL-VENT & PVP-SCB-00002 & PVP-VESSEL-VENT_PVP-SCB-00002 \\
\hline PVP-VESSEL-VENT & PVP-VESSEL-VENT & PVP-VESSEL-VENT_PVP-VESSEL-VENT \\
\hline PVP-OXID-00001 & PVP-ABS-00001AB & PVP-OXID-00001_PVP-ABS-00001AB \\
\hline PVP-OXID-00001 & PVP-OXID-00001 & PVP-OXID-00001_PVP-OXID-00001 \\
\hline PJM-RFD-VENT & PJV-VSL-00001 & PJM-RFD-VENT_PJV-VSL-00001 \\
\hline PJM-RFD-VENT & PJM-RFD-VENT & PJM-RFD-VENT_PJM-RFD-VENT \\
\hline PVP-ABS-00001AB & PVP-STACK & PVP-ABS-00001AB_PVP-STACK \\
\hline PVP-ABS-00001AB & PVP-ABS-00001AB & PVP-ABS-00001AB_PVP-ABS-00001AB \\
\hline PJV-VSL-00001 & PWD-VSL-00043 & PJV-VSL-00001_PWD-VSL-00043 \\
\hline PJV-VSL-00001 & PJV-HEPA & PJV-VSL-00001_PJV-HEPA \\
\hline
\end{tabular}




\begin{tabular}{|c|c|c|}
\hline Upstream Equipment & Downstream Equipment & Totalizer File \\
\hline PVP-VSL-00001 & PWD-VSL-00044 & PVP-VSL-00001_PWD-VSL-00044 \\
\hline DIW-TK-00004 & PVP-HEME-00001 & DIW-TK-00004_PVP-HEME-00001 \\
\hline DIW-TK-00004 & PJV-VSL-00001 & DIW-TK-00004_PJV-VSL-00001 \\
\hline AIR-VENT-LUMP-SUM & PVP-VESSEL-VENT & AIR-VENT-LUMP-SUM_PVP-VESSEL-VENT \\
\hline SHR-TK-00007 & PVP-SCB-00002 & SHR-TK-00007_PVP-SCB-00002 \\
\hline PVP-SCB-00002 & PVP-HEME-00001 & PVP-SCB-00002_PVP-HEME-00001 \\
\hline PVP-SCB-00002 & PWD-VSL-00044 & PVP-SCB-00002_PWD-VSL-00044 \\
\hline PVP-HEPA-00001-2 & PVP-OXID-00001 & $\begin{array}{l}\text { PVP-HEPA-00001-2_PVP-OXID-00001 } \\
\end{array}$ \\
\hline PVP-HEPA-00001-2 & PVP-HEPA-00001-2 & PVP-HEPA-00001-2_PVP-HEPA-00001-2 \\
\hline PJV-HEPA & PVP-STACK & PJV-HEPA_PVP-STACK \\
\hline PJV-HEPA & PJV-HEPA & PJV-HEPA_PJV-HEPA \\
\hline $\begin{array}{l}\text { UFV-HLP-3HR- } \\
\text { ROTATION }\end{array}$ & PVP-VESSEL-VENT & UFV-HLP-3HR-ROTATION_PVP-VESSEL-VENT \\
\hline RLD-VSL-00004 & RLD-VSL-00005 & RLD-VSL-00004_RLD-VSL-00005 \\
\hline RLD-VSL-00005 & TLP-VSL-00009A & RLD-VSL-00005_TLP-VSL-00009A \\
\hline RLD-VSL-00005 & TLP-VSL-00009B & RLD-VSL-00005_TLP-VSL-00009B \\
\hline RLD-VSL-00005 & $\begin{array}{l}\text { SUPPLEMENTAL-LAW- } \\
\text { RECYCLE }\end{array}$ & $\begin{array}{l}\text { RLD-VSL-00005_SUPPLEMENTAL-LAW- } \\
\text { RECYCLE }\end{array}$ \\
\hline LVP-TK-00001 & LVP-SCB-00001 & LVP-TK-00001_LVP-SCB-00001 \\
\hline LVP-TK-00001 & RLD-VSL-00017A & LVP-TK-00001_RLD-VSL-00017A \\
\hline LVP-TK-00001 & RLD-VSL-00017B & LVP-TK-00001_RLD-VSL-00017B \\
\hline LVP-VSL-00001-NAOH & LVP-TK-00001 & LVP-VSL-00001-NAOH_LVP-TK-00001 \\
\hline $\begin{array}{l}\text { LVP-VSL-00001- } \\
\text { WATER }\end{array}$ & LVP-TK-00001 & LVP-VSL-00001-WATER_LVP-TK-00001 \\
\hline LMP-MLTR-00002-AIR & LMP-MLTR-00002 & LMP-MLTR-00002-AIR_LMP-MLTR-00002 \\
\hline LOP-WESP-00002 & RLD-VSL-00004 & LOP-WESP-00002_RLD-VSL-00004 \\
\hline LOP-WESP-00002 & LAW-VESSEL-VENT & LOP-WESP-00002_LAW-VESSEL-VENT \\
\hline LOP09 & LOP-WESP-00002 & LOP09_LOP-WESP-00002 \\
\hline $\begin{array}{l}\text { LOP-SCB-00002- } \\
\text { WATER }\end{array}$ & LOP-SCB-00002 & LOP-SCB-00002-WATER_LOP-SCB-00002 \\
\hline LOP-SCB-00002 & RLD-VSL-00005 & LOP-SCB-00002_RLD-VSL-00005 \\
\hline LOP-SCB-00002 & LOP-WESP-00002 & LOP-SCB-00002_LOP-WESP-00002 \\
\hline $\begin{array}{l}\text { LMP-MLTR-00002- } \\
\text { WATER }\end{array}$ & LMP-MLTR-00002 & LMP-MLTR-00002-WATER_LMP-MLTR-00002 \\
\hline LMP-MLTR-00002 & LOP-SCB-00002 & LMP-MLTR-00002_LOP-SCB-00002 \\
\hline LMP-MLTR-00002 & LAW-CONTAINERS-2 & LMP-MLTR-00002_LAW-CONTAINERS-2 \\
\hline HFP-VSL-00002 & HMP-MLTR-00001 & HFP-VSL-00002_HMP-MLTR-00001 \\
\hline HFP-VSL-00001 & HFP-VSL-00002 & HFP-VSL-00001_HFP-VSL-00002 \\
\hline HFP-VSL-00006 & HMP-MLTR-00002 & HFP-VSL-00006_HMP-MLTR-00002 \\
\hline HFP-VSL-00005 & HFP-VSL-00006 & HFP-VSL-00005_HFP-VSL-00006 \\
\hline RHEOLOGY-HLW & HFP-VSL-00005 & RHEOLOGY-HLW_HFP-VSL-00005 \\
\hline RHEOLOGY-HLW & HFP-VSL-00001 & RHEOLOGY-HLW_HFP-VSL-00001 \\
\hline LVP-SCB-00001 & LVP-TK-00001 & LVP-SCB-00001_LVP-TK-00001 \\
\hline LVP-SCB-00001 & LAW-STACK & LVP-SCB-00001_LAW-STACK \\
\hline LVP-SCR-00001-2 & LVP-SCB-00001 & LVP-SCR-00001-2_LVP-SCB-00001 \\
\hline LVP-SCR-00001-2 & LVP-SCR-00001-2 & LVP-SCR-00001-2_LVP-SCR-00001-2 \\
\hline AMR-VSL-00001-2-NH3 & LVP-SCR-00001-2 & AMR-VSL-00001-2-NH3_LVP-SCR-00001-2 \\
\hline LAW-VESSEL-VENT- & LAW-VESSEL-VENT & LAW-VESSEL-VENT-AIR_LAW-VESSEL-VENT \\
\hline
\end{tabular}




\begin{tabular}{|c|c|c|}
\hline Upstream Equipment & Downstream Equipment & Totalizer File \\
\hline \multicolumn{3}{|l|}{ AIR } \\
\hline LAW-VESSEL-VENT & LVP-HEPA-00001AB & LAW-VESSEL-VENT_LVP-HEPA-00001AB \\
\hline LAW-VESSEL-VENT & LAW-VESSEL-VENT & LAW-VESSEL-VENT_LAW-VESSEL-VENT \\
\hline LVP-ADBR-00001AB & LVP-SCR-00001-2 & LVP-ADBR-00001AB_LVP-SCR-00001-2 \\
\hline LVP-ADBR-00001AB & LVP-ADBR-00001AB & LVP-ADBR-00001AB_LVP-ADBR-00001AB \\
\hline AMR-VSL-00001-2-AIR & LVP-SCR-00001-2 & AMR-VSL-00001-2-AIR_LVP-SCR-00001-2 \\
\hline LVP-HEPA-00001AB & LVP-ADBR-00001AB & LVP-HEPA-00001AB_LVP-ADBR-00001AB \\
\hline LVP-HEPA-00001AB & LVP-HEPA-00001AB & LVP-HEPA-00001AB_LVP-HEPA-00001AB \\
\hline LMP-MLTR-00001-AIR & LMP-MLTR-00001 & LMP-MLTR-00001-AIR_LMP-MLTR-00001 \\
\hline LOP-WESP-00001 & RLD-VSL-00004 & LOP-WESP-00001_RLD-VSL-00004 \\
\hline LOP-WESP-00001 & LAW-VESSEL-VENT & LOP-WESP-00001_LAW-VESSEL-VENT \\
\hline LOP09 & LOP-WESP-00001 & LOP09_LOP-WESP-00001 \\
\hline $\begin{array}{l}\text { LOP-SCB-00001- } \\
\text { WATER }\end{array}$ & LOP-SCB-00001 & LOP-SCB-00001-WATER_LOP-SCB-00001 \\
\hline LOP-SCB-00001 & RLD-VSL-00005 & LOP-SCB-00001_RLD-VSL-00005 \\
\hline LOP-SCB-00001 & LOP-WESP-00001 & LOP-SCB-00001_LOP-WESP-00001 \\
\hline $\begin{array}{l}\text { LMP-MLTR-00001- } \\
\text { WATER }\end{array}$ & LMP-MLTR-00001 & LMP-MLTR-00001-WATER_LMP-MLTR-00001 \\
\hline LMP-MLTR-00001 & LOP-SCB-00001 & LMP-MLTR-00001_LOP-SCB-00001 \\
\hline LMP-MLTR-00001 & LAW-CONTAINERS-1 & LMP-MLTR-00001_LAW-CONTAINERS-1 \\
\hline HMP-MLTR-00001 & HOP-SCB-00001 & HMP-MLTR-00001_HOP-SCB-00001 \\
\hline HMP-MLTR-00001 & HLW-CANISTERS-1 & HMP-MLTR-00001_HLW-CANISTERS-1 \\
\hline HMP-MLTR-00001-AIR & HMP-MLTR-00001 & HMP-MLTR-00001-AIR_HMP-MLTR-00001 \\
\hline HOP-WESP-00001 & HOP-SCB-00001 & HOP-WESP-00001_HOP-SCB-00001 \\
\hline HOP-WESP-00001 & HLW-VESSEL-VENT-1 & HOP-WESP-00001_HLW-VESSEL-VENT-1 \\
\hline DIW-TK-00002 & HOP-WESP-00001 & DIW-TK-00002_HOP-WESP-00001 \\
\hline HOP08-1 & HOP-SCB-00001 & HOP08-1_HOP-SCB-00001 \\
\hline HOP-SCB-00001 & PJV-HEPA-00004-5 & HOP-SCB-00001_PJV-HEPA-00004-5 \\
\hline HOP-SCB-00001 & RLD-VSL-00007 & HOP-SCB-00001_RLD-VSL-00007 \\
\hline HOP-SCB-00001 & HOP-WESP-00001 & HOP-SCB-00001_HOP-WESP-00001 \\
\hline HOP-HEME-00001 & HOP-SCB-00001 & HOP-HEME-00001_HOP-SCB-00001 \\
\hline HOP-HEME-00001 & HOP-HEPA-00001-2-AB & HOP-HEME-00001_HOP-HEPA-00001-2-AB \\
\hline DIW-TK-00002 & HOP-HEME-00001 & DIW-TK-00002_HOP-HEME-00001 \\
\hline HLW-VESSEL-VENT-1 & HOP-HEME-00001 & HLW-VESSEL-VENT-1_HOP-HEME-00001 \\
\hline HLW-VESSEL-VENT-1 & HLW-VESSEL-VENT-1 & HLW-VESSEL-VENT-1_HLW-VESSEL-VENT-1 \\
\hline HOP-SCR-00001 & HLW-STACK & HOP-SCR-00001_HLW-STACK \\
\hline HOP-SCR-00001 & HOP-SCR-00001 & HOP-SCR-00001_HOP-SCR-00001 \\
\hline HOP-ABS-00002 & HOP-SCR-00001 & HOP-ABS-00002_HOP-SCR-00001 \\
\hline HOP-ABS-00002 & HOP-ABS-00002 & HOP-ABS-00002_HOP-ABS-00002 \\
\hline HOP-SCR-00001-NH3 & HOP-SCR-00001 & HOP-SCR-00001-NH3_HOP-SCR-00001 \\
\hline $\begin{array}{l}\text { HLW-VESSEL-VENT-1- } \\
\text { AIR }\end{array}$ & HLW-VESSEL-VENT-1 & $\begin{array}{l}\text { HLW-VESSEL-VENT-1-AIR_HLW-VESSEL- } \\
\text { VENT-1 }\end{array}$ \\
\hline HOP-ADBR-00001AB & HOP-ABS-00002 & HOP-ADBR-00001AB_HOP-ABS-00002 \\
\hline HOP-ADBR-00001AB & HOP-ADBR-00001AB & HOP-ADBR-00001AB_HOP-ADBR-00001AB \\
\hline HOP-SCR-00001-AIR & HOP-SCR-00001 & HOP-SCR-00001-AIR_HOP-SCR-00001 \\
\hline HOP-HEPA-00001-2-AB & HOP-ADBR-00001AB & HOP-HEPA-00001-2-AB_HOP-ADBR-00001AB \\
\hline HOP-HEPA-00001-2-AB & HOP-HEPA-00001-2-AB & HOP-HEPA-00001-2-AB_HOP-HEPA-00001-2-AB \\
\hline
\end{tabular}




\begin{tabular}{|c|c|c|}
\hline Upstream Equipment & Downstream Equipment & Totalizer File \\
\hline PJV-HEPA-00004-5 & HLW-PJV-STACK & PJV-HEPA-00004-5_HLW-PJV-STACK \\
\hline PJV-HEPA-00004-5 & PJV-HEPA-00004-5 & PJV-HEPA-00004-5_PJV-HEPA-00004-5 \\
\hline HMP-MLTR-00002 & HOP-SCB-00002 & HMP-MLTR-00002_HOP-SCB-00002 \\
\hline HMP-MLTR-00002 & HLW-CANISTERS-2 & HMP-MLTR-00002_HLW-CANISTERS-2 \\
\hline HMP-MLTR-00002-AIR & HMP-MLTR-00002 & HMP-MLTR-00002-AIR_HMP-MLTR-00002 \\
\hline HOP-WESP-00002 & HOP-SCB-00002 & HOP-WESP-00002_HOP-SCB-00002 \\
\hline HOP-WESP-00002 & HLW-VESSEL-VENT-2 & HOP-WESP-00002_HLW-VESSEL-VENT-2 \\
\hline DIW-TK-00002 & HOP-WESP-00002 & DIW-TK-00002_HOP-WESP-00002 \\
\hline HOP08-2 & HOP-SCB-00002 & HOP08-2_HOP-SCB-00002 \\
\hline HOP-SCB-00002 & PJV-HEPA-00004-5 & HOP-SCB-00002_PJV-HEPA-00004-5 \\
\hline HOP-SCB-00002 & RLD-VSL-00007 & HOP-SCB-00002_RLD-VSL-00007 \\
\hline HOP-SCB-00002 & HOP-WESP-00002 & HOP-SCB-00002_HOP-WESP-00002 \\
\hline HOP-HEME-00002 & HOP-SCB-00002 & HOP-HEME-00002 HOP-SCB-00002 \\
\hline HOP-HEME-00002 & HOP-HEPA-00007-8-AB & HOP-HEME-00002_HOP-HEPA-00007-8-AB \\
\hline DIW-TK-00002 & HOP-HEME-00002 & DIW-TK-00002_HOP-HEME-00002 \\
\hline HLW-VESSEL-VENT-2 & HOP-HEME-00002 & HLW-VESSEL-VENT-2_HOP-HEME-00002 \\
\hline HLW-VESSEL-VENT-2 & HLW-VESSEL-VENT-2 & HLW-VESSEL-VENT-2_HLW-VESSEL-VENT-2 \\
\hline HOP-SCR-00002 & HLW-STACK & HOP-SCR-00002_HLW-STACK \\
\hline HOP-SCR-00002 & HOP-SCR-00002 & HOP-SCR-00002_HOP-SCR-00002 \\
\hline HOP-ABS-00003 & HOP-SCR-00002 & HOP-ABS-00003_HOP-SCR-00002 \\
\hline HOP-ABS-00003 & HOP-ABS-00003 & HOP-ABS-00003 HOP-ABS-00003 \\
\hline $\begin{array}{l}\text { HLW-VESSEL-VENT-2- } \\
\text { AIR }\end{array}$ & HLW-VESSEL-VENT-2 & $\begin{array}{l}\text { HLW-VESSEL-VENT-2-AIR_HLW-VESSEL- } \\
\text { VENT-2 }\end{array}$ \\
\hline HOP-ADBR-00002AB & HOP-ABS-00003 & HOP-ADBR-00002AB_HOP-ABS-00003 \\
\hline HOP-ADBR-00002AB & HOP-ADBR-00002AB & HOP-ADBR-00002AB_HOP-ADBR-00002AB \\
\hline HOP-SCR-00002-NH3 & HOP-SCR-00002 & HOP-SCR-00002-NH3_HOP-SCR-00002 \\
\hline HOP-SCR-00002-AIR & HOP-SCR-00002 & HOP-SCR-00002-AIR_HOP-SCR-00002 \\
\hline HOP-HEPA-00007-8-AB & HOP-ADBR-00002AB & HOP-HEPA-00007-8-AB_HOP-ADBR-00002AB \\
\hline HOP-HEPA-00007-8-AB & HOP-HEPA-00007-8-AB & HOP-HEPA-00007-8-AB_HOP-HEPA-00007-8-AB \\
\hline HDH-VSL-00001 & HDH-VSL-00003 & HDH-VSL-00001_HDH-VSL-00003 \\
\hline NAR-TK-00001 & HDH-VSL-00002 & NAR-TK-00001_HDH-VSL-00002 \\
\hline HDH-VSL-00002 & HDH-VSL-00003 & HDH-VSL-00002_HDH-VSL-00003 \\
\hline $\begin{array}{l}\text { HDH-VSL-00001- } \\
\text { WATER }\end{array}$ & HDH-VSL-00001 & HDH-VSL-00001-WATER_HDH-VSL-00001 \\
\hline HDH-TK-00002 & HDH-VSL-00002 & HDH-TK-00002_HDH-VSL-00002 \\
\hline DIW-TK-00002 & HDH-VSL-00002 & DIW-TK-00002_HDH-VSL-00002 \\
\hline HDH-VSL-00003 & RLD-VSL-00007 & HDH-VSL-00003_RLD-VSL-00007 \\
\hline SHR-TK-00002 & HDH-VSL-00003 & SHR-TK-00002_HDH-VSL-00003 \\
\hline HPS & HDH-VSL-00003 & HPS_HDH-VSL-00003 \\
\hline HDH-TK-00003 & HDH-VSL-00003 & HDH-TK-00003_HDH-VSL-00003 \\
\hline HDH-VSL-00004 & HDH-VSL-00003 & HDH-VSL-00004_HDH-VSL-00003 \\
\hline NAR-TK-00001 & HDH-VSL-00004 & NAR-TK-00001_HDH-VSL-00004 \\
\hline HDH-TK-00002 & HDH-VSL-00004 & HDH-TK-00002_HDH-VSL-00004 \\
\hline DIW-TK-00002 & HDH-VSL-00004 & DIW-TK-00002_HDH-VSL-00004 \\
\hline HDH-VSL-00003-FLUSH & RLD-VSL-00007 & HDH-VSL-00003-FLUSH_RLD-VSL-00007 \\
\hline PSA & HLP-VSL-00028 & PSA_HLP-VSL-00028 \\
\hline PSA & HLP-VSL-00027B & PSA_HLP-VSL-00027B \\
\hline
\end{tabular}




\begin{tabular}{|c|c|c|}
\hline Upstream Equipment & Downstream Equipment & Totalizer File \\
\hline PSA & HLP-VSL-00027A & PSA HLP-VSL-00027A \\
\hline PSA & UFP-VSL-00062B & PSA_UFP-VSL-00062B \\
\hline PSA & UFP-VSL-00062C & PSA_UFP-VSL-00062C \\
\hline PSA & UFP-VSL-00062A & PSA_UFP-VSL-00062A \\
\hline PSA & UFP-VSL-00002B & PSA_UFP-VSL-00002B \\
\hline PSA & UFP-VSL-00002A & PSA_UFP-VSL-00002A \\
\hline PSA & UFP-VSL-00001A & PSA_UFP-VSL-00001A \\
\hline PSA & UFP-VSL-00001B & PSA_UFP-VSL-00001B \\
\hline PSA & RLD-VSL-00007 & PSA_RLD-VSL-00007 \\
\hline PSA & RDP-VSL-00002C & PSA_RDP-VSL-00002C \\
\hline PSA & RDP-VSL-00002A & PSA_RDP-VSL-00002A \\
\hline PSA & RDP-VSL-00002B & PSA_RDP-VSL-00002B \\
\hline PSA & PWD-VSL-00044 & PSA_PWD-VSL-00044 \\
\hline PSA & PWD-VSL-00016 & PSA_PWD-VSL-00016 \\
\hline PSA & PWD-VSL-00015 & PSA_PWD-VSL-00015 \\
\hline PSA & PWD-VSL-00043 & PSA_PWD-VSL-00043 \\
\hline PSA & PWD-VSL-00033 & PSA_PWD-VSL-00033 \\
\hline PSA & HOP-SCB-00002 & PSA_HOP-SCB-00002 \\
\hline PSA & HOP-SCB-00001 & PSA_HOP-SCB-00001 \\
\hline PSA & FRP-VSL-00002D & PSA_FRP-VSL-00002D \\
\hline PSA & FRP-VSL-00002C & PSA_FRP-VSL-00002C \\
\hline PSA & FRP-VSL-00002B & PSA_FRP-VSL-00002B \\
\hline PSA & FRP-VSL-00002A & PSA_FRP-VSL-00002A \\
\hline PSA & HLP-VSL-00022 & PSA_HLP-VSL-00022 \\
\hline PSA & FEP-VSL-00017A & PSA_FEP-VSL-00017A \\
\hline PSA & FEP-VSL-00017B & PSA_FEP-VSL-00017B \\
\hline PSA & CXP-VSL-00026C & PSA_CXP-VSL-00026C \\
\hline PSA & TCP-VSL-00001 & PSA_TCP-VSL-00001 \\
\hline PSA & CXP-VSL-00026B & PSA_CXP-VSL-00026B \\
\hline PSA & CXP-VSL-00026A & PSA_CXP-VSL-00026A \\
\hline PSA & TLP-VSL-00009B & PSA_TLP-VSL-00009B \\
\hline PSA & TLP-VSL-00009A & PSA_TLP-VSL-00009A \\
\hline PSA & CXP-VSL-00005 & PSA_CXP-VSL-00005 \\
\hline PSA & CNP-VSL-00003 & PSA_CNP-VSL-00003 \\
\hline PSA & CNP-VSL-00004 & PSA_CNP-VSL-00004 \\
\hline RFD-AIR & HLP-VSL-00028 & RFD-AIR_HLP-VSL-00028 \\
\hline RFD-AIR & HLP-VSL-00027B & RFD-AIR_HLP-VSL-00027B \\
\hline RFD-AIR & HLP-VSL-00027A & RFD-AIR_HLP-VSL-00027A \\
\hline RFD-AIR & UFP-VSL-00062B & RFD-AIR_UFP-VSL-00062B \\
\hline RFD-AIR & UFP-VSL-00062C & RFD-AIR_UFP-VSL-00062C \\
\hline RFD-AIR & UFP-VSL-00062A & RFD-AIR_UFP-VSL-00062A \\
\hline RFD-AIR & UFP-VSL-00002B & RFD-AIR_UFP-VSL-00002B \\
\hline RFD-AIR & UFP-VSL-00002A & RFD-AIR_UFP-VSL-00002A \\
\hline RFD-AIR & UFP-VSL-00001A & RFD-AIR_UFP-VSL-00001A \\
\hline RFD-AIR & UFP-VSL-00001B & RFD-AIR_UFP-VSL-00001B \\
\hline RFD-AIR & RLD-VSL-00007 & RFD-AIR_RLD-VSL-00007 \\
\hline RFD-AIR & RDP-VSL-00002C & RFD-AIR_RDP-VSL-00002C \\
\hline
\end{tabular}




\begin{tabular}{|c|c|c|}
\hline Upstream Equipment & Downstream Equipment & Totalizer File \\
\hline RFD-AIR & RDP-VSL-00002A & RFD-AIR_RDP-VSL-00002A \\
\hline RFD-AIR & RDP-VSL-00002B & RFD-AIR_RDP-VSL-00002B \\
\hline RFD-AIR & PWD-VSL-00044 & RFD-AIR_PWD-VSL-00044 \\
\hline RFD-AIR & PWD-VSL-00016 & RFD-AIR_PWD-VSL-00016 \\
\hline RFD-AIR & PWD-VSL-00015 & RFD-AIR_PWD-VSL-00015 \\
\hline RFD-AIR & PWD-VSL-00043 & RFD-AIR_PWD-VSL-00043 \\
\hline RFD-AIR & PWD-VSL-00033 & RFD-AIR_PWD-VSL-00033 \\
\hline RFD-AIR & HOP-SCB-00002 & RFD-AIR_HOP-SCB-00002 \\
\hline RFD-AIR & HOP-SCB-00001 & RFD-AIR_HOP-SCB-00001 \\
\hline RFD-AIR & FRP-VSL-00002D & RFD-AIR_FRP-VSL-00002D \\
\hline RFD-AIR & FRP-VSL-00002C & RFD-AIR_FRP-VSL-00002C \\
\hline RFD-AIR & FRP-VSL-00002B & RFD-AIR_FRP-VSL-00002B \\
\hline RFD-AIR & FRP-VSL-00002A & RFD-AIR_FRP-VSL-00002A \\
\hline RFD-AIR & HLP-VSL-00022 & RFD-AIR_HLP-VSL-00022 \\
\hline RFD-AIR & FEP-VSL-00017A & RFD-AIR FEP-VSL-00017A \\
\hline RFD-AIR & FEP-VSL-00017B & RFD-AIR_FEP-VSL-00017B \\
\hline RFD-AIR & CXP-VSL-00026C & RFD-AIR_CXP-VSL-00026C \\
\hline RFD-AIR & TCP-VSL-00001 & RFD-AIR_TCP-VSL-00001 \\
\hline RFD-AIR & CXP-VSL-00026B & RFD-AIR_CXP-VSL-00026B \\
\hline RFD-AIR & CXP-VSL-00026A & RFD-AIR_CXP-VSL-00026A \\
\hline RFD-AIR & TLP-VSL-00009B & RFD-AIR_TLP-VSL-00009B \\
\hline RFD-AIR & TLP-VSL-00009A & RFD-AIR_TLP-VSL-00009A \\
\hline RFD-AIR & CXP-VSL-00005 & RFD-AIR_CXP-VSL-00005 \\
\hline RFD-AIR & CNP-VSL-00003 & RFD-AIR_CNP-VSL-00003 \\
\hline RFD-AIR & CNP-VSL-00004 & RFD-AIR_CNP-VSL-00004 \\
\hline FEP-VSL-00017A & PJM-RFD-VENT & FEP-VSL-00017A_PJM-RFD-VENT \\
\hline FEP-VSL-00017A & FEP-SEP-00001A & FEP-VSL-00017A_FEP-SEP-00001A \\
\hline FEP-VSL-00017A & FEP-SEP-00001B & FEP-VSL-00017A_FEP-SEP-00001B \\
\hline FEP-VSL-00017B & PJM-RFD-VENT & FEP-VSL-00017B_PJM-RFD-VENT \\
\hline FEP-VSL-00017B & FEP-SEP-00001A & FEP-VSL-00017B_FEP-SEP-00001A \\
\hline FEP-VSL-00017B & FEP-SEP-00001B & FEP-VSL-00017B_FEP-SEP-00001B \\
\hline FEP-VSL-00005A & RLD-TK-00006A & FEP-VSL-00005A_RLD-TK-00006A \\
\hline FEP-DMST-00001A-AIR & FEP-DMST-00001A & FEP-DMST-00001A-AIR_FEP-DMST-00001A \\
\hline FEP-DMST-00001A & FEP-VSL-00005A & FEP-DMST-00001A_FEP-VSL-00005A \\
\hline FEP-DMST-00001A & PVP-VESSEL-VENT & FEP-DMST-00001A_PVP-VESSEL-VENT \\
\hline $\begin{array}{l}\text { FEPA-INTER-AFTER- } \\
\text { CONDENSER-WATER }\end{array}$ & FEP-VSL-00005A & $\begin{array}{l}\text { FEPA-INTER-AFTER-CONDENSER- } \\
\text { WATER FEP-VSL-00005A }\end{array}$ \\
\hline FEP-SEP-00001B & FEP-DMST-00001B & FEP-SEP-00001B_FEP-DMST-00001B \\
\hline FEP-SEP-00001B & HLP-VSL-00027A & FEP-SEP-00001B_HLP-VSL-00027A \\
\hline FEP-SEP-00001B & UFP-VSL-00001A & FEP-SEP-00001B_UFP-VSL-00001A \\
\hline FEP-SEP-00001B & UFP-VSL-00001B & FEP-SEP-00001B_UFP-VSL-00001B \\
\hline FEP-SEP-00001B & FRP-VSL-00002A & FEP-SEP-00001B_FRP-VSL-00002A \\
\hline FEP-VSL-00005B & RLD-TK-00006A & FEP-VSL-00005B_RLD-TK-00006A \\
\hline FEP-DMST-00001B-AIR & FEP-DMST-00001B & FEP-DMST-00001B-AIR_FEP-DMST-00001B \\
\hline FEP-DMST-00001B & FEP-VSL-00005B & FEP-DMST-00001B_FEP-VSL-00005B \\
\hline FEP-DMST-00001B & PVP-VESSEL-VENT & FEP-DMST-00001B_PVP-VESSEL-VENT \\
\hline FEPB-INTER-AFTER- & FEP-VSL-00005B & FEPB-INTER-AFTER-CONDENSER- \\
\hline
\end{tabular}


24590-WTP-MDD-PR-01-002, Rev 12 Dynamic (G2) Model Design Document

\begin{tabular}{|l|l|l|}
\hline Upstream Equipment & Downstream Equipment & Totalizer File \\
\hline CONDENSER-WATER & & WATER_FEP-VSL-00005B \\
\hline FEP-SEP-00001A & FEP-DMST-00001A & FEP-SEP-00001A_FEP-DMST-00001A \\
\hline FEP-SEP-00001A & HLP-VSL-00027A & FEP-SEP-00001A_HLP-VSL-00027A \\
\hline FEP-SEP-00001A & UFP-VSL-00001A & FEP-SEP-00001A_UFP-VSL-00001A \\
\hline FEP-SEP-00001A & UFP-VSL-00001B & FEP-SEP-00001A_UFP-VSL-00001B \\
\hline FEP-SEP-00001A & FRP-VSL-00002A & FEP-SEP-00001A_FRP-VSL-00002A \\
\hline
\end{tabular}

